Final Report

\title{
SUBGRADE RESILIENT MODULUS FOR PAVEMENT DESIGN AND EVALUATION
}

\author{
by \\ Woojin Lee, Nihal C. Bohra, \\ Adolph G. Altschaeffl, and Thomas D. White \\ Joint Highway Research Project \\ Project No.: C-36-52N \\ File No.: 6-20-14 \\ Prepared for an Investigation \\ Conducted by the \\ Joint Highway Research Project \\ Engineering Experiment Station \\ Purdue University \\ in cooperation with the \\ Indiana Department of Transportation \\ and the \\ U. S. Department of Transportation \\ Federal Highway Administration
}

The contents of this report reflect the views of the authors who are responsible for the facts and the accuracy of the data presented herein. The contents do not necessarily reflect the official views or policies of the Federal Highway Administration. This report does not constitute a standard, specification, or regulation.

Purdue University

West Lafayette, Indiana 47907

Auguist 16, 1993 


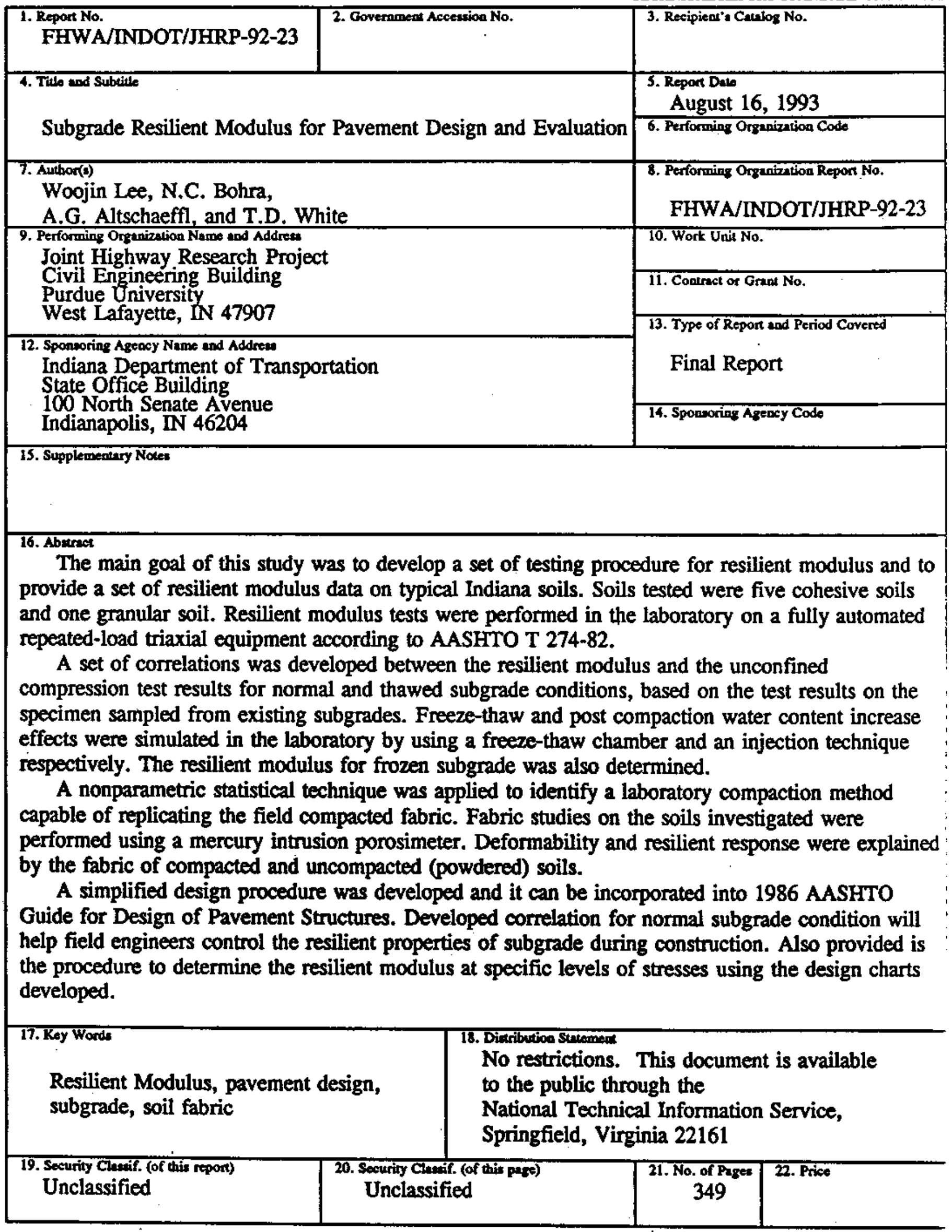


TABLE OF CONTENTS

Page

LIST OF TABLES

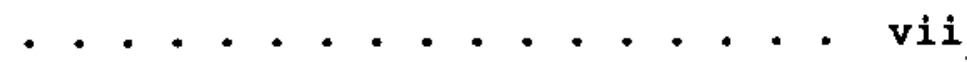

LIST OF FIGURES

............... . ix

LIST OF NOTATIONS

xvi

1. INTRODUCTION . . . . . . . . . . . . 1

1.1 Objectives of the Project............. 1

1.2 Expected Benefits ................. 2

1.3 Work Plan ................. 2

2. SOIL INVESTIGATED . . . . . . . . . . . 5

2.1 Sampling sites ............ 5

2.2 Undisturbed Soils Investigated ........ 5

2.3 Disturbed Solls Investigated . . . . . . 17

3. DEVELOPMENT OF TESTING PROCEDURE

FOR RESILIENT MODULUS . . . . . . . . . . . 20

3.1 Introduction . . . . . . . . . . 20

3.2 System for Resilient Modulus Test:..... 21

3.2.1 Testing Equipment ........... 21

3.2.2 Resilient Modulus Testing Software . . 24

3.2.3 Data Acquisition and Processing . . . . 28

3.3 Test Procedure for Resilient Modulus . . . . 29

3.4 Reliability of Test Result . . . . . . . . 31

3.4.1 Performance of Testing System . . . . . , 31

3.4.2 Accuracy of Test Result . . . . . . . . 32

3.4.3 Repeatability of Test Result . . . . . 38 
4. RESILIENT MOdULUS OF COHESIVE SOILS . . . . . . . 41

4.1 Introduction ..............41

4.2 Factors Affecting Resilient Modulus of

Cohesive Solls . . . . . . . . . . . . . 42

4.2.1 Number of Stress Applications . . . . . 42

4.2.2 Thixotropy ............... 42

4.2 .3 Level of Stresses............ . 43

4.2.4 Matrix Suction ............ . 45

4.2 .5 Method of Compaction ........ 46

4.2 .6 Compaction Water content

and Dry Density .............. 47

4.2 .7 Stabilization ............48 48

4.3 Resilient Modulus Test on

Field Compacted Cohesive Soils . . . . . . 49

4.3.1 Sampling of Soils .......... 49

4.3.2 Laboratory Experimental Program . . . : 50

4.3.3 Reduction of Test Results . . . . . . 51

4.3.3.1 Unconfined Compression Test . . . 51 4.3.3.2 Resilient Modulus Test . . . . 54

4.3.4 Test Results and Discussions . . . . 56

4.4 Resilient Modulus Test on

Laboratory Compacted Cohesive Solis . . . . . 73

4.4.1 Preparation of Specimens. . . . . . . . 73

4.4.2 Laboratory Tests ......:.: : 79

4.4.3 Test Results and Discussions :.:. 82

4.5 Resilient Properties of Laboratory and

Field Compacted Soll ............ . 96

4.5.1 Comparison of Laboratory and

Field Compaction . . . . . . . . . 96

4.5.2 Resillent Modulus of Laboratory and . . 96
Field Compacted Solls . . . . . 100

4.6 Concluding Remarks . . . . . . . . . 106

5. EFFECTS OF ENVIRONMENTAL FACTORS ON RESILIENT

MODULUS OF COHESIVE SOILS . . . . . . . . . 114

5.1 Introduction .............. 114

5.2 Resilient characteristics of $\cdot{ }^{*} \cdot{ }^{-} \cdot{ }^{-} 114$

Frozen-Thawed Cohesive Soils . . . . . . 115

5.2.1 Mechanism of Freezing and Thawing . . 115 
5.2.2 Effect of Freeze-Thaw on

Resilient Modulus . . . . . . . . . 119

5.2.3 Laboratory Testing Program . . . . . . 121

5.2.4 Test Results and Discussions . . . . . 127

5.3 Variation in $H_{R}$ Characteristics due to

Water Content Change . . . . . . . . . . . 139

5.3.1 Effect of Water Content Change

on Resilient Modulus . . . . . . . . 139

5.3.2 Laboratory Testing Program . . . . . 140

5.3.3 Test Results and Discussions . . . . . 141

5.4 Resilient Characteristics of

5 Frozen Cohesive Solls . . . . . . . . . 146

5.5 Concluding Remarks . . . . . . . . . . . 148

6. RESILIENT MODULUS OF GRANULAR SOIL . . . . . . . . 151

6.1 Introduction .............. 151

6.2 Factors Influencing Resilient Properties

of Granular Soil . . . . . . . . . . . . . 152

6.2 .1 Loading Pulse . . . . . . . . . . . 152

6.2 .2 Level of Stresses . . . . . . . . . . 153

6.2.3 Initial Dry Density . . . . . . . . . 157

6.2.4 Degree of Saturation . . . . . . . . . 157

6.2 .5 Gradation .. . . . . . . . . . . 159

6.3 Laboratory Experimental Program . . . . . . 161

6.3.1 Preparation of Specimen . . . . . . . 161

6.3.2 Resilient Modulus Test on Dune Sand . . 167

6.4 Reduction of Test Results . . . . . . . . . 169

6.5 Test Results and Discussions . . . . . . . . 169

6.6 Concluding Remarks . . . . . . . . . . . . . 184

7. FABRIC OF SOIL - THEORETICAL BACKGROUND . . . . . 186

7.1 Definition of Soil Fabric . . . . . . . . 186

7.2 Previous studies ................ 187

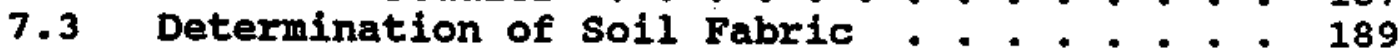

7.4 Pore Size Distribution Using Porosimetry . . 190

8. REPLICATION OF FIELD COMPACTED FABRIC . . . . . . 193

8.1 Introduction . . . . . . . . . . . 193

8.2 Replication Technique . . . . . . . . . . 194

8.3 Input Data Preparation . . . . . . . . . . . 196

8.4 Computational Process ............ . . 202

8.5 Computational stages . . . . . . . . . . 206 
8.6 Variability within the Laboratory Groups . . 208

8.7 Variability within the Field Groups ..... 211

8.8 Comparison of the Laboratory and Field

8.9 Comparison of the Laboratory and ${ }^{\circ} \dot{0}^{\circ} \cdot{ }^{*} \cdot 211$

8.9.1 Match Reversal .. . . . . . . . 215

8.9.2 Multiple Best-matches:. . . . . . :.${ }^{-} 215$

8.9.3 The Worst Matches .............. . 217

8.9.4 Frequently Matched Groups . . . . . . . 218

8.9.5 The Two-way Match............. . 220

8.10 Concluding Remarks ........... 220

9. FABRIC OF SOIL - EXPERIMENTAL STUDIES . . . . . 225

9.1 Introduction ............ 225

9.2 Fabric of Uncompacted Soll . . . . . . . 226

9.4 Laboratory Compaction and Fabric of Soil • 231

9.5 Fabric

9.6 Replication

9.7 Concluding or Fleld Compacted Fabric . . . 253

10. DEVELOPMENT OF SIMPLIFIED DESIGN PROCEDURE . . . 263

10.1 Introduction ............. 263

10.2 Procedure for Cohesive soil : : : : : : 263

10.2.1 Newly Constructed Pavement . . . . . 264

10.2.2 Reconstructed Pavement...:.: 268

10.3 Procedure for Cohesive soll subjected to

10.4 Change in water Content after Construction . 269

10.5 Procedure for Frozen-Thawed Cohesive Soll • 276

10.6 Seasedure for Frozen Cohesive Soll . . . . 277

10.7 Resilient Modulus at Resilient Modulus . . 278

Level of stresses............. 280

10.7.1 Procedure for Normal Cohesive soil. . 280

10.7.2 Procedure for Thawed Cohesive Soil : 282

10.8 Development of Specification to control

In-Service Subgrade Deformability..... 286

10.9 Procedure for Granular Soll . : : : : : 287

10.10 Numerical Examples ......:. : : 287

10.10.1 Newly Constructed Pavement . . . . 288

10.10.2 Reconstructed Pavement . : : : 290

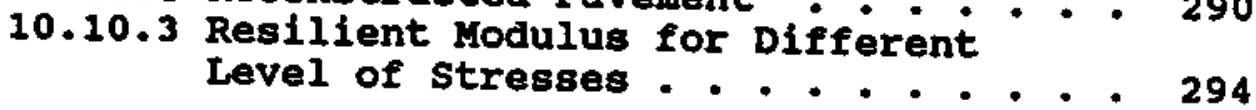


11. CONCLUSIONS AND RECOMMENDATIONS . . . . . . . 300 11.1 Conclusions . . . . . . . . . . . . . 300 11.2 Recommendations for Future Research . . . . . 303

LIST OF REFERENCES . . . . . . . . . . . 305 APPENDICES

Appendix A Information on Tested Specimens .... 320 Appendix B Unconfined Compression Test Results : : 326 Appendix C Resilient Modulus Test Results . . . . 334 Appendix D Group Matrix ............ 343 


\section{LIST OF FIGURES}

Figure

Page

2.1 Classification of Tested Soils . . . . . . . . . . 7

2.2 In-Service and As-Compacted condition

of the South Bend site . . . . . . . . . . . . 9

2.3 In-Service and As-Compacted condition

of the Fort Wayne site (Station 56+00) . . . . . 10

2.4 In-Service and As-Compacted Condition

of the Fort Wayne Site (Station 73+00) . . . . . . 11

2.5 In-Service and As-compacted Condition

of the Fort Wayne Site (Station 181+00) . . . . . . 12

2.6 In-Service and As-Compacted Condition

of the Washington Site . . . . . . . . . . . . 14

2.7 In-Service Condition of the Bedford site . . . . . 15

2.8 In-Service Condition of the Bloomington site . . . 16

2.9 Particle size Distribution of Tested Soils . . . . 18

3.1 Typical Output Format of Resilient Modulus

Test Result .. . . . . . . . . . . . . . . 27

3.2 Stress Pulse Measured During

Resilient Modulus Test . . . . . . . . . . 33

3.3 Variation of Deviator stress

During Conditioning stage . . . . . . . . . . 34

3.4 Variation of strains During Conditioning stage . . 35

3.5 Variation of Resilient Modulus

During Conditioning Stage .. . . . . . . . . 36

3.6 Result of Round Robin Test on Granular Soil . . . . 39

4.1. Stress-strain Relation and Transformed Hyperbolic

Relation of Unconfined Compression Test . . . . . . 53 
4.2 Typical Result of Resilient Modulus Test on Cohesive Soil

4.3 Profile of Resilient Modulus with Depth for the South Bend site . . . . . . . . . . 57

4.4 Relationship of $M_{R}$ with w/c and $\gamma_{d}$ for the South Bend Soil

4.5 Profile of Resilient Modulus with Depth for the Fort Wayne Site............ 60

4.6 Relationship of $M_{R}$ with $w / c, \gamma_{d}, E_{i j}$, and $S_{u 1.0 x}$ for the Fort Wayne Soil.............. 61

4.7 Profile of Resilient Modulus with Depth for the Washington site .............. 62

4.8 Relationship of $K_{k}$ with $w / c, \gamma_{d}, E_{i}$, and $S_{u 1.0 x}$ for the Washington Soil ..............63

4.9 Profile of Resilient Modulus with Depth for the Bedford Site . . . . . . . . . . 64

4.10 Relationship of $M_{\mathrm{g}}$ with $w / C, \gamma_{d}, E_{j}$, and $S_{\mathrm{u} 1.0 x}$ for the Bedford Soil

4.11 Profile of Resilient Modulus with Depth for the Bloomington site

4.12 Relationship of $\mathrm{M}_{\mathrm{k}}$ with w/C, $\gamma_{d}, E_{\mathrm{i}}$, and $S_{\mathrm{ul.0}}$ for the Bloomington Soil

4.13 Relationship between $M_{R}$ at $\sigma_{d}=6$ psi and $\sigma_{3}=3$ psi and $S_{v 1.0 x}$ for All sites ............. 70

4.14 Relationship between $M_{R}$ at $\sigma_{0,6}$ psi and $\sigma_{3}=6$ psi and $s_{\text {w..0x }}$ for All sites ............. 71

4.15 Relationship between $k_{R}$ at $\sigma_{2}-6 \mathrm{psi}$ and $\sigma_{3}=0$ psi and $S_{\text {a. } .0 x}$ for All sites ............. 72

4.16 Comparison between Measured and Predicted In-Service Resilient Modulus 76

4.17 Effect of Mold and Rammer on Compaction . . . . . 78

4.18 Laboratory Compaction Curves of the South Bend Soil

4.19 Laboratory Compaction Curves of the Washington Soil 
4.20 Relationship between $S_{\mathrm{ul} .0 *}$ and Water Content of the Laboratory Compacted South Bend Soll . . . 85

4.21 Relationship between $S_{\mathrm{v1} .0 \times}$ and water Content of the Laboratory Compacted Washington Soll . . . 86

4.22 Relationship between $\mathrm{K}_{\mathrm{R}}$ and water content of the Laboratory Compacted South Bend Soil . . . 87

4.23 Relationship between $M_{R}$ and water content of the Laboratory Compacted Washington Soil . . . 88

4.24 Contour of Resilient Modulus for the Laboratory Compacted South Bend Soil . . . . 89

4.25 Contour of Resilient Modulus for the Laboratory Compacted Washington Soil . . . . 90

4.26 Relationship between $M_{R}$ and $S_{a 1.0 x}$ for the Laboratory Compacted South Bend Soil . . . . 92

4.27 Relationship between $M_{R}$ and $S_{u 1.0 \%}$ for the Laboratory Compacted Washington Soil . . . . 93

4.28 Relationship between $M_{k}$ and $S_{41.05}$ for the Laboratory Compacted Soils . . . . . . . 95

4.29 Comparison between Measured and Predicted $M_{R}$ of Laboratory Compacted Soils . . . . . . . . . 97

4.30 Comparison of Resilient Modulus between Field and Laboratory Compacted Soil of AASHO Test Road . . . . . . . . . . 101

4.31 Resilient Modulus of AASHO Test Road . . . . . 102

4.32 As-Compacted and In-Service Resilient Modulus of the South Bend Soll ............ 104

4.33 As-Compacted and In-Service Resilient Modulus of the Washington Soil 105

4.34 Relationship between $\mathrm{K}_{\mathrm{R}}$ and $\mathrm{S}_{\mathrm{ul} .0 \mathrm{x}}$ for Field and Laboratory Compacted Washington Soil . . . 107

4.35 Relationship between $K_{k}$ and $s_{w 1.0 \%}$ for Field and Laboratory Compacted Solls $\left(\sigma_{3}=0 \mathrm{psi}\right)$. . . 108

4.36 Relationship between $\boldsymbol{M}_{R}$ and $S_{\text {w1.0x }}$ for Field and Laboratory Compacted Soils $\left(\sigma_{3}=3 \mathrm{psi}\right)$. . . 109

4.37 Relationship between $M_{2}$ and $s_{\text {w1.0 }}$ for Field and Laboratory Compacted Solls $\left(\sigma_{3}=6\right.$ psi) 
5.1 Profile of Water Content, Dry Density, and Resilient Modulus of the South Bend Site

5.2 Effect of Freeze-Thaw Cycles on Laboratory Compacted South Bend Soil............

5.3 Cycles Used for Closed-System Freeze-Thaw Test . 125

5.4 Effect of Freeze-Thaw on Resilient Modulus of the Fort Wayne Soil . . . . . . . . . . 128

5.5 Effect of Freeze-Thaw on Resilient Modulus of the Washington soil

5.6 Effect of Freeze-Thaw on Resilient Modulus of the Bedford Soil

5.7 Effect of Freeze-Thaw on Resilient Modulus of the Bloomington Soil

5.8 Relationship between Thawed $M_{R}$ and $S_{v 1.0 \%}$ of All Tested Solls

5.9 Comparison between Measured and Predicted $M_{p}$ of Frozen-Thawed soils

5.10 Relationship between $M_{R}$ and $S_{v 1.0 x}$ of Thawed Soil and Non Frozen-Thawed Soil

5.11 Effect of Water Content Change after Compaction on Resilient Modulus of Laboratory Compacted Soils

5.12 Effect of Molding Water Content on the Resilient Modulus due to Subsequent Change in Water Content

5.13 Relationship between $\mathrm{H}_{\mathrm{R}}$ and $\sigma_{\text {, }}$ of Frozen Soils $\quad 147$

6.1. Relationship between $k_{1}$ and $k_{2}$ (after Rada and witczak, 1981)

6.2 Compaction Curves of Dune sand ......... 162

6.3 Relationship between Peak to Peak Amplitude of Vertical vibration and Dial setting of the vibrating Table... . . . . . . . . 164

6.4 Change of Dry Density due to vibration ..... 165

6.5 Typical Stress Pulse Measured During $M_{\mathrm{R}}$ Test on Dune Sand 
6.6 Resilient Characteristics of Vibratory Compacted and Impact Compacted Dune Sand . . . . . . . 171

6.7 Relationship between Repeated Deviator Stress and Resilient Strain of Dune Sand . . . . 172

6.8 Variation of Total and Permanent strains with Stress Applications on Dune Sand . . . . . . 174

6.9 Effect of Water Content on Parameters A and B . . 176

6.10 Effect of Water Content and Dry Density on Permanent Deformation of Dune Sand . . . . . 178

6.11 Effect of Dry Density on the Relationship between $M_{\mathrm{k}}$ and $\theta$.............. 180

6.12 Variation of Mean Square Error with Dry Density . 181

6.13 Relationship of Parameters $A$ and $B$ with Relative Compaction............. 183

7.1 Sketch of Soil Macro Fabric ........... 188

8.1 Computation of the K-S D Parameter ....... 195

8.2 Field Compaction curve for the Caterpillar compactor . . . . . . . . . . 197

8.3 Field Compaction Curve for the Rascal Compactor ............. 198

8.4 Laboratory Compaction Curves for Impact compaction . . . . . . . . . . 199

8.5 Laboratory Compaction Curves for Kneading Compaction ............ 200

8.6 Sample coding for Laboratory and Field Samples . 203

8.7 Laboratory and Field Group Variability . . . . 209

8.8 Frequently Watched Laboratory and Field Groups . 219

8.9 The First Five Best Matched Groups . . . . . . 222

9.1 Pore size Distribution of Uncompacted Soils . . 227

9.2 Correlation between Macroporosity of Uncompacted Soils and the Maximum Attainable Dry Unit Weight 
9.3 Correlation between Microporosity of Uncompacted Soils and clay praction............ 232

9.4 Laboratory Compacted Fabric-Bedford Soil . . . 234

9.5 Laboratory Compacted Fabric-Bloomington Soil . 235

9.6 Laboratory Compacted Fabric-Fort Wayne Soil . . 236

9.7 Laboratory Compacted Fabric-South Bend Soil . . 237

9.8 Laboratory Compacted Fabric-Washington Soil . . 238

9.9 Comparison of Laboratory Compacted Fabric at Optimum Water Content............ 240

9.10 Field Compacted Fabric-Bedford Soil . . . . . 243

9.11 Field Compacted Fabric-Bloomington Soil . . . . 244

9.12 Field Compacted Fabric-Fort wayne Soil . . . 245

9.13 Field Compacted Fabric-South Bend Soil . . . . 246

9.14 Field Compacted Fabric-Washington Soil . . . 247

9.15 Correlation between Clay Fraction and Resilient Modulus.............. 252

9.16 Replication of Bedford Field Compacted Fabric . . . . . . . . 255

9.17 Replication of Bloomington Field Compacted Fabric ........... 256

9.18 Replication of Fort wayne Field Compacted Fabric . . . . . . . . . . 258

9.19 Replication of South Bend Field Compacted Fabric . . . . . . . . 259

9.20 Replication of Washington Field Compacted Fabric . . . . . . . . 261

10.1 Moisture Characteristic Curve and Relationship between $\Delta \mathrm{M}_{\mathrm{R}}$ and $\Delta \mathrm{w} / \mathrm{c}$ for South Bend Site . . . 271

10.2 Moisture Characteristic Curve and Relationship between $\Delta \mathrm{M}_{\mathrm{R}}$ and $\Delta \mathrm{w} / \mathrm{c}$ for Fort Wayne site . . . 272

10.3 Moisture Characteristic Curve and Relationship between $\Delta \mathrm{M}_{\mathrm{R}}$ and $\Delta \mathrm{w} / \mathrm{c}$ for Washington Site ... 273 
10.4 Molsture Characteristic Curve and Relationship between $\Delta \mathrm{M}_{\mathrm{R}}$ and $\Delta \mathrm{w} / \mathrm{c}$ for Bedford site . . . . . 274

10.5 Moisture Characteristic Curve and Relationship between $\Delta y_{R}$ and $\Delta w / c$ for Bloomington Site . . . 275

10.6 Seasonal Variation of Resilient Modulus . . . . 279

10.7 Chart for Estimation of Parameters in Equation 4.4..... ............... . 281

10.8 Relationships between $M_{R}$ and $\sigma_{d}$ for Each Group of $\mathrm{s}_{\mathrm{v1:0}}$. . . . . . . . . . . . . . 283

10.9 Chart for Estimation of Parameters in Equation 5.1. . . . . . . . . . . . . . . 285

10.10 Chart for Estimation of Resilient Modulus of Vibratory Compacted Dune Sand . . . . . . . 289

10.11 Estimation of $\mathrm{K}_{\mathrm{RT}}$ for Example in section 10.10 .1

10.12 Seasonal Variation of Resilient Modulus for Example in Section 10.10.1

10.13 Estimation of Effective Subgrade $M_{R}$ for Example in Section 10.10.1

10.14 Estimation of $\mathrm{M}_{\mathrm{RT}}$ for Example in Section 10.10.2 295

10.15 Seasonal Variation of Resilient Modulus for Example in Section 10.10.2 . . . . . . . . . 296

10.16 Estimation of Effective Subgrade $M_{R}^{*}$ for Example in Section 10.10.2 . . . . . . . . 297

10.17 Estimated Regression Parameters and Predicted Resilient Modulus... . . . . . . 298

10.18 Measured and Predicted Relationship between $M_{R}$ and $\sigma_{d}$ for specimen FW05 


\section{LIST OF NOTATIONS}

\begin{tabular}{|c|c|}
\hline$\gamma_{d}$ & dry unit weight \\
\hline$\theta$ & sum of principal stresses $\left(\sigma_{1}+\sigma_{2}+\sigma_{3}\right)$ \\
\hline$\mu$ & micrometer \\
\hline$\sigma_{1}, \sigma_{2}, \sigma_{3}$ & principal stresses \\
\hline$\sigma_{d}$ & deviator stress \\
\hline$\tau_{\text {ot }}$ & octahedral shear stress \\
\hline $\mathbf{a}$ & regression parameter \\
\hline$A / D$ & Analog/Digital \\
\hline $\begin{array}{l}\text { AScrI } \\
b\end{array}$ & $\begin{array}{l}\text { American Standard code for Information Interchange } \\
\text { regression parameter }\end{array}$ \\
\hline d & limiting pore diameter \\
\hline D/A & Digital/Analog \\
\hline D & Kolmogorov-Smirnov $(K-S)$ statistic \\
\hline $\mathbf{E}_{\boldsymbol{i}}$ & breakpoint resilient modulus \\
\hline $\boldsymbol{F}$ & field group \\
\hline $\begin{array}{l}F_{\text {L }} \\
F_{\text {, }}\end{array}$ & $\begin{array}{l}\text { variability between the field and laboratory group } \\
\text { cumulative distribution function }\end{array}$ \\
\hline $\mathbf{F}_{\mathbf{w}}$ & average variability within the field group \\
\hline$F_{\text {vm }}$ & maximum variability within the field group \\
\hline $\mathbf{G}_{\mathbf{8}}$ & $\begin{array}{l}\text { specific gravity } \\
\text { cycles per second }\end{array}$ \\
\hline $\begin{array}{l}\mathrm{Hz} \\
\mathrm{I} / \mathrm{O}\end{array}$ & $\begin{array}{l}\text { cycles per second } \\
\text { Input/output }\end{array}$ \\
\hline $\mathbf{J}_{2}$ & second invariant of stresses \\
\hline $\mathrm{k}-\mathbf{s}$ & Kolmogorov-Smirnov $D$ statistic \\
\hline$k_{1}, k_{2}$ & regression parameters \\
\hline $\mathbf{L}$ & laboratory group \\
\hline $\begin{array}{l}\mathbf{L} \mathbf{F}_{\mathbf{v}} \\
\mathbf{L}_{\mathrm{ve}}\end{array}$ & $\begin{array}{l}\text { variability between the laboratory and field group } \\
\text { average variability within the laboratory group }\end{array}$ \\
\hline
\end{tabular}




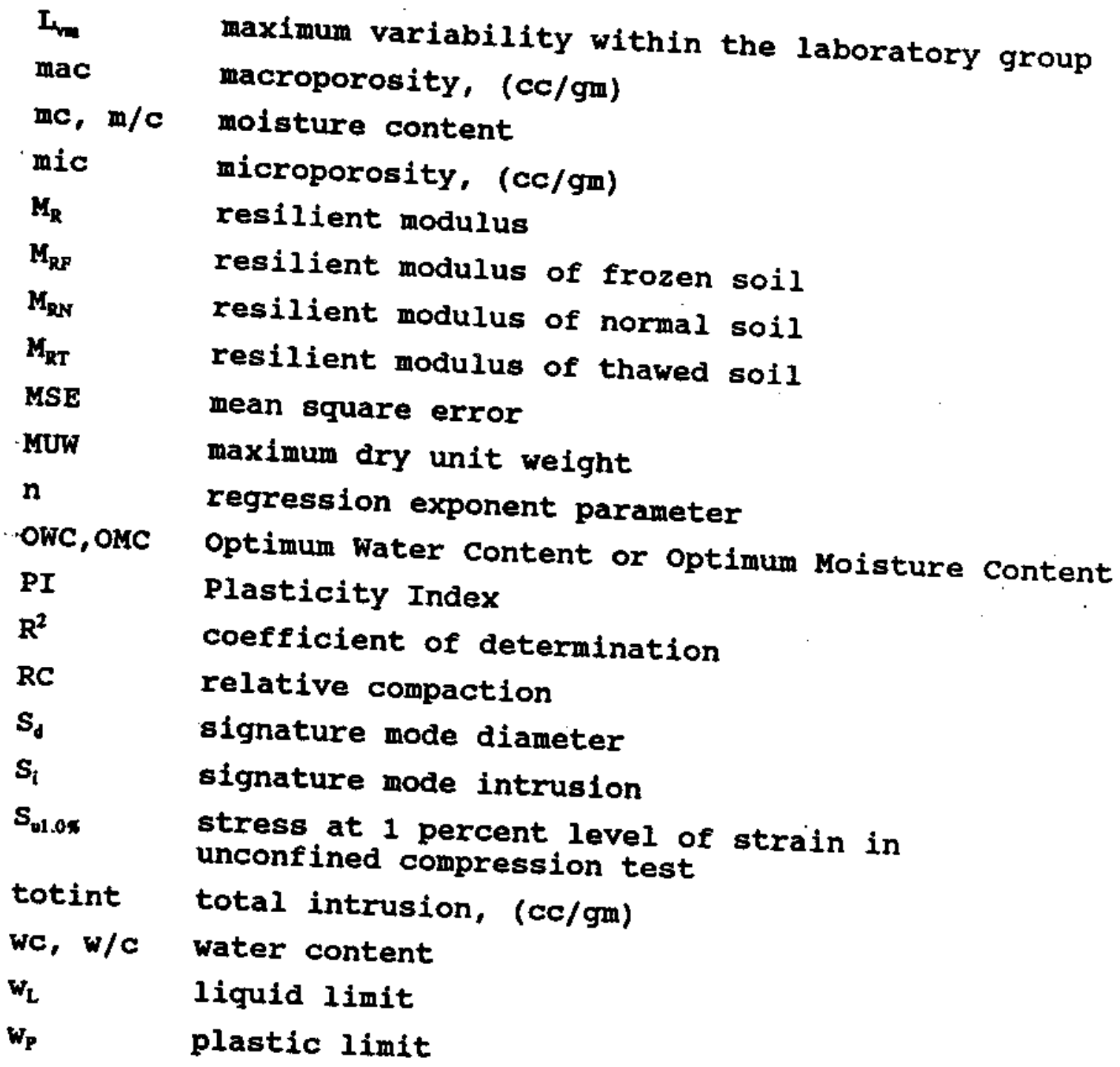


1. INTRODUCTION

1.1 objectives of the Project

There are several goals for this research project. In the short term, one goal is to develop the testing capabilities to conduct the resilient modulus test. With these capabilities the data base can be developed to permit fulfillment of the requirements of the 1986 AASHTO Guide for Design of Pavement structures to utilize resilient modulus in design. The data base will represent a range of soil classes encountered in Indiana. The data base can be used to assign resilient modulus values to soils in the design process, or to check values of resilient modulus estimated by other procedures.

The long term goal of the study is to participate in a more basic approach to the role of the subgrade in pavement design and performance. Consideration will be given to the possibility of establishing limiting criteria for subgrade deformability. This project contributes to this goal by creating a procedure to allow creation of subgrade preparation special provisions that will assure the presence of a limiting resilient modulus in this portion of the pavement system. What should be the magnitude of the limiting modulus must come from pavement analysts for it requires data on what are excessive deformations of the materials for the various components of the pavement system. The criteria are not now available nationally. There is also no back-calculation procedure that can translate, reliably, deformation data into subgrade modulus requirements. When these are developed and accepted, then the procedure outlined in this report will allow creation of the compaction specifications to assure a limiting subgrade modulus. 
For this project, existing electro-hydraulic loading systems and automatic data acquisition system are utilized. A limited number of undisturbed samples from in-situ subgrade are collected. The appropriate physical properties of these samples are determined. In addition, the resilient modulus test is conducted on specimens of the undisturbed samples.

To allow more widespread testing, procedures are developed that permit specimens to be created in the laboratory that simulate the fabric of the field-created soil fabric. The laboratory testing program is prosecuted to develop the expanded data base. Efforts are made to allow estimation of resilient modulus without the need for use of the extensive complex testing techniques now required.

\subsection{Expected Benefits}

Successful completion of this project will mean INDOT can better assess and utilize the 1986 AASHTO Pavement Design Procedure. If control of subgrade deformability can be accomplished, then INDOT can re-examine its pavement design procedures with a view to improvement. The ability to control the deformability of each component of the pavement system is a powerful tool which would lead to less pavement cracking and less early deterioration. Longer pavement life offers countless benefits that have been sought for some time. Results of this project can be combined with the results of previous and on-going projects to enhance this longer pavement life.

\subsection{Work Plan}

The focus of this work is the resilient modulus, as determined by AASHTO Test Method 274. The details of this plan are based on the presumption that the results of the test will be related to the state of the soil and its fabric at the 
initiation of the test procedure. The state of the soil fabric is characterized by the pore-size distribution. Those initial conditions must simulate the conditions present in the field, in-service. Problems are expected because it is believed impossible to simulate the changes that occur in environmental condition, in-service.

The foregoing served as the context for a work plan of 6 tasks:

Task 1: Literature search for:

a) other resilient modulus data for comparison with Indiana data;

b) relations between soil fabrics, laboratory vs. field, and means of producing field fabric in the laboratory;

c) possible correlations between resilient modulus and other, more easily obtained, soil characteristics;

d) effects of additives on subgrade performance.

Task 2: Selection of soils

a) the type of soils to be tested and locations for sampling are to be selected. Mention is made of separating soil horizons in the profile.

Task 3: Testing one soil

a) field location is to be sampled. At conditions found in-service, dry density, water content, resilient modulus, and pore-size distribution are to be determined. Inspection test data are to provide the as-compacted conditions;

b) in the laboratory, using laboratory compaction on the same soil sampled in bulk from borrow area: 1) compaction curves and associated resilient modulus and pore-size data; 
2) varying the water content from the ascompacted condition and the associated resilient modulus and pore-size data;

3) simulation of freeze-thaw upon the ascompacted condition and the associated resilient modulus and pore-size data;

c) the following questions are to be answered by the testing on this one soil:

1) can laboratory compaction simulate the fabric created in the field?

2) is resilient modulus related to soil fabric?

3) can differences in resilient modulus and pore-size data be related to each other and to water content and other physical conditions?

4) how significant are horizon data?

d) Answers to these questions would be included in the work plan for the other soils

Task 4: Testing the other soils

Task 5: Analyze the Data
a) tabulations for direct use of resilient modulus in pavement design
b) design charts to allow control of subgrade deformability

Task 6: Final Report

The final report will be prepared, tendered for review, corrected, and prepared for final publication. Upon acceptance of the report, a workshop can be planned for the potential users. 
2. SOILS INVESTIGATED

\subsection{Sampling sites}

six soils, typically found in Indiana, were included in the present study to investigate the resilient characteristics. Five cohesive soils and one granular soil were obtained from six different sites. Cohesive soils sampled are $C L$ and $C H$ in the Unifled Soll Classification System, and A-4, A-6, A-7-5 and A-7-6 in AASHTO classification. The granular soil investigated is a dune sand classified as A-3 or SP. - Information on sampling sites is given in Table 2.1 .

\subsection{Undisturbed Soils Investigated}

To determine the field resilient characteristics of cohesive soils, undisturbed samples were obtained by pushing Shelby tubes into the compacted subgrades. Undisturbed samples were collected from under-construction and in-service pavements. Detailed information on the undisturbed samples for all sites is given in Appendix $A$.

Data for in-service subgrade were obtained by measuring the weight, moisture content and dimension of specimens after opening the shelby tubes. Atterberg Limits tests were also performed on each specimen after testing for resilient modulus. Atterberg Limits of undisturbed samples tested for resilient modulus are plotted in the plasticity chart shown in Figure 2.1.

Figures 2.2 to 2.8 show the dry unit weight and moisture content in the subgrade during construction and in-service for sampled sites, with the compaction curve for standard proctor 
Table 2.1 Information on Sampling sites

\begin{tabular}{|c|c|c|c|c|c|c|}
\hline sites & Abbr & Location & station & soil class. & $\begin{array}{c}\text { Date } \\
\text { Compacted }\end{array}$ & Date Sampled \\
\hline South Bend & SB & $\begin{array}{c}\text { US20 } \\
\text { Bypass } \\
\end{array}$ & $140-141$ & $\begin{array}{c}A-4 / A-6 \\
(\mathrm{CL}) \\
\end{array}$ & Aug 89 & Jan;May;July 90 \\
\hline Fort Wayne & FW & $\begin{array}{r}\text { Us2 } 4 \\
\text { Bypass } \\
\end{array}$ & $\begin{array}{c}56,73, \\
181 \\
\end{array}$ & $\begin{array}{c}A-6 \\
(C L) \\
\end{array}$ & Aug 87 & Sept 90 \\
\hline Washington & WA & $\begin{array}{c}\text { US50 } \\
\text { Bypass } \\
\end{array}$ & 290 & $\begin{array}{c}A-6 / A-4 \\
(C L) \\
\end{array}$ & June 89 & $\operatorname{Jan} 91$ \\
\hline Bedford & IA & SR-37 & $530-532$ & $\begin{array}{c}A-7-5 / A-7-6 \\
(\mathrm{CH})\end{array}$ & Aug 70 & Apr; Sept 91 \\
\hline Bloomington & BL & SR-37 & 849 & $\begin{array}{c}A-7-6 \\
(\mathrm{CH})\end{array}$ & Oct 72 & Sept 91 \\
\hline E. Chicago & 194 & $I-94$ & 1076 & $\begin{array}{l}A-3 \\
(S P) \\
\end{array}$ & & Apr 91 \\
\hline
\end{tabular}




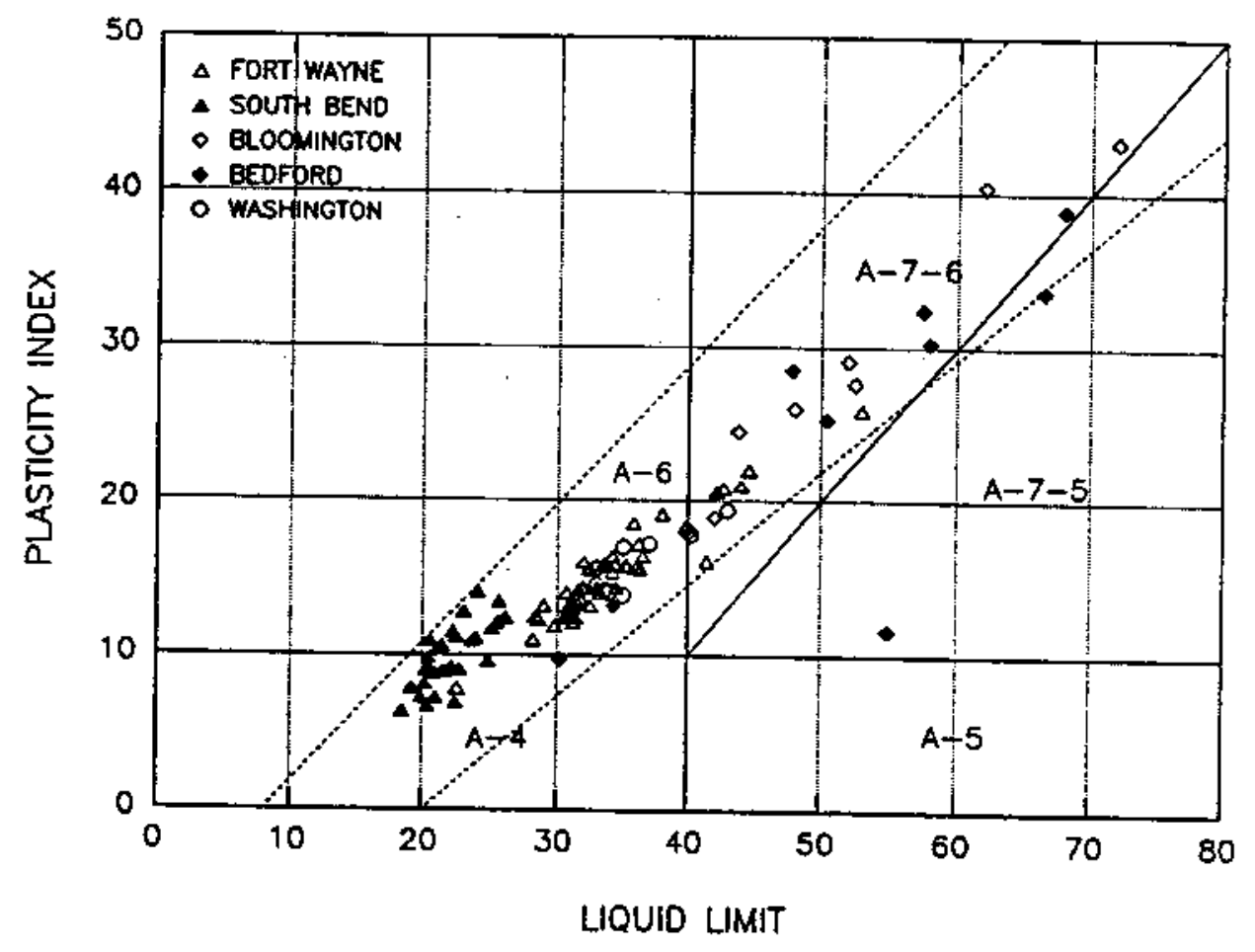

Figure 2.1 Classification of Tested Soils 
energy. Data for as-compacted condition were taken from the field inspection data as measured by a Troxler 3440 moisturedensity gauge or by the sand-cone method during construction. South Bend was the only site where the soil was sampled before the construction of pavement was completed. Embankment fill was compacted with four passes of a Caterpillar CAT 815 sheepsfoot roller. By comparing the field compaction in Figure 2.2 with laboratory compaction curves in Figure 4.18 , it appears that field compacted density is similar to laboratory compacted density achieved with enexgy between 4500 and 6700 $1 \mathrm{~b} \cdot \mathrm{ft} / \mathrm{ft}^{3}$, which is less than half of the standard Proctor energy. However, comparison of the South Bend laboratory and field compacted soil fabric in chapter 9 suggests that the laboratory compaction with standard Proctor energy at about optimum water content gives a closer match to the field compacted fabric. This suggests that the same compaction result by different methods of compaction does not necessarily mean the same soil fabric. At this site, there were increases in dry density and decreases of water content after construction of embankment. Since the subgrade at south Bend had not been covered by the pavement structure during the period between construction and sampling, the reduction of water content may be due to the drying of the soil during and after construction. The increase in density may be attributed to construction traffic for an overpass bridge located at the end of the embankment.

The Fort wayne soil was sampled at three different stations; all stations were compacted with a Caterpillar CAT 825 sheepsfoot roller during construction. The number of passes of this roller were four to six for station $56+00$ and four for station $181+00$. At station $73+00$, the top 2 feet of subgrade was compacted with eight to nine passes while the lower embankment was compacted with four to six passes. As shown in Figures 2.3 to 2.5 , station $56+00$ and $73+00$ were compacted near optimum, and station $181+00$ was compacted dry 


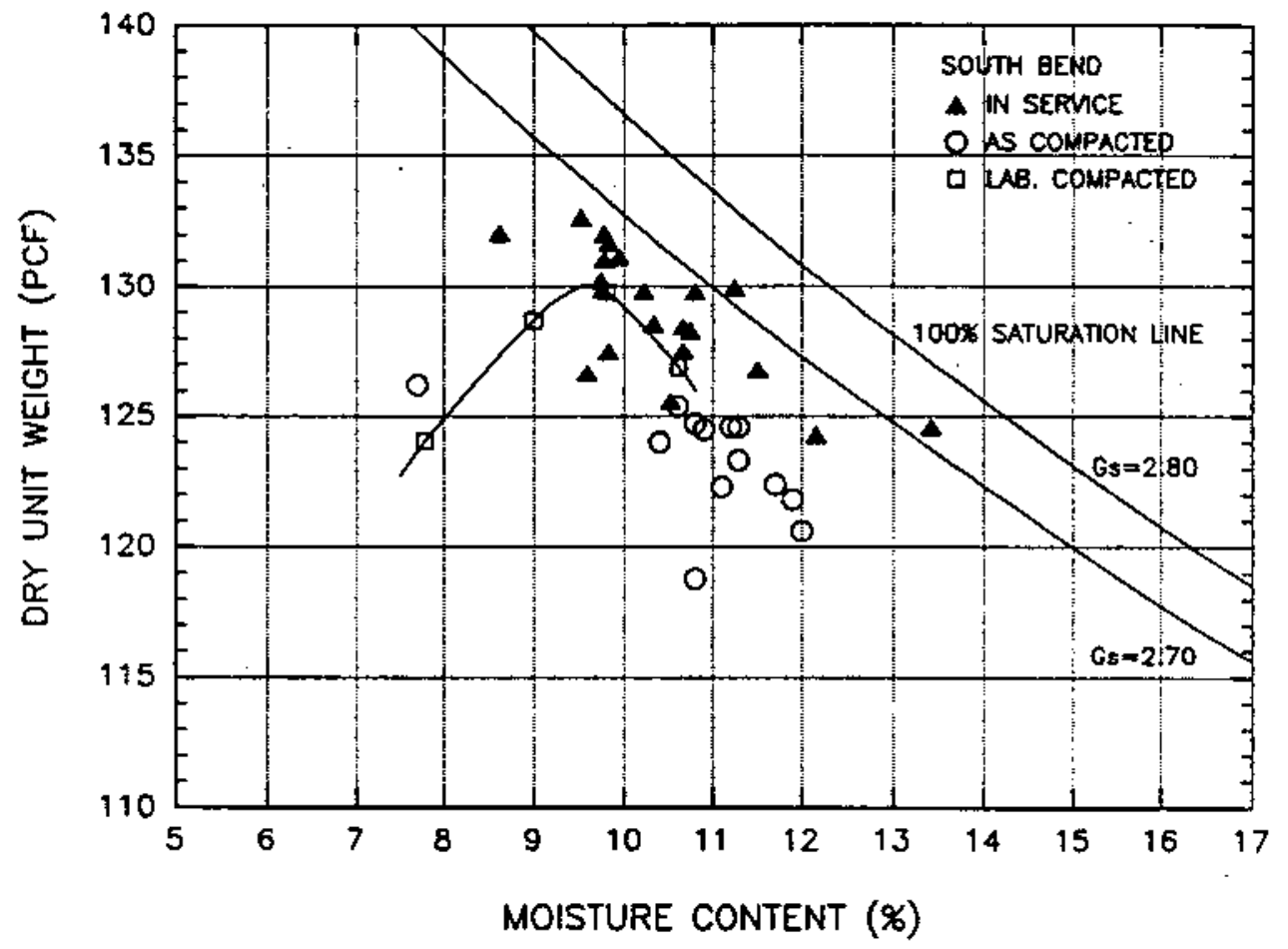

Figure 2.2 In-Service and As-Compacted Condition of the South Bend Site 


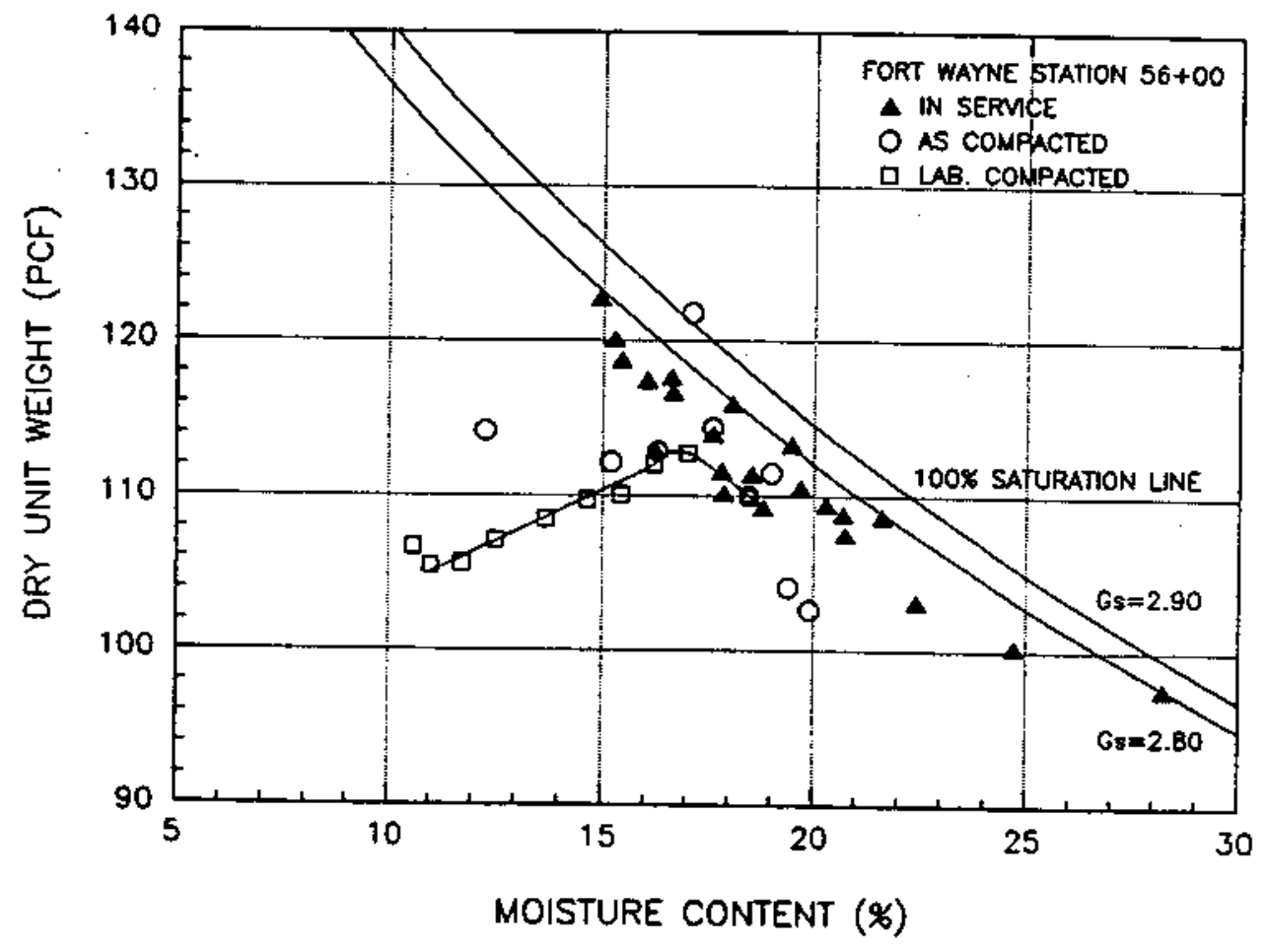

Figure 2.3 In-Service and As-Compacted Condition of the Fort
Wayne Site (Station 56+00) 


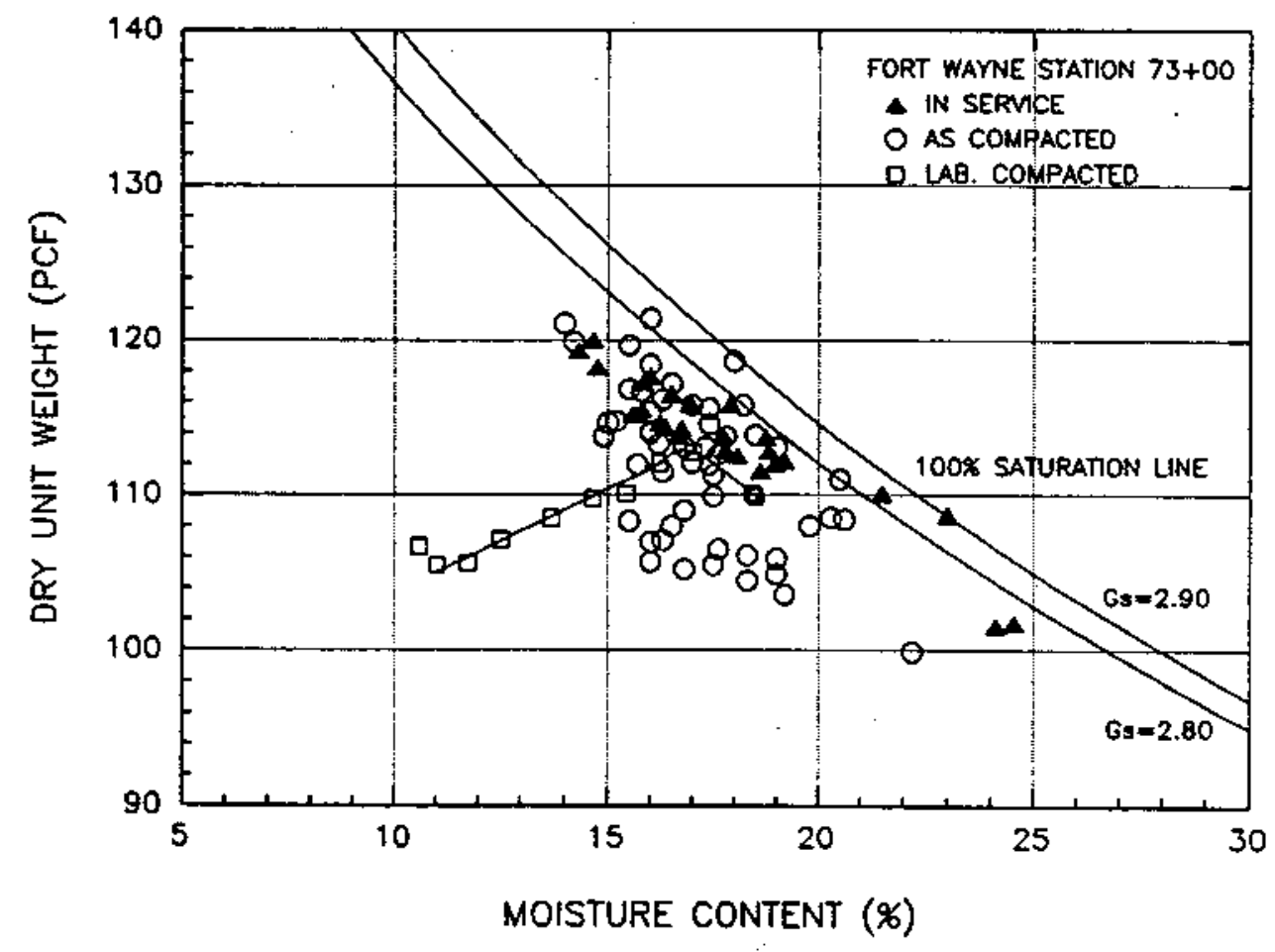

Figure 2.4 In-Service and As-Compacted Condition of the Fort Wayne Site (Station $73+00$ ) 


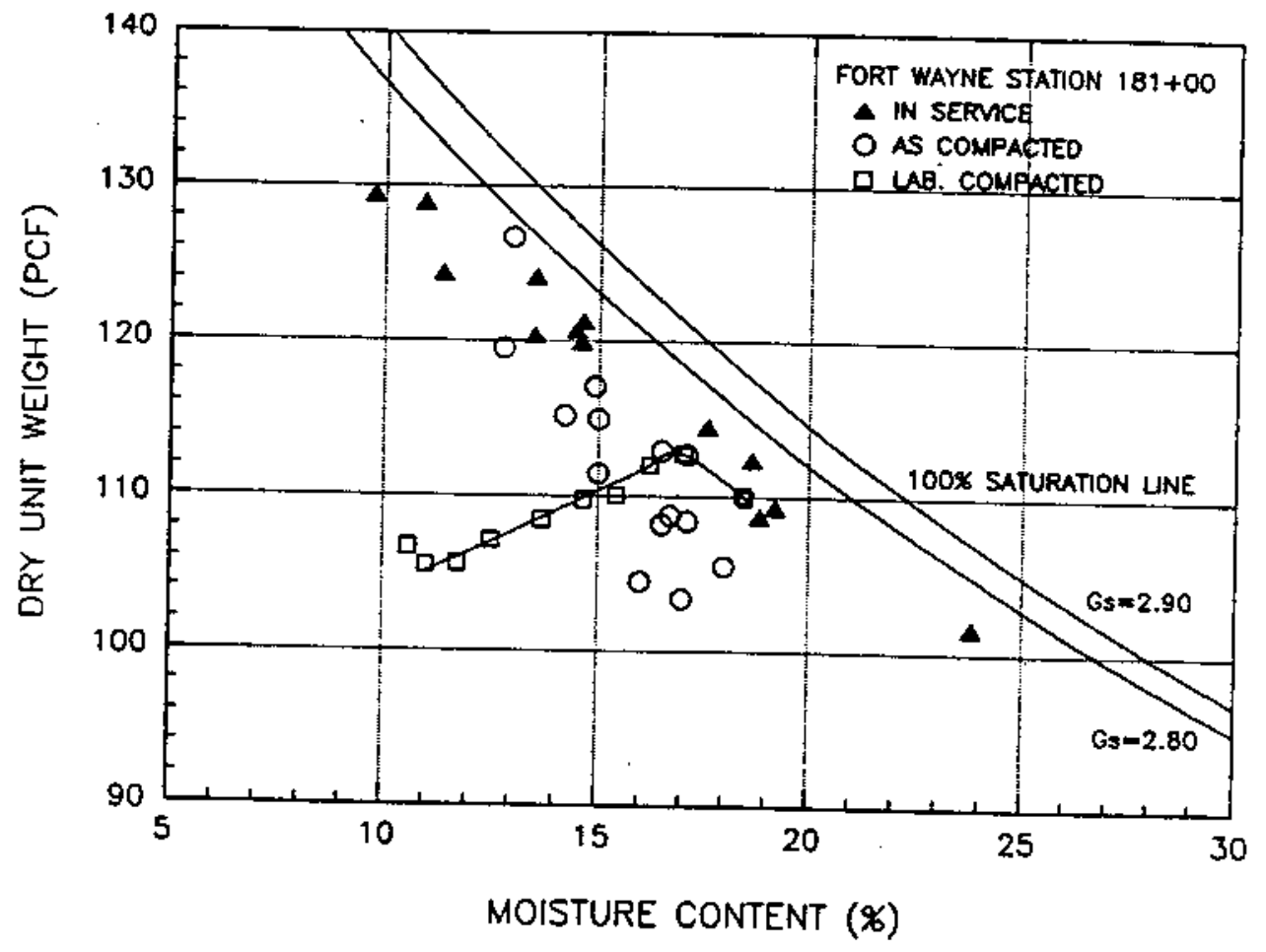

Figure 2.5 In-Service and As-Compacted Condition of the Fort
Wayne Site (Station 181+00) 
side of laboratory optimum moisture content. All three stations show insignificant change in dry density after construction while the water content varied more widely and the degree of saturation increased slightly after construction. For these stations, field compacted density appears to be similar with laboratory compacted density achieved with Standard Proctor energy. Similar to the South Bend site, laboratory compaction at standard Proctor energy at about optimum water content gives a comparable match for the field compaction in terms of soil fabric. However, there is no significant difference between the laboratory compacted fabric at Standard and Modified Proctor energies as explained later in Section 9.6 .

As shown in Figure 2.6, compaction of the Washington site was well controlled during construction. One group of data having higher density and lower water content is for the top 2 feet of compacted layer, the so-called special subgrade treatment. This site was compacted with three passes of a Caterpillar CAT 553 sheepsfoot roller and two passes of a Raygo 404 vibrating sheepsfoot roller. Some of the special subgrade treatment was subjected to 3 passes of the Raygo 404 roller. Since the compactive efforts for the special subgrade treatment and embankment are almost same, the line connecting the two groups of field compaction data can be considered as a wet side portion of field compaction. This implies that the line of optimums for field compaction locates on the dry side of the line of optimum of laboratory compaction. It is also noted that there was a significant increase in the degree of saturation after construction. The in-service condition is formed in a narrow range parallel to the 100 percent saturation line; the in-service density and water content vary over a wider range than the as-compacted condition.

since the Bedford and Bloomington sites were constructed in the early 1970's, information on in-place inspection tests was not available. As shown in Figures 2.7 and 2.8 , in-service 


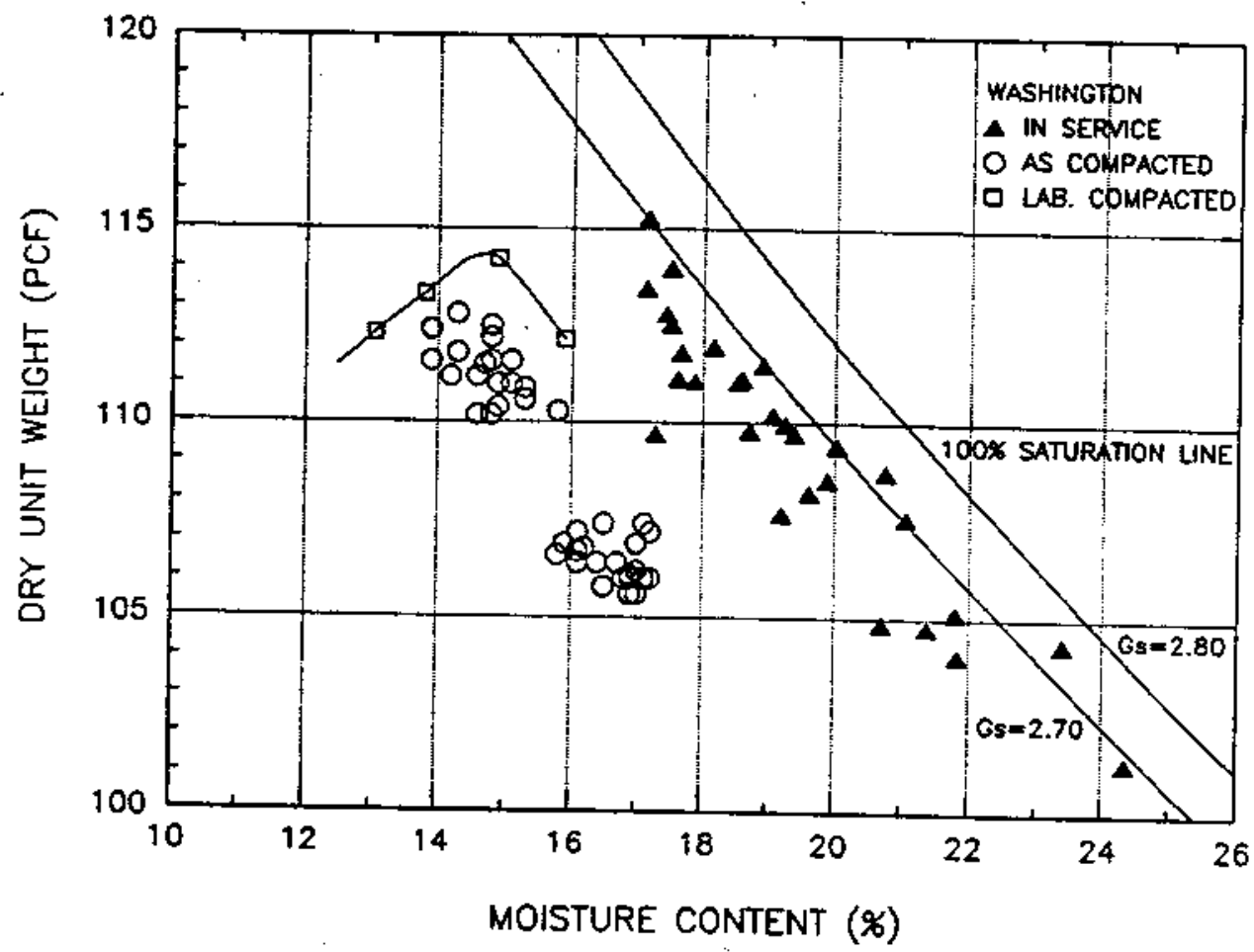

Figure 2.6 In-Service and As-Compacted Condition of the 


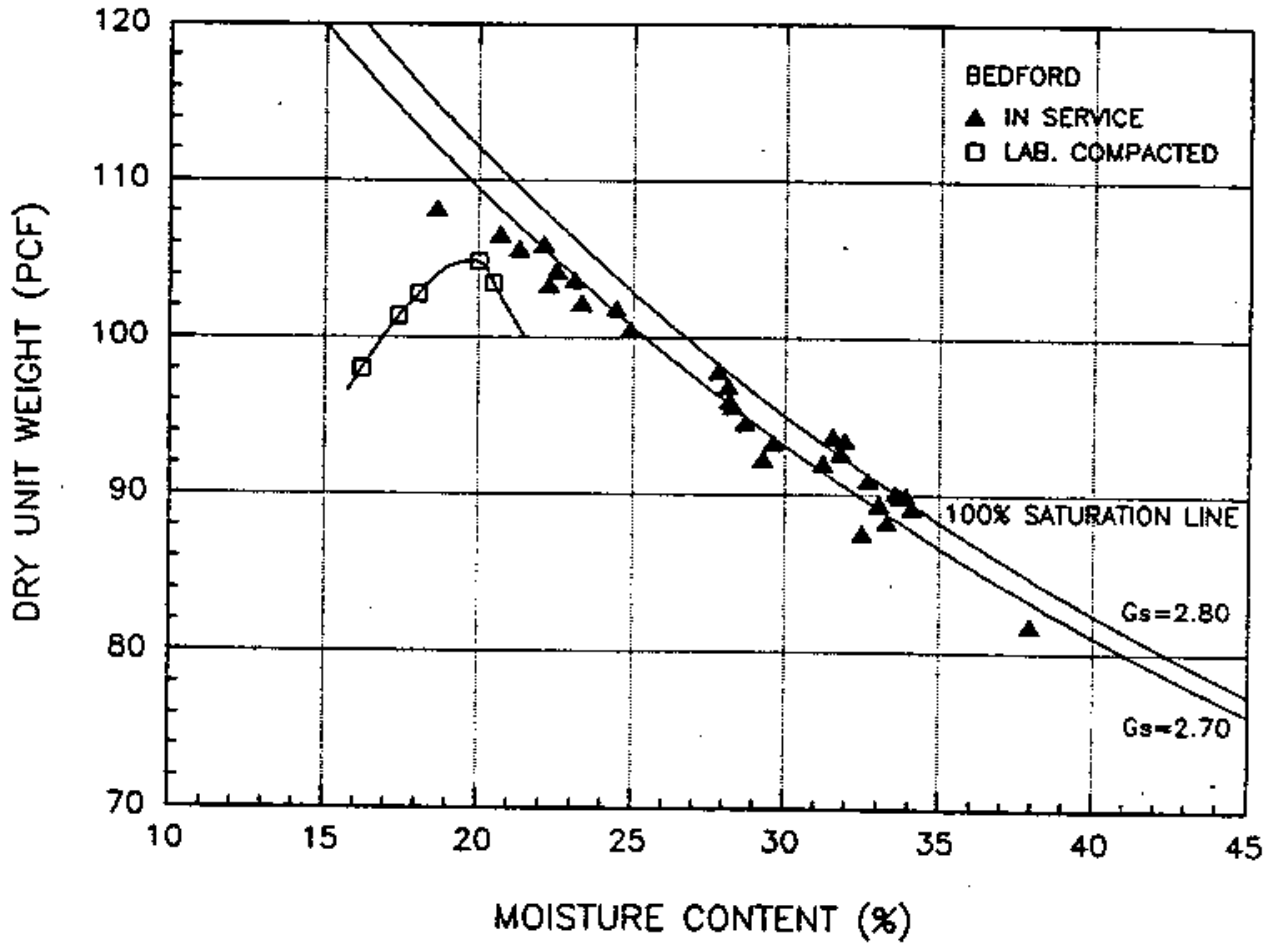

Figure 2.7 In-Service Condition of the Bedford site 


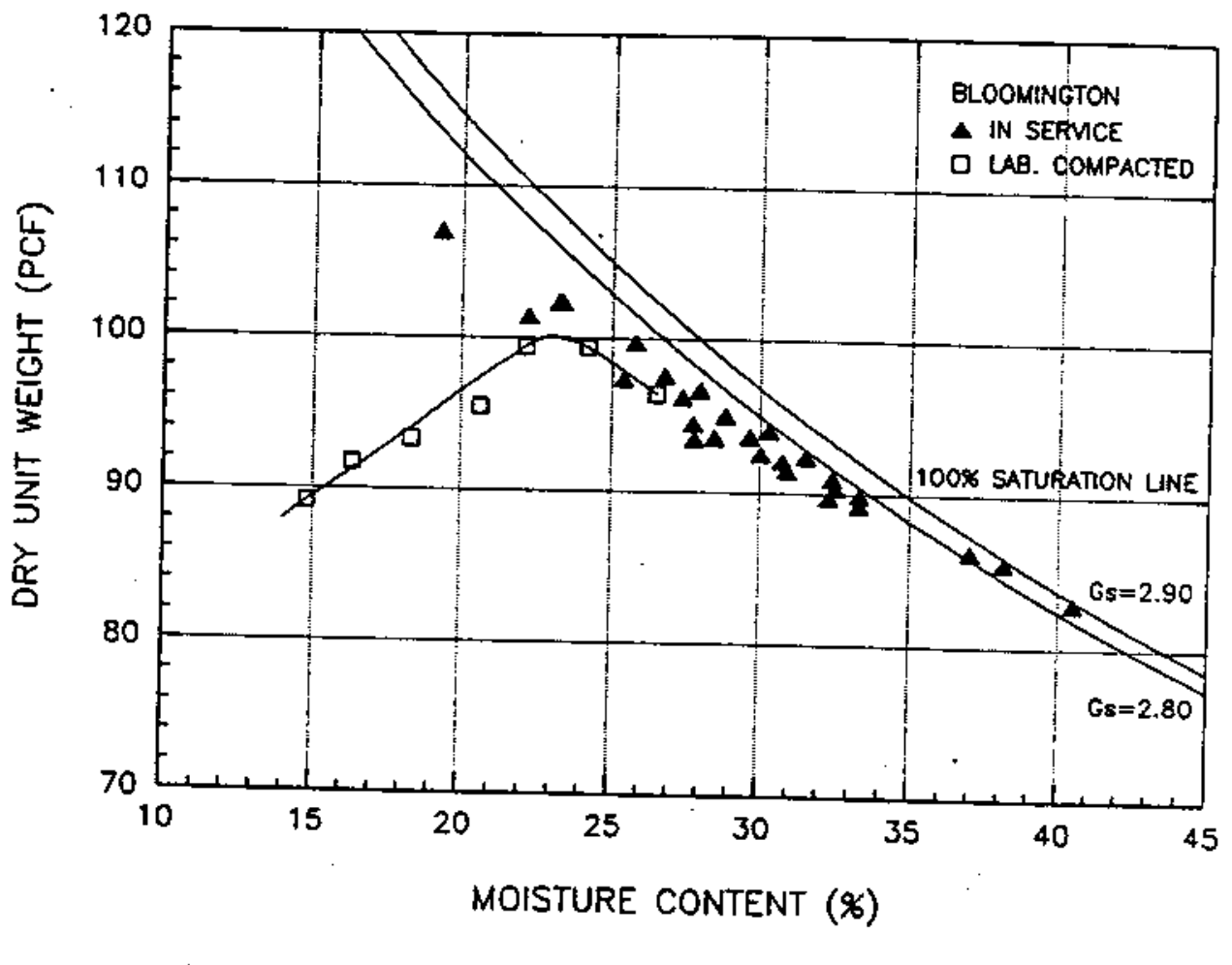

Figure 2.8 In-Service Condition of the Bloomington site 
water content for both sites ranges from $18 \%$ to $40 \%$ and the in-service dry density ranges from 80 pcf to 110 pcf. It is noted that the degree of saturation for these sites appears to be almost 100 percent.

\subsection{Disturbed Soils Investigated}

Disturbed cohesive soils were collected from all five sites for the preparation of laboratory samples. Disturbed soils were air-dried and processed for laboratory compaction. Laboratory specimens were compacted in five layers of approximately equal height with a rammer specially designed for this study. The compaction mold of about 2.8 inch diameter was used for laboratory compaction to get the required sample size similar to that of the field samples. Laboratory compaction curves of each sites for standard proctor energy are given in Figures 2.2 to 2.8 . Properties of the processed cohesive soils are shown in Table 2.2. Particle size distributions for these soils are shown in Figure 2.9 .

Table 2.2 Properties of Processed Soils

\begin{tabular}{c|c|c|c|c|c|c}
\hline Site & $\begin{array}{c}\gamma_{\text {dmax }} \\
(\text { pCf })\end{array}$ & $\begin{array}{c}w_{\text {OMC }} \\
(8)\end{array}$ & $G_{1}$ & $w_{L}$ & $W_{P}$ & $P I$ \\
\hline SB & 129.5 & 9.4 & 2.76 & 21 & 14 & 7 \\
\hline FW & 112.8 & 16.8 & 2.67 & 34 & 19 & 15 \\
\hline WA & 114.0 & 14.9 & 2.74 & 30 & 21 & 9 \\
\hline IA & 105.3 & 19.5 & 2.70 & 52 & 24 & 28 \\
\hline BL & 100.0 & 23.5 & 2.79 & 51 & 26 & 25 \\
\hline I94 & & & 2.65 & NP & NP & NP \\
\hline
\end{tabular}




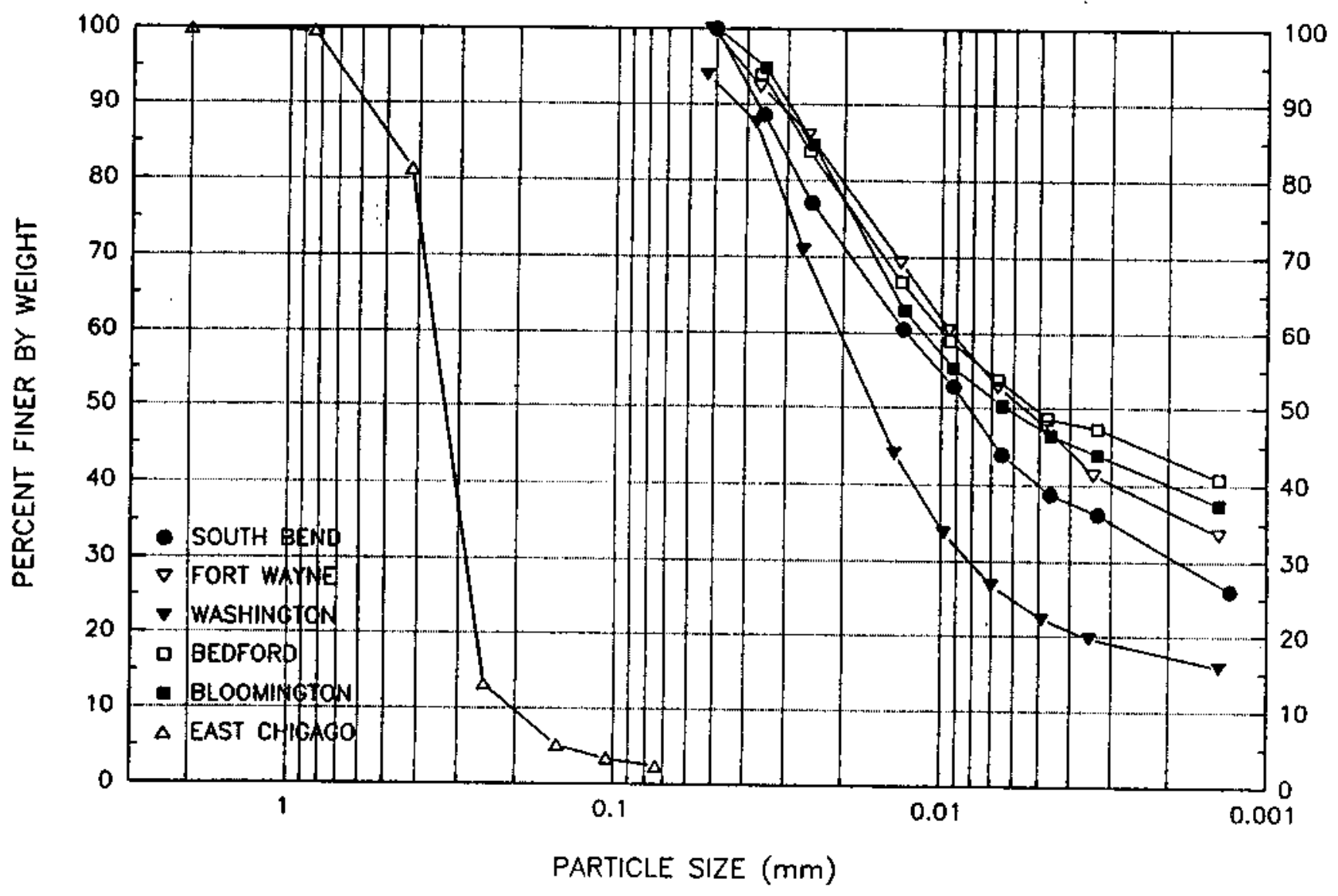

Figure 2.9 Particle Size Distribution of Tested Soils 
Since there is no satisfactory sampling method for undisturbed granular soil, sand specimens were prepared by laboratory compaction. Granular soil obtained from the field was air-dried in the laboratory and was processed for laboratory compaction. Granular soil tested is a very uniform sand, as shown in Figure 2.9. To match the specification in use for field compaction, one series of specimens was compacted by impact hammer with standard proctor energy into a split mold of 4-inch diameter. Another series of specimens was prepared by vibratory compaction. Results of both series of compaction are shown in Figure 6.2. 
3. DEVELOPMENT OF TESTING PROCEDURE FOR RESILIENT MODULUS

3.1 Introduction

It has been recognized that an intolerably uneven pavement surface will develop if there exists excessive plastic deformation of the pavement components and that extensive cracking of asphaltic pavements results from excessive elastic deflections without any significant plastic deformation. The primary cause of failures of the latter type has been attributed to the fatigue of the pavement surface resulting from repeated stress applications over a long period of time.

Recognition of the prevalence of fatigue failure has demonstrated the need to predict recoverable transient deformations in order to assess the magnitude of the strains developed in the pavement surface and, thus, estimate the fatigue life of the pavement. Since a substantial part of transient deflections of pavement system results from compression of the subgrade, the resilient modulus of subgrade would appear to be an important characteristic of the multilayer pavement system.

In order to determine resilient properties, it is necessary to develop an appropriate test method. Reliable measurement of resilient characteristics can only be obtained under test conditions simulating as closely as possible the loading conditions existing in the field. Therefore, it is essential to use a dynamic-loading type of test procedure. In this chapter, the features of a resilient-modulus testing system, including testing software and data acquisition, as well as the developed testing procedure will be described. 
Reliability of test data obtained by developed testing procedure and the performance of testing system will also be discussed.

3.2 System for Resilient Modulus Test

\subsubsection{Testing Equipment}

Equipment used for the resilient modulus test is composed of the loading frame with hydraulic power supply unit, controlling and conditioning unit including wave-form generator, an oscilloscope monitoring the input and output stress pulse, and a Personal computer (PC) which controls the resilient modulus test and performs data acquisition.

The load frame is an MTS-810 Soll Test system with a capacity of 22 kips and is a closed-loop servo-hydraulic system. Load is applied by an actuator moving the piston against the crosshead of the frame. This system has the ability to apply load at high rate and to follow command signal input changes. However, dynamic response of the complete system with feedback must be tuned for the materials tested. This is done by adjusting the active gain to a value known by experience to be stable for the particular specimen under testing. Performance of an improperly adjusted system can range from sluggish to violently unstable.

Several AC and DC types of transqucers were used to measure the changes during the test. Adequate signal scaling and uniformity of the test results are obtained by matching the expected range of measurand so as not to exceed the upper or lower limit of the operating range. Generally a range cartridge rated at the closest value larger than the expected value is used. Detailed information on the instruments used for the resilient modulus test is given in Table 3.1 .

A load transducer to measure the load on the specimen is located between the loading piston and the triaxial cell. Two 
Table 3.1 Instruments for Resilient Modulus Test

\begin{tabular}{|c|c|c|c|c|c|c|}
\hline Instrument & Model & Capacity & Cartridge & output & Resolution & Note \\
\hline Load cell & MTS $661.19 \mathrm{C}-04$ & $55001 \mathrm{bs}$ & $550 \quad 1 \mathrm{bs}$ & $10 \mathrm{~V}$ & $0.1343 \mathrm{lbs}$ & DC \\
\hline Ioad cell & MTS $661,18 \mathrm{~A}-01$ & $220 \mathrm{lbs}$ & $200 \quad 1 \mathrm{bs}$ & $10 \mathrm{~V}$ & $0.0488 \quad 1 \mathrm{bs}$ & DC \\
\hline LVDT & MTS 244.12 & $150 \mathrm{~mm}$ & $100 \mathrm{~mm}$ & $10 \mathrm{~V}$ & $1.922 \times 10^{-4} \mathrm{in}$ & $A C$ \\
\hline $\begin{array}{l}\text { Displacement } \\
\text { gage }\end{array}$ & MTS $632.06 \mathrm{C}-20$ & $4 \mathrm{~mm}$ & $4 \mathrm{~mm}$ & $10 \mathrm{~V}$ & $3.845 \times 10^{-5} \mathrm{in}$ & $D C$ \\
\hline $\begin{array}{l}\text { Pressure } \\
\text { transducer }\end{array}$ & $\begin{array}{l}\text { Sensotec } \\
\text { TJE/708-15-02 }\end{array}$ & $300 \mathrm{psi}$ & $29 \mathrm{psi}$ & $10 \mathrm{~V}$ & $0.0071 \mathrm{psi}$ & DC \\
\hline $\begin{array}{l}\text { Pressure } \\
\text { regulator }\end{array}$ & Bellofram 1001 & $30 \mathrm{psi}$ & & $10 \mathrm{~V}$ & & \\
\hline
\end{tabular}


load transducers of different capacity have been used for different sizes of specimen. For specimens obtained from 2.8inch diameter shelby tube, a load transducer of 220 lbs capacity was used with a 100 lbs range cartridge, since the maximum load applied during the resilient modulus test is about 64 lbs. Another transducer of a $5.5 \mathrm{kip}$ capacity with 550 lbs range cartridge was used for the 4-inch diameter sand specimens which are subjected to maximum loading of about 250 lbs during the test.

To monitor the input and output signals of load, an oscilloscope was connected to the load conditioning unit. By comparing signals visually during the test, it can be known if the specimen responds well to the input signal. If the stiffness of specimen matches well to the response of machine, almost identical input and output signals are observed. If not, gain and rate in the control module are adjusted until both signals match.

A DC type displacement gage is mounted on the supporting block fixed on top of the triaxial cell so that an extended sensing arm from the displacement gage contacts the lower side of the concentric bracket attached to the loading piston. By attaching the concentric bracket below the load cell, the measured deformation is not affected by the deflection of the load cell itself. Deformation of specimen causes the movement of the sensing arm and bends the metallic element, changing the resistance of strain gages. The change in the balance of the Wheatstone bridge produces an electrical output proportional to the displacement of the sensing arm.

During the resilient modulus test, it is required to change the magnitude of deviator stress and confining pressure frequently. Confining pressure is controlled by supplying air through a Bellofram pressure regulator actuated by the voltage signal sent from the controlling computer. This change of confining pressure is monitored by a pressure transducer at the port of the triaxial cell. 
Triaxial cells used in this test can support specimens up to 4 inches in diameter. A loading ram screwed to the top endcap extends through the triaxial cell and can be connected to the load cell. Friction between the cell and loading ram is minimized by low-friction ball bearings. The upper and lower plates supported by three stainless steel columns are enclosed by a removable translucent pressure shell which allows specimen setup without disassembly of the triaxial cell. The cell is sealed by two pressurization o-rings on support platens.

Controllers, signal conditioning units, and waveform generator are contained in a stand-alone cabinet. Controllers enable the test control in force or displacement mode, and feature easy and fast transfer between control modes. For the resilient modulus test, one AC controller and three DC controllers were used. Two active controllers include the valve driver, and all four controllers contain the transducer and command conditioning unit. Each controller has several plug-in cartridges allowing multiple range capabilities. The waveform generator is a microprocessor-controlled digital unit and can be used to program any shape of load or displacement input. The remote mode operation enables the personal computer to create segment parameters for any kind of waveform and to send them to the waveform generator through the interface during the resilient modulus test.

\subsubsection{Resilient Modulus Testing Software}

Since the resilient modulus test follows a sophisticated testing procedure and fast dynamic loading is applied during test, it is not possible to control the test and acquire the test data without sophisticated testing software. The software package originally developed by kTS corporation was modified and used in this study. This test software is composed of four main sections: parameter input, execution of test, analysis, and data conversion. 
Test parameters are entered by the operator using a filepreparation program. The information entered is used in both test execution and analysis portions of the test. The test parameters include:

a. general information: specimen ID and sampling location etc.

b. measurement of specimen: height, diameter, w/c and density etc.

c. test control parameters: waveform to be used, load duration, frequency, sequence of test etc.

d. station parameters: full scale ranges of instruments etc.

Test execution consists of station verification, conditioning stage, main test, and data storage. station verification includes the confirmation of readiness, such as turning on hydraulics and enabling the interlocks. It also checks the displacement transducer to ensure that it is within a 20 millivolt noise limit. After verification is complete, test is begun with a command by operator.

since the resilient modulus test is stress-controlled and consists of applications of several different combinations of stress states, it is necessary to control the magnitude of deviator stress and confining pressure automatically without interference. Confining pressure is controlled by a pressure regulator actuated by a voltage corresponding to a desired level of confining pressure. A calibration equation for the pressure regulator is contained in testing program, and the target voltage calculated from that equation is sent to the regulator through a Digital to Analog converter in the data acquisition board. After confining pressure reaches the target value, the test proceeds to waveform generation with generated waves sent to the DC load controller. The resilient modulus test software used has a capacity to generate four different shapes of stress pulse, such as haversine, square, triangle, and special triangle. Each waveform consists of applied 
deviator stress and release. Duration of load ranges from 0.05 second to 0.5 second, and cyclic duration from 0.75 second to 3 seconds.

The test is performed following the sequence specified in AASHO $T$ 274-82. Test sequence involves two stages of testing: conditioning, and the main test. Conditioning is intended to minimize the effects of the interval between compaction (or sampling) and loading, and of initially imperfect contact between the platens and the specimen. Resilient modulus test for cohesive soil consists of 20 different combinations of confining pressures and deviator stresses, and 200 stress pulses are applied for each stress combination. The test for cohesive soil takes about 80 minutes. The test for granular soil consists of 33 different states of stresses, and it takes about 2 hours when a stress pulse of $1 \mathrm{~Hz}$ is used.

While the test is running, the program provides an online data display which shows current testing status continuously and measured resilient modulus every 100 stress pulses. Data obtained during the test are stored in binary data files. One data file stores the deviator stress, resilient strain, confining pressure and calculated length of specimen for the 100th and 200th cycle for each test sequence. If the option is chosen for extra data, the entire stress and displacement data for the 200th cycle of each state of stresses are stored in a separate binary file. These two binary files can be converted to ASCII text format or spreadsheet form using a conversion program. Figure 3.1 shows a typical summary of test result converted into AscII text format. Another data file is a sequential file containing the confining pressure, current number of the applied cycles, calculated deviator stress, total deformation, resilient deformation, specimen length and resilient modulus, for every 4 cycles. 


\begin{tabular}{|c|c|c|c|c|c|c|}
\hline $\begin{array}{l}\text { start Date } \\
\text { End Date } \\
\text { soil sample }\end{array}$ & $\begin{array}{l}: \\
: \\
:\end{array}$ & $\begin{array}{l}\text { BL15 } \\
01-10-195 \\
01-10-195 \\
\text { BL8491, D }\end{array}$ & $14-20=$ & $\begin{array}{l}\text { Start Times } \\
\text { End Tine : }\end{array}$ & $\begin{array}{l}00: 02: 19 \\
01: 20: 53\end{array}$ & \\
\hline $\begin{array}{l}\text { Denired } \\
\text { Deviator } \\
\text { stress } \\
\text { (p\$i) }\end{array}$ & $\begin{array}{l}\text { Desired } \\
\text { Confining } \\
\text { Pressure } \\
\text { (pei) }\end{array}$ & $\begin{array}{l}\text { Data } \\
\text { Cycla }\end{array}$ & $\begin{array}{c}\text { Calc } \\
\text { Deviator } \\
\text { stross } \\
\text { (psi) }\end{array}$ & $\begin{array}{c}\text { Calc } \\
\text { Resilient } \\
\text { strain } \\
\text { (in/tn) }\end{array}$ & $\begin{array}{l}\text { Calc } \\
\text { Gage } \\
\text { Iongth } \\
\text { (in) }\end{array}$ & $\begin{array}{l}\text { Resilient } \\
\text { Modulun } \\
\text { (psi) }\end{array}$ \\
\hline 1.00 & 6.00 & .100 & $9.898-01$ & $9.1296 \mathrm{z}-05$ & $5.896 \mathrm{E}+00$ & $1.093 E+04$ \\
\hline 1.00 & 6.00 & 200 & $1.00 E+00$ & $1.0434 E-04$ & $5.896 E+00$ & $9.620 \mathrm{E}+03$ \\
\hline 2.00 & 6.00 & 100 & $1.96 \mathrm{E}+00$ & $1.9567 \mathrm{E}-04$ & $5.095 E+00$ & $1.003 E+04$ \\
\hline 2.00 & $6.00^{\circ}$ & 200 & $1.92 \mathrm{E}+00$ & $1.8263 \mathrm{E}-04$ & $5.895 E+00$ & $1.049 E+04$ \\
\hline 4.00 & 6.00 & 100 & $3.85 E+00$ & $4.6975 \mathrm{E}-04$ & $5.8938+00$ & $8.191 E+03$ \\
\hline 4.00 & 6.00 & 200 & $3.83 \mathrm{E}+00$ & $4.69778-04$ & $5.8938+00$ & g. $1582+03$ \\
\hline 9.00 & 6.00 & 100 & $7.638+00$ & $1.24098-03$ & $5.8872+00$ & $6.1532+03$ \\
\hline 9.00 & 6.00 & 200 & $7.65 E+00$ & $1.2542 \mathrm{E}-03$ & $5.886 \mathrm{~B}+00$ & $6.100 \mathrm{E}+03$ \\
\hline 10.00 & 6.00 & 100 & $9.52 \mathrm{E}+00$ & $1.72598-03$ & $5.8811+00$ & $5.516 E+03$ \\
\hline 10.00 & 6.00 & 200 & $9.60 \mathrm{E}+00$ & $1.7267 \mathrm{E}-03$ & $5.9788+00$ & $5.558 \mathrm{E}+03$ \\
\hline 1.00 & 6.00 & 100 & $1.06 \mathrm{E}+00$ & $9.15618-05$ & $5.879 E+00$ & 1. $163 E+04$ \\
\hline 1.00 & 6.00 & 200 & $1.05 E+00$ & $9.15602-05$ & $5.879 E+00$ & $1.146 E+04$ \\
\hline 1.00 & 3.00 & 100 & $9.58 z-01$ & $1.04618-04$ & $5.881 E+00$ & $9.1598+03$ \\
\hline 1.00 & 3.00 & 200 & $1.06 \mathrm{E}+00$ & $1.1768 \mathrm{E}-04$ & $5.881 E+00$ & $9.046 E+03$ \\
\hline 1.00 & 0.00 & 100 & $9.738-01$ & $1.4375 z-04$ & $5.884 E+00$ & $6.771 E+03$ \\
\hline 2.00 & 0.00 & 200 & $9.28 \mathrm{E}-01$ & $1.4375 E-04$ & $5.884 E+00$ & $6.454 E+03$ \\
\hline 2.00 & 6.00 & 100 & $1.998+00$ & $1.9617 \mathrm{E}-04$ & $5.890 \mathrm{E}+00$ & $9.613 \mathrm{E}+0 \mathrm{3}$ \\
\hline 2.00 & 6.00 & 200 & $1.958+00$ & $1.96178-04$ & $5.880 E+00$ & $9.923 \mathrm{E}+03$ \\
\hline 2.00 & 3.00 & 100 & $1.958+00$ & $2.35348-04$ & $5.081 z+00$ & $8.272 E+03$ \\
\hline 2.00 & 3.00 & 200 & $1.95 E+00$ & $2.4842 E-04$ & $5.881 E+00$ & $7.936 E+03$ \\
\hline 2.00 & 0.00 & 100 & $1.84 E+00$ & $3.00588-04$ & $5.884 \mathrm{E}+00$ & $6.122 E+03$ \\
\hline 2.00 & 0.00 & 200 & $1.92 \mathrm{E}+\infty 0$ & $3.2671 E-04$ & $5.884 E+00$ & $5.8658+03$ \\
\hline 4.00 & 6.00 & 100 & $3.82 \mathrm{~B}+00$ & $4.97018-04$ & $5.879 B+00$ & $7.681 \mathrm{E}+03$ \\
\hline 1.00 & 6.00 & 200 & $3.83 E+00$ & $4.83942-04$ & $5.879 E+00$ & $7.919 \mathrm{E}+03$ \\
\hline 4.00 & 3.00 & 100 & $3.928+00$ & $5.7535 E-04$ & $5.891 z+00$ & $6.635 E+03$ \\
\hline 4.00 & 3.00 & 200 & $3.86 \mathrm{E}+00$ & $5.88422-04$ & $5.8918+00$ & $6.565 E+03$ \\
\hline 4.00 & 0.00 & 100 & $3.761+00$ & $7.58088-04$ & $5.883 E+00$ & $4.955 E+03$ \\
\hline 1.00 & 0.00 & 200 & $3.80 x+00$ & $7.58075-04$ & $5.883 z+00$ & $5.0152+03$ \\
\hline 8.00 & 6.00 & 100 & $7.662+00$ & $1.22981-03$ & $5.877 \mathrm{~B}+\infty 0$ & $6.2328+03$ \\
\hline 8.00 & 6.00 & 200 & $7.65 z+00$ & $1.2299 x-03$ & $5 . \$ 77 z+00$ & $6.2208+03$ \\
\hline 8.00 & 3.00 & 100 & $7.605+00$ & $1.43898-03$ & $5.878 z+00$ & $5.285 E+03$ \\
\hline 8.00 & 3.00 & 200 & $7.54 E+00$ & $1.4389=-03$ & $5.978 z+00$ & $5.2428+03$ \\
\hline 8.00 & 0.00 & 100 & $7.56 \mathrm{E}+00$ & $1.7262 x-03$ & $5.890 z+00$ & $4.379 E+03$ \\
\hline 8.00 & 0.00 & 200 & $7.488+00$ & $1.73948-03$ & $5.880 \mathrm{z}+00$ & $4.302 E+03$ \\
\hline 10.00 & 6.00 & 100 & $9.662+00$ & $1.62365-03$ & $5.8738+00$ & $5.948 E+03$ \\
\hline 10.00 & 6.00 & 200 & $9.602+00$ & $1.63708-03$ & $5.8721+00$ & $5.862 \mathrm{E}+03$ \\
\hline 10.00 & 3.00 & 100 & $9.55 E+00$ & $1.9969 z-03$ & $5.8728+00$ & $5.030 \mathrm{E}+03$ \\
\hline 10.00 & 3.00 & 200 & $9.61 E+00$ & $1.96472-03$ & $5.8712+00$ & $4.892 \mathrm{E}+03$ \\
\hline 10.00 & 0.00 & 100 & $9.848+00$ & $2.27945-03$ & $5.870 z+00$ & $4.317 z+03$ \\
\hline 10.00 & 0.00 & 200 & $9.858+00$ & $2.3325 z-03$ & $5.869 z+00$ & $1.225 E+03$ \\
\hline
\end{tabular}

Figure 3.1 Typical Output Format of Resilient Modulus Test Result 


\subsubsection{Data Acquisition and Processing}

The data acquisition system for the resilient modulus test is composed of a DT 2801 board, RS-232 interface, PCLAB software and resilient modulus testing software. The DT 2801 board contains two 12-bit D/A converters, a 12-bit A/D converter and 16-line digital I/O ports. This board is plugged into a system expansion slot in a personal computer and connected to the controlling modules through the RS-232 interface. PCLAB software consists of libraries of routines supporting analog and digital data transfers for the DT board both into and out of the personal computer, and allows manipulation of the interface board.

The Analog to Digital (A/D) converter changes the analog voltage from transducers into a binary code which is intelligible to the computer. During the resilient modulus test, signals from the load cell and displacement gauge are obtained simultaneously over two channels through the interface board. An on-board programmable clock in the DT board is set to take 2000 sets of data over one cycle duration of the stress pulse.

The Digital to Analog (D/A) converter changes a digital code into a continuously varying analog signal. The binary code corresponding to a desired level of confining pressure generated by test software is converted to an analog signal by the $D / A$ converter.

stress pulses generated by resilient modulus testing software are sent to the waveform generator through a digital I/O port. The waveform generator containing an 18-bit D/A converter changes stress pulses in digital code into analog signals, and sends them to a DC load controller which drives the hydraulic actuator to apply the desired load to the specimen.

Measured loads and displacements are transferred through the A/D converter and are used to calculate the deviator 
stress, resilient strain and resilient modulus. Resilient strain is obtained by dividing the difference between maximum and minimum displacement during a cycle duration by the length of the specimen. Permanent displacement is calculated by subtracting the initial displacement reading at the start of the resilient modulus test from the minimum displacement measured.

Due to the displacement caused during the test, the cross sectional area of the specimen is corrected as follows;

$$
A_{\text {corx }}=\frac{A_{\text {init }}}{1-\frac{d_{\text {peII }}}{L_{\text {In } 1 t}}}
$$

where $A_{\text {corr }}$ and $A_{\text {ink }}$ are the corrected and initial cross-sectional area of specimen, respectively; $d_{\text {perm }}$ is the permanent displacement; $L_{\text {min }}$ is initial specimen length at the start of test. Deviator stress is calculated by dividing the obtained difference between maximum and minimum load with corrected cross-sectional area of specimen. Resilient modulus is, then, obtained as follows;

$$
M_{R}=\frac{\sigma_{d}}{\epsilon_{x}}
$$

where $\sigma_{d}$ is the deviator stress and $\epsilon_{\mathrm{r}}$ is resilient strain.

3.3 Test Procedure for Resilient Modulus

This section explains the procedure for setting up and testing the prepared specimen for determination of resilient modulus. Summarized testing procedure is as follows:

1. Turn on the power of the main console and auxiliary equipment, and wait until all electrical self-checks are passed. Adjust span to 10 , and reduce gain and rate for controllers to minimum value; 
2. Check the full scale setting for each transducer. Full scale setting of transducer should be identical to the scale of the range cartridge. Make sure that the displacement controller is active and adjust the set point control on the displacement controller to make the DC error zero;

3. Turn on hydraulic power. Move the crosshead of loading frame to have enough clearance for loading piston and triaxial cell, and lock the crosshead. Adjust the location of loading piston by rotating set point control in displacement controller;

4. Check the parameters of warm-up waves. A sinusoidal warm-up wave having $10 \mathrm{~mm}$ peak to peak amplitude and $1 \mathrm{~Hz}$ frequency is already programmed and stored in the waveform generator. Run this program, and the loading piston starts to move following sinusoidal action. By running this program until the hydraulic system is warmed up, smoother loading is applied to the specimen during the test;

5. Measure the dimensions and weight of specimen. Place the specimen in the triaxial cell and put the rubber membrane on the specimen. Put o-rings on the membrane and connect the drainage lines to the specimen;

6. Get into the resilient modulus testing program in the PC to prepare the input file. Type in the input parameters following the instructions from the screen;

7. Stop running the warm-up program. Locate the triaxial cell containing the specimen and align the loading ram of triaxial cell and the loading piston connected to the actuator;

8. Adjust the zero control in the force controller to zero the conditioned signal of the load cell. Make the DC error of force controller zero and transfer active control to the force controller. Adjust carefully the set point control in the force controller to make the loading piston contact the loading ram of the triaxial cell, and apply about 2 lbs seating load on the specimen. Couple the loading piston to the loading ram by tightening the screws. Fix the triaxial cell tightly to the supporting deck of the loading frame by clamps. since the system is in force control, the loading piston wili move up if the force transducer feels no compressive seating load. Therefore, it is necessary to monitor the change in magnitude of the seating load;

9. Adjust the zero control in the displacement controller to zero the conditioned signal from LVDT. Connect the air supply line from the confining pressure regulator to the coupling on top of the triaxial cell. Connect the coaxial 
signal cable to the confining pressure transducer, and adjust the zero control in the confining pressure controller to zero its output signal;

10. Install the displacement gage on the supporting block fixed on top of the cell so that the extended sensing arm contacts the concentric bracket attached to the loading piston. Connect the signal cable to the displacement gage. Rotate the height adjustment handle to move the displacement gage to the location giving an output signal near zero. Adjust the zero control in the displacement gage controller to zero its output signal;

11. Activate the interlocks. Switch the mode of Microprofiler to the remote mode to enable the computer to control the testing system;

12. Run the test program to start the resilient modulus test. Follow the instructions in the monitor to verify the status of the system. Once the verification is over, the test procedure is fully controlled by the testing software. Confining pressure is increased to a desired level and the repetitive stress pulse is applied to the specimen;

13. To achieve the desired deviator stress level and to get better response between testing equipment and the specimen, command conditioning units in force controller are adjusted during the test while observing the calculated deviator stress in monitor or by observing the input and output signals of force transducer on the oscilloscope;

14. After the test is over, disconnect the signal cables from transducers and detach the displacement gage from supporting block. Uncouple the air supply line from triaxial cell. Disconnect the triaxial cell from the loading piston. Move up the loading piston slowly by adjusting the set point control in the force controller. Quickly transfer the active control to the displacement controller. Remove the triaxial cell from loading frame.

\subsection{Reliability of Test Result}

\subsubsection{Performance of Testing System}

Since the resilient modulus testing system is a sophisticated one, it is necessary to evaluate the performance of the system by checking the response measured during the test. The haversine waveform of 0.1 second load duration and 
1.0 second cyclic duration was used for the tests presented in this report. Figure 3.2 is the typical plot of measured deviator stress for a single cycle of stress pulse during the test on cohesive soil. It can be seen that the testing system generates the intended waveform almost perfectly.

Figures 3.3 to 3.5 show the deviator stresses, strains, and resilient moduli measured during the conditioning stage on one of the sand specimens prepared by vibratory compaction in the laboratory. It is shown in Figure 3.3 that the testing system maintains the magnitudes of deviator stress closely to the target values throughout the whole conditioning stage. Figure 3.4 is the plot of the variation of total strain, resilient strain and permanent strain during the conditioning stage. It shows that resilient strain remains almost constant while the permanent strain increases continuously. The permanent strain increases at a relatively rapid rate for the first 50 applications of loading after the magnitude of deviator stress is changed, and it approaches a constant value as the number of stress application increases. It appears that the displacement gage used gives rellable measurement of specimen deformation. Figure 3.5 is the plot of resilient moduli calculated from the measured deviator stresses and resilient strains shown in Figure 3.3 and 3.4. It appears that the resilient modulus is consistent during each stage of the test (200 applications of stress pulse).

\subsubsection{Accuracy of Test Result}

Since the 1960's when researchers started to realize the necessity of repeated loading type of test to simulate the actual pavement behavior due to a moving wheel, many resilient modulus test results have been reported. However, there has been no chance to compare the variation of resilient modulus test results between laboratories until a Round Robin test and the SHRP (Strategic Highway Research Project) program were carried out. 


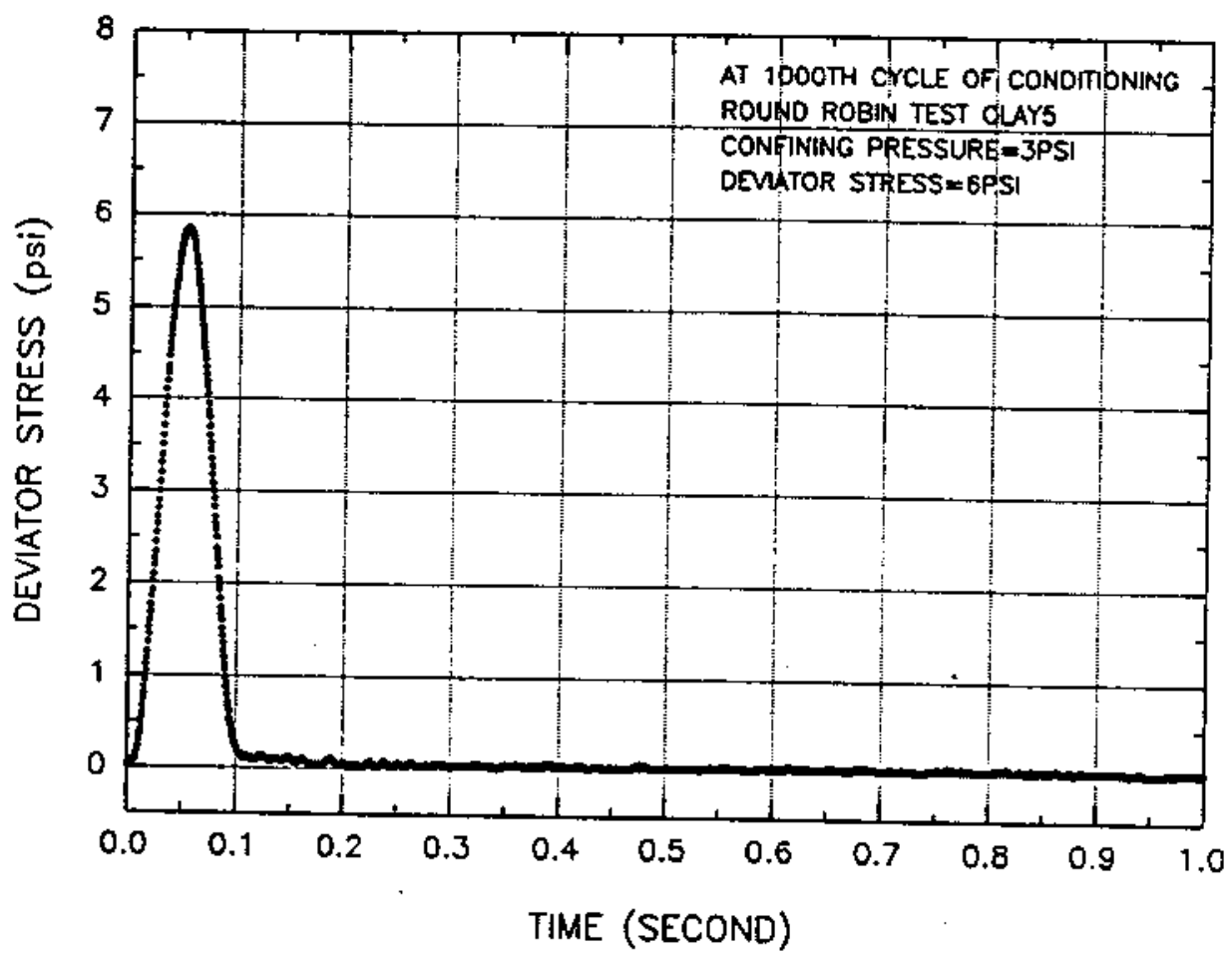

Figure 3.2 $\begin{aligned} & \text { Stress Pulse Measured During Resilient Modulus } \\ & \text { Test }\end{aligned}$ 


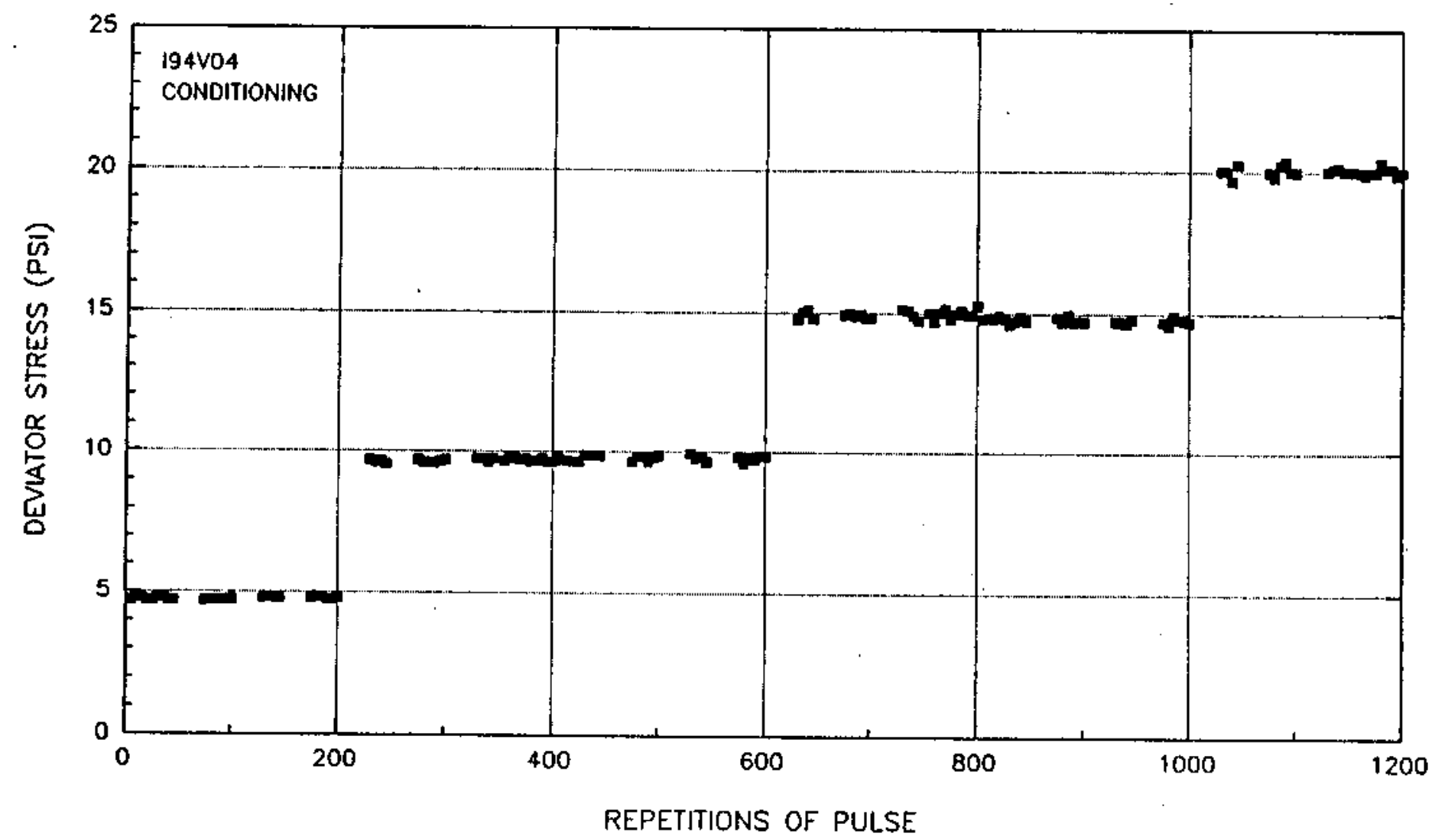

Figure 3.3 Variation of Deviator stress During Conditioning Stage 


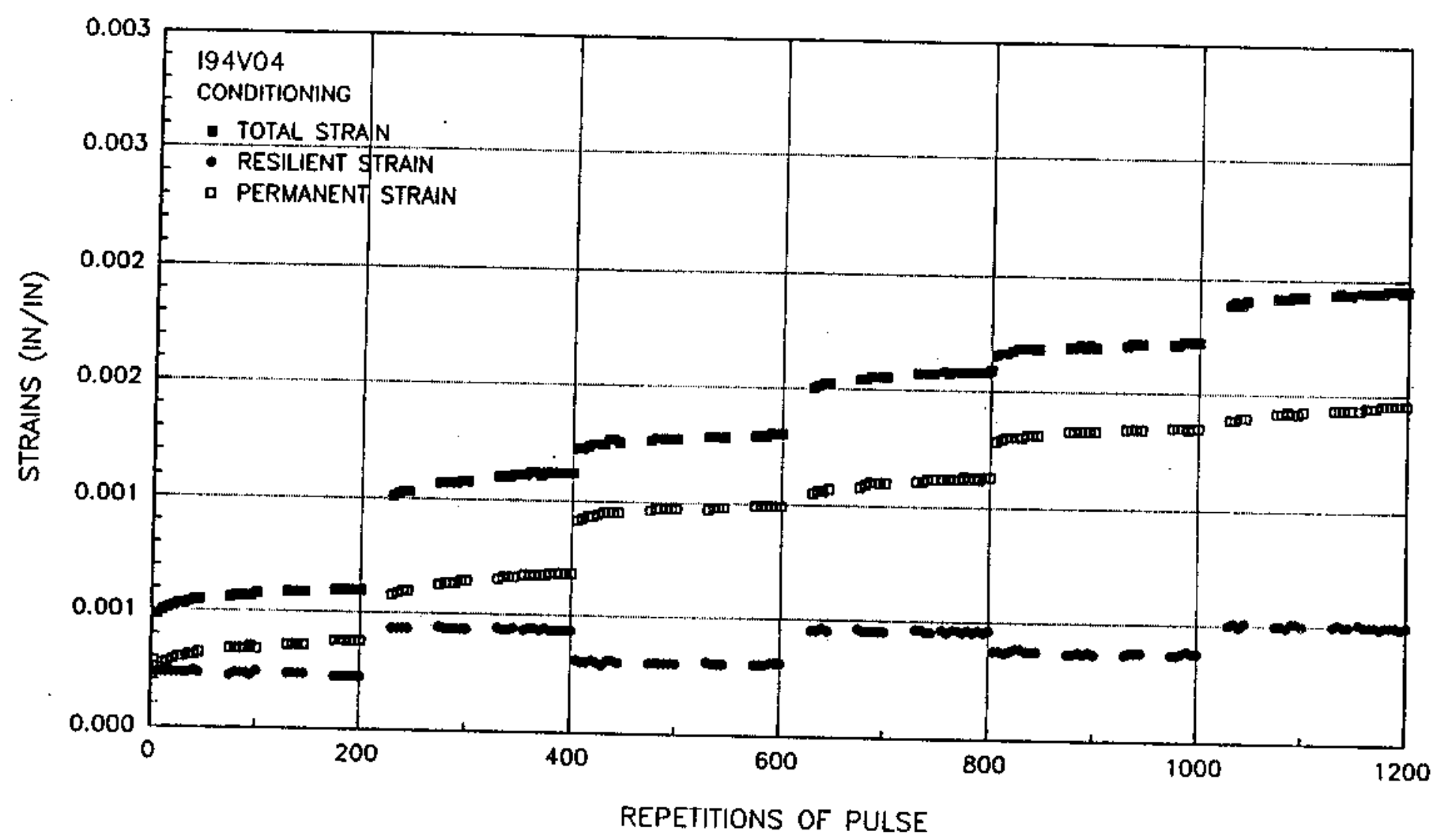

Figure 3.4 Variation of Strains During Conditioning stage 


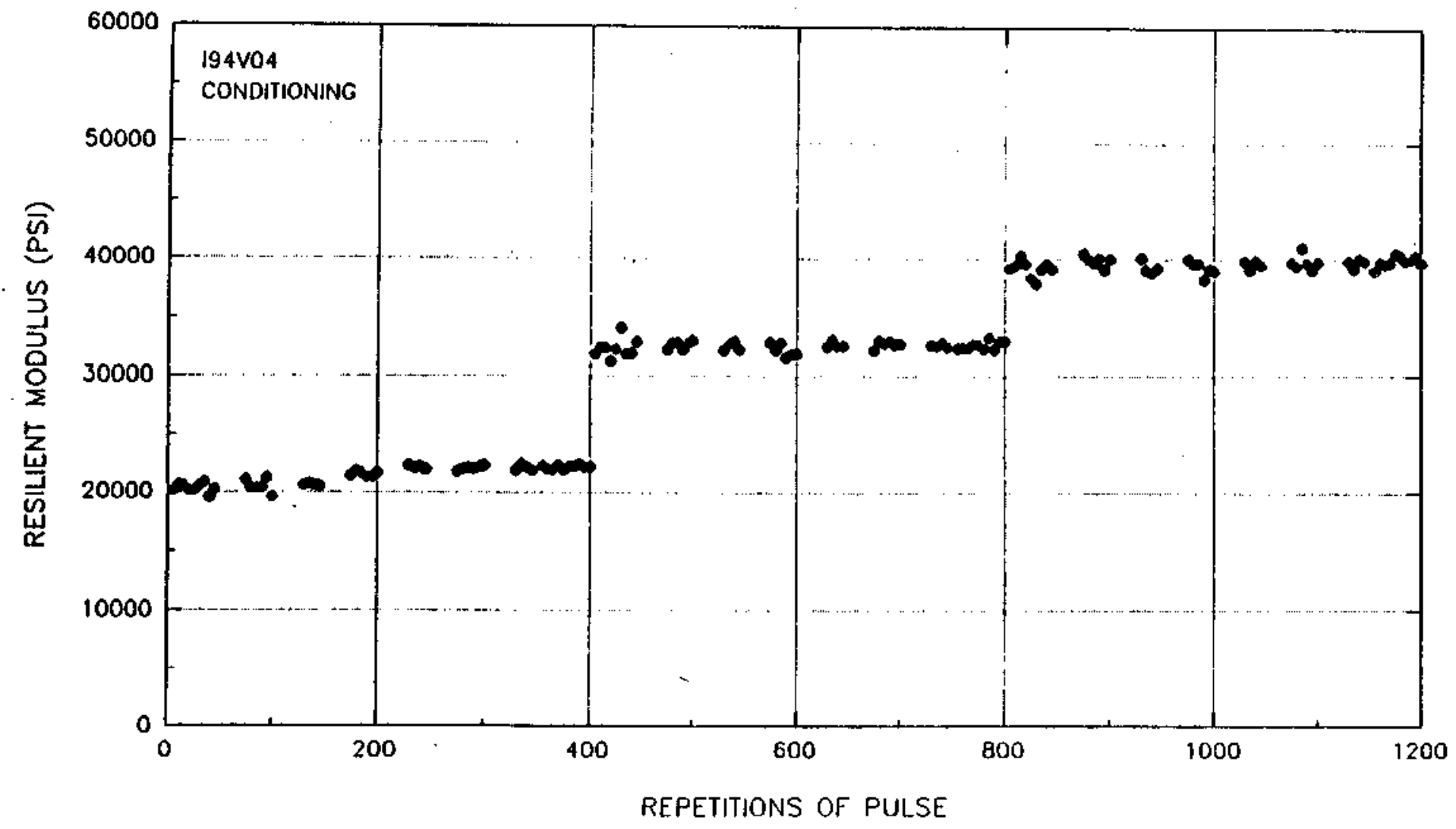

Figure 3.5 Variation of Resilient Modulus During Conditioning stage 
Purdue University participated in the Round Robin test hosted by the New York Department of Transportation. Identical sand and clay soils were distributed to 10 participating laboratories throughout the United states, and resilient modulus tests were performed following a specification provided by the N.Y. DOT. The granular soil tested was a uniform sand from Albany (New York) having an average 3.9 percent finer than $\$ 200$ sieve by weight. Cohesive soil tested was a medium plastic clay with $\mathrm{L} L=38$ percent and $\mathrm{PL}=19.3$ percent. Both materials were compacted in a 4-inch diameter and 8-inch height split mold with Modified Proctor energy. compaction water content for clay and sand were 14 percent and 12 percent, respectively.

Summarized test results (Lenker, 1992) from eight laboratories indicated that the test results on clay show significant variability between laboratories. The test results in the same laboratory show large difference in measured resilient modulus. According to the test results obtained at Purdue University, resilient modulus varies significantly even though the dry density and water content for five compacted clay specimens are almost identical. Large variability of test results on clay soil may be attributed to the quality of compacted specimen. Compaction water content requested by N.X. DOT was about 5 percent lower than the plastic limit of the given soil, and defects between the compaction layers were observed during the test. These defects have a large influence on resilient modulus value since the resilient strain measured for this clay was about $10^{-1}-10^{-5}$ in/in. Therefore, it appears that, in Round Robin test on the clay soil, the quality of compacted specimen has a larger influence on the measured resilient modulus than the performance of the testing system.

on the other hand, resilient modulus test results on the granular soll show far smaller, but still moderate, variation between laboratories and good repeatability within each laboratory. Considering the repeatability of the test results 
in each laboratory, it is reasonable to assume that each laboratory produced almost identical compacted specimens for resilient modulus test. No visible defects was observed in compacted sand specimens. Therefore, the variation between laboratories may be attributed to the performance of testing systems, e.g. test equipment, test control and data acquisition system, calibration of system etc.

From the above discussions, it can be concluded that differences in test results exist between laboratories, and this may be due to the different configuration of testing system and the testing experience. The resilient modulus test result on clay appears to be influenced more by the sample preparation procedure than the performance of the testing system. It is believed that the evaluation of the accuracy of test is difficult at this stage and remains inconclusive.

\subsubsection{Repeatability of Test Result}

Among participating laboratories in the Round Robin test, Purdue University shows the smallest variation in the test results on five compacted sand specimens. For the equation $M_{R}=k_{1} \theta^{k}$ used to fit the test result, coefficients of variation for parameters $k_{1}$ and $k_{2}$ are 4.3 percent and 2.2 percent, respectively. For other laboratories, coefficient of variation for $k_{1}$ ranges from 5.2 percent to 55.7 percent and coefficient of variation of $k_{2}$ from 2.9 percent to 48.9 percent.

Table 3.2 shows the Round Robin test results on the granular soil by Purdue University. As can be seen, water content and dry density are very close for all the specimen. Therefore, all five specimen prepared can be considered as identical specimens. It is noted that the model $M_{R}=k_{1} \theta^{k}$ fits test result perfectly. All the test results are plotted in Figure 3.6 for comparison, and resilient response of specimens are almost identical. Therefore, it can be concluded that the resilient modulus testing system developed shows good repeatability while the accuracy of test is inconclusive. 


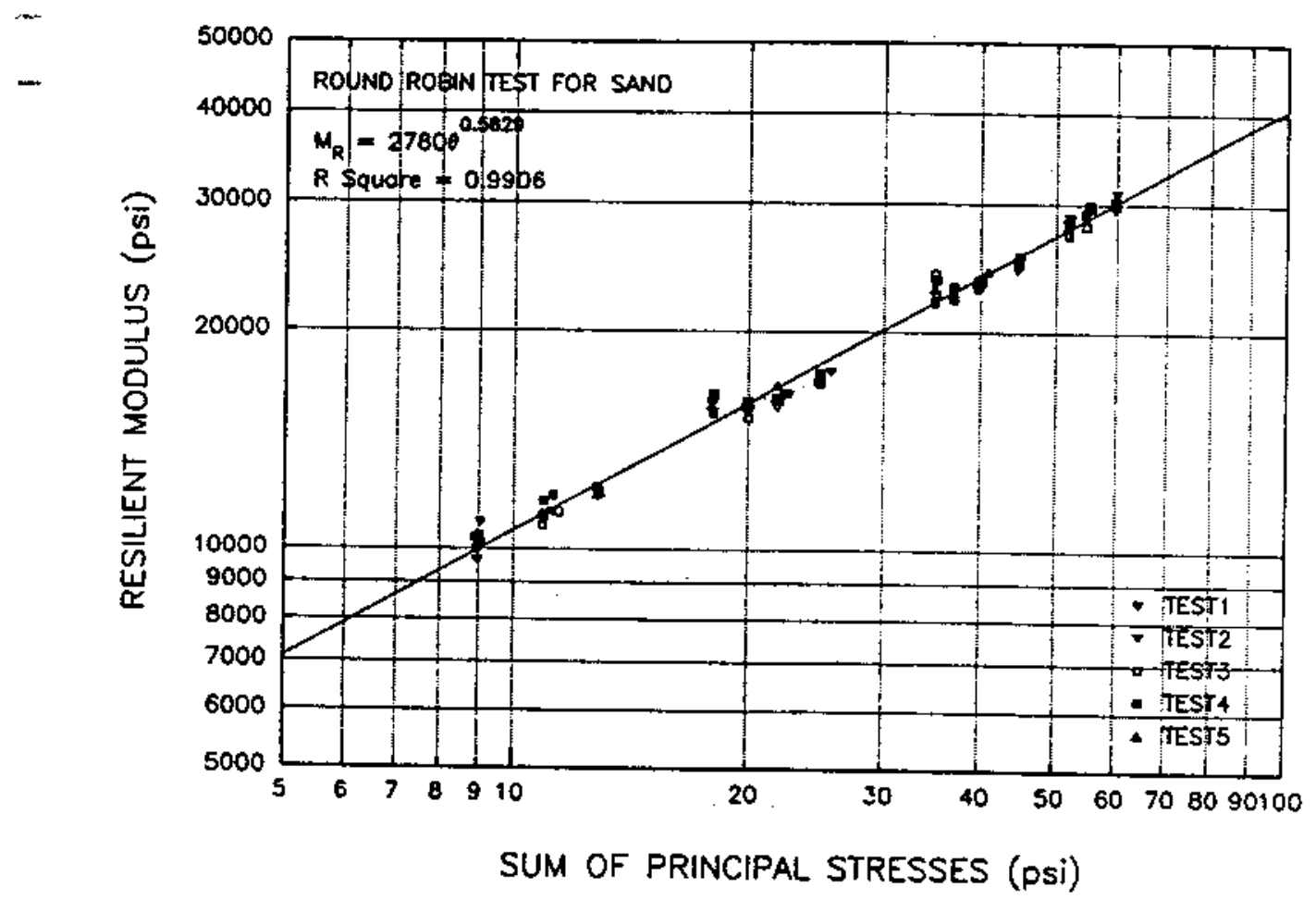

Figure 3.6 Result of Round Robin Test on Granular Soil 
Table 3.2 Result of Round Robin Test on Granular Soil

\begin{tabular}{c|c|c|c|c}
\hline Test ID & W/C (\%) & $\gamma_{d}$ (pcf) & Regr. Eqn. & $R^{2}$ \\
\hline 1 & 10.64 & 111.63 & $M_{R}=2592 \theta^{0.6027}$ & 0.995 \\
\hline 2 & 11.35 & 109.91 & $\mathbf{M}_{R}=2850 \theta^{0.5761}$ & 0.987 \\
\hline 3 & 11.22 & 110.12 & $M_{R}=2774 \theta^{0.5817}$ & 0.987 \\
\hline 4 & 11.33 & 110.64 & $M_{R}=2883 \theta^{0.572}$ & 0.993 \\
\hline 5 & 11.12 & 110.55 & $\mathbf{M}_{R}=2811 \theta^{0.5813}$ & 0.993 \\
\hline
\end{tabular}


4. RESILIENT MODULUS OF COHESIVE SOILS

4.1 Introduction

Resilient modulus is a basic material property that can be included in mechanistic analysis of multilayered systems for predicting behavior of pavement. The 1986 AASHTO Guide for the Design of Pavement structures introduced the resilient modulus as a definitive material property to characterize roadbed soil. Although the incorporation of resilient modulus represents a significant advance in pavement design practice, most highway engineers are not familiar with the resilient modulus and most highway agencies do not have experience with the resilient modulus test.

Therefore, at first, development of proper testing equipment and technique is required. It is also necessary to develop a broad data base on resilient modulus for the soils expected to be encountered in pavement construction. Finally, since the resilient modulus test is a complex and timeconsuming test, it is beneficial to try to develop reliable correlations between resilient properties and the parameters from more simple routine tests.

To develop the data base on the resilient properties of typical clayey subgrade soils in Indiana, undisturbed samples were obtained from five in-service subgrades. Resilient modulus tests on samples prepared by impact compaction were performed to evaluate the relationship between the resilient characteristics of field compacted soils and those of laboratory compacted soils. 
4.2 Factors Affecting Resilient Modulus of Cohesive Soils

4.2.1 Number of Stress Applications

When repetitive loadings are applied, the soil undergoes a plastic deformation plus a recoverable deformation. Many studies on the effects of repeated loading on the plastic deformation of cohesive soils (Brown et al., 1975; Raymond et al., 1979; Seed et al., 1955; 1958; 1960; 1961; 1962; 1967; Monismith et al., 1967; and Grainger and Lister, 1962) have been performed. It was shown that the plastic deformation increases continuously with repetitions of loading as function of stress level, stress history and sensitivity of soil, and frequency and duration of loading.

Seed et al. (1962) and Tanimoto and Nishi (1970) showed that the resilient strain of laboratory compacted soil on the wet side of the optimum water content varies markedly at the first several thousands of stress repetitions and then decreases gradually; soll compacted on the dry side shows relatively small variation in resilient strain. Tanimoto and Nishi (1970) also showed that changes of resilient strains of undisturbed specimens obtained from in-service subgrade are similar to those of specimens compacted wet of optimum in the laboratory; the variation of resilient strains of undisturbed specimens is comparatively small in spite of the higher water content. Monismith et al. (1967) reported test results on natural subgrade soil that indicated resilient strains tend to be stable or decrease slightly with repeated loading up to approximately 1000 applications and then remain constant or increase slightly.

\subsubsection{Thixotropy}

It was shown by Mitchell (1960) and seed and Chan (1957) that samples compacted to a high degree of saturation exhibit 
a significant increase in strength if they are allowed to rest before tested. similar effects were observed by seed et al. (1962) in the studies of the resilient characteristics of AASHO Test Road subgrade soil prepared by kneading compaction. The effect of thixotropy on resilient strains varies with the number of stress applications but a marked reduction in total deformation due to thixotropic strength gain is apparent. Up to 40,000 repetitions, an increase in storage time reduces the resilient strain, but for larger numbers of stress repetitions the resilient deformations are not significantly affected by thixotropy. This is probably due to the fact that the deformations induced by the repeated load progressively destroy the thixotropic strength gain in aged samples.

Tanimoto and Nishi (1970) showed that the storage period has little effect on the resilient characteristics of the sample compacted about 4 percent dry of optimum while the storage time causes a large change in the resilient characteristics of specimens compacted wet of optimum.

\subsubsection{Level of Stresses}

The resilient modulus of cohesive soil is significantly affected by the magnitude of the deviator stress and confining stress. Several studies (Seed et al., 1962; Monismith et al., 1967; Thompson and Robnett, 1979; Tanimoto and Nishi, 1970; Brown et al., 1975) have shown that, at low levels of repeated deviator stress, the resilient modulus decreases rapidly with increasing deviator stress; the rate of decrease is smaller at higher deviator stresses.

A simple expression relating resilient modulus to the deviator stress has been widely used:

$$
M_{R}=k_{1} \sigma_{d} k_{1}
$$

where $k_{1}$ and $k_{2}$ are material constants determined from test. It 
is noted that this equation does not include the confining stress.

Brown et al. (1975) studied the effect of overconsolidation ratio on the resilient characteristics of reconstituted Keuper marl, and concluded that the resilient modulus is a function of initial effective confining stress together with the cyclic deviator stress. By normalizing the deviator stress with respect to the initial stress state of the sample, a unique relationship was obtained as follows:

$$
M_{R}=\frac{K}{\left(\frac{\sigma_{d}}{\sigma_{3}{ }^{\prime}}\right)^{n}}
$$

where $\sigma_{3}{ }^{\prime}$ is initial effective confining stress; $\sigma_{d}$ is the deviator stress; and $\mathrm{K}$ and $\mathrm{n}$ are material parameters.

In an extensive study on the Illinois soils prepared by kneading compaction, Robnett and Thompson (1976) found that the stress dependency of resilient modulus could be adequately characterized as two intersecting straight lines in the plot between resilient modulus and repeated deviator stress. The deviator stress at the point of intersection was approximately 6 psi and was defined as the break-point deviator stress, $\sigma_{\text {di }}$. The resilient modulus at $\sigma_{d i}$ was defined as the break-point resilient modulus, $\mathrm{M}_{\mathrm{n}}$.

From tests on Leda clay, Raymond et al. (1979) showed a linear relationship between resilient modulus and stress difference factor at $10^{5}$ cycles, which is expressed as

$$
M_{R}=10^{(1.60-1.37 x)}
$$

where $x$ is a stress difference factor defined as the repeated deviator stress divided by the axial deviator stress that caused failure in a static drained test at the same confining pressure; $\mathrm{M}_{R}$ is resilient modulus in $\mathrm{MPa}$.

Drumm et al. (1990) conducted repeated-load triaxial tests on laboratory compacted specimens from 11 Tennessee 
soils. The relationship between resilient modulus and deviator stress was expressed as an hyperbolic relation:

$$
M_{R}=\frac{a^{\prime}+b^{\prime} \sigma_{d}}{\sigma_{d}}
$$

where $a^{\prime}$ and $b^{\prime}$ are functions of parameters from the unconfined compression test, percent clay, Atterberg limits, dry unit weight, degree of saturation, and percent finer than $\$ 200$ sieve.

\subsubsection{Matrix suction}

Dehlen (1969) and Finn et al. (1972) showed that a linear relationship exists between the resilient modulus measured by laboratory repeated-load tests and soil moisture suction. It was also shown that laboratory prepared specimens exhibit the same stiffness characteristics as field compacted specimens when specimens are compared at the same values of suction.

Frediund et al. (1975) proposed from a stress analysis standpoint that the resilient modulus is a function of three stress variables;

$$
M_{R}=f\left[\left(\sigma_{3}-u_{a}\right),\left(\sigma_{1}-\sigma_{3}\right),\left(u_{a}-u_{w}\right)\right]
$$

where $M_{R}$ is the resilient modulus; $\sigma_{3}$ is the confining stress; $\sigma_{1}$ is the major principal stress; $u_{2}$ is the pore air pressure; $u_{w}$ is the pore water pressure; $\left(\sigma_{3}-u_{1}\right)$ is the net confining stress; $\left(\sigma_{1}-\sigma_{3}\right)$ is the deviator stress, and $\left(u_{n}-u_{w}\right)$ is the matrix suction. The resilient modulus tests were performed on glacial till compacted statically in the laboratory, and the following equation was proposed:

$$
\log _{R}=C_{1 d^{-}}-m_{1 d}\left(\sigma_{1}-\sigma_{3}\right)
$$

where $c_{1 d}$ and $m_{1 d}$ are the intercept and slope of the semilog plot, respectively. The slopes and intercepts of the semilog 
plots of resilient modulus versus deviator stress were, then, expressed as a function of matrix suction. A family of curves of resilient modulus versus matrix suction was obtained. To correct the resilient modulus for different levels of confining stress, the following equation was suggested:

$$
\Delta M_{R}=m_{c} \Delta\left(\sigma_{3}-u_{a}\right)
$$

where $\Delta \mathrm{M}_{\mathrm{R}}$ is the change in resilient modulus, $\mathrm{m}_{c}$ is slope of the plot for confining stress versus resilient modulus, and $\Delta\left(\sigma_{3}-u_{2}\right)$ is the change in confining stress from that used to define the plot for resilient modulus versus deviator stress.

\subsubsection{Method of Compaction}

Previous studies of compacted clays showed that the method of compaction has a profound effect on soil structure and strength characteristics, and that the molding water content and the change of water content after compaction have significant effects on the strength characteristics of compacted clay. Lambe (1958) stated, based on colloidal theory, that compaction of clay soil at dry of optimum tends to produce a flocculated arrangement of clay particles, while compaction of a same soil at wet of optimum tends to produce a dispersed arrangement of particles. Considering the effect of shear strain induced during compaction, seed and chan (1959) concluded that for samples compacted to low degrees of saturation, the method of compaction has little effect on the structure and properties of soil while for samples compacted to high degrees of saturation, variations in method of compaction cause large changes in structure and properties. Lines of optimum on a compaction plot were recommended as a boundary line between high and low degrees of saturation. Generally, it has been known that the kneading and impact methods produce a flocculent structure dry of optimum and a dispersed structure at wet of optimum, while static compaction 
tends to produce the flocculated structure at any molding water content.

Seed et al. (1962) suggested that the foregoing conclusions were also applicable to the resilient characteristics of compacted clays. From the tests on samples from the AASHO test road subgrade prepared by kneading and static compaction, it was shown that resilient strains of samples compacted at water content below optimum were about the same for both methods of compaction; the resilient strain of the kneading compacted specimens was markedly larger than that of the static compacted specimens when the compaction water content was above optimum.

Elliott and Thornton (1988) reported test results on five Arkansas cohesive soils compacted using both kneading and static methods. Two of the five soils tested were found to be affected by compaction method and the other soils showed virtually the same resilient response. For soils affected by the compaction method, the resilient modulus of that prepared by static compaction was higher than that by kneading compaction for the entire range of water content.

\subsubsection{Compaction Water Content and Dry Density}

The influence of compaction water content and density on the resilient characteristics of subgrade soils is important since the placement of compacted fill is specified and controlled by these factors.

Seed et al. (1962) and Tanimoto and Nishi (1970) showed that resilient strains were relatively small for specimens prepared dry of optimum and increased rapidly as the water content exceeded the optimum. Seed et al. (1962) and Monismith et al. (1967) developed contours of resilient strain as functions of water content and dry density which could be used to determine the influence of compaction conditions on ascompacted resilient strains. It was shown that, at high degree 
of saturation, small changes in water content and density caused large change of resilient strain. The desirability of compacting soil to low degrees of saturation in order to minimize resilient strain was also apparent.

Thompson and Robnett (1979) showed that the resilient modulus decreased as the degree of saturation increased. The relationship between resilient modulus and the degree of saturation was different for $95 \%$ and $100 \%$ compaction relative to maximum density by standard Proctor compaction, and the difference was reduced at increased degrees of saturation. Regression equations obtained were:

$$
\begin{aligned}
& M_{x i}=45.2-0.428 S_{x} \\
& M_{x i}=32.9-0.334 S_{x}
\end{aligned}
$$

where $M_{n i}$ is break-point resillent modulus in $k s i$, and $s_{r}$ is degree of saturation (as-compacted) in percent. The first equation is for $100 \%$ relative compaction and the second is for 958 relative compaction.

\subsubsection{Stabilization}

From chemical stabilization studies, lime has been the only additive studied relative to the resilient behavior of cohesive subgrade soil. The addition of lime to a fine-grained -soil initiates several reactions. Cation exchange and agglomeration-flocculation reactions cause immediate improvement in soil plasticity, workability, uncured strength, and volume change. Plasticity and swell are reduced and workabillty is substantially improved because of the low plasticity and the friable nature of the mixture. Depending on the characteristics of the soll being stabilized, a soil-lime pozzolanic reaction may also occur to form various cementing agents that increase compacted mixture strength and durability. 
Robnett and Thompson (1976) tested Illinois soils treated with lime and compacted in the laboratory, and showed that resilient response of uncured lime-treated soil was substantially different from the response of untreated soil. It was also found that the detrimental effects of freeze-thaw cycles on resilient behavior appeared to be minimized or eliminated when the soils were treated with 5 percent calciumhydrated lime. The resilient modull of untreated soils ranged from 3000 to $6000 \mathrm{psi}$ after freeze-thaw cycles while those of lime-treated soils ranged from 14000 to 20000 psi after 10 freeze-that cycles, showing the remarkable strengthening effect of lime treatment.

4.3 Resilient Modulus Test on Field Compacted cohesive Soils 4.3.1 Sampling of soils

Typical cohesive soils in Indlana were obtained from five sites. Soils sampled are classified as $C L$ and $C H$ in the Unified Soil classification System, and as A-4, A-6, A-7-5, and A-7-6 in the AASHTO classification as shown in Figure 2.1 . Information on sampling sites is given in Table 2.1 .

The pavement surface was cored by a 12-inch diameter coring machine, and gravels in the base and subbase layer vere removed by a hollow-stem auger to expose the top of subgrade. After removing the debris at the bottom of hole, a shelby tube lubed with silicone oll was pushed slowly by a drill rig. The Shelby tube was usually pushed 24 inches into the subgrade, unless it hit gravels, and was left for about two to three minutes to allow the soil to stick to the tube. Then, the tube was rotated slowly 180 degrees and lifted from the hole. If the sample was not recovered with the lubed tube, an unlubed tube was used for next sampling.

Tubes containing soils were sealed with a plastic cap, packer and sealing tape immediately after they were removed 
from the drilled hole. Each tube was marked with location and depth for identification. Sealed tubes were brought and stored in an environmental chamber controlled to a temperature of $10^{\circ} \mathrm{C}$ and an humidity of 95 percent.

It was shown in Figures 2.2 to 2.8 that the degree of saturation increases after construction and approached 90 percent to 100 percent as the service period increased after construction. Field compaction appears to match laboratory compaction with the compactive effort equal to or less than standard Proctor energy. Some of the data implies that the Iine of optimums for field compaction is different from that of laboratory compaction.

\subsubsection{Laboratory Experimental Program}

The laboratory experimental program consists of unconfined compression tests, repeated-loading triaxial test for resilient modulus, mercury intrusion porosimetry, and conventional index tests. Since specimens from the field are not identical, unlike the specimens compacted in the laboratory, all the tests mentioned above need to be performed on the same specimen. Detailed information on each specimen used for laboratory tests is given in Appendix A.

Since the soils in the shelby tube stick to the tube wall during storage, it is difficult to extrude the soil from the tube without disturbance. Soils compacted in the field were observed to have invisible cracks at compacted lift boundaries approximately every 6 inches. Therefore, each specimen was prepared for testing by cutting the tube longitudinally and splitting it to remove the soil from the tube. All specimens were trimmed to have heights of 5.8 to 6.1 inches. The periphery of specimen was not trimmed and the diameter ranged from 2.85 to 2.88 inches. Several sizes of gravel typically less than $10 \mathrm{~mm}$ in diameter were found in the Fort wayne and South Bend soils. Because these gravels were insignificant by 
weight and were imbedded in a cohesive soil matrix in specimens, it is believed that they did not affect the laboratory test results appreciably.

After measuring dimensions and weight of specimen, and putting on the rubber membrane, an unconfined compression test was carried out up to 0.06 -inch deformation with 1 percent strain per minute $(0.001$ inch/second). Since the resilient modulus test has a conditioning stage and the deformation during the unconfined compression test is only $0.06 \mathrm{inch}$, performing the unconfined compression test before the resilient modulus test is not believed to have a significant effect on the resilient modulus.

After the unconfined compression test, the resilient modulus test was conducted on the same specimen, following the procedure described in AASHTO $T$ 274-82. The haversine stress pulse with a load duxation of 0.1 second and a cycle duration of 1 second was used to apply the repeated load to specimen. After the resilient modulus test was completed, specimens for the mercury intrusion porosimetry were cut with a sharp razor blade. The rest of the specimen was used for the measurement of moisture content and for Atterberg Limits tests.

\subsubsection{Reduction of Test Results}

\subsubsection{Unconfined Compression Test}

Kondner (1963) and Duncan and Chang (1970) have shown that the nonlinear stress-strain relationship of soil can be approximated by an hyperbola. The hyperbolic equation proposed by them was;

$$
o_{d}=\frac{\epsilon}{a+b \epsilon}
$$

in which $\sigma_{d}$ is deviator stress; $\epsilon$ is axial strain; and a and b are constants whose values are determined experimentally. 
The above equation can be rewritten in the following form:

$$
\frac{\epsilon}{\sigma_{d}} \times a+b \epsilon
$$

where $a$ and $b$ are, respectively, the intercept and the slope of the transformed hyperbolic stress-strain curve. By plotting stress-strain data on transformed axes, the parameters $a$ and $b$ corresponding to the best fit of the test data are easily determined. Both of these constants $a$ and $b$ have visualized physical meanings. Parameter $a$ is the reciprocal of the initial tangent modulus, $E_{i}$, and $b$ is the reciprocal of the asymptotic value of deviator stress which the stress-strain curve approaches at infinite strain, $\sigma_{d \text { uk }}$. Typical plots of stress-strain relationship and transformed hyperbolic stressstrain curve of unconfined compression tests are shown in Figure 4.1.

since the level of stress applied to the specimen during repeated loading triaxial test for cohesive soils is less than $10 \mathrm{psi}$ and the deformation by dynamic loading is significantly smaller than that by static loading (Seed et al., 1962), it seems reasonable to characterize the resilient behavior by the parameters from the stress-strain relationship in unconfined compression test for strains less than 1 percent. Stresses at different strain levels and secant moduli at different stress levels were calculated to develop the empirical correlations -between resilient modulus and parameters from the unconfined compression test. Parameters obtained from the unconfined compression test are;

(1) Stresses at $0.25,0.5,0.75$, and 1.0 axial strain

$$
\left(S_{\infty 0.25 *}, S_{\infty 0.5 *}, S_{\infty 0.75 *}, S_{w 1.0 x}\right)
$$

(2) Secant modulus at 2, 4, 6, and 10 psi

$$
\text { ( } \left.E_{2 p \text { pir }}, E_{\text {ppoi, }} E_{\text {oppi }}, E_{10 \text { pai }}\right)
$$

(3) Initial tangent modulus and asymptotic deviator stress $\left(E_{\mathrm{j}}, 1 / \mathrm{b}\right)$

Summaries of test results of unconfined compression test for each site are given in Appendix $B$. 

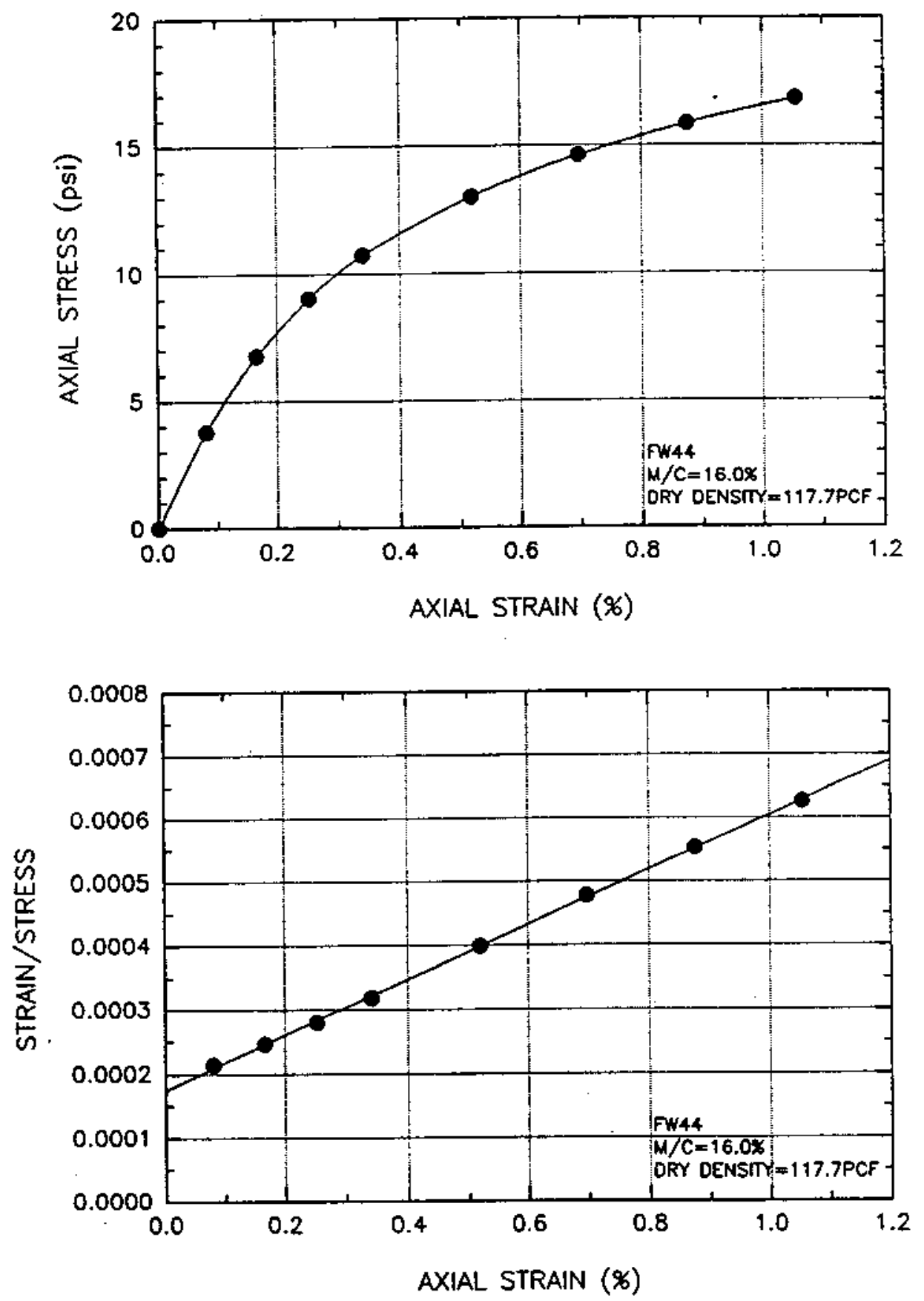

Figure 4.1 Stress-Strain Relation and Transformed Hyperbolic Relation of Unconfined Compression Test 
4.3.3.2 Resilient Modulus Test

The resilient modulus test for cohesive soil consists of repeated applications of the striess pulse, changing the magnitude of deviator stress and confining stress every 200 cycles. A typical result of the test is plotted as three curves corresponding to different confining stresses, as shown in Figure 4.2. Also shown is the relationship between deviator stress and resilient strain.

To model the relationship between the resilient modulus and deviator stress, the following equation is used;

$$
M_{R}=A \sigma_{d}^{B}
$$

where $\sigma_{1}$ is repeated deviator stress; $A$ and $B$ are constants determined experimentally. Three sets of $A$ and $B$ corresponding to each confining stress level are obtained by linear regression after taking the logarithm of $\mathrm{M}_{\mathrm{R}}$.

Thompson and Robnett (1979) suggested that the breakpoint resilient modulus, $M_{i i}$ obtained from the bilinear representation of the resilient modulus versus deviator stress is a representative modulus which can be used for correlation purposes. They used this single modulus to characterize the entire $M_{R}$ versus $\sigma_{d}$ response and recommended $M_{i}$ as a resilient modulus at a deviator stress of $6 \mathrm{psi}$ for practical purpose. since they performed repeated-load tests without confining stress, the breakpoint resilient modulus is at $\sigma_{3}$ of 0 psi. In present study, the resilient modulus at a deviator stress of 6 psi and at a confining stress of $3 \mathrm{psi}$ is used as a representative.

since the resilient modulus versus deviator stress response varies with the confining pressure, the equation suggested by Janbu (1963) to describe the relationship between initial tangent modulus and confining pressure is adopted. However, this expression can not consider the modulus at zero confinement. Therefore, the equation by Janbu (1963) is modifled as; 

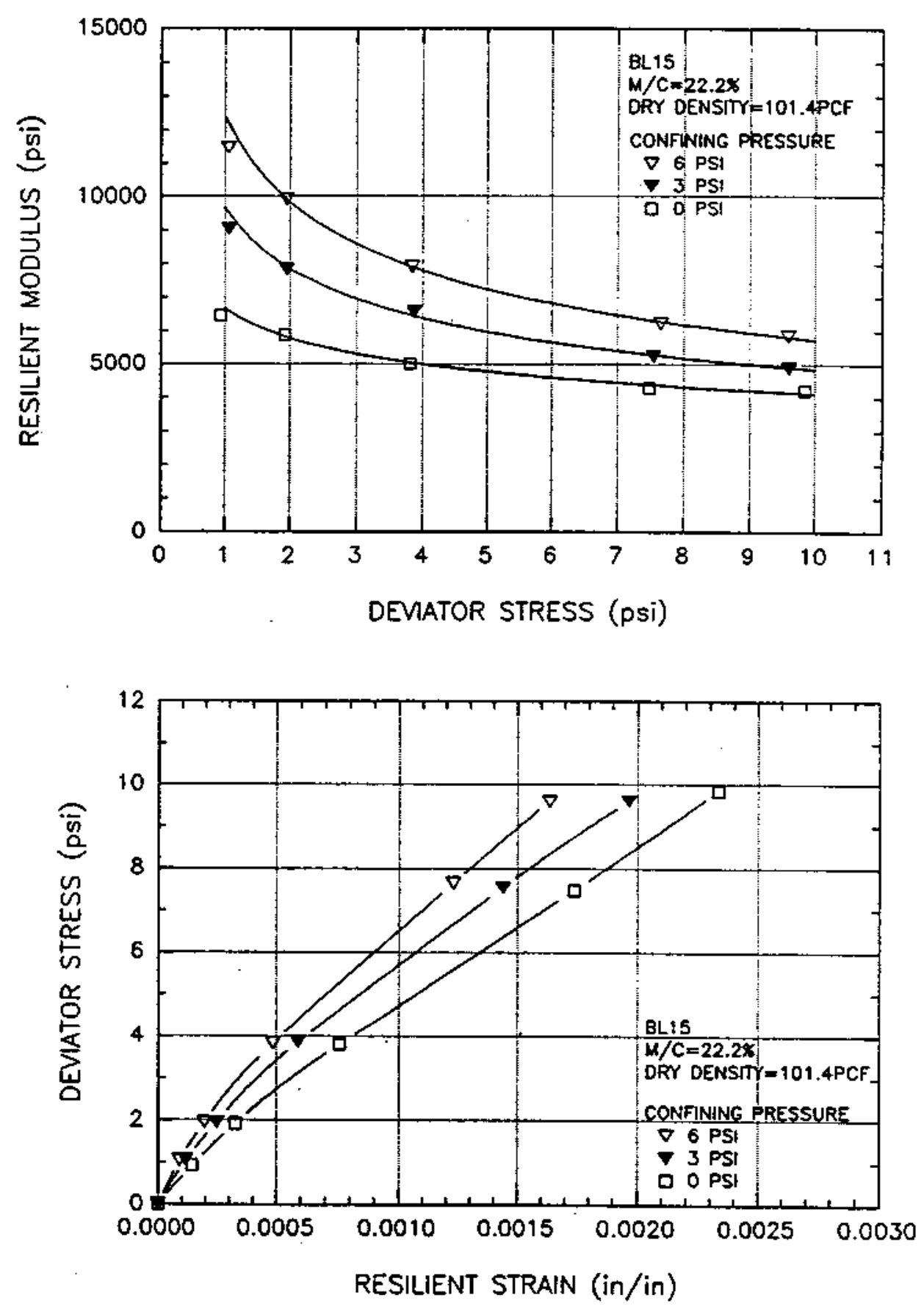

Figure 4.2 Typical Result of Resilient Modulus Test on Cohesive Soll 


$$
M_{r i}=k\left(\frac{P_{\mathrm{a}}+\sigma_{3}}{P_{a}}\right)^{D}
$$

where $M_{n}$ is resilient modulus at deviator stress of $6 \mathrm{psi} ; \sigma_{3}$ is confining stress; $P$, is atmospheric pressure expressed in the same unit as $\sigma_{3}(14.696 \mathrm{psi}) ; \mathrm{k}$ is a modulus number which is equal to the resilient modulus at a deviator stress of 6 psi and at zero confining pressure; and $n$ is the exponent determining the rate of variation of $M_{i}$ with confining stress. values of $k$ and $n$ are determined by plotting the values of $M_{r i}$ versus $\sigma_{3}$ on $\log -\log$ scales and fitting straight line on the data points. Reduced data on resilient modulus test are presented in Appendix $c$.

\subsubsection{Test Results and Discussions}

Variations of resilient moduli with depth are presented in Figures from 4.3 to 4.7, with in-service moisture content, dry density, and Atterberg Limits. The resilient modulus plotted is the modulus at $\sigma_{3}$ of $3 \mathrm{psi}$ and $\sigma_{\mathrm{d}}$ of $6 \mathrm{psi}$.

In-service moisture content at the South Bend site ranges from 8.62 to 13.42 percent, and dry density from 124.28 pcf to 132.65 pcf. Moisture contents are approximately 2 or 3 percent lower than Plastic Limits. The south Bend site shows the largest scatter in resilient modulus among the five sites investigated. Resilient modulus ranges from 7500 psi to 40000 psi with an average value of about $22000 \mathrm{psi}$. As shown in Figure 4.3, the resilient modulus varies about $13000 \mathrm{psi}$ at a depth of 20 to 30 inch, while the changes of water content and dry density with depth are not significant. To look at the relationship of resilient modulus with in-service water content and dry density, resilient moduli are plotted in Figure 4.4. Even though large scatters exist in the plots, it appears that the resilient modulus decreases with water content and increases with increasing dry density. 


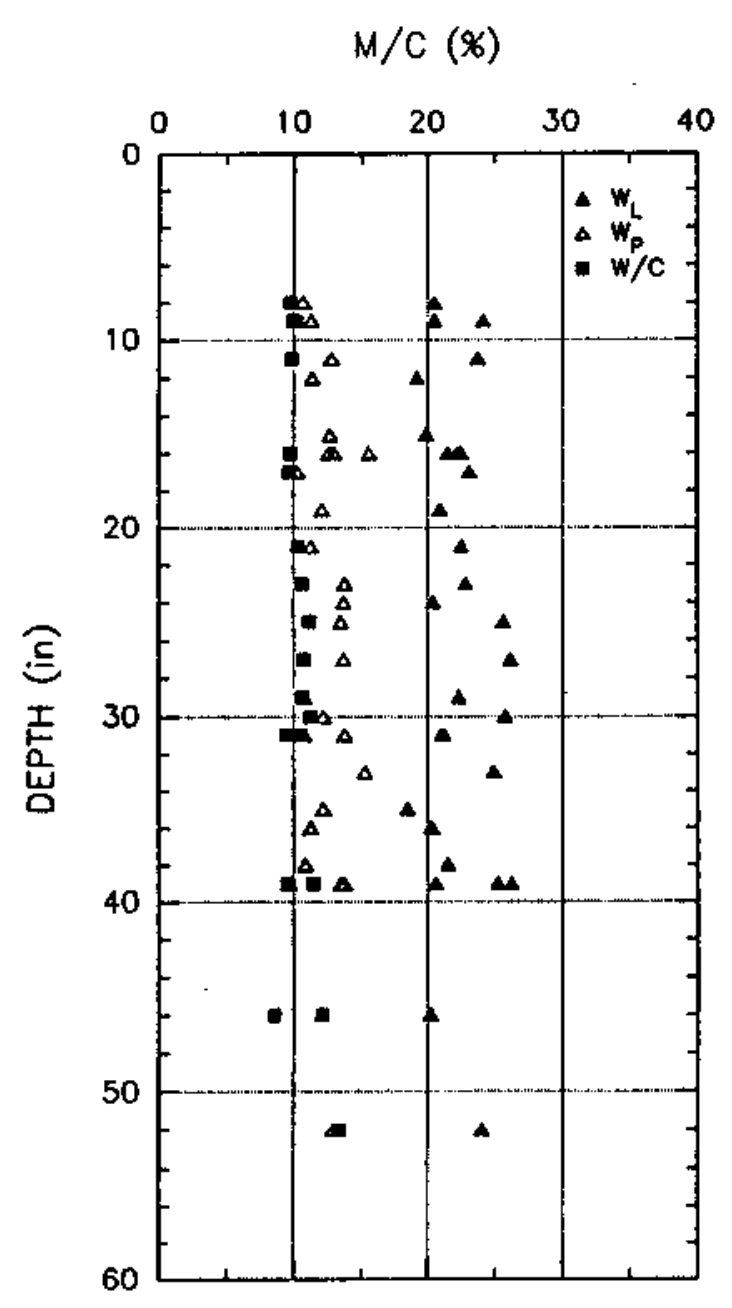

DRY UNIT WEIGHT (pcf)

RESILIENT MODULUS (psi)
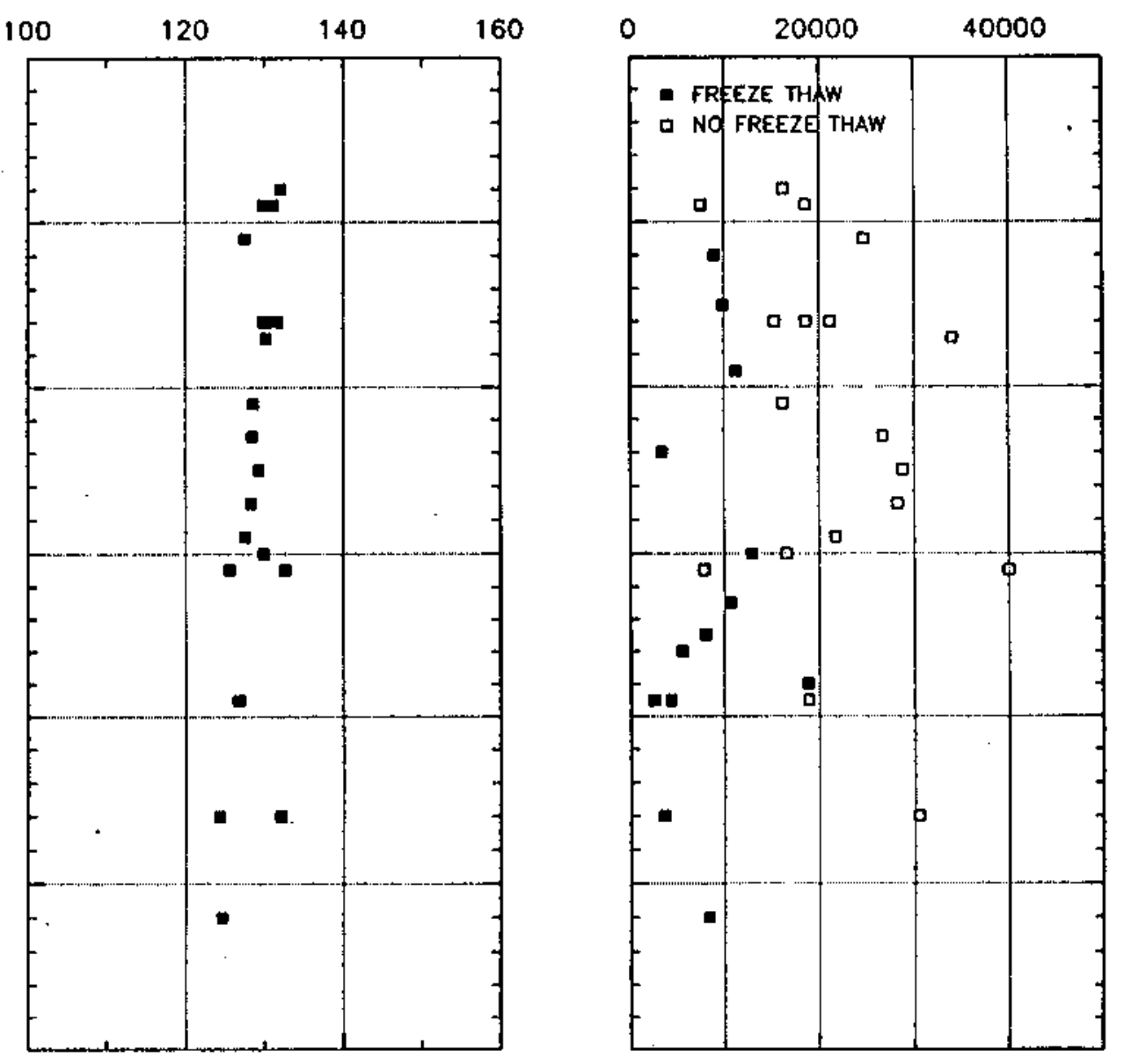

Figure 4.3 Profile of Resilient Modulus with Depth for the South Bend Site 

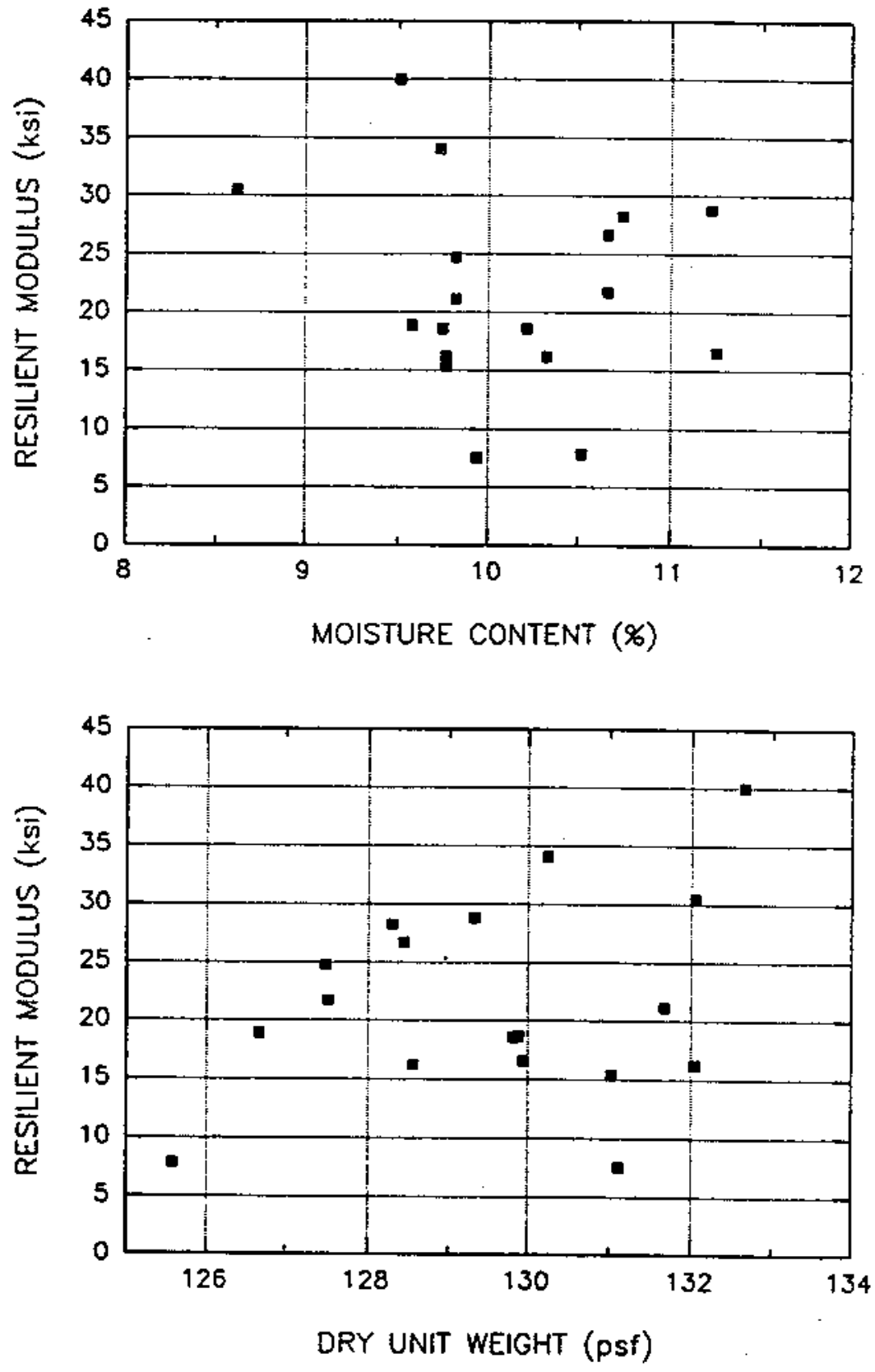

Figure 4.4 Relationship of $M_{R}$ with $w / c$ and $\gamma_{d}$ for the south Bend Soil 
For the Fort Wayne site, the in-service moisture content ranges from 9.74 to 28.26 percent and the average is slightly lower than the Plastic Limits. Dry density ranges from 97.56 pcf to 129.45 pcf with an average of about 114 pcf. As shown in Figure 4.5, this site also shows large scatter in resilient modulus ranging from $2200 \mathrm{psi}$ to $24000 \mathrm{psi}$. The average value of resilient modulus is about $12000 \mathrm{psi}$. To search for the indicators of $M_{R}$, Figure 4.6 shows plots with the water content, dry density, initial tangent modulus and stress causing 1 percent axial strain in unconfined compression test. It can be seen that $\mathrm{E}_{\mathrm{i}}$ and $s_{\mathbf{1 . 0 \times}}$ give a good correlation with resilient modulus while water content and dry density are moderately correlated, but with large scatter. This implies that $S_{u 1.0 x}$ and $E_{i}$ can be used as good indicators of $M_{R}$.

The in-service moisture at the washington site ranges from 17.14 to 24.35 percent and the dry density from 101.33 pcf to 115.28 pcf. Plastic limits are almost the same as the water content. Resilient modulus at this site ranges from 3800 psi to 11000 psi with an average value of about 8000 psi. It is shown in Figure 4.8 that moisture content and dry density give no indication on the magnitude of resilient modulus while $E_{i}$ and $S_{t_{1.0 x}}$ give excellent relationships.

As shown in Figure 4.9, in-service water content at the Bedford site ranges from 18.65 to 37.93 percent and dry density from 81.76 pcf to 108.19 pcf. Average water content is slightly higher than Plastic Limits. Resilient modulus varies between $2300 \mathrm{psi}$ and $13000 \mathrm{psi}$ with an average of $5500 \mathrm{psi}$. This site also shows $S_{\text {u. } .0 x}$ as a good indicator of $\mathbf{M}_{R}$.

The in-service moisture content at the Bloomington site is significantly larger than Plastic Limits and the average value of resilient modulus is the smallest among the sites tested. Water content ranges from 19.30 to 38.14 percent and the dry density from 86.26 pcf to 107.0 pcf. Resilient modulus varies between $3700 \mathrm{psi}$ and $8000 \mathrm{psi}$, with an average value of $5500 \mathrm{psi}$. Since the resilient modulus of this site varies in 

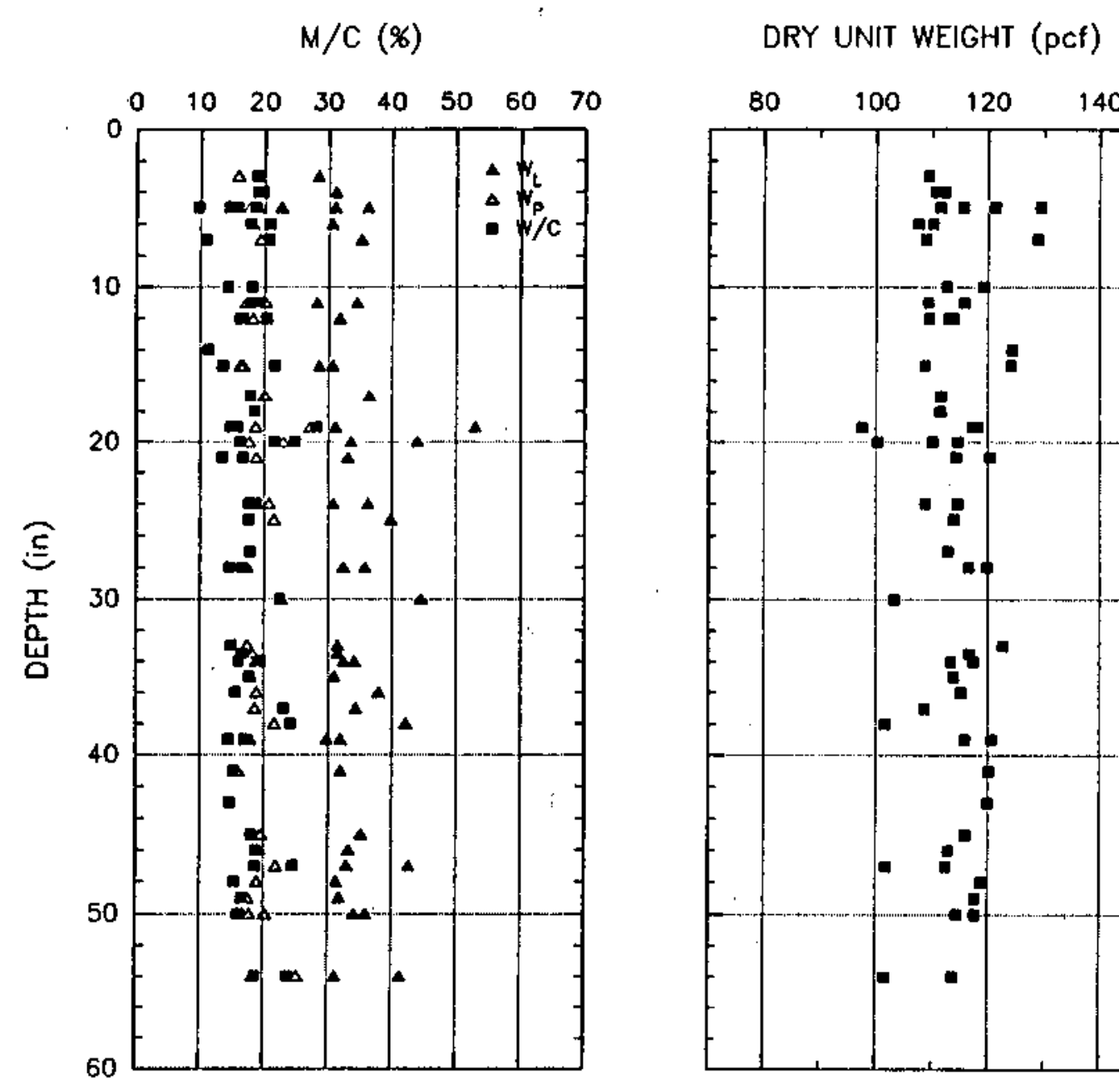

\section{RESILIENT MODULUS (psi)}
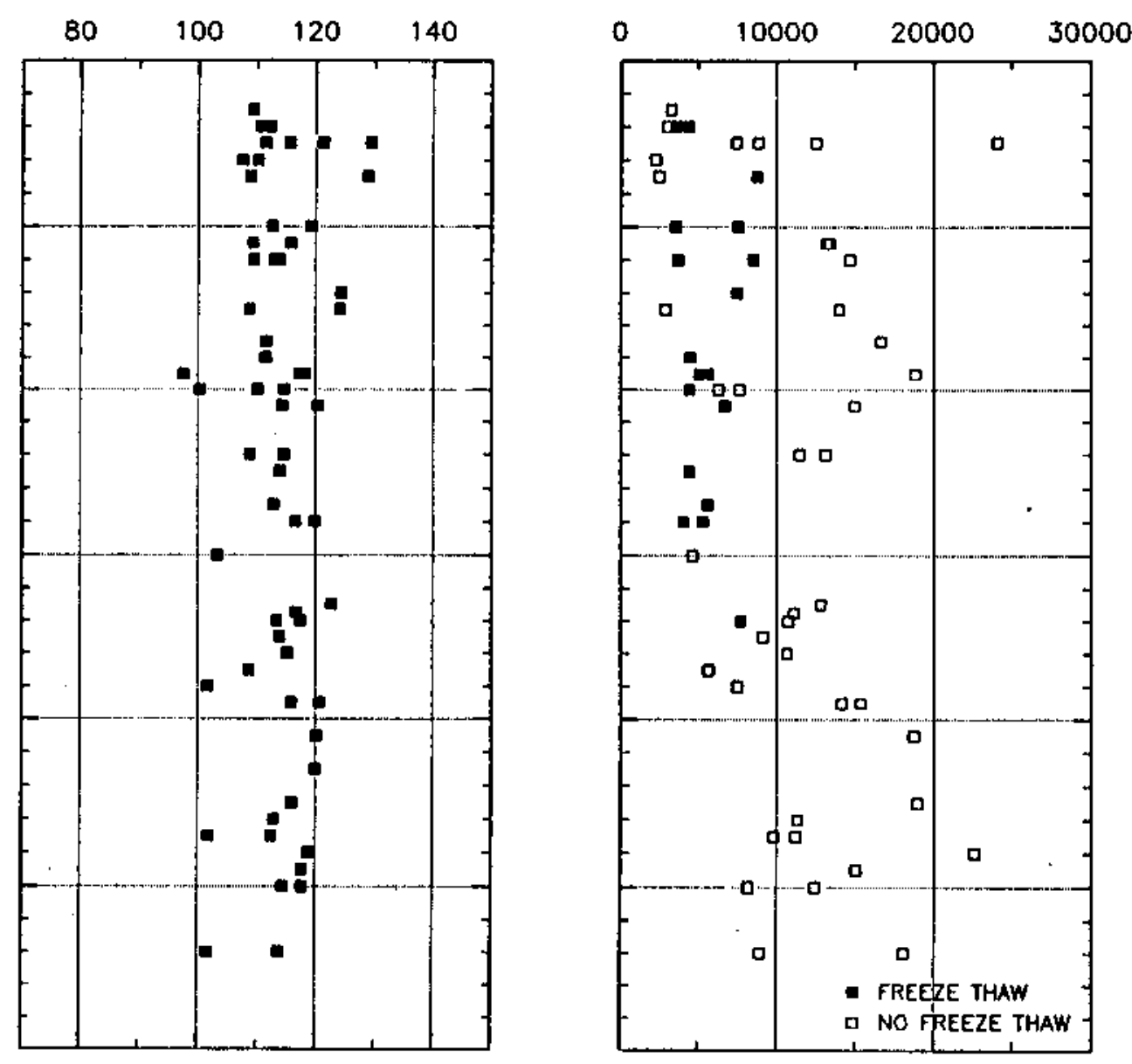

Figure 4.5 Profile of Resilient Modulus with Depth for the Fort wayne site 

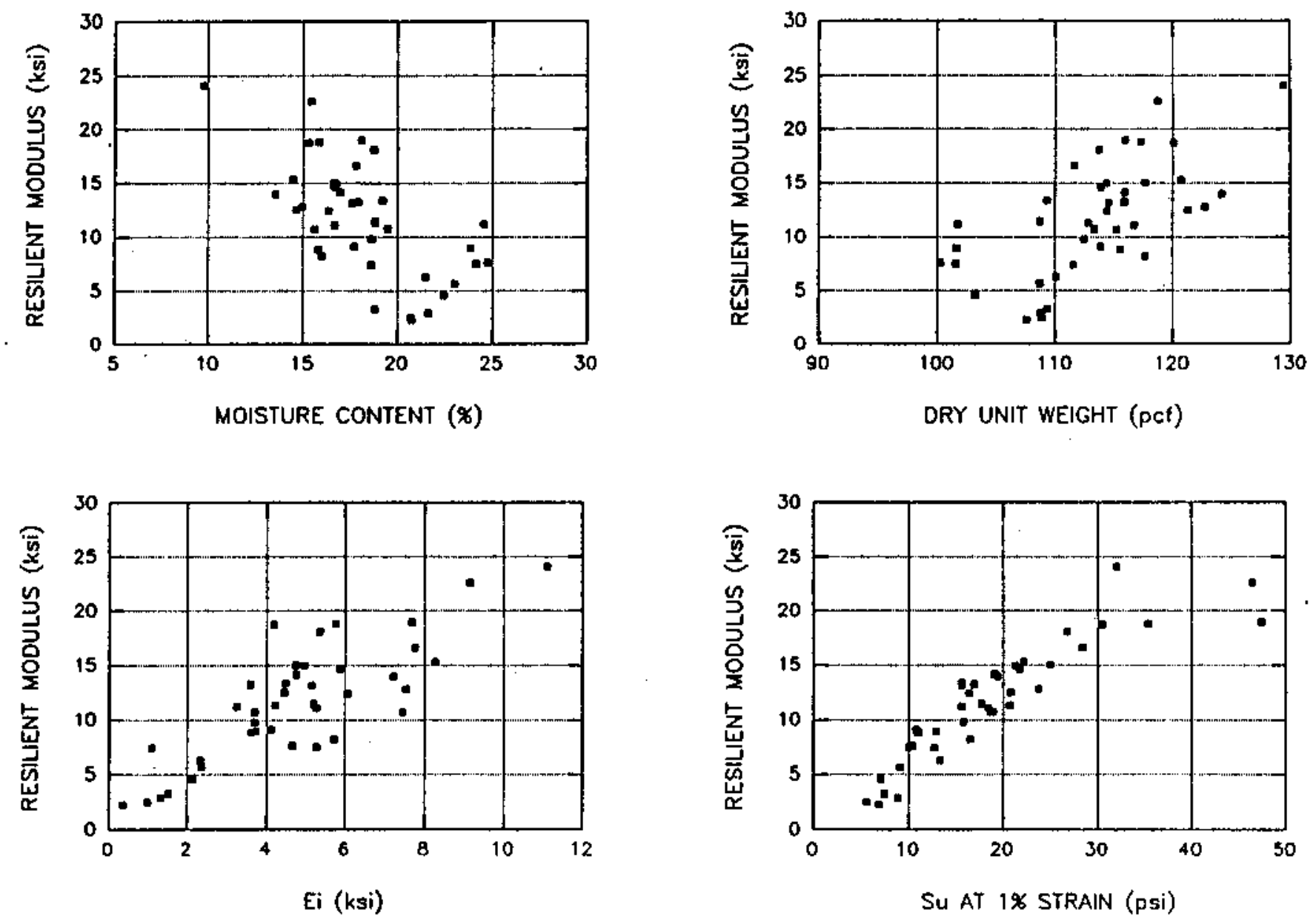

Figure 4.6 Relationship of $M_{R}$ with $w / C, \gamma_{d}, E_{i}$, and $s_{u 1,0 \%}$ for the Fort Wayne soil 


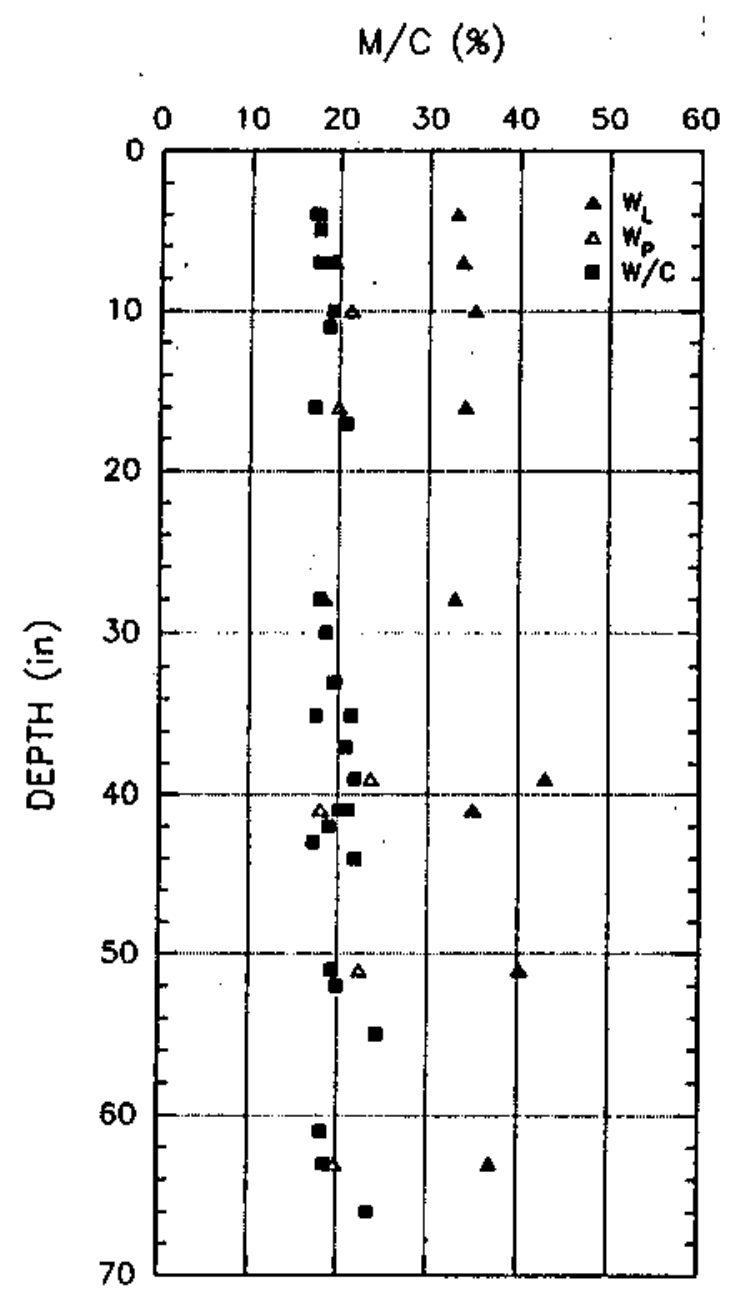

DRY UNIT WEIGHT (pcf)

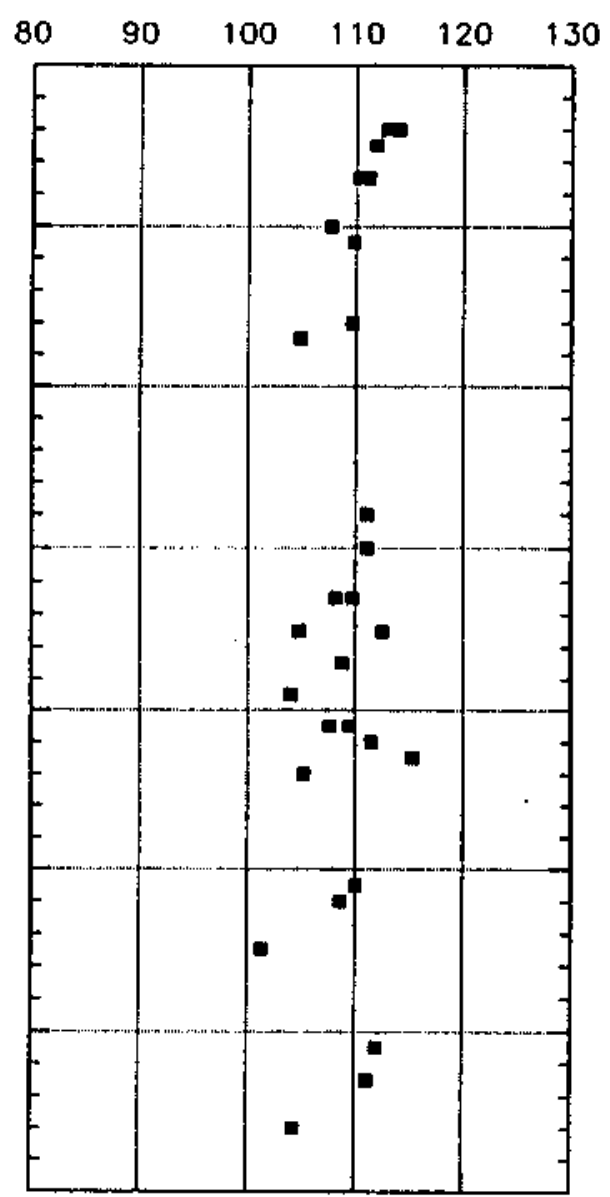

RESILIENT MOOULUS (psi)

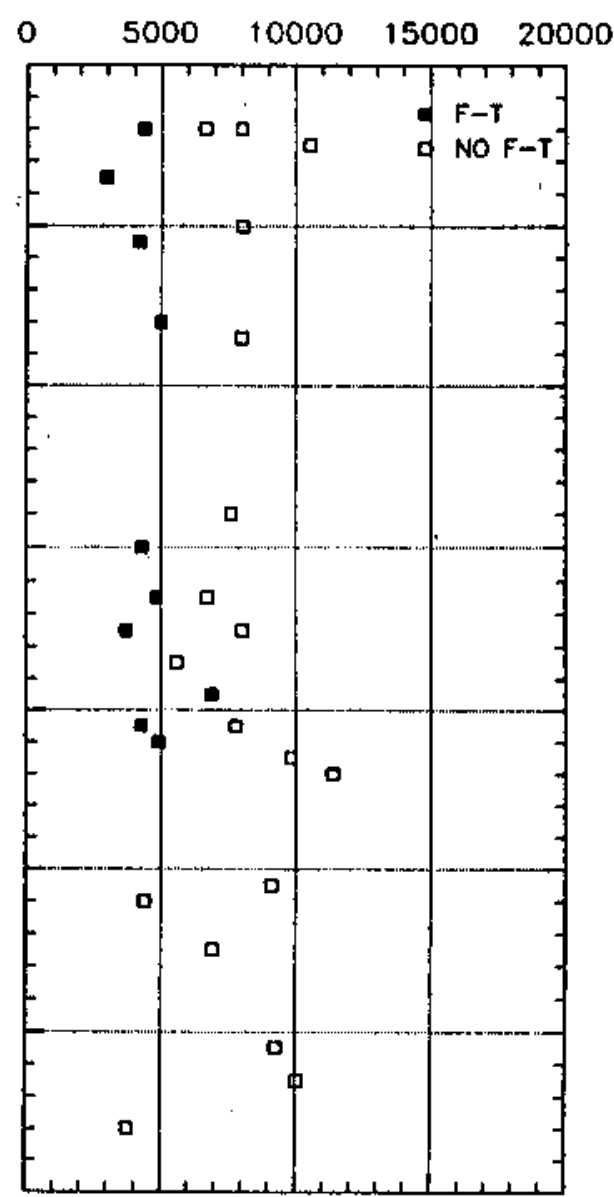

Figure 4.7 Profile of Resilient Modulus with Depth for the Washington site 


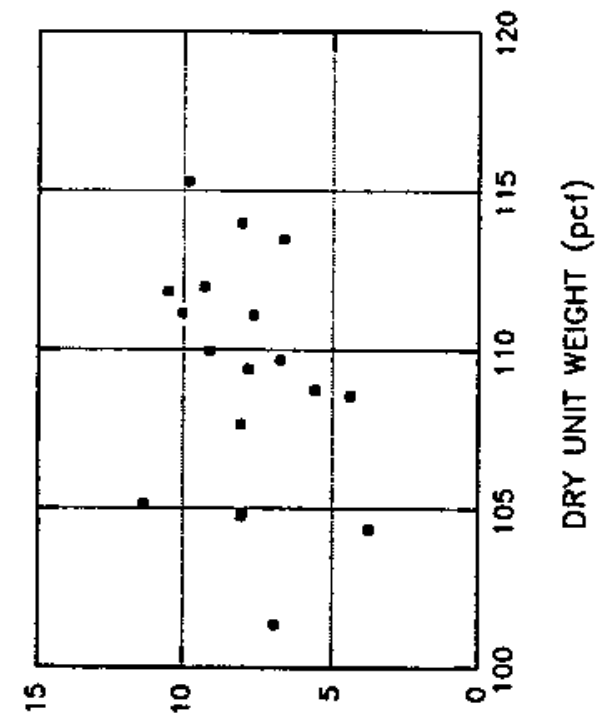

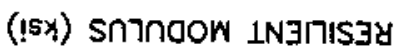

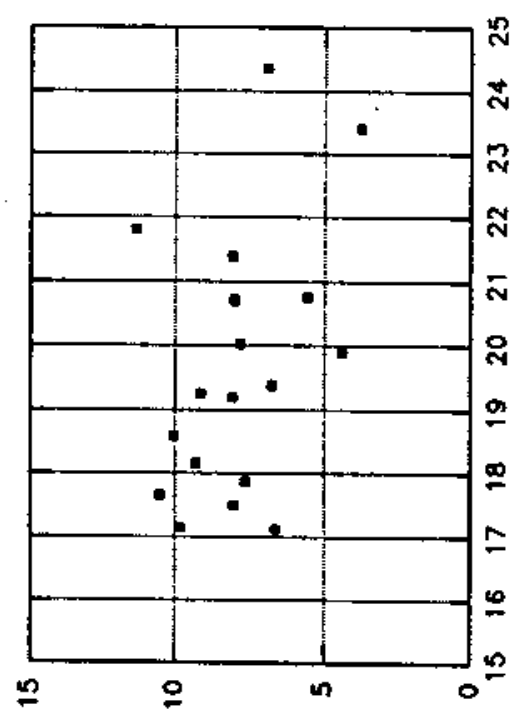

(!sx) sกากoow 1N3/7IS3y

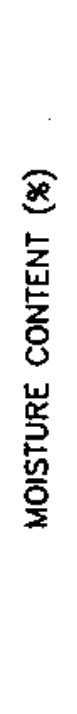

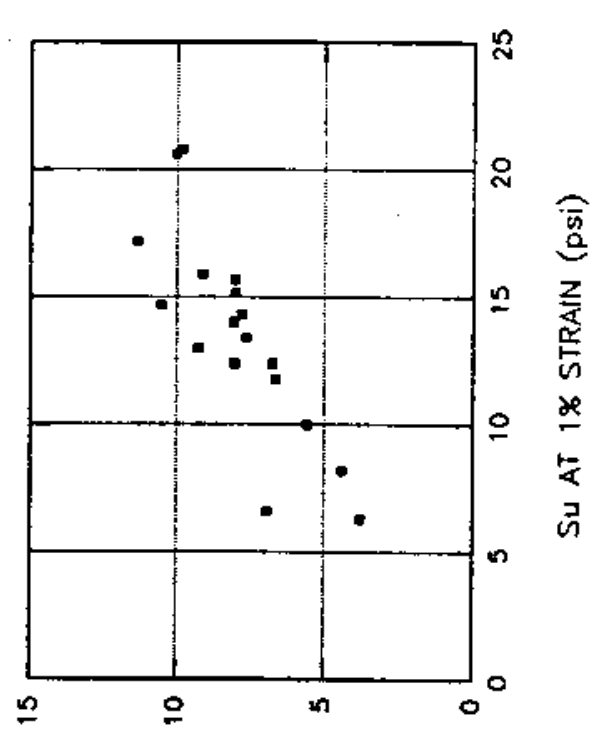

(IS) Sกากดow IN3)

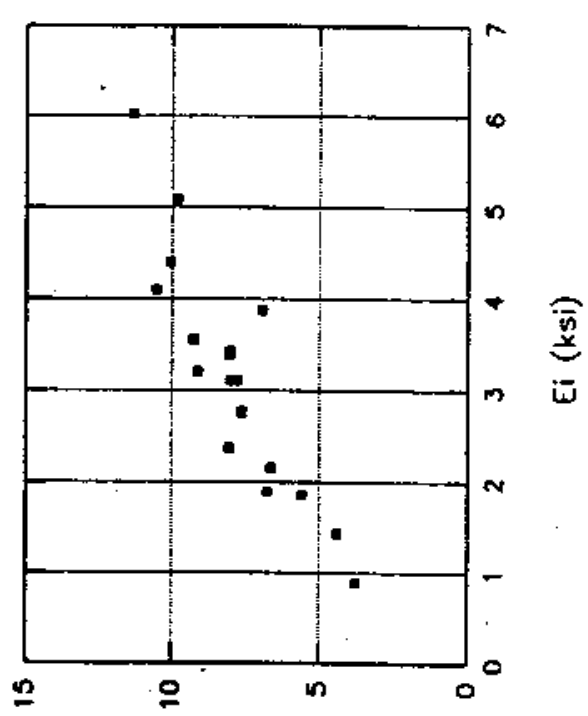

(!5y) Sก7ก0OW IN3mISJy

क्षे

iे

0

돈

$\pm$

岁

足

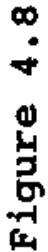




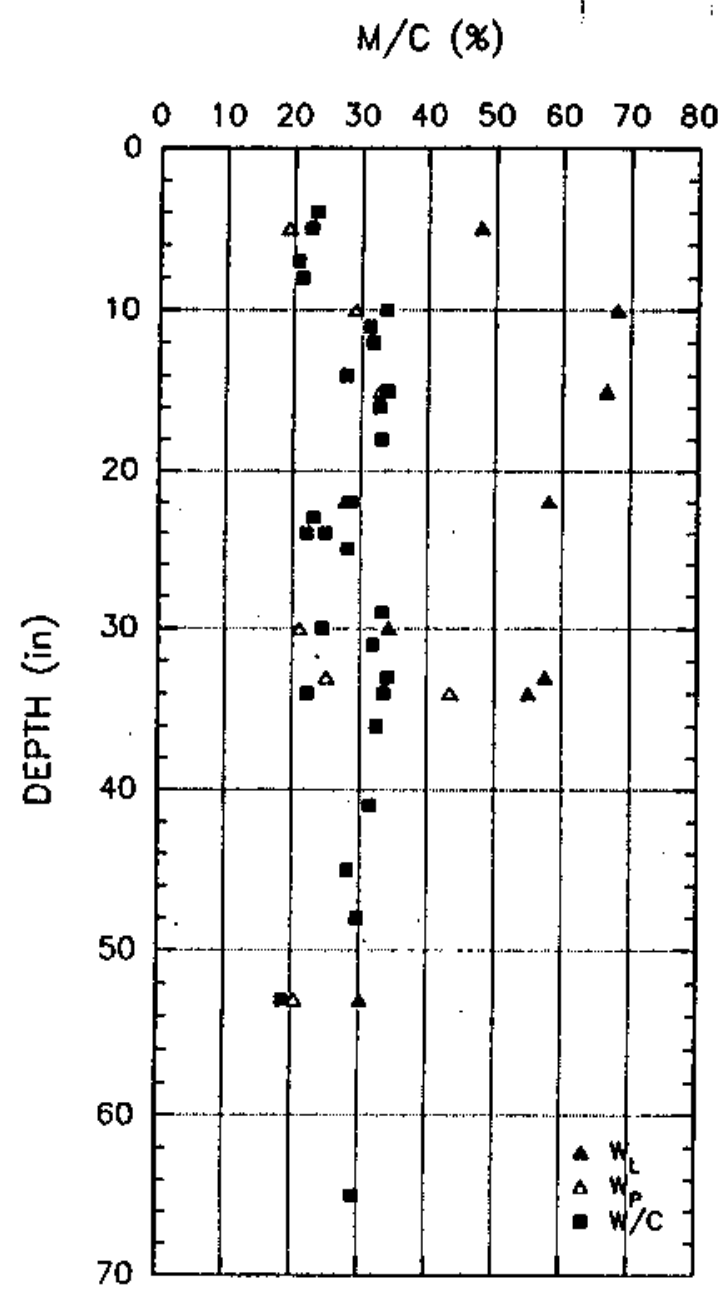

DRY UNIT WEIGHT (pcf)

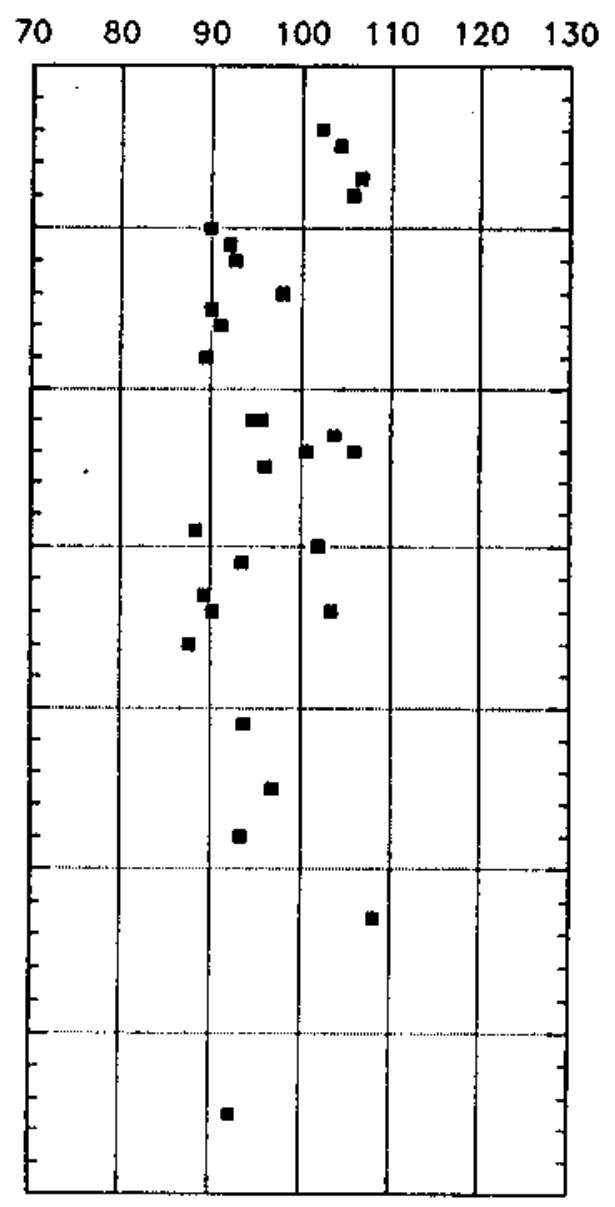

RESILIENT MODULUS (psi)

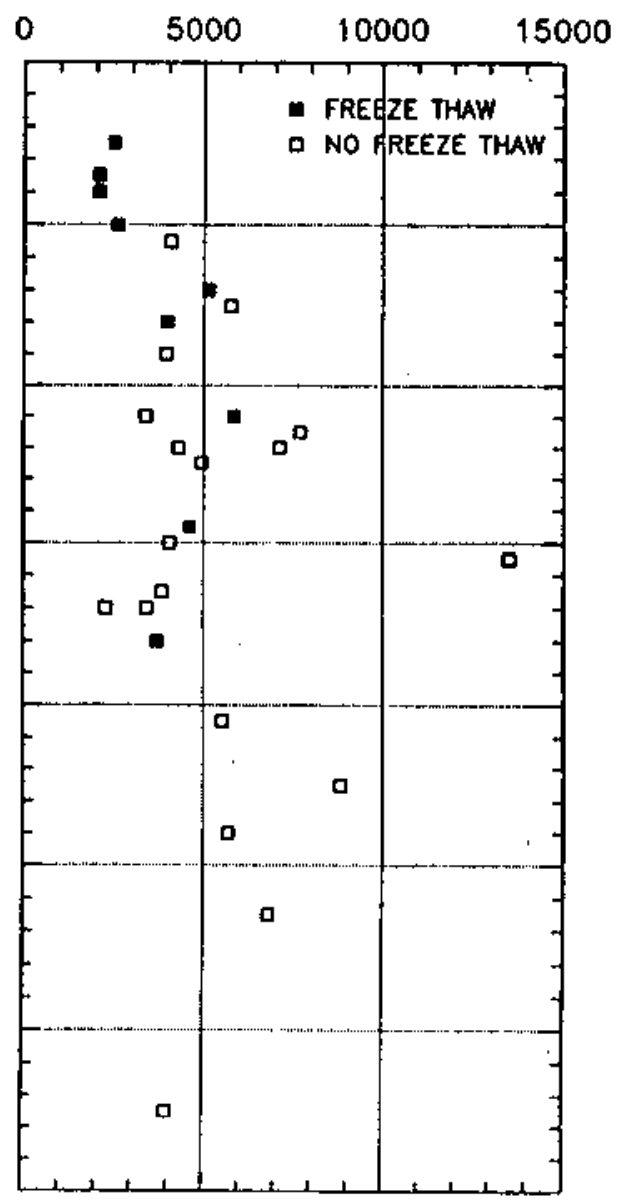



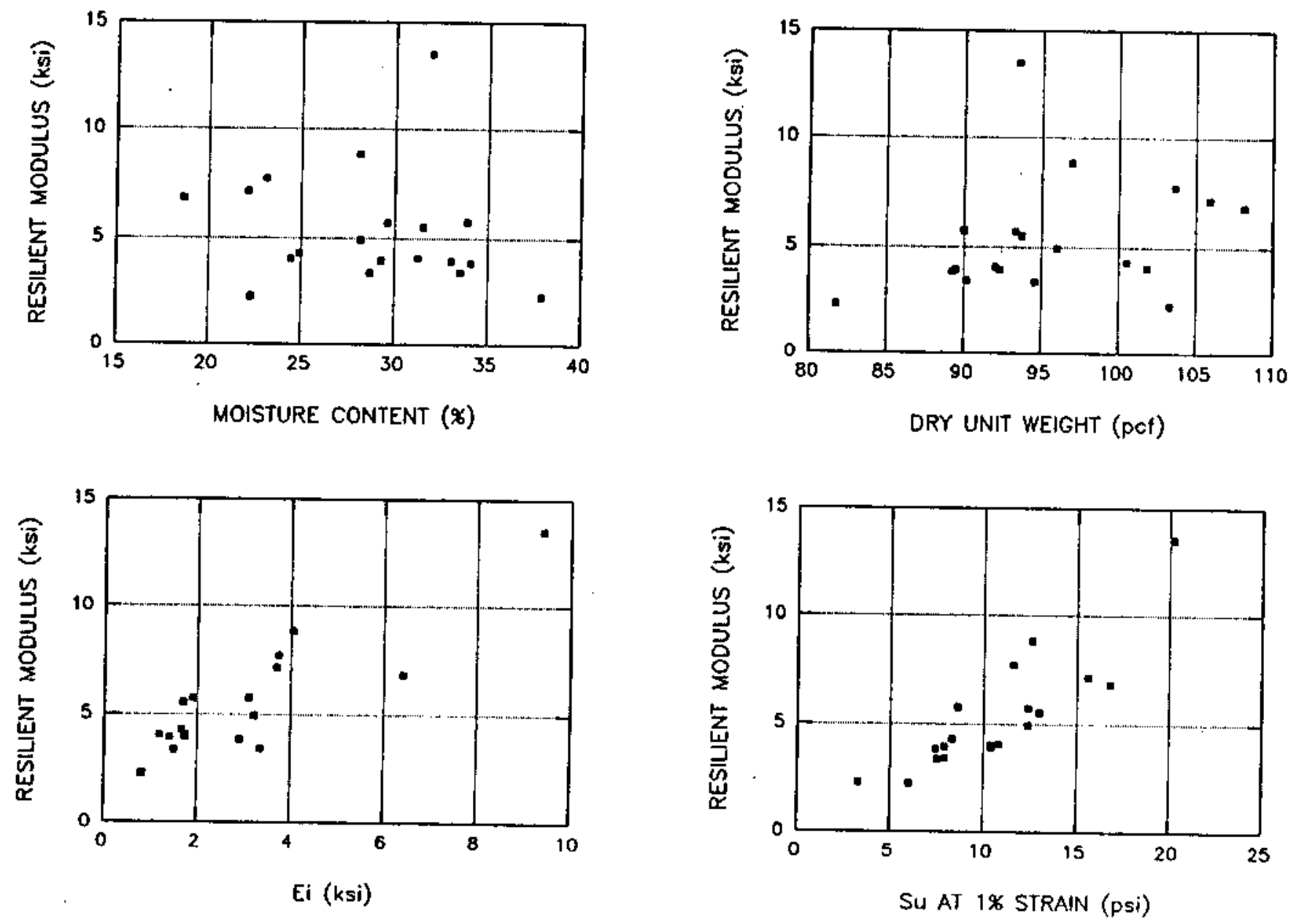

Figure 4.10 Relationship of $M_{R}$ with $w / C, \gamma_{d}, E_{i}$, and $S_{u 1.0 x}$ for the Bedford Soil 

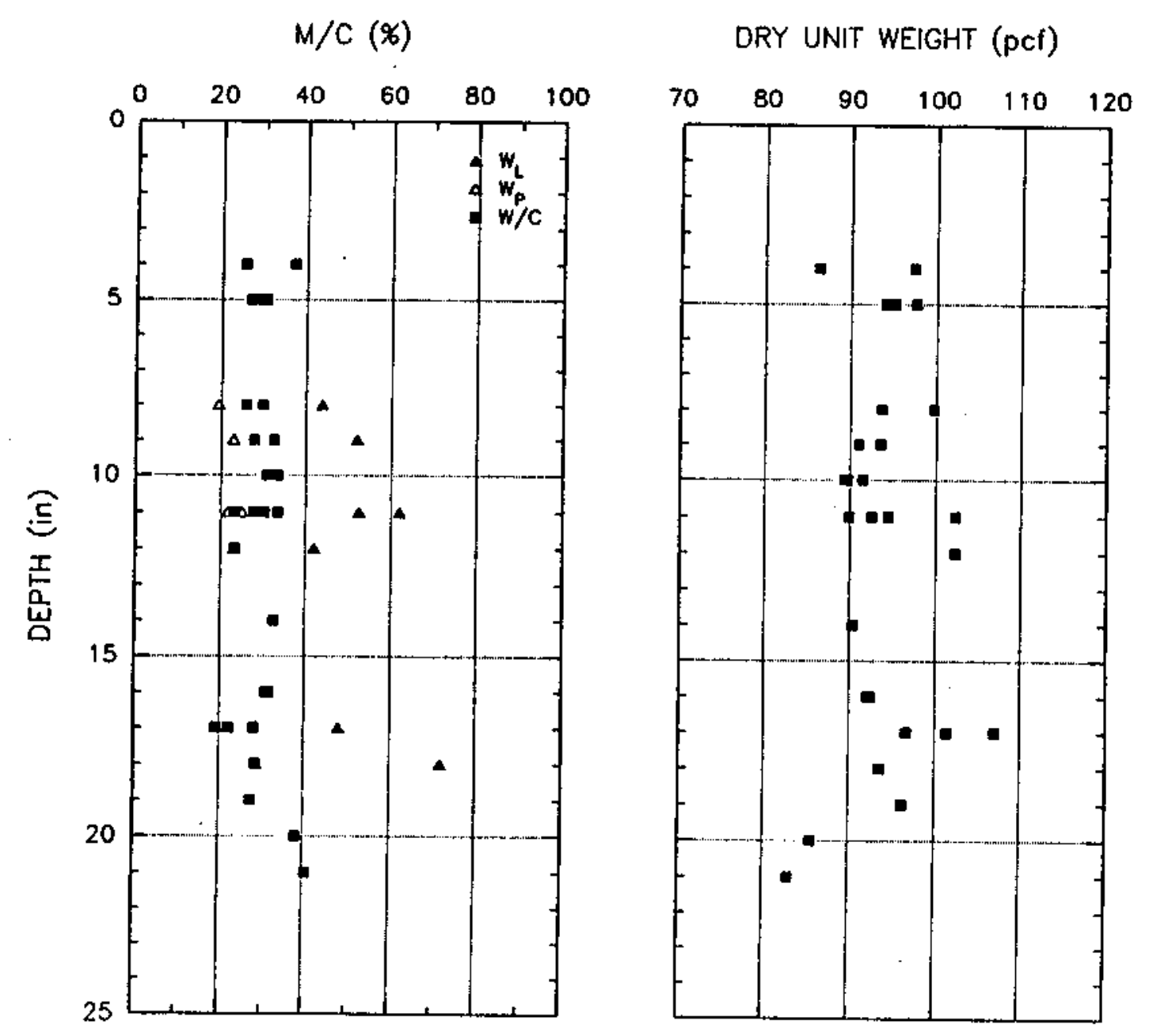

RESILIENT MODULUS (psi)

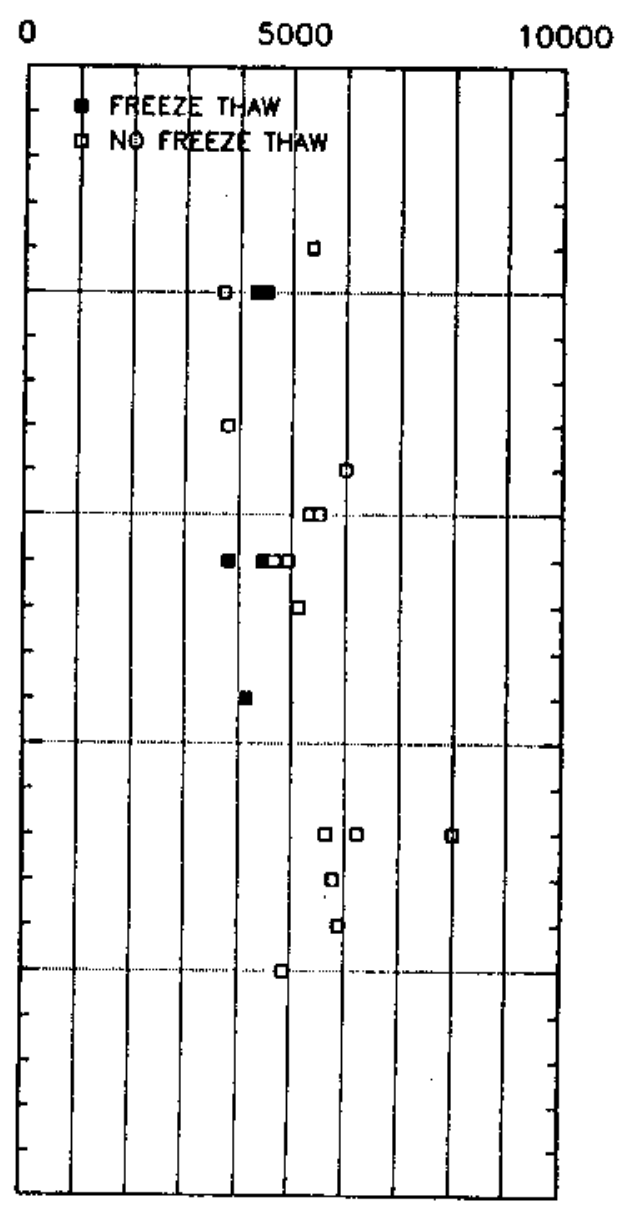

Figure 4.11 Profile of Resilient Modulus with Depth for the Bloomington Site 

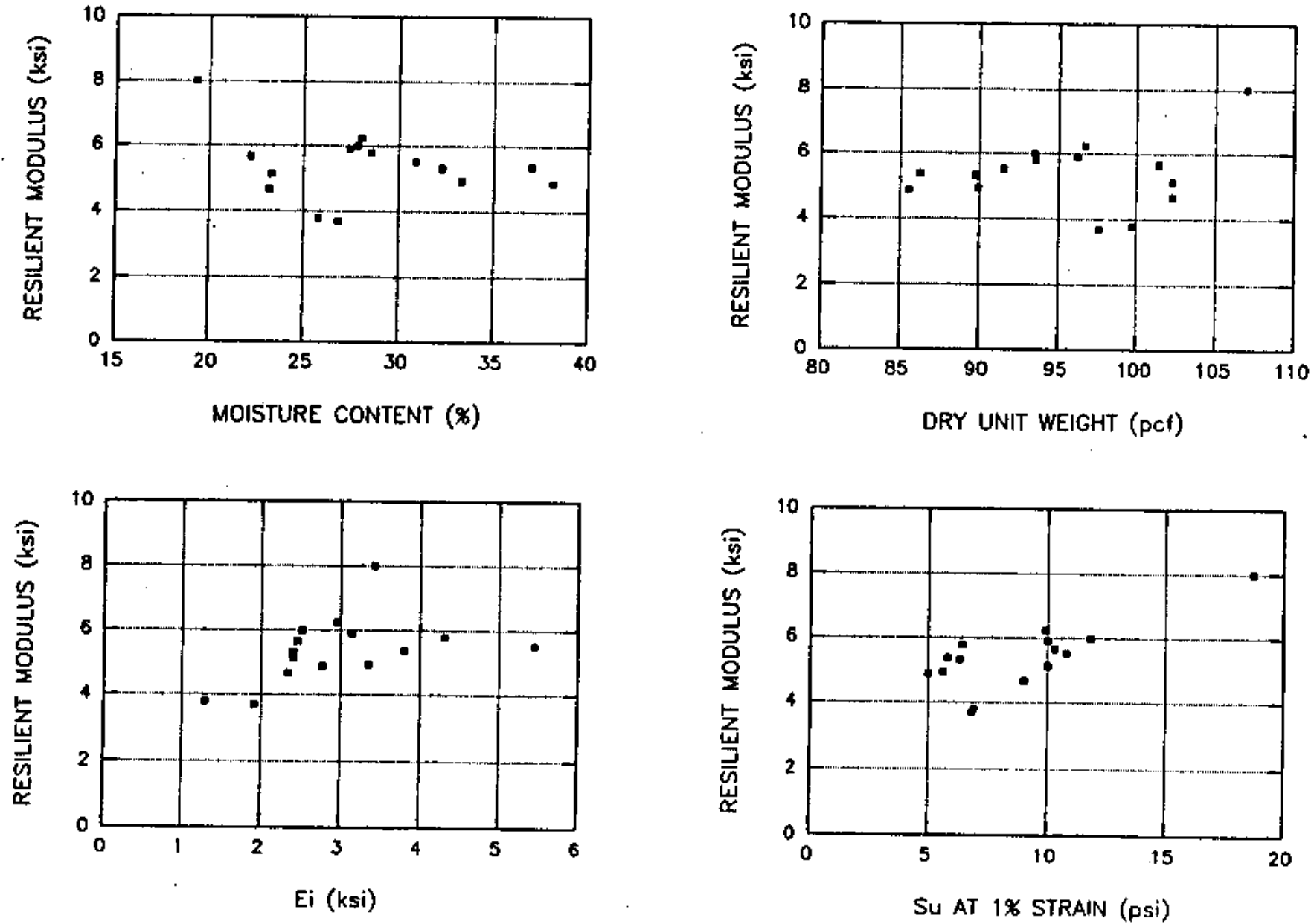

Figure 4.12 Relationship of $M_{R}$ with $w / c, \gamma_{d}, E_{i}$, and $S_{u 1.0 \pi}$ for the Bloomington Soil 
a narrow range, $E_{i}$ and $S_{w 1.0 *}$ seem to show only slight relation with resilient modulus, as shown in Figure 4.12 .

It was shown by Lambe (1958), seed and Chan (1959), Barden and sides (1970), and Hodek and Lovell (1979) that the structure and engineering properties of compacted clay depend on molding water content and compactive effort. Seed et al. (1962) and Monismith et al. (1967) also showed that the resilient behavior of soil compacted dry of optimum and subjected to an increase of water content to wet of optimum is different from behavior of soil compacted directly to wet of optimum. Therefore, it seems that the in-service condition is not a good basis for relating structure and engineering properties of compacted cohesive soils. It is also reasonable to conclude that the resilient modulus of in-service subgrade may not be predicted well by water content and dry density alone or by the test results on as-compacted specimen. This may be the reason why in-service moisture condition and dry density did not provide a good indication of $\mathrm{M}_{R}$, as observed in Figures $4.4,4.6,4.8,4.10$ and 4.12 . Even at the Fort Wayne site showing strong relation of in-service condition with resilient modulus, they are too scattered to give a good correlation.

The results of repeated-load triaxial tests and unconfined compression tests are expected to be affected by the same factors; relating both test results directly may exclude the influence of water content, dry density and other basic indices. Because the resilient strain caused by repeated-loading triaxial tests is very $\operatorname{sinall}\left(10^{-3}-10^{-5}\right.$ in./in.), the stress to cause 1 percent strain instead of the unconfined compressive strength would give a better indication of resilient modulus. As can be seen in Figures 4.6, 4.8, 4.10 and $4.12, S_{w 1.0 x}$ shows a good correlation with resilient modulus for all sites tested, except for south Bend. The South Bend site has no unconfined compression data because this relation grew after south Bend was tested. Therefore, the resilient modulus was correlated to $s_{m .0 x}$. It is also noted that all 
sites show a similar relationship between $M_{R}$ and $S_{u 1.0 \%}$.

To investigate the possibility of an unified correlation, applicable to all soils tested, data from four sites are plotted together in a single plot, as shown in Figure 4.13. It can be seen that there exists an unique relationship between $M_{R}$ at $\sigma_{3}$ of 3 psi and $\sigma_{d}$ of $6 \mathrm{psi}$ and $s_{1.0 \pi}$, regardless of sites. To see whether $M_{R}$ at different levels of stress show similar relations, $M_{R}$ at $\sigma_{3}$ of $6 \mathrm{psi}$ and at $\sigma_{3}$ of $0 \mathrm{psi}$, both with $\sigma_{d}$ of $6 \mathrm{psi}$, are plotted with respect to $s_{u 1.0 x}$, as shown in Figures 4.14 and 4.15. These relationships also appear to be unique, regardless of origin of soils. Therefore, it is concluded that resilient modulus at a specific level of stress can be uniquely related to $s_{v 1.0 x}$, regardless of the soil type:

It can be also seen in Figures from 4.13 to 4.15 that the resilient modulus at higher confining stress is larger than that with lower confining stress and the difference becomes larger as $S_{w 1.0 \pi}$ increases. For soils with $s_{\mathrm{w1.0}}$ of $30 \mathrm{psi}$, resilient modulus at $\sigma_{3}$ of $6 \mathrm{psi}$ is about 50 percent larger than that at $\sigma_{3}$ of $0 \mathrm{psi}$. Therefore, consideration of the effect of confining stress on $K_{R}$ is important when the subgrade is a relatively stiff clay.

To develop the prediction equations, stepwise multiple regression was performed on resilient moduli at different levels of confining stress and repeated deviator stress with independent variables, such as $s_{w 0.75 x}, S_{u 1.0 x}, E_{i}, E_{2}, 1 / b$, water content and dry density. since slight curvature was observed in $\mathrm{M}_{\mathrm{k}}$ versus stress causing a certain percent of strain in the unconfined compression test, second-order terms of $s_{w 0.75 *}$ and $S_{u 1.0 x}$ are also added to independent variables. From regression analysis, two prediction equations are developed:

$$
\begin{gathered}
M_{R}=a+b S_{u 1.0 t}+c S_{u 1.0 t^{2}} \\
M_{R}=a+b S_{u 0.75 t}+c S_{u 1.08}+d S_{u 1.0 t^{2}}+e E_{t}+f \gamma_{d}
\end{gathered}
$$

where $M_{R}$ is resilient modulus at a specific level of stress (psi); $S_{w .75 *}$ and $S_{1.0 \%}$ are stress causing 0.75 percent and 1 


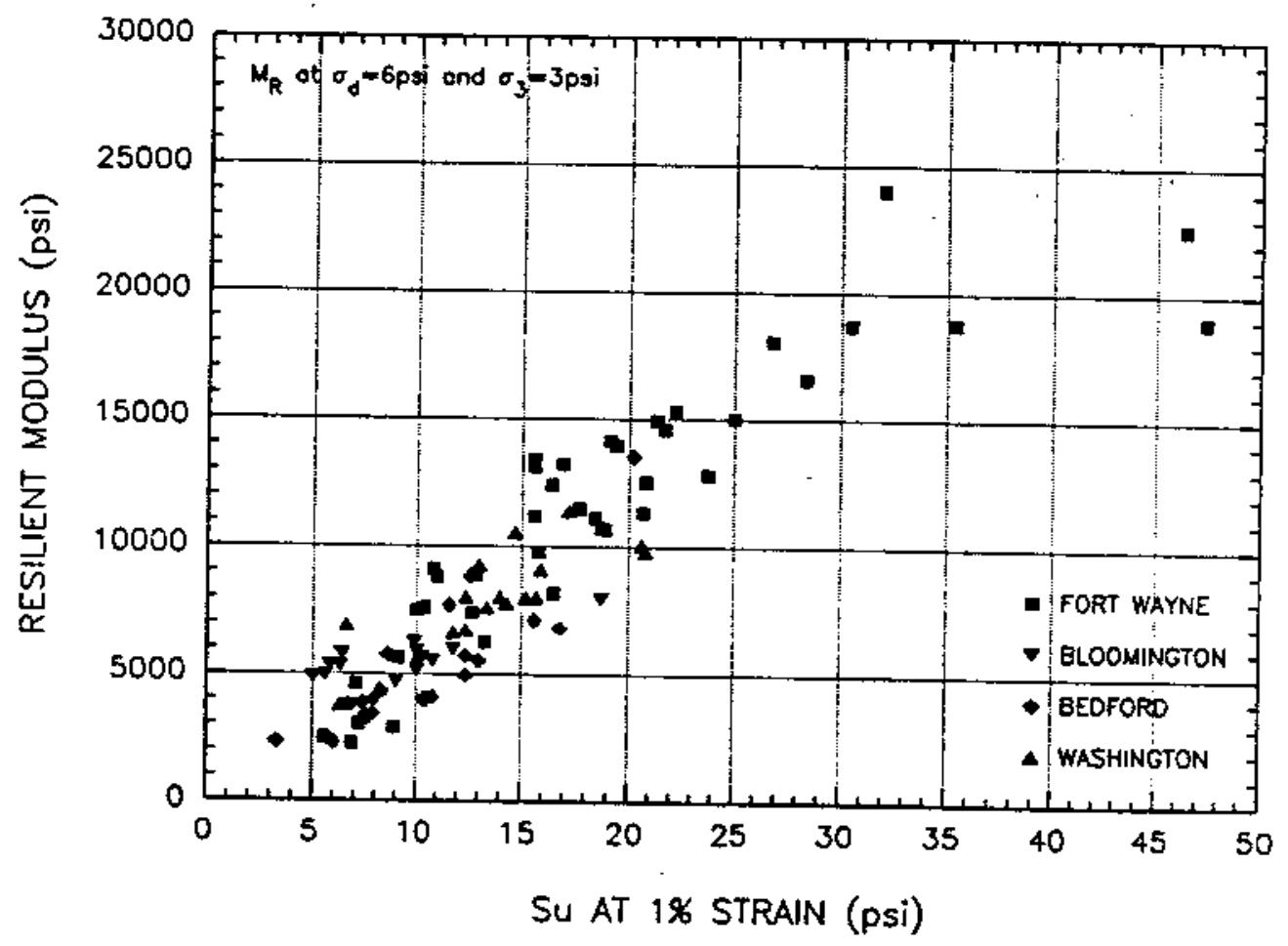
Figure 4.13 Relationship between $M_{R}$ at $\sigma_{d}=6$ psi and $\sigma_{3}=3$ psi
and $S_{u 1.0 \text { for All sites }}$ 


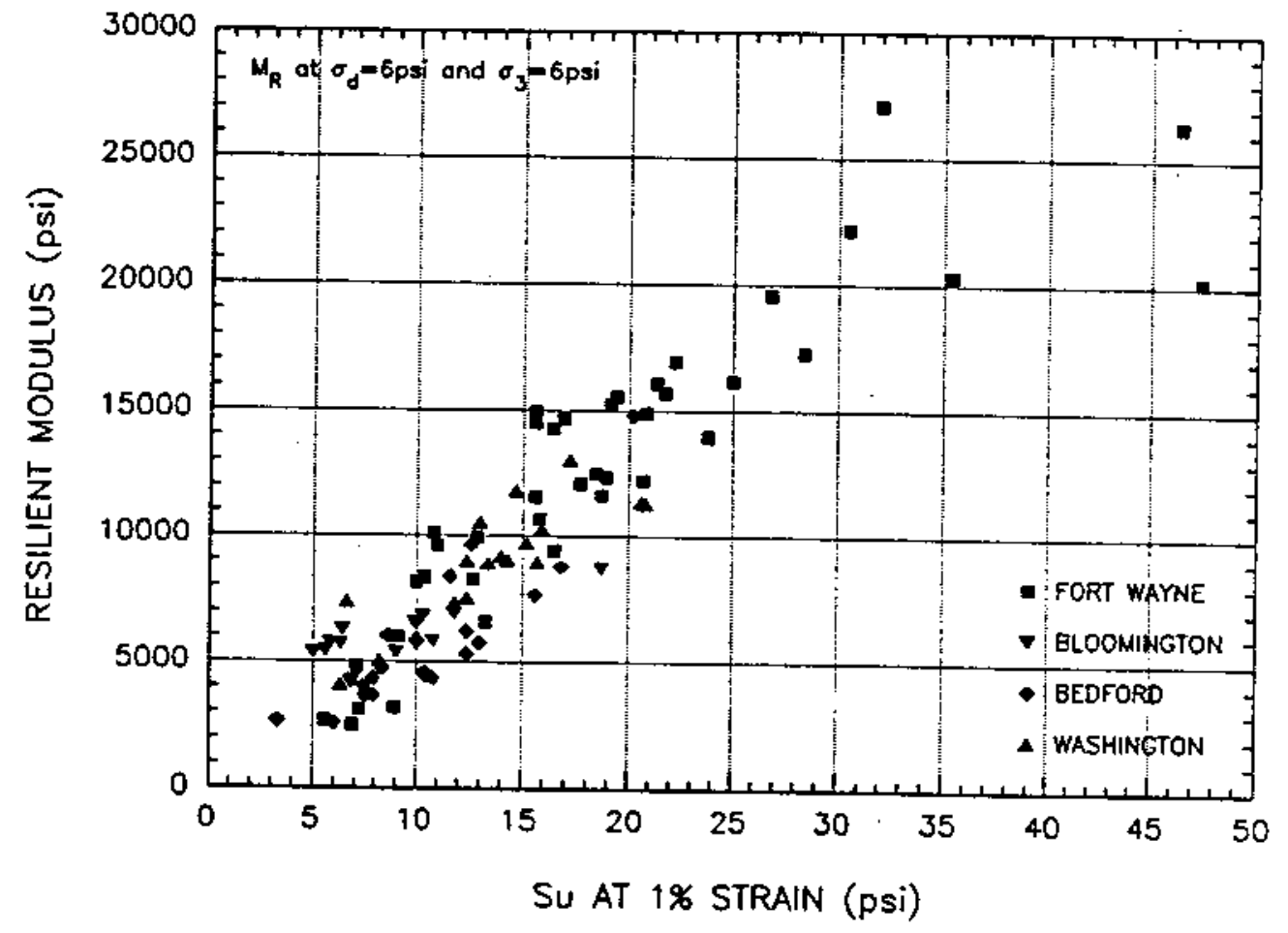

Figure 4.14 Relationship between $M_{R}$ at $\sigma_{d}=6$ psi and $\sigma_{3}=6$ psi and $S_{u 1.0 \%}$ for All sites 


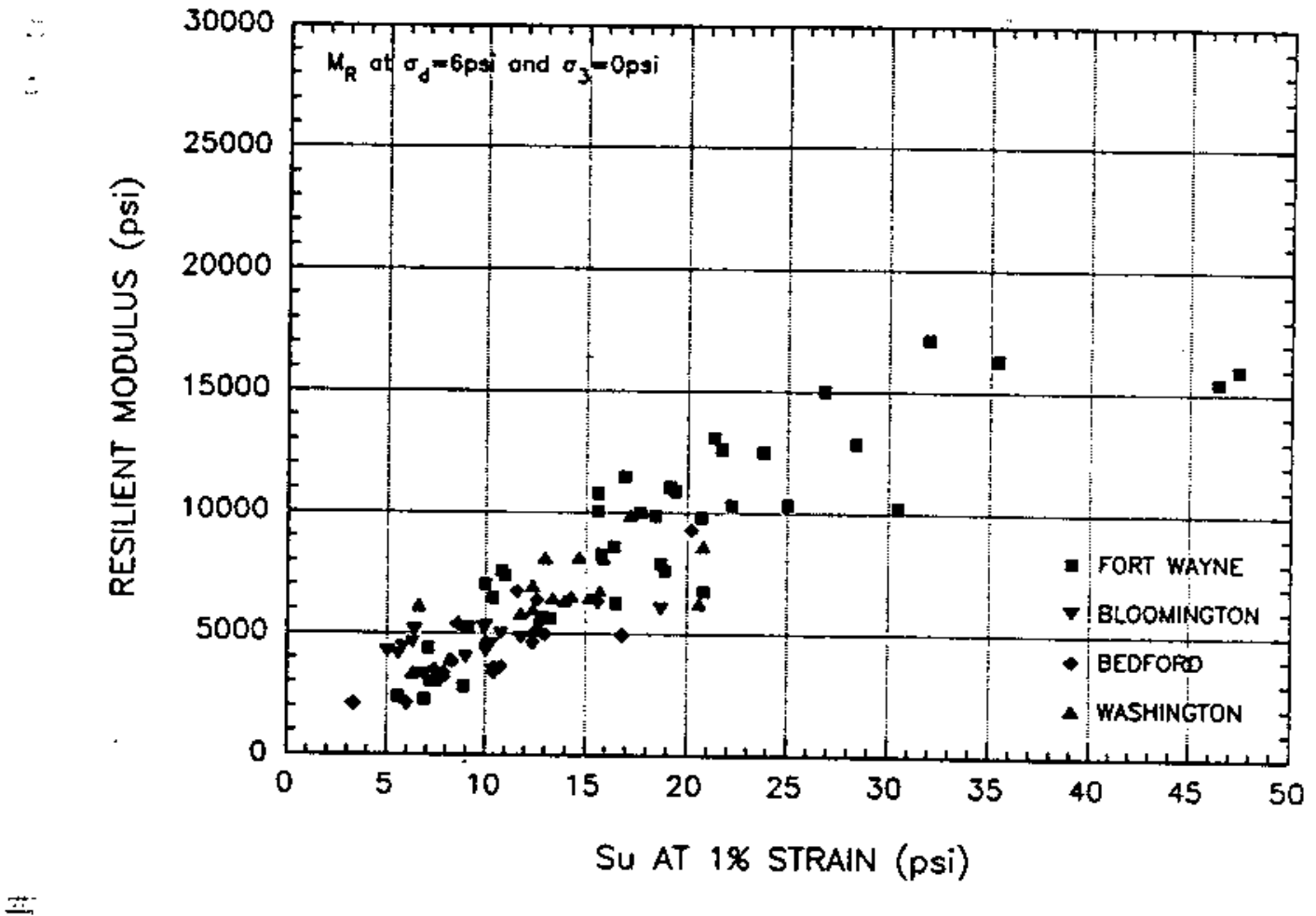
Figure 4.15 Relationship between $M_{R}$ at $\sigma_{d}=6$ psi and $\sigma_{3}=0$ psi
and $S_{u 1.0 \times}$ for All sites 
percent axial strain during the unconfined compression test, respectively (psi); $E_{i}$ is initial tangent modulus from the unconfined compressive stress-strain curve (psi); $\gamma_{\mathrm{d}}$ is dry unit weight (pcf); $a, b, c, d, e$ and $f$ are regression parameters. Equation 4.4 is based on only $S_{u 1.0 x}$ for practical use, and Equation 4.5 is selected because it gives the smallest error of estimation. Regression parameters for these prediction equations, with correlation coefficient and mean square error, are presented in Tables 4.1 and 4.2. Equation 4.4 gives the coefficient of determination, $R^{2}$, ranging from 0.51 to 0.85 and, in most cases, the coefficient of variation (Root MSE over mean value of resilient modulus) does not exceed 25 percent. For Equation 4.5, $\mathrm{R}^{2}$ ranges from 0.55 to 0.91 and, in most cases, the coefficient of variation is less than 21 percent. Generally, prediction equation of resilient modulus at deviator stress level less than 4 psi shows smaller $R^{2}$ and larger mean square error while the reverse is true at deviator stress larger than 4 psi.

To check the reliability of the proposed prediction equations, the resilient moduli at $\sigma_{d}$ of $6 \mathrm{psi}$ and $\sigma_{3}$ of $3 \mathrm{psi}$ calculated by the proposed equations are compared with measured $M_{R}$. As shown in Figure 4.16 , both equation give good predictions of resilient modulus. It is noted that adding three more variables improves predictability only slightly. 4.4 Resilient Modulus Test on Laboratory Compacted Cohesive
Soils

4.4.1 Preparation of Specimens

Disturbed soils obtained from South Bend and Washington site were air dried until the soils became friable, and then broken up with a mallet. After measuring the water content of the processed soil, a calculated amount of water to obtain a desirable compaction water content was mixed with air-dried 
Table 4.1 Regression Parameters for Equation 4.4

\begin{tabular}{|c|c|c|c|c|c|c|}
\hline $\begin{array}{c}\sigma_{3} \\
(p s i)\end{array}$ & $\begin{array}{c}\sigma_{d} \\
(p s i)\end{array}$ & $\begin{array}{l}\text { Regr } \\
\text { a }\end{array}$ & $\begin{array}{c}\text { sion param } \\
\text { b }\end{array}$ & ters & $R^{2}$ & VMSE \\
\hline 6 & 2 & -3289.92 & 1808.998 & -21.4597 & 0.74 & 4506 \\
\hline 6 & 3 & -2658.54 & 1413.663 & -15.0910 & 0.80 & 3211 \\
\hline 6 & 4 & -2287.64 & 1187.214 & -11.5754 & 0.83 & 2598 \\
\hline 6 & 5 & -2038.04 & 1037.146 & -9.3147 & 0.84 & 2266 \\
\hline 6 & 6 & -1856.14 & 928.921 & -7.7260 & 0.85 & 2070 \\
\hline 6 & 7 & -1716.41 & 846.421 & -6.5427 & 0.85 & 1949 \\
\hline 6 & 8 & -1604.98 & 781.012 & -5.6239 & 0.85 & 1870 \\
\hline 6 & 9 & -1513.59 & 727.612 & -4.8881 & 0.84 & 1818 \\
\hline 6 & 10 & -1436.98 & 683.016 & -4.2847 & 0.84 & 1783 \\
\hline 3 & 2 & -2545.68 & 1542.024 & -17.9856 & 0.73 & 4018 \\
\hline 3 & 3 & -2138.68 & 1227.384 & -12.9396 & 0.79 & 2868 \\
\hline 3 & 4 & -1892.92 & 1044.798 & -10.1121 & 0.83 & 2314 \\
\hline 3 & 5 & -1724.34 & 922.624 & -8.2730 & 0.85 & 2011 \\
\hline 3 & 6 & -1599.66 & 833.832 & -6.9683 & 0.85 & 1832 \\
\hline 3 & 7 & -1502.75 & 765.705 & -5.9885 & 0.85 & 1723 \\
\hline 3 & 8 & -1424.67 & 711.390 & -5.2223 & 0.85 & 1654 \\
\hline 3 & 9 & -1360.08 & 666.828 & -4.6048 & 0.85 & 1610 \\
\hline 3 & 10 & -1305.52 & 629.449 & -4.0953 & 0.85 & 1581 \\
\hline 0 & 2 & 842.19 & 854.758 & -9.5604 & 0.51 & 3707 \\
\hline 0 & 3 & 319.24 & 744.848 & -7.5743 & 0.63 & 2688 \\
\hline 0 & 4 & 18.57 & 678.014 & -6.4021 & 0.71 & 2137 \\
\hline 0 & 5 & -181.51 & 631.725 & -5.6084 & 0.76 & 1799 \\
\hline 0 & 6 & -326.44 & 597.153 & -5.0265 & 0.80 & 1579 \\
\hline 0 & 7 & -437.42 & 570.019 & -4.5769 & 0.82 & 1432 \\
\hline 0 & 8 & -525.81 & 547.966 & -4.2165 & 0.84 & 1334 \\
\hline 0 & 9 & -598.29 & 529.566 & -3.9195 & 0.85 & 1268 \\
\hline 0 & 10 & -659.10 & 513.901 & -3.6695 & 0.85 & 1225 \\
\hline
\end{tabular}


Table 4.2 Regression Parameters for Equation 4.5

\begin{tabular}{|c|c|c|c|c|c|c|c|c|c|}
\hline \multirow{2}{*}{$\begin{array}{c}\sigma_{3} \\
\left(p^{8 i}\right)\end{array}$} & \multirow[b]{2}{*}{ psi) } & \multirow[b]{2}{*}{ a } & \multicolumn{3}{|c|}{ Regre日sion parameters } & \multirow[b]{2}{*}{ e } & \multirow[b]{2}{*}{$f$} & \multirow[t]{2}{*}{$\mathbf{R}^{2}$} & \multirow[t]{2}{*}{ VMSE } \\
\hline & & & b & c & d & & & & \\
\hline 6 & 2 & -21442 & -290 & 1337 & -13.118 & 0.8965 & 217.17 & 0.78 & 4195 \\
\hline 6 & 3 & -14741 & -825 & 1578 & -10.013 & 0.9759 & 142.08 & 0.84 & 2905 \\
\hline 6 & 4 & -11221 & -1123 & 1702 & -8.243 & 1.0168 & 103.03 & 0.87 & 2270 \\
\hline 6 & 5 & -9043 & -1315 & 1775 & -7.079 & 1.0409 & 79.07 & 0.89 & 1909 \\
\hline 6 & 6 & -7561 & -1450 & 1823 & -6.247 & 1.0561 & 62.88 & 0.90 & 1688 \\
\hline 6 & 7 & -6487 & -1551 & 1856 & -5.618 & 1.0661 & 51.23 & 0.91 & 1545 \\
\hline 6 & 8 & -5672 & -1628 & 1880 & -5.123 & 1.0729 & 42.45 & 0.91 & 1451 \\
\hline 6 & 9 & -5035 & -1690 & 1898 & -4.723 & 1.0774 & 35.63 & 0.91 & 1387 \\
\hline 6 & 10 & -4522 & -1740 & 1911 & -4.392 & 1.0805 & 30.17 & 0.91 & 1343 \\
\hline 3 & 2 & -14702 & 224 & 812 & -11.192 & 0.6481 & 147.54 & 0.76 & 3859 \\
\hline 3 & 3 & -10390 & -350 & 1106 & -8.644 & 0.7616 & 98.22 & 0.83 & 2681 \\
\hline 3 & 4 & -8108 & -681 & 1269 & -7.188 & 0.8243 & 72.35 & 0.86 & 2088 \\
\hline 3 & 5 & -6688 & -900 & 1374 & -6.228 & 0.8641 & 56.38 & 0.89 & 1745 \\
\hline 3 & 6 & -5717 & -1057 & 1447 & -5.540 & 0.8915 & 45.54 & 0.90 & 1532 \\
\hline 3 & 7 & -5011 & -1177 & 1501 & -5.019 & 0.9114 & 37.70 & 0.91 & 1395 \\
\hline 3 & 8 & -4475 & -1271 & 1543 & -4.609 & 0.9263 & 31.79 & 0.91 & 1303 \\
\hline 3 & 9 & -4054 & -1347 & 1577 & -4.277 & 0.9379 & 27.18 & 0.91 & 1242 \\
\hline 3 & 10 & -3715 & -1410 & 1604 & -4.001 & 0.9470 & 23.48 & 0.91 & 1200 \\
\hline 0 & 2 & -7530 & 1659 & -957 & $-2,198$ & 0.0630 & 108.31 & 0.55 & 3622 \\
\hline 0 & 3 & -5643 & 991 & -425 & -2.254 & 0.2324 & 76.51 & 0.67 & 2603 \\
\hline 0 & 4 & -4585 & 579 & -99 & -2.298 & 0.3365 & 58.42 & 0.75 & 2041 \\
\hline 0 & 5 & -3892 & 290 & 129 & -2.337 & 0.4092 & 46.45 & 0.80 & 1685 \\
\hline 0 & 6 & -3396 & 72 & 300 & -2.373 & 0.4636 & 37.81 & 0.84 & 1445 \\
\hline 0 & 7 & -3020 & -101 & 436 & -2.407 & 0.5065 & 31.20 & 0.86 & 1277 \\
\hline 0 & 8 & -2721 & -242 & 547 & -2.438 & 0.5414 & 25.95 & 0.88 & 1157 \\
\hline 0 & 9 & -2478 & -361 & 640 & -2.468 & 0.5707 & 21.64 & 0.89 & 1071 \\
\hline 0 & 10 & -2274 & -463 & 720 & -2.495 & 0.5956 & 18.02 & 0.90 & 1011 \\
\hline
\end{tabular}



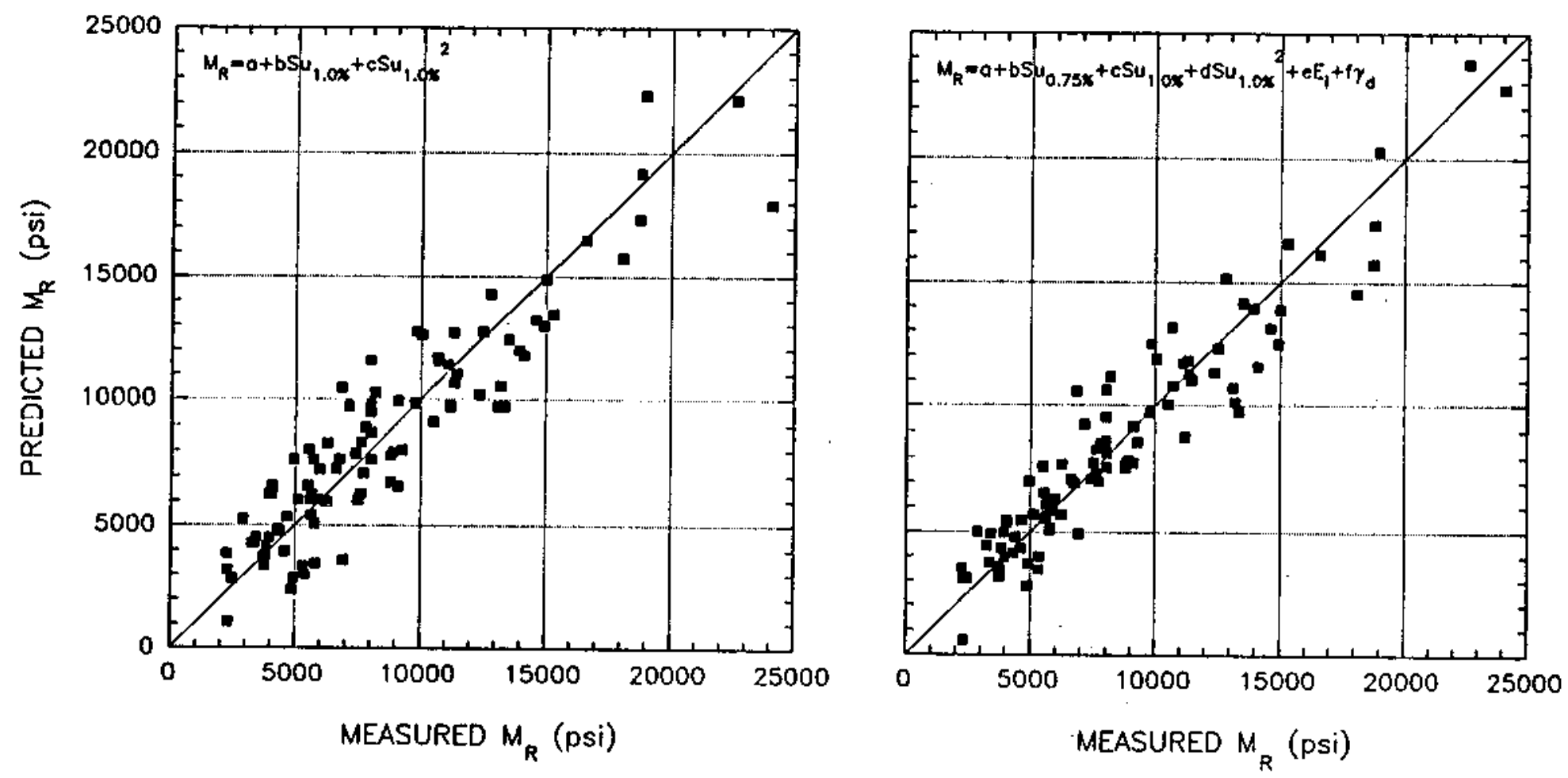

Figure 4.16 Comparison between Measured and Predicted In-Service Resilient Modulus 
soil. Mixed soil was wrapped in a plastic bag and stored in the humidity room for two days before compaction.

Each specimen was compacted in five layers of approximately equal height with a rammer of 3.531 lbs and drop height of 12 inches. The compaction mold of 2.879 inch in diameter and 6.292 inch in height was used. To validate the compaction method used in this study, three different compactions using different size of mold and hammer were carried out with similar compactive efforts. Result of these compactions are shown in Figure 4.17. It can be seen that similar compaction curves were created with almost identical maximum dry density; the optimum moisture conterit of standard mold compaction is about 0.6 percent larger than the other compactions. Therefore, it is concluded that the compaction used in this study is compatible to both existing standard and Modified Proctor compaction.

Before adding a new layer of soil, the surface of the previously compacted layer was scarified in order to prevent separation at the contact between layers. Four different levels of compaction energy were used and a series of at least four specimens was compacted for each level of compactive effort. Compactive efforts and blows per layer used are given in Table 4.3 .

Table 4.3 Details of Laboratory Compaction

\begin{tabular}{c|c|c}
\hline $\begin{array}{c}\text { Compaction Energy } \\
\left(1 \mathrm{~b} \cdot \mathrm{ft} / \mathrm{ft}^{3}\right)\end{array}$ & $\begin{array}{c}\text { Total } \\
\text { Blows }\end{array}$ & Blows per layer \\
\hline 12364 & 82 & $16,16,16,17,17$ \\
\hline 8938 & 60 & $12,12,12,12,12$ \\
\hline 6703 & 45 & $9,9,9,9,9$ \\
\hline 4469 & 30 & $6,6,6,6,6$ \\
\hline
\end{tabular}




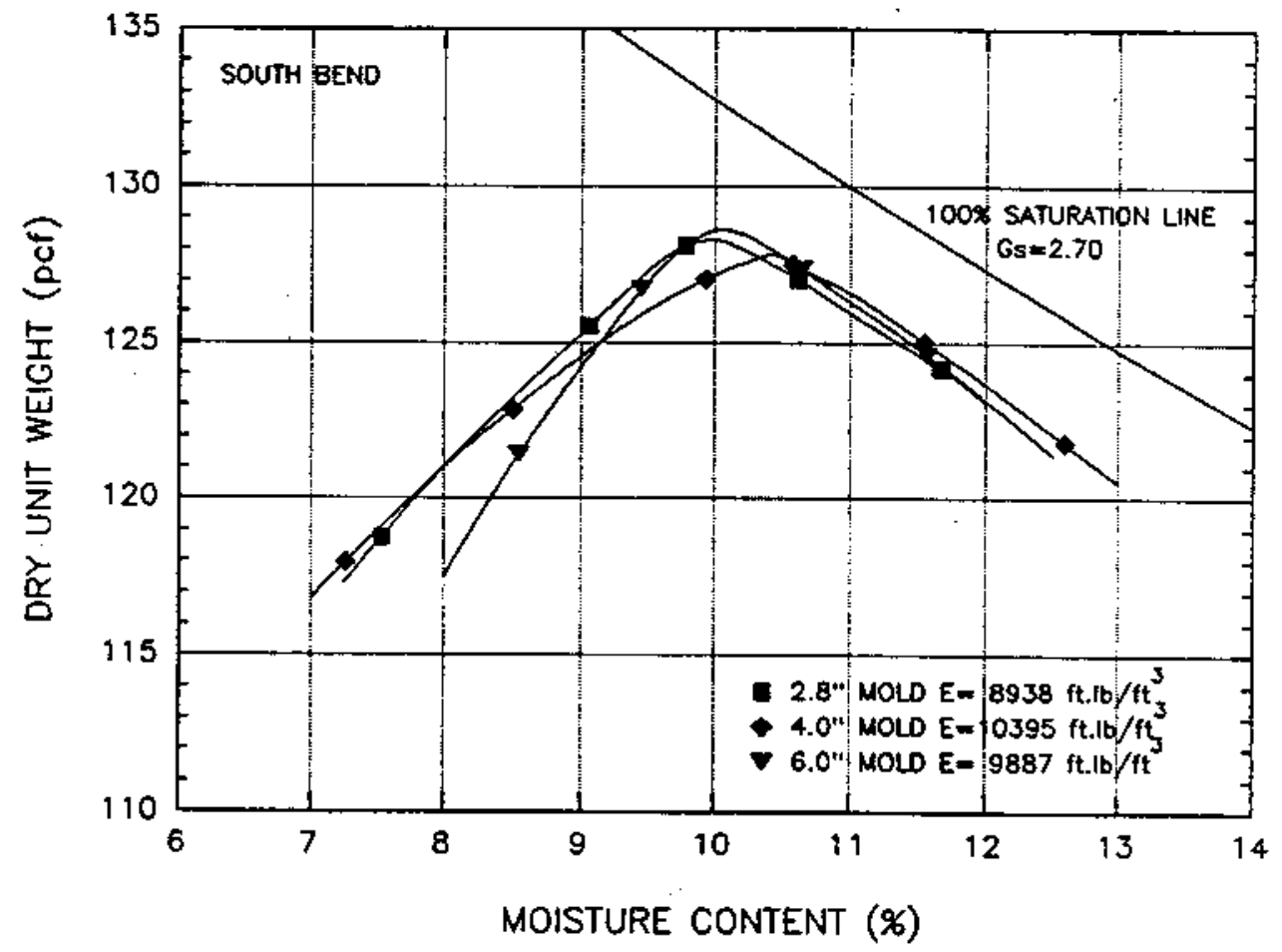

Figure 4.17 Effect of Mold and Rammer on Compaction 
Following compaction, the extension collar was removed and the compacted specimen was carefully trimmed even with the top of the mold by means of a straightedge. After measuring the weight of mold and soil, the specimen was extruded using a hydraulic jack. The extruded specimen was wrapped in a plastic bag and stored in the humidity room for two days before tested. Results of the laboratory compaction for South Bend and Washington soil are shown in Figure 4.18 and 4.19 .

\subsubsection{Laboratory Tests}

A rubber nembrane was applied after a specimen was removed from the plastic bag. The unconfined compression test was performed up to 1 percent axial strain with a strain rate of 1 percent per minute before the resilient modulus test was performed. Details of the unconfined compression test and data reduction are same as those for undisturbed specimens explained previously. Since it takes a significant amount of time to perform the resilient modulus test following the sequence described in AASHTO T-274 (82), a reduced number of stress combinations was used for laboratory compacted specimen. The sequence used is presented in Table 4.4.

Table 4.4 Resilient Modulus Test Sequence on Laboratory Compacted Cohesive Soils

\begin{tabular}{c|c|c|c}
\hline $\begin{array}{c}\text { Test } \\
\text { Sequence }\end{array}$ & $\begin{array}{c}\text { Confining } \\
\text { Stress (psi) }\end{array}$ & $\begin{array}{c}\text { Deviator } \\
\text { Stress (psi) }\end{array}$ & $\begin{array}{c}\text { No. of Stress } \\
\text { Repetitions }\end{array}$ \\
\hline 1 & 6 & 2 & 200 \\
\hline 2 & 6 & 4 & 200 \\
\hline 3 & 6 & 6 & 200 \\
\hline 4 & 3 & 6 & 200 \\
\hline 5 & 0 & 6 & 200 \\
\hline
\end{tabular}




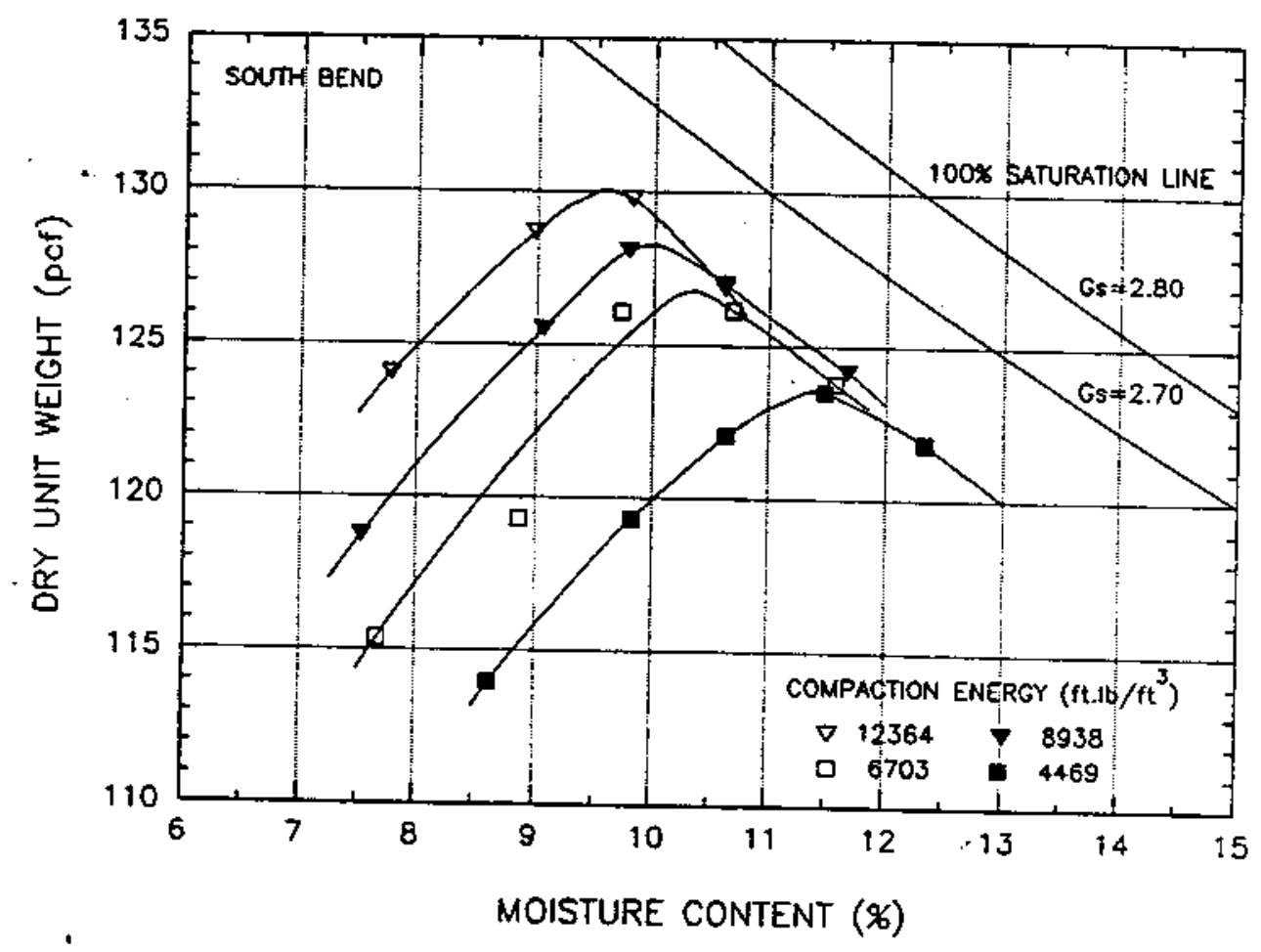

Figure 4.18 Laboratory Compaction Curves of the South Bend
Soil 


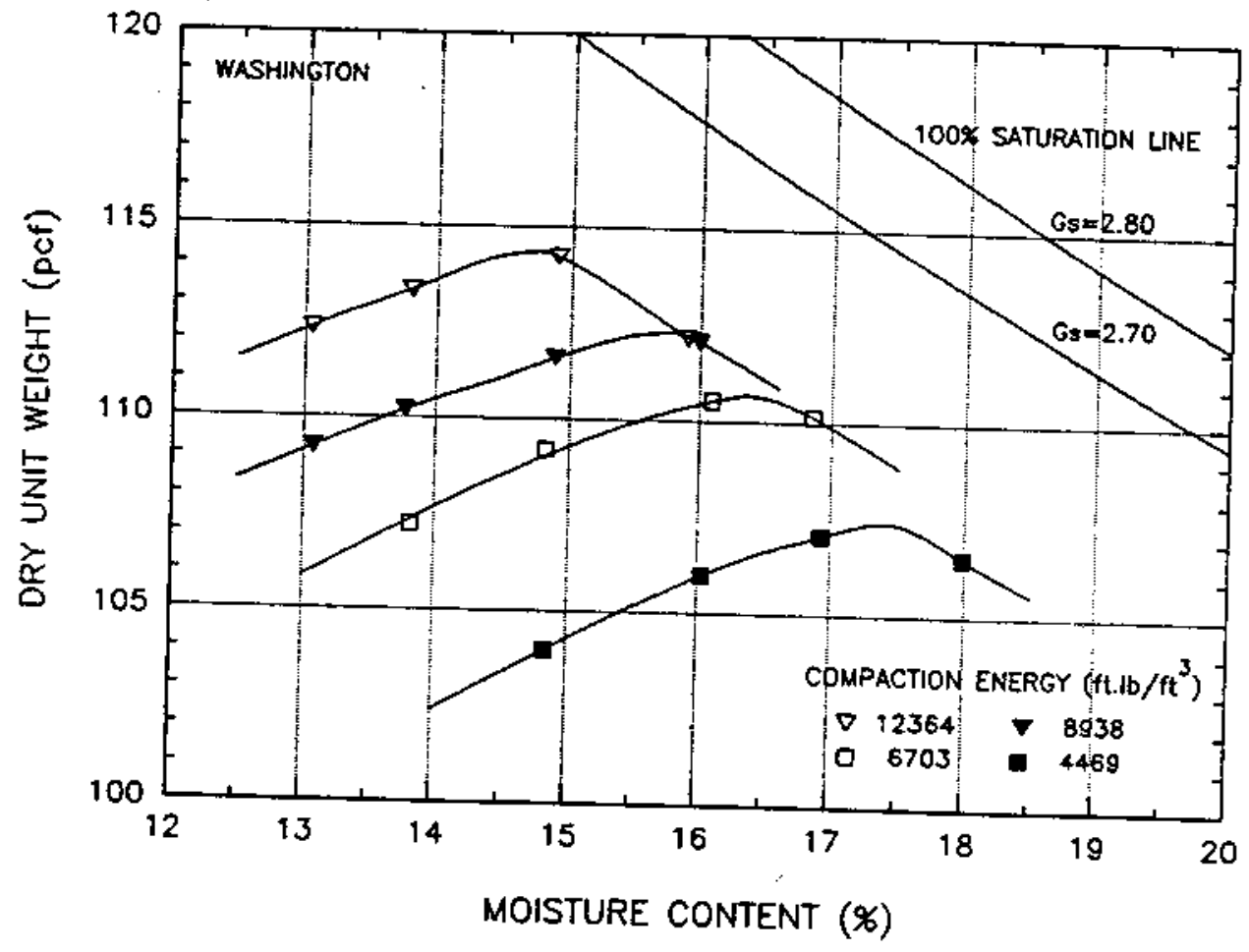

Figure 4.19 Laboratory Compaction curves of the Washington
Soil 


\subsubsection{Test Results and Discussions}

Summarized results of laboratory compaction, unconfined compression test and resilient modulus test for South Bend and washington sites are presented in Table 4.5 and 4.6, respectively.

Figures 4.20 and 4.21 are the plots of test results showing the relationship between stress causing 1 percent axial strain in the unconfined compression test and compaction water content. It is shown that the stress at 1 percent axial strain starts to decrease at an increasing rate as water content approaches 1.0 percent to 1.5 percent dry of optimum. The stress at 1 percent strain decreases more slowly near the optimum water content, beyond which it is essentially constant. A larger compactive effort produces a greater $S_{\mathrm{ul} .0 x}$ at water contents dry of optimum, while reverse is true on wet of optimum.

Figures 4.22 and 4.23 are plots showing the relationship between the resilient modulus at $\sigma_{\mathrm{d}}$ of $6 \mathrm{psi}$ and $\sigma_{3}$ of $3 \mathrm{psi}$ and the compaction moisture content of the specimen. It is noted that the change of resilient modulus versus moisture content is almost identical to the change of stress required to cause 1 percent axial strain in unconfined compression test versus water content.

To get more convenient presentation of resilient modulus, the data in Figures 4.22 and 4.23 are transferred to a standard plot of dry density versus water content. By finding the point of intersection of the curves with any horizontal line on the graph, it is possible to read off a series of corresponding values of water content and dry density which produce equal resilient moduli. These points defining a contour of resilient modulus are, then, plotted in a plot of dry density versus water content. Repeating this procedure for different values of resilient modulus results in the family of curves, as shown in Figures 4.24 and 4.25 . 
Table 4.5 Summary of Test Results on Laboratory Compacted South Bend Soil

\begin{tabular}{|c|c|c|c|c|c|c|c|c|c|c|c|c|}
\hline \multirow{2}{*}{$\begin{array}{c}\text { rest } \\
\text { ID }\end{array}$} & \multirow{2}{*}{$\begin{array}{l}w / c \\
(8)\end{array}$} & \multirow[t]{2}{*}{$\boldsymbol{\gamma}_{\boldsymbol{d}}$} & \multirow[b]{2}{*}{$\begin{array}{r}s_{0.25} \\
(\mathrm{psi})\end{array}$} & \multicolumn{2}{|c|}{ Unconfined } & \multicolumn{3}{|c|}{ Compression Test } & \multicolumn{3}{|c|}{$M_{R}$ at $\sigma_{d}=6 \mathrm{psi}$} & \multirow{2}{*}{$\begin{array}{l}\text { Compact } \\
\text { Energy } \\
\text { lbft/ft })\end{array}$} \\
\hline & & & & $\begin{array}{r}s_{0.5} \\
\text { (psi) }\end{array}$ & $\begin{array}{r}s_{\text {wo.7s }} \\
\text { (psi) }\end{array}$ & $\begin{array}{r}S_{w 1.0 x} \\
(p g i)\end{array}$ & $\begin{array}{c}E_{1} \\
(p s i)\end{array}$ & $1 / \mathrm{b}$ & $\begin{array}{r}\sigma_{3}=6 \mathrm{pai} \\
(\mathrm{psi})\end{array}$ & $\begin{array}{r}\sigma_{3}=3 \mathrm{psi} \\
(\mathrm{pgi})\end{array}$ & $\begin{array}{l}\sigma_{3}=0 \mathrm{ps} 1 \\
(\mathrm{ps} 1)(1\end{array}$ & \\
\hline SBLC01 & 7.78 & 124.04 & 17.4 & 29.5 & 38.3 & 45.0 & 8514 & 95.6 & 26024 & 21990 & 12938 & 12364 \\
\hline SBLCO2 & 8.99 & 128.70 & 9.0 & 14.8 & 20.4 & 25.2 & 3569 & 85.5 & 16835 & 13620 & 8456 & 12364 \\
\hline SBLCO3 & 9.8 & 129.79 & 1.7 & 2.7 & 3.5 & 4.1 & 794 & 8.3 & 4539 & 3710 & 3505 & 12364 \\
\hline SBLCO4 & 10.61 & 126.89 & 0.8 & 1.2 & 1.5 & 1.7 & 372 & 3.1 & 2050 & 1405 & 1315 & 12364 \\
\hline SBLC17 & 7.53 & 118.76 & 17.0 & 27.0 & 32.0 & 34.0 & 9335 & 57.3 & 21826 & 18951 & 12922 & 8938 \\
\hline SBLCO5 & 9.06 & 125.53 & 8.8 & 15.0 & 19.6 & 23.1 & 4306 & 49.6 & 16318 & 13830 & 9794 . & 8938 \\
\hline SBLC06 & 9.78 & 128.12 & 3.0 & 5.0 & 6.8 & 8.3 & 1238 & 24.8 & 9455 & 6646 & 4491 & 8938 \\
\hline SBLC07 & 10.62 & 127.03 & 0.9 & 1.3 & 2.8 & 2.0 & 408 & 4.1 & 2907 & 2993 & 3016 & 8938 \\
\hline SBLCO8 & 11.67 & 124.17 & 0.6 & 0.8 & 1.1 & 1.3 & 257 & 2.4 & 1826 & 1496 & 1305 & 8938 \\
\hline $\operatorname{seLC18}$ & 7.67 & 115.39 & 12.0 & 19.5 & 23.5 & 25.5 & 6499 & 45.0 & 17830 & 15263 & 10408 & 6703 \\
\hline SBLC12 & 8.87 & 119.31 & 10.8 & 17.0 & 21.0 & 23.8 & 5958 & 39.7 & 16619 & 13450 & 9265 & 6703 \\
\hline sBLC09 & 9.72 & 126.08 & 4.0 & 6.4 & 8.7 & 10.6 & 1639 & 29.8 & 11270 & 8254 & 5523 & 6703 \\
\hline SBLC10 & 10.69 & 126.15 & 1.4 & 2.0 & 2.5 & 3.0 & 601 & 5.8 & 3913 & 3510 & 3530 & 6703 \\
\hline SBLC11 & 11.57 & 123.76 & 0.7 & 1.0 & 1.3 & 1.5 & 334 & 2.6 & 1955 & 1800 & 1450 & 6703 \\
\hline SBLC19 & 8.63 & 113.98 & 8.2 & 12.6 & 15.5 & 17.0 & 4665 & 27.3 & 12699 & 10212 & 6720 & 4469 \\
\hline $\operatorname{seLC13}$ & 9.82 & 119.32 & 5.4 & 8.5 & 10.6 & 12.1 & 2909 & 20.7 & 11601 & 8716 & 5578 & 4469 \\
\hline SBLCI4 & 10.64 & 122.05 & 3.0 & 4.9 & 6.2 & 7.1 & 1575 & 12.9 & 9568 & 6891 & 4591 & 4469 \\
\hline SBLC15 & 11.48 & 123.48 & 1.2 & 1.7 & 2.1 & 2.4 & 542 & 4.4 & 3378 & 3015 & 2824 & 4469 \\
\hline SBLC16 & 12.34 & 121.82 & 0.5 & 0.7 & 0.9 & 1.0 & 206 & 2.0 & 1500 & 1500 & 1500 & 4469 \\
\hline
\end{tabular}


Table 4.6 Sumary of Test Results on Laboratory Compacted Washington Soil

\begin{tabular}{|c|c|c|c|c|c|c|c|c|c|c|c|c|}
\hline $\begin{array}{c}\text { Test } \\
\text { ID }\end{array}$ & $\begin{array}{l}w / c \\
\text { (*) }\end{array}$ & $\begin{array}{c}\gamma_{d} \\
\text { (pcf) }\end{array}$ & $\begin{array}{r}s_{w 0.25} \\
(p=1)\end{array}$ & $\begin{array}{r}\text { Unce } \\
s_{\infty, 5} \\
(p=1)\end{array}$ & $\begin{array}{c}\text { nf Ined } \\
s_{w .75} \\
\text { (pei) }\end{array}$ & $\begin{array}{c}\text { Compres } \\
s_{\mathrm{ul} .0 *} \\
\text { (pei) }\end{array}$ & $\begin{array}{c}\text { Bion } T \\
E_{i} \\
\text { (psi) }\end{array}$ & $1 / b$ & $\begin{array}{r}\sigma_{3}=6 \mathrm{ps} 1 \\
(\mathrm{pBI})\end{array}$ & $\begin{array}{c}M_{k} \text { at } \sigma_{d}=6 \\
\sigma_{3}=3 \mathrm{psi} \\
\text { (psi) }\end{array}$ & $\begin{array}{l}\sigma_{3}=0 p 81 \\
\quad(p a 1)(1\end{array}$ & $\begin{array}{l}\text { Compact } \\
\text { Energy } \\
\text { (bft/ft3) }\end{array}$ \\
\hline WALCO1 & 13.05 & 112.32 & 12.0 & 20.9 & 27.7 & 32.0 & 5628 & 80.7 & 14835 & 12303 & 7646 & 12364 \\
\hline WALCO2 & 13.81 & 113.32 & 8.7 & 15.9 & 22.0 & 27.1 & 3849 & 91.8 & 14542 & 12017 & 6969 & 12364 \\
\hline WALCO3 & 14.90 & 114.23 & 2.6 & 4.4 & 6.3 & 7.9 & 988 & 40.1 & 9860 & 7397 & 4608 & 12364 \\
\hline WALCO4 & 15.90 & 112.15 & 1.0 & 1.5 & 1.9 & 2.3 & 448 & 4.5 & 3163 & 2643 & 2100 & 12364 \\
\hline WALC05 & 13.07 & 109.20 & 10.3 & 17.6 & 23.0 & 27.0 & 4953 & 60.4 & 14614 & 12460 & 8174 & 8938 \\
\hline WALC06 & 13.77 & 110.20 & 9.5 & 16.1 & 20.9 & 24.0 & 4682 & 51.6 & 13228 & 10359 & 6323 & 8938 \\
\hline WALCO 7 & 14.89 & 111.59 & 6.5 & 11.7 & 15.9 & 19.4 & 2932 & 57.7 & 12344 & 9953 & 6540 & 8938 \\
\hline WALCO8 & 15.99 & 112.06 & 1.5 & 2.4 & 3.2 & 3.8 & 632 & 9.5 & 6023 & 3993 & 2966 & 8938 \\
\hline WALCO9 & 13.82 & 107.23 & 9.6 & 15.3 & $19.0^{\circ}$ & 21.7 & 5157 & 37.4 & 12505 & 10610 & 7064 & 6703 \\
\hline WALCIO & 14.83 & 109.19 & 8.2 & 13.0 & 16.2 & 18.5 & 4391 & 32.0 & 12582 & 10221 & 6301. & 6703 \\
\hline WALC11 & 16.09 & 110.54 & 2.7 & 4.8 & 6.8 & 8.6 & 1073 & 42.4 & 9793 & 7345 & 4603 & 6703 \\
\hline WALC12 & 16.86 & 110.15 & 1.2 & 1.8 & 2.4 & 2.9 & 469 & 7.7 & 4577 & 3068 & 2549 & 6703 \\
\hline WALC13 & 14.84 & 104.00 & 6.9 & 10.5 & 12.5 & 13.9 & 4379 & 20.3 & 10170 & 8217 & 5402 & 4469 \\
\hline WALC14 & 26.03 & 105.99 & 5.9 & 9.1 & 11.0 & 12.3 & 3460 & 19.1 & 10232 & 7915 & 5140 & 4469 \\
\hline WALC̣15 & 16.93 & 107.05 & 3.7 & 6.3 & 8.2 & 9.7 & 1776 & 21.2 & 9060 & 6863 & 4459 & 4469 \\
\hline WALC19 & 17.99 & 106.51 & 1.6 & 2.6 & 3.6 & 4.6 & 602 & 18.7 & 6701 & 4625 & 3036 & 4469 \\
\hline
\end{tabular}




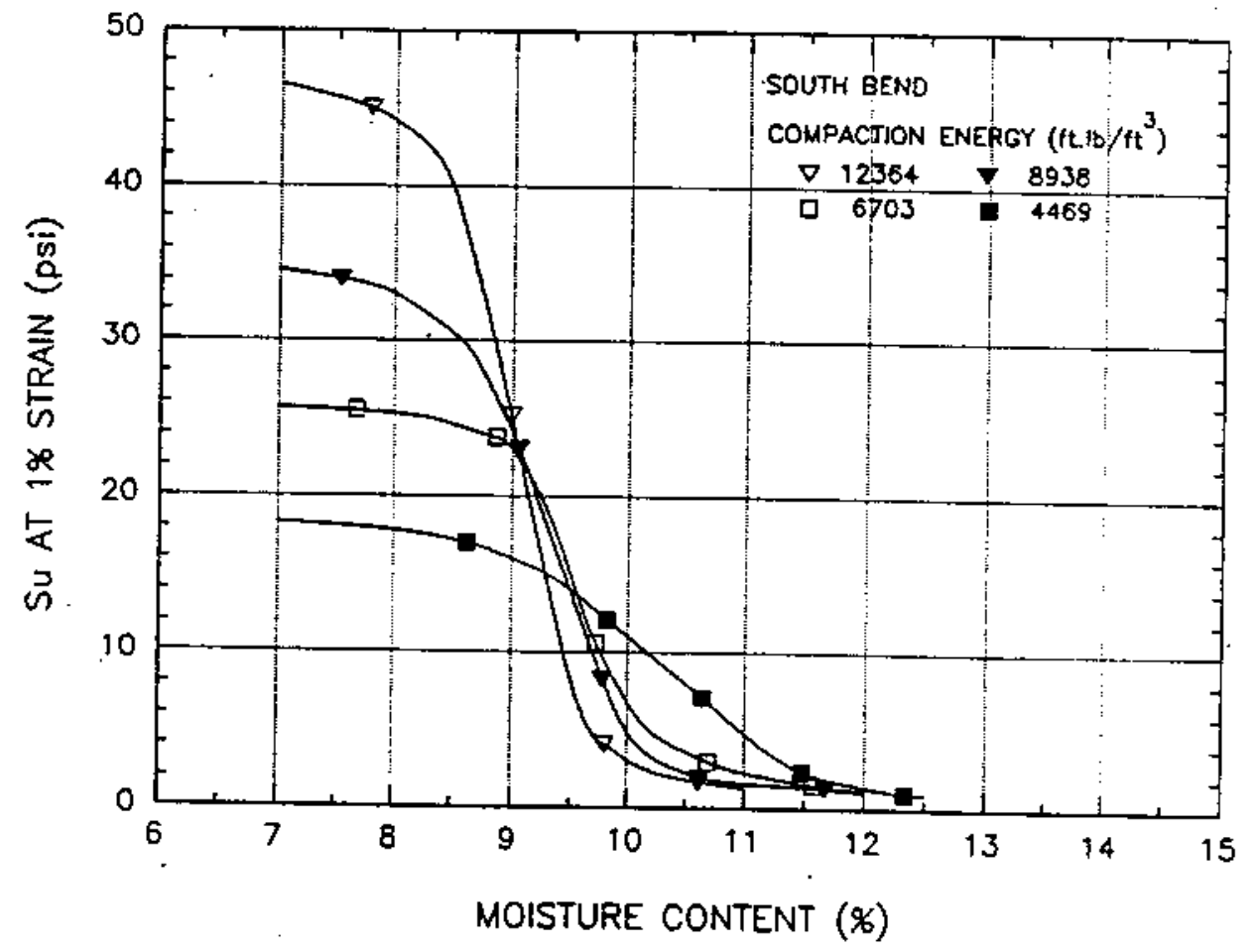

Figure 4.20 Relationship between $S_{w 1.0 \%}$ and water Content of the Laboratory Compacted South Bend soil 


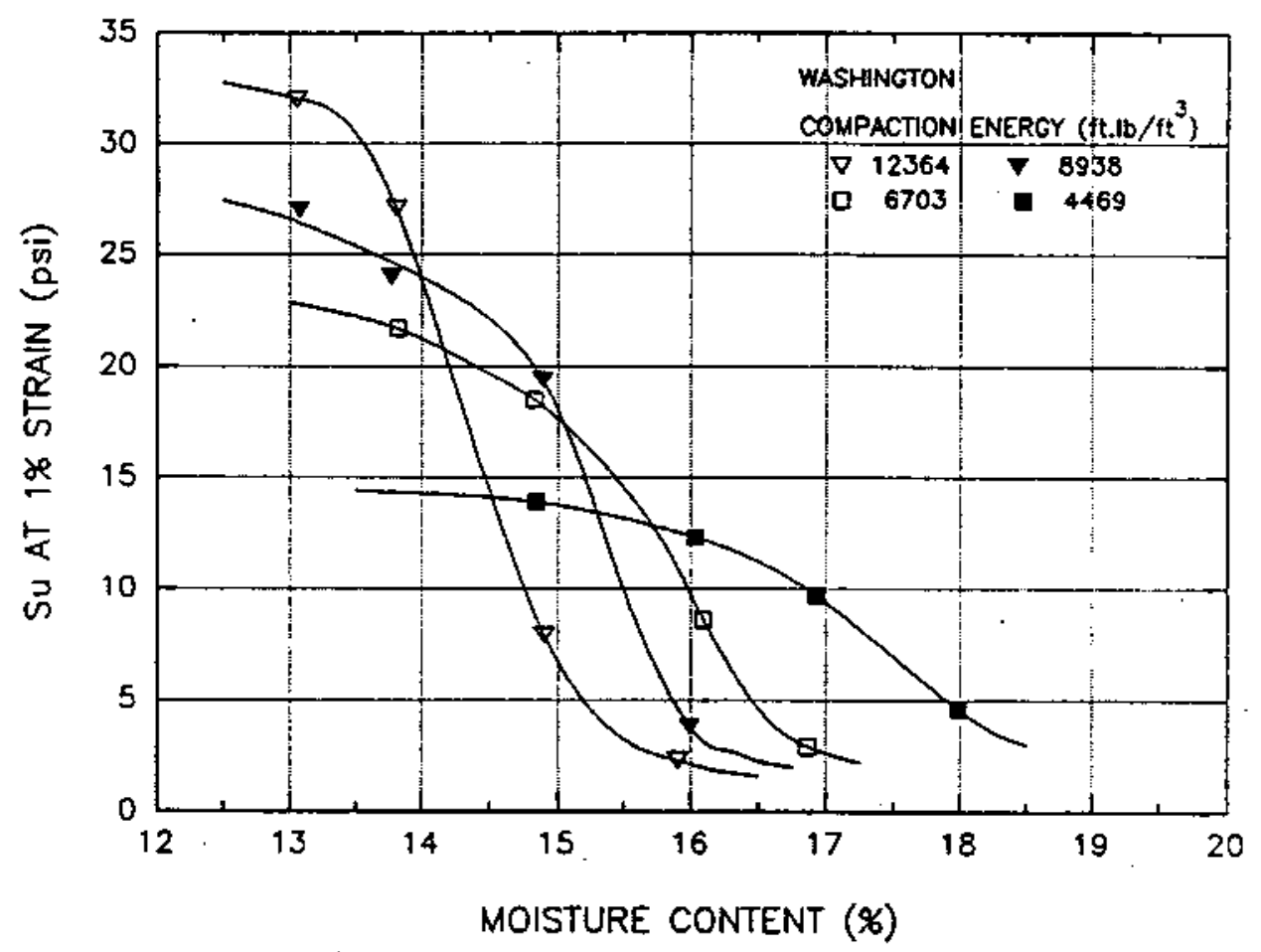

Figure 4.21 Relationship between $S_{n 1.0 x}$ and Water Content of the Laboratory Compacted Washington Soil 


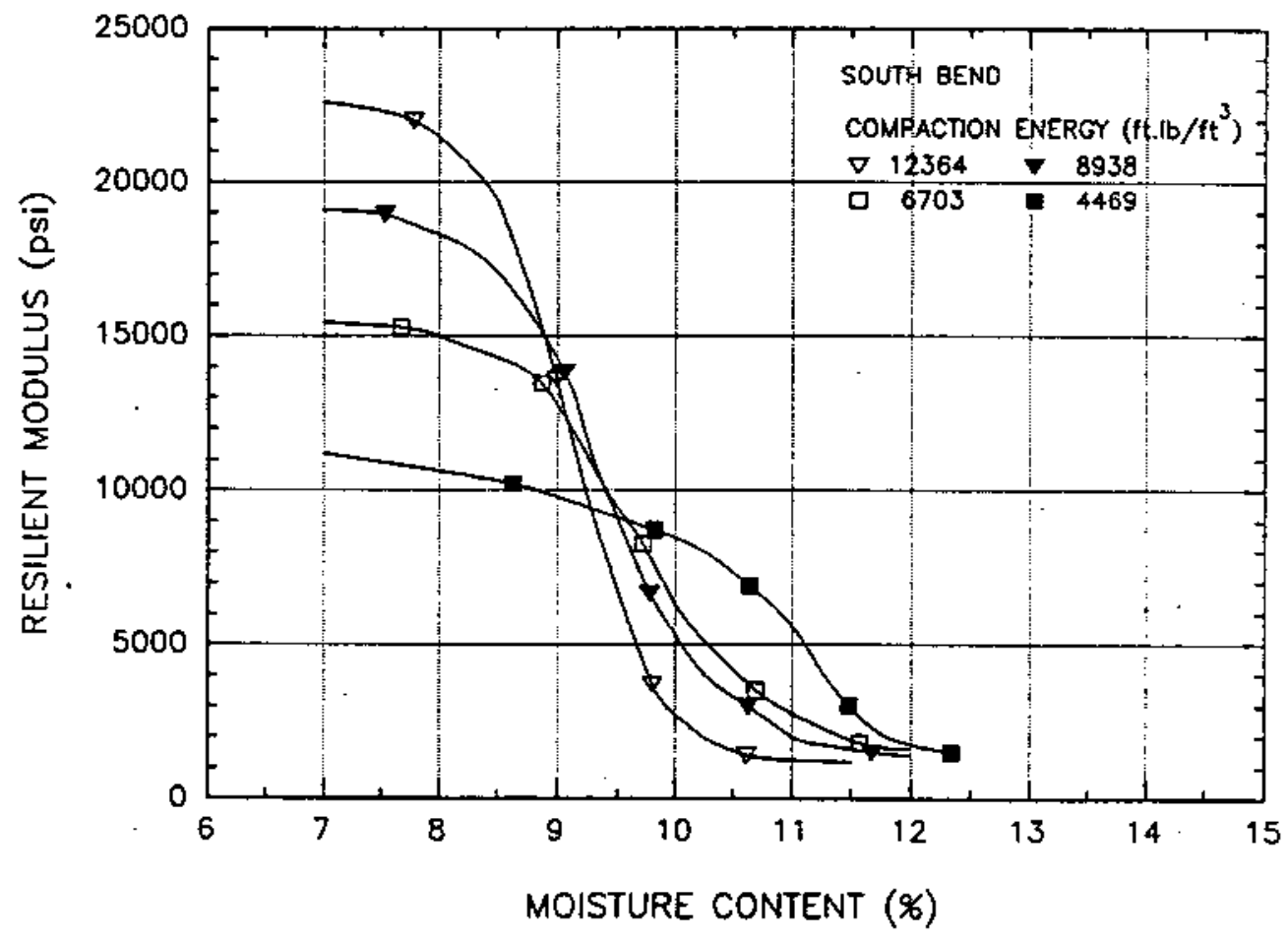

Figure 4.22 Relationship between $M_{R}$ and water content of the Laboratory Compacted South Bend Soil 


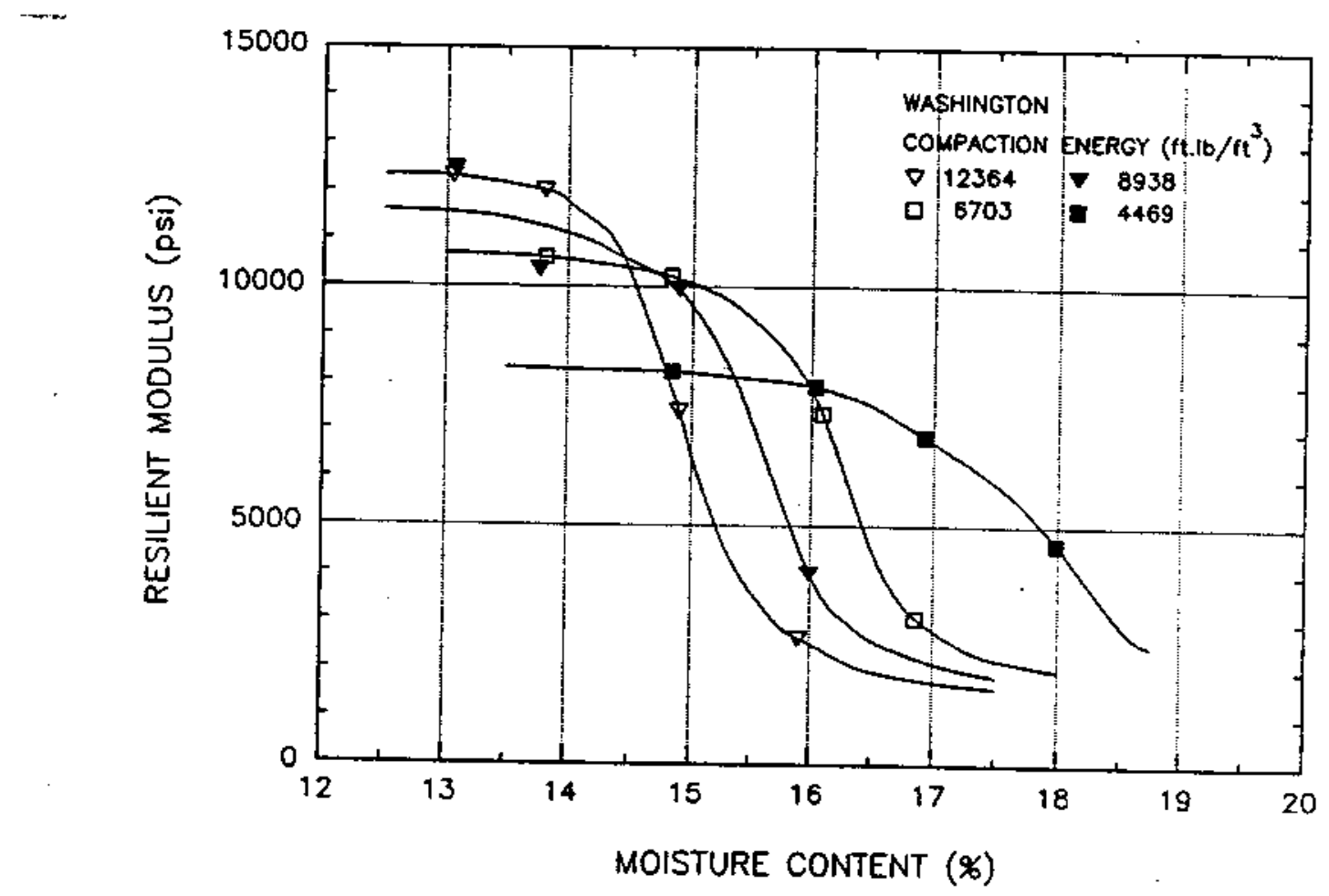

Figure 4.23 Relationship between $M_{R}$ and water content of the Laboratory Compacted washington Soil 


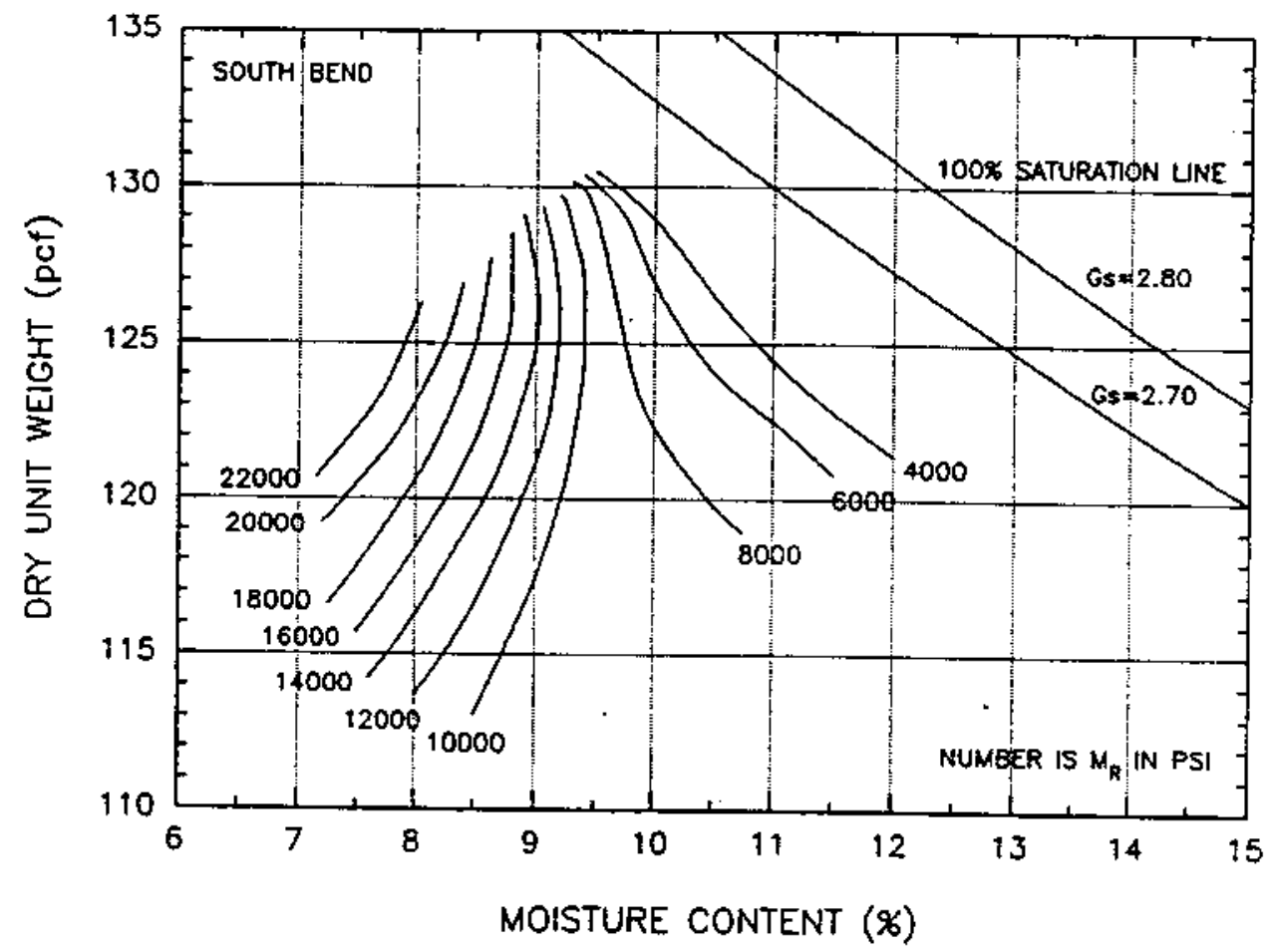

Figure 4.24 Contour of Resilient Modulus for the Laboratory
Compacted South Bend Soil 


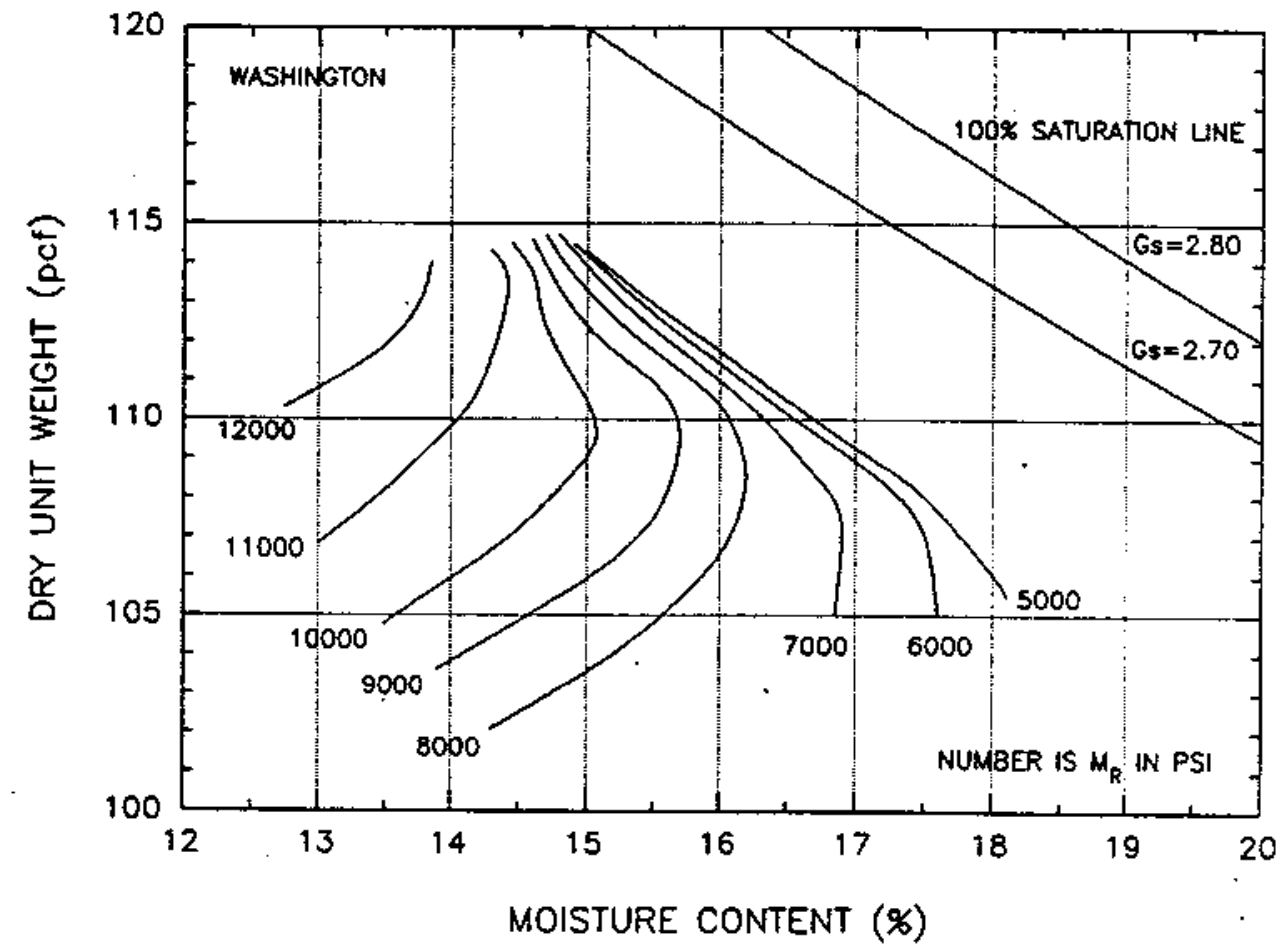
Figure 4.25 Contour of Resilient Modulus for the Laboratory
Compacted Washington Soil 
From this type of plot, the influence of compaction conditions on as-compacted resilient modulus can readily be determined. For conditions on the wet side of optimum, the contour of resilient modulus is approximately parallel to the 100 percent saturation line. This implies that compaction on wet side of the optimum would give a low value of resilient modulus regardless of compactive effort and, therefore, the degree of saturation may be a good indicator of resilient modulus. It is also shown that a change of compaction condition has the smaller influence on resilient modulus when the soil is compacted with lower compactive effort. The Washington site shows that small changes of water content and dxy density produce large effects on the resilient characteristics when the soil is compacted wet of optimum. Comparison of soil fabrics at different compactive efforts for the South Bend and Washington sites (Bohra, 1993) reveals that the compactive efforts have little effect on the fabric created at about optimum water content for the South Bend site and for a wider range of water content for the washington site. Insensitivity of fabric changes for these sites causes water content to be a significant factor influencing resilient modulus values. It is also noted that, for some region of water content and ary density, higher compactive efforts gives lower resilient modulus at same water content.

since it was observed that the resilient modulus and stress required to cause 1 percent strain in unconfined compression test vary with water content in a similar manner, both variables are belleved to offer good correlation. Figures 4.26 and 4.27 are the plots of resilient modulus versus $S_{u 1.0 \times}$ for the South Bend and Washington sites. Plots for both sites are identical up to $s_{w 1.0 *}$ of $20 \mathrm{psi}$ while the resilient modulus of South Bend soil is slightly larger than that of Washington soil as $S_{\text {ul.0x }}$ increases. It is noted that test results on specimens compacted with four different compactive efforts fit well in a single relationship between $M_{R}$ and $s_{01.0 x}$, and that 


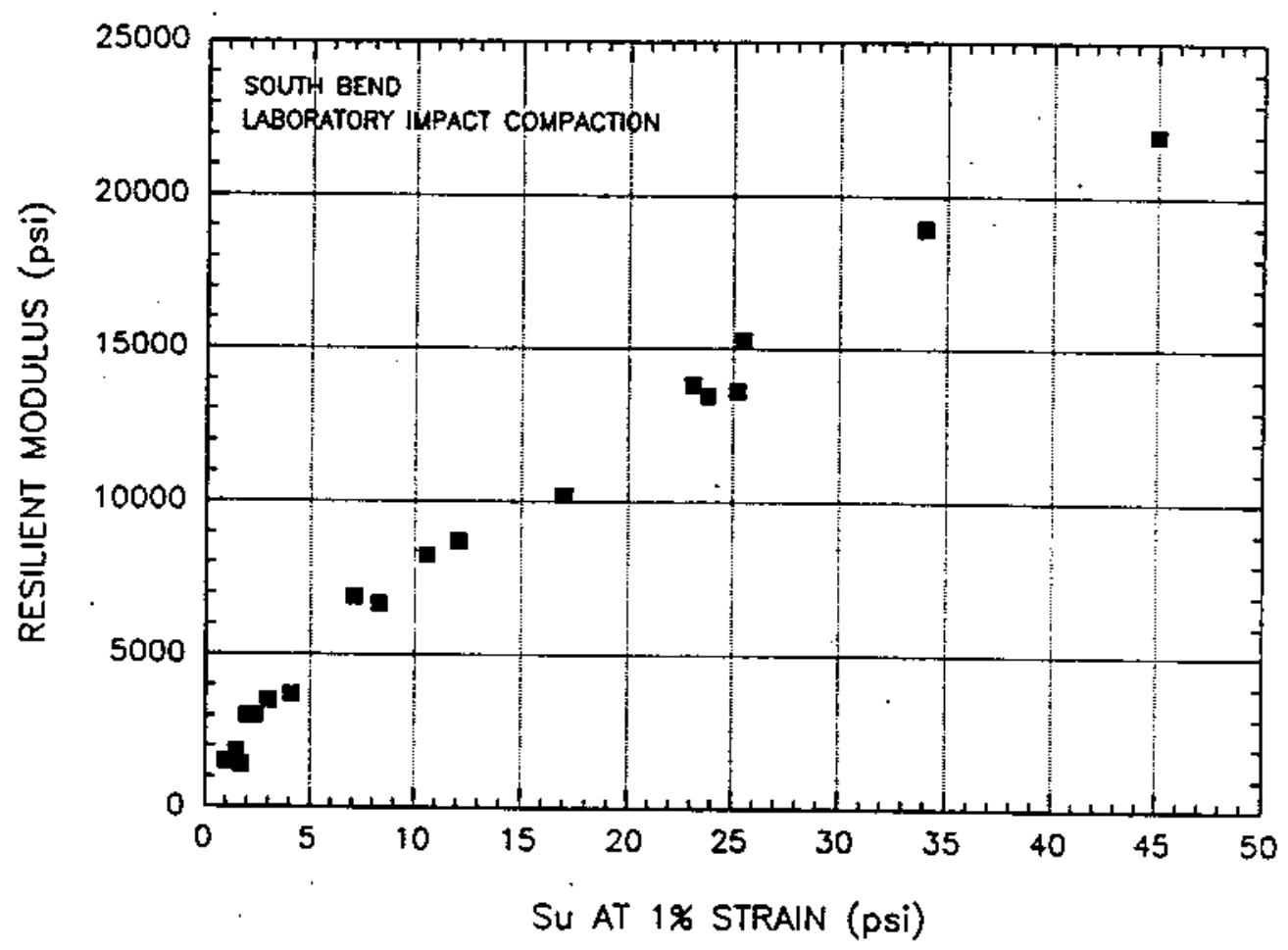

Figure 4.26 Relationship between $M_{R}$ and $S_{\mathrm{ut}, 0 x}$ for the Laboratory Compacted South Bend Soil 


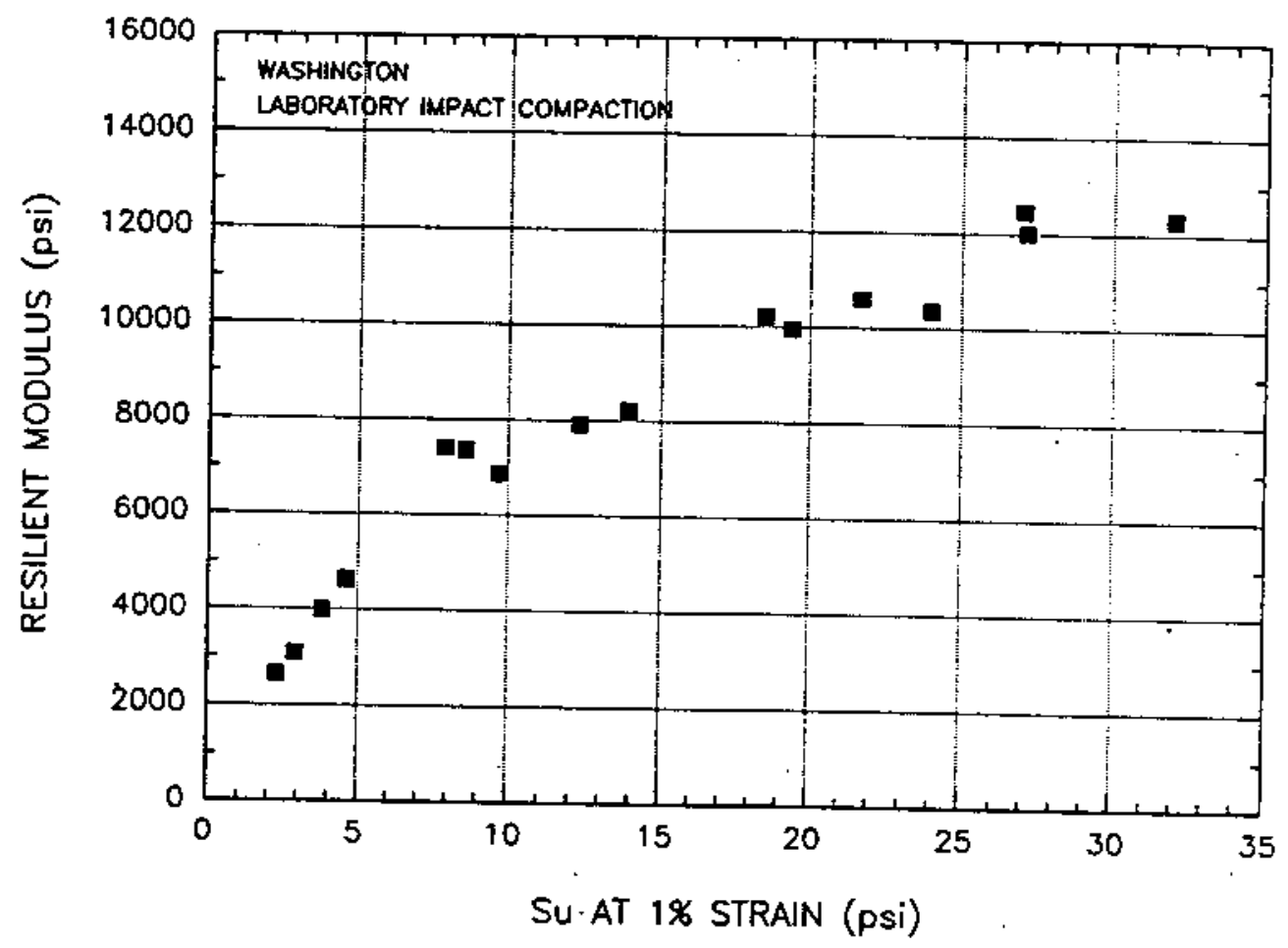

Figure 4.27 Relationship between $M_{R}$ and $s_{u t .0 x}$ for the Laboratory Compacted Washington soil 
the relationship appears unique for a given soil. Therefore, it is concluded that the relationship between $M_{R}$ and $S_{u l .0 x}$ for a given soil can be obtained by conducting resilient modulus tests on a series of four. or five specimens compacted at different molding water content with the same compactive effort.

To look at the possibility of a unified relationship between the resilient modulus and the stress at 1 percent strain in unconfined compression test, four specimens of the Bloomington soil were compacted with standard Proctor energy, and tested for the resilient modulus. All the test results from 3 sites are plotted in Figure 4.28. It can be seen that the relationship between $M_{R}$ and $S_{1.0 x}$ of the Bloomington soil is similar to that of South Bend and Washington sites. Three different soils $(A-4, A-6$, and $A-7-6)$ are found to have a similar relationship on laboratory compacted soil, and the relationships between $H_{R}$ and $S_{u 1.0 \pi}$ of four field compacted soils were observed similar. Therefore, it is expected that the other two sites have a similar relationship for the laboratory compacted soils.

The relationship between $M_{k}$ and $s_{* 1.0 *}$ applicable to all the soils studied is developed by multiple regression as follows:

$$
M_{R}=a S_{u 1.08}+b S_{u 1: 08}{ }^{2}
$$

where $M_{R}$ is resilient modulus at deviator stress of 6 psi (psi); $s_{\text {ot.ox }}$ is stress causing 1 percent unconfined compressive strain (psi); a and $b$ are regression parameters given in Table 4.7. $R^{2}$ and Root MSE are also presented in Table 4.7. It should be noted that the $S_{v 1.0 x}$ refers to same specimen for which the resilient modulus is wanted. 


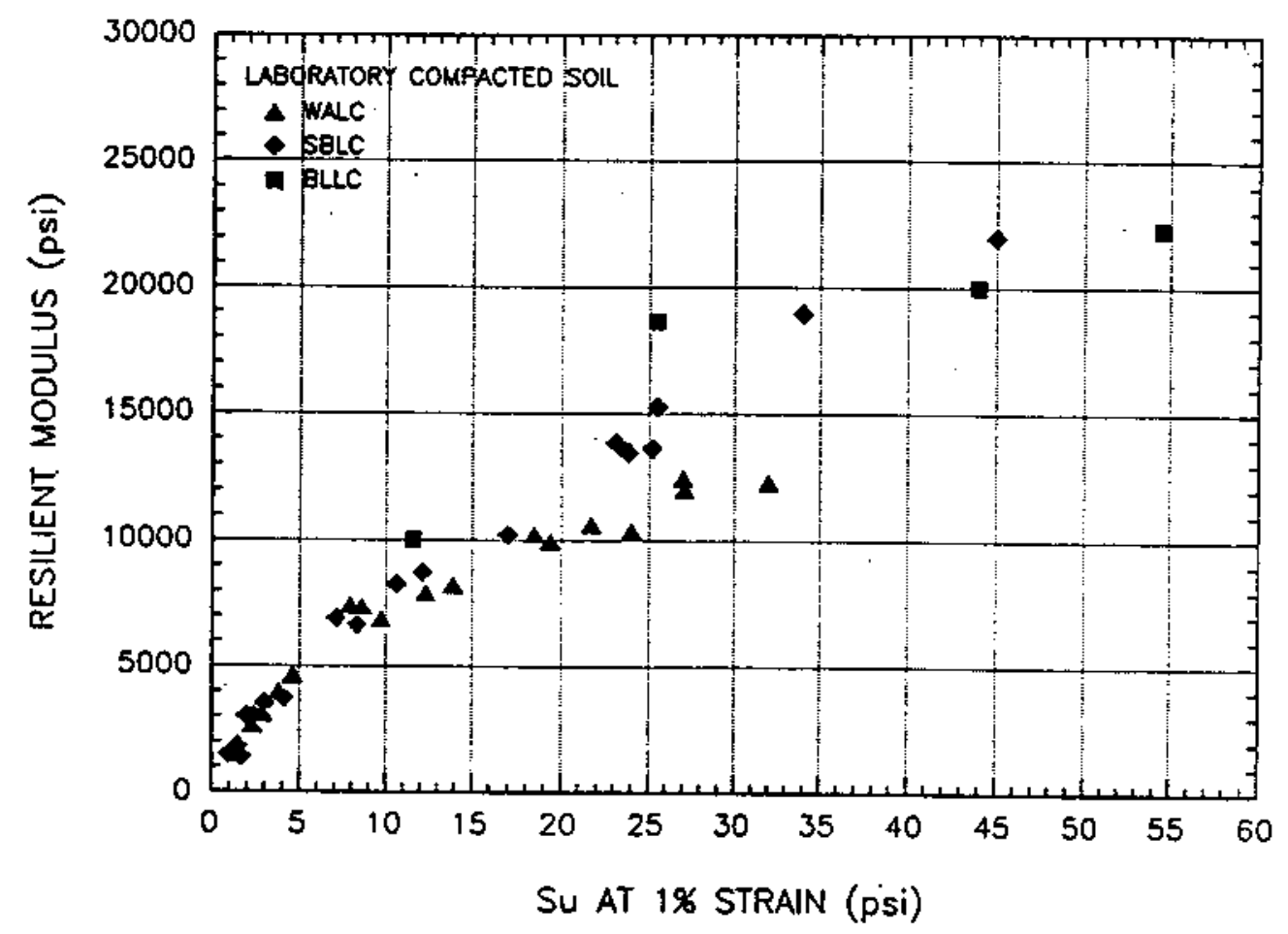

Figure 4.28 . Relationship between $M_{R}$ and $S_{\mathrm{w1.0 \%}}$ for the
Laboratory Compacted Soils 
Table 4.7 Regression Parameters for Equation 4.6

\begin{tabular}{c|c|c|c|c}
\hline $\mathrm{M}_{\mathrm{R}}$ at & $\mathrm{a}$ & $\mathrm{b}$ & $\mathrm{VMSE}$ & $\mathrm{R}^{2}$ \\
\hline$\sigma_{\mathrm{d}}=6, \sigma_{3}=6 \mathrm{psi}$ & 905.6074 & -9.35894 & 2211 & 0.967 \\
\hline$\sigma_{\mathrm{d}}=6, \sigma_{3}=3 \mathrm{psi}$ & 695.3604 & -5.92966 & 1642 & 0.973 \\
\hline$\sigma_{d}=6, \sigma_{3}=0 \mathrm{psi}$ & 487.0301 & -5.18183 & 1365 & 0.956 \\
\hline
\end{tabular}

To check the reliability of proposed prediction equation, the resilient moduli at $\sigma_{d}$ of $6 \mathrm{psi}$ and $\sigma_{3}$ of $3 \mathrm{psi}$ calculated by the proposed equation are compared with measured resilient modulus. As shown in Figure 4.29, Equation 4.6 gives reasonable estimation of resilient modulus for Washington, South Bend, and Bloomington soils. The Equation 4.6 may be used to estimate the resilient modulus of the other two sites.

4.5 Resilient Properties of Laboratory and Field Compacted
Soil

4.5.1 Comparison of Laboratory and Field Compaction

The primary purpose of laboratory compaction is to produce the compacted specimen having identical properties with the field compacted soil. Presently, it seems that no satisfactory laboratory compaction method exists that can exactly duplicate field compaction although many different laboratory methods have been developed in an attempt to simulate field compaction.

Waterways Experimental station (1949) conducted comprehensive laboratory and field investigations on a compacted vicksburg silty clay. It was shown that the strength of the laboratory compacted samples were quite different from the strength of the field compacted samples for both soaked 


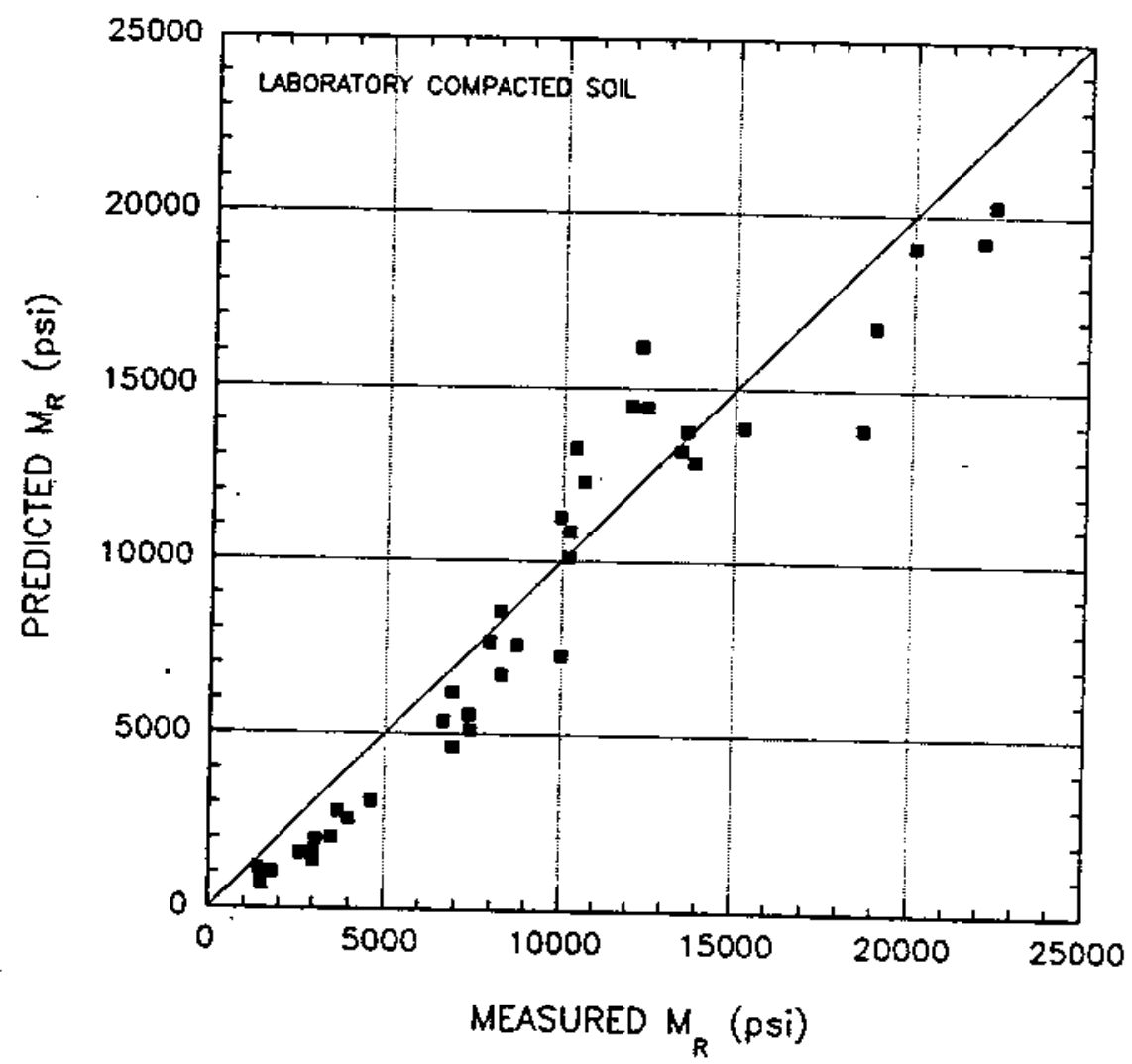
Figure 4.29 Comparison between Measured and Predicted $M_{R}$ of
Laboratory Compacted Soils 
and unsoaked conditions. Because the moisture-density conditions were in general quite different for the field compacted samples and the laboratory compacted samples, it was difficult to isolate the effect of the method of compaction. Therefore, it was concluded that shear strength of laboratory compacted samples may or may not represent the strength of the field compacted samples, and that the differences in strength must be attributed to the differences in the material rather than differences in the dry density and molding water content. Johnson and Sallberg $(1960,1962)$ and wahls et al. (1966) reviewed previous studies as an effort to correlate laboratory and field compaction results, and showed that the attempt to devise a laboratory compaction test method that simulates field compaction was unsuccessful. It was noted that the line of optimums for field and laboratory compaction often do not coincide and the relative position of the lines with respect to the line of 100 percent saturation varied for various laboratory and field compaction methods and for various soil types.

For the purpose of predicting field compactor performance, selig (1971) developed a set of equations defining compactive effort of the four basic classes of roller. Equations were derived for the compactive effort per unit volume of soil, production rate in volume of soil compacted per unit time, and horsepower required to provide the towing force at desired speed. Though further study is required for better estimation of the coefficients in the proposed equations, the approach is suitable for a preliminary compactor analysis and it is believed to be the best approach available.

Numerous investigators, such as Lambe (1958), Olson (1963), and seed and Chan (1959) have recognized that, in case of compacted cohesive soils, similarities in molding water content and dry density do not necessarily imply similarities in engineering properties of the soil. When a soil is 
compacted, whether in a laboratory or in the field, the type of compaction procedure used would introduce different amount of shearing strains as the compaction energy is added. Differences in soil structures due to shearing strains is believed to be the cause of variations in engineering properties of the material of similar water content and dry density.

A series of studies on the compacted soil has been performed at Purdue University to create a better predictability of behavior of soils compacted in the field. Nwaboukei and Lovell (1984) summarized the studies on St. croix clay and expressed shear strength parameters as functions of the relevant initial compaction variables. From the statistical analysis between field and laboratory parameters, the procedure for predicting the shear strength behavior of field compacted soil from laboratory compacted data was also developed.

White (1980) conducted pore-size distribution measurements on the st. Croix clay to investigate a possible source of the differences in soil fabric between the laboratory and field compacted soil. It was concluded that the fabric of the laboratory compacted soil was significantly different from that of the field compacted soil. The difference was more pronounced at water contents on the dry side of optimum. It was also pointed out that laboratory impact and kneading compaction produced similar soil fabric at wet of optimum. Differences in fabric. appear to exist between the soils compacted on the dry side of optimum in the field by Rascal and Caterpillar compactors. Prapaharan et al. (1991) examined the data of White (1980) and concluded that no laboratory procedure appears capable of always reproducing the fabric of field compacted soil. According to these data, laboratory compaction by impact and kneading procedures produced the same fabric, and this fabric might simulate that produced in the field when compacted on the wet side of optimum at the same energy level. 
4.5.2 Resilient Modulus of Laboratory and Field Compacted Soils

To evaluate the relationship between the resilient characteristics of soils compacted in the field and the laboratory, seed et al. (1962) took undisturbed samples from the subgrade of AASHO test road three to five years after construction. By comparing the resilient modulus of undisturbed samples with the ranges of modulus values for samples prepared by laboratory compaction, they concluded that the properties of clays compacted by rubber-tire rollers are very similar to those prepared in the laboratiory by kneading compaction and, therefore, these two compaction methods induce similar structures in the compacted soil. However, Lee et al. (1992) reinterpreted the above test results by comparing the field resilient modulus with resilient modulus estimated from test results on laboratory compacted soils for same water content and dry density as in-service subgrade; they showed that the estimated resilient modulus was considerably larger than that of the field resilient modulus, as shown in Figure 4.30. Therefore, it appears reasonable to conclude that the $M_{R}$ of in-service subgrade can not be estimated from the test result of laboratory compacted specimen without considering the effect of water content change after construction.

Due to the lack of data on as-compacted resilient modulus for field compacted soils, the possibilities for prediction of as-compacted field resilient modulus from the data on laboratory compacted soil are investigated indirectly by analyzing test data on three different sites which have available resilient modulus data.

The resilient modulus is estimated for average ascompacted conditions of six loops of the AASHO test road and compared with in-service resilient modulus reported by seed et al. (1962) and Monismith et al. (1967). As can be seen in Figure 4.31, in-service condition of untrafficked loops moved 


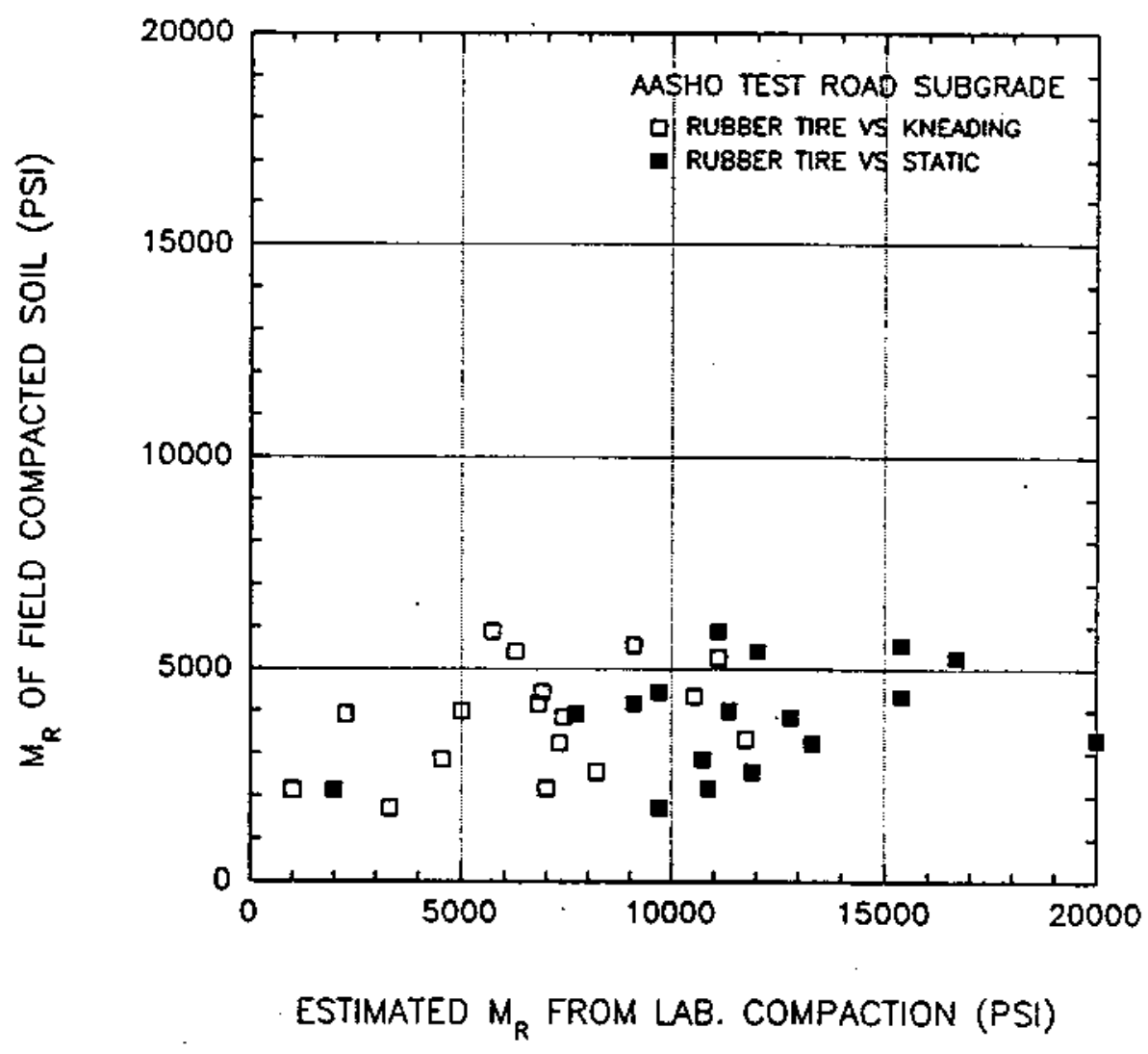

Figure 4.30 Comparison of Resilient Modulus between Field and Laboratory Compacted Soil of AASHO Test Road 

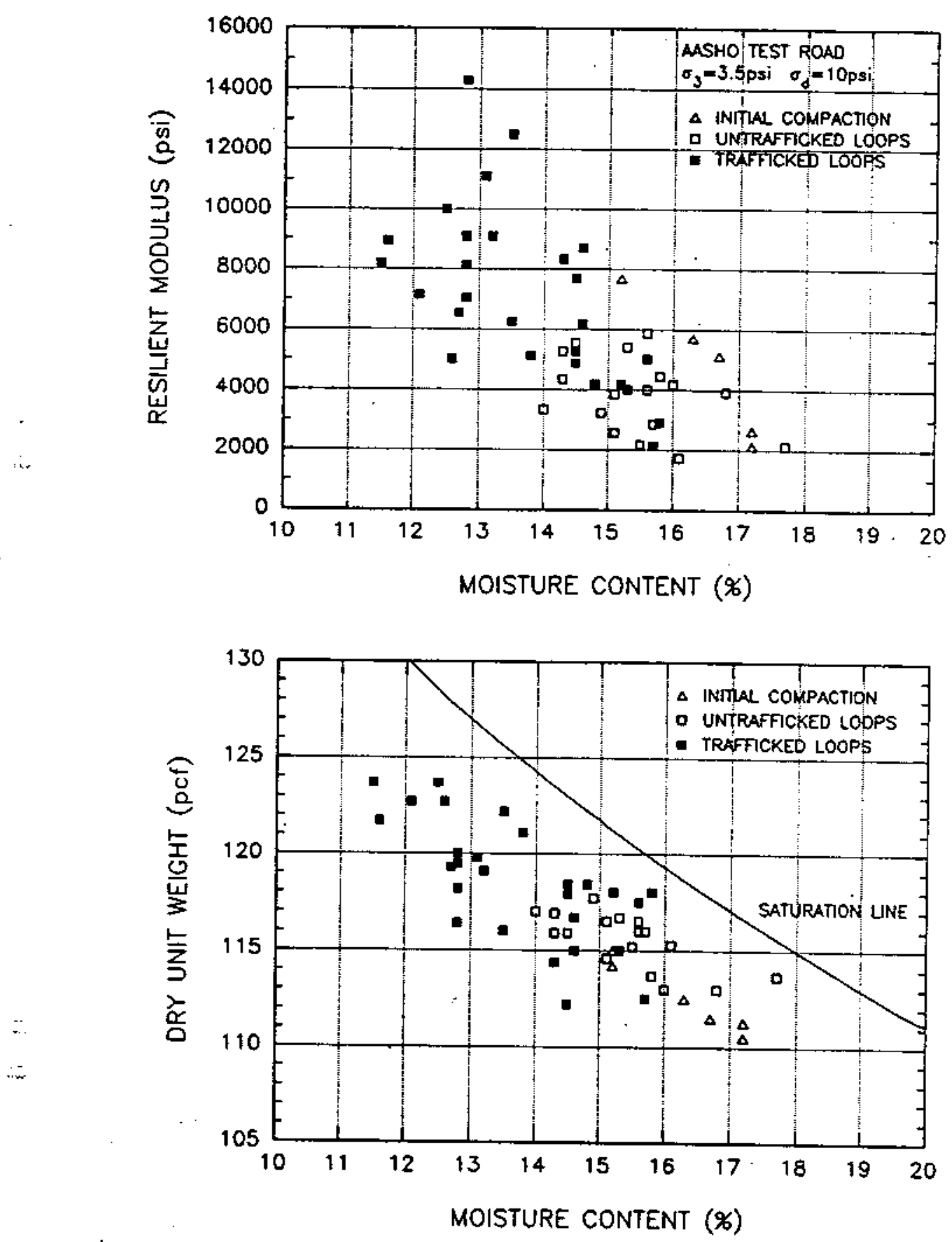

Figure 4.31 Resilient Modulus of AAsHO Test Road 
to slightly lower water content and higher dry density from as-compacted condition, while trafficked loops were subjected to larger decrease in water content and increase of dry density after construction. Estimated as-compacted resilient modulus appears slightly larger than or equal to in-service modulus of untrafficked loops, while it is significantly lower than in-service modulus of trafficked loops.

A similar trend is observed on the data of the South Bend site where water content decreased and density increased after construction. As shown in Figure 4.32, in-service resilient modulus measured by repeated load triaxial test is significantly larger than as-compacted modulus estimated from Figure 4.24 with average water content and dry density for each lift during construction.

At the Washington site where there was a significant increase in water content, a different trend is evident. As shown in Figure 4.33, in-service resilient modulus is about 20 to 30 percent lower than estimated as-compacted modulus up to depth of 40 inches where special subgrade treatment was done during construction with relatively lower water content. Below 40 inch in depth, the trend in changes of resilient modulus is not clear due to the lack of data and large scatter.

From the above observations, it can be seen that the increment of water content after construction causes the reduction in resilient modulus, and that the decrease of water content with increase of dry density increases the resilient modulus. Since this observed trend is appropriate in the engineering sense, it can be concluded indirectly that the resilient modulus of laboratory compacted specimen may represent the as-compacted field resilient modulus. As mentioned before, however, laboratory resilient modulus is significantly different from in-service resilient modulus if there has been a change in water content and dry density after construction. Prediction of resilient modulus based on fabric parameters and water content (Chapter 9) also suggests that 


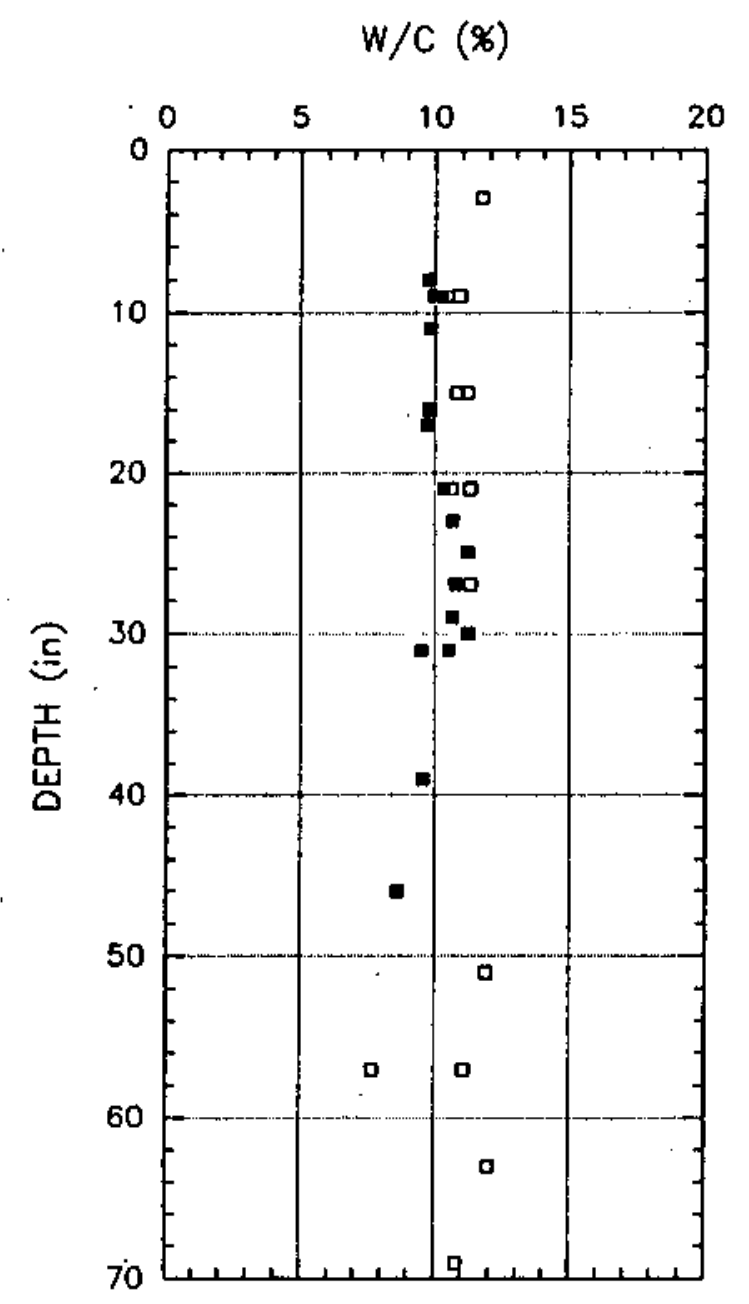

DRY UNIT WEIGHT (pcf)

RESILIENT MODULUS (psi)
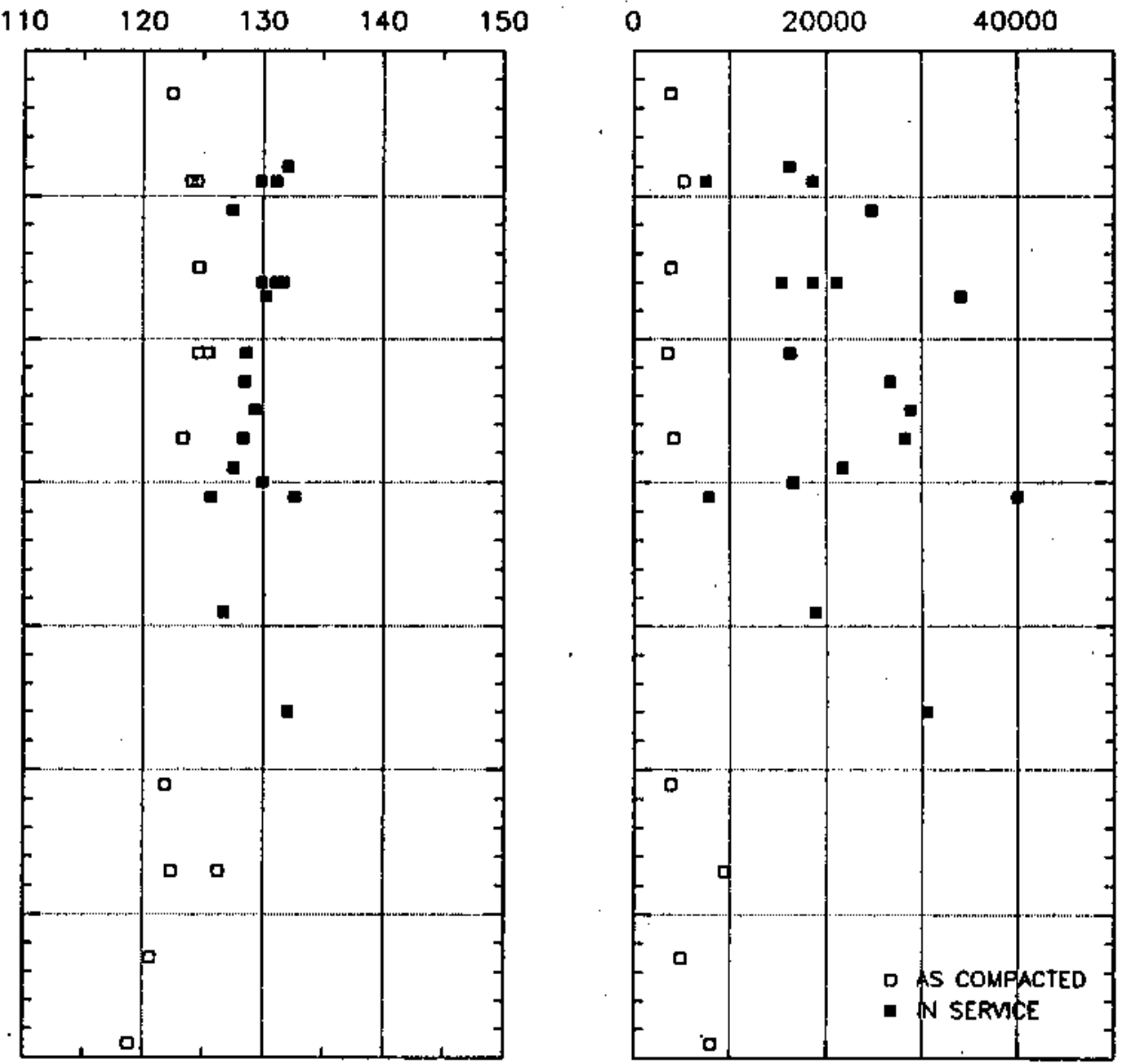

Figure 4.32 As-Compacted and In-Service Resilient Modulus of the South Bend Soil 

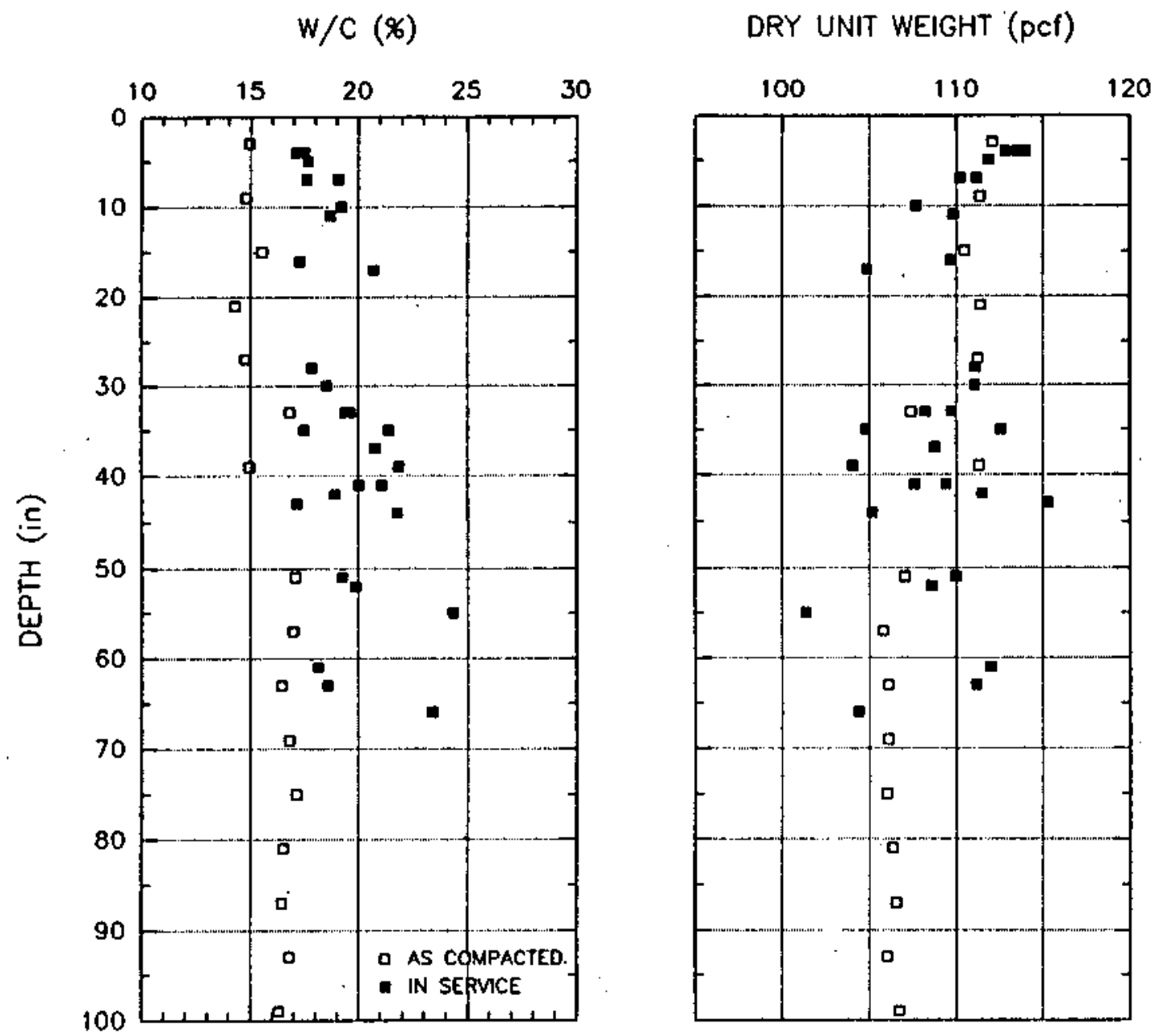

RESILIENT MODULUS (psi)

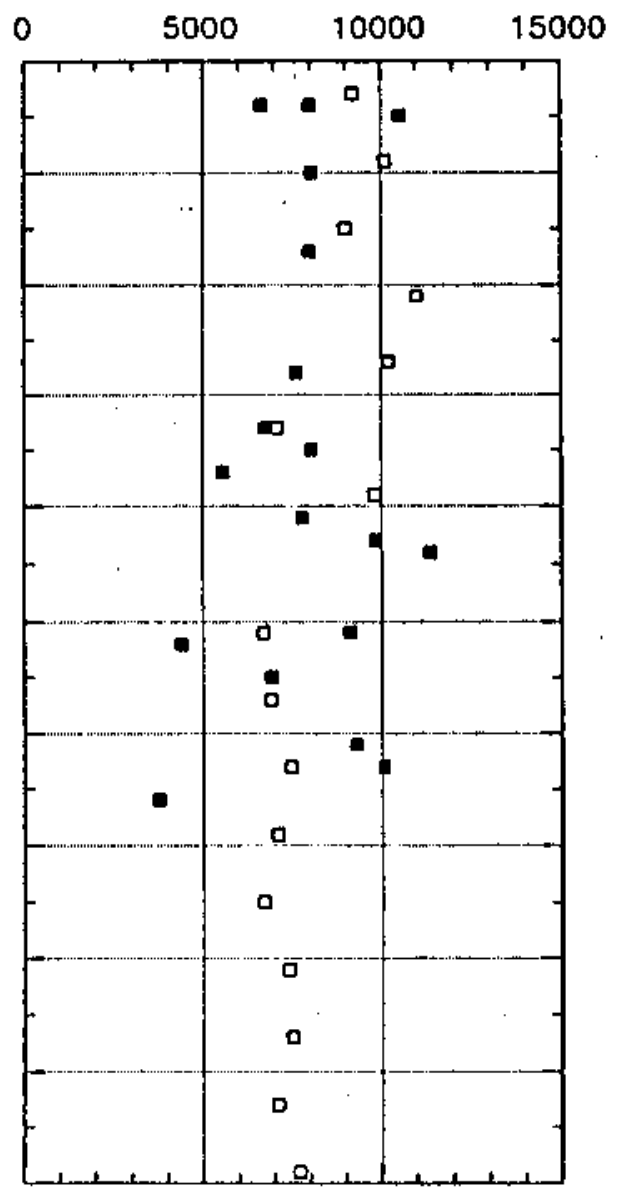

Figure 4.33 As-Compacted and In-Service Resilient Modulus of the Washington Soil 
water content has a significant influence on resilient modulus.

To compare the relationship of $M_{k}$ and $S_{41.0 *}$ between field and laboratory compacted solls, data of the Washington site are plotted together in Figure 4.34. It is observed that relationship between $N_{R}$ and $S_{u 1.0 \%}$ is similar in the tested range for both laboratory and field compacted soil. To see whether this observation is true in general, data for all tested sites are plotted together in Figures 4.35 to 4.37 . It -is found that relationship at no confining stress is slightly different between the field and the laboratory compacted soil while relationships with confining stress are somewhat similar between two compactions. Laboratory compacted soil has a nonlinear relationship at $s_{\text {ul.0* }}$ less than $10 \mathrm{psi}$ and field soil shows less curvature in relationship. For lower confining stress condition, resilient modulus of laboratory compacted soil is moderately smaller than that of field compacted soil at $S_{u 1.0 *}$ larger than $20 \mathrm{psi}$ and they are similar at lower $S_{u 1.0 x}$. For higher confining stress condition; laboratory compacted soil shows slightly smaller $M_{R}$ than field compacted soil at $S_{u 1.0 *}$ larger than $25 \mathrm{psi}$ and slightly larger $\mathrm{M}_{\mathrm{R}}$ than field compacted soil at $S_{u l .0 x}$ smaller than $15 \mathrm{psi}$. For soils having $S_{\mathrm{ul} .0 \times}$ ranging from $5 \mathrm{psi}$ to $20 \mathrm{psi}$, field resilient modulus is approximately same as laboratory resilient modulus.

\subsection{Concluding Remarks}

From the inspection data and in-service conditions of sites studied, it was observed that the condition of subgrade approaches 90 to 100 percent degree of saturation after several years of service, regardless of as-compacted condition. The site where the in-service water content is lower than the plastic Limit shows the higher resilient modulus than the site where the water content is larger than the Plastic Iimit. Field compaction of south Bend and 


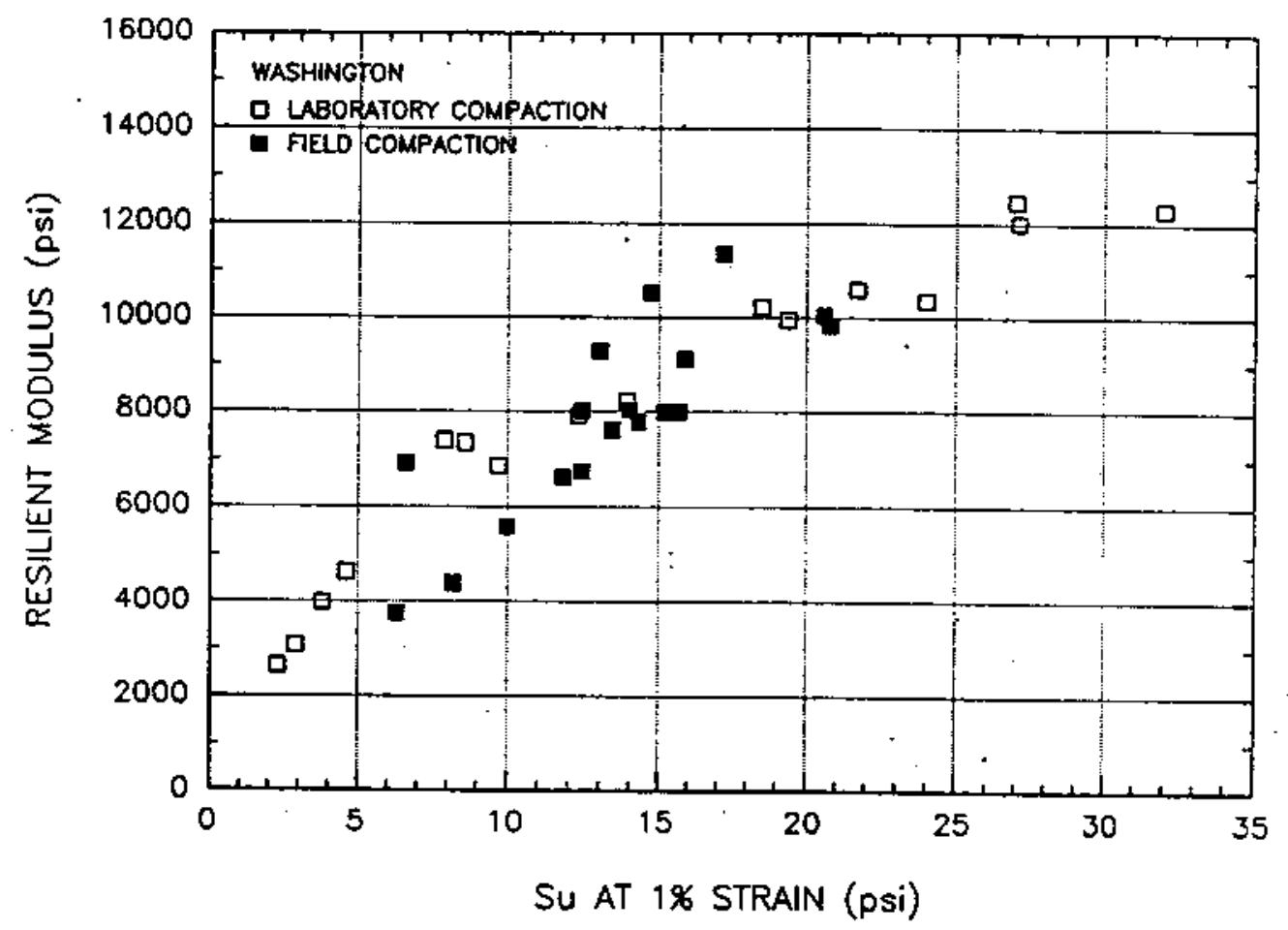

Figure 4.34 Relationship between $M_{R}$ and $S_{u 1.0 \%}$ for Field and Laboratory Compacted Washington Soil 


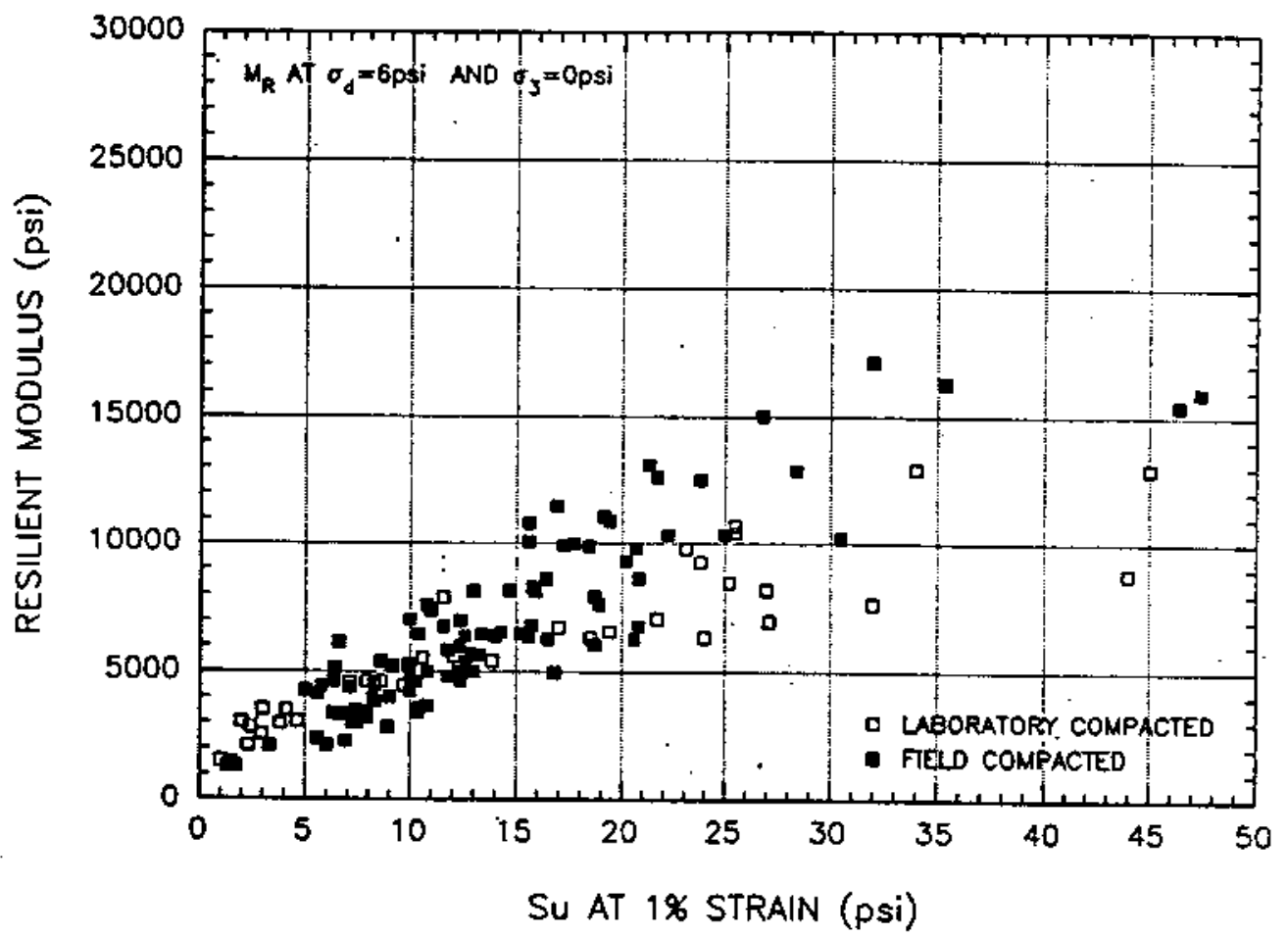

Figure 4.35 Relationship between $M_{R}$ and $S_{01.0 x}$ for Field and Laboratory Compacted Soils $\left(\sigma_{3}=0\right.$ psi) 


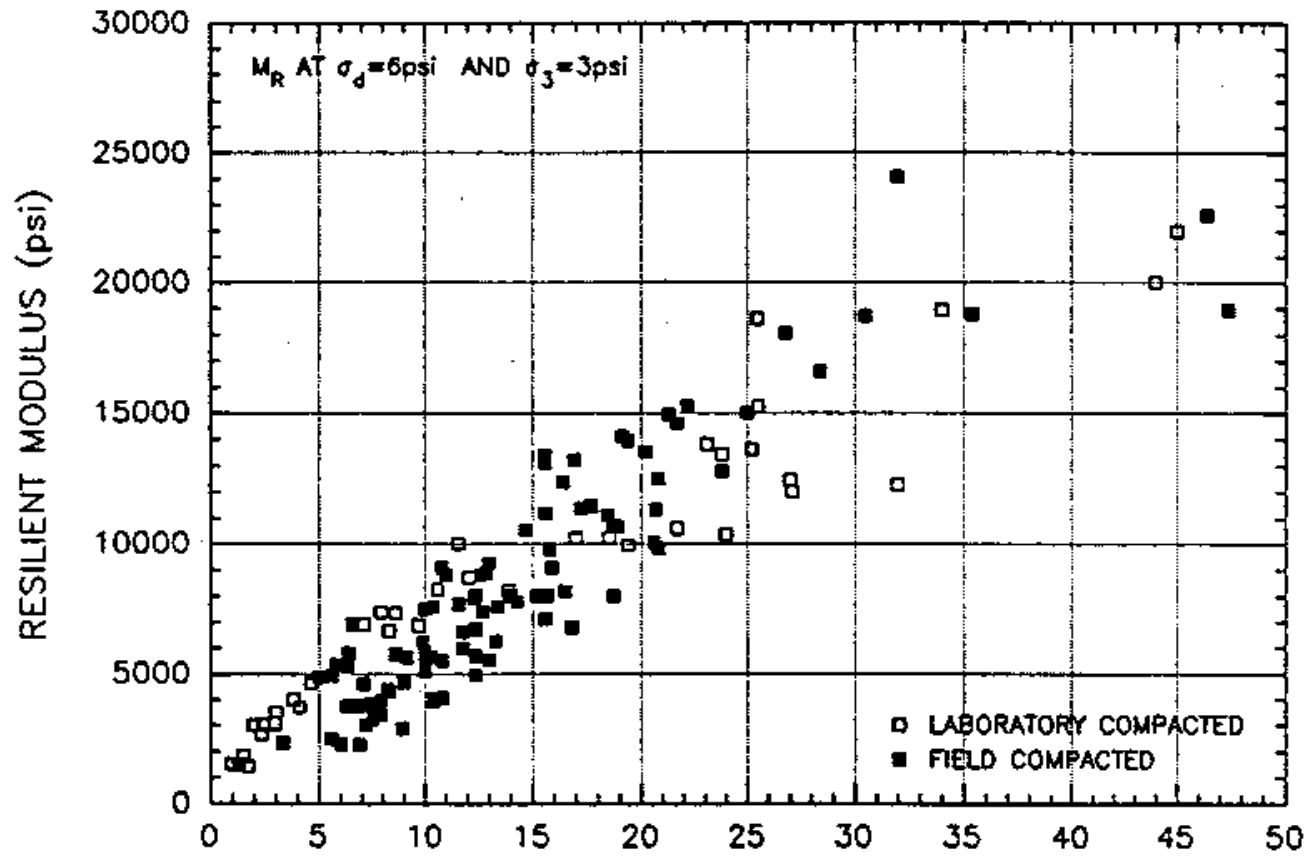

SU AT 1\% STRAIN (psi)

Figure 4.36 Relationship between $M_{R}$ and $S_{u 1.0 *}$ for Field and Laboratory Compacted Soils $\left(\sigma_{3}=3\right.$ psi) 


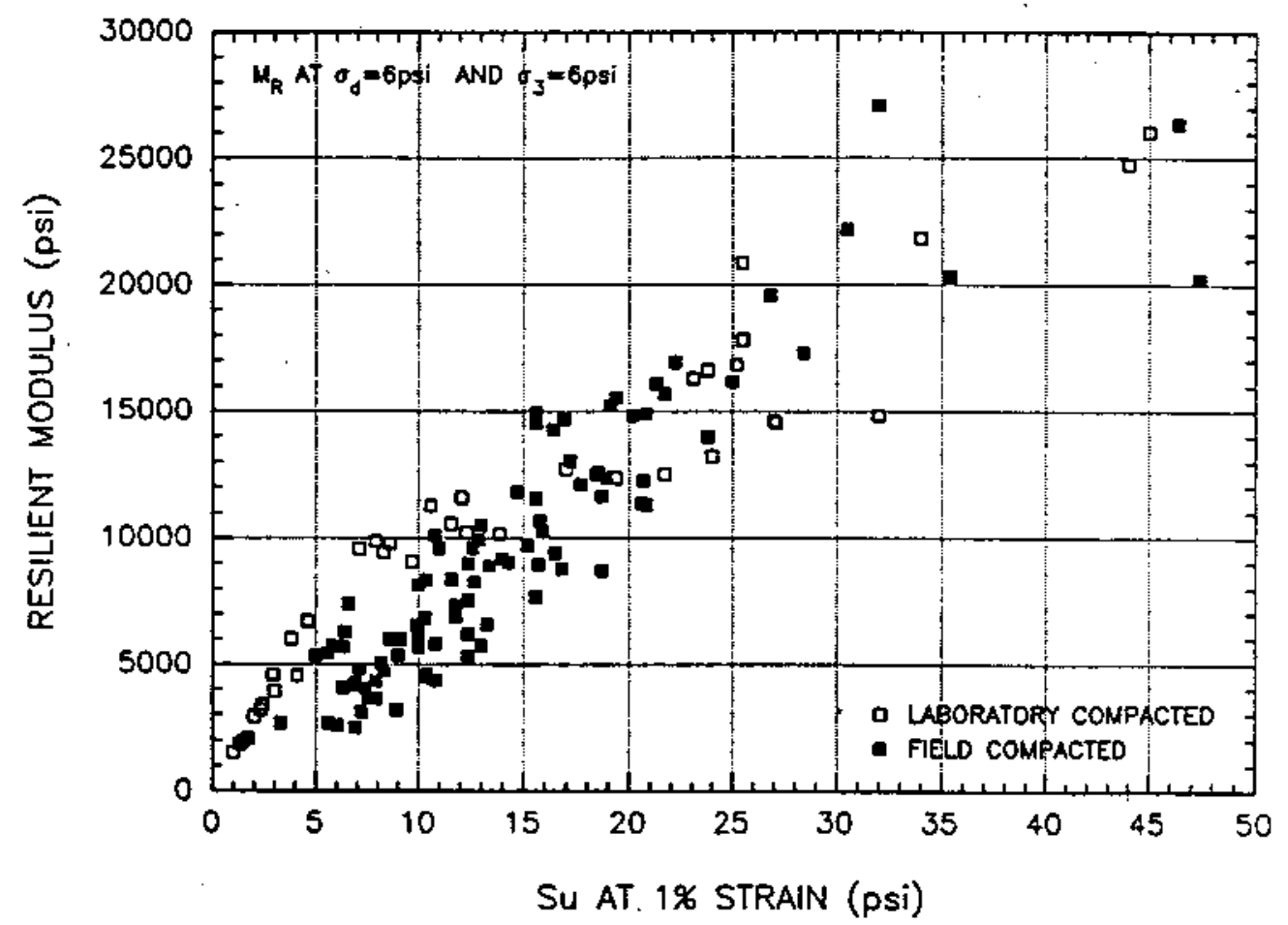

Figure 4.37 Relationship between $M_{R}$ and $S_{u 1.0 \pi}$ for Field and Laboratory compacted Soils $\left(\sigma_{3}=6\right.$ psi) 
Washington sites gives lower dry density than that obtained by the laboratory compaction with energy lower than standard Proctor, while field compaction of the Fort wayne site gives similar density with the laboratory compaction with standard Proctor energy. However, fabric studies on the South Bend and Washington soils suggest that the compactive effort does not significantly influence the soil fabrics. For the Fort wayne site, higher compactive efforts (higher than or equal to standard Proctor energy) do not influence the fabric when compacted at about optimum water content.

Generally, in-service resilient modulus increases with increase in dry density or decrease in water content. However, these relationships are highly scattered since the soil structure is affected by molding water content rather than inservice condition. on the other hand, relationship of resilient modulus with $s_{v 1.0 x}$ or $z_{i}$ clearly shows that $s_{v 1.0 x}$ and $E_{i}$ are good indicators of the resilient modulus. This implies that the results of repeated-load triaxial test and unconfined compression test are affected by the same factors, and, therefore, direct comparison of both test results excludes the scatter due to in-service water content and dry density.

For laboratory compacted soils, it was observed that the relationship between $\mathrm{M}_{\mathrm{R}}$ and $S_{\mathrm{ul} .0 \mathrm{x}}$ for a given soil is unique regardless of water content and compactive effort. The relationship is similar for three sites tested and is believed to be similar for the other two sites. The prediction equation applicable to all five sites is developed as follows:

$$
M_{R}=695.36 S_{u 1.08}-5.9297 S_{u 1.0 t^{2}}
$$

where $M_{R}$ is the resilient modulus at $\sigma_{3}=3$ psi and $\sigma_{d}=6$ psi (psi); and $S_{u 1.0 x}$ is the stress causing 1 percent strain in the unconfined compression test (psi).

In-service resilient modulus of the field compacted soil can be uniquely related to $s_{\text {zi.0\%, }}$ regardless of soil type. since the addition of three more independent variables in the 
prediction equation only slightly improves predictability, the following simple prediction equation is recommended for practical purpose:

$$
M_{R}=-1599.66+833.83 S_{u 1.04}-6.9683 S_{u 1.00^{2}}
$$

where $M_{R}$ is the resilient modulus at $\sigma_{3}=3$ psi and $\sigma_{d}=6$ psi (psi); and $S_{v 1.0 x}$ is the stress causing 1 percent strain in the unconfined compression test (psi). The equation is recommended for estimation of the in-service resilient modulus from the unconfined compression test result on an undisturbed specimen obtained from the subgrade.

By comparing the in-service resilient modulus and an estimated as-compacted resilient modulus, it was shown that in-service field resilient modulus can not be estimated from the resilient modulus of laboratory compacted specimen without considering the effect of water content change after construction; the as-compacted resilient modulus for field compacted soil may be represented by the resilient modulus of laboratory compacted soil. However, the relationship itself between $M_{R}$ and $S_{01.0 \%}$ is believed not to be affected by the change of water content and dry density after construction; and it was shown that the relationship between $M_{R}$ and $S_{u 1.0 x}$ is only slightly different for both laboratory and field compactions. Therefore, Equation 4.8 may be applicable to estimate as-compacted as well as in-service resilient modulus.

Replication of density and water content by laboratory compaction does not give good estimation of field resilient characteristics from laboratory data. Since the soil fabric is largely affected by molding water content and it does not appear to change due to the variation of moisture condition after construction, the replication of field compacted soil fabric in the laboratory may allow the estimation of ascompacted resilient modulus. Details about the replication of the field compacted fabric for the different sites are given in Section 9.6. In-service resilient modulus can, then, be 
estimated from the laboratory compacted modulus by considering the decrease in the resilient modulus due to the postcompaction increase in water content as described in sections 5.3 and 10.3 . 
5. EFFECTS OF ENVIRONMENTAL FACTORS ON RESILIENT MODULUS OF
COHESIVE SOILS 5.1 Introduction

Engineering response of compacted soll is said to be controlled by soil fabric (or structure), which is determined by water content at the time of compaction and the manner in which soil is placed. Since water content and dry density during field compaction are often difficult to control, variation in the resilient response of a given subgrade soll is expected. In addition, changes of moisture content due to climatic conditions and traffic contribute to variations of subgrade resilient response and pavement deflection.

Studies by Seed et al. $(1962,1967)$, Bergan and Monismith (1973) have shown that there is a change in compaction condition after construction. For the AASHO test road examined by seed et al. (1962), there was a significant change of resilient response due to an increase of dry density and a decrease of water content after construction. Bergan and Monismith (1973) showed a most significant increase in water content after construction for the subgrade initially placed on the dry side of optimum.

Studies by Bergan and Fredlund (1973), Cully (1971), Johnson et al. (1979), Bergan and Culley (1973), Bergan and Monismith (1973), Robnett and Thompson (1976), and Elliott and Thornton (1988) reported that the resilient behavior of cohesive soil is significantly affected by freeze-thaw action. The studies revealed that a small number of freeze-thaw cycles cause a substantial increase in resilient deformation even though no moisture change occurred. 
It appears obvious that change of moisture content subsequent to construction and freeze-thaw action significantly affect the resilient characteristics of the subgrade. The engineer should be able not only to predict variations of such conditions but also to design a pavement that can accommodate the changes in soil support conditions due to moisture change and freeze-thaw. The purpose of this chapter is to evaluate the effect of moisture change after construction and freeze-thaw on the resilient modulus.

5.2 Resilient Characteristics of Frozen-Thawed Cohesive Soils

5.2.1 Mechanism of Freezing and Thawing

Detrimental effects associated with frost action in pavement are frost heaving and subgrade shrinkage in winter, and the loss of soil strength by thaw weakening in spring. In the specific area of soil volume change due to freezing, the majority of the published studies deal with the frost heaving phenomena. However, the shrinkage of clays on freezing has not been investigated to any great extent although it may also result in deterioration of pavement structures.

The physical changes occurring as a soil freezes or thaws are described by Tsytovich (1975), Andersland and Anderson (1978), Kaplar (1970), and Konrad (1991). When air temperature drops below freezing, the moisture in the upper soil layer starts to freeze in place. If a fine-grained soil freezes, ice first forms in the largest pores where the water potential and freezing point are highest. As the temperature drops continuously, the pore water freezes in progressively smaller pores, and films of water coating the soil particles gradually freeze. Free water flows from unfrozen regions through the unfrozen water film to the growing ice grains to form small ice lenses. Upon freezing to a given temperature, two opposing forces develop tending to cause volume change in the soil 
mass. An expansionary tendency is created by the volume increase when soil water freezes. Shrinkage forces also are present as freezing of soil water reduces the thickness of adsorbed water films around individual soil particles and packets of soil particles, and this increases the soil suction in a manner similar to drying. The drying can also happen due to the moisture migration to the freezing front if replenishment does not occur. In general, the tendency to expansion will predominate in soils at high degree of saturation, while overall shrinkage occurs at lower degrees of saturation.

If existing conditions are favorable for frost heaving (a soil-moisture supply, sufficiently cold temperatures to cause soil freezing, and frost-susceptible soil), ice lenses continue to grow and expand, and the ground surface moves upward. When the air temperature increases above $0^{\circ} \mathrm{C}$ during spring, the thawing isotherm progresses downward below the roadway surface. Ice lenses in the upper soil layer start to melt and the melt water is trapped above the impervious frozen layer. The trapped melt water in the upper soil layer is associated with reduced load-carrying capacity of pavements.

From field observation in northern Canada, Hamilton (1966) suggested that partially saturated clay subgrades may undergo considerable shrinkage during freezing. Elevation measurements of flexible pavement surfaces over highly plastic clay subgrades have shown that downward movements may occur during winter months, resulting in elevation differentials of up to 1.5 inches on transverse sections with negligible changes in moisture content.

Leroueil et al. (1991) conducted closed-system freezingthawing on nine champlain sea clays from seven different sites. Except for one clay taken in the clay crust at shallow depth, soils have apparently not been frozen in the past and the water content of these soils ranges from 54 percent to 92 percent. During freezing of soil, it was observed that 
freezing of clays creates a new macroscopic fabric made up of modules with edges measuring a few millimeters. Isotropic consolidation with effective stress of $25 \mathrm{KPa}$ showed that the clays that were subjected to a freeze-thaw cycle for the first time displayed volume change varying between 12.2 percent and 26 percent while, for soil which had already experienced a large number of freeze-thaw cycles, the volumetric strain after an additional cycle in the laboratory was only 0.8 . percent. The volumetric consolidation of intact clay was smali and less than 2.0 percent.

Chamberlain and Gow (1979) studied the effect of freezing and thawing on the permeability and structure of fine grained soils prepared in the form of a slurry in the consolidometer. The specimens were frozen one-dimensionally with the side of the specimen at the opposite end of the freeze or thawing surface exposed to water. It was observed that in all cases freezing and thawing caused a reduction in void ratio and an increase in permeability. The changes were greatest for the soil with the largest plasticity index. For soils in which clay particles predominate, the large negative pore water pressure during freezing causes vertical shrinkage cracks and horizontal ice lenses, formed perpendicular to the direction of freezing. The shrinkage cracks become linked together to form columns with polygonal cross-sections that resemble the shrinkage structures observed in desiccated muds. It was found that the thicknesses of ice lenses decrease and the frequency of occurrence increases with increasing applied stress on specimen during freezing-thawing. The average polygon diameter also decreased with. increasing applied stress level. For coarser-grained soil in which more angular silt or sand particles control compressibility, vertical shrinkage cracks were not observed though the permeability was increased due to freeze-thaw. It was concluded that clay packets in the pores of the coarse fraction collapsed to a more dispersed and denser structure during freezing, and less void space is, then, occupied with clay solids and associated bound water. 
Ice segregation during freezing is not always a necessary prerequisite for thaw-weakening of pavement. This subtle type of thaw weakening has been confirmed in the laboratory closedsystem freeze-thaw test by several researchers, such as Bergan and Fredlund (1973), Kim and Daniel (1992), and Zimmie and La Plante (1990). Chamberlain (1973) referred to studies by cook (1963) and Titov (1965) which observed module or nugget structures having higher surface moisture contents and loss of cohesion without visible ice heaving. Mikhailov and Bredyuk (1971) attributed the reduced cohesion in clay to the conversion of bound water into free water. Chamberlain (1973) concluded that closed-system freeze-thaw induces a strength reduction in clays with or without visible ice segregation. It was also noted that the rate of strength reduction decreases as the number of freeze-thaw cycles increases.

Hamilton (1966) first noted a relationship between volume change on freezing and degree of saturation for partially saturated cohesive soils compacted in the laboratory. It was observed that soils compacted below 90 percent degree of saturation underwent a shrinkage during freezing while those compacted at higher degree of saturation showed increase in volume on freezing. Maximum shrinkage for tested soils occurred at degrees of saturation between 60 and 70 percent. The least plastic clay underwent a maximum shrinkage of 4 percent in volume at 61 percent degree of saturation while highly plastic clay shrank 10 percent at 67 percent degree of saturation. It was also noted that a large portion of the total volume change due to freezing occurred over the temperature range $+30^{\circ} \mathrm{F}$ to $+20^{\circ} \mathrm{F}$. A net increase in volume was observed upon thawing. Increasing compactive effort resulted in a reduction in the maximum freezing shrinkage and increases the degree of saturation at which the maximum shrinkage occurs.

Penner et al. (1975) also observed shrinkage in the freezing. of laboratory samples in preparation for thermal- 
conductivity measurements. The reduction in thermal conductivity is the evidence that the adsorbed surface water on the soil particles is withdrawn by crystallization inside the air-filled pores. When thawing takes place, the adsorbed films thicken, causing swelling. It was suggested that this shrinkage and swelling phenomenon induced by the freeze-thaw process might contribute to thaw weakening in unsaturated subgrades when no ice lensing occurred.

Fredlund et al. (1975) suggested that the deformation occurring as a result of freezing and thawing of partially saturated soil in a closed system can be examined in terms of changes in the stress state variables. The net total stress, $\sigma-u_{a,}$ and matrix suction, $u_{2}-u_{w}$, were proposed as independent stress state variables. Since the total and air pressures are essentially unchanged before and after freezing, only the changes in water pressure need to be observed. From suction test results on Regina clay, Bergan and Frediund (1973) reported that undisturbed samples which have already been subjected to several freeze-thaw cycles in the field showed considerably.lower matrix suction than the remolded samples did. Significant arop in suction was observed after freezethaw cycle on the remolded samples, while a smaller but significant drop in suction occurred after one freeze-thaw cycle on undisturbed samples. A well-defined secondary structure was present in the undisturbed samples and definite ice segregation was evident in the samples. It was concluded that the free water accumulated in the secondary structure during freezing controls the matrix suction and causes the reduction in matrix suction when solls thaw.

\subsubsection{Effect of Freeze-Thaw on Resilient Modulus}

Culley (1971) studied the effect of closed-system freezethaw cycling on the resilient modulus of Qu'Appelle glacial till compacted in the laboratory. It was observed that 
substantial reductions in resilient modulus occurred as a result of freeze-thaw, and the amount of reduction was dependent on the density and moisture content of the specimens. At optimum moisture content corresponding to Standard Proctor energy, the reduction in resilient modulus ranged from 41 percent to 61 percent. At water content lower than optimum, the reduction in resilient modulus due to freeze-thaw decreased as density increased, and at water content higher than optimum, freeze-thaw effect increased as density increased. It was concluded that an increase in compactive effort at optimum water content improved resilient characteristics while detrimental volume change caused by freeze-thaw cycle would increase. For improvement in both resilient characteristics and volume change characteristics due to freeze-thaw, a decrease in water content must accompany an increase in density.

Mickleborough (1970) investigated the effect of freezethaw on the resilient properties of highly plastic glacial lake clay compacted to the maximum standard density. Resilient moduli were determined for samples subjected to zero, one, two, and four freeze-thaw cycles, and that closed-system freezing was at temperatures chosen to cause freezing in 24 hours. Typical results showed reduction of resilient modulus by 58,63 , and 74 percent after one, two, and four cycles of freeze-thaw, respectively.

Bergan and Monismith (1973) tested the same lacustrine clay tested by Mickleborough (1970). Resilient moduli of undisturbed specimen (at $\sigma_{3}$ of $2 \mathrm{psi}$ and $\sigma_{4}$ of $5 \mathrm{psi}$ ) were in most cases between $5000 \mathrm{psi}$ and $10000 \mathrm{psi}$, averaging $8400 \mathrm{psi}$ for fall samples and $6300 \mathrm{psi}$ for spring samples. After freeze-thaw, the resilient modulus ranged from 4000 psi to $6000 \mathrm{psi}$. After about 10000 load repetitions it recovered its original value prior to freeze-thaw. Test of remolded samples, compacted to in-situ moisture content and dry density; showed resilient moduli of $15000 \mathrm{psi}$ before freeze-thaw and $6500 \mathrm{psi}$ after two cycles of closed-system freeze-thaw. 
Robnett and Thompson (1976) conducted repeated load triaxial tests on silty clay and lean clay compacted in the laboratory. Tests were performed after 1 to 10 cycles of closed-system freeze-thaw. Even though the clayey soil had substantially higher resilient modulus than silty clay before freeze-thaw, both soils showed similar resilient response after freeze-thaw, ranging from approximately 1000 psi to 4200 psi at varying levels of deviator stress. It was also shown that detrimental effects of freeze-thaw cycles on resilient modulus was significantly reduced by treating the soils with hydrated lime.

Johnson et al. (1979) and Chamberlain et al. (1979) reported the test results on silt and clay soils taken from test pavements in the frozen condition and tested in thawed condition for varying deviator stress and moisture content. Upon thawing, most Hanover silt samples were too soft and weak to test and had to be either partially or fully consolidated before the repeated load was applied. Test data showed that resilient modulus decreased significantly with increasing deviator stress and ranged from 1200 to $13000 \mathrm{psi}$. Thawed Morin clay had sufficient integrity to allow handling after thawing and the resilient modulus of specimen with water content of 25.4 percent ranged from 700 to 8000 psi at confining stress of $0.5-8 \mathrm{psi}$.

\subsubsection{Laboratory Testing Program}

To develop the procedures for freeze-thaw test, the type of freeze-thaw test, temperature and duration for freeze and thaw, and number of required freeze-thaw cycles must be determined. By examining the data of water content for the South bend site where the soil was sampled during winter and summer, it was found that the change of water content was negligible even though two data points of water content of specimen sampled in January shows 3 or 4 percent higher values 
than others, as shown in Figure 5.1. Therefore, closed-system freeze-thaw test appeared appropriate to simulate the field condition.

Temperatures for freezing and thawing are determined from the data by Thompson and Dempsey (1970) which reported the frost action parameters for a typical pavement section for five locations in Illinois from 30 years of climatic data. since the South Bend and Fort Wayne sites are expected to be subjected to climatic condition similar to that of northern Illinois, the possible lowest freezing temperature and possible highest above-freezing temperature for chicago in January are chosen as freezing and thawing temperature, respectively. For the Washington, Bedford and Bloomington sites, those are determined from the data for st. Louis in the same way. Other features, such as cooling rate, warming rate, and duration of freezing and thawing are impractical to be simulated in the laboratory with reasonable time. Therefore, each freeze-thaw cycle consisting of 32 hours of thawing, 10 hours of cooling, 32 hours freezing, and 10 hours of warming is used for the laboratory freeze-thaw test in this project.

To determine the effect of freeze-thaw cycles, four identical specimens compacted in the laboratory with standard Proctor energy were tested by repeated load after they were subjected to $0,1,2,3$ cycles of freeze-thaw. As can be seen in Figure 5.2, a single cycle of freeze-thaw caused a 30 to 50 percent reduction in resilient modulus at deviator stress less than 6 psi; subsequent freeze-thaw cycles increase the resilient modulus. Since specimens were compacted and put into the freeze-thaw chamber together, specimens subjected to more freeze-thaw cycles may have the increase in resilient modulus due to thixotropy. Considering this pilot test result and test data reported in literature, it was concluded that one or two cycles of freeze-thaw are enough to simulate the effect of freeze-thaw on resilient characteristics of cohesive soils. Therefore, two cycles of freeze-thaw shown in Figure 5.3 were used for the laboratory closed-system freeze-thaw test. 


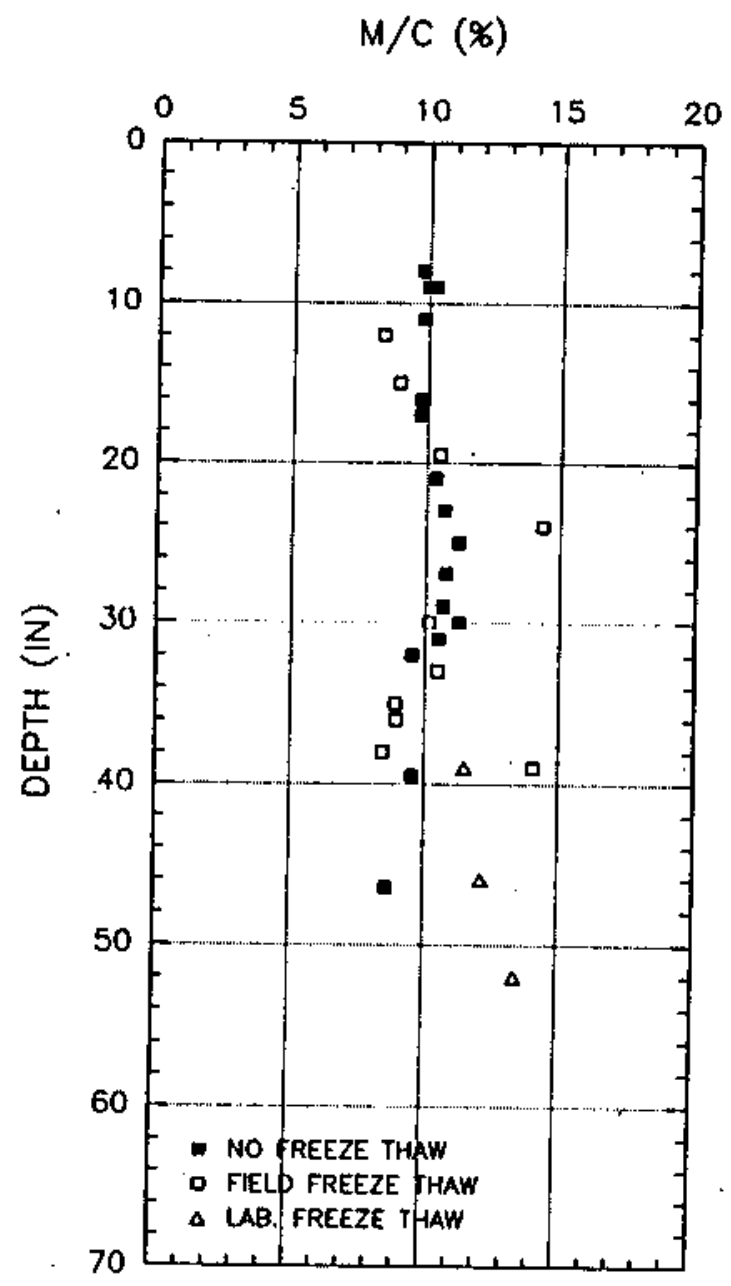

DRY UNIT WEIGHT (PCF)

RESILIENT MODULUS (PSI)
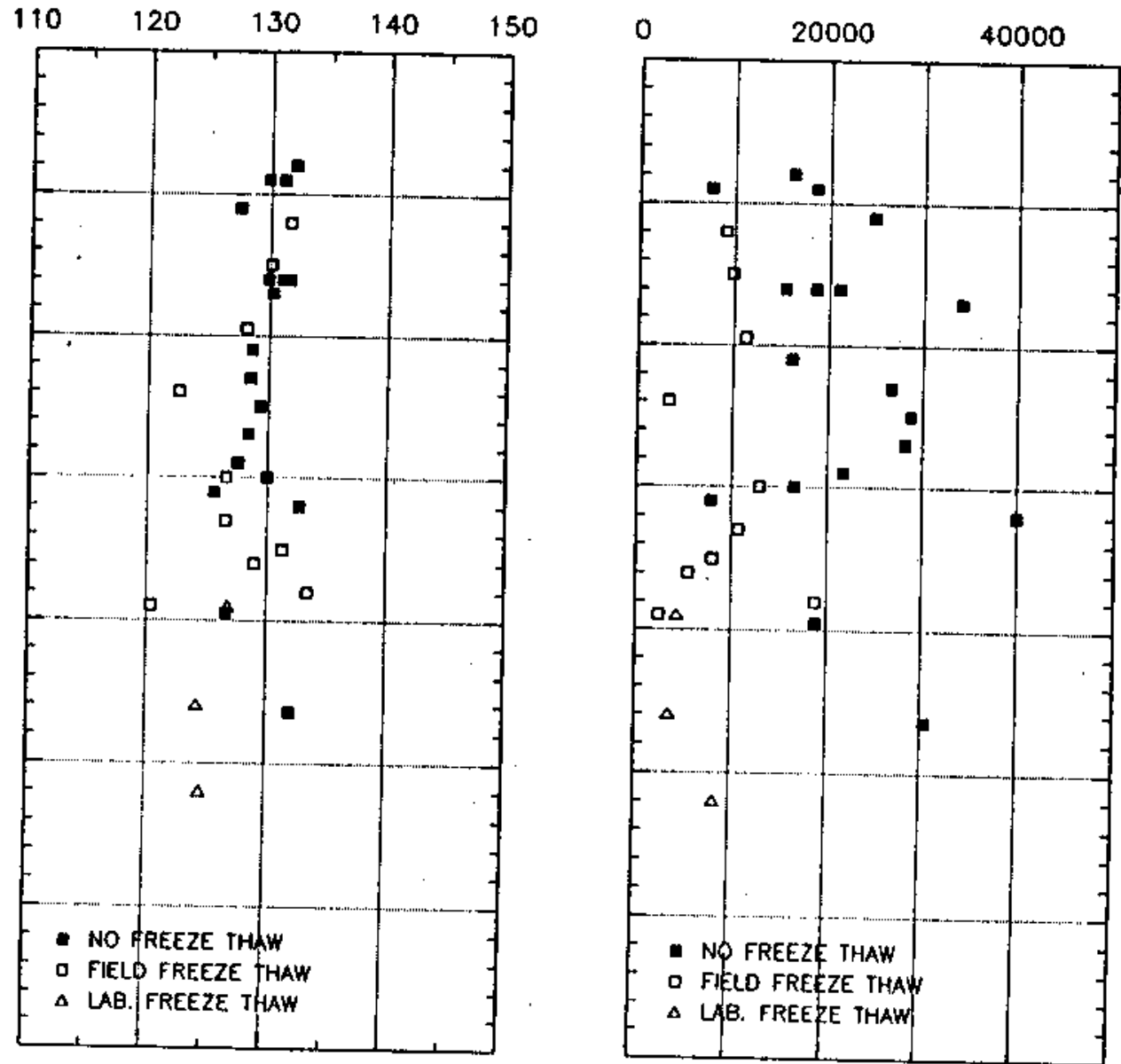

Figure 5.1 Profile of Water Content, Dry Density, and Resilient Modulus of the
South Bend Site 


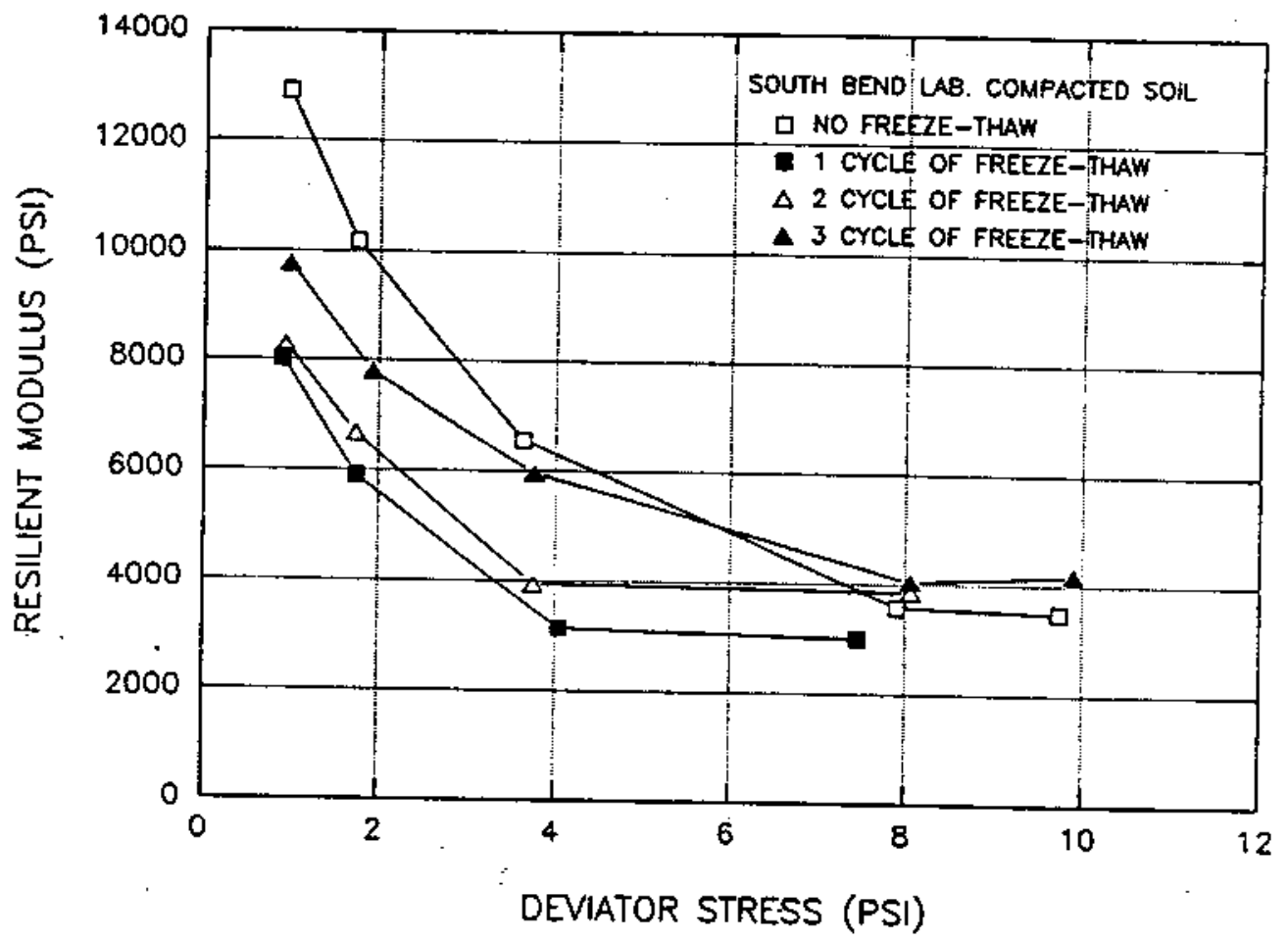

Figure 5.2 Effect of Freeze-Thaw Cycles . on Laboratory
Compacted South Bend Soil 


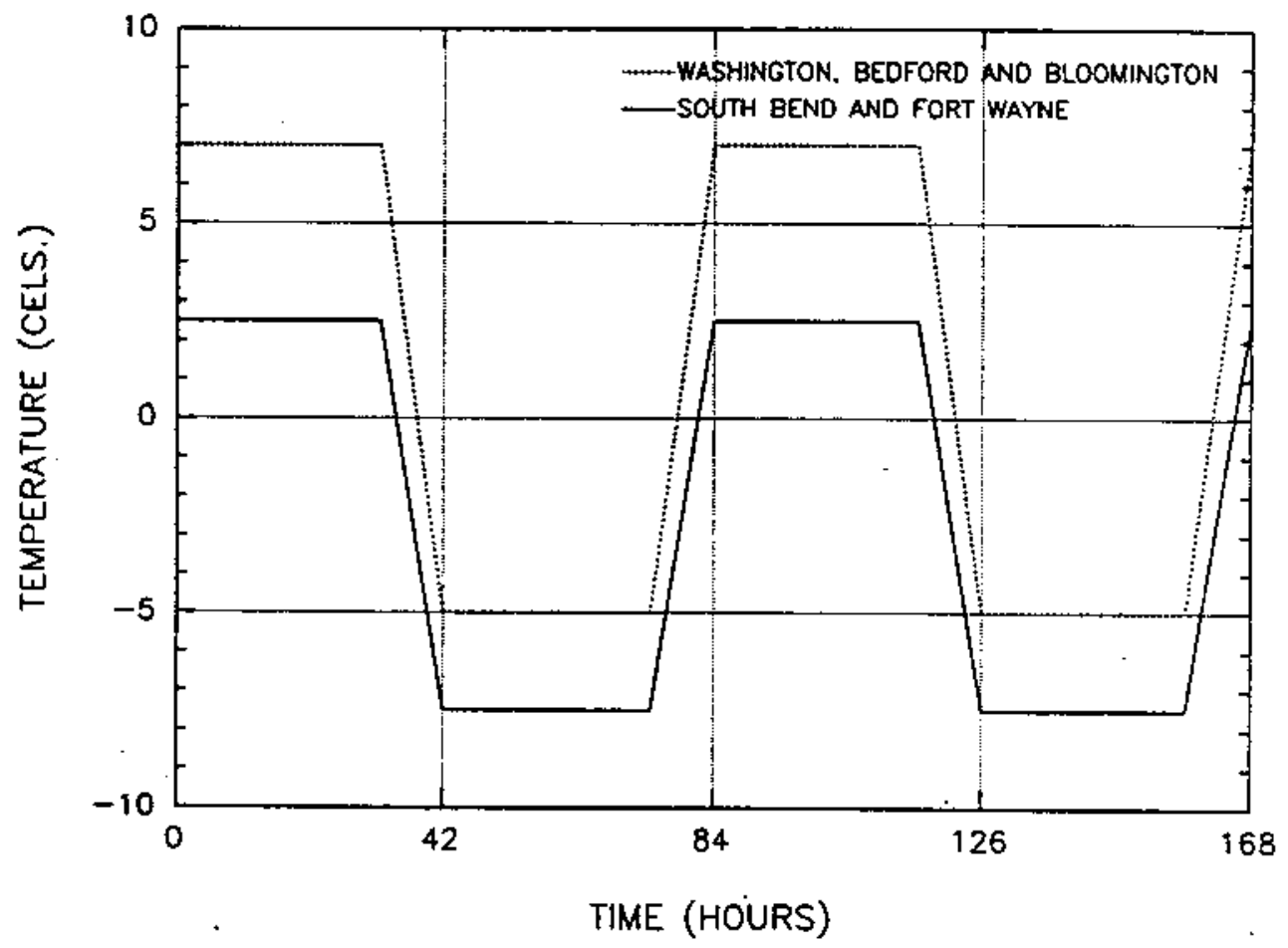

Figure 5.3 Cycles Used for closed-System Freeze-Thaw Test 
Undisturbed soils sampled from five in-service subgrades in Indiana were used, and specimens were prepared in the same manner as explained in section 4.3.2. Detailed information on each specimen tested for freeze-thaw is given in Appendix $A$. After the unconfined compression test was performed up to 1 percent axial strain, each specimen was sealed in a rubber membrane with half inch thick top and bottom copper endcaps and o-rings to prevent change of water content during the freeze-thaw process. The sealed specimen was then placed in a 7-inch high by 8-inch wide styrofoam box. A mixture of wood and saw dust was lightly compacted around the specimen up to the top endcap to promote uniaxial freezing. This box was then closed with a styrofoam cover having a 3 -inch circular hole to expose the top endcap to air.

The styrofoam box containing the specimen was put into a large freeze-thaw chamber whose temperature is controlled by a pre-programmed schedule. No attempt was made to measure either the rate of temperature change within the specimen or the volume change of specimen. After 2 cycles of freeze-thaw, the specimen was removed from the freeze-thaw chamber and allowed to thaw at room temperature. After 24 hours of thaw, the specimen was removed from the styrofoam box and was subjected to repeated loading following the sequence described in AASHTO $T$ 274-82.

It was found that the specimens lost about 2 to 5 grams of water during the freeze-thaw test. Visual examination of frozen specimens revealed no ice lenses although small ice crystals were observed occasionally at the surface of the specimens. It was also observed that cracks on the surface of specimen due to sampling disturbance and invisible cracks between compaction layers were weakened by the freeze-thaw process. 
5.2.4 Test Results and Discussions

Results of unconfined compression and resilient modulus tests were reduced as explained in section 4.3.3, and summaries of the reduced data are presented in Appendices $B$ and $c$, respectively. Some of the specimens were too weak to be tested after thaw and data on resilient modulus for these specimens remains blank in the table. Note that unconfined compression tests were performed only on three specimens for South Bend soil.

Specimens from SBFTOI to SBFT10 were sampled in January, 1990 and ice lenses were observed during the sampling operation. Sampled soil was stored in an humidified room for thawing, and then tested for resilient modulus after extrusion and trimming of the specimens. On the other hand, specimens from SBFT11 to SBFT13 were sampled in July and tested after closed-system freeze-thaw. Test results of both series of specimens are plotted with depth in Figure 5.1. It can be seen from this figure that resilient modulus of field frozen-thawed soil is slightly larger than that of laboratory frozen-thawed soil. However, the difference in resilient modulus is not significant, compared with the difference between frozenthawed soil and the unfrozen soil. Therefore, it may be concluded that closed-system freeze-thaw test in the laboratory can simulate the freeze-thaw occurring in the field.

To see the effect of freeze-thaw, the resilient modulus at confining stress of $3 \mathrm{psi}$ and deviator stress of $6 \mathrm{psi}$ is plotted with stress causing 1 percent strain in unconfined compression test, as shown in Figures 5.4 to 5.7 . It can be seen that freeze-thaw causes a significant reduction in resilient modulus for all sites tested. There is a negligible effect of freeze-thaw for soils having. $S_{u 1.0 *}$ less than 8 psi while the effect of freeze-thaw on resilient modulus becomes larger as $S_{v 1.0 x}$ increases. For $S_{\text {ui. }}$ of $10 \mathrm{psi}$, the reduction of 


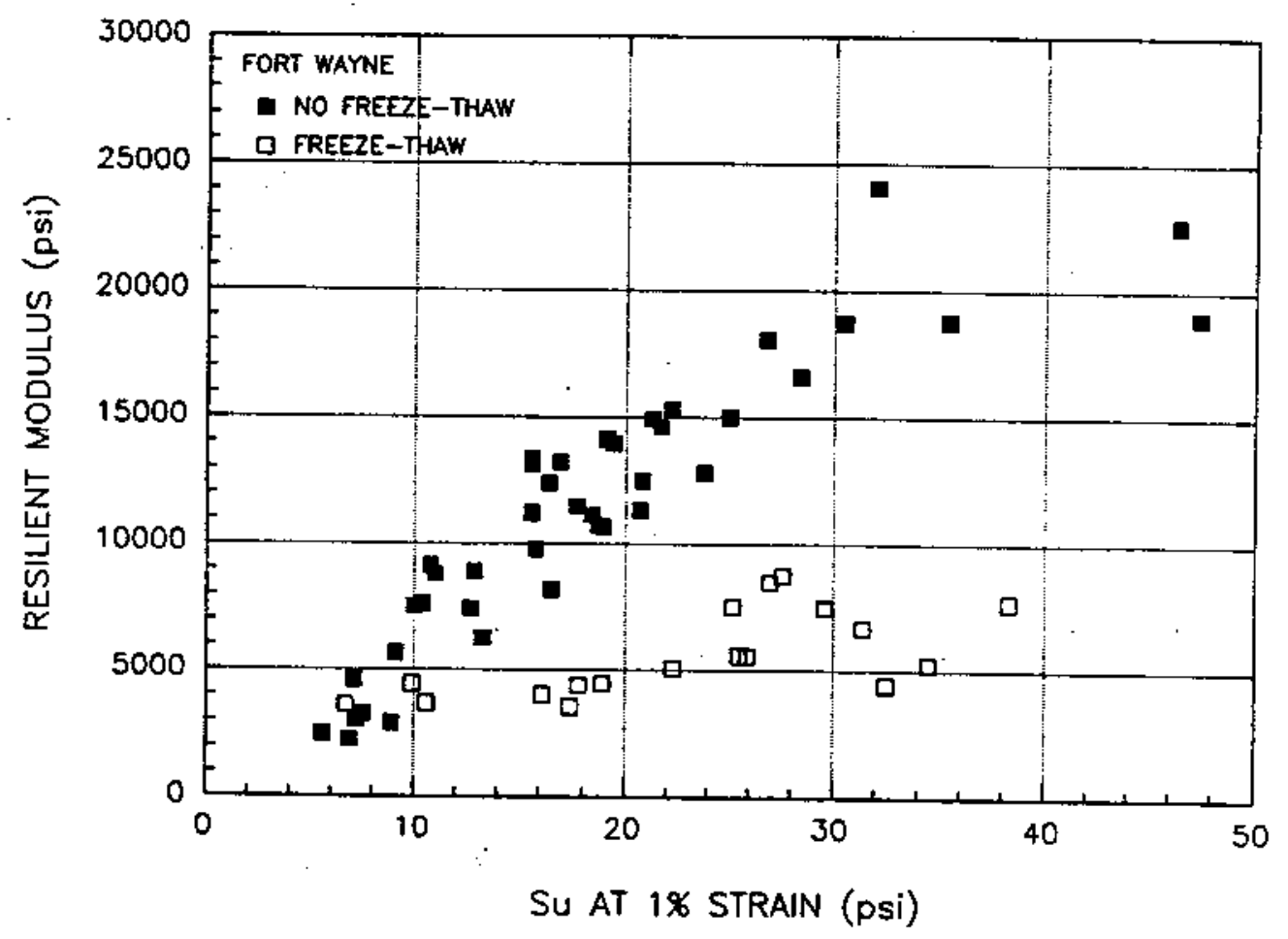

Figure 5.4 Effect of Freeze-Thaw on Resilient Modulus of the Fort Wayne Soil 


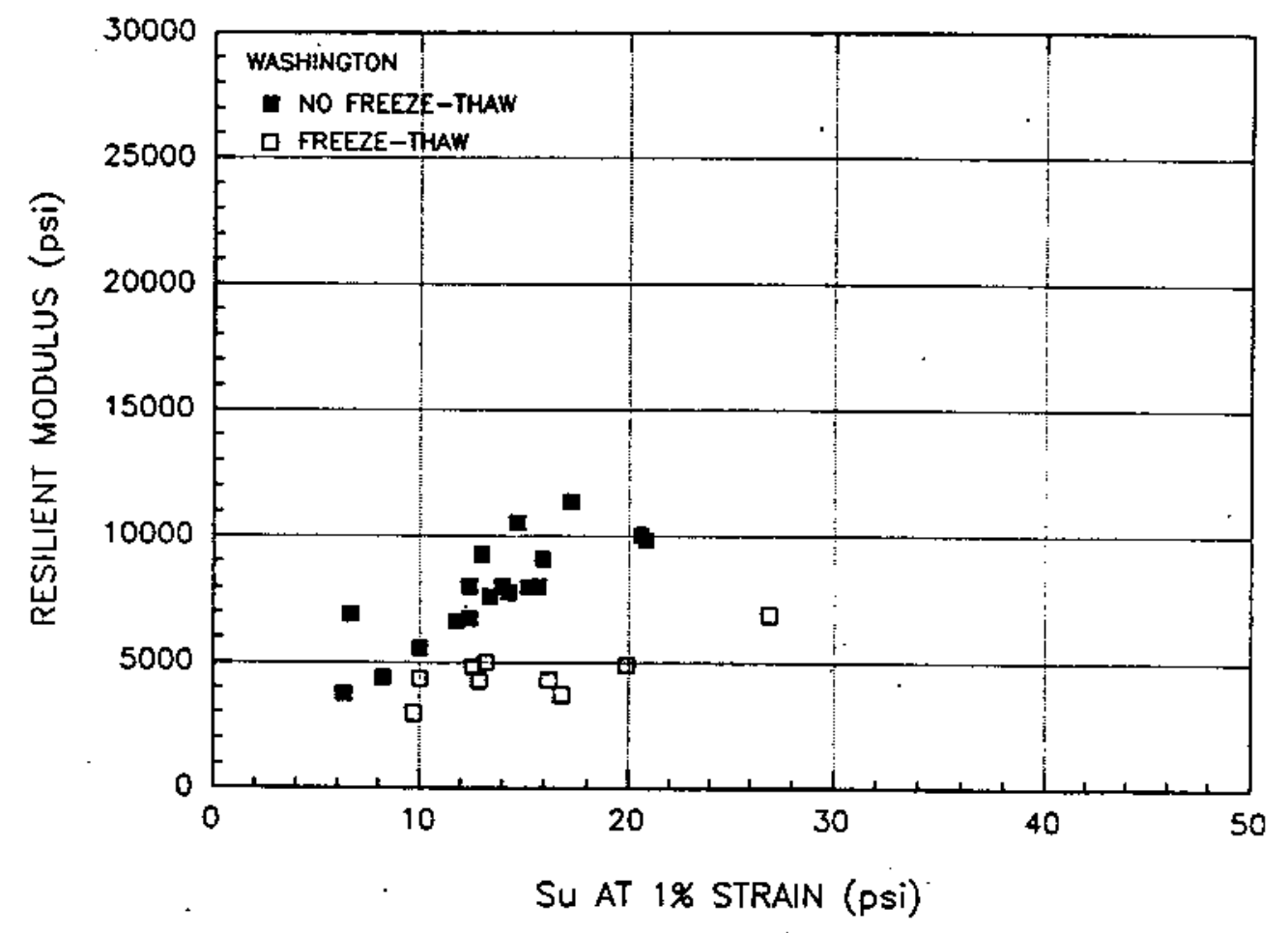

Figure 5.5 Effect of Freeze-Thaw on Resilient Modulus of the Washington Soil 


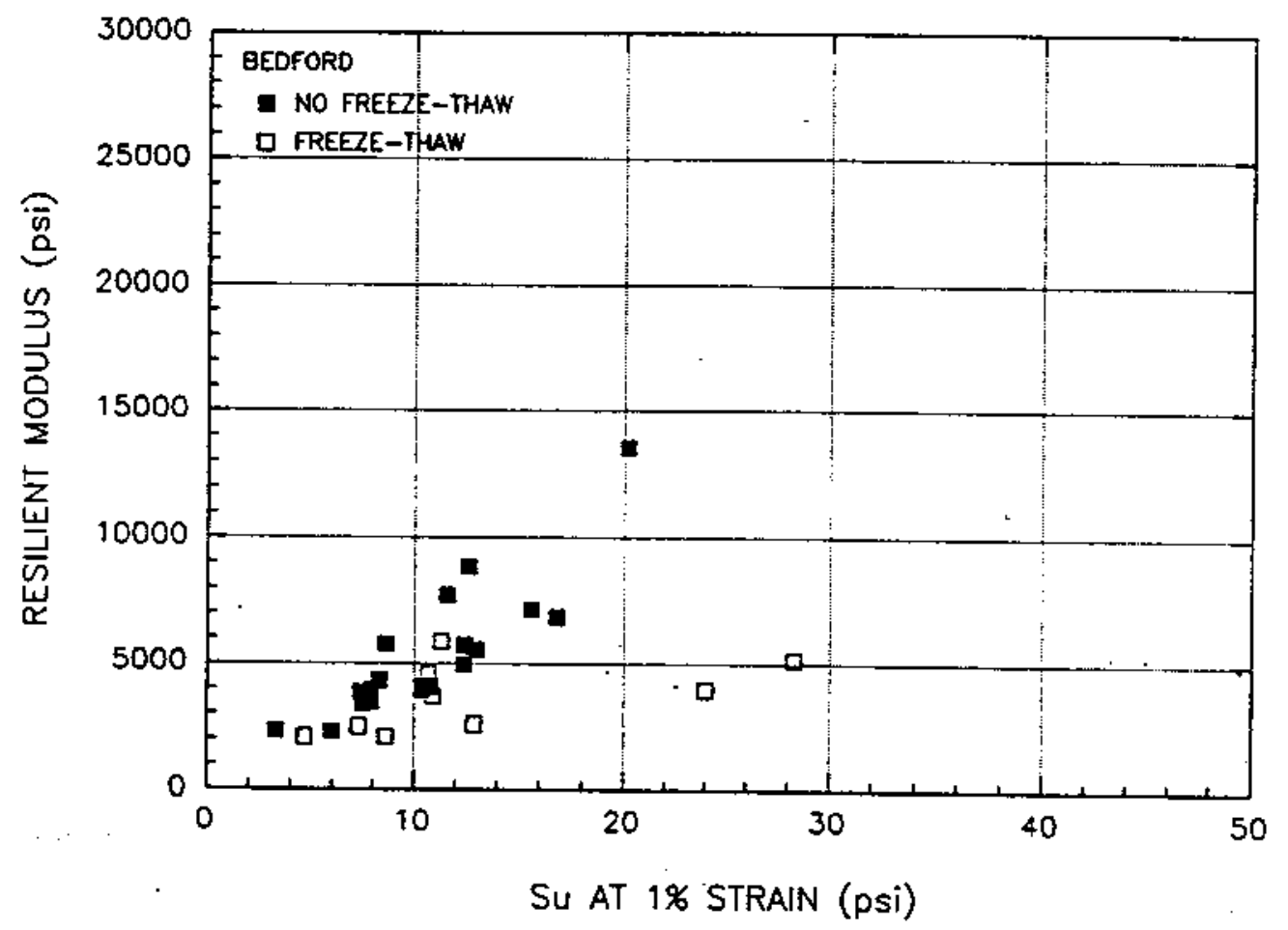

Figure 5.6 Effect of Freeze-Thaw on Resilient Modulus of the Bedford Soil 


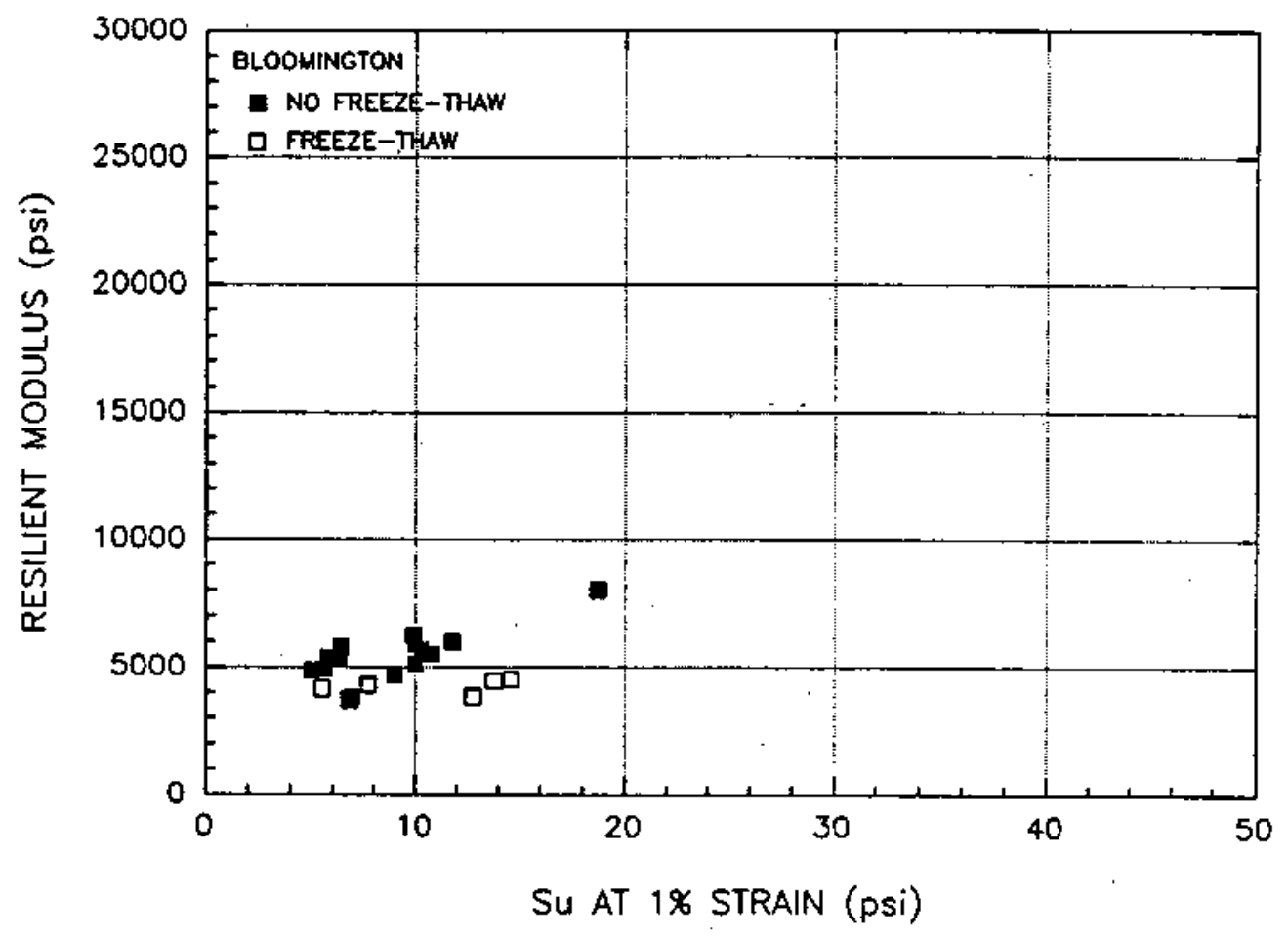

Figure 5.7 Effect of Freeze-Thaw on Resilient Modulus of the Bloomington' Soil 
resilient modulus due to freeze-thaw is about 30 percent; for $S_{u 1.0 x}$ of 40 psi, it is about 60 percent.

To compare the relationships between thawed resilient modulus and $S_{\mathrm{ul} .0 \%}$ for each site, data are plotted together in Figure 5.8. Though some scatter is observed, the relationships for five different sites appear to fit one single relationship. Therefore, it seems possible to predict the reduction of the resilient modulus due to freeze-thaw from unconfined compression tests performed before freeze-thaw. This prediction equation is applicable only where insignificant change of water content occurred during freezing and thawing. Since soils tested have different frost susceptibility and they are found to have similar

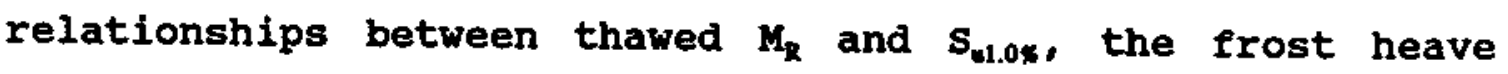
criteria may not be applicable to the estimation of thawweakening effect on resilient modulus.

To develop the prediction equations, test results on all frozen-thawed field soils were analyzed by multiple regression. Stepwise regression was performed on resilient moduli at different levels of confining stress and repeated deviator stress. From this analysis, two prediction equations were selected; one for simple use and another showing the smallest prediction error:

$$
\begin{gathered}
M_{R}=a+b S_{u 1.01} \\
M_{R}=a+b S_{u z .08}+c S_{u 1.08}{ }^{2}+d E_{1}+e E_{2}+f W C
\end{gathered}
$$

where $M_{R}$ is resilient modulus of frozen-thawed soil (psi); $S_{u 1.0 x}$ is the stress causing 1 percent strain in unconfined compression test (psi); $E_{i}$ and $E_{2}$ are initial tangent modulus and tangent modulus at 2 psi stress in unconfined compressive stress-strain relation, respectively (psi); wC is water content (percent). It should be noted that $s_{w 1.0 x}, E_{i}$, and $E_{2}$ are obtained from unconfined compression tests performed before freeze-thaw. Regression parameters, with coefficient of 


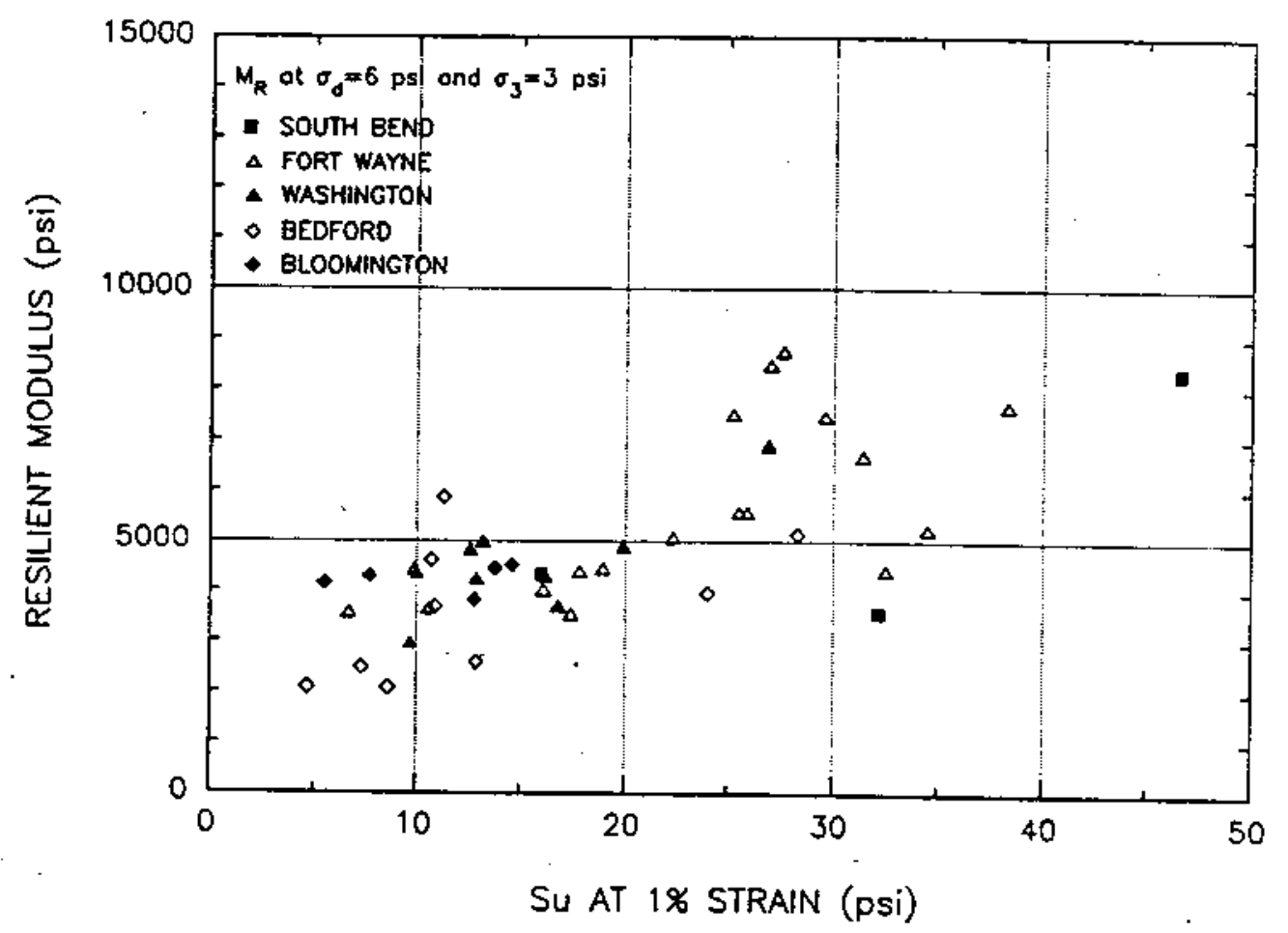

Figure 5.8 $\begin{aligned} & \text { Relationship between Thawed } M_{R} \text { and } S_{u s .0 x} \text { of Ail } \\ & \text { Tested Solls }\end{aligned}$ 
determination $\left(R^{2}\right)$ and square root of MSE, for specific conditions of stresses are presented in Table 5.1 and 5.2. For Equation 5.1, $R^{2}$ is in the range of $0.42-0.62$ and $\mathrm{MSE}$ is 693 - 1770 psi. For Equation 5.2, $R^{2}$ ranges in $0.52-0.74$ and MSE is 623 - 1509 psi. Generally, MSE is larger for larger average value of resilient modulus.

To check the reliability of the proposed prediction equations, resilient moduli at deviator stress of $6 \mathrm{psi}$ and confining stress of 3 psi calculated by the proposed equations are compared with measured resilient moduli at same stress condition. As shown in Figure 5.9, neither equation has good predictability. This may be due to the fact that the independent variables contained in prediction equations are measured before freeze-thaw while the resilient modulus is measured after freeze-thaw.

For specimens from the Fort wayne site, the unconfined compression test was performed before and after freeze-thaw. For comparison, relationship of thawed resilient modulus with thawed $S_{u 1.0 x}$ is plotted with the relationship of $M_{R}$ and $S_{u t .0 x}$ for soils which have not been subjected to freeze-thaw, as shown in Figure 5.io. It can be seen that there is a slight difference in the relationship for $M_{p}$ less than 5000 psi while no difference exists for $K_{R}$ greater than $5000 \mathrm{psi}$. Therefore, the resilient modulus of thaw-weakened soil may be estimated by a relationship between $w_{R}$ and $s_{w 1.0 x}$ of the non-frozen-thawed soil if $s_{u 1.0 x}$ of frozen-thawed soil is known. Above discussion imply that the relationship between $k_{R}$ and $s_{w 1.0 \%}$ may be similar for a given soll under any conditions if initial soil fabric is same. Therefore, the resilient modulus for the as-compacted condition, the in-service condition, and the thaw-weakened condition may be estimated from the relationship presented in Figures 4.13 to 4.15 or Equation 4.4 , if $s_{w .0 \%}$ is measured on the specimen under the corresponding condition. 
Table 5.1 Regression Parameters for Equation 5.1

\begin{tabular}{|c|c|c|c|c|c|}
\hline $\begin{array}{c}\sigma_{3} \\
(\mathrm{psi})\end{array}$ & $\begin{array}{r}\sigma_{d} \\
(p s i)\end{array}$ & $\begin{array}{c}\text { Regressi } \\
\text { a }\end{array}$ & $\begin{array}{c}\text { parameters } \\
\text { b }\end{array}$ & $\mathrm{R}^{2}$ & VMSE \\
\hline 6 & 2 & 5960.11 & 226.2040 & 0.57 & 1770 \\
\hline 6 & 3 & 4488.60 & 193.2672 & 0.56 & 1528 \\
\hline 6 & 4 & 3671.62 & 172.5947 & 0.54 & 1438 \\
\hline 6 & 5 & 3141.87 & 157.9982 & 0.51 & 1388 \\
\hline 6 & 6 & 2766.13 & 146.9489 & 0.49 & 1352 \\
\hline 6 & 7 & 2483.51 & 138.1896 & 0.47 & 1324 \\
\hline 6 & 8 & 2261.93 & 131.0131 & 0.45 & 1299 \\
\hline 6 & 9 & 2082.76 & 124.9863 & 0.43 & 1278 \\
\hline 6 & 10 & 1934.37 & 119.8266 & 0.42 & 1258 \\
\hline 3 & 2 & 4767.35 & 213.9579 & 0.62 & 1492 \\
\hline 3 & 3 & 3721.81 & 178.1807 & 0.60 & 1289 \\
\hline 3 & 4 & 3127.75 & 156.4477 & 0.58 & 1206 \\
\hline 3 & 5 & 2735.66 & 141.4299 & 0.55 & 1158 \\
\hline 3 & 6 & 2453.48 & 130.2410 & 0.52 & 1124 \\
\hline 3 & 7 & 2238.60 & 121.4811 & 0.50 & 1097 \\
\hline 3 & 8 & 2068.29 & 114.3772 & 0.48 & 1074 \\
\hline 3 & 9 & 1929.24 & 108.4625 & 0.46 & 1054 \\
\hline 3 & 10 & 1813.07 & 103.4365 & 0.44 & 1036 \\
\hline 0 & 2 & 4257.07 & 124.8181 & 0.50 & 1113 \\
\hline 0 & 3 & 3351.18 & 112.3153 & 0.58 & 862 \\
\hline 0 & 4 & 2829.44 & 103.8894 & 0.59 & 769 \\
\hline 0 & 5 & 2481.88 & 97.6582 & 0.59 & 730 \\
\hline 0 & 6 & 2230.01 & 92.7786 & 0.58 & 711 \\
\hline 0 & 7 & 2037.14 & 88.8060 & 0.56 & 702 \\
\hline 0 & 8 & 1883.59 & 85.4797 & 0.55 & 697 \\
\hline 0 & 9 & 1757.76 & 82.6345 & 0.53 & 695 \\
\hline 0 & 10 & 1652.28 & 80.1597 & 0.52 & 693 \\
\hline
\end{tabular}


Table 5.2 Regression Parameters for Equation 5.2

\begin{tabular}{|c|c|c|c|c|c|c|c|c|c|}
\hline \multirow{2}{*}{$\begin{array}{c}\sigma_{3} \\
(\mathrm{pgi})(\mathrm{pg}\end{array}$} & \multirow{2}{*}{$\begin{array}{l}\sigma_{\mathrm{d}} \\
\text { i) }\end{array}$} & \multicolumn{6}{|c|}{ Regression parameters } & \multirow[t]{2}{*}{$R^{2}$} & \multirow[t]{2}{*}{ VMSE } \\
\hline & & $\mathbf{a}$ & b & c & $d$ & e & $\mathbf{f}$ & & \\
\hline 6 & 2 & 1370 & 887 & -9.817 & 9.828 & -10.909 & -104.47 & 0.72 & 1509 \\
\hline 6 & 3 & 200 & 775 & -8.464 & 8.333 & -9.308 & -74.14 & 0.70 & 1341 \\
\hline 6 & 4 & -385 & 706 & -7.671 & 7.443 & -8.348 & -58.35 & 0.66 & 1298 \\
\hline 6 & 5 & -731 & 657 & -7.131 & 6.834 & -7.688 & -48.60 & 0.63 & 1278 \\
\hline 6 & 6 & -955 & 620 & -6.730 & 6.381 & -7.195 & -41.93 & 0.60 & 1263 \\
\hline 6 & 7 & -1110 & 591 & -6.416 & 6.027 & -6.809 & -37.08 & 0.57 & 1248 \\
\hline 6 & 8 & -1222 & 567 & -6.160 & 5.740 & -6.494 & -33.37 & 0.55 & 1234 \\
\hline 6 & 9 & -1306 & 546 & -5.945 & 5.500 & -6.231 & -30.44 & 0.54 & 1220 \\
\hline 6 & 10 & -1369 & 529 & -5.761 & 5.296 & -6.006 & -28.06 & 0.52 & 1206 \\
\hline 3 & 2 & 68 & 740 & -7.017 & 8.903 & -9.831 & -53.46 & 0.73 & 1324 \\
\hline 3 & 3 & -464 & 653 & -6.316 & 7.579 & -8.427 & -41.32 & 0.72 & 1152 \\
\hline 3 & 4 & -720 & 597 & -5.866 & 6.776 & -7.568 & -34.81 & 0.69 & 1095 \\
\hline 3 & 5 & -865 & 557 & -5.539 & 6.219 & -6.970 & -30.68 & 0.65 & 1065 \\
\hline 3 & 6 & -955 & 526 & -5.286 & 5.802 & -6.520 & -27.79 & 0.63 & 1044 \\
\hline 3 & 7 & -1015 & 502 & -5.081 & 5.475 & -6.165 & -25.64 & 0.60 & 1026 \\
\hline 3 & 8 & -1056 & 481 & -4.909 & 5.208 & -5.876 & -23.95 & 0.58 & 1011 \\
\hline 3 & 9 & -1085 & 464 & -4.762 & 4.984 & -5.633 & -22.60 & 0.57 & 997 \\
\hline 3 & 10 & -1105 & 449 & -4.635 & 4.794 & -5.425 & -21.48 & 0.55 & 983 \\
\hline 0 & 2 & -192 & 503 & -4.874 & 7.071 & -7.695 & -10.11 & 0.66 & 975 \\
\hline 0 & 3 & -377 & 452 & -4.453 & 5.975 & -6.545 & -12.72 & 0.73 & 728 \\
\hline 0 & 4 & -461 & 420 & -4.184 & 5.319 & -5.853 & -14.35 & 0.74 & 651. \\
\hline 0 & 5 & -507 & 396 & -3.990 & 4.869 & -5.377 & -15.46 & 0.73 & 628 \\
\hline 0 & 6 & -535 & 378 & -3.842 & 4.536 & -5.024 & -16.26 & 0.71 & 623 \\
\hline 0 & 7 & -553 & 364 & -3.722 & 4.276 & -4.748 & -16.86 & 0.69 & 625 \\
\hline 0 & 8 & -565 & 352 & -3.622 & 4.066 & -4.525 & -17.33 & 0.67 & 629 \\
\hline 0 & 9 & -573 & 342 & -3.537 & 3.892 & -4.339 & -17.70 & 0.65 & 633 \\
\hline 0 & 10 & -579 & 333 & -3.464 & 3.744 & -4.181 & -18.00 & 0.63 & 637 \\
\hline
\end{tabular}



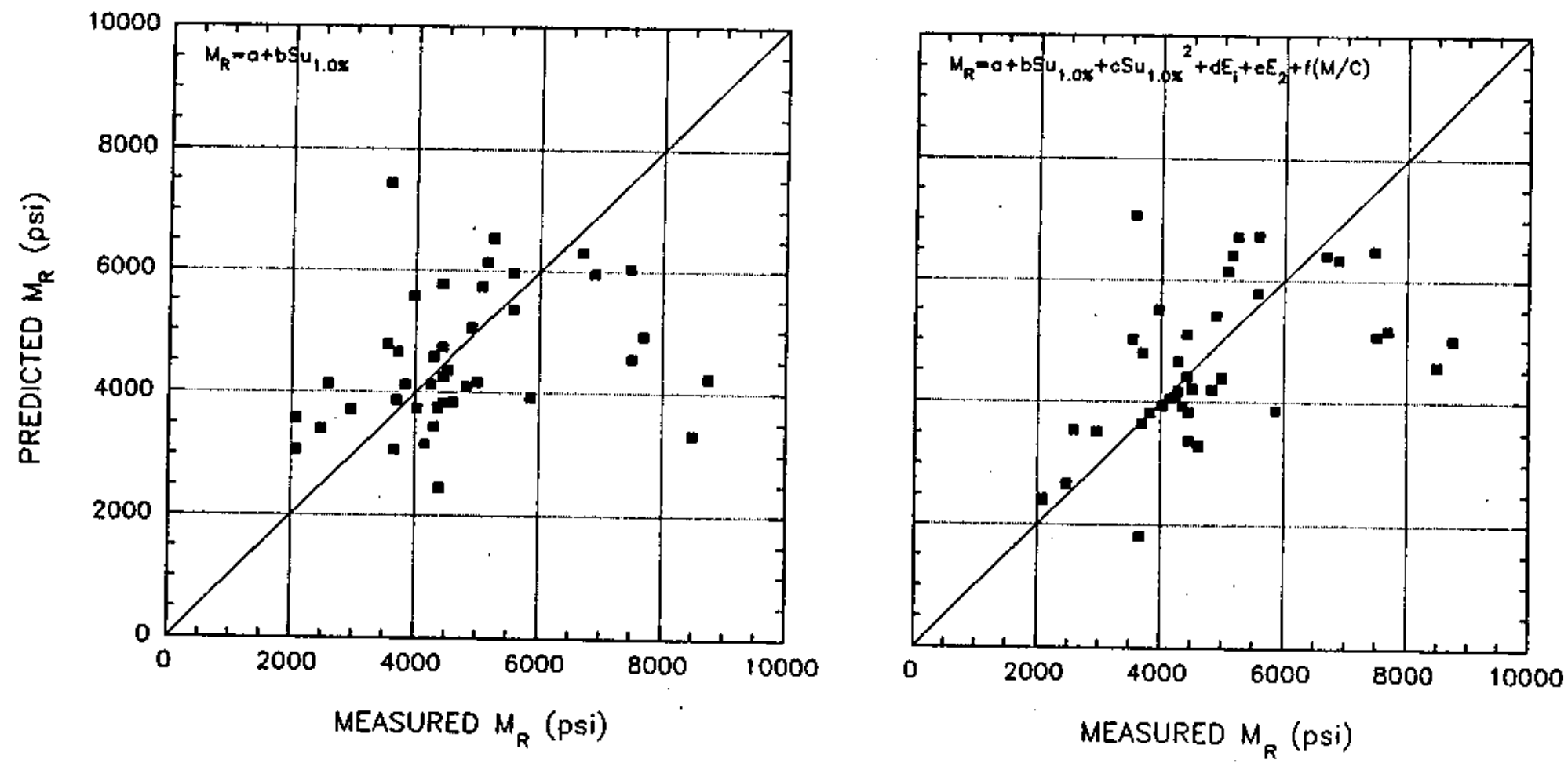

Figure 5.9 Comparison between Measured and Predicted $M_{R}$ of Frozen-Thawed Soils 


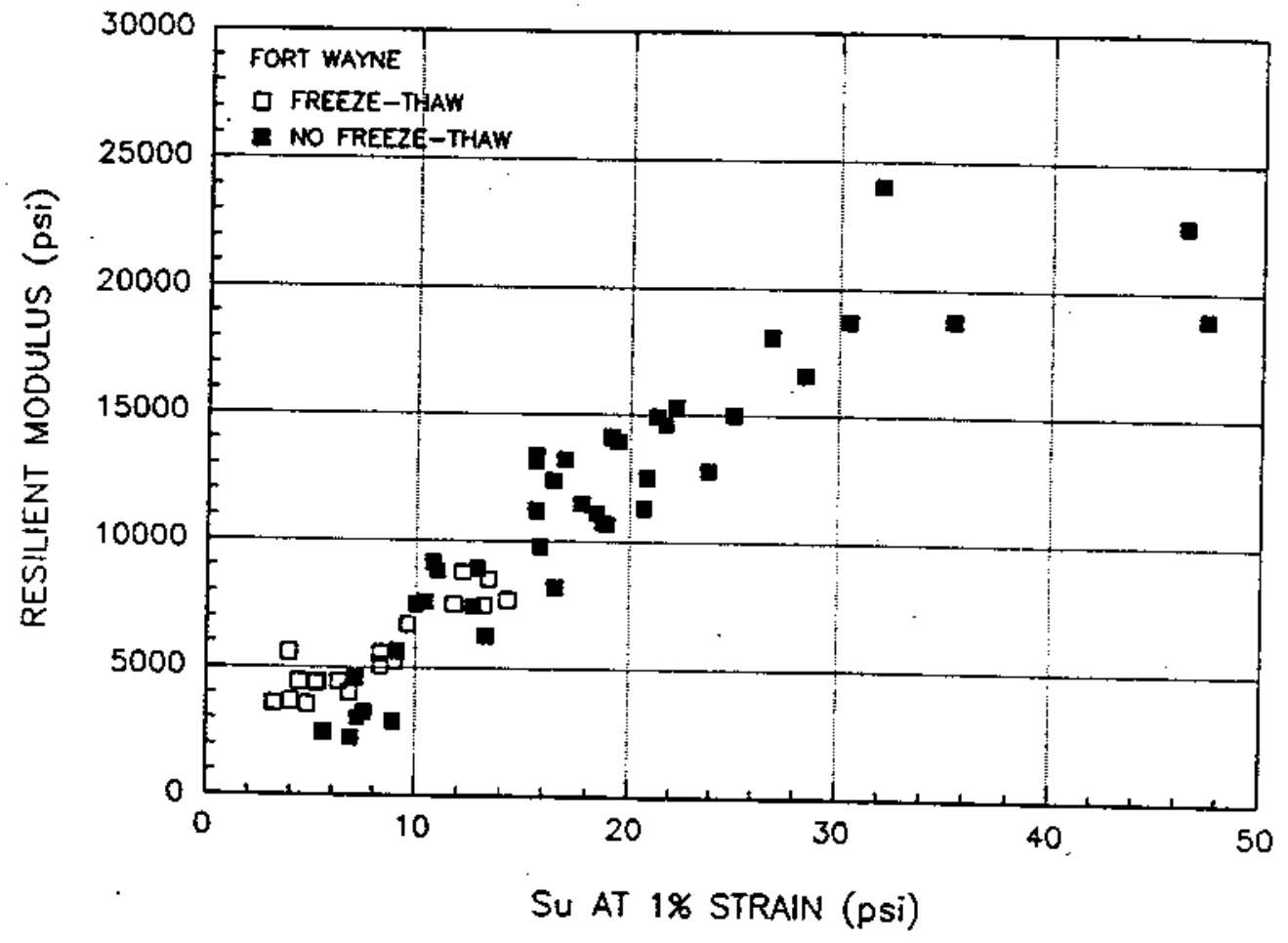

Figure 5.10 Relationship between $M_{R}$ and $S_{v 1.0 \%}$ of Thawed Soil and Non Frozen-Thawed Soil 
5.3 Variation in $M_{R}$ Characteristics due to water content Change

5.3.1 Effect of Water Content Change on Resilient Modulus

It has been known that the water content of subgrades changes with time after construction. Generally, the subgrade is compacted initially to a degree of saturation of about 75 to 85 percent and, after some time, the soil may absorb water to raise the degree of saturation to more than 90 to 95 percent. Therefore, the resilient modulus of in-service subgrade can be significantly different from the as-compacted resilient modulus.

By performing repeated-loading triaxial tests on specimens soaked after compaction, seed et al. (1967) and Tanimoto and Nishi (1970) showed that the resilient strains of specimens compacted on the dry side of optimum increased significantly due to soaking to a high degree of saturation. However, the resilient strains were much smaller than those of specimens compacted directly to the final condition.

since it requires considerable amount of time and effort to prepare a specimen by compaction and to allow it to approach the desirable degree of saturation, seed et a.1. (1962, 1967) and Monismith et al (1967) took an interesting approach with the assumption that the samples compacted wet of optimum by static compaction have similar resilient characteristics to those compacted dry of optimum by kneading compaction and subsequently soaked to a similar degree of saturation. By extrapolating the results on specimens prepared by static compaction, it was shown that, after increasing water content at constant volume to a degree of saturation of 90 percent, the resilient strain of a sample compacted initially to 100 percent relative compaction was only one fifth of that of a sample compacted initially to 96 percent relative compaction and soaked to the same degree of 
saturation. This indicates the beneficial effect of higher density in minimizing the resilient deformation.

\subsubsection{Laboratory Testing Program}

water content of a subgrade may change after construction by several environmental and other factors, such as infiltration of surface runoff, capillary rise from the ground water table, plant growth, and seasonal changes. For better prediction of subgrade behavior it is important to investigate the effect of water content change after construction on resilient characteristics of subgrade soil.

Conventionally, water content of compacted sample is increased by percolation or back pressure techniques (Lowe et al., 1960; Ran and clough, 1984). The magnitude of back pressure and the time required to achieve a desired degree of saturation largely depend on the permeability, pore structure, and the type of soil. Any increase in the water content is achieved by the reduction of the pore air volume. During the process of back pressure saturation, the volume of pore aix in soil is decreased by compressing and pushing out the pore air, and by dissolving additional amount of pore air in the pore water. Compressibility and solubility of the pore air are governed by Boyle's and Henry's law, respectively. For fine grained soils, the back-pressure technique requires high pressure over a long period of time. Moreover, it is required to perform the tests under the applied back pressure, without removing the sample from the cell.

It is difficult to perform resilient modulus tests with high confining pressure and back pressure together using water as a confining fluid. The confining pressure and the compensating back pressure applied on the sample are required to be changed during resilient modulus testing. The change of confining pressure and back pressure causes the change in the degree of saturation during the test, not a desirable matter 
for data interpretation. In order to avoid these changes to occur, a technique has been developed to achieve small increments in the water content of specimen at a faster rate.

Laboratory samples were prepared by impact compaction in a 2.8-inch diameter mold with standard proctor energy. After compacting the samples, a small (about $1 \mathrm{~mm}$ diameter) hole is made at the center of the sample along its length, without taking the sample out of the compaction mold. A small quantity of water is then slowly injected into the hole using an hypodermic needle and the mold is wrapped in a plastic bag to prevent moisture loss from the sample. In order to achieve uniformity of water content in the sample, the mold is rotated by means of an electrical motor to apply a small centrifugal force on the injected water. Slow rotation of a sample induces the flow of water away from the injection hole. Multiple injections at an interval of 4 to 5 hours may be necessary to achieve the required degree of saturation. Uniform injection of water along the length of the sample is achieved by injecting water from both ends of the sample. An increase in water content up to 2.5 percent and the corresponding increase in the degree of saturation of 2 to 9 percent were achieved by this technique. Samples were prepared with a final degree of saturation of up to 95 percent.

Laboratory compacted fine-grained samples for all the sites were injected by this technique. Most of the samples were compacted at 0.5 to 2 percent dry of optimum water content and injected to different final water content.

\subsubsection{Test Results and Discussions}

In genexal, the cohesive soils investigated show a decrease in resilient modulus with increase in the water content. Test results on all five sites are compared in Figure 5.11. The variation of resilient modulus with increase in water content for the each site is discussed in section 10.4. 


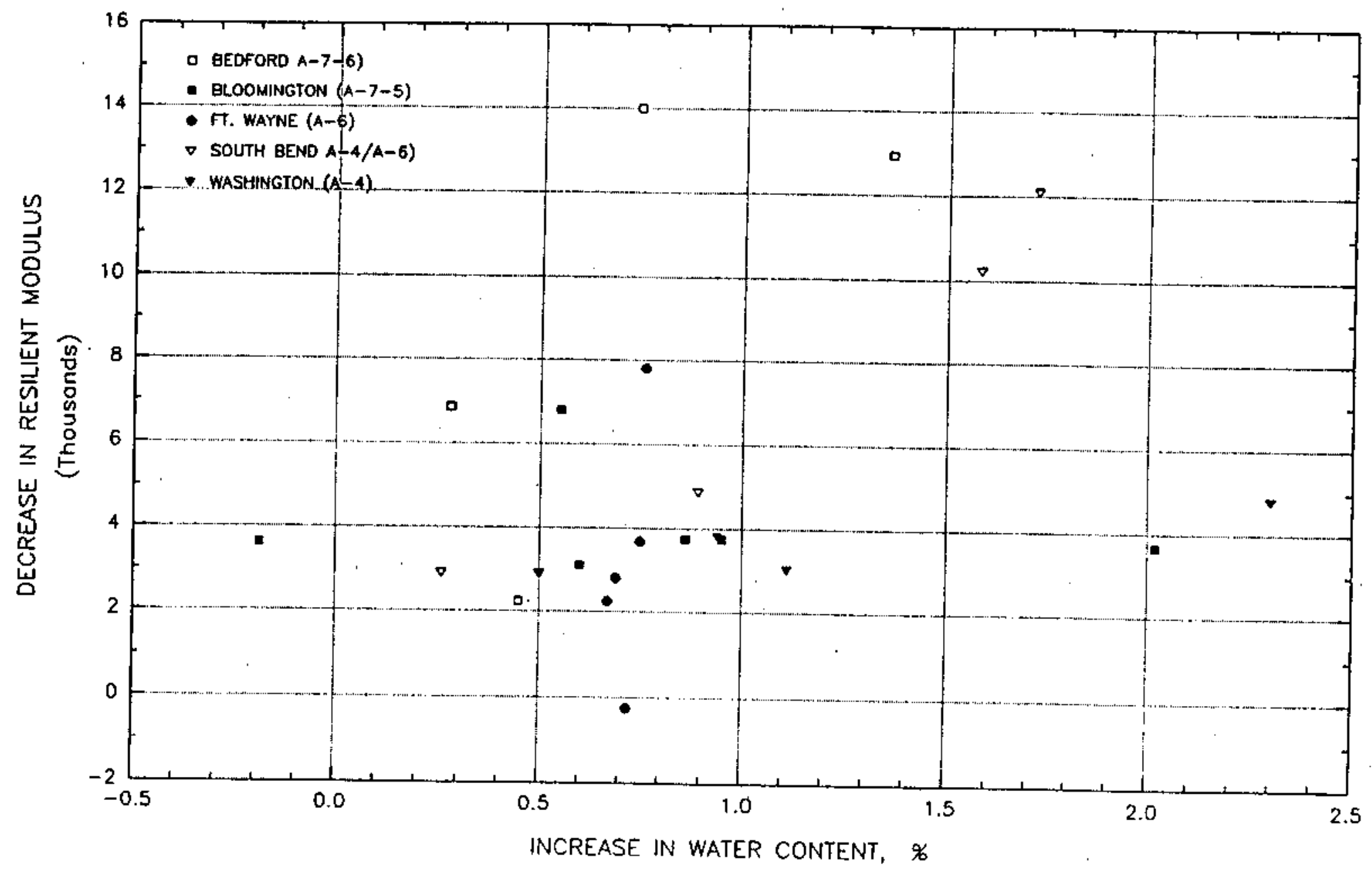

Figure 5.11 Effect of Water Content Change after Compaction on Resilient Modulus 
It can be seen that the reduction in resilient modulus is in the range of $2000 \mathrm{psi}$ to $14000 \mathrm{psi}$; the Bedford soil shows the largest decrease in resilient modulus due to increase in water content. The large scatter may be due to the inconsistent quality of the compacted specimens. Even with the scatter, a general trend in the reduction of resilient modulus with increase in water content is clear.

Bedford soil was compacted at about 1.5 percent dry of optimum. The increase in water content due to injection ranges between 0.28 to 1.36 percent, and causes a decrease in the resilient modulus up to about $14000 \mathrm{psi}$.

Bloomington soil was initially compacted at about 2 percent dry of optimum. The increase in water content ranges between 0.55 to 2.0 percent and causes a decrease in resilient modulus between $3000 \mathrm{psi}$ to $7000 \mathrm{psi}$. Due to the arying of sample during test, one of the data point shows negative value of increase in water content. Results in Figure 5.11 indicate that resilient response of Bloomington soil is relatively less sensitive to changes of water content after compaction.

Fort Wayne soil samples were compacted at about 2 percent dry of optimum. All the specimens subjected to water injection show similar amount of increase in water content. The average increase in water content is about 0.76 percent while resilient modulus shows a wide range of decrement ranging from 0 to $8000 \mathrm{psi}$.

South Bend soil samples displayed the lowest value of optimum water content, and samples for this site have been compacted at about 0.5 to 1 percent dry of optimum. It can be seen that this site shows an exponential decrease in resilient modulus due to increase in water content after compaction.

Washington soll samples were compacted at about 2 percent dry of optimum and the increase in water content due to injection ranges between 0.5 to 2.3 percent. This site shows a nominal decrease in the resilient modulus with the increase in the water content after compaction. 
To compare the effect of the dry side compaction with the wet side compaction on the reduction in resilient modulus due to water content change after compaction, the reduction in resilient modulus of South Bend samples compacted ary side of optimum is plotted with that compacted wet of optimum, as shown in Figure 5.12. As expected, samples compacted wet side of optimum show significantly smaller reduction in resilient modulus than those compacted dry of optimum do.

In order to investigate the effect of molding water content on the change of resilient modulus due to subsequent increase of water content, samples of Fort wayne site were prepared at different molding water contents. As shown in Figure 5.12, the influence of water content change after compaction is diminished with increase in the initial molding water content. It also shows increasing difficulty to increase water content of samples compacted at higher molding water contents. One set of data (at 14.5 percent water content) shows the effect of wetting and drying cycle. This was achieved by injecting water in the sample and leaving the mold unwrapped overnight. Result shows that the resilient modulus at the stage of drying is larger than that of as-compacted condition, at the same water content. The increase in the modulus may be caused by the development of tension in the water menisci at the contact boundaries of soil particles.

Though the current technique is effective in increasing the water content moderately, there are practical problems in achieving a larger increase in water content using this technique. A subgrade eventually reaches the equilibrium water content due to the capillary rise from the ground water table. Also, there is high probability of having near full saturation due to infiltration of water from sources other than the ground water table. To predict the reduction in the resilient modulus due to increase in the water content after construction, it is necessary to develop the equipment and technique which can increase a specific amount of water content effectively. 

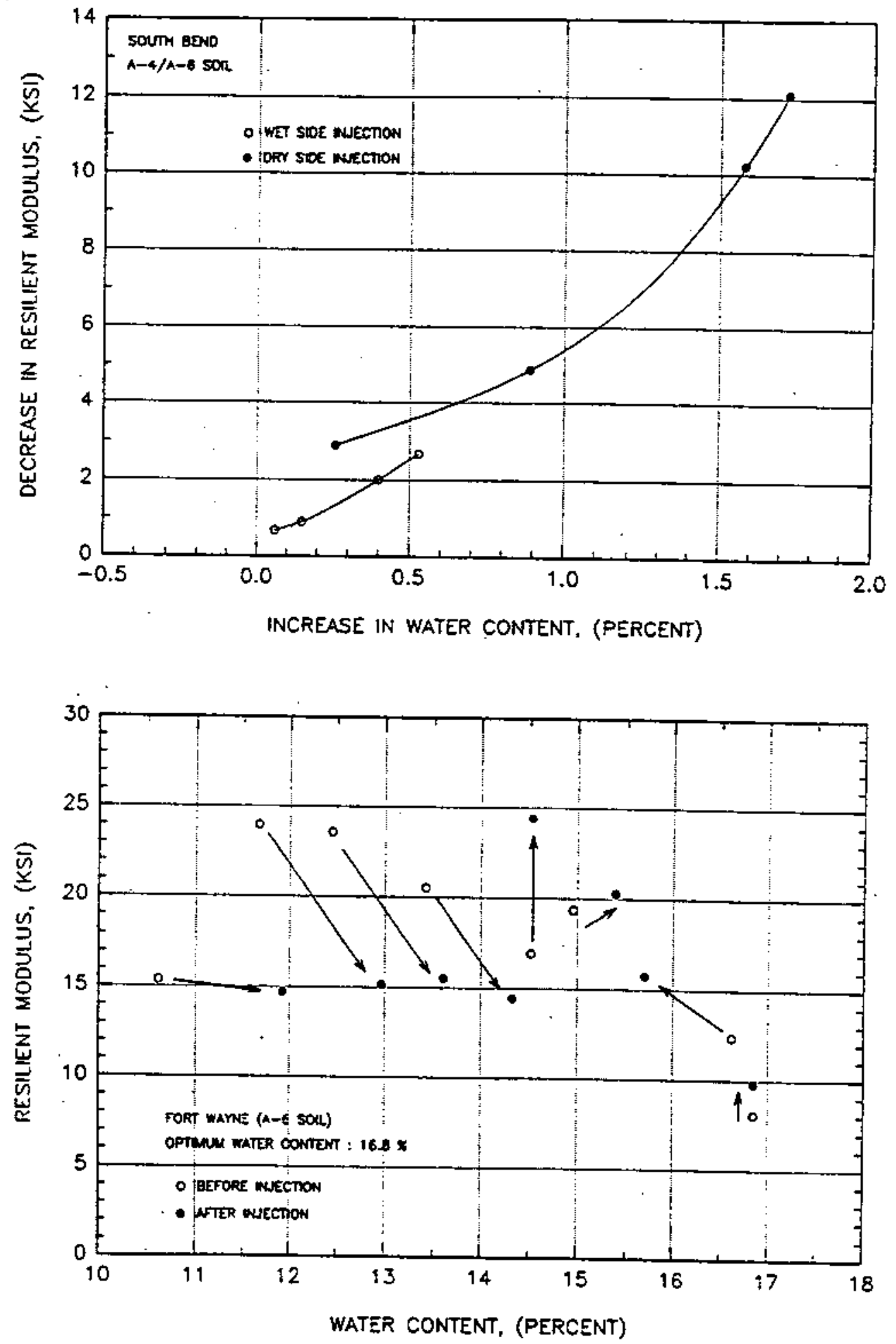

Figure 5.12 Effect of Molding Water Content on the Resilient Modulus due to subsequent Change in Water 
5.4 Resilient Characteristics of Frozen Cohesive Soils

Johnson et al. (1979) tested the Hanover silt and Morin clay specimens at several subfreezing temperatures, taken from the test pavements in the frozen condition. The resilient modulus of frozen Hanover silt was observed to decrease more than one order of magnitude as the temperature approaches the thawing point. Increasing $\sigma_{d}$ influenced the resilient modulus to a lesser but still significant degree. The factors of confining stress, water content and dry density did not appear to significantly affect the results for the range of conditions imposed. The resilient modulus ranges from 126,000 psi at $-0.5^{\circ} \mathrm{C}$ to $5,800,000^{\circ} \mathrm{psi}$ at $-6.5^{\circ} \mathrm{C}$. The resilient modulus of frozen Morin clay was also found to decrease with increasing temperature and increasing deviator stress. It was shown that range in resilient modulus is about $36,000 \mathrm{psi}$ to $2,170,000 \mathrm{ps} 1$, and that the specimen of higher water content resulted in higher resilient modulus.

In this study, six specimens were removed from freezethaw chamber at the end of freezing period and tested by repeated load to study the resilient modulus for frozen cohesive soil. Since no cooling system was used to maintain the frozen condition during repeated-loading test, the resilient modulus was observed to decrease significantly after conditioning stage. Therefore, resilient modulus only during conditioning stage $\left(\sigma_{3}=6 \mathrm{psi}\right)$ is presented in Figure 5.13 . Conditions of each specimen before freeze-thaw are given in Table 5.3. It is shown that the resilient moduli of frozen soils are almost identical, except washington soil, and are almost constant in the range of deviator stress 1 to 10 psi even though the conditions of solls before freezing are considerably different. Average resilient modulus of 27000 psi is reasonable for Bloomington, Fort wayne and south Bend soils. Washington soil shows an average resilient modulus of 46000 psi. 


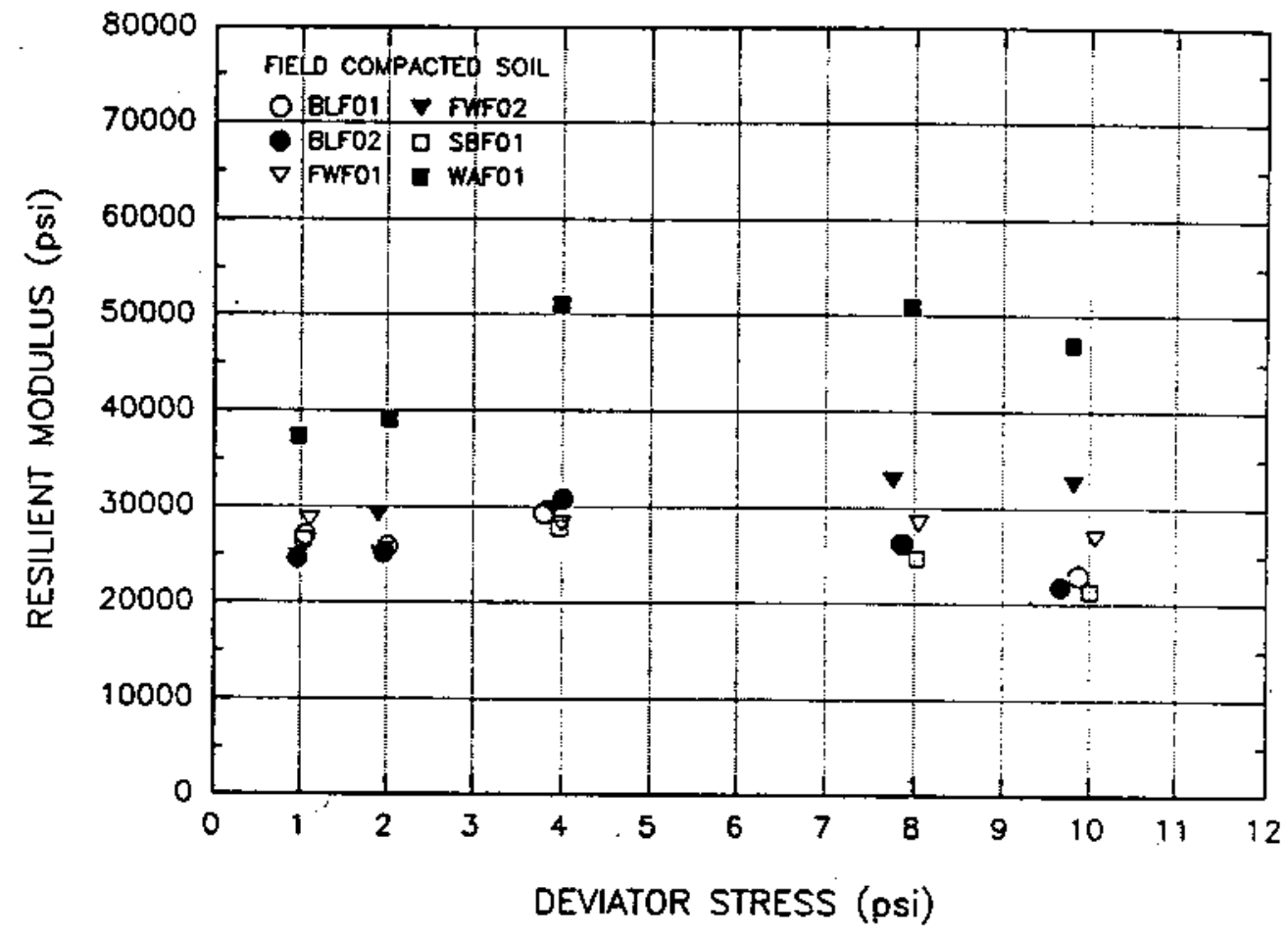

Figure 5.13 Relationship between $M_{R}$ and $\sigma_{d}$ of Frozen soils 
Table 5.3 Properties of Frozen Specimens

\begin{tabular}{c|c|c|c|c|c}
\hline ID & $\begin{array}{c}\text { M/C } \\
(\%)\end{array}$ & $\begin{array}{c}\gamma_{d} \\
(\text { pcf })\end{array}$ & $\begin{array}{c}S_{\mathrm{u} 1.0 \times} \\
(\mathrm{psi})\end{array}$ & $\begin{array}{c}w_{L} \\
(\delta)\end{array}$ & $\begin{array}{c}W_{\mathrm{P}} \\
(\delta)\end{array}$ \\
\hline BLF01 & 32.9 & 89.6 & 8.9 & & \\
\hline BLF02 & 24.9 & 98.4 & 10.9 & & \\
\hline FWF01 & 11.5 & 124.6 & 24.7 & 29.0 & 13.2 \\
\hline FWF02 & 17.0 & 117.8 & 23.4 & 33.5 & 15.8 \\
\hline SBF01 & 8.3 & 135.3 & 36.3 & 21.7 & 8.9 \\
\hline WAF01 & 19.0 & 110.0 & 14.0 & 40.0 & 18.0 \\
\hline
\end{tabular}

\subsection{Concluding Remarks}

Ice segregation during freezing is not always a necessary condition for thaw-weakening of pavement subgrade. It was observed in this study that the closed-system freeze-thaw induces a significant reduction in resilient modulus of cohesive soils without visible ice segregation. It was also shown that only one or two cycles of freeze-thaw is enough to reduce the resilient modulus significantly.

From the comparison of the resilient modulus between laboratory frozen-thawed soil and field frozen-thawed soil, the closed-system freeze-thaw testing procedure developed in this study was found to simulate the freeze-thaw occurring in the field reasonably well. There is a negligible effect of freeze-thaw for soils having $S_{\text {ul.ox }}$ less than 8 psi, while the effect of freeze-thaw on resilient modulus becomes larger as $S_{\text {ul. } 0 x}$ increases. The soil whose $S_{01.0 \pi}$ is greater than 15 psi would have a more than 50 percent reduction in resilient modulus due to freeze-thaw. 
The relationship between thawed resilient modulus and the $S_{u 1.0 *}$ for five sites appear to fit one single relationship. If an insignificant change of water content occurs during freezing-thawing, the following equation is suggested to predict the thawed resilient modulus of in-service subgrade:

$$
M_{R}=2453.48+130.24 S_{u 1.0 *}
$$

where $M_{R}$ is the resilient modulus at $\sigma_{3}=3$ psi and $\sigma_{d}=6$ psi (psi); and $S_{u 1.0 \%}$ is the stress to cause 1 percent strain in unconfined compression test performed before the freeze-thaw of specimen. It was shown in chapter 4 that the relationship between $M_{R}$ and $S_{v 1.0 \pi}$ for laboratory and field compacted soil is only slightly different, and, therefore, the effect of freezethaw on laboratory compacted soil is expected similar with that of field compacted soil. So, the thawed resilient modulus of as-compacted soil may be estimated from the unconfined compression test on laboratory compacted specimen using Equation 5.3 .

For in-service conditions, the relationship of thawed $M_{R}$ with thawed $S_{u 1.0 *}$ is found similar with that between $M_{R}$ and $S_{u 1.0 \%}$ of soils which have not been subjected to freeze-thaw. Therefore, the thawed resilient modulus of in-service soil can be estimated from the unconfined compression test result on the specimen obtained from thawed subgrade using following equation:

$$
M_{R}=-1599.66+833.83 S_{u 1.08}-6.9683 S_{u 1.0 t^{2}}
$$

where $M_{R}$ is the thaw-weakened resilient modulus at $\sigma_{3}=3$ psi and $\sigma_{d}=6 \mathrm{psi}$ (psi); and $S_{\mathrm{vl} .0 \mathrm{x}}$ is the stress to cause 1 percent strain in unconfined compression test performed on the undisturbed soil obtained from thawed subgrade. The resilient modulus of the as-compacted condition, the in-service condition, and the thaw-weakened condition may be estimated from the above equation if $S_{a 1.0 *}$ is measured on the specimen under corresponding conditions. 
In general, cohesive soils show a decrease in resilient modulus with increase in the water content after compaction. Relationship between the reduction in resilient modulus due to the increase in water content after compaction for each sites is given in Section 10.4. Estimation of the in-service water content can be made by using the moisture characteristics curves prepared by the pore-size data. However, reliable estimate of change in in-service water content can only be made by collecting samples in different seasons, and hence considered beyond the scope of this work. It is desirable to develop a technique capable of increasing the water content of specimen more effectively, and to study further the effect of change in water content after construction on the resilient modulus.

It was observed that the resilient modulus of frozen soil is independent of the deviator stress and is almost identical for Bloomington, Fort Wayne, and South Bend soils. A recommended resilient modulus of frozen condition is $27000 \mathrm{psi}$ for Bloomington, Fort Wayne, and South Bend sites, and 46000 psi for the Washington site. The modulus of frozen soil does depend on the magnitude of the in place temperature. We have used the data obtained in Illinois for subgrade temperatures, when testing procedures were established (see pages 120-122 of the report). Thus, we believe the data of this report are directly applicable to Indiana conditions. It could be useful, in long-term studies of pavement performance, to include subgrade temperature variation as basis for possible refinements of the details of this report. 
6. RESILIENT MODULUS OF GRANULAR SOIL

\subsection{Introduction}

The rational approach to pavement design requires that the resilient characteristics of each component of the pavement system be known. As dune sand has been widely used as a subgrade material in northern Indiana, it is necessary to investigate its resilient property for the mechanistic analysis of the pavement structure and for use with the 1986 AASHTO Guide for the Design of Pavement structures.

For this purpose, sand was sampled from the subgrade of I-94 at station 1076+00. This dune sand is very uniform, and its particle size distribution is shown in Figure 2.9. About 65 percent of particles are between $0.425 \mathrm{~mm}$ and $0.250 \mathrm{~mm}$. More than 99.8 percent of particles are smaller than $2.0 \mathrm{~mm}$ and the percent passing No. 200 sieve is less than 2.5 percent by weight. since there exists no satisfactory method to sample the undisturbed sand with reasonable effort and time, disturbed sand was compacted in the laboratory and tested for resilient modulus by a repeated-load triaxial test.

According to the previous studies, the most important factors influencing resilient behavior of granular materials are the state of stress, degree of saturation, initial density, and gradation. Since previous studies are mainly for aggregates used as base and subbase materials, it is necessary to study the influences of those factors on the resilient characteristics of dune sand. In this study, the effects of compaction method, moisture content, and dry density on the resilient characteristics and plastic deformation of dune sand are studied. Regression equations and design chart to predict 
the resilient modulus of dune sand are also developed to help pavement design engineers.

6.2 Factors Influencing Resilient Properties of Granular Soil

6.2.1 Loading pulse

Hicks and Monismith (1971) tested two aggregates, one a well-graded, subgranular, partially crushed gravel and another a well-graded crushed rock, to study the effect of density, gradation and degree of saturation on the resilient modulus. Through preliminary test to establish the influence of number of stress repetitions and sequence of test, it was found that as long as the stresses are representative of those found in a pavement structure, the resilient modulus determined after 50 to 100 axial stress applications could be used to properly characterize the behavior of granular materials; one sample could be used to determine the resilient response for stresses of different magnitudes. Some of the tests were conducted over a range in load duration of 0.1 to 0.25 second and no observable influence on resilient modulus was found.

Monismith et al. (1967) tested Monterey sand by repeated loading of 5 to $60 \mathrm{psi}$ with a stress ratio $\left(\sigma_{1} / \sigma_{3}\right)$ of 1.5 up to 100000 repetitions; it was shown that resilient strain increased rapidly up to 10 repetitions and reached a constant value at fewer than 100 repetitions.

Tanimoto and Nishi (1970) showed from the tests on the residual soil derived from decomposed granite with two frequencies, 20 and 120 applications per minute, that the higher the frequency of stress application, the smaller the resilient modulus. In this test, the maximum possible increase in resilient strain was about 30 percent. For dry sand, the resilient strain decreased as the number of stress applications increased. This decrease in resilient strain was attributed to an increase in density of the specimen during repeated loading. 
Kalcheff and Hicks (1973) tested four crushed stones which had 5.6 to 12.6 percent fines passing $\$ 200$ sieve. Test results indicated that the number of stress repetitions and sequence of stress had little effect on the resilient behavior. There was no evidence of a change in resilient modulus with changes in load duration or frequency. It was also shown that the resilient response determined after 150 to 200 repetitions of stress would provide a reasonable indication of resilient properties and the response to stresses of different intensities could be measured in any sequence on a single specimen. It was noted that there was a considerable effect of stress sequence on the plastic deformation of the specimen though there was little effect of stress sequence on the resilient properties.

Allen and Thompson (1974) performed double cyclic loading test on two aggregates, crushed stone and gravel; they showed that both materials yielded results similar to those reported by Hicks and Monismith (1971). The resilient response of these materials after 25 to 100 stress applications represented the response determined after several thousand stress repetitions. One specimen could be used to measure the resilient response over a wide range of stress levels, and these stresses could be applied in any order. The resilient modulus of these materials was found to be affected slightly by variations in pulse duration from 0.04 to 1.0 second.

\subsubsection{Level of Stresses}

Hicks and Monismith (1971), Kalcheff and Hicks (1973), Monismith et al. (1967), Allen and Thompson (1974), Brown and Pell (1967), and Khedr (1985) have reported a significant increase in resilient modulus for untreated granular base course materials with an increase in confining stress. Smaller increases in resilient modulus are associated with an increase in repeated deviator stress at a constant confining stress. 
These effects of confining stress and deviator stress upon resilient modulus have been approximated by:

$$
M_{R}=k_{1} \theta^{k_{2}}
$$

where $\theta$ is first invariant of stress $\left(\sigma_{1}+\sigma_{2}+\sigma_{3}\right)$, and $k_{1}$ and $k_{2}$ are constants determined experimentally. This equation has been widely used and is supported by data obtained from repeated load triaxial tests. Rada and Witczak (1981) analyzed 271 resilient modulus test results and found that there appeared to be an inverse relationship between $k_{1}$ and $k_{2}$ for all granular materials. As shown in Figure 6.1, a definite correlation exists between them; the regression equation is as follows:

$$
\log _{1}=4.657-1.807 k_{2}
$$

The results shown include a very wide range of granular materials ranging from fine silty sands to slag and lime-rock aggregates.

The model discussed above has been extremely useful and simple, but has some deficiencies. First, the equation $M_{R}=$ $k_{1} \theta^{k 2}$ is dimensionally unsatisfactory. Second, there is evidence that the relationship between $M_{R}$ and $\theta$ varies with the magnitude of the repeated deviator stress. Repeated load triaxial testing of a crushed granite by Brown (1974) indicated that the relationship between $M_{R}$ and $\theta$ can not be represented by a single straight line. Furthermore, Johnson et al. (1986) showed the dependency of this relationship on stress ratio, and proposed the model involving the second invariant of stress $\left(J_{2}\right)$ and the octahedral shear stress $\left(\tau_{\alpha c}\right)$ as:

$$
M_{R}=k_{1}\left(\frac{J_{2}}{\tau_{\text {oct }}}\right)^{k_{2}}
$$




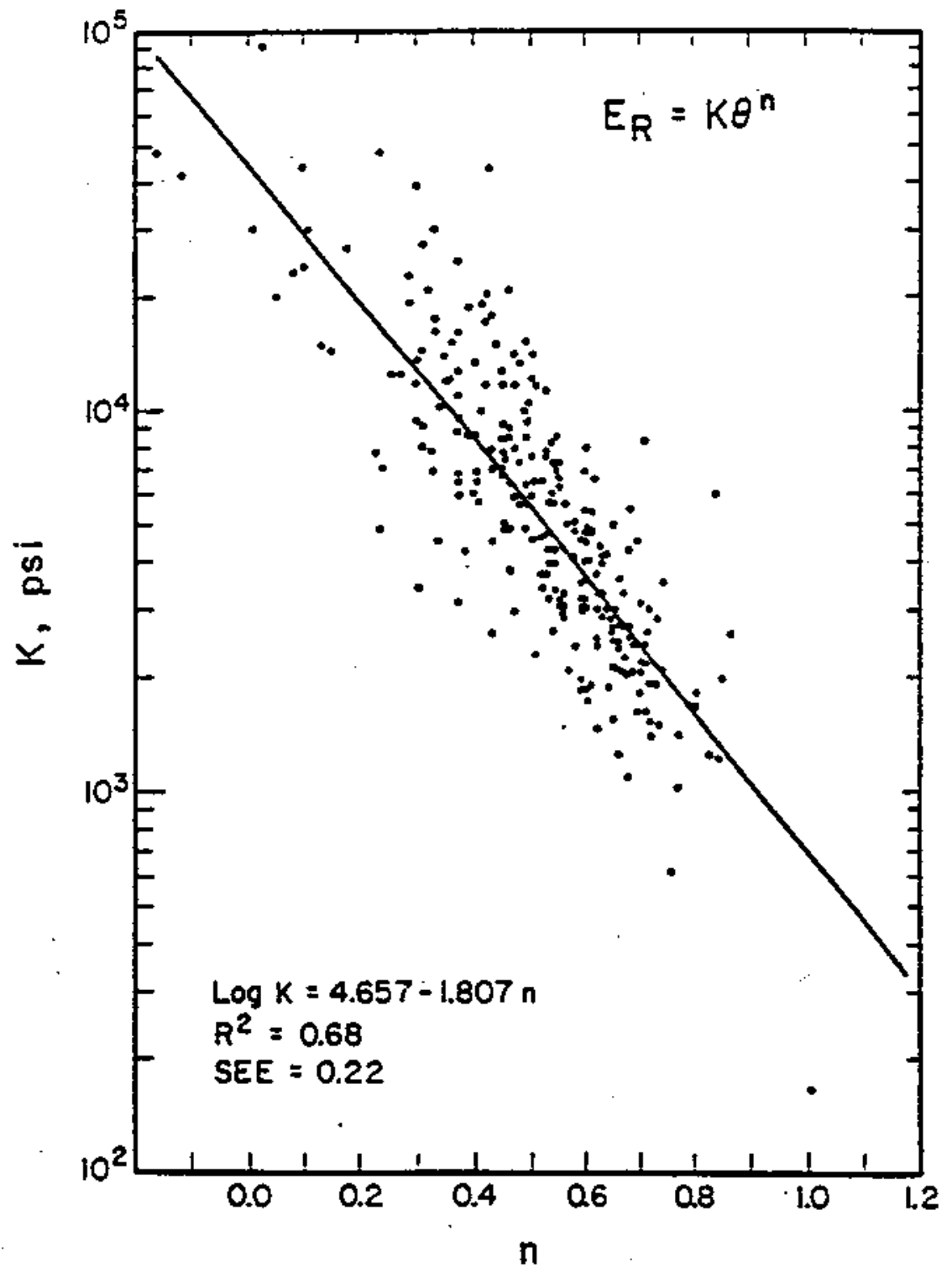

Figure 6.1 Relationship between $k_{1}$ and $k_{2}$ (after Rada and 
where $J_{2}=\sigma_{1} \sigma_{2}+\sigma_{2} \sigma_{3}+\sigma_{3} \sigma_{1} ; \tau_{\alpha t}=(\sqrt{2} / 3) \sigma_{d} ;$ and $k_{1}$ and $k_{2}$ are constants determined experimentally. Lastly, Equation 6.1 implies that, for a constant confining stress, the resilient modulus should increase with an increase in repeated deviator stress. However, Khedr (1985) showed that the resilient modulus decreased with increasing deviator stress, for deviator stress less than 10 psi. Uzan (1985) also noticed the reversed trends of resilient modulus of a dense graded aggregate. It was shown that the resilient modulus predicted by Equation 6.1 at constant confining stress increased monotonically as the vertical strain increases, whereas the test results indicated that the resilient modulus first decreased as the vertical resilient strain increased and then increased as the strain increased further. The increase in the modulus values occurred at principal stress ratios $\left(\sigma_{1} / \sigma_{3}\right)$ larger than 2 or 3 . Therefore, the following equation including deviator stress in the model was suggested:

$$
M_{R}=k_{1} \theta^{k_{2}} \sigma_{d}^{k_{3}}
$$

where $k_{1}, k_{2}$ and $k_{3}$ are constants determined experimentally. Equations 6.2 and 6.3 clearly underline the influence of repeated deviator stress on the resilient modulus of granular materials. However, they are dimensionally unsatisfactory and have not been generally accepted for characterization of granular materials. On the other hand, Equation 6.1 remains popular because of its simplicity.

Nataatmadja and Parkin (1989) proposed the following model which is dimensionally satisfactory and incorporates confining stress:

$$
\frac{M_{R} \sigma_{d}}{\theta}=E+F \sigma_{d}
$$

A plot of $\left(M_{R} \sigma_{d}\right) / \theta$ versus $\sigma_{d}$ allows $E$ and $F$ to be determined as the intercept value and the slope of the straight line, 
respectively. Comparison between predicted resilient modulus and the experimental data reported by Hicks and Monismith (1971) and Uzan (1985) indicated that the proposed model was able to predict the $M_{R}-\epsilon_{R}$ and $\sigma_{d}-\epsilon_{R}$ relation of material. The predicted $\sigma_{\mathrm{d}}-\epsilon_{\mathrm{R}}$ curves were initially concave downwards, indicating decreasing resilient modulus; for deviator stress values greater than 10 to $13 \mathrm{psi}$, the predicted curves were concave upwards, indicating increasing modulus.

\subsubsection{Initial Dry Density}

Hicks and Monismith (1971) showed that the coefficient $k_{1}$ increased with increasing density while $k_{2}$ remained relatively constant or decreased slightly. For partially crushed aggregate, the modulus increased with relative density; for the crushed aggregate, relative density had only a small influence on the resilient modulus. For both type of aggregates, the effect of density decreased as the percentage of fines increased.

Rada and Witczak (1981) performed repeated loading triaxial tests on six aggregates and showed the effect of compaction energy on the resilient characteristics. It was observed that $k_{1}$ increased gradually with increasing compaction energy, and that the $k_{2}$ showed no distinctive pattern and remained essentially constant. The average increase in $k_{1}$, by increasing effort from Standard to Modified Proctor energy, was about 48 percent.

\subsubsection{Degree of Saturation}

The degree of saturation of most untreated granular base course material has generally been found to affect its resilient characteristics. It should be noted that for typical gradations and densities of untreated granular base course materials a small change in water content corresponds to a large change in degree of saturation. 
Haynes and Yoder (1963) performed repeated load triaxial tests on both gravel and crushed stone base course material. It was found that the resilient modulus of the gravel at a degree of saturation of 97 percent was about one half of that at a degree of saturation of 70 percent; tests on crushed stone had small random variations not exceeding 20 percent within the range of 70 to 80 percent degree of saturation. The decrease in modulus with increase in degree of saturation appeared to be approximately Iinear. Kallas and Riley (1967) reported a linear decrease in the regression constant $k_{1}$ with increase in degree of saturation when test results were analyzed on a total stress basis, while the constant $\mathbf{k}_{2}$ remained approximately constant with changes in degree of saturation.

Hicks and Monismith (1971) concluded from their test results that resilient modulus of untreated granular material decreased with increase in degree of saturation if test results were analyzed on the basis of total stress, but remains approximately unchanged if analyzed on the basis of effective stress. Seed et al. (1967) subjected a prototype pavement to repeated loading using circular loading plates. Test results indicated that when the granular base course was saturated, the resilient deformation within the base course layer increased by approximately 50 percent.

Smith and Nair (1973) tested well graded, partially crushed gravel ( $\frac{3}{4}$ inch maximum, class 2 aggregate base, $2-3$ percent passing $\$ 200$ sieve, $\gamma_{d}$ of 139.3 paf, water content of 5.2 percent) with single and double cyclic test. Data from both tests indicated that for a given value of $\theta$, the resilient modulus for single cyclic triaxial tests was somewhat greater than that from double cyclic tests. The difference in resilient behavior from both type of tests was attributed to the difference in generated pore water pressure. since the double cyclic tests would induce the greater pore water pressures, the effective value of $\theta$ would be smaller for 
double cyclic tests. Thus, the resilient modulus at a given total stress value of $\theta$ would be smaller for double cyclic tests. By analyzing the data reported by Hicks and Monismith (1971) for undrained single cyclic repeated load tests on saturated specimens, it was shown that the reduction in modulus due to saturation based on total stress was approximately 10 percent if only axial stress was repeated; dry untreated granular base course material would have a modulus 50 percent greater than that of highly saturated material if the specimen was subjected to simultaneous increases in axial and radial stresses.

Rada and witczak (1981) showed the marked reduction in the magnitude of $k_{1}$ with increasing degree of saturation from the 159 separate test results gathered from 10 agencies. In general, the effects of moisture can change $k_{1}$ from about 30000 for the dry state to 1000 for the saturated state, with resultant changes in modulus from 40000 to $10000 \mathrm{psi}$ or less. By analyzing test results on six aggregates performed at the University of Maryland, it was shown that the influence of degree of saturation on $k_{1}$ appeared significant and depended on type of aggregate, while $k_{2}$ was little changed. The two crushed stone materials exhibited less sensitivity in $k_{1}$ to the degree of saturation while $k_{1}$ of the bank-run gravel (poorly graded sand-gravel) decreased significantly at degrees of saturation larger than 80 percent.

\section{2 .5 Gradation}

Hicks and Monismith (1971) showed that the regression constants $k_{1}$ and $k_{2}$ were slightly affected by the fines content. The manner in which $k_{1}$ changes was dependent on the type of aggregate. The constant $k_{1}$ of partially crushed aggregate generally decreased while $k_{1}$ of crushed aggregate increased as fines content increased. The same trends were observed for the partially saturated and saturated test 
series. Although the effect of gradation on $k_{2}$ was not well defined, it appeared that $k_{2}$ decreased slightly as fines content increased. For the ary test series, $k_{1}$ was always larger for the crushed aggregate than in the partially crushed material, regardless of aggregate gradation. The percent change of $k_{1}$, however, appeared to be a function of aggregate gradation. For coarse grading, the difference was about 5 percent, while for the medium and fine grading the differences were 31 and 64 percent, respectively. The actual effect on the resilient modulus was not nearly as great because there were also differences in $k_{2}$.

Rada and witczak (1981) showed from the test results on six aggregates that there was no general trend applicable for aggregate types. For angular base materials, there appeared to be little change in either $k_{1}$ or $k_{2}$ for percent passing $\$ 200$ sieve of 7 to 17 percent. For bank-run gravel, an optimum $k_{1}$ value was apparent near the dense condition and a marked decrease in $k_{1}$ occurs as the percent passing 200 sieve increased. Although no pronounced changes occurred in resilient modulus for the base materials, it was suggested that an increase in percent passing $\$ 200$ sieve beyond 16 to 18 percent would eventually have pronounced changes in the resilient response for these materials.

Knutson and Thompson (1977) tested five typical open graded aggregate materials prepared by vibratory compaction with three levels of compaction energy. It was found that the resillent moduli of No. 4 and No. 5 ballast gradation aggregates (American Railway Engineering Association) were usually slightly lower than that of a well-graded aggregate. The resilient moduli of open-graded ballast materials were virtually insensitive to changes in gradation or compaction level.

Allen and Thompson (1974) showed that the effects of type of material on the resilient parameters were insignificant compared with the effects of changes in stress level. In 
general, crushed stone yielded slightly higher values of resilient modulus than gravel.

\subsection{Laboratory Experimental Program}

6.3.1 Preparation of Specimen

Sand obtained from the field was air-dried in the laboratory and tapped by wood hammer to break any clods. After measuring the water content of the air-dried sand, amounts of air-dried sand and water to be mixed were calculated. considering the loss of water during mixing and curing, an amount of water corresponding to 0.5 percent of water content was added to the target value. Soil was mixed manually while spraying water into a mixing pan. Mixed soil was put into a plastic bag and stored at room temperature for 24 hours.

since there were no data on the as-compacted conditions of this sand, the project advisory committee suggested that disturbed sand in the laboratory be compacted to the same specifications as field compaction specifications and to test for resilient modulus by repeated-load triaxial tests. Sand was compacted by impact hammer into a split mold of 4-inch diameter and 8-inch height. To achieve standard proctor energy, each specimen was compacted in five layers with total of 128 blows by 5.5 lbs hammer. Compaction results by impact hammer are summarized in Figure 6.2 ; as shown, the dry density increases slightly when compaction water content increases from 3.5 percent to 7 percent, while dry density of about 106.4 pcf is almost constant at water content ranging from 7 percent to 13.5 percent. It has been known that uniform sands, containing little or no silt and clay, respond poorly to impact compaction but respond very well to vibratory compaction. Johnson and Sallberg (1962) showed that some sands display straight-line impact compaction curves throughout the range from the dry to saturated condition. 


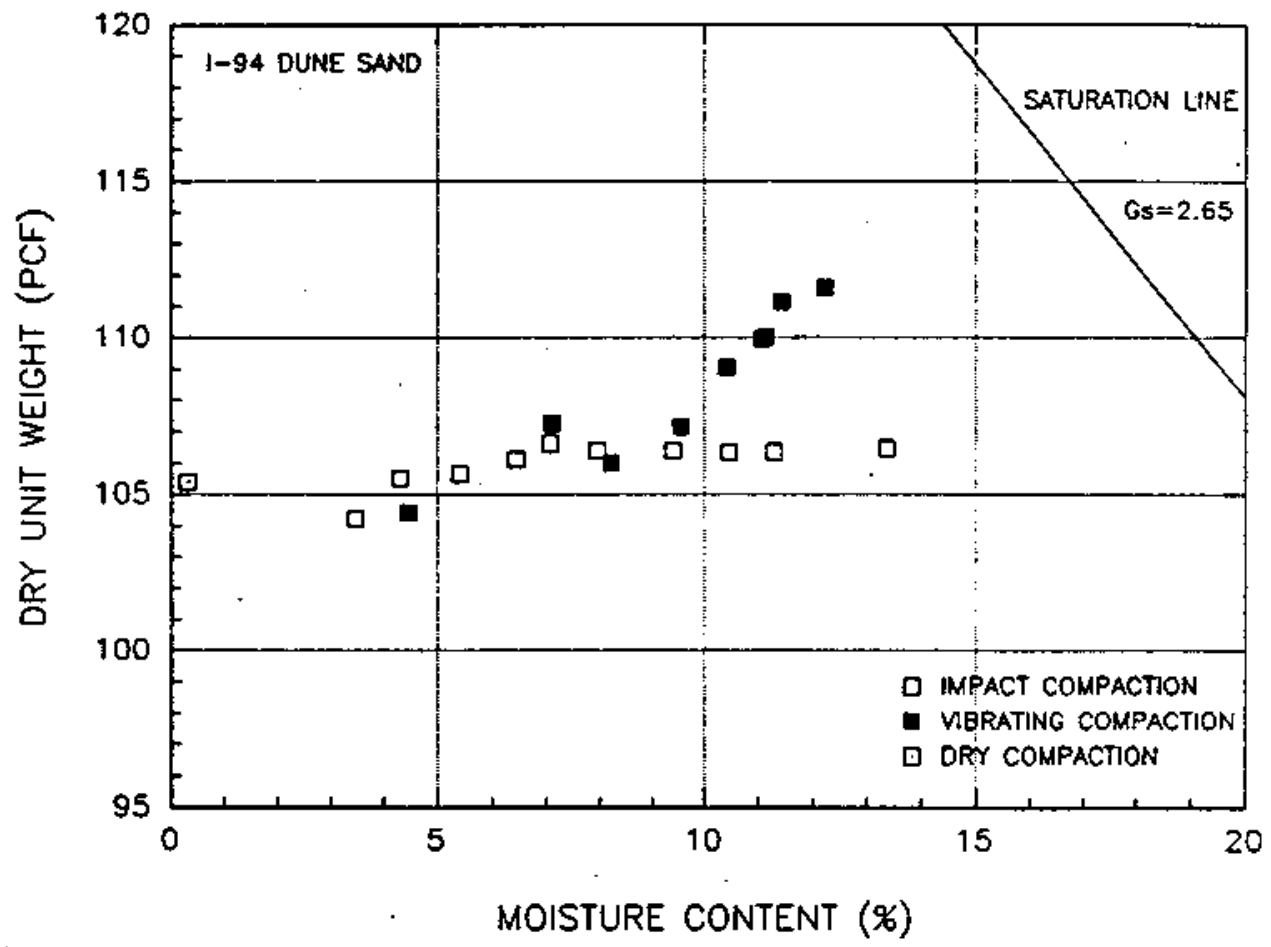

Figure 6.2 Compaction Curves of Dune Sand 
Another series of sand specimen was prepared by vibratory compaction. A table generating vibrations of $60 \mathrm{~Hz}$ was used for compaction and it had 10 dial settings to control the magnitude of vertical amplitude. The relationship between peak to peak amplitude of vertical vibration and the dial settings of the vibrating table was established, as shown Figure 6.3, by measuring amplitude using a PCB 308B piezoelectric accelerometer. It was observed during measurement that the harmonic frequencies of $60 \mathrm{~Hz}$ were present with $120 \mathrm{~Hz}$ being dominant at dial settings less than 4. As can be seen in Figure 6.3, peak to peak amplitude is less than 0.0005 inch up to dial settings of 4 and increases rapidly for dial settings larger than 4 .

To determine the optimum amplitude of vibration, four specimens were prepared by placing air-dried soil as loosely as possible in the mold by pouring the soil from the spout, adjusting the height of the spout to maintain a free fall of sand of about $\frac{1}{2}$ inch. After levelling the excess soil with the top of the mold with a straight edge, the weight of mold and soil was measured to calculate the dry density. Dry density of four prepared specimens ranged from 89.03 pcf to 90.27 pcf. The side of mold was, then, struck several times using a wood hammer to settle the soil so that the surcharge base could be easily placed into position. Dry density after tapping was between 96.54 pcf and 97.86 pcf. After placing a convenient surcharge of 13.3 lbs on the surface of sand, the mold containing sand was attached to the vibrating table and the change of density during vibration was measured at different times. Changes of ary unit weight of sand with time due to vertical vibration of four different amplitudes are shown in Figure 6.4. For all four compactions, the density increases significantly with 60 second vibration, and five minutes of vibration appears enough to obtain full densification. Continued increase of dry density after 5 minutes may be due to crushing of sand particles. To account for the effect of 


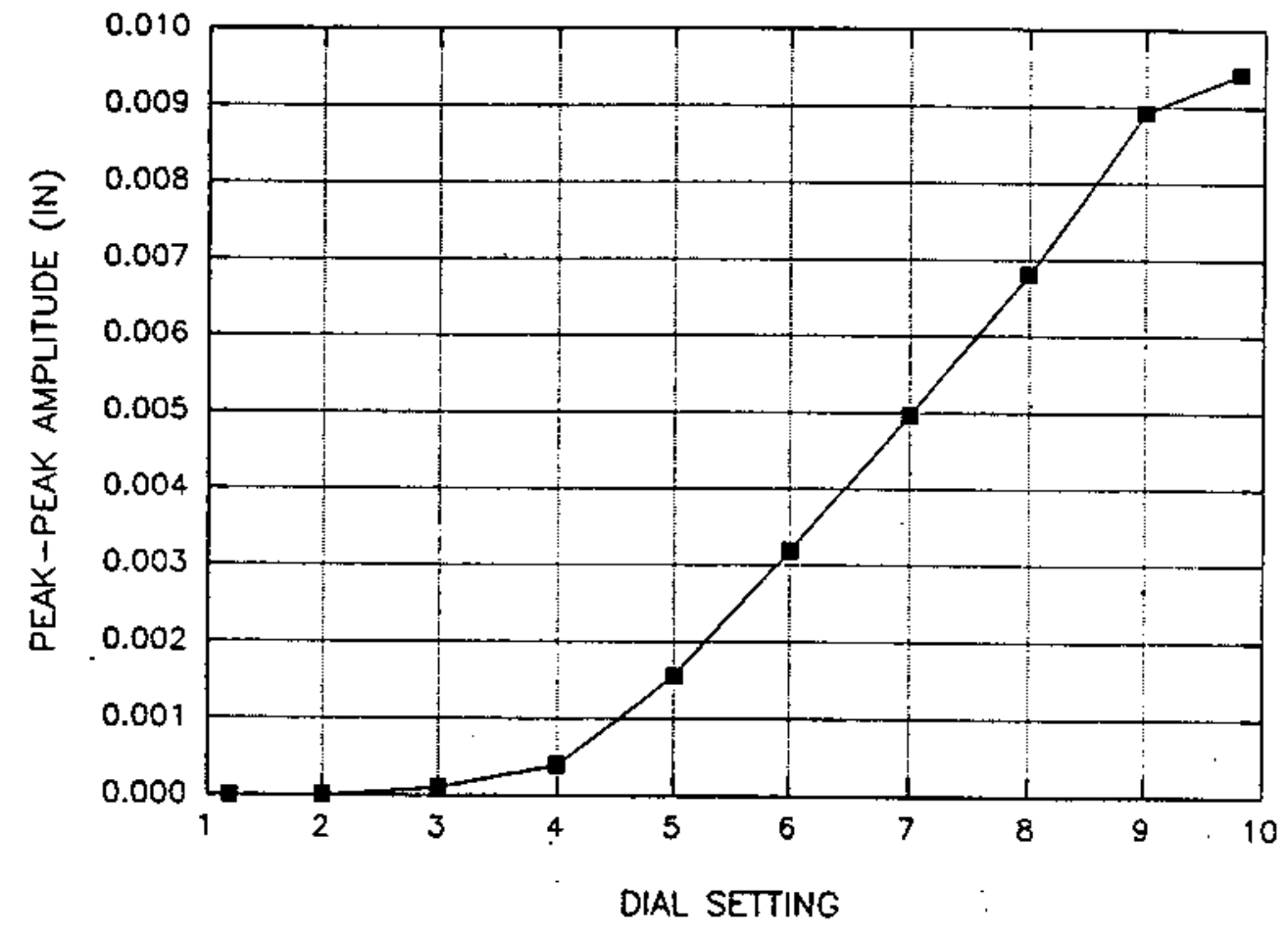

Figure 6.3 Relationship between Peak to Peak Amplitude of Vertical vibration and Dial setting of the vibrating Table 

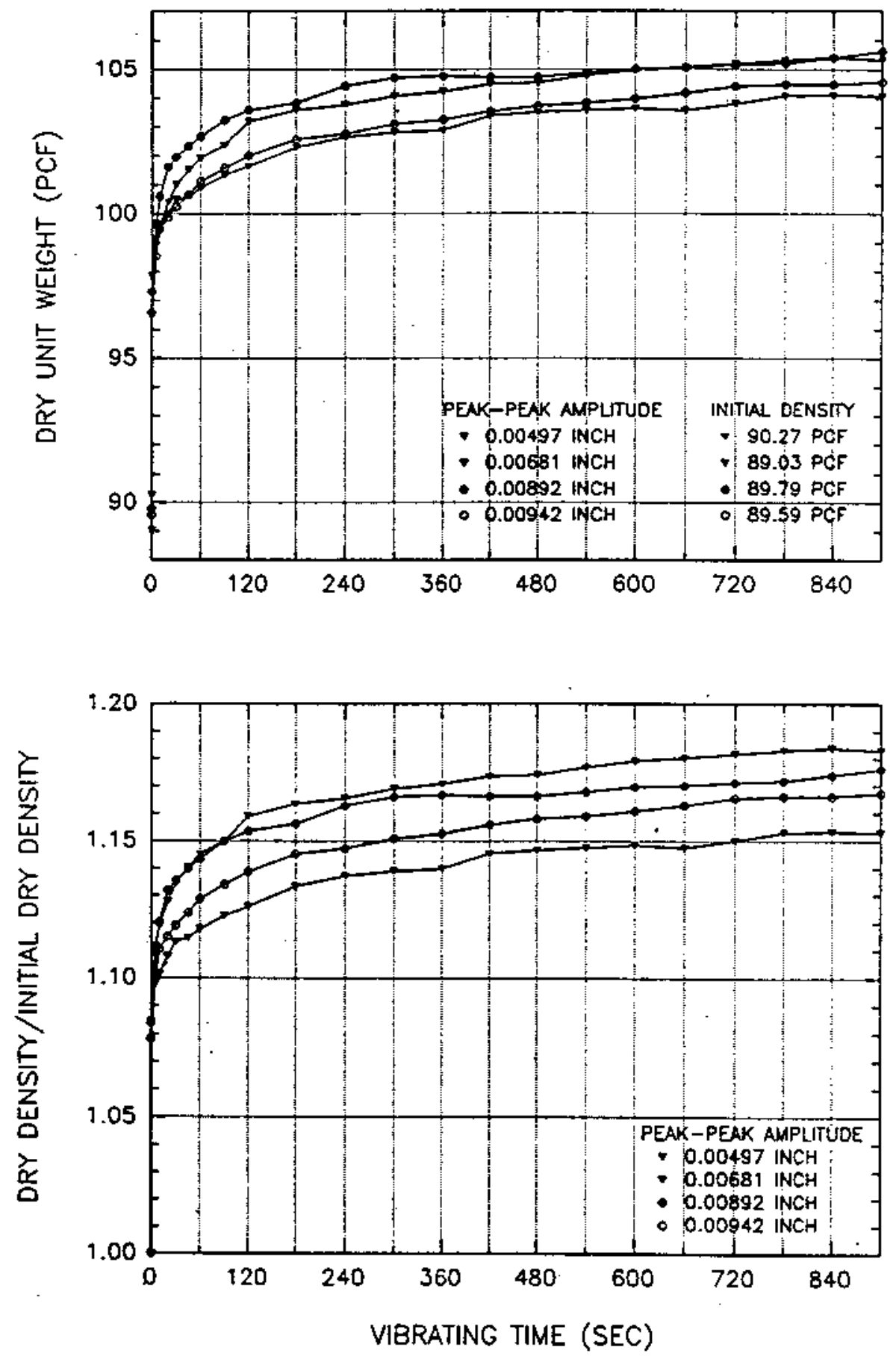

Figure 6.4 Change of Dry Density due to vibration 
differences in initial dry density, measured density was divided by initial density and plotted with time. As shown in the lower plot in Figure 6.4, a dial setting of 8 (peak to peak amplitude of 0.0681 inch) generates the most efficient amplitude for the given sand materials. Therefore, vertical peak to peak amplitude of 0.0681 inch was used to prepare the specimen for resilient modulus test.

Mixed soil was compacted on the vibrating table in a mold of 4-inch diameter and 8-inch height. Each specimen was compacted in five layers and each layer was subjected to 5 minute vibration with 13.3 lbs surcharge. Compaction results by vibrating table are summarized in Figure 6.2. As can be seen, compaction curves by impact and vibratory compaction are almost identical at water content less than 9 percent while vibratory compaction shows an increase of dry density at water content greater than 9 percent. For molding water content larger than 11.5 percent, water squeezing out of the mold during compaction was observed. When soils with the two highest water contents were compacted, the reduction of water content during vibration was about 2 percent. Therefore, compaction. with water content higher than 13 percent was not conducted.

For both impact and vibrating compaction, the rubber membrane, which is typically used for the preparation of granular specimen, was not used. At water content less than 7 percent, the specimen could stand alone due to capillary tension after the split mold was removed. However, at water content higher than 7 percent, the adhesion between soil and mold was large enough to fail the specimen when the split mold was removed. To reduce the attraction force between soil and mold, the mold was put into a drying oven and removed after 2 or 3 minutes. Evaporation of water in contact with the mold reduced the attraction force and the split mold could be removed without failing the specimen. The change in water content due to this procedure was negligible. 
6.3.2 Resilient Modulus Test on Dune Sand

After compaction, the split mold containing compacted specimen was placed on the bottom platen in triaxial cell, and the mold was split and removed from the soil specimen. The top endcap was placed on the top of the specimen, and the rubber membrane and o-rings were put on the specimen. After connecting drainage lines to the top endcap, vacuum was applied to lines connected to top and bottom of the specimen. The loading piston was then carefully screwed into the top endcap and upper support plate of the cell was tightened to the columns of the triaxial cell by screw caps. Pressure cell was then slid down and locked by clamping blocks.

The assembled triaxial cell was moved into the loading frame and the loading piston of the triaxial cell was joined to the load cell by tightening cap screws through adapter plates. An extensometer was installed between load cell and triaxial cell to measure the deformation of specimen during test. The air supply line was also connected to the triaxial cell. Signal cables from load cell, extensometer and confining pressure transducer were connected to control and signal conditioning units.

The resilient modulus test was carried out following the procedure specified in AASHTO $T$ 274-82. As mentioned in section 3.2, the test for granular soil consists of 6 conditioning stages and 27 testing stages, which have combinations of different magnitude of $\sigma_{3}$ and $\sigma_{d}$. During the resilient modulus test, it was observed that, for all tests performed in this study, the total deformation exceeds the limit of the extensometer (4 mm) at confining stress of 1 psi. The test was terminated at repeated deviator stress of $7.5 \mathrm{psi}$ for all impact compacted specimens and at repeated deviator stress of $10 \mathrm{psi}$ for all vibratory compacted specimens. Typical stress pulse and plot of axial stress versus deformation measured during resilient modulus test are shown
in Figure 6.5. 

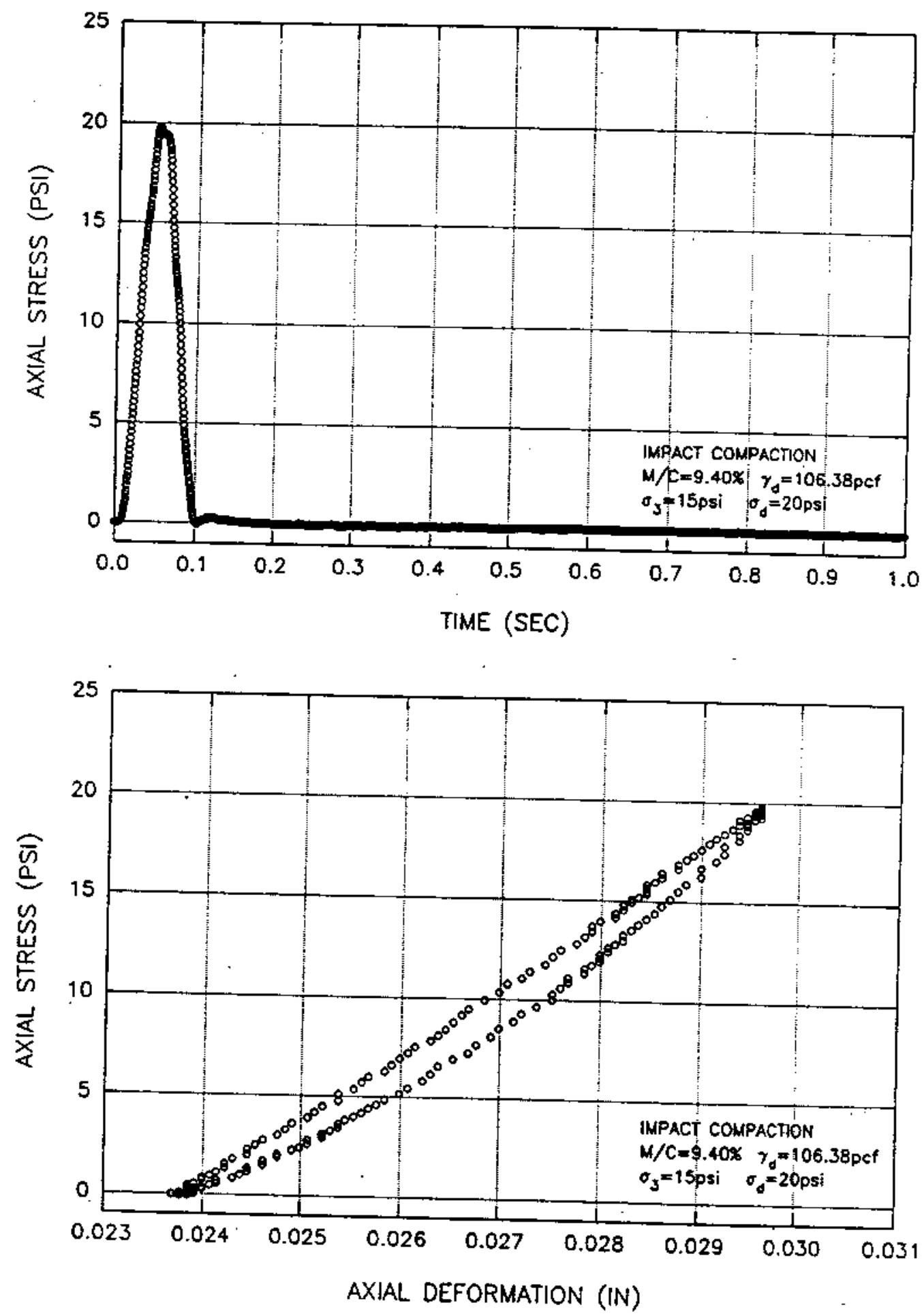

Figure 6.5 Typical Stress Pulse Measured During $M_{R}$ Test on
Dune Sand 
6.4 Reduction of Test Results

As discussed in section 6.2.2, previous studies of resilient response of granular soils have indicated that the resilient modulus can be expressed in terms of bulk stress:

$$
M_{R}=A \theta^{B}
$$

where $\theta$ is the first invariant of stress; $A$ and $B$ are equal to $k_{1}$ and $k_{2}$ in Equation 6.1, respectively. Regression parameters are determined by linear regression after taking logarithm of $M_{R}$ and $\theta$. Results of resilient modulus tests reduced by above equation for both impact and vibratory compacted specimens are presented in Table 6.1.

\subsection{Test Results and Discussions}

Typical plots of test results are shown in Figure 6.6, one curve for soil compacted with the impact hammer and the other for that compacted with the vibrating table. Solid lines are the linear regression results based on Equation 6.5, and this equation appears to fit test results well. It is noted that the resilient modulus for specimens compacted with the vibrating table is higher than that for impact compacted specimen even though the impact compacted specimen has slightly higher ary density and lower water content. It is -also shown in Figure 6.7 that the repeated deviator stress resilient strain relationships for soils. compacted by impact and vibration are significantly different and the vibratory compacted soil behaves as if it is far stiffer than impact compacted soil. It is noted that the resilient strain varies not only with the repeated deviator stress but also with the confining stress. Nonlinearities in the stress-strain relationships are apparent in all cases with greater degrees of nonlinearity at lower levels of confining pressure. 
Table 6.1 Resilient Modulus Test Results of Laboratory Compacted Dune sand

\begin{tabular}{crrcccccc}
\hline $\begin{array}{c}\text { TEST } \\
\text { ID }\end{array}$ & $\begin{array}{r}\text { W/C } \\
\text { (8) }\end{array}$ & $\begin{array}{c}\text { Yd } \\
\text { (pcf) }\end{array}$ & A & B & VMSE & $R^{2}$ & NOTE \\
\hline I94I01 & 3.38 & 104.23 & 2337.59 & 0.5984 & 2221.82 & 0.95 & impact \\
I94I02 & 4.31 & 105.51 & 2215.91 & 0.5996 & 1349.09 & 0.98 & impact \\
I94I04 & 6.17 & 106.12 & 2209.69 & 0.6064 & 1409.81 & 0.98 & impact \\
I94I09 & 7.95 & 106.39 & 2040.19 & 0.6399 & 1530.04 & 0.98 & impact \\
I94I10 & 9.40 & 106.38 & 2176.60 & 0.5973 & 1771.46 & 0.98 & impact \\
I94I11 & 10.46 & 106.33 & 2476.92 & 0.5664 & 1655.92 & 0.96 & impact \\
I94I13 & 11.31 & 106.34 & 2270.19 & 0.5910 & 1389.22 & 0.98 & impact \\
I94V04 & 4.46 & 104.42 & 3165.03 & 0.6017 & 2513.09 & 0.96 & vibrate \\
I94V07 & 7.11 & 107.24 & 3042.70 & 0.6245 & 2201.73 & 0.97 & vibrate \\
I94V11 & 11.08 & 109.95 & 3315.59 & 0.5795 & 1769.33 & 0.96 & vibrate \\
I94V12 & 11.15 & 110.01 & 3286.55 & 0.5630 & 1876.42 & 0.95 & vibrate \\
I94V13 & 11.45 & 111.14 & 2770.17 & 0.6059 & 1793.67 & 0.97 & vibrate \\
I94V14 & 12.24 & 111.59 & 3174.41 & 0.5629 & 1518.18 & 0.96 & vibrate \\
\hline
\end{tabular}




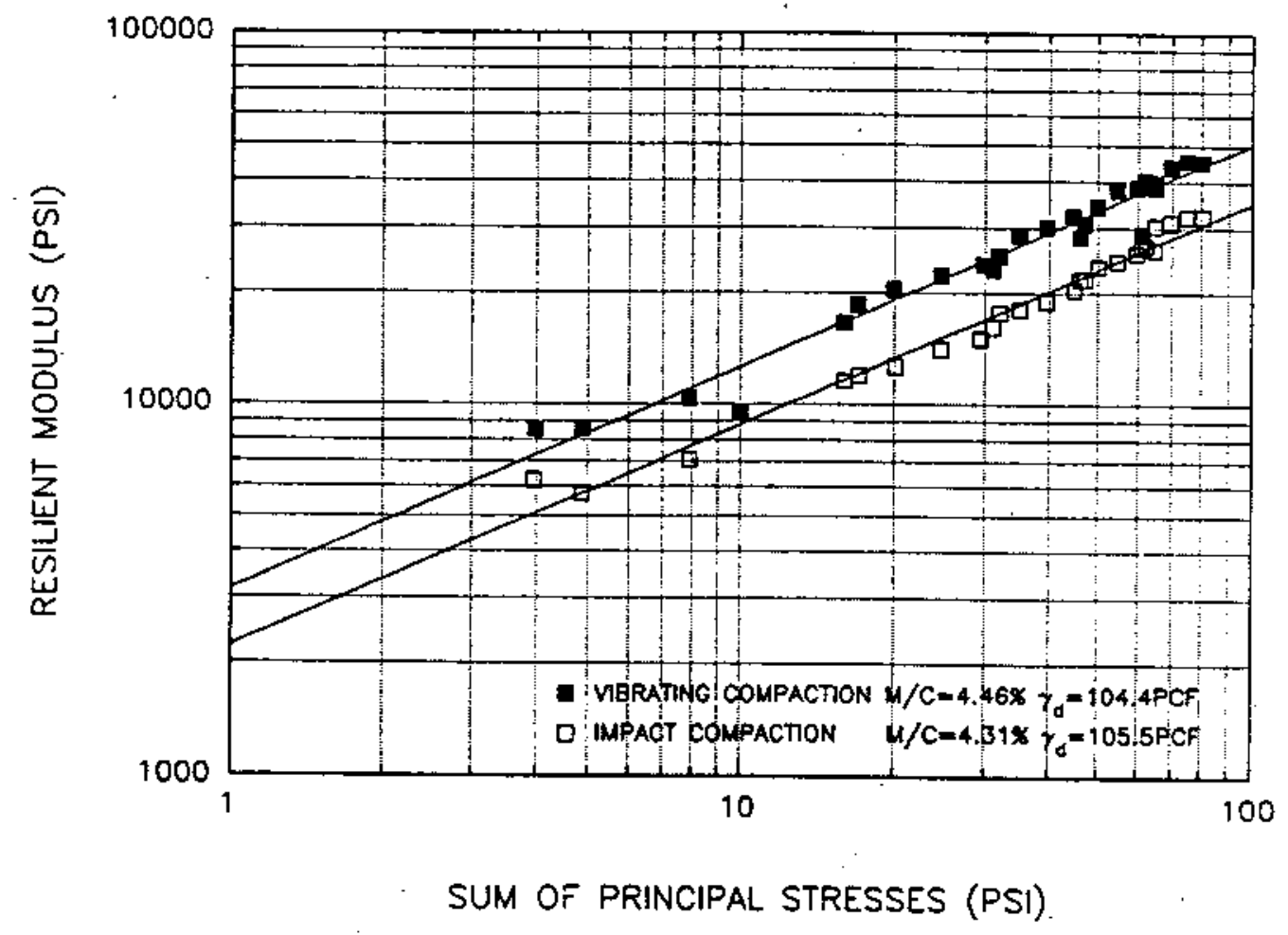

Figure 6.6 Resilient Characteristics of vibratory Compacted and Impact Compacted Dune sand 

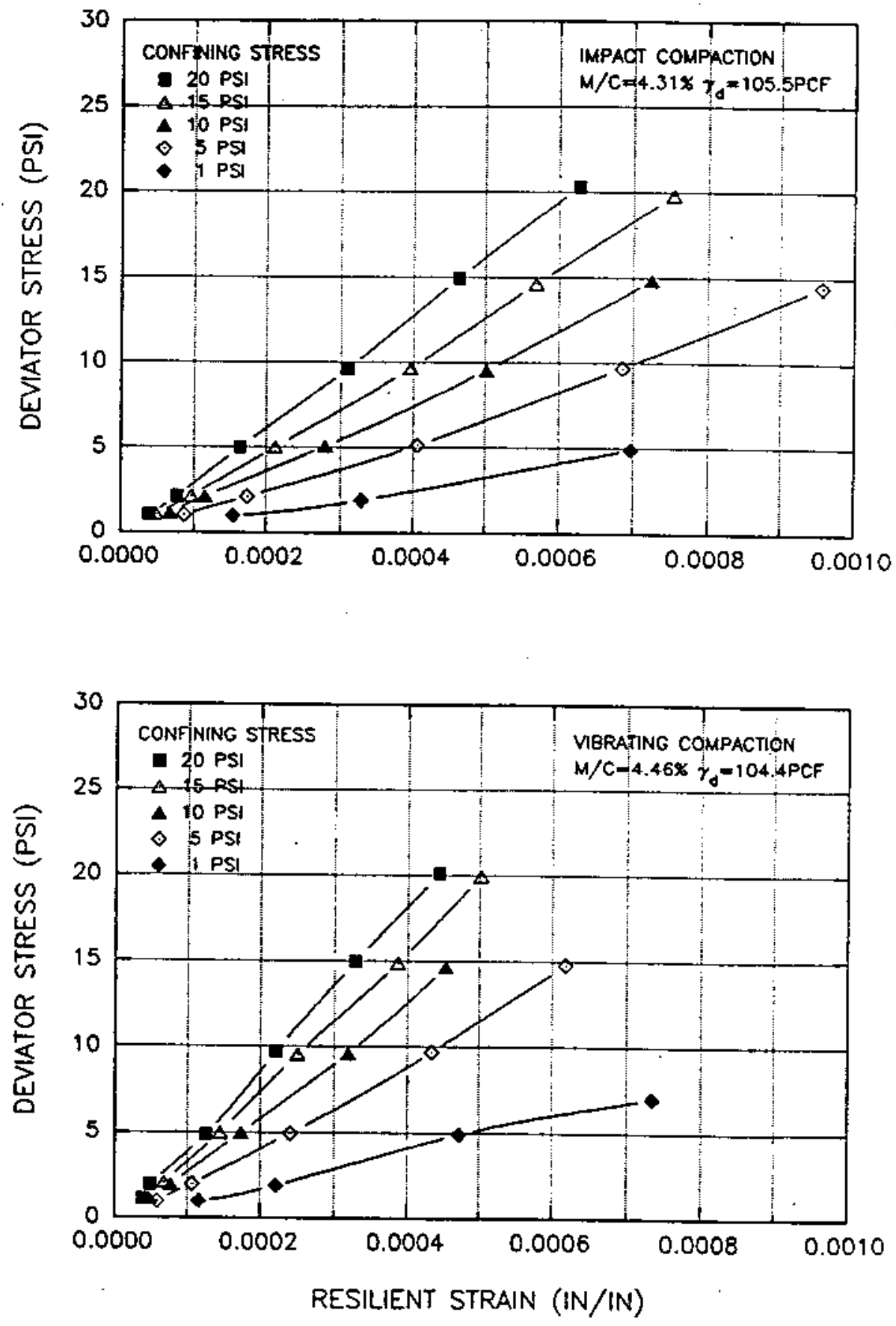

Figure 6.7 Relationship between Repeated Deviator Stress and Resilient Strain of Dune Sand 
Measured total and permanent strains with repetitions of stress pulse at confining pressure 1 psi are shown in Figure 6.8. It can be seen that the permanent strain of impact compacted specimen is about 2.5 times larger than that of vibratory compacted specimen at same stage of testing. Resilient strain is the difference between total strain and permanent strain. Resilient strain of impact compacted specimen is about 20 to 40 percent larger than that of vibratory compacted specimen. It is also interesting that the resilient strain is almost constant when the permanent strain is increasing rapidly before the test is terminated. Therefore, it is concluded that vibratory compacted specimens produce significantly less permanent deformation and moderately larger resilient modulus than impact compacted specimens.

The difference in the behavior during the resilient modulus test may be due to nonuniform densification of impact compacted specimen. specimens compacted by vibration are densified uniformly by means of surcharge on top of soil during compaction while specimens compacted by impact are densified less uniformly. During impact compaction, disturbance due to impact hammer in the upper half portion of each compacted layer was observed. This disturbance may cause nonuniformity in density in compacted specimen. Therefore, the larger resilient deformation occurs in less densified layers when stress pulses are applied, and results in smaller resilient modulus.

Another possible explanation on the different resilient response of sands compacted by vibration and impact is a different fabric and stress history of compacted soils. It was shown by Holtz and Kovacs (1981), referring to personal communication with Leonards (1976), that it is possible for sands to have identical relative density, but significantly different fabrics and, thus, significantly different engineering behavior. Lambrechts and Leonards (1978) and 


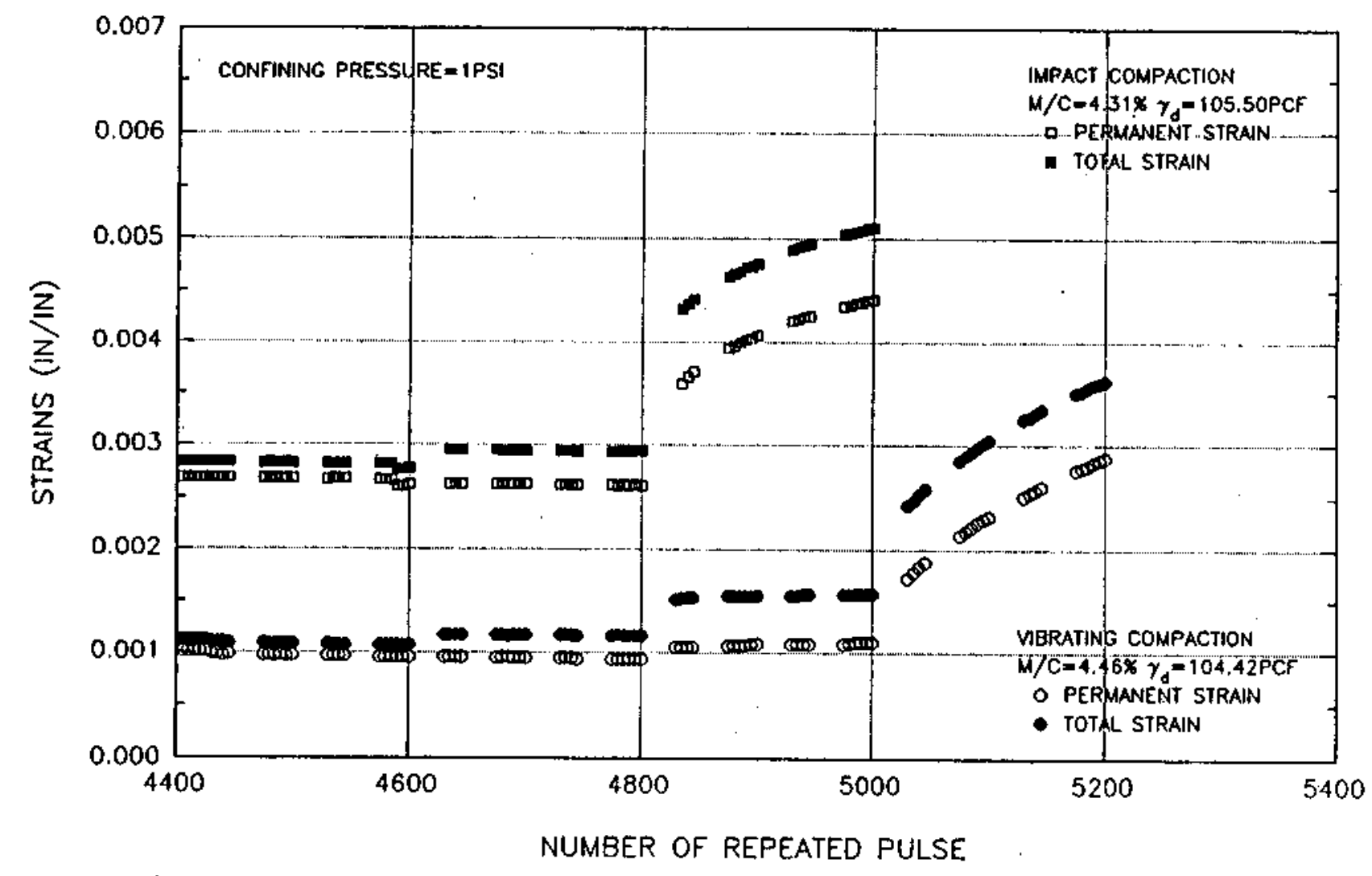

Figure 6.8 Variation of Total and Permanent Strains with Stress Applications 
Schmertmann (1991) also showed that deposits of granular materials which have been prestressed have a very different stress-strain property from that of deposits subjected to no prestress. Since the specimen compacted by vibration had been subjected to surcharge of $13.3 \mathrm{lbs}$ during compaction, there is a possibility that the vibratory compacted specimens are preloaded. Therefore, granular soils compacted by different methods may produce different soil fabrics even though they have the same water content and dry density; and that soils compacted by vibration with surcharge have preloading effects due to surcharge and respond better than impact compacted soils in terms of resilient and permanent deformations.

To investigate the effect of compaction water content on the resilient characteristics of dune sand, the constants $A$ and $B$ are plotted with water content, as shown in Figure 6.9. Parameters $A$ and $B$ appear not to be affected by change of water content for both compaction methods. This implies that the resilient behavior of soils compacted at different water content by same compaction mechanism and energy is identical. It is noted that the parameter A of vibratory compacted soil is about 40 percent larger than that of impact compacted soil while parameter $B$ is almost same for specimens prepared by both compaction methods. This means that log-log plots of resilient modulus for soils compacted by both methods are parallel to each other with different intercepts, as shown in FIgure 6.6. The average value of A for vibrating compaction is 3126 and for impact compaction 2247. The average value of $B$ for both compaction is 0.595 . Therefore, the resilient modulus of dune sand compacted by vibration is defined by:

$$
M_{R}=3126 \theta^{0.595}
$$

where $M_{R}$ is resilient modulus in psi and $\theta$ is sum of principal stresses in psi. For dune sand compacted by impact hammer with standard Proctor energy, resilient modulus is predicted by following equation: 

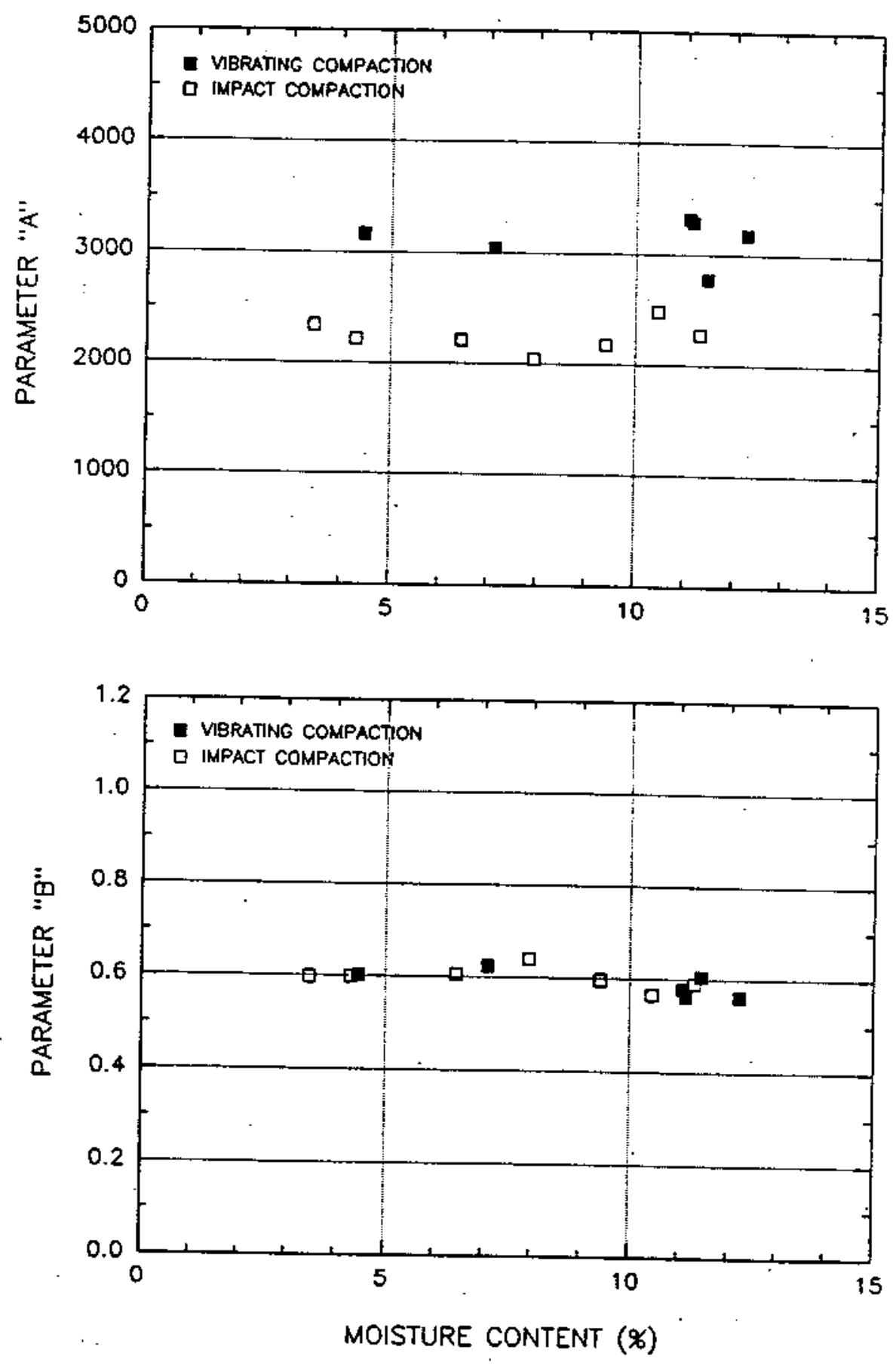

Figure 6.9 Effect of Water Content on Parameters $A$ and $B$ 


$$
M_{R}=2247 \theta^{0.595}
$$

From the above discussion, it can be concluded that the change of compaction water content has no effect on the resilient characteristics of dune sands compacted with same compactive effort. Since the standard resilient modulus test is a drained type of test, the pore pressure generated during the test dissipates quickly and water may have no effect on behavior. This conclusion agrees with the previous test results reported by Hicks and Monismith (1971) and smith and Nair (1973). Therefore, it is believed that the resilient behavior of sand in the field is not affected by change of water content if adequate subdrainage is provided to not allow a build-up of pore pressure from traffic loading.

To investigate the effects of compaction water content and dry density on the permanent deformation, permanent strain at the end of 22 nd stage of testing $\left(\sigma_{3}=5 \mathrm{psi}\right.$ and $\left.\sigma_{d}=15 \mathrm{psi}\right)$ was calculated from measured deformation and plotted with water content. As can be seen in Figure 6.10 , permanent strain of impact compacted sand increases about two times as water content increases from 3.47 percent to 11.31 percent. It is also noted that permanent strain of vibratory compacted sand is less than half of that of impacted compacted sand at same water content. Also shown in lower plot of Figure 6.10 is effect of dry unit weight on the permanent strain. Magnitude of permanent strain increases as dry density decreases. Moisture content of these soils ranges from 9.52 percent to 9.91 percent. The permanent strain of specimen of 109.64 pcf is $0.135 \mathrm{in} / \mathrm{in}$ while the permanent strain of specimen of 102.0 pef is 0.511 in/in.

Field compaction is usually controlled by relative compaction to the maximum dry density and, therefore, it is necessary to study the influence of dry density on. the resilient behavior of sand. Since resilient modulus of dune sand compacted with same vibrator was observed not to be 

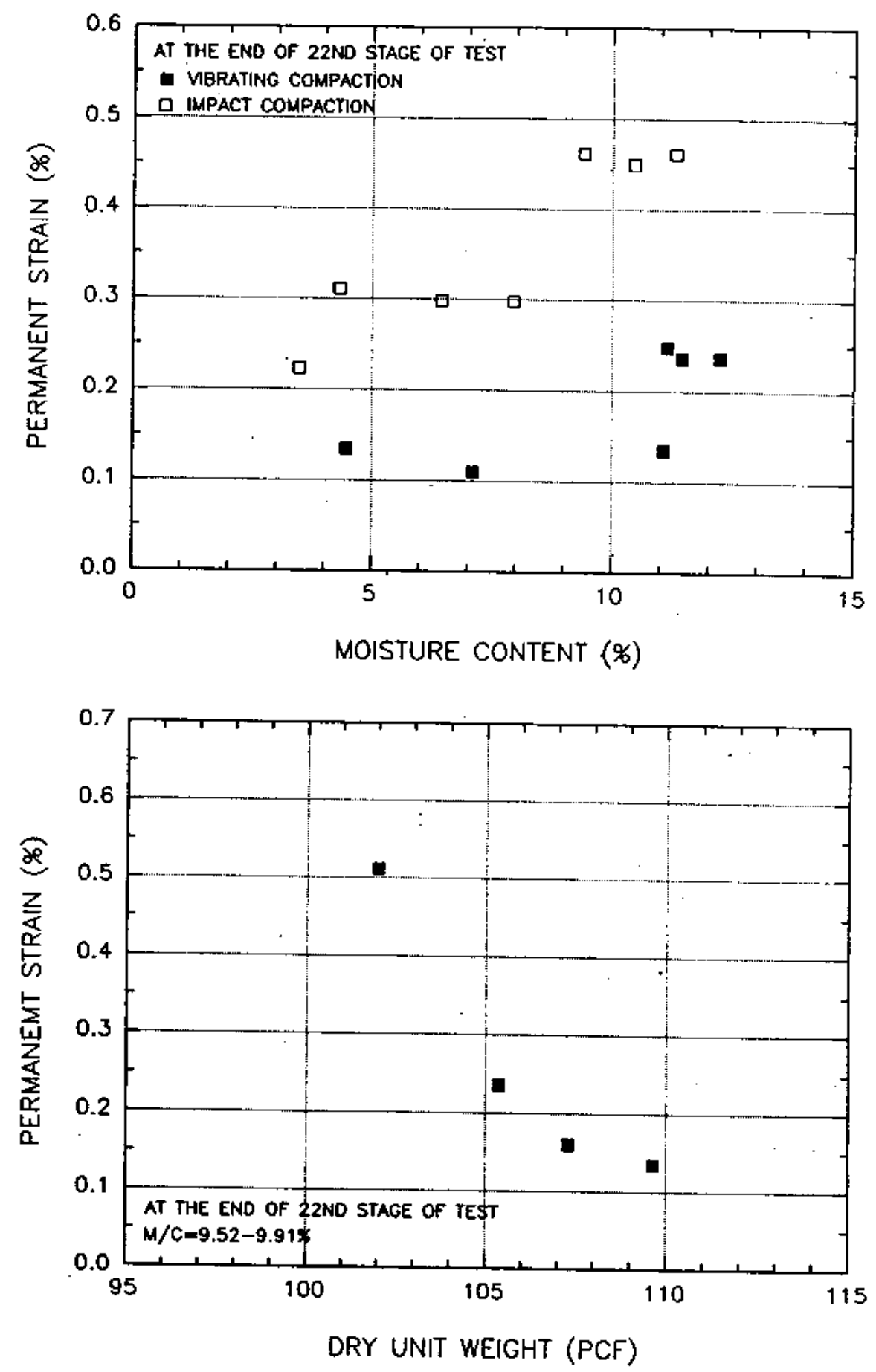

Figure 6.10

Effect of Water Content and Dry Density on Permanent Deformation of Dune Sand 
affected by water content and since the maximum dry density is not clearly defined due to the difficulty explained before, four specimens were compacted at water content of 9.52 percent to 9.91 percent with different duration of vibration to study the effect of dry density on resilient modulus. Duration of vibration used for these specimens were 5 minutes, 1 minute, 15 seconds and 5 seconds per layer. Due to shortage of soil, the sand once used was dried and reused for this series of compaction. As expected, the dry density of specimen compacted with vibration of 5 minutes per layer is about 3 pef larger than that of specimen compacted with fresh soil.

Test results on these specimens with linear regression lines are presented in Figure 6.11. For two specimens with larger dry density, resilient modulus increases slightly as the magnitude of repeated deviator stress increases. On the other hand, two specimens of lower density shows a decrease in resilient modulus at deviator stress less than 2 psi and a slight increase at higher deviator stress. It is also seen that the test results on specimen with smallest density (102.0 pcf) shows large scatter around the regression line while the results of other three specimens fit well to the regression line. This is shown in Figure 6.12, which is a plot of square root of Mean Square Error in the regression equation versus dry density. Square root of MSE for the specimen of smallest dry density is more than $4000 \mathrm{psi}$ while error for other specimens of higher density is about $2000 \mathrm{psi}$. Therefore, the regression equation appears inadequate to fit the resilient modulus test result of specimens compacted with dry density less than 104 pcf at water content about 9.75 percent.

To express the resilient modulus in terms of relative magnitude of axy densities, relative compaction for recompacted specimens is defined as the ratio of dry density of each specimen to the expected density by compacting fresh sand with 5 minutes vibration per layer at same water content. By comparing Figures 6.11 and 6.6 , it can be observed that the 

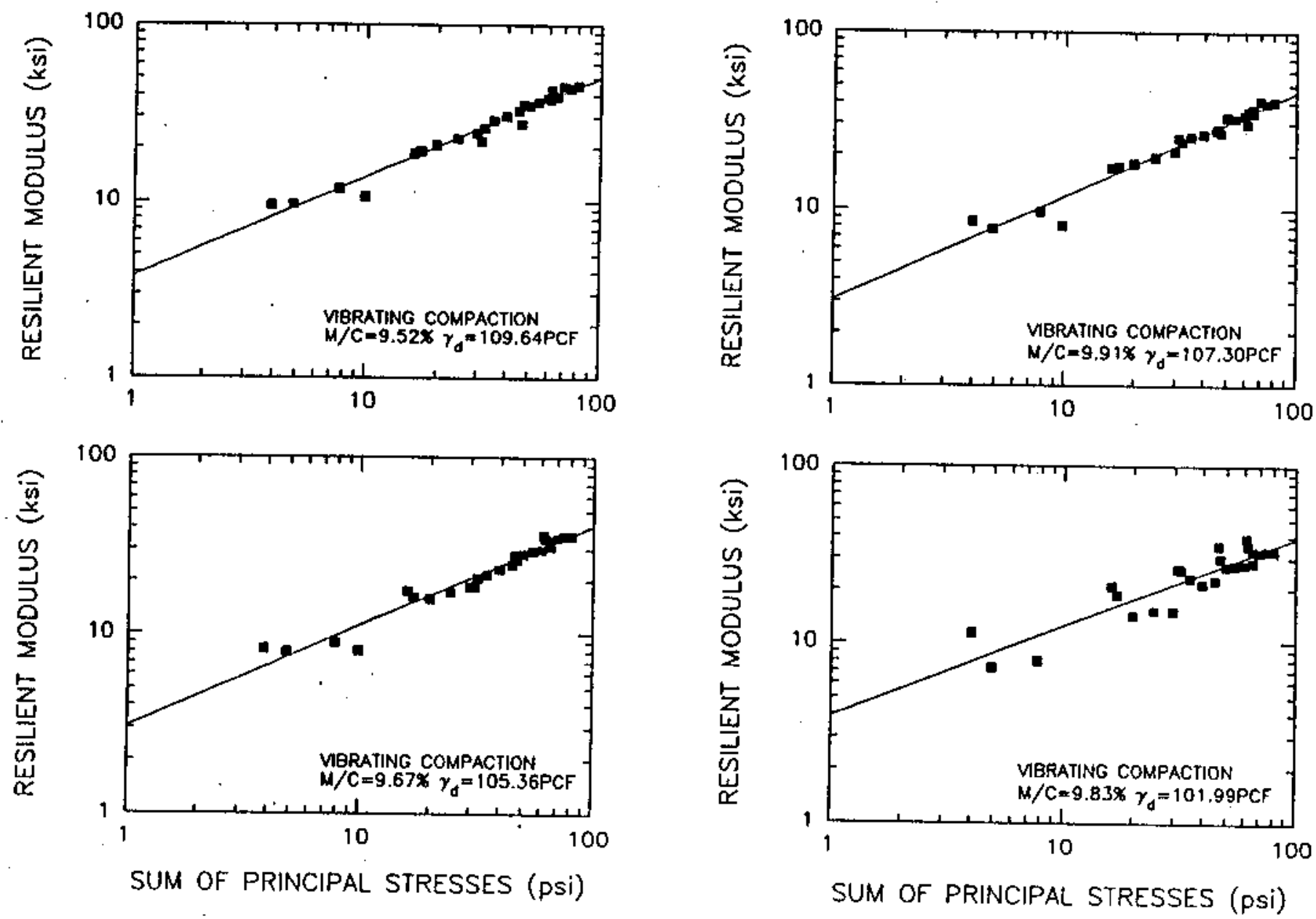

Figure 6.11 Effect of Dry Density on the Relationship between $M_{R}$ and $\theta$ 


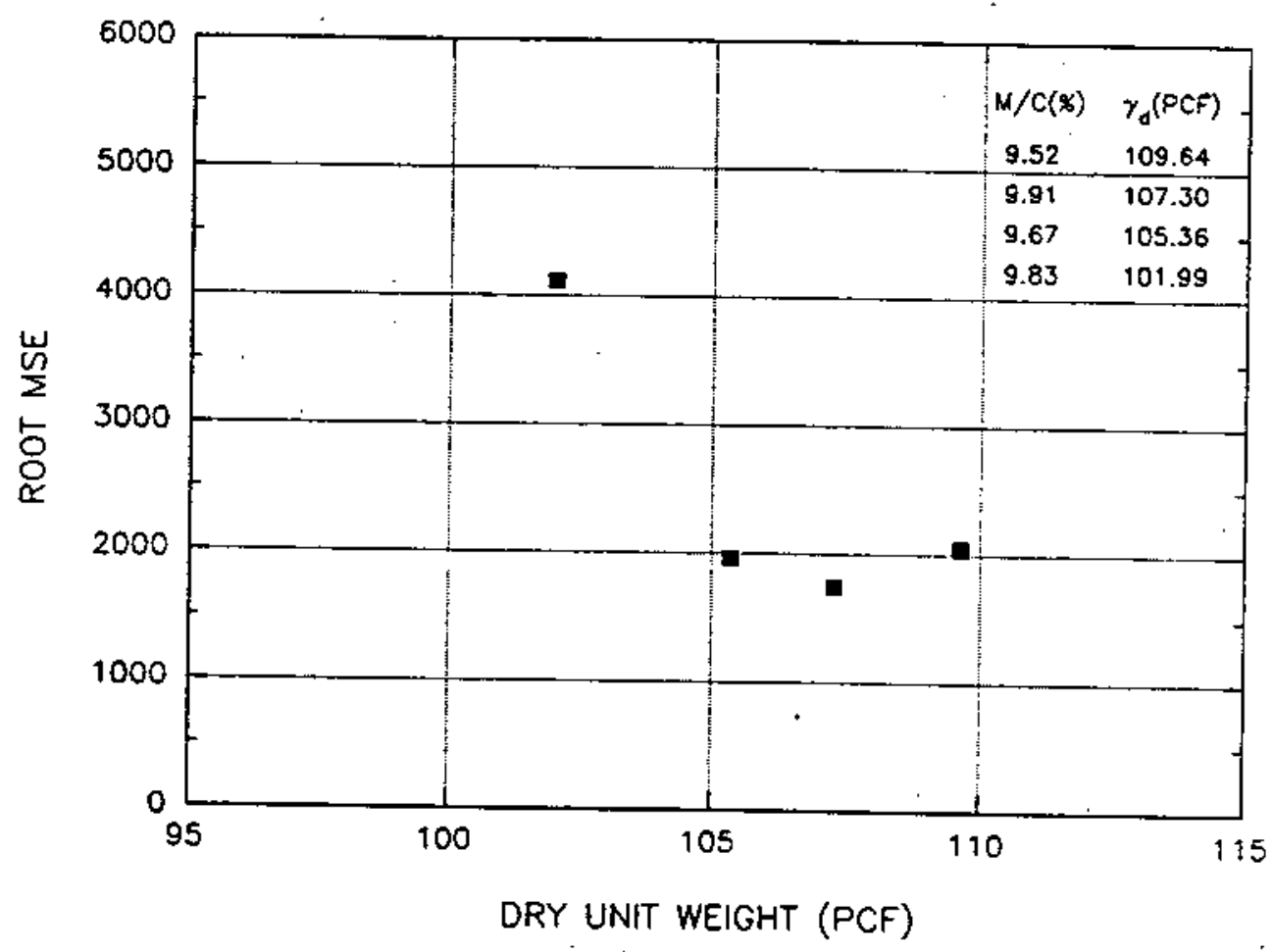

Figure 6.12 Variation of Mean Square Error with Dry Density 
recompacted sand with vibration of 1 minute per layer has similar dry density with fresh sand compacted with 5 minutes per layer, and that they show similar resilient characteristics. Therefore, relative compaction for recompacted specimens are calculated by dividing dry densities with 107.3 pcf. In addition, parameters of specimen compacted with 1 minute vibration per layer at water content of 5.42 percent is included to validate the effect of relative compaction on resilient modulus at different water content. Relative compaction of this specimen is determined by dividing density of 101.7 pcf by 105.65 pcf, expected dry density by 5 minutes vibration per layer. As discussed before, parameters $A$ and $B$ for specimen with $w / c=9.83$ percent and $\gamma_{d}=102.0$ pcf are not adequate due to large scatter of test results around the regression line: For conservative results, parameters A and $B$ for this specimen are obtained by the regression on resilient modulus test results at deviator stress levels larger than 2 psi.

The parameters $A$ and $B$ of these specimens are plotted with relative compaction, as shown in Figure 6.13. It is observed that the parameter $A$ increases almost linearly as relative compaction increases while parameter $B$ is almost constant at relative compaction ranging from 95 percent to 103 percent. Parameter $A$ is expressed as a function of relative compaction by linear regression. Since the average magnitude of A for vibratory compacted sand at relative compaction of 100 percent is 3126 , the regression equation is calibrated to this condition and the result is:

$$
A=-20163+232.886 R C
$$

where RC is relative compaction defined as ratio of dry density of specimen to expected dry density by 5 minutes vibration per layer (percent). Since it was observed that the parameter. B is almost constant with changes of relative compaction and that magnitude of $B$ is about the same as that 


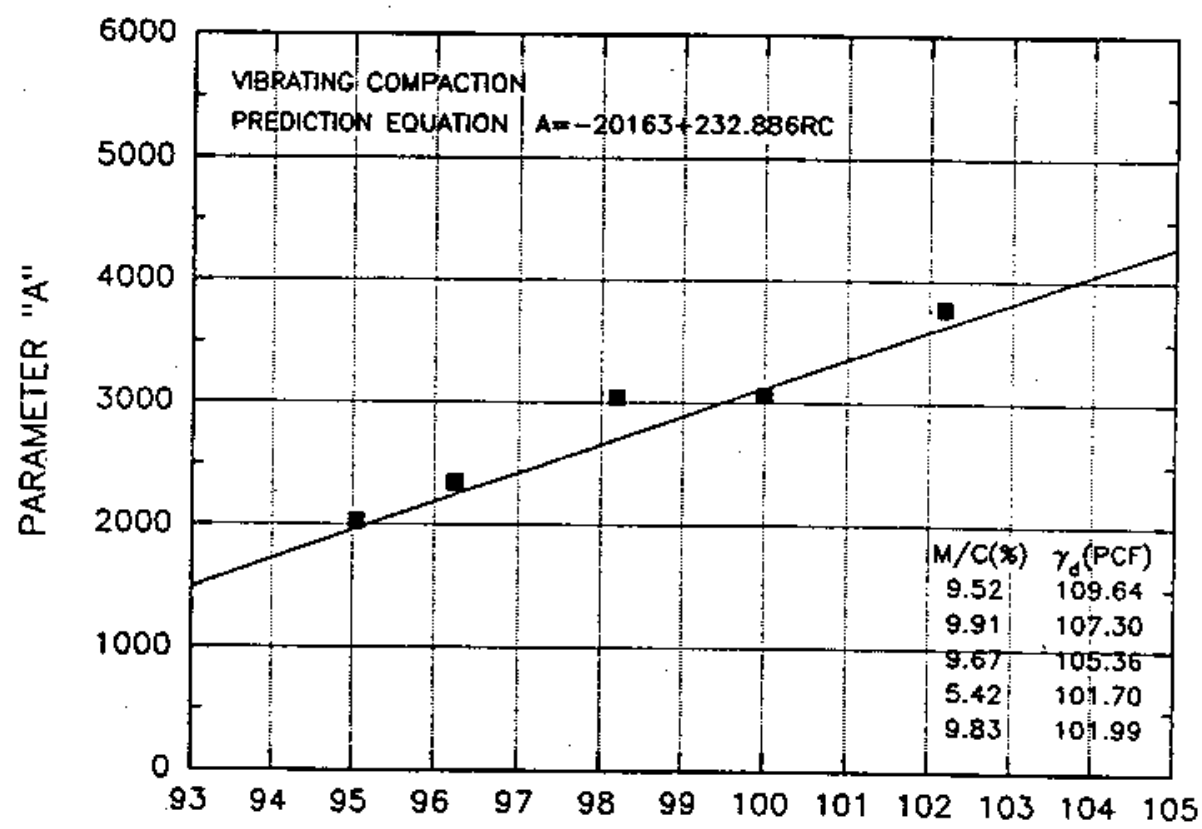

$-$

$=$

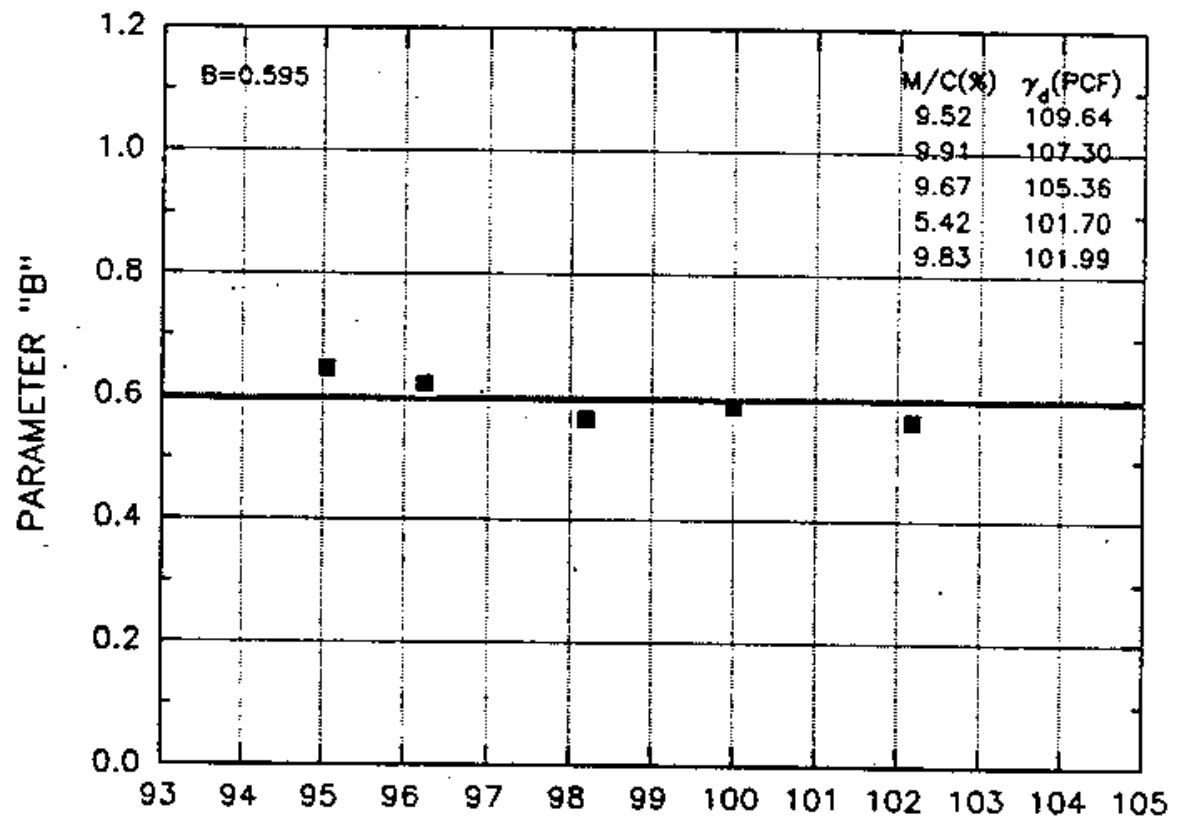

RELATIVE COMPACTION (\&)

Figure 6.13 Relationship of Parameters A and B with Relative 
for fresh specimens, B value of 0.595 is believed reasonable. Therefore, the resilient modulus of dune sands compacted by vibration is given as:

$$
M_{R}=(-20163+232.886 R C) \theta^{0.595}
$$

where $M_{R}$ is resilient modulus in psi and $\theta$ is the sum of principal stresses in psi.

\subsection{Concluding Remarks}

At the same water content and dry density, the resilient modulus of vibratory compacted specimen is higher than that of impact compacted specimen. The repeated deviator stress resilient strain relationship showed that the vibratory compacted specimen behaves as if it is far stiffer than the impact compacted specimen. It was also shown that the permanent strain of impact compacted specimen is far larger than that of vibratory compacted specimen at same stage of testing. Therefore, it can be concluded that the dune sand be compacted by vibratory compactor for better resilient response
in the field.

The resilient moduli of specimens compacted with the same compactive effort is identical, regardless of water content, while the permanent strain increases as the water content increases. This is true for both methods of compaction. Therefore, the resilient behavior of sand is not affected by the change of water content if adequate subdrainage is provided not to allow a build-up of pore pressure due to traffic loading. The resilient modulus of dune sand compacted with vibratory energy, producing the dry density similar to 100 percent standard proctor density at water content lower than 9.5 percent, is defined by:

$$
M_{R}=3126 \theta^{0.595}
$$

where $M_{R}$ is the resilient modulus in psi; and $\theta$ is the sum of 
principal stresses in psi.

For specimens with relative compaction larger than 96 percent, the resilient modulus increases as the magnitude of repeated deviator stress increases. Specimens of lower density show a decrease in resilient modulus at deviator stress less than 2 psi and a slight increase at higher deviator stress. Scattex around the regression line increases as the density of specimen decreases. Parameter "B" is not affected by water content and dry density, while parameter "A" is affected by dry density. For vibratory compacted sand, the resilient modulus is estimated by the following equation:

$$
M_{R}=(-20163+232.886 R C) \theta^{0.595}
$$

where $M_{R}$ is the resilient modulus in psi; $\theta$ is the sum of principal stresses in psi; and $R C$ is the relative compaction in percent defined as a ratio of dry density of specimen to the expected dry density by 5 minute vibration per layer. 
7. FABRIC OF SOIL - THEORETICAL BACKGROUND

7.1 Definition of Soil Fabric

Historically, soil structure and fabric have been studied by pedologist and geologists for agricultural, colloidal, physical, and chemical behavior. Several different variations of the definitions of structure and fabric have been proposed (Jenny, 1941; U.S.D.A., 1951; American Geological Institute, 1957; Baver, 1964). The terms structure and fabric have been defined in different ways and have been interchanged for the same concept. Brewer (1964) discussed different shades of these definitions and suggested the following general definition for soll fabric:

The physical constitution of a soil material as expressed by the spatial arrangement of the solid particles and associated voids.

Similarly, Brewer (1964) defined the soil structure:

The physical constitution of a soil material as expressed by the size, shape and arrangement of the solid particles and voids including both the primary particles to form compound particles and the compound particles themselves; fabric is the element of structure which deals with arrangement.

Collins and McGown (1974) extended the definition of soil structure to include other components: the mineralogy, size, physical arrangement and relative proportions, by weight; of the soil particles present; the pore size, pore water and pore air distribution; the chemistry of the solid, liquid and gaseous phase.

In short, fabric is a measure of the arrangement of the soil particles. The term soil fabric includes the particle 
spacing and the pore size distribution (Yong and sheeran, 1973). It is important to note that the structure and the fabric of a soil do not include the mineralogical and chemical composition of the soll material.

\subsection{Previous studies}

Fabric of a soil can be classified into different categories based on the level of viewing. A fabric unit may represent just a single particle or a group of particles (Yong and Sheeran, 1973). Size of a fabric unit may vary from the size easily visible to the naked eyes $(0.1 \mathrm{~mm})$ to a size only visible through an electron microscope. Figure 7.1 illustrates different components of a fabric unit - cluster and domain. As shown in Figure 7.1, a macropore is the space between the fabric units (peds) and the micropore is the space between the clusters and domains existing in a ped. Arrangements of clusters and domains in a ped and the orientation of peds have been defined using different models. Following is a summary of the proposed models:

(a) Honeycomb (Terzaghi, 1925; Casagrande, 1932)

(b) Non-salt/salt flocculated (Lambe, 1958)

(c) Dispersed (Lambe, 1958)

(d) Edge-to-face (EF) and edge-to edge (EE) flocculated and aggregated (van Olphen, 1977)

(e) Stair-step cardhouse (O'Brian, 1971)

(f) Bookhouse, turbo-static (Barden and sides, 1970)

(g) Ring buttress (Dudley, 1970)

Earlier studies were mainly concerned with either the study of the overall microfabric using polarizing microscope (low magnifications) or the study of individual particle arrangements using transmission and scanning electron microscopes (high magnifications). Studies based on the electron microscope suggested that single particle interaction is extremely rare and it is difficult to determine the extreme ends of a clay particle (Barden, 1972). 


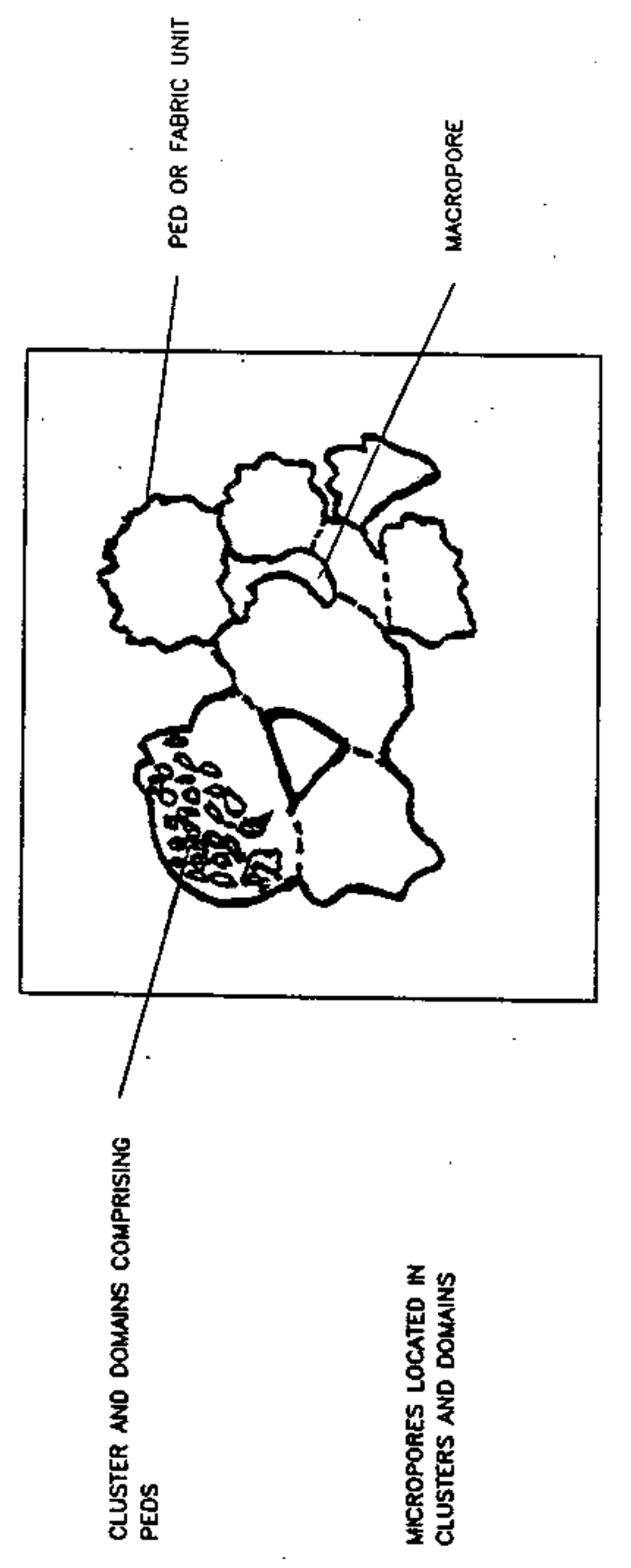

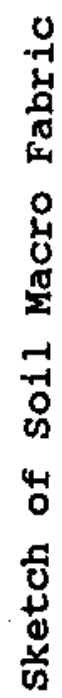

r. 
7.3 Determination of Soil Fabric

Prior to the application of $x$-ray diffraction, electron microscopy, and scanning electron microscopy, the study of the soil particle arrangements were of a speculative nature by indirect measurement of some parameters. Methods of measurement of soil fabric can be classified into two categories: (i) direct and (ii) indirect methods. Following is a summary of the methods of measurement of soil fabric:

Direct Methods :

(a) Optical Microscope

(b) Electron Microscope:

(1) Scanning Electron Microscope (SEM)

(2) Transmission Electron Microscope (TEM)

(c) X-Ray Diffraction

(d) Pore Size Distribution (PSD)

Indirect Methods:

(e) Acoustical Velocity

(f) Dielectric Dispersion and Electrical Conductivity

(g) Thermal conductivity

(h) Magnetic Susceptibility

(i) Mechanical Properties

A summary of these methods, their limitations, and sample preparation for different methods can be found in Mitchell (1976). All of the above mentioned techniques give different types of information about the pore space but, finally, all the data reduce to almost the same result. A comparison of different techniques may lead to discrepancies. For example, a two dimensional micrograph will have a high probability of disagreement with a pore size curve - a non-vector quantity obtained by intrusion from all around the soil facades. Differences between the techniques for a real porous medium are still a subject of research. 
7.4 Pore Size Distribution Using Porosimetry

Pore space and the orientation of particles are complementary to each other. Characteristics of the pore space component of soll fabric-size, shape, and orientation - have been investigated by several researchers. One of the widely used experimental techniques to determine the pore space is mercury intrusion porosimetry. (MIP). Theoretical background for the experimental technique of MIP was prepared by Washburn (1921). Ritter and Drake (1945) used the washburn equation to measure pore diameters up to $100 \AA$ ( 0.01 microns) by applying a maximum of 10,000 psi pressure. Use of MIP became widespread in the 1960 's after the introduction of hydraulically pressured commercial instruments. Diamond $(1970,1971)$ and Sridharan et al. (1971) were first to study the fabric of compacted clays. The topic became so popular that a whole issue of Powder Technology (1981; Volume 29, No. 13) was devoted to porosimetry. In the same journal, Modry et al. (1981) published a bibliography listing 998 papers on the topic.

MIP is a simpler and faster method compared to other techniques providing similar information. Mercury porosimetry has become a useful method to determine the pore structure of several different materials from paper to nylon. Most publications on the use of mercury porosimetry are concerned with building materials including soil. Results are presented as a pore size curve. It covers a large range of pore diameters that can be determined. MIP is based on the capillary law governing penetration of liquid into small pores. Washburn suggested the following equation for the pressure required to force mercury into the pores in the most materials:

$$
p=-4 \gamma \cos \theta / d
$$

where $p$ is a pressure required to cause the intrusion; $d$ is a 
diameter of the pore being intruded; $\gamma$ is a surface tension of mercury; $\theta$ is a contact angle between the mercury and the pore wall.

Following is a list of the factor which may affect the reliability of the pore size results:

(a) Pore spaces in most of the materials are rarely cylindrical. Thus, use of Equation 7.1 will give an equivalent pore diameter of the intruded cross section of the pore area.

(b) Real pore space is too complicated to fit into any hypothetical model. The diameter evaluated at a certain pressure in Equation 7.1 is a measure of the corresponding entrance to the void space. Entrance to a void may lead to an area larger than the entrance equivalent diameter. This effect is termed as bottle neck or ink bottle effect. Hence, the term $d$ in Equation 7.1 gives a limiting pore diameter.

(c) Surface tension of mercury has been assumed as a constant value at all pressure levels. This value depends on the purity of the intruded mercury and the material in contact with mercury.

(d) Contact angle between mercury and the soil walls has been assumed as constant. Value of the contact angle depends on solid composition of the material.

(e) Intruded pore diameters are limited by the minimum and maximum pressure that can be used in a certain equipment.

(f) Boundary conditions of a small finite soil sample may not be representative of a semi-infinite porous media.

Even with the factors and limitations cited above, mercury porosimetry is often a rellable technique and good reproducibility of results can be achieved. significance of the errors involved due to the several assumptions and limitations has been discussed by Ritter and Drake (1945) and Brakel et al. (1981). The only serious limitation of the test is due to the bottle-neck effect. This effect is a function of the fabric being measured. Higher frequency of bottle necks in a material will result in an overestimation of finer pores using MIP. 
Finally, it is important to mention the applicability of a pore size curve as a measure of soil fabric. The pore-size curve gives a measure of fabric as a non-vector quantity. Fabric of a soil is non-isotropic but little is available to get tensor data. As discussed above, there are several limitations to the MIP technique but good reproducibility has been achieved on the soil samples. The pore-size curve, in an absolute sense, to represent fabric of a soil and to predict soil behavior should be used with caution. However, comparative studies eliminate these limitations while the fabrics of two different solls are being compared. The present study uses PSD to suggest that if two soil samples have the same PSD, then they have the same fabric. 
8. REPLICATION OF FIELD COMPACTED FABRIC

\subsection{Introduction}

Characterization of fabric as a pore-size curve presents data which can be analyzed quantitatively. Comparison of two pore-size curves provides a good measure of the difference between the fabric of the soils being compared. Pore-size curves for compacted soils generally cover about six orders of diameters ( $10^{-3}$ to $10^{3}$ microns) and do not fit in conventional probability distributions.

Previous studies have attempted to represent a pore-size curve by fabrlc descriptors. A fabric descriptor is an arbitrary point on the pore-size curve believed to represent certain characteristics of the pore-size curve. A summary of the fabric descriptors can be found in White (1980). White (1980) compared two pore-size curves using fabric descriptors. He found that the assumptions of the normality of the distributed data and the homogeneity of the variances among the sets of data (required for the parametric studies) were not met by the majority of the descriptors. He also found that only three fabric descriptors ( $D_{50}, D_{75}$ and $\left.P V\right)$, out of 35 selected, were found to satisfy the normality and homogeneity assumptions. Hence, conventional parametric methods are not effective in comparing two pore-size curves. The present stuay uses a nonparametric approach to compare two pore-size curves. A program has been developed to find a comparable match between the laboratory and the field compacted fabric. 


\subsection{Replication Technique}

classical statistical techniques are based on specific assumptions about the population sampled and are termed as parametric methods. on the other hand, nonparametric techniques require more general assumptions about the population. The assumption most frequently required is that the population be continuous. Nonparametric inferences are based on some function of the actual observations of the random variables in the sample. Several techniques are available which use nonparametric approaches. Details about different nonparametric techniques can be found in standard texts such as Gibbons, 1985; and wadsworth, 1990.

Comparison of two cumulative pore-size distribution curves can be done by the kolmogorov-Smirnov (K-S) method. The only assumption required in this method is that the sample be drawn from a continuous population. The test statistic that is used to measure the difference between two cumulative distribution curves is:

$$
D=\max \cdot\left|F_{s_{1}}(x)-F_{s_{2}}(x)\right|
$$

where $D$ is the $K-S$ statistic; $F_{S}$ is a sample cumulative distribution function (CDF) from the first curve; $F_{S_{2}}$ is a sample CDF from the second curve.

The parameter $D$ is the largest absolute vertical deviation between the two cumulative functions as shown in Figure 8.1. The sampling distribution of $D$ under the hypothesis that the two aistributions are identical is given as a two-sample $K-S D$ statistic (Gibbons, 1985).

In order to compare a large number of pore-size curves, a computational technique for determining the $\pi-s$ test statistic ( $D$ parameter) has been developed. Interactive Matrix Language (IML feature of statistical Analysis system) has been used on an IBM 3090 to perform the required computations. 


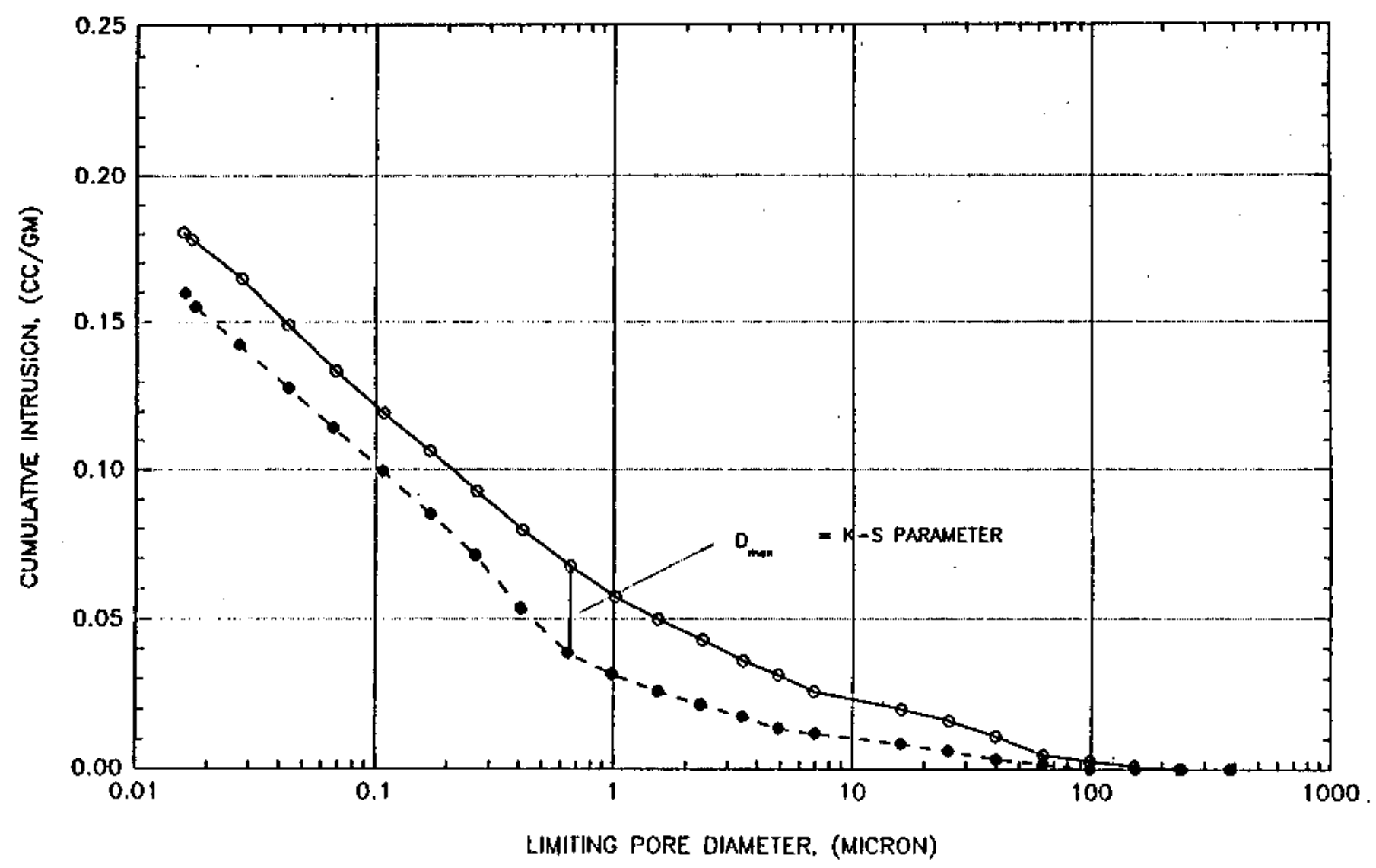

Figure 8.1 Computation of the K-S D Parameter 


\subsection{Input Data Preparation}

The analysis has been performed on the pore-size results obtained in a previous study (White, 1980). The soil was an A6 (CL) medium plastic clay. other soil parameters can be found in White (1980). Four compaction methods (impact and kneading for the laboratory, and Caterpillar and Rascal compactor for the field) were used. Field and Laboratory compaction curves are shown in Figures 8.2 through 8.5 .

Most of the pore-size curves have about 24-30 uniformly spaced (on logarithmic scale) observation points. A total of 262 such curves (125 for laboratory and 137 for field compacted samples) were included in the study. Each curve has been given an unique seven-digit code, and each digit represents a different parameter. First three digits (starting from left) represent the method of compaction, molding water content, and energy level. They determine a particular group representing that sample. Digits four through seven represent sample number and replication number, and are useful only to identify different samples in a particular group.

The first digit represents the method of compaction. Compaction methods in alphabetical order (Caterpillar, Impact, Ineading, and Rascal) are represented by numbers 1 through 4 respectively.

The second digit represents the molding water content. Molding water content is considered as the relative value to the optimum moisture content rather than the absolute value. Molding water content is represented by five ranges (digit 1 through 5) as shown in Table 8.1 .

The third digit represents the energy level. Three energy levels (less than Proctor, standard Proctor, and Modified Proctor) are used for the laboratory compaction. Comparative energy levels, based on the number of passes of the compactor, are selected for the fleld compaction. Details on compaction energy are summarized in Table 8.2 . 


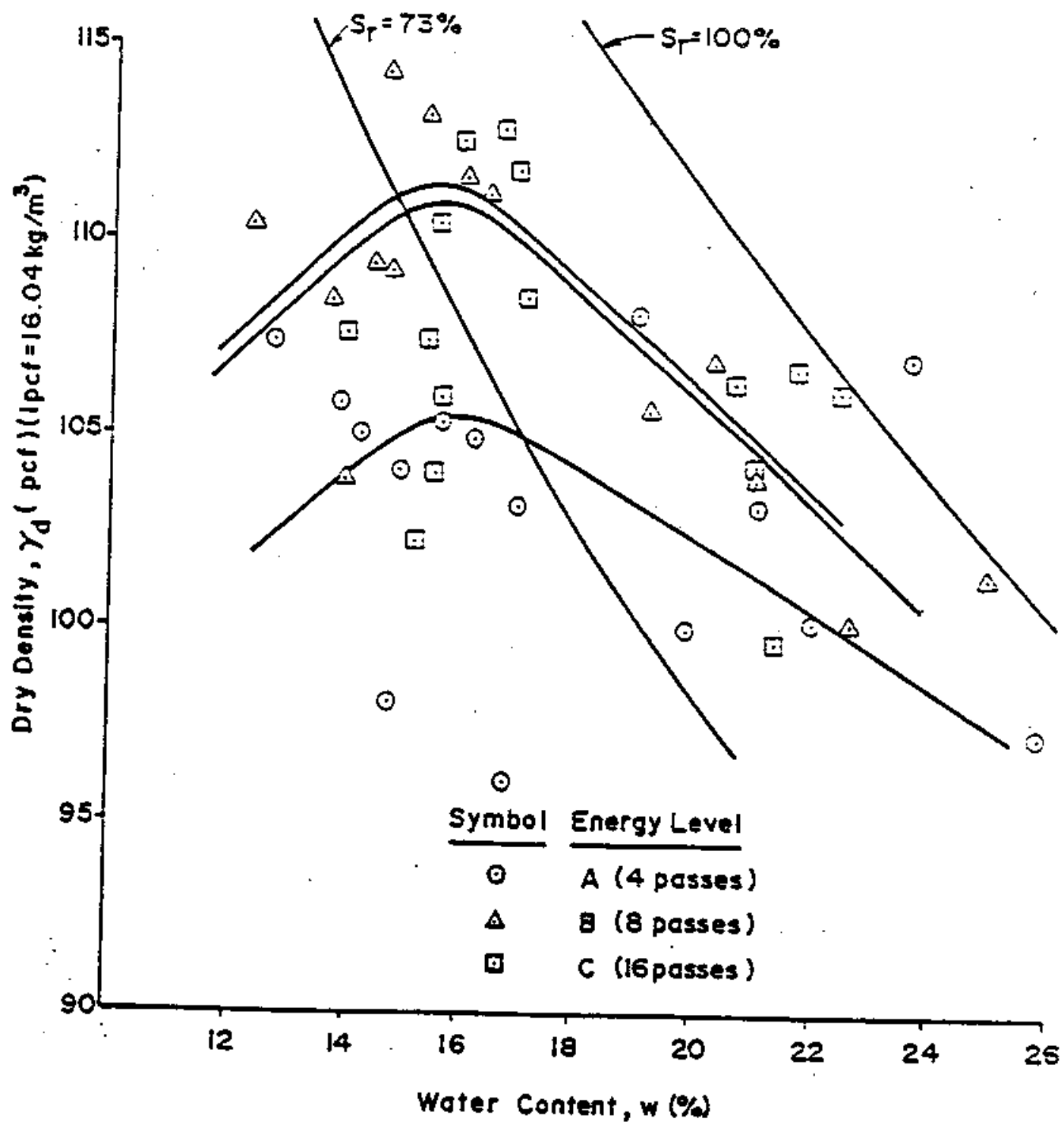

Figure 8.2 Field Compaction curve for the Caterpillar
Compactor 


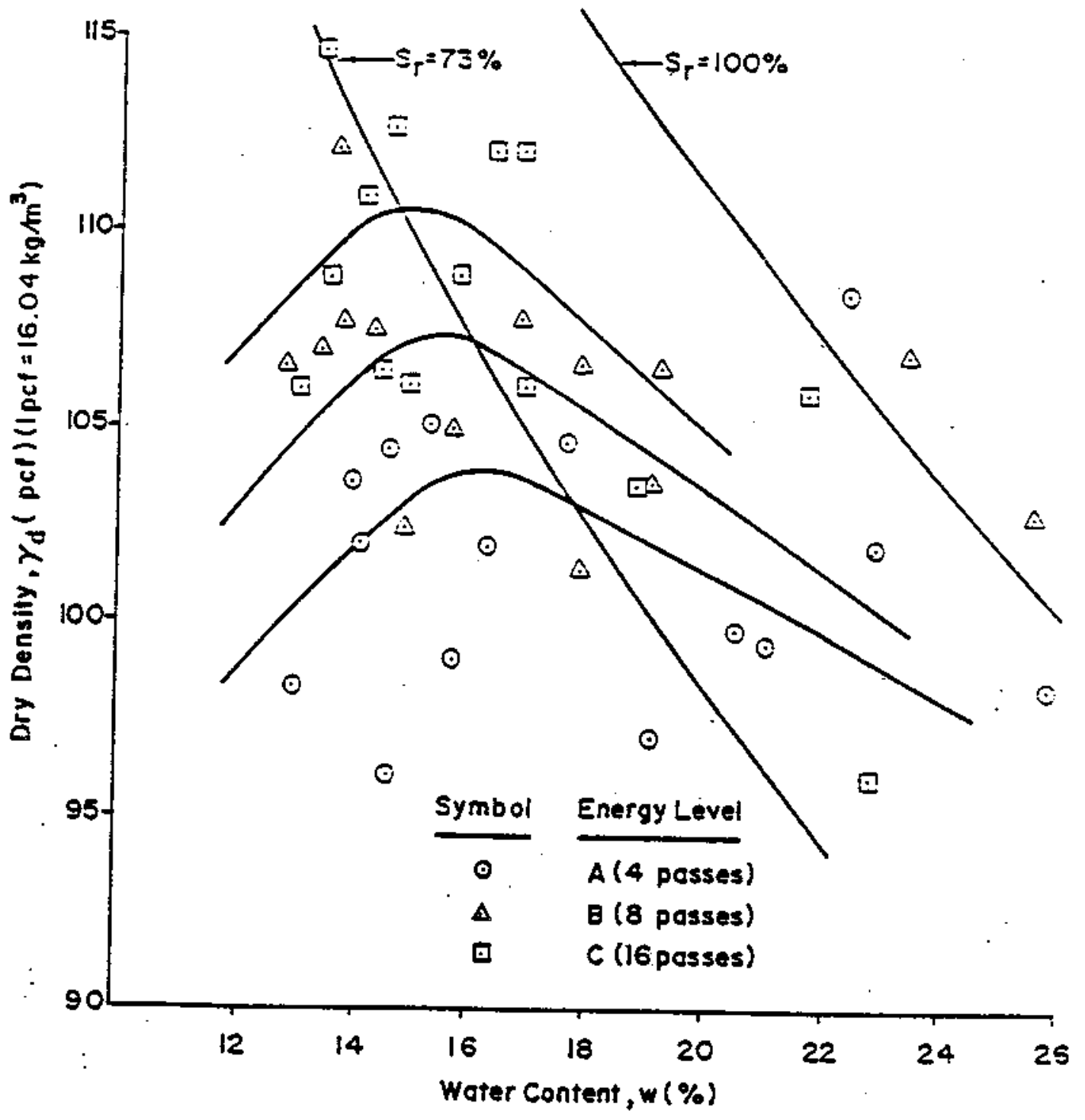

Figure 8.3 Field Compaction curve for the Rascal compactor 


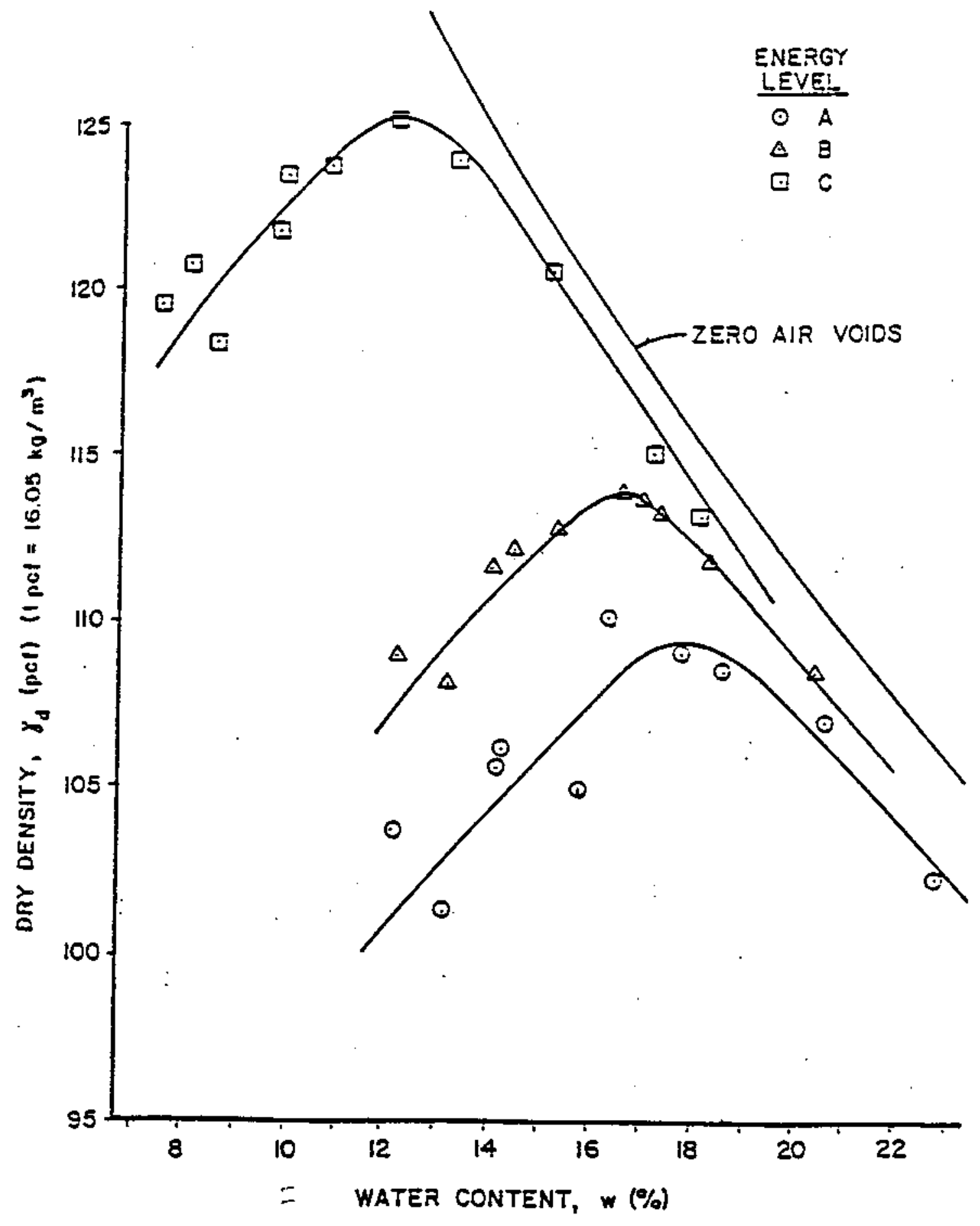

Figure 8.4 $\begin{aligned} & \text { Iaboratory compaction curves for Impact } \\ & \text { Compaction }\end{aligned}$ 


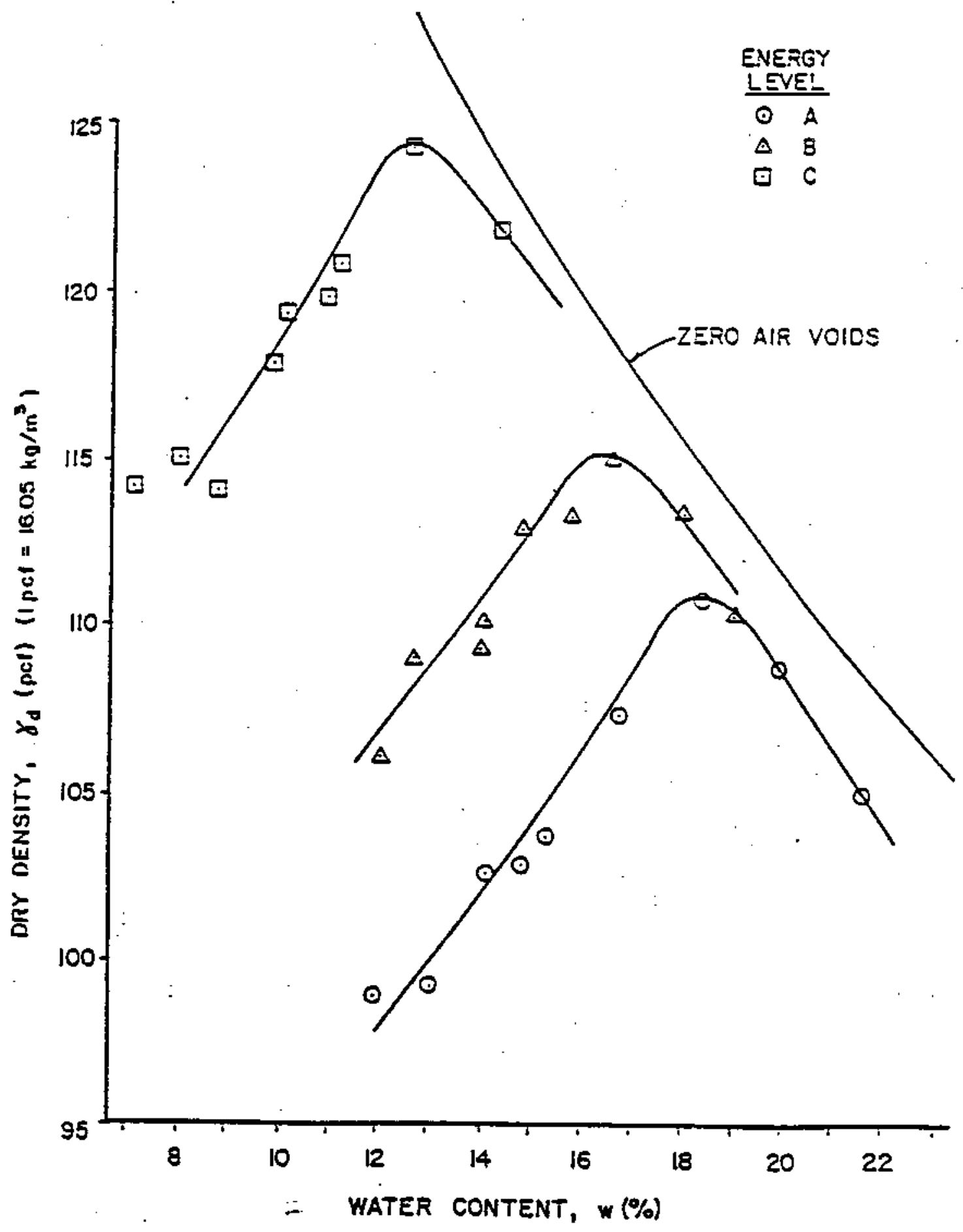

Figure 8.5 Laboratory Compaction curves for Kneading 
Table 8.3 Description of Laboratory and Field Groups

\begin{tabular}{|c|c|c|c|}
\hline $\begin{array}{l}\text { Energy Level/ } \\
\text { Water Content } \\
\end{array}$ & $\mathbf{A}$ & B & c \\
\hline \multicolumn{4}{|l|}{ IXPACT COMPACTION } \\
\hline $\begin{array}{l}\text { very dry ( } Z 1.6 \% \text { dry of opt.) } \\
\text { dry ( } 1.5 \% \text { dry of opt.) } \\
\text { optimum } \\
\text { wet ( } 1.4 \% \text { wet of opt.) } \\
\text { very wet ( } Z 1.5 \% \text { wet of opt.) } \\
\text { ToTAL SAMPLES }\end{array}$ & $\begin{array}{l}- \\
- \\
4 \\
3 \\
4 \\
11\end{array}$ & $\begin{array}{l}8 \\
4 \\
4 \\
7 \\
0 \\
23\end{array}$ & $\begin{array}{l}3 \\
8 \\
4 \\
7 \\
8 \\
30\end{array}$ \\
\hline \multicolumn{4}{|l|}{ KNEADING COMPACTION } \\
\hline $\begin{array}{l}\text { very dry ( } \geq 1.6 \% \text { dry of opt.) } \\
\text { dry ( } 1.5 \% \text { dry of opt.) } \\
\text { optimum } \\
\text { wet (s } 1.4 \% \text { wet of opt.) } \\
\text { very wet ( } 1.5 \% \text { wet of opt.) } \\
\text { ToTAL sAMPLES }\end{array}$ & $\begin{array}{l}5 \\
4 \\
4 \\
4 \\
4 \\
21\end{array}$ & $\begin{array}{l}4 \\
4 \\
4 \\
4 \\
4 \\
20\end{array}$ & $\begin{array}{l}4 \\
8 \\
4 \\
4 \\
- \\
20\end{array}$ \\
\hline
\end{tabular}

CATERPIIIAAR COMPACTOR

very dry ( $21.6 \%$ dry of opt.)

dry ( $\leq 1.5 t$ dry of opt.)

optimum

wet ( $\leq 1.48$ wet of opt.)

very wet ( 21.58 wet of opt.)

TOTAL SAMPLES

$\begin{array}{lll}4 & 7 & 4 \\ 4 & 8 & 4 \\ 4 & 4 & 4 \\ 3 & 3 & 3 \\ 8 & 7 & 4 \\ 23 & 29 & 19\end{array}$

RASCAL COMPACTOR

very dry ( $\geq 1.6 \%$ dry of opt.)

dry ( $\leq 1.58$ dry of opt.)

optimum

wet (s 1.4t wet of opt.)

very wet ( $21.5 \%$ west of opt.)

TOTAL SAMPLES

$\begin{array}{lll}4 & 4 & 4 \\ 4 & 4 & 4 \\ 4 & 5 & 4 \\ 9 & 4 & 4 \\ 4 & 4 & 4 \\ 25 & 21 & 20\end{array}$

\section{TOTAL FIELD SAYPLES-137; TOTAL NUMBER OF GROUPS: ${ }^{1} 35$}

1 Groups with more than 6 replications are divided in two groups-each representing a different sample. 
Table 8.4 Laboratory and Field Group codes and Number of Replications

\begin{tabular}{|c|c|c|c|c|c|}
\hline \multicolumn{3}{|c|}{ LABORATORY GROUPS- } & \multirow{2}{*}{$\overline{-}$} & \multirow{2}{*}{$\begin{array}{c}\text { FIELD GROUPS } \\
\text { code } \\
I^{\prime} \mathrm{J}^{\prime} \mathrm{K}^{\prime} \mathrm{L}^{\prime} \mathrm{M}^{\prime} \mathrm{O} \\
\end{array}$} & \multirow{2}{*}{ TrF. } \\
\hline 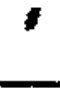 & $\begin{array}{c}\text { code } \\
\text { I KL O M O }\end{array}$ & rep. & & & \\
\hline 1 & 2221010 & 8 & 1 & $\$ 114010$ & 4 \\
\hline 2 & 2231060 & 3 & 2 & 1121010 & 3 \\
\hline 3 & 2321050 & 4 & 3 & 1126010 & 4 \\
\hline 4 & 2331010 & 8 & 4 & 1132010 & 4 \\
\hline 5 & 2411010 & 4 & 5 & 1214010 & 4 \\
\hline 6 & 2421010 & 4 & 6 & 1221010 & 4 \\
\hline 7 & 2431050 & 4 & 7 & 1223010 & 4 \\
\hline 8 & 2511020 & 3 & 8 & 1231020 & 4 \\
\hline 9 & 2521010 & 7. & 9 & 1314010 & 4 \\
\hline 10 & 2531010 & 7 & 10 & 1326010 & 4 \\
\hline 11 & 2611010 & 4 & 11 & 1332010 & 4 \\
\hline 12 & 2631010 & 8 & 12 & 1411020 & 3 \\
\hline 13 & 3211010 & 5 & 13 & 1421010 & 3 \\
\hline 14 & 3221010 & 4 & 14 & 1433020 & 3 \\
\hline 15 & 3231050 & 4 & 15 & 1511010 & 4 \\
\hline 16 & 3311010 & 4 & 16 & 1516010 & 4 \\
\hline 17 & 3321010 & 4 & 17 & 1522020 & 3 \\
\hline 18 & 3331010 & 8 & 18 & 1525010 & 4 \\
\hline 19 & 3411010 & 4 & 19 & 1535010 & 4 \\
\hline 20. & 3421010 & 4 & 20 . & 4113010 & 4 \\
\hline 21 & 3431010 & 4 & 21 & 4126010 & 4 \\
\hline 22 & 3511010 & 4 & 22 & 4134010 & 4 \\
\hline 23 & 3521010 & 4 & 23 & 4212010 & 4 \\
\hline 24 & 3531010 & 4 & 24 & 4221010 & 4 \\
\hline 25 & 3611010 & 4 & 25 & 4232010 & 4 \\
\hline \multirow[t]{10}{*}{26} & 3621010 & 4 & 26 & 4312010 & $4^{\circ}$ \\
\hline & 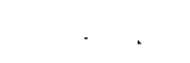 & & 27 & 4321010 & 5 \\
\hline & & & 28 & 4334010 & 4 \\
\hline & & & 29 & 4412010 & 5 \\
\hline & & & 30 & 4413010 & 4 \\
\hline & & & 31 & 4422010 & 4 \\
\hline & & & 32 & 4434010 & 4 \\
\hline & & & 33 & 4511010 & 4 \\
\hline & & - & 34 & 4526010 & 4 \\
\hline & & & 35 & 4534010 & 4 \\
\hline
\end{tabular}

I. I': COMPACTION METHOD

$1=$ Caterpillar

$2=$ Impect

$3=$ Kneading

$4=$ Raceal

J, I: MOLDING WATER CONTENT

1 =very dry of optimum

2=dry of optinum

3 =optimum

4 ewet of optimum

$5=$ very wet of optinum

$J=\left(J^{\prime}+1\right)$

K, R': ENERGY LEVEL

$1=L$ ess than Proctor; (4 passes)

$2=$ Proctor, (8 patses)

$3=$ Modified Proctor; (16 passes)

L, L': SAMPLE NUMBER

$M, M^{\prime}:$ REPLICATION NUMBER 


\subsection{Computational stages}

Replication analyses have been performed in three major parts:

(a) variability within each laboratory group

(b) variability within each field group

(c) comparison between laboratory and field groups

A group is a collection of replicated test results. Most of the groups have four replicated results as shown in Table 8.4. Variability within a group is defined on the basis of the $D$ parameter. Each curve in a group is compared with the rest of the curves to determine respective $D$ parameters. The computational process outlined in section 8.3 is used to determine the $D$ parameters. The methodology of the determination of the within a group variability is explained by an example. This example considers a group with four replications. In this example group, the curve 1 will have four $D$ parameters $D_{11}, D_{12}, D_{19}$, and $D_{1 s}$. The first subscript represents curve 1 and the second subscript represents the curve (within the group) with which the curve 1 is being compared. For example, the parameter $D_{11}$ represents comparison of the curve 1 with itself and is equal to zero. Continuation of this process results in $16\left(n^{2}\right) \quad D$ parameters (4 for each curve) as shown in Table 8.5. For efficient processing of the data, only the upper part of the symmetric matrix in Table 8.5, with $6(n[n-1] / 2) D$ parameters, is evaluated in the actual computations. Note that the diagonal terms representing comparison of a curve with itself are zero.

Average variability for the group is computed by taking the average of the non-zero $D$ parameters. Maximum variability of the group is computed by taking the maxima of the four ( $n$ ) Dmax parameters obtained for each curve as shown in Table 8.5. These two parameters define the variability of a particular group. 
Table 8.5 Computation of $D$ Parameter for a Group with Four Replications

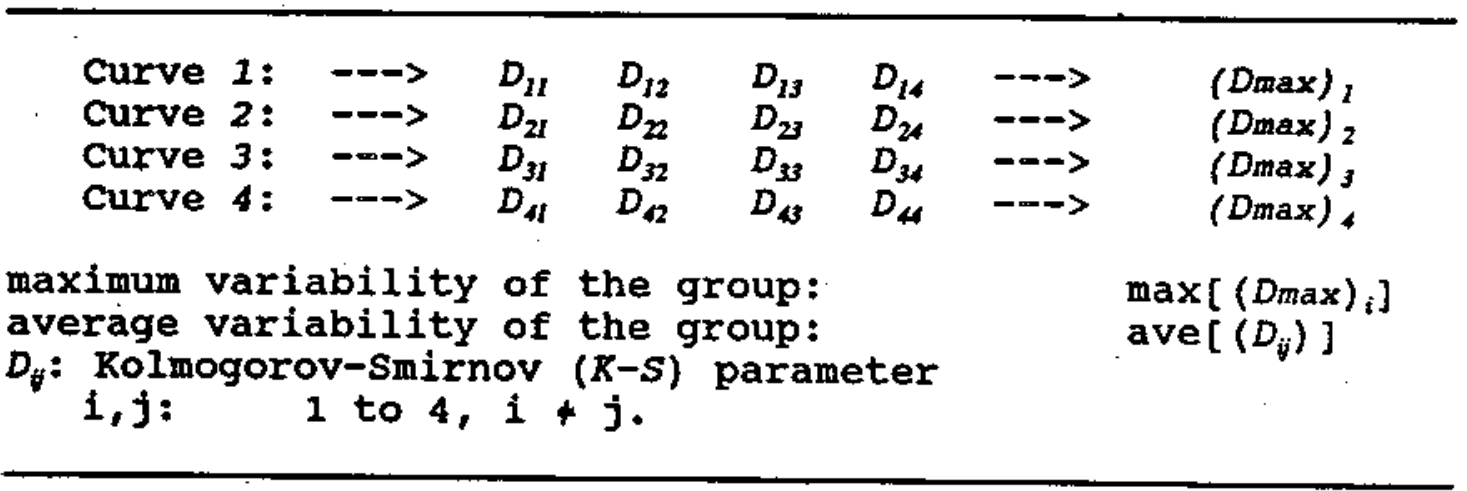

The group variability in the form of the average and the maximum value of $D$ is computed for both the laboratory and the field samples. A total number of 26 laboratory and 35 field groups are included in this study.

In the final stage of the analyses all the laboratory curves are compared with all the field curves, irrespective of their groups, to determine the $D$ parameters for the each of the comparisons. Computations in this stage of the analysis are independent of the previous two stages. The present study includes comparison of 125 laboratory and 137 field curves. Results are obtained in a matrix form representing 17,125 (125 $x 137$ matrix) $D$ parameters. This matrix is defined as the curve matrix.

The curve matrix $(125 \times 137)$ is reduced to a smaller matrix (26 x 35), defined as the group matrix, representing comparison of laboratory and field groups. This reduction is achieved by averaging the individual $D$ parameters over the replicated samples.

The reduction process transforming the curve matrix into the group matrix is explained by an example. Suppose we are interested in the variability between the laboratory group 5 (L5) and the field group 12 (F12). Table 8.4 shows that the group $L_{5}$ has 4 replications and the group F12 has 3 
replications. Twelve $D$ parameters from the curve matrix are used to find the group variability between $\mathrm{L} 5$ and F12. These twelve parameters represent comparison of the first curve in the group I5 with all the curves in the group F12 and so on. These twelve parameters are averaged to obtain a single $D$ value representing comparison between the groups $\mathrm{L} 5$ and F12.

Details of the results of the analyses performed on the 26 laboratory and 35 field compacted soil groups are discussed in the following sections.

8.6 Variability within the Laboratory Groups

The numbers of replications included in the different laboratory groups are shown in Table 8.4. Samples compacted at a very dry (w/c: $\geq 1.6 \%$ dry of optimum) and dry of optimum (w/c: $\leq 1.5 \%$ dry of optimum). water content and with less than standard Proctor energy (level A) were not available for the analyses. The group variability of the laboratory compacted samples is plotted in Figure 8.7. Figure 8.7 shows the average and the maximum variability within the each group-based on the replicated samples. Values of the variability results shown in Figure 8.7 are tabulated in Table 8.6. Higher number of replications in a group (for example: groups L1, L4, L9, and L18) tend to increase the group variability as shown in Figure 8.7. One reason for this higher variability is the increased likelihood of inclusion of samples with water content values on the extreme ends of the range represented by the group.

Samples with higher molding water content (optimum or higher) and energy levels ( $Z$ standard Proctor) tend to have a smaller variability. This can be seen in groups L7, L10, L12, L21, and L26 plotted in Figure 8.7. One probable reason for this low variability is the increased uniformity achieved by the compaction at higher molding water content and energy levels. The compaction at higher water content and energy levels results in a significant reduction in the macropores. 


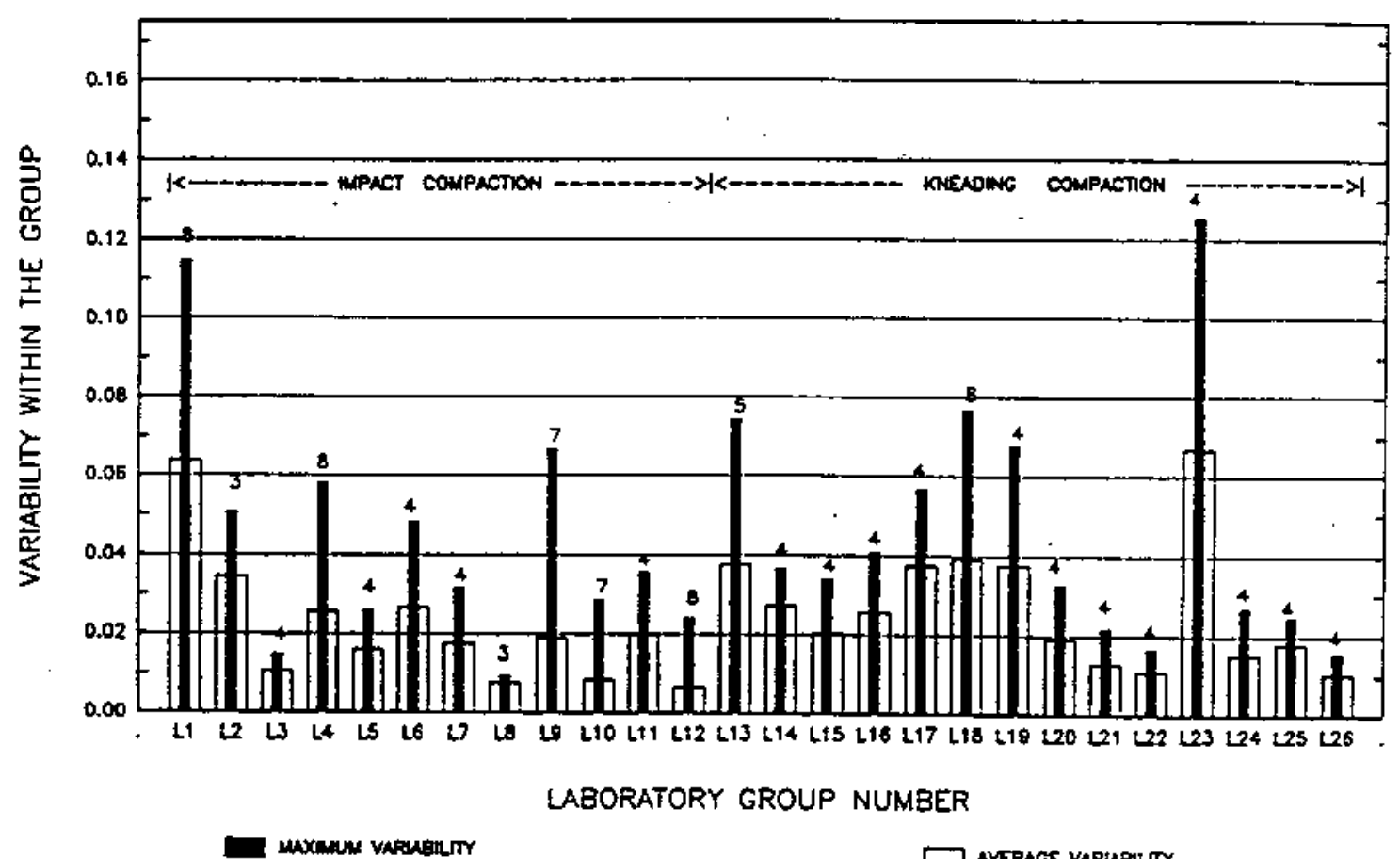

NULGERS AT THE TOP OF THE BARS ARE THE NUMBER OF REPUCATONS.

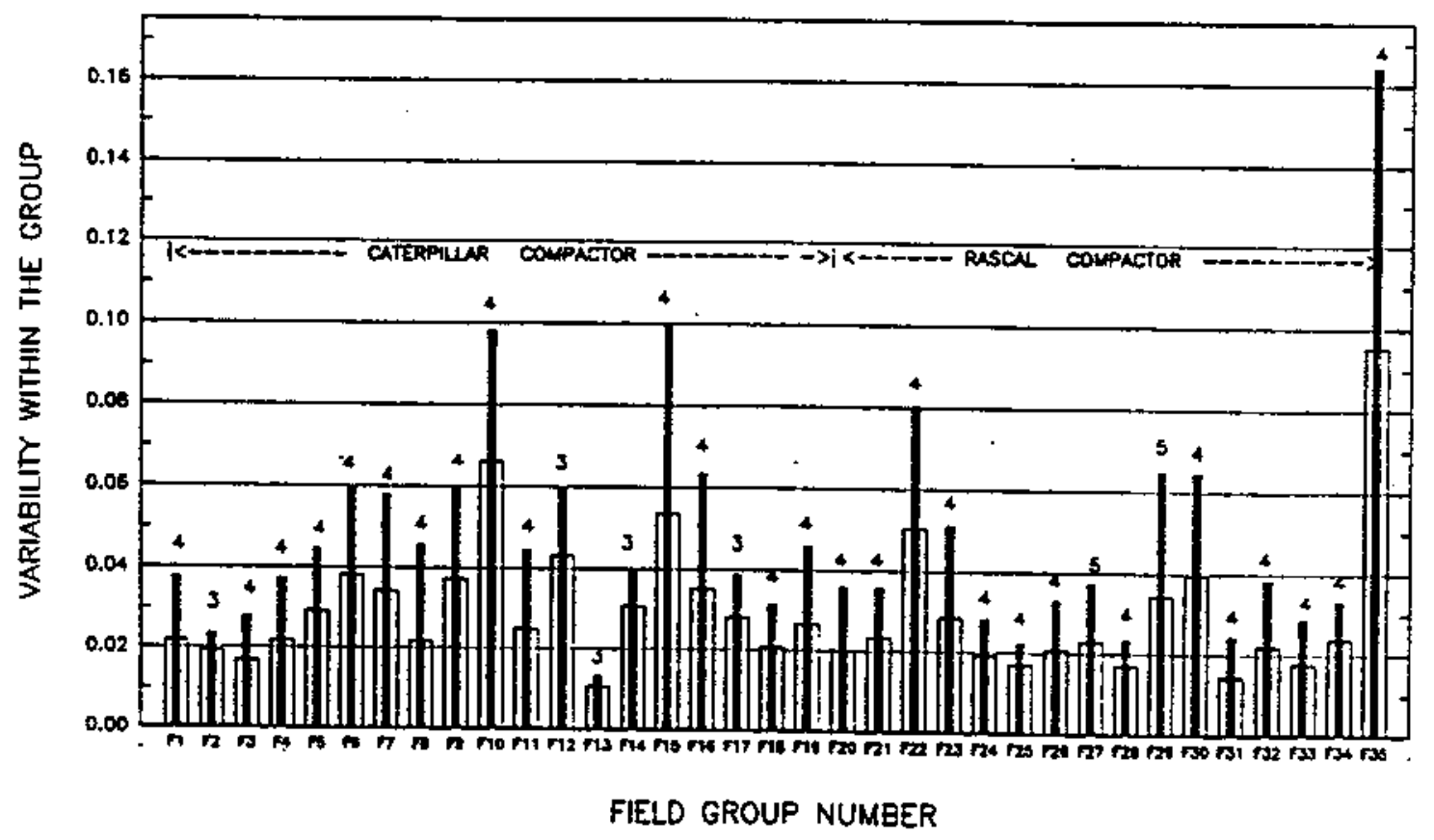

Figure 8.7 Laboratory and Field Group Variability 
Table 8.6 Variability within the Laboratory Groups

\begin{tabular}{|c|c|c|c|c|c|c|c|c|c|}
\hline \multirow{3}{*}{$\begin{array}{l}\text { Sorted by } \\
\text { GROUP } \\
\text { group } \\
\#\end{array}$} & \multirow{3}{*}{$\begin{array}{l}\text { baboritory } \\
\text { code }\end{array}$} & \multirow{2}{*}{\multicolumn{2}{|c|}{ variability }} & \multirow{2}{*}{\multicolumn{3}{|c|}{$\begin{array}{l}\text { Sortod by: } \\
\text { AVERAGE VARIABILITY }\end{array}$}} & \multicolumn{3}{|c|}{$\begin{array}{l}\text { Sorted by: } \\
\text { MAXIMUM VARIABIITY }\end{array}$} \\
\hline & & & & & & & \multirow{2}{*}{$\begin{array}{c}\text { group } \\
\# \\
\end{array}$} & \multicolumn{2}{|c|}{ variability } \\
\hline & & ave. & $\max$. & $\begin{array}{c}\text { group } \\
\#\end{array}$ & ave. & max. & & ave. & $\max$. \\
\hline 1 & 2221010 & 0.0638 & 0.1146 & 12 & 0.0066 & 0.0238 & 8 & 0.0075 & 0.0093 \\
\hline 2 & 2231060 & 0.0345 & 0.0507 & 8 & 0.0075 & 0.0093 & 3 & 0.0106 & 0.0147 \\
\hline 3 & 2321050 & 0.0106 & 0.0147 & 10 & 0.0085 & 0.0283 & 26 & 0.0104 & 0.0156 \\
\hline 4 & 2331010 & 0.0256 & 0.0581 & 26 & 0.0104 & 0.0156 & 22 & 0.0110 & 0.0166 \\
\hline 5 & 2411010 & 0.0160 & 0.0259 & 3 & 0.0106 & 0.0147 & 21 & 0.0130 & 0.0217 \\
\hline 6 & 2421010 & 0.0265 & 0.0483 & 22 & 0.0110 & 0.0166 & 12 & 0.0066 & 0.0238 \\
\hline 7 & 2431050 & 0.0176 & 0.0316 & 21 & 0.0130 & 0.0217 & 25 & 0.0179 & 0.0247 \\
\hline 8 & 2511020 & 0.0075 & 0.0093 & 24 & 0.0151 & 0.0270 & 5 & 0.0160 & 0.0259 \\
\hline 9 & 2521010 & 0.0190 & 0.0665 & 5 & 0.0160 & 0.0259 & 24 & 0.0151 & 0.0270 \\
\hline 10 & 2531010 & 0.0085 & 0.0283 & 7 & 0.0176 & 0.0316 & 10 & 0.0085 & 0.0283 \\
\hline 11 & 2611010 & 0.0199 & 0.0355 & 25 & 0.0179 & 0.0247 & 7 & 0.0176 & 0.0316 \\
\hline 12 & 2631010 & 0.0066 & 0.0238 & 9 & 0.0190 & 0.0665 & 20 & 0.0192 & 0.0325 \\
\hline 13 & 3211010 & 0.0375 & 0.0743 & 20 & 0.0192 & 0.0325 & 15 & 0.0204 & 0.0340 \\
\hline 14 & 3221010 & 0.0271 & 0.0365 & 11 & 0.0199 & 0.0355 & 11 & 0.0199 & 0.0355 \\
\hline 15 & 3231050 & 0.0204 & 0.0340 & 15 & 0.0204 & 0.0340 & 14 & 0.0271 & 0.0365 \\
\hline 16 & 3311010 & 0.0255 & 0.0409 & 16 & 0.0255 & 0.0409 & 16 & 0.0255 & 0.0409 \\
\hline 17 & 3321010 & 0.0373 & 0.0565 & 4 & 0.0256 & 0.0581 & 6 & 0.0265 & 0.0483 \\
\hline 18 & 3331010 & 0.0391 & 0.0768 & 6 & 0.0265 & 0.0483 & 2 & 0.0345 & 0.0507 \\
\hline 19 & 3411010 & 0.0376 & 0.0677 & 14. & 0.0271 & 0.0365 & 17 & 0.0373 & 0.0565 \\
\hline 20 & 3421010 & 0.0192 & 0.0325 & 2 & 0.0345 & 0.0507 & 4 & 0.0256 & 0.0581 \\
\hline 21 & 3431010 & 0.0130 & 0.0217 & 17 & 0.0373 & 0.0565 & 9 & 0.0190 & 0.0665 \\
\hline 22 & 3511010 & 0.0110 & 0.0166 & 13 & 0.0375 & 0.0743 & 19 & 0.0376 & 0.0677 \\
\hline 23 & 3521010 & 0.0668 & 0.1253 & 19 & 0.0376 & 0.0677 & 13 & 0.0375 & 0.0743 \\
\hline 24 & 3531010 & 0.0151 & 0.0270 & 18 & 0.0391 & 0.0768 & 18 & 0.0391 & 0.0768 \\
\hline 25 & 3611010 & 0.0179 & 0.0247 & 1 & 0.0638 & 0.1146 & 1 & 0.0638 & 0.1146 \\
\hline 26 & 3621010 & 0.0104 & 0.0156 & 23 & 0.0668 & 0.1253 & 23 & 0.0668 & 0.1253 \\
\hline
\end{tabular}


Hence, compaction at higher water contents and energy levels generates an uniform pore-size curve resulting in a reduced variability in the replicated tests.

Comparison of the laboratory compaction methods (impact and kneading) reveals that the impact compaction produces lower variability in the pore-size data than does the kneading compaction. Figure 8.7 provides a visual comparison and Table 8.6 gives the variability range for the impact (average range: $0.0066-0.0637$ ) and the kneading (average range: $0.0104-0.0668$ ) compaction.

\subsection{Variability within the Field Groups}

The number of replications included in the field groups are given in Table 8.4. The field compacted samples are highly variable in nature due to less control over the molding water content and the field compaction process in general. Molding water content in the field may vary 6 per cent from the target value. The field group variability results are plotted in Figure 8.7 and the values are given in Table 8.7. Figure 8.7 does not show any pattern in the field variability results. Samples compacted with the Rascal compactor (sample number F20 through F35) show comparatively smaller variability (average range: 0.0145-0.0505, excluding group 35) than those with the Caterpillar compactor (sample number F1 through F19; average range: 0.0107-0.0662). However, no conclusions can be drawn from this difference.

8.8 Comparison of the Laboratory and Field Group Variability

Comparison of the field and the laboratory groups (Figure 8.7) shows that the fleld compacted groups have higher varlability (average range: 0.0066-0.0668) than that of the laboratory compacted samples (average range: 0.0133-0.0982). As shown in Figure 8.7, about 70 percent (25 out of 36 ) of the 
Table 8.7 Variability within the Field Groups

\begin{tabular}{|c|c|c|c|c|c|c|c|c|c|}
\hline $\begin{array}{c}\text { Sorted by: } \\
\text { GROUP } \\
\text { group } \\
\end{array}$ & $\begin{array}{l}\text { field } \\
\text { code }\end{array}$ & $\begin{array}{c}\text { variability } \\
\text { ave. }\end{array}$ & max. & $\begin{array}{c}\text { Sorted bs } \\
\text { AVERA } \\
\text { group } \\
\#\end{array}$ & $\begin{array}{l}\text { GE VARIAB } \\
\text { variability } \\
\text { ave. } \\
\end{array}$ & max. & $\begin{array}{c}\text { Sorted } \\
\text { MAXI } \\
\text { group } \\
\#\end{array}$ & $\begin{array}{l}\text { by: } \\
\text { MUM VARI } \\
\text { variability } \\
\text { ave. }\end{array}$ & $\begin{array}{l}\text { ABILITY } \\
\text { max. }\end{array}$ \\
\hline 1 & 1114010 & 0.0218 & 0.0376 & 13 & 0.0107 & 0.0133 & 13 & 0.0107 & 0.0133 \\
\hline 2 & 1121010 & 0.0193 & 0.0236 & 31 & 0.0145 & 0.0244 & 25 & 0.0172 & 0.0226 \\
\hline 3 & 1126010 & 0.0169 & 0.0280 & 3 & 0.0169 & 0.0280 & 28 & 0.0170 & 0.0234 \\
\hline 4 & 1132010 & 0.0219 & 0.0371 & 28 & 0.0170 & 0.0234 & 2 & 0.0193 & 0.0236 \\
\hline 5 & 1214010 & 0.0293 & 0.0446 & 25 & 0.0172 & 0.0226 & 31 & 0.0145 & 0.0244 \\
\hline 6 & 1221010 & 0.0382 & 0.0598 & 33 & 0.0176 & 0.0288 & 3 & 0.0169 & 0.0280 \\
\hline 7 & 1223010 & 0.0341 & 0.0578 & 24 & 0.0193 & 0.0284 & 24 & 0.0193 & 0.0284 \\
\hline 8 & 1231020 & 0.0218 & 0.0456 & 2 & 0.0193 & 0.0236 & 33 & 0.0176 & 0.0288 \\
\hline 9 & 1314010 & 0.0371 & 0.0598 & 20 & 0.0201 & 0.0359 & 18 & 0.0209 & 0.0315 \\
\hline 10 & 1326010 & 0.0662 & 0.0982 & 26 & 0.0208 & 0.0331 & 26 & 0.0208 & 0.0331 \\
\hline 11 & 1332010 & 0.0249 & 0.0445 & 18 & 0.0209 & 0.0315 & 34 & 0.0240 & 0.0333 \\
\hline 12 & 1411020 & 0.0431 & 0.0595 & 8 & 0.0218 & 0.0456 & 21 & 0.0235 & 0.0357 \\
\hline 13 & 1421010 & 0.0107 & 0.0133 & 1 & 0.0218 & 0.0376 & 20 & 0.0201 & 0.0359 \\
\hline 14 & 1433020 & 0.0309 & 0.0399 & 4 & 0.0219 & 0.0371 & 27 & 0.0231 & 0.0371 \\
\hline 15 & 1511010 & 0.0536 & 0.1003 & 32 & 0.0222 & 0.0384 & 4 & 0.0219 & 0.0371 \\
\hline 16 & 1516010 & 0.0352 & 0.0633 & 27 & 0.0231 & 0.0371 & 1 & 0.0218 & 0.0376 \\
\hline 17 & 1522020 & 0.0283 & 0.0388 & 21 & 0.0235 & 0.0357 & 32 & 0.0222 & 0.0384 \\
\hline 18 & 1525010 & 0.0209 & 0.0315 & 34 & 0.0240 & 0.0333 & 17 & 0.0283 & 0.0388 \\
\hline 19 & 1535010 & 0.0266 & 0.0460 & 11 & 0.0249 & 0.0445 & 14 & 0.0309 & 0.0399 \\
\hline 20 & 4113010 & 0.0201 & 0.0359 & 19 & 0.0266 & 0.0460 & 11 & 0.0249 & 0,0445 \\
\hline 21 & 4126010 & 0.0235 & 0.0357 & 17 & 0.0283 & 0.0388 & 5 & 0.0293 & 0.0446 \\
\hline 22 & 4134010 & 0.0505 & 0.0802 & 23 & 0.0287 & 0.0512 & 8 & 0.0218 & 0.0456 \\
\hline 23 & 4212010 & 0.0287 & 0.0512 & 5 & 0.0293 & 0.0446 & 19 & 0.0266 & 0.0460 \\
\hline 24 & 4221010 & 0.0193 & 0.0284 & 14 & 0.0309 & 0.0399 & 23 & 0.0287 & 0.0512 \\
\hline 25 & 4232010 & 0.0172 & 0.0226 & 7 & 0.0341 & 0.0578 & 7 & 0.0341 & 0.0578 \\
\hline 26 & 4312010 & 0.0208 & 0.0331 & 29 & 0.0344 & 0.0645 & 12 & 0.0431 & 0.0595 \\
\hline 27 & 4321010 & 0.0231 & 0.0371 & 16 & 0.0352 & 0.0633 & 6 & 0.0382 & 0.0598 \\
\hline 28 & 4334010 & 0.0170 & 0.0234 & 9 & 0.0371 & 0.0598 & 9 & 0.0371 & 0.0598 \\
\hline 29 & 4412010 & 0.0344 & 0.0645 & 6 & 0.0382 & 0.0598 & 16 & 0.0352 & 0.0633 \\
\hline 30 & 4413010 & 0.0396 & 0.0645 & 30 & 0.0396 & 0.0645 & 29 & 0.0344 & 0.0645 \\
\hline 31 & 4422010 & 0.0145 & 0.0244 & 12 & 0.0431 & 0.0595 & 30 & 0.0396 & 0.0645 \\
\hline 32 & 4434010 & 0.0222 & 0.0384 & 22 & 0.0505 & 0.0802 & 22 & 0.0505 & 0.0802 \\
\hline 33 & 4511010 & 0.0176 & 0.0288 & 15 & 0.0536 & 0.1003 & 10 & 0.0662 & 0.0982 \\
\hline 34 & 4526010 & 0.0240 & 0.0333 & 10 & 0.0662 & 0.0982 & 15 & 0.0536 & 0.1003 \\
\hline 35 & 4534010 & 0.0952 & 0.1643 & 35 & 0.0952 & 0.1643 & 35 & 0.0952 & 0.1643 \\
\hline
\end{tabular}


average field variability results are higher than 0.02 compared to 43 percent (11 out of 26) of the laboratory groups. Another comparison is made in Table 8.8. Table 8.8 shows the mean and standard deviation of the results presented in Tables 8.6 and 8.7 . Table 8.8 also presents data by excluding the groups showing very high variability, considering them as outlier. In all the cases, the laboratory average and the maximum variability results are smaller compared to the field groups.

8.9 Comparison of the Laboratory and Field compacted Fabric

Most of the present analyses deal with cross-comparison of all the laboratory and field compacted pore-size curves, irrespective of their groups. This is achleved by performing a total of 17,125 (125 $x$ 137 matrix; curve matrix) comparisons. Computational technique involved in this process is same as that used for the laboratory and the field within comparisons. Results of these curve-to-curve comparisons, defined as the curve matrix (125 x 137), are too big to report here. The curve matrix has been reduced to a smaller matrix (26 $\times 35)$, presented as group matr1x. Group matrix provides comparisons between the laboratory and the field groups. The computational steps involved in this reduction process implement the averaging technique discussed in section 8.5.

Group matrix results are arranged in ascending order of variability. The two different formats are used to report the results. In the first format (Appendix D, 26x35), a laboratory group is compared with all the fleld groups. For example, the laboratory group $L 1$ has the best match with field group $F 2$ $(L I F 2,=0.0781)$ and the worst match with the field group $F 32$ $(L 1 F 32 v=0.1584)$. In the second format (Appendix VIII, 35x26), a field group is compared with all the laboratory groups. For example, the field group $F 1$ has the best match with the laboratory group $L 26(F 1 L 26,=0.0191)$ and the worst 
Table 8.8 Comparison of Laboratory and Field Variability in Pore-size Data

\begin{tabular}{lllll}
\hline $\begin{array}{l}\text { Variabllity } \\
\text { wthin }\end{array}$ & $\begin{array}{l}\text { Lab- } \\
\text { oratory }\end{array}$ & Field & Lab- & Field \\
oratory & & (excluding groups) \\
Group & (all) & (all) & 1 \& 23 & 35 \\
\hline
\end{tabular}

Average Variability

$\begin{array}{llllll}\begin{array}{l}\text { mean } \\ \text { standard } \\ \text { deviation }\end{array} & 0.02439 & 0.02996 & 0.02098 & 0.02804 \\ & 0.01559 & 0.01643 & 0.01032 & 0.01206\end{array}$

Kaximum Variability

\begin{tabular}{lllll}
$\begin{array}{l}\text { mean } \\
\text { standard } \\
\text { deviation }\end{array}$ & 0.04452 & 0.04844 & 0.03823 & 0.04503 \\
\hline
\end{tabular}


match with the laboratory group $L 13\left(F 1 L 26_{v}=0.1372\right)$. Tables $D .1$ and $D .2$ are given in Appendix D.

The first format helps identify a laboratory group capable of replicating the field compacted fabric; and the second format helps identify a field group having a similar laboratory compacted fabric. Several observations can be made from the results provided in Tables D.1 and D.2. The following paragraphs discuss the observations and the findings:

\subsubsection{Match Reversal}

A certain laboratory group best matches with a particular field group but that particular field group may best match with some other laboratory group. For example, Table D. 1 shows that the laboratory group $L 1$ has the best match with the field group $F 2(L 1 F 2,=0.0781)$ but the field group $F 2$ has the best match (Table D.2) with the laboratory group L6. $\left(F 2 L \sigma_{v}=\right.$ 0.0270 ). It suggests that the laboratory group $L_{1}$, at best, matches fabric of $F 2$ but $F 2$ creates a fabric closer to that of group $L 6$ than to group $L 1$. Not surprisingly, a majority of the comparisons shown in Tables D.1 and D.2 shows this trend because the comparison technique tries to find a match for the each group considered in the analyses. Hence, fabric matching is not necessarily a reversible process.

A fabric is considered to be replicated when the matching process is reversible.

\subsubsection{Multiple Best-matches}

A particular laboratory group can best match with more than one field groups and vice versa. This multiple matching is explained by an example. In Table D.2 (row 1), the laboratory group 210 has the best match with 10 field groups: $F 31, F 4, F 28, F 19, F 34, F 13, F 8, F 9, F 10$, and $F 32$, in that order. This order can be seen in Table D.1 (column L10). Six 
out of these ten multiple best matched groups $(F 31, F 4, F 28$, $F 19, F 34$ and $F 13$ ) lie in the first 10 best-matched groups with L10 (Table D.1, column L10). Laboratory group L10 (code: 2531010) represents a group having a wet of optimum molding water content and Modified Proctor energy. Almost all of the $L 10$ best-matched multiple field groups have optimum or higher molding water content and compacted with 8 or 16 passes. Though no comparison has been made between the field groups, this observation shows that there is no significant difference between the field compacted groups on the wet side of optimum, particularly with higher compactive energies. Additionally, the laboratory and the field compacted groups on the wet side of optimum with higher compactive energies show similarity in the fabric.

Similarly, data in Table $D .1$ (row 1) indicate that the field group F2 has multiple best matches with 9 laboratory

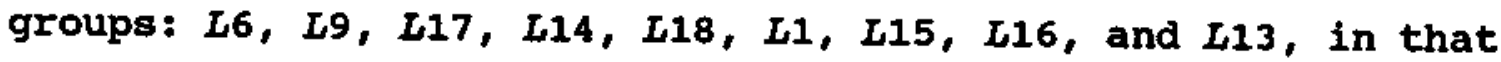
order. This order can be seen in Table D.2 (column F2). OnIy three (L6, L9, and $L 17$ ) of these nine multiple best-matched groups lie in the first 10 best-matched groups with $F 2$, as shown in Table 0.2 (column F2). In fact, groups L15, L16, and $L 13$ are at the bottom of the $F 2$ column. It suggests that $I 1$, L15, and L13, at their best, can come close to F2, but F2 generates a fabric similar to $L 6$ and other groups higher up in the column $F 2$ (Table D.2).

Group 72 (1121010) represents a sample compacted in the fleld using 8 passes of Caterpillar compactor at very dry of optimum water content range. Due to the large scatter in the field samples, actual values of the molding water content for the samples in the group $F 2$ are higher (close to optimum) than those represented by the group code (very dry of optimum). This scatter is visible in the field compaction curves shown in Figures 8.2 and 8.3. High scatter in the field groups makes it difficult to make any conclusive statements based on the above observation. But in general, it can be said that the 
laboratory and field compacted fabric at or above optimum water content with Proctor or higher energy do not differ much with respect to pore-size data.

\subsubsection{The Worst Matches}

Table D. 2 shows that laboratory groups $L 1, L 13, L 15, L 16$, $L 18$, and $L 25$ occur frequently in the bottom five matches. It indicates that these groups have a low probability of matching a fabric generated by any of the field compacted groups. These groups except $L 25$ represent laboratory samples compacted on the dry to very dry of optimum water content. Group 225 (3611010) represents kneading compaction at very wet of optimum with less than standard proctor energy.

It is concluded that the laboratory groups with very dry to dry of optimum water content, particularly with less than standard Proctor energy, are less likely to create a fabric obtained by any of the compactors used in the field. Interestingly, most of the worst matched laboratory groups belong to the kneading compaction method.

The worst matched groups are now discussed by turning to Table D.1 to find which of the field fabrics is difficult to create in the laboratory. Field groups $F 8, F 9, F 10, F 32$ and F35 are frequent groups in the last five matches in Table D.1. These field groups represent fabrics which are difficult to replicate by any of the laboratory methods included in the study. These groups represent caterpillar compacted samples at ary of optimum, and Rascal compacted samples at wet to very wet of optimum, with high compactive effort of 16 passes. These are also field compaction conditions that specifications try to avoid (INDOT, 1993). Since the fleld groups have high internal variability as shown in Figure 8.7 , a caution is advised in this conclusion. 


\subsubsection{Frequently Matched Groups}

The first five matches from Tables $D .1$ and 0.2 were picked to represent the frequently matched groups as shown in Figure 8.8. Figure 8.8 clearly shows that the frequently matched laboratory groups have concentrated occurrences (high frequency in a few groups). On the other hand, field groups show a spread of frequently occurring groups over a wide range of groups. This again confirms an overlap (high variability) in the field compacted groups - the different characteristics of the laboratory and the field variability.

Laboratory frequency plot in Figure 8.8 shows that, in general, impact groups at very dry of optimum are less likely to replicate any of the field compacted group fabric. Samples on the wet and very wet side, with less than standard Proctor energy (group $L 8$ and $L 11$ ), are also less likely to replicate any of the field compacted group fabric.

Kneading compacted samples have low overall frequency $(68 / 175)$ of matching in the first five matched groups as shown in Figure 8.8. Not a single sample compacted with kneading compaction at less than optimum water content (Samples $L_{13}$ through $L 19$ ) was able to fall in the first five matched groups. Later, samples compacted at wet to very wet with less than Standard Proctor energy ( $L 23$ and $L 25$ ) were also not able to match in the first five matched groups.

In summary, Figure 8.8 shows that impact compaction is more likely to produce a fabric similar to that created in the field with Caterpillar and Rascal compactors. Also field compacted fabric is less likely to be replicated by laboratory impact compaction: (a) on dry side of optimum; and (b) with less than standard Proctor energy on the wet side of optimum. 

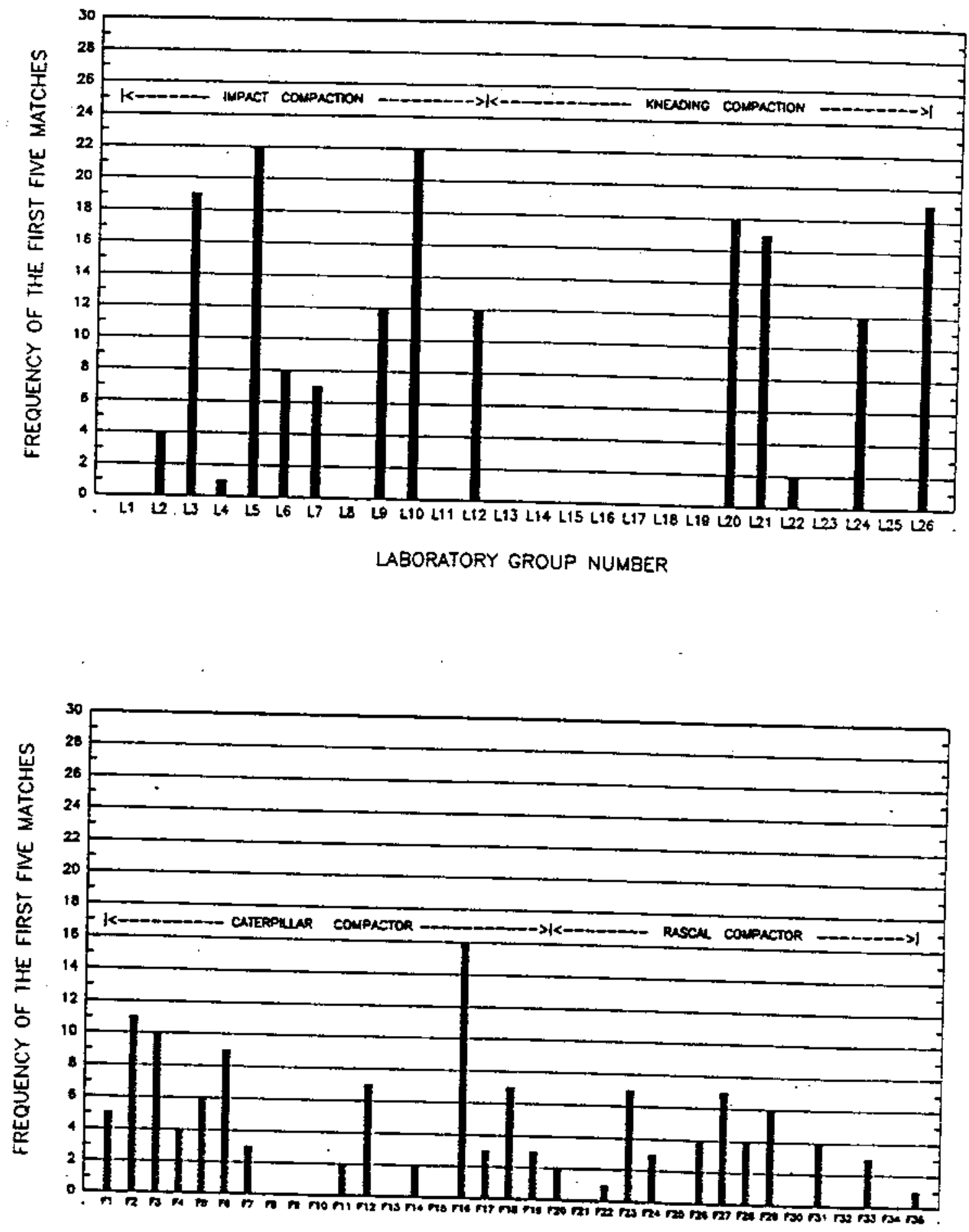

FIELD GROUP NUMEER

Figure 8.8 Frequently Matched Laboratory and Field Groups 


\subsubsection{The Two-way Match}

Table 8.9 and Figure 8.9 have been prepared by picking up the five absolute minimum values from Tables $D .1$ and $D .2$. Note that these values are minimum in two-way comparison, satisfying the replication reversal criterion. For example, L10 has the minimum variability with F31 (Table D.1) and F31 has the minimum variability with L10 (Table D.2). In other words, $L 10$ best matches $F 31$ and $F 31$ best matches 210 . Table 8.9 suggests that the best matched laboratory groups represent the optimum or higher molding water contents. However, on the field side, groups $F 2$ and $F 3$ (basically the same groups with different sample numbers, see Table 8.4) belong to the dry side of optimum. As mentioned earlier, the actual values of the water contents in these groups (F2 and F3) suggest that the water contents of the samples tested have higher values (close to optimum) than those represented by the group.

Table 8.9 suggests that the best-matched groups represent a majority of the samples (both the field and the laboratory compacted) at the optimum or higher water content. Moreover, most of the laboratory samples in Table 8.9 represent standard proctor or higher energy level.

\subsection{Concluding Remarks}

Statistical methods, such as the nonparametric method applied for the present study, can be used as a tool in getting a direction towards finding a match between the laboratory and the field compacted fabric. It is difficult to assign a single laboratory group capable of replicating the field compacted fabric and vice versa. However, the present analyses provide several important conclusions regarding the laboratory and the field compacted fabric. These findings are specifically applicable to the soil in question, a medium plastic A-6 (CL) soil. However, general conclusions about the 
Table 8.9 The Best Matched Laboratory and Field Groups

\begin{tabular}{|c|c|c|c|}
\hline $\begin{array}{l}\text { Group } \\
\text { Match }\end{array}$ & $L F_{v}$ & $\begin{array}{l}\text { Variability within } \\
\text { laboratory and the } \\
L_{\text {may }} L_{\mathrm{m}} \\
\text { (ave. max.) }\end{array}$ & $\begin{array}{l}\text { the } \\
\text { field groups } \\
F_{\text {wag }} F_{m \text { m }} \\
\text { aave. max.) }\end{array}$ \\
\hline $\begin{array}{l}\text { Match I } \\
\text { L10/F31 }\end{array}$ & 0.0160 & $(0.0085,0.0283)$ & $(0.0145,0.0244)$ \\
\hline${ }_{L 5 / F 33}^{\text {Match }}$ II & 0.0176 & $(0.0160,0.0259)$ & $(0.0176,0.0288)$ \\
\hline $\begin{array}{l}\text { Match III } \\
\text { L26/F26 }\end{array}$ & 0.0181 & $(0.0104,0.0156)$ & $(0.0208,0.0331)$ \\
\hline $\operatorname{Match}_{\mathrm{L} 20 / \mathrm{F} 3}$ IV & 0.0240 & $(0.01921,0.0325)$ & $(0.0169,0.0280)$ \\
\hline $\begin{array}{l}\text { Match V } \\
\text { L6/F2 }\end{array}$ & 0.0270 & $(0.0265,0.0483)$ & $(0.0193,0.0236)$ \\
\hline
\end{tabular}

Description of the Best Matched Groups

Best Match Laboratory Method Field Method

Match I

Impact compaction wet of optimum Modified Proctor

Rascal Compactor wet of optimum 8 passes

Match II

Impact compaction optimum w/c

$<$ Proctor.

Match III

Kneading Compaction very wet of optimum standard Proctor

Match IV

Kneading Compaction

optimum w/c

standard proctor

Match V

Impact compaction

optimum w/C standard Proctor

Rascal Compactor very wet of optimum 4 passes

Rascal Compactor optimum w/C 4 passes

Caterpillar Compactor very dry of optimum 8 passes

Caterpillar Compactor very ary of optimum 8 passes

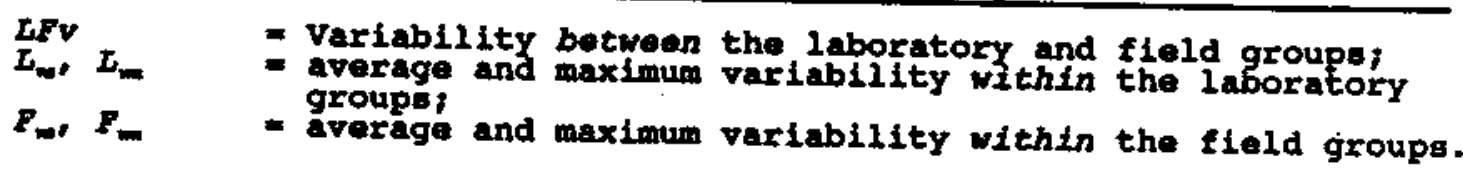



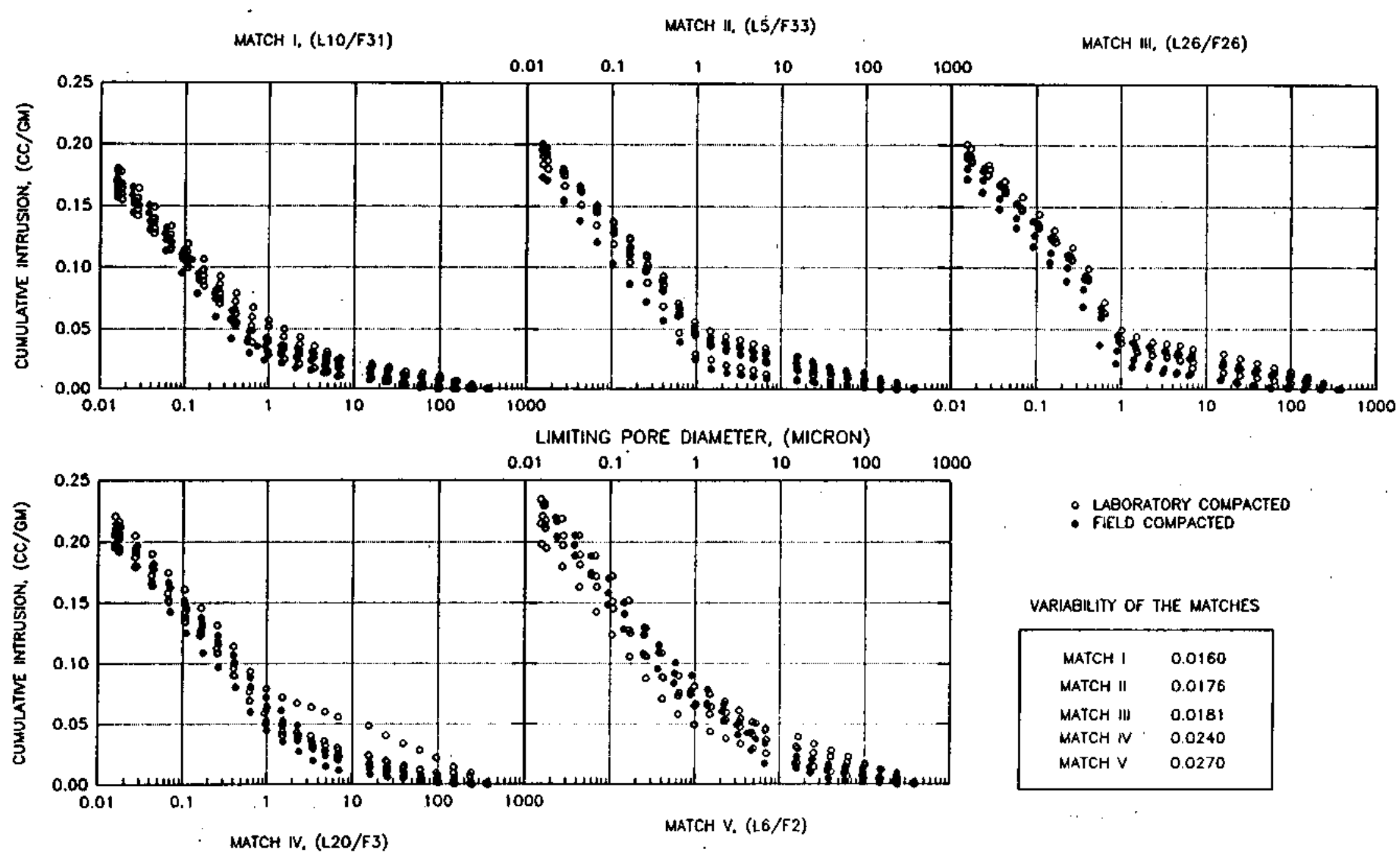

- Lagoratory compacteo

- FIELD COMPACTED

VARIABILITY OF THE MATCHES

$\begin{array}{ll}\text { MATCH I } & 0.0160 \\ \text { MATCH II } & 0.0176 \\ \text { MATCH III } & 0.0181 \\ \text { MATCH N } & 0.0240 \\ \text { MATCH V } & 0.0270\end{array}$

Figure 8.9 The First Five Best Matched Groups 
fabric of the laboratory and the field compacted fabric for the fine-grained soils can be made from this study:

(a) Fabric of a compacted fine-grained soil is significantly influenced by the molding water content;

(b) Fabrics on the dry and the wet slde of the optimum are significantly different. The differences in the dry and wet side fabric are mainly due to the difference in the macropore zone;

(c) Compaction has a significant influence on the macropore zone and the micropore zone is relatively insensitive to the compaction process;

(d) Field compacted samples show higher variability in the pore-size data on the replicated samples;

(e) Impact compaction gives less variability in pore-size data compared to the kneading compaction;

(f) In the laboratory, a group (with certain molding water content and energy level) can be represented by a distinct pore-size curve. Field compacted samples, even under highly controlled conditions, show significant scatter and overlap between aifferent;

(g) Wet side compaction, especially with higher compactive efforts, gives a uniform fabric which is less sensitive to the compaction method involved;

(h) Dry side laboratory fabric is significantly influenced by the compaction method and the energy level;

(i) In general, variability of pore-size data decreases with increase in the molding water content. It suggests that the fabric on the wet side of optimum is comparatively uniform and consistent;

(j) Kneading compaction is less likely to replicate a field compacted fabric than impact compaction;

(k) The best matches between the laboratory and the field compacted fabric almost always come from the optimum or the wet side compaction.

Based on the results of the present analysis, it can be concluded that a field compacted fabric can be created in the laboratory by the impact compaction at optimum and slightly wet of optimum (about 1 to 2 wet of optimum) water content 
using Standard Proctor energy. Comparison of the energy levels in Table 8.2 also suggests that the energy required in the laboratory is smaller than the energy applied in the field. Kneading compaction (at standard Proctor energy level) is also capable of replicating the field compacted fabric at water content higher than optimum values. Hence, at comparable energy levels, impact and kneading compacted fabric on the wet side of optimum do not differ significantly. 


\section{FABRIC OF SOIL - EXPERIMENTAL STUDIES}

\subsection{Introduction}

For the purpose of design and analysis, a soil mass has almost always been considered as a continuum though, in reality, it is composed of discrete soil particles arranged in some fashion. Predicted soil behavior, using the theories of continuum soil mechanics, is greatly dependent on the particle characteristics such as shape, size, texture, arrangement, forces between the particles, etc. Hence it is important to understand this property clearly for a better prediction and understanding of soil behavior.

Fabric of soil has been correlated to other engineering behavior such as permeability (Marshall, 1958; GarciaBengochea et al, 1979; Juang and Holtz, 1986); consolidation (Griffiths and Joshi, 1989); compressibility (Osipov, 1982); collapse mechanism (Clark and Gillott, 1985); suction (Nagpal, 1972; Prapaharan et al., 1985), shear strength (Ahmed et al., 1974); and frost heave (Reed et al., 1979) etc.

Pore-size studies reported in the literature mostly deal ... with the fabric of a compacted soil mass. The present investigation starts with the investigation of fabric of uncompacted (fraction finer than U.S. 16 sieve) soil mass. Five fine-grained soil classes (A-7-6, A-7-5, A-6, A-4/A-6, and $A-4)$ are included in the present study. It is believed that the determination of the fabric of uncompacted soil will provide inherent soil characteristics and a reference from which the effect of compaction on the fabric can be evaluated. Two terms, macroporosity and microporosity, are defined to represent the distribution of porosity as determined by the 
pore-size curves. Correlations are reported between macroporosity and the maximum attainable dry density, and between microporosity and clay fraction. Further, pore-size results for the laboratory and field compacted samples for the selected soil classes are investigated. Finally, pore-size results on the compacted soils are correlated with the resilient modulus of the soil.

9.2 Fabric of Uncompacted Soil

At first, borrow soil samples for the pore-size determination (prior to oven drying) were air dried. The airdried samples are then oven dried prior to the determination of pore-size distribution. Pore-size distribution of uncompacted soil was determined on the soll fraction passing a U.S. * 16 (opening $1.18 \mathrm{~mm}$ ) sieve. Fabric changes of airdried uncompacted samples during oven drying were ignored. Pore-size procedure for the uncompacted (soil powder) samples was similar to that for the compacted soil samples. An especially designed penetrometers with an inverted cone-shaped bulb was used for the uncompacted soils to prevent the soil particles from sliding into the penetrometer stem. Due to the higher total intruded volume required for the uncompacted samples, a smaller quantity of samples $(0.5-1.0 \mathrm{gm})$ was used. Pore-size curves on the fine grained soil types are shown in Figure 9.1. Results axe plotted as both cumulative and differential curves. Comparisons of uncompacted pore-size curves give insight about the soil type, plasticity limits, and clay content. Pore-size distribution on uncompacted soil also provides information about the maximum dry unit weight that can be attained at certain energy level.

A cumulative curve provides total intruded volume during porosimetry. On the other hand, a differential curve provides intruded volume between two consecutive pore diameters. The pore-size distribution curve is divided into two parts at one 

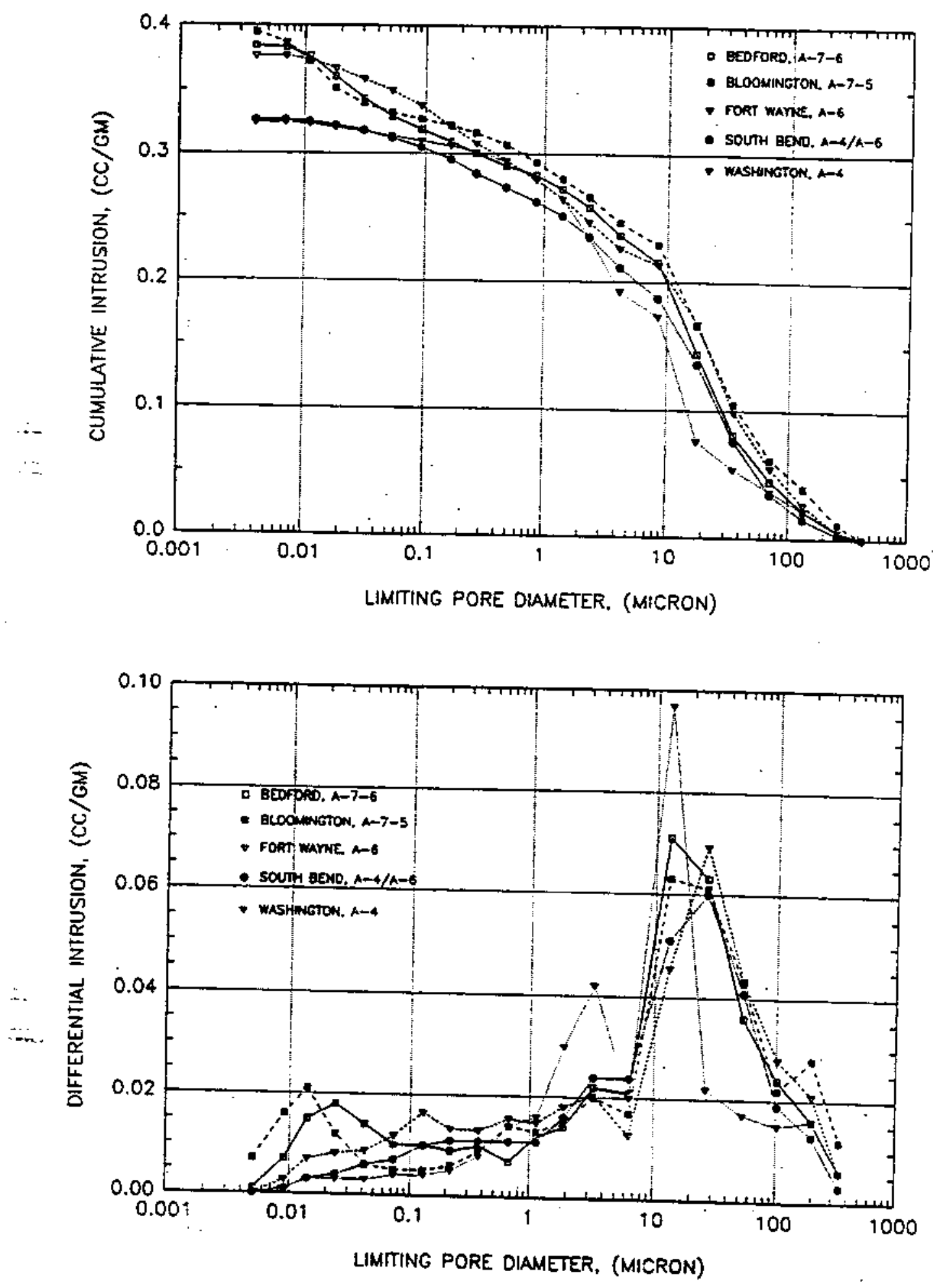

Figure 9.1 Pore Size Distribution of Uncompacted Soils 
micron limiting pore diameter. The part of the curve representing the pore diameters larger than one micron is termed as the macropore zone. similarly, the part of the curve where pore diameters are finer than one micron is termed as the micropore zone.

Differential intrusion curves for uncompacted clayey soil classes (A-7-5, A-7-6, and A-6) display bimodal distributions. One peak occurs in the macropore zone and the other in the micropore zone as shown in Figure 9.1. The macropore mode has a comparatively higher peak than the micropore mode. The micropore mode, termed as the signature mode (Garcia-Bengochea et al., 1979), represents inherent properties of a clayey soil. It is important to note here that only soils with relatively high clay fraction display a signature mode. Signature mode parameters such as diameter ( $S_{d}$, micron), and intrusion $\left(S_{1}, c c / g m\right)$ on a differential curve, are related to the clay content and the plasticity limits of the soil. signature mode occurs towards finer pore diameters as the clay contents of soil increases. Signature mode parameters for clayey soil classes $(A-7-5, A-7-6$, and $A-6)$ are given in Table 9.1 .

The volume of mercury intruded in the micropore zone (pore diameter $\leq 1 \mu$ ) is defined as the microporosity (cc/gm). similarly, the volume of mercury intruded in the macropore zone (pore diameter $>1 \mu$ ) is defined as the macroporosity $(\mathrm{gm} / \mathrm{cc})$. Microporosity is a function of the characteristics of the finer soil particles and the clay fraction present in the soil. Based on the pore-size data on the uncompacted soils (with different clay fractions), a correlation between clay fraction (particles <2 $\mu$; Mitchell, 1976) and microporosity has been developed for different soil classes. This correlation is given in Figure 9.2. It can be seen that a soil with higher clay fraction displays higher microporosity and vice versa. Values of the micro and the macroporosity with other soil parameters are given in Table 9.1. 
Table 9.1 Fabric Parameters of the Uncompacted and Compacted Solls

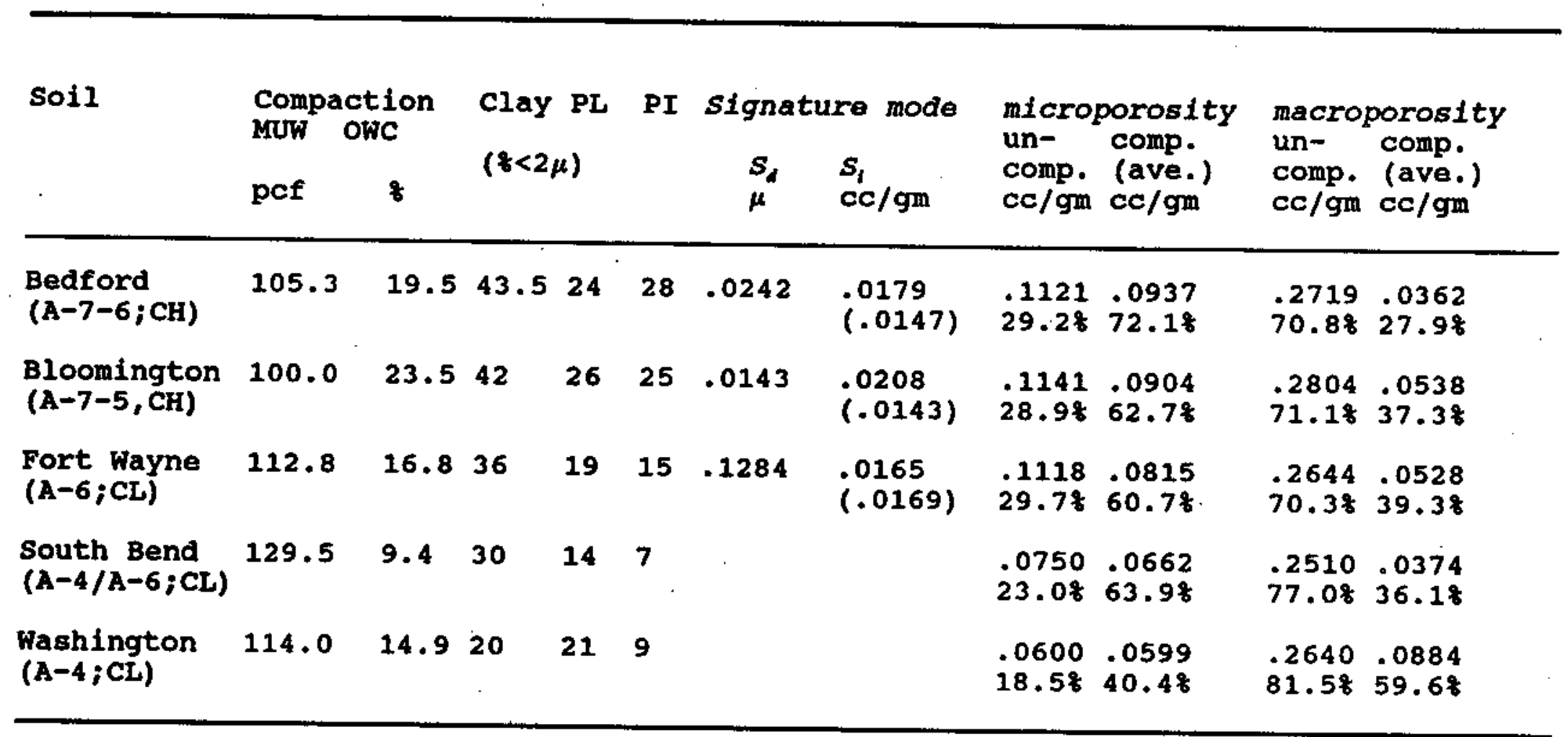

MUW: Maximum Dry Unit Weight

OWC: Opt Imum Water Content

$S_{1}$ in parenthesis is the average value of compacted eamples 


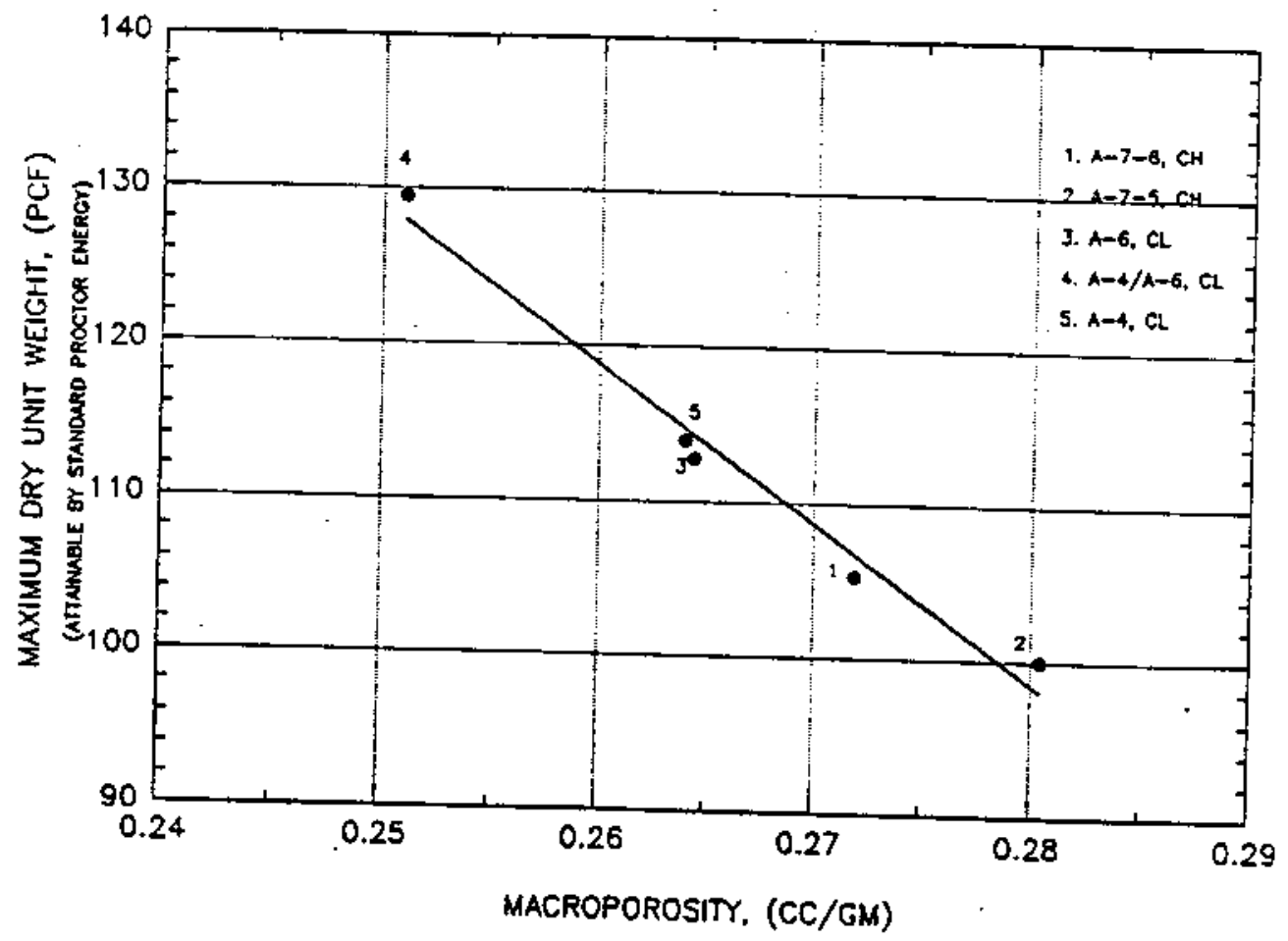

Figure 9.2 Correlation between Macroporosity of Uncompacted Solls and the Maximum Attainable Dry Unit Weight 
Macroporosity of an uncompacted soil sample is a function of particle characteristics such as shape, angularity, and size. Macroporosity of an uncompacted soil is generally reduced by compaction. The maximum dry unit weight that can be attained by certain compactive effort is a function of macroporosity. A correlation between the maximum attainable dry unit weight at standard proctor energy and macroporosity is given in Figure 9.3. The correlation shown in Figure 9.3 indicates that soil with lower macroporosity attains higher dry unit weight during compaction.

\subsection{Laboratory Compaction and Fabric of soil}

When water is added to a soil mass, part of it first wets the soil surface to form an adsorbed water layer. Part of this water is then drawn into the fine capillaries. soils compacted at lower water contents (ary of optimum) will have a majority of the macropores dry as most of the water will be drawn into the micropores. A majority of the micropores in the samples compacted at higher water contents (wet of optimum) is filled with water and the pore water starts to occupy the macropores. At higher water contents the capillary tension in the vater held in the finer pores breaks.

Pore-size curves on the samples compacted in the laboratory at different molding water contents were obtained. Samples were compacted to cover the entire range of the laboratory compaction curves. Samples were compacted in a $2.8-$ inch diameter mold using standard proctor energy. Samples were first tested for resilient modulus. After the resilient modulus test, pore-size samples were obtained from the middle third of the sample. Pore-size samples were freeze-dried with liquid nitrogen and kept under vacuum for at least one day before pore-size determination. To account for variability, at least two pore-size tests were performed for the each compacted sample and average results are reported. The 


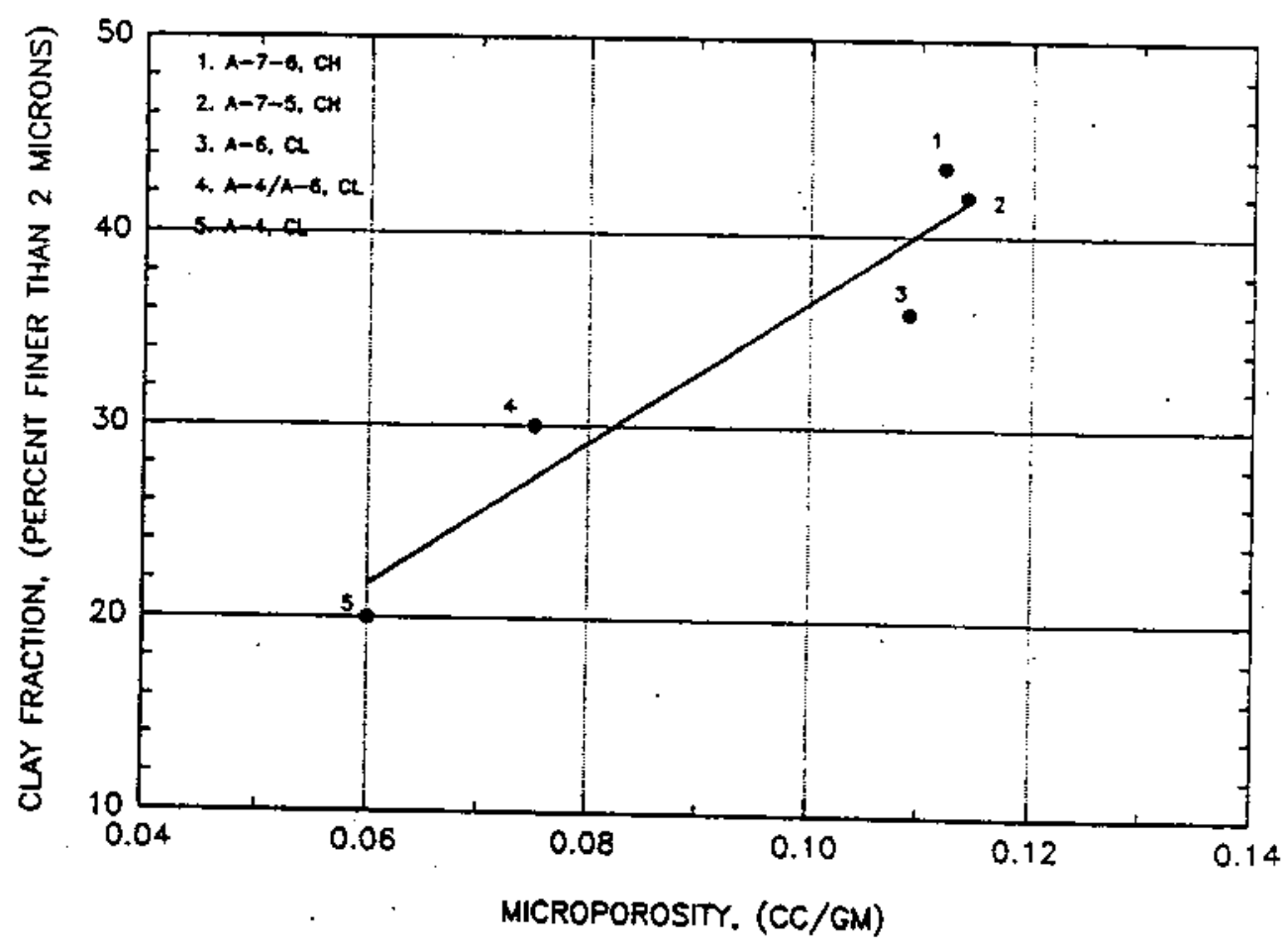

Figure 9.3 Correlation between Microporosity of Uncompacted Soils and Clay Fraction 
laboratory. compacted pore-size curves for the five soil classes are given in Figures 9.4 through Figure 9.8. Effect of compaction on the fabric of soil can be evaluated by comparing pore-size curves for the uncompacted and the compacted samples.

Comparison of uncompacted (Figure 9.1) and compacted cumulative pore-size curves (Figure 9.4 through 9.8) suggests that compaction causes a decrease in the total intruded volume. A decrease in the total intruded volume, particularly for high plastic soils, is concentrated in the macropore zone. Pore-size data show that the differential curves in the micropore zone are practically unaffected by molding water content. This is particularly true for high-plastic soils (A7-6 and A-7-5). Compaction first reduces the frequency of the larger pores and then progressively shifts the frequency of the larger pores towards the finer pores.

Reduction in the larger pore frequency can be seen by comparing the peak intrusion values of the uncompacted and the compacted samples in the macropore zone. Increase in molding water content reduces peak intrusion in the macropore zone. There is a significant difference in the macropore peaks on the dry side and the wet side samples for Bloomington (A-7-5) and Fort Wayne $(A-6)$ soil samples, as shown in Figures 9.5 and 9.6 .

Data reported here are obtained from the specimens compacted with standard Proctor energy. Sridharan et al. (1971) reported that the compactive energy has virtually no effect on the frequency of intrusion in the micropore zone.

Pores for compacted samples in the micropore zone are relatively unaffected by compaction and give almost the same distribution at different molding water contents. This is particularly true for high-plastic soils (A-7-6, A-7-5, and A6 soil). Signatuxe mode diameters $\left(S_{d}\right)$ for soils $(A-7-6, A-7-$ 5, and $\mathrm{A}-6$ ) are not changed by compaction; $i . e$. the signature mode occurs at the same pore diameter for both the uncompacted 

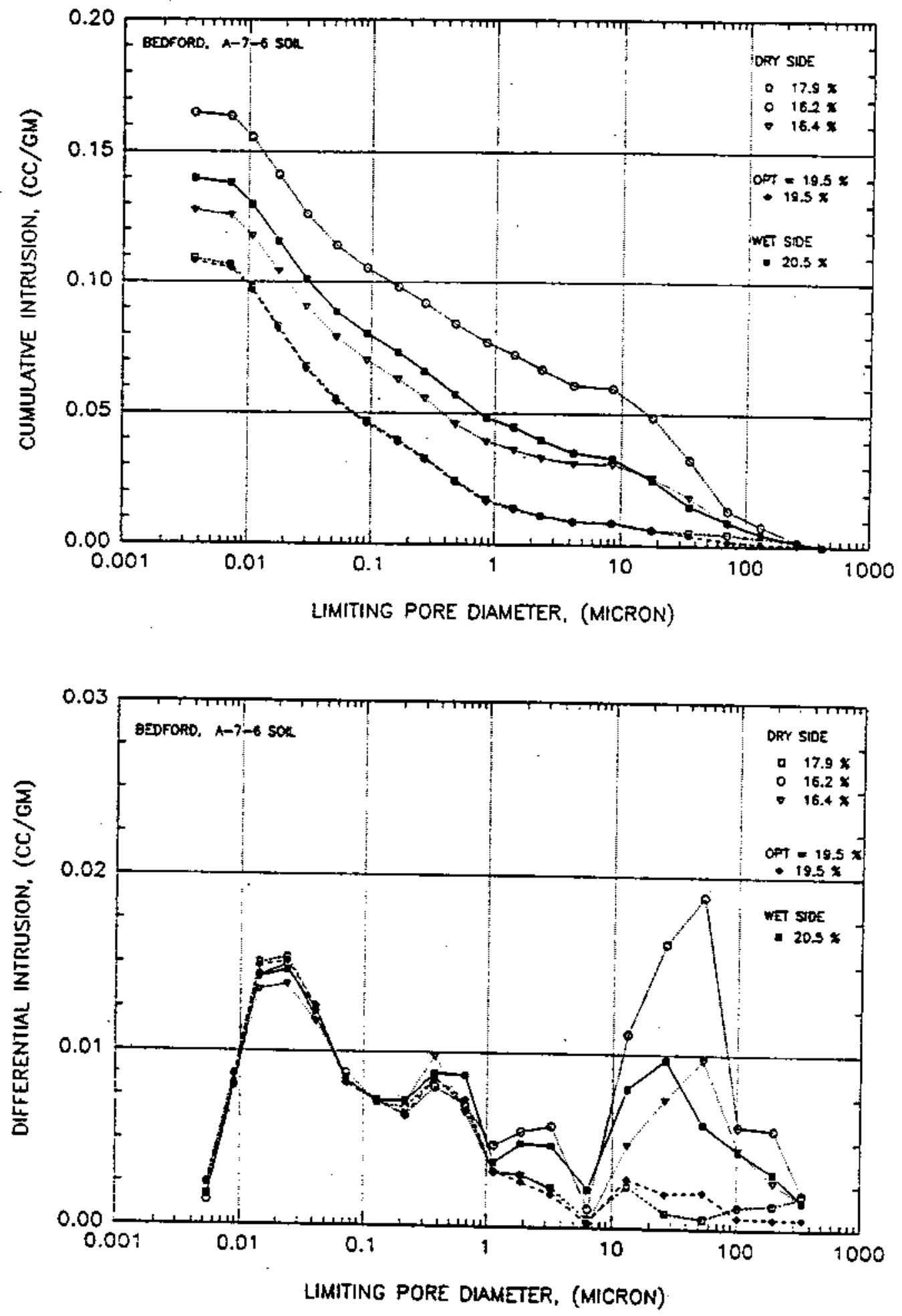

Figure 9.4 Laboratory Compacted Fabric-Bedford Soil 

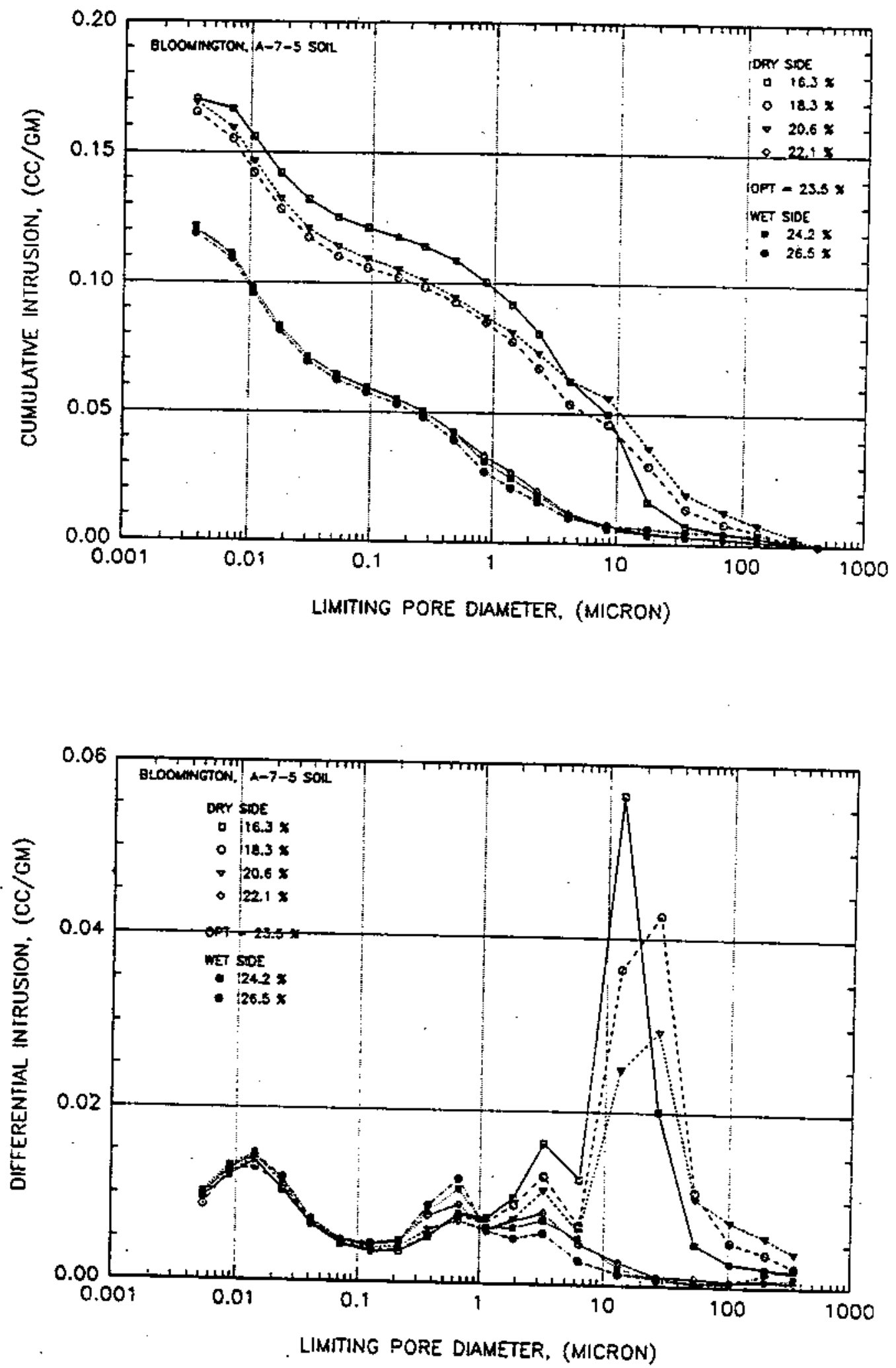

Figure 9.5 Laboratory Compacted Fabric-Bloomington Soil 

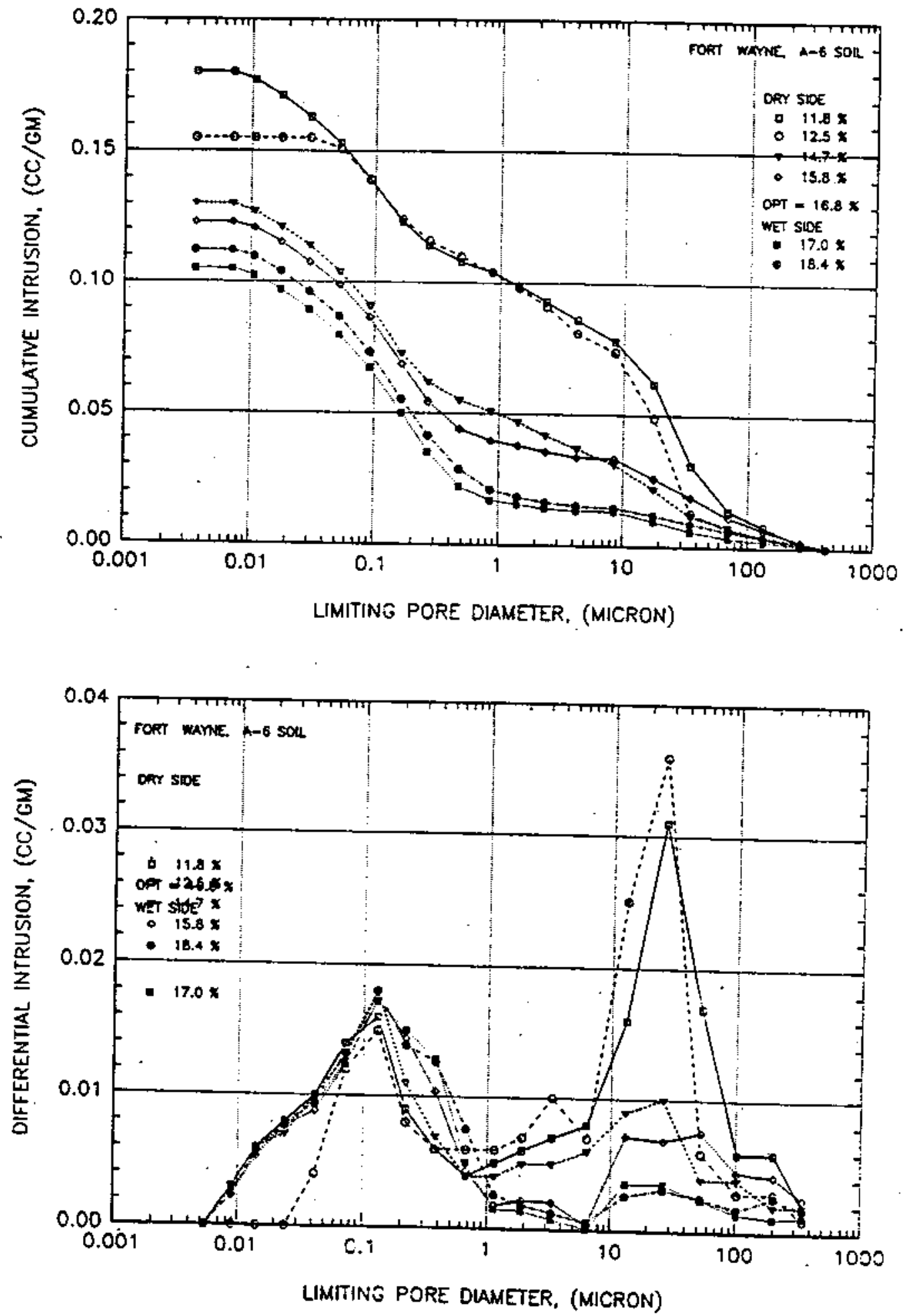

Figure 9.6 Laboratory Compacted Fabric-Fort Wayne Soil 

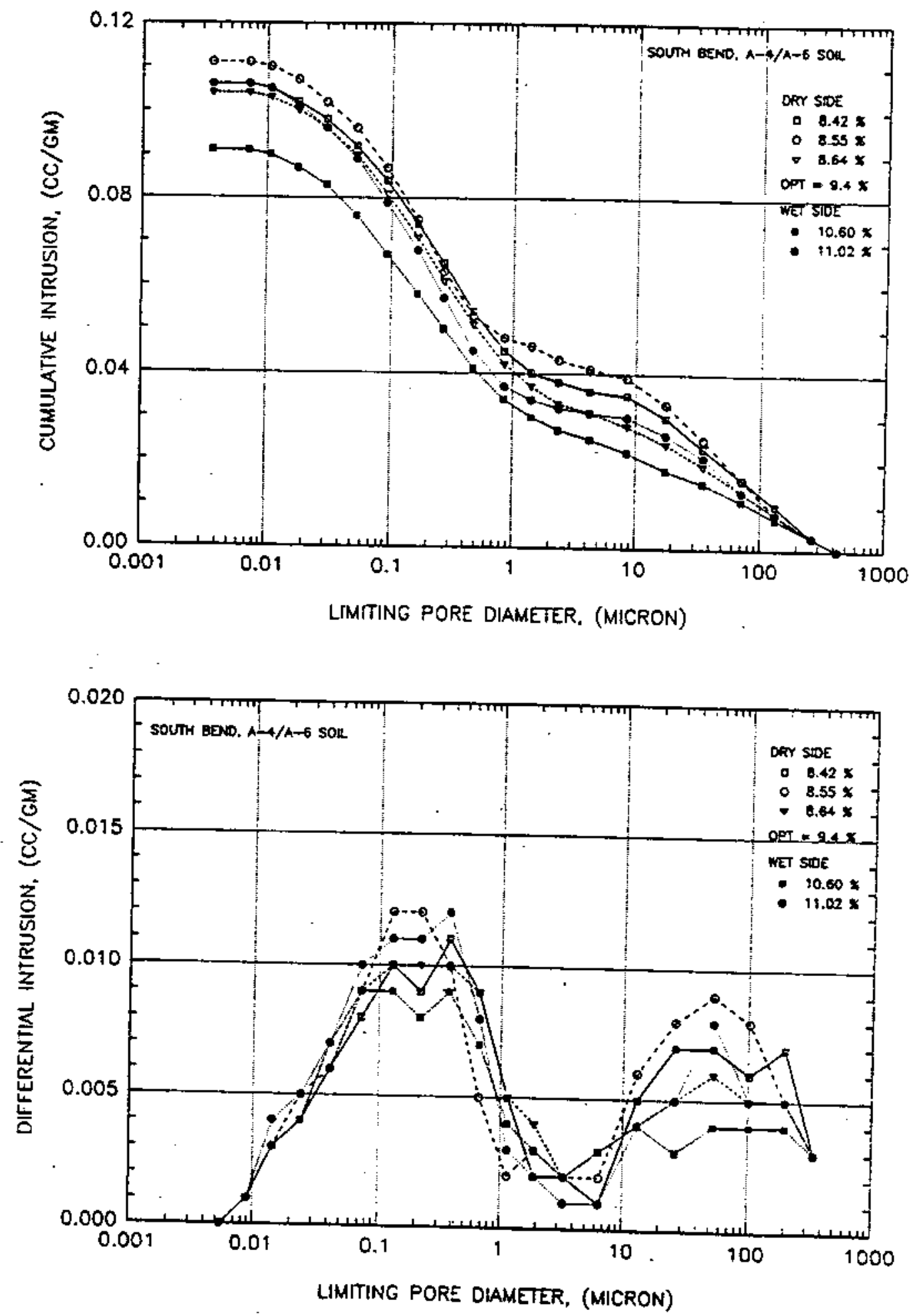

Figure 9.7 Laboratory Compacted Fabric-South Bend Soil 

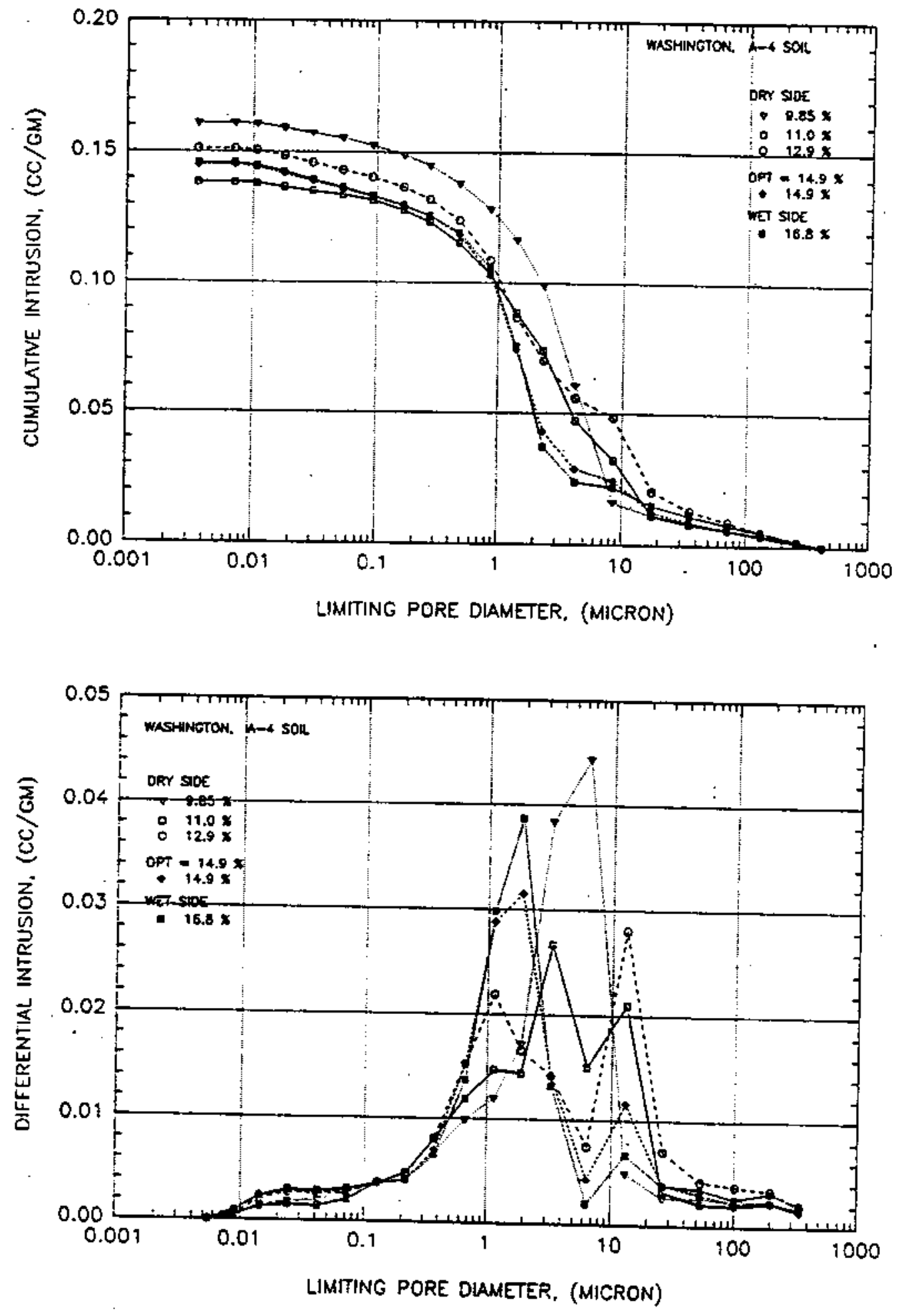

Figure 9.8 Laboratory Compacted Fabric-Washington Soil 
and compacted samples. Parameter $s_{l}$ is changed by the compaction of soil. Soils with larger clay fraction (A-7-6 and A-7-5) show slight reduction in $S_{i}$ due to denser packing of clay fraction. Soils with moderate clay fraction (A-6) show a slight increase in $s_{i}$. This increase for soils with smaller fraction of clay is due to the shifting of the larger pores towards the finer pores. This trend can be seen in the signature mode parameters given in Table 9.1.

Average values of macroporosity and microporosity for the compacted samples are given in Table 9.1. Macroporosity and microporosity values are also reported as the percent of the total intruded volume of the samples. Absolute value of microporosity (cc/gm) of the samples decreases due to compaction. The decrease in microporosity due to compaction is higher in high-plastic soils $(A-7-6, A-7-5$, and A-6) and smaller in the soils with smaller cláy fraction. For example, soil A-4 (CL) has practically no change in the microporosity due to compaction. This is generally due to the reduction of pores and due to the progressive shifting of larger pores towards smaller pores. Average microporosity of compacted samples, as a percent of the total compacted intrusion, is significantly larger compared to the uncompacted samples as given in Table 9.1. This indicates that compaction eliminates a majority of macroporosity and most of the pores in a compacted soil belong to the micropore zone.

Effect of compaction on the fabric of different soil classes can be studied by plotting the pore-size curves on a single plot. Pore-size curves for different soil classes at their respective optimum molding water contents are shown in Figure 9.9. Comparison of cumulative curves at optimum water content with curves in Figure 9.1 (uncompacted) shows that there is a significant decrease in the total intruded volume for all the soil types. Also there is a significant decrease in the macropore peak intrusion. In most of the samples, except Washington $(A-4)$ soil, the macropore peaks are totally 


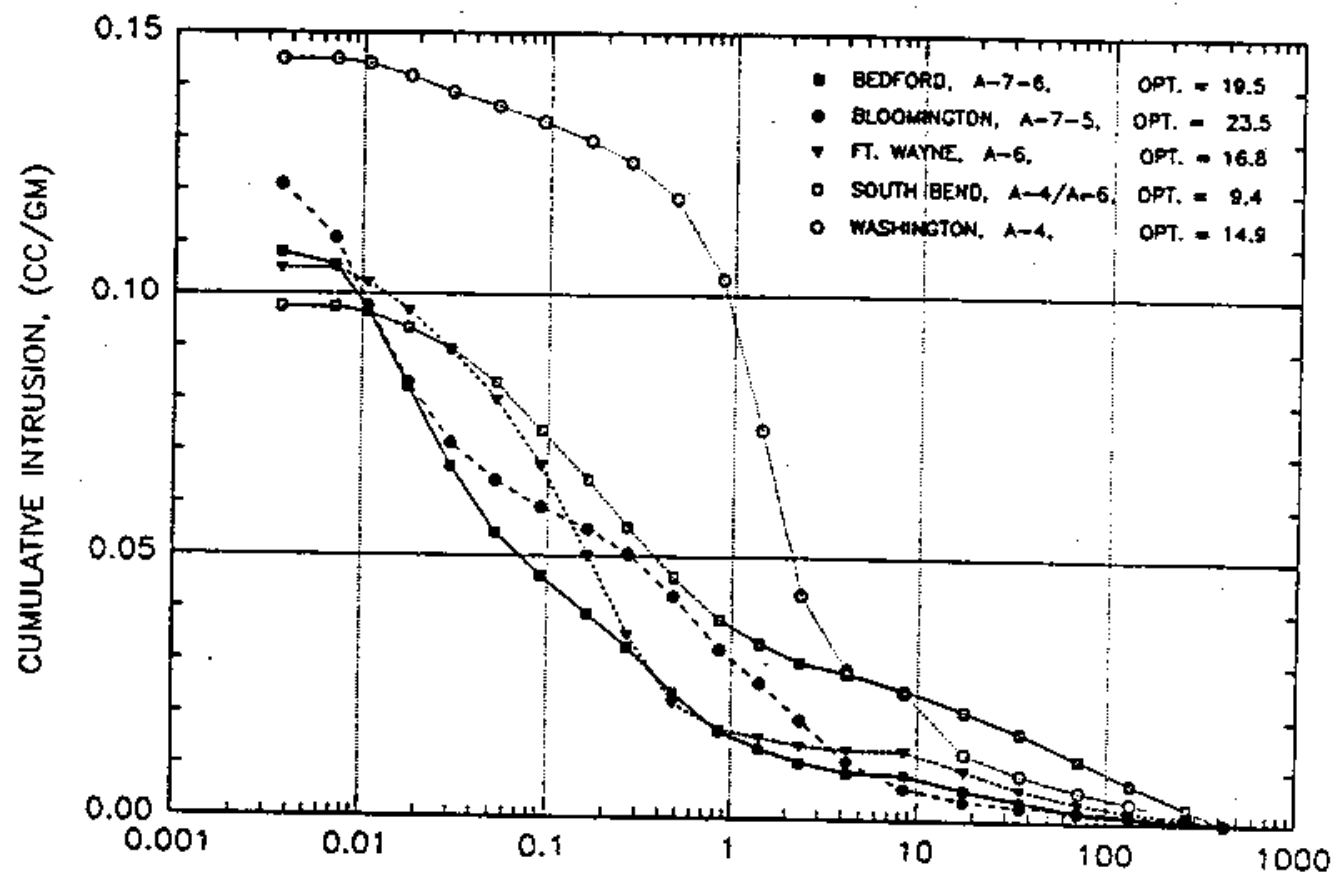

- LIMTING PORE DIAMETER, (MICRON)

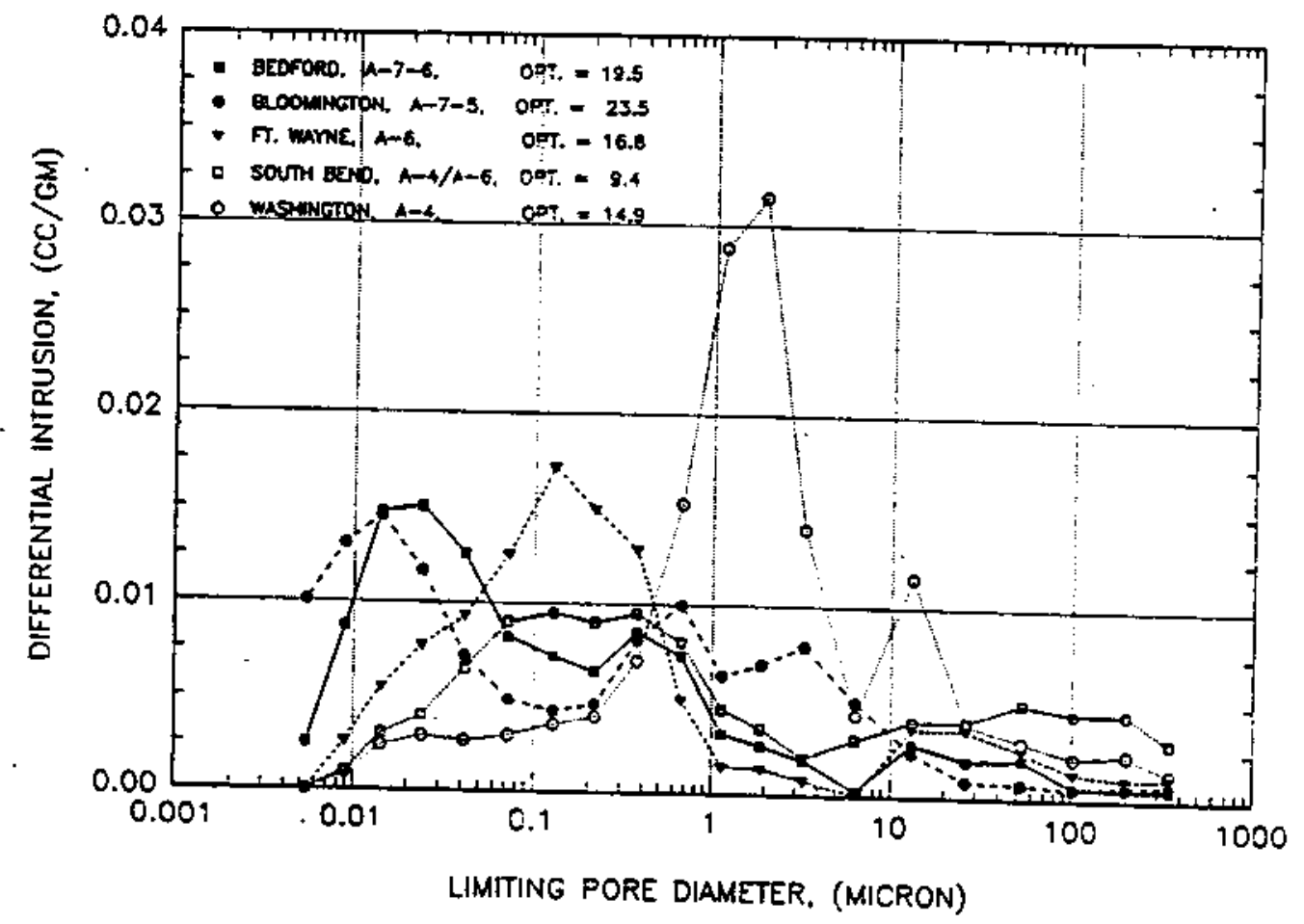

Figure 9.9 Comparison of Laboratory Compacted Fabric at 
eliminated by compaction. Another important observation is the change of the slope of the differential curves between pore diameter 1 and 10 microns. Pore frequency from 10 to 1 micron of uncompacted samples slopes downward as shown in Figure 9.1. Compaction reverses the corresponding slope as shown in Figure 9.9. This upward movement of frequency is caused by the shifting of the larger pores towards the finer pores. The upward movement for A-7-6, A-7-5, and A-6 soils continues up to the signature mode without altering the position of the signature mode. Hence, compaction contributes to a significant reduction in the macroporosity and causes progressive movement of the larger pores towards the finer pores, without affecting microporosity significantly.

\subsection{Field Compacted Fabric}

Field compacted fabrics of the soils from all the sites were determined from the samples collected for resilient modulus testing using Shelby tubes. Similar to the laboratory studies, pore-size samples for field fabric studies were obtained from the samples after performing the resilient modulus tests. Pore-size tests were repeated at least once to account for the variability. Pore-size curves for field compacted samples were reduced to represent microporosity and macroporosity.

Laboratory compacted fabric showed that the molding water content has a significant influence on the created fabric. Differences in the fabric mainly come from the collapse of the macropores and progressive shifting of the macropores towards the finer pores. Degree of saturation rather than water content may be a better parameter to study the variation of compacted fabric. Degree of saturation includes dry unit weight and specific gravity of soil in addition to the water content. Field compacted samples generally display higher values of specific gravity, due to the presence of bigger size 
particles, than those determined in the laboratory. Use of the laboratory determined specific gravity with the field water content values may result in a degree of saturation higher than 100 percent. In the absence of a reliable measurement of specific gravity determination on each of the field compacted samples, the variation of the fabric is reported with water content. Determination of the field compacted pore-size distribution was performed by ignoring the presence of any pebbles and by randomly selecting samples from the middle third of a resilient modulus test sample.

Field compacted fabric is reported as the variation of the total intruded volume, microporosity, and macroporosity with the water content. Water content was measured at the end of the resilient modulus test. The water content includes the as-compacted water content and the subsequent changes. Field compacted fabric results are plotted in Figures 9.10 through 9.14. For the purpose of comparison, laboratory compacted results are also given on the same plots. Laboratory results on the injected samples are also plotted in Figures 9.10 through 9.14. In the majority of the soil types increases in the post-compacted water content do not significantly change the fabric of the laboratory compacted samples. In almost all of the cases, water content for the field compacted samples was higher than the highest values reported for the laboratory compacted samples. Results for the different soil classes are discussed below:

Bedford, $A-7-6$ Soil

water contents compacted samples for the Bedford site have higher Comparison of the inan the laboratory compacted samples. with the laboratory compaction curves as-compacted field data the samples tested are on the wet shows that almost all of compaction curves. There was an increase in the water content 


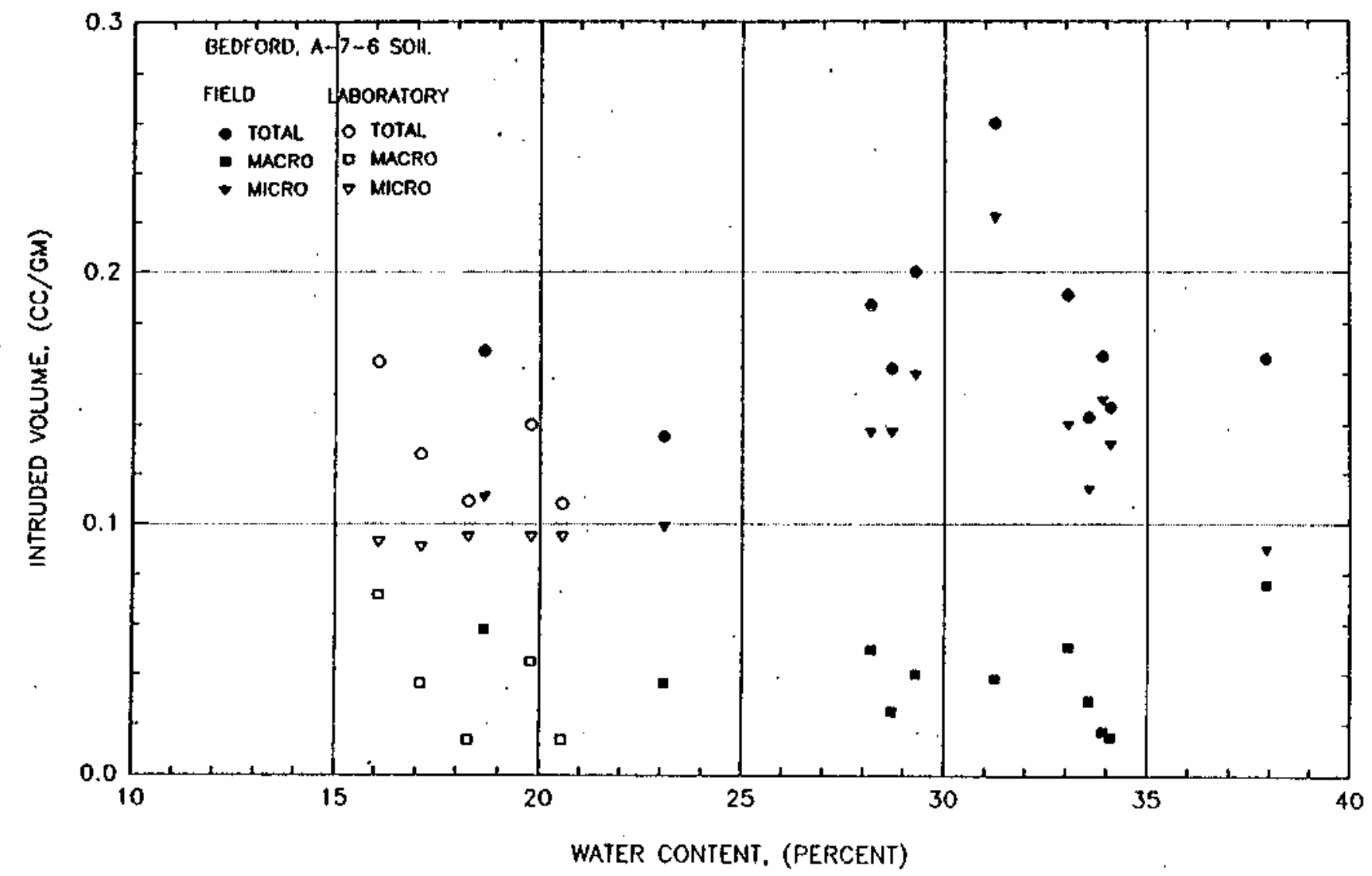

Figure 9.10 Field Compacted Fabric-Bedford soil 


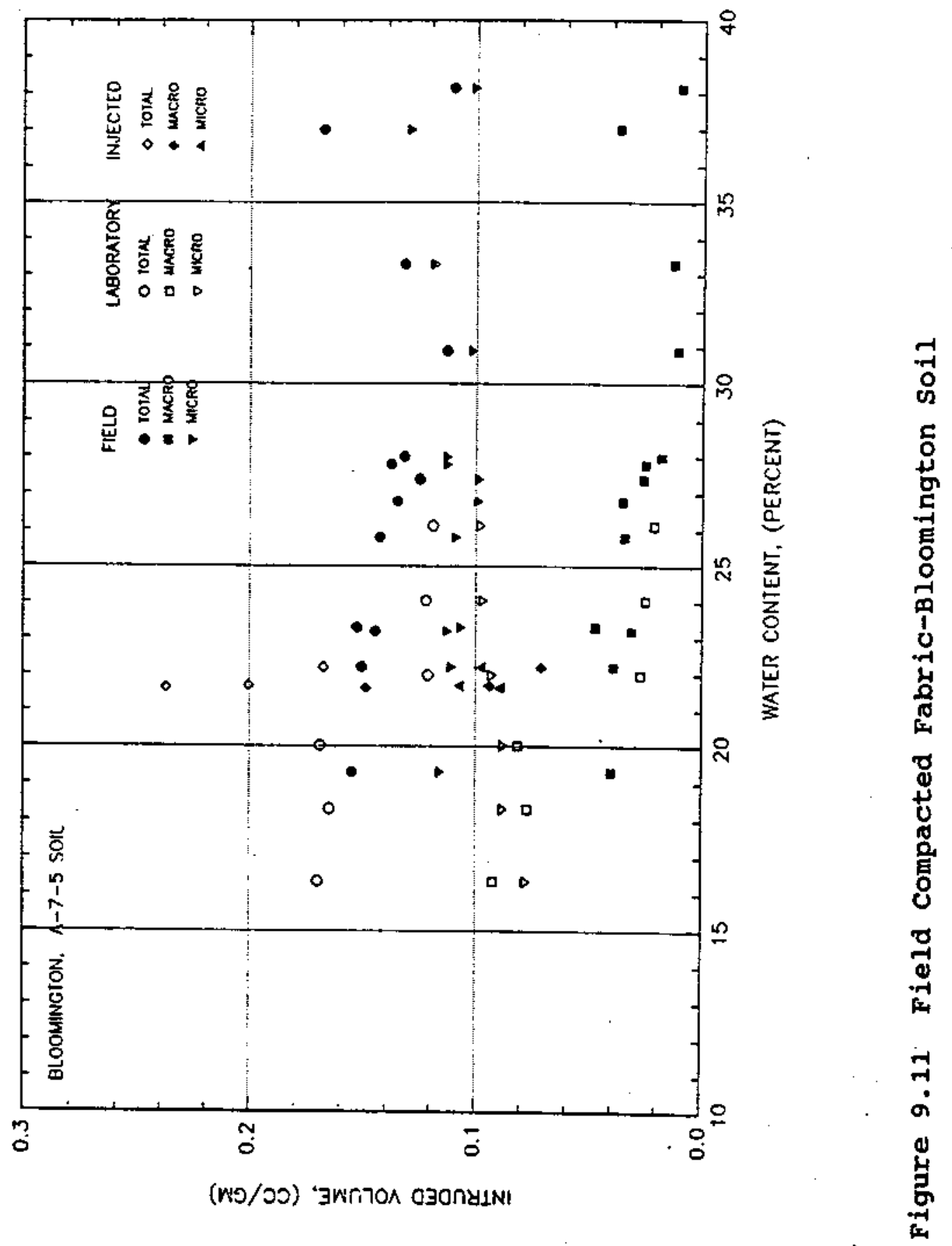




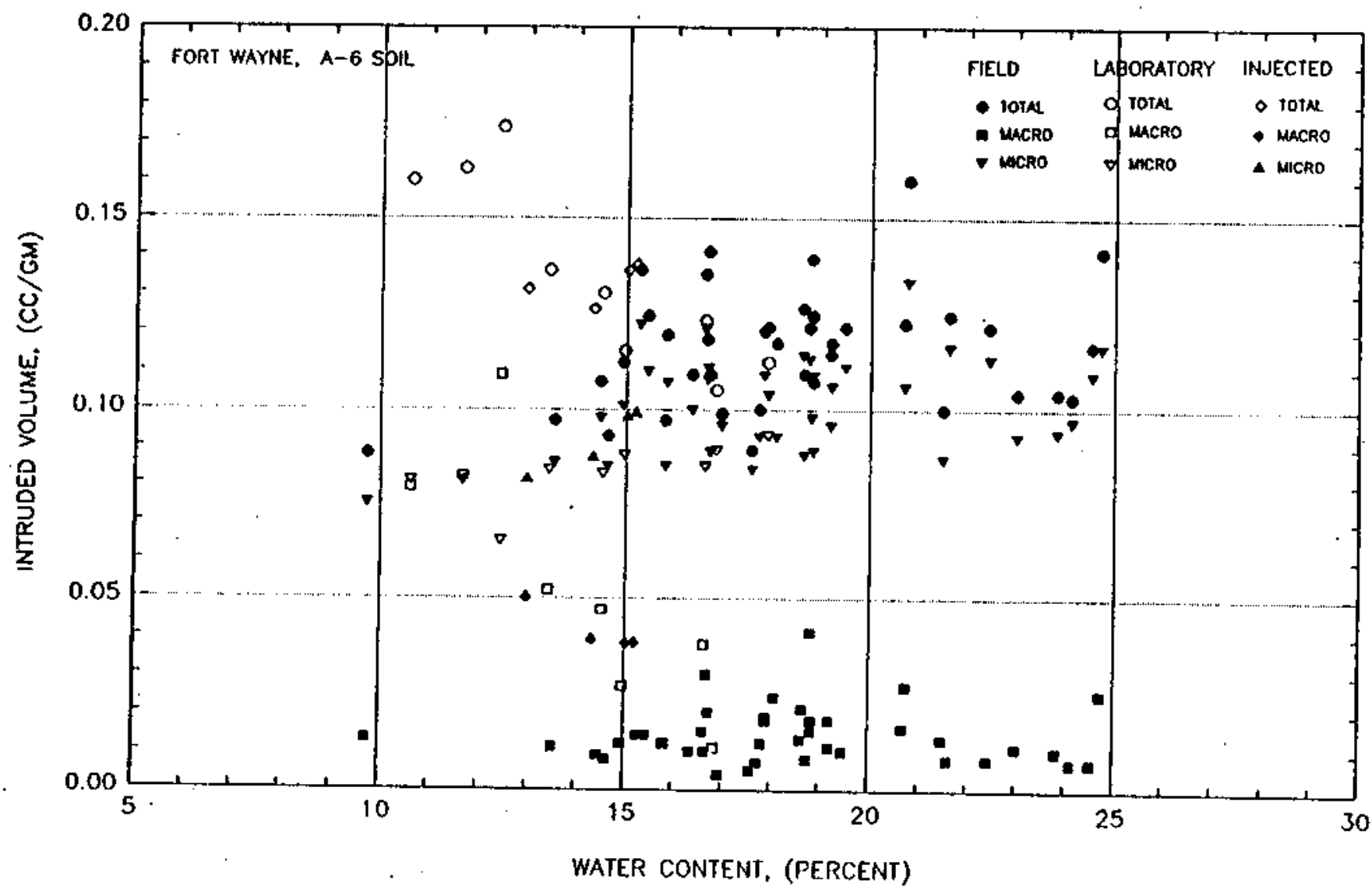

Figure 9.12 Field Compacted Fabric-Fort wayne soil 


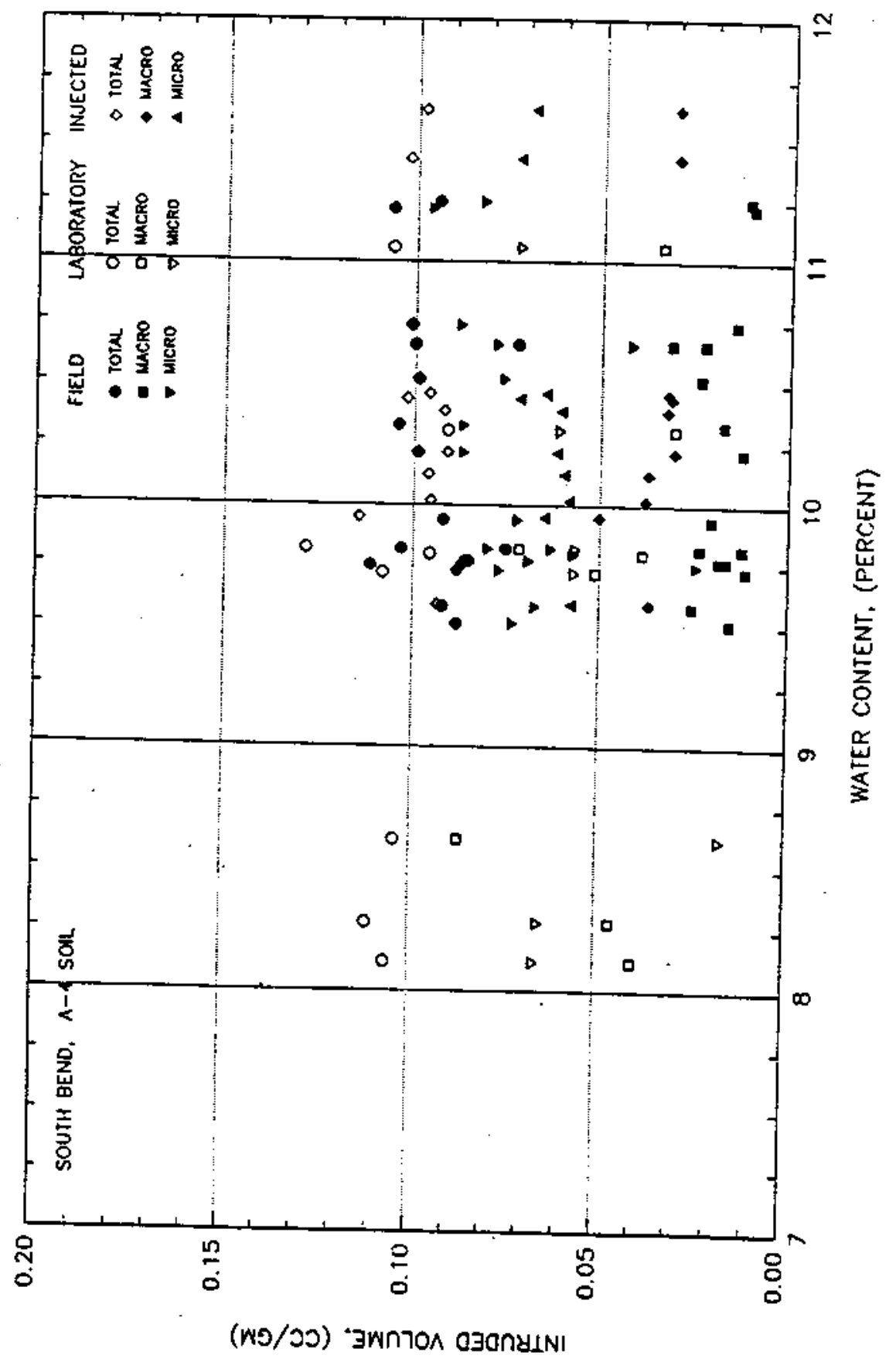

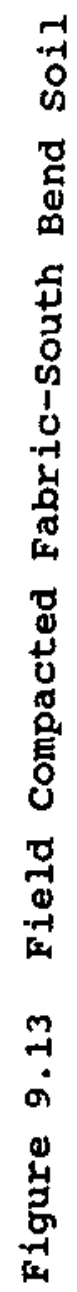




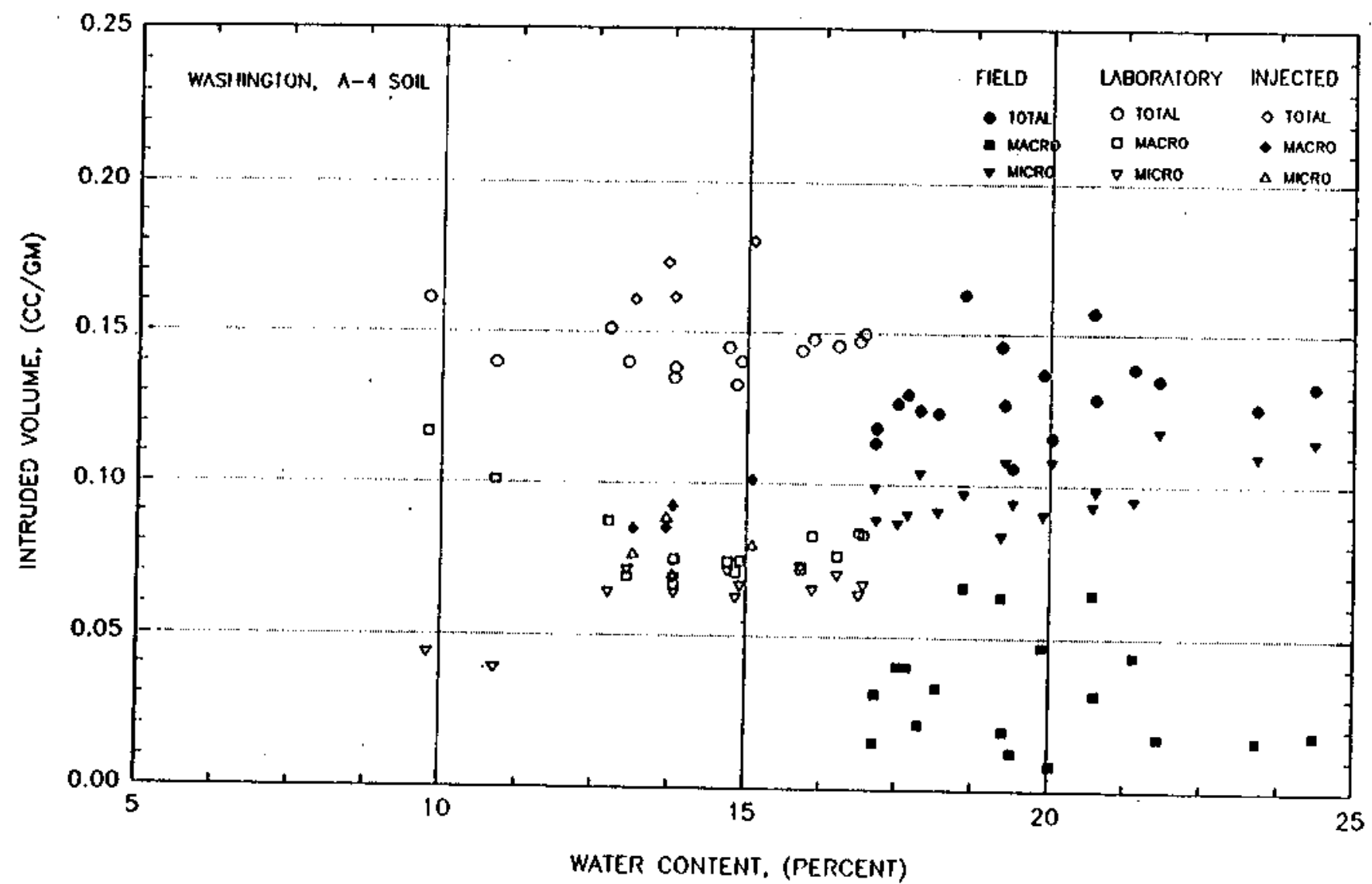

Figure 9.14 Field Compacted Fabric-Washington Soil 
after the placement of the subgrade. Total intruded volume and microporosity of the field compacted samples are higher than those of the laboratory compacted samples as shown in Figure 9.10. Also, there is a larger difference between the micro and macroporosity of the field compacted samples. The macroporosity of the field compacted sample is relatively uniform. Unlike laboratory compacted samples, microporosity of the field compacted samples varies significantly from one sample to another.

\section{Bloomington, A-7-5 soil}

Pore-size data for the Bloomington site are shown in Figure 9.11. Most of the field compacted samples show higher water content but there is an overlap between the laboratory and the field water content. Water contents of a few of the field samples have not been changed significantly from its ascompacted value. For all the results, there is a general trend of decrease in the total intruded volume and macroporosity with increase in the water content. Microporosity' shows slight increase with increase in the water content. Microporosity of the field compacted samples is higher than that of the laboratory samples.

\section{Fort Wayne, A-6 so11}

Fabric data for Fort Wayne soil are given in Figure 9.12. Water content and dry density values for the field samples are higher than the as-compacted and the laboratory compacted values. Fort Wayne field compacted samples displayed uniform fabric which is less sensitive to water content changes. Fort Wayne field samples contain a smaller macroporosity and almost all the macroporosity values fall between 0 and $0.015 \mathrm{cc} / \mathrm{gm}$. Majority of the total porosity for the field compacted samples resides in the micropores. This trend was not observed in the laboratory compacted samples as can be seen in Figure 9.12. 
South Bend, $A-4 / A-6$ soil

South Bend soil has been compacted comparatively recently (August 1989) in the field and the water content does not show much deviation from the as-compacted value. Pore-size data for the South Bend site show the lowest total intruded volume among all the soil types investigated. Lower value of the total intrusions provides the highest ary unit weight (129.5 pcf, by Standard Proctor Energy) among all the soil classes investigated. Fabric data for the laboratory compacted, and the injected samples are also plotted in Figure 9.13 . It is shown that the increase in water content after compaction does not affect the fabric. Unlike other soil classes, field compacted sample for this site shows lower macroporosity and higher microporosity. This is due to the low plasticity and the clay fraction present in the soil and the collapse and the progressive shifting of the macroporosity during compaction.

Washington, A-4 Soil

Pore-size data for Washington soil, shown in Figure 9.14, show much higher water content values for the field compacted samples. Study of the inspection data reveals that there was a significant increase in water content after compaction. similar to South Bend soll, Washington soil represents lower microporosity and higher macroporosity due to lower plastic limits and clay fraction present in the soil. Macroporosity in the field compacted samples shows large variability and the majority of the porosity resides in the micropore zone.

\subsection{Fabric and Resilient Kodulus}

Determination of resilient modulus of the fine-grained soils in the laboratory involves application of repeated deviator stresses. Externally applied forces are resisted by 
the rigidity and the structure of the soil skeleton. Internal forces are transferred at the grain contact boundaries. Fabric of the soil largely determines the configuration of the soil skeleton and the contact boundaries. Creation of fabric by compaction is largely a function of molding water content, clay fraction, type of soil, and plasticity.

Presence of water at the grain contact boundaries plays an important role in the way soil respond to an externally applied loading. On the dry side, water mostly resides in the micropore zone and is held in capillary tension in the finer pores at the contact boundaries. Tension in the capillary water offers an additional resistance to the applied force. As the water content is increased, water in the soil pore structure moves to the macropore zone and starts filling larger pores. As the molding water content approaches saturation, most of the pore space is filled with water and water menisci in the finer pores loose capillary tension. Knowledge about the distribution of the macro and microporosity helps understand the behavior of soil under applied loading. Distribution of porosity alone can not explain the soil behavior. Two soils might have similar distribution of porosity but a very different distribution of pore water resulting in a very different response to the applied loading.

Resilient response of a compacted soil can be predicted by pore-size distribution and the water present in the pore structure. Pore-size data alone can not predict the response of soil due to the significant interaction of the pore structure with the molding water content. Regression techniques can be used as a tool to predict resilient modulus based on the molding water content and the pore-size distribution.

Resilient modulus and pore-size results on the laboratory and field compacted samples for different soil types were used for regression analysis using statistical Analysis system 
(SAS) on IBM 3090. The macroporosity, microporosity and the total intruded volume were used to represent the pore structure of the soils. Decisions about the inclusion of the independent parameters in the regression equations was made by using stepwise regression. Stepwise regression gives the coefficients of determination $\left(R^{2}\right)$ for the different combinations of the independent parameters. Different combinations of the variables with higher $R^{2}$ values were used in a further attempt to improve the coefficient of determination. The following regression equations were developed for two soil classes:

For Bedford $(A-7-6)$ soil $\left(R^{2}=0.833\right)$ :

$$
\begin{aligned}
M_{R} & =121.465-3.743(w c)-474.939(m i c) \\
& -35.525 \text { (totint) }+0.525(m i c)(w c)^{2}
\end{aligned}
$$

For Bloomington $(A-7-5)$ soll $\left(R^{2}=0.798\right)$ :

$$
\begin{gathered}
M_{R}=98.118-2.391(w C)-535.388(m i c) \\
+30.816 \text { (totint) }-0.336 \text { (mic) (wC) }
\end{gathered}
$$

where $M_{k}$ is a predicted resilient modulus (ksi); wC is molding water content (percent); mic is microporosity (cc/gm); totint is total intrusion (cc/gm).

Regression equations for the other soil types (A-6, A4/A-6, and A-4) could not give a coefficient of determination higher than about 0.60 . Interaction between the molding water content and the microfabric of soils with high clay fraction can be observed in the above regression equations. Reliable prediction of resilient modulus in terms of the molding water content and pore-size can only be done for soils with higher
clay fraction.

In order to study the effect of the clay fraction on the resilient modulus, modulus values for different soil classes 


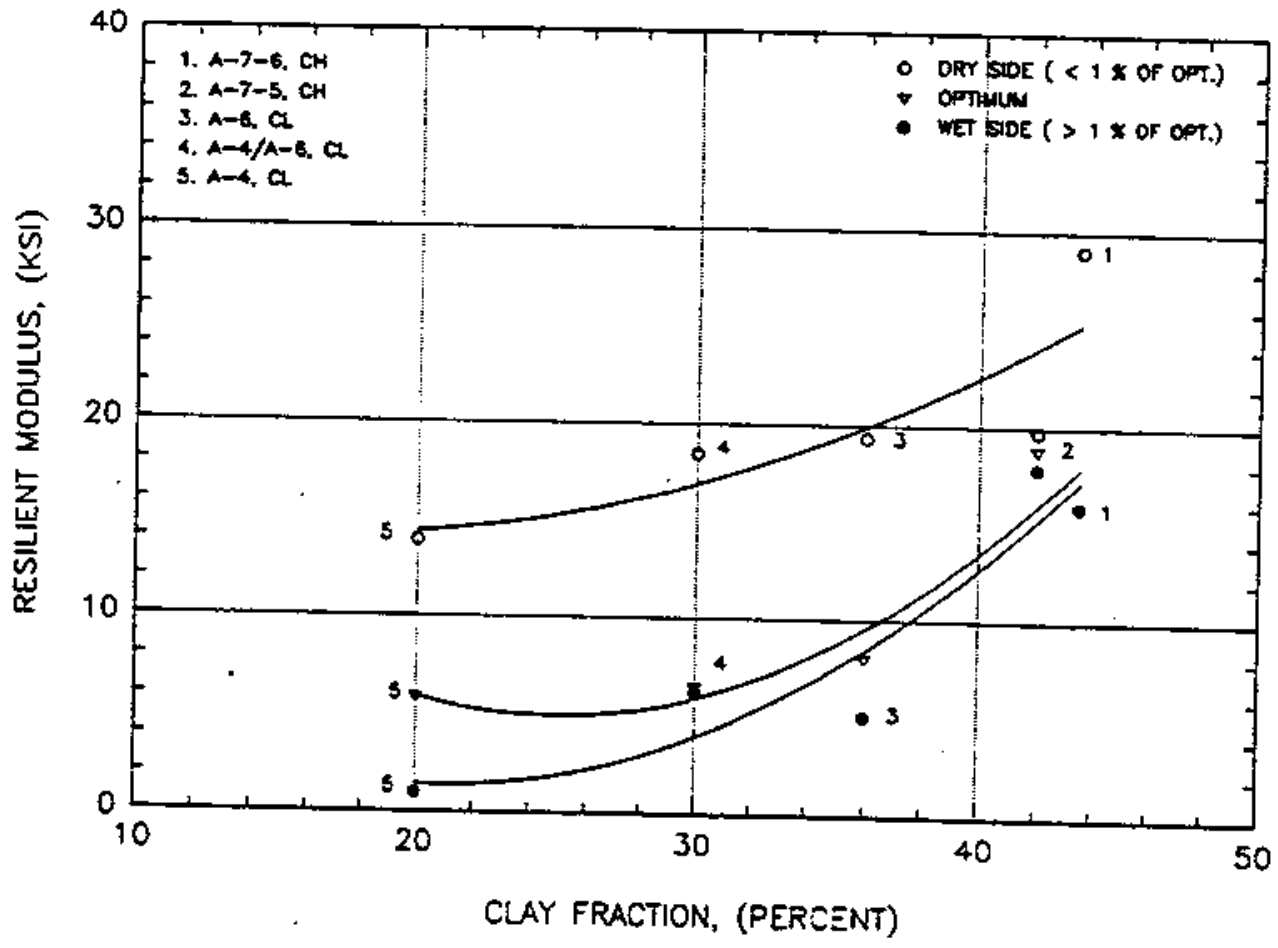
Figure 9.15 Correlation between clay Fraction and Resilient
Modulus 
at optimum water content, about one percent dry of optimum, and about one percent wet of optimum were determined. variation of resilient modulus with clay content is given in Figure 9.15. The dry side resilient modulus values are much higher than the optimum and wet side values. For the soils with higher clay fractions, difference between the optimum and the wet side resilient modulus is not significant. Effect of the clay fraction on the resilient modulus values increases as the molding water content of the soils are increased. For dry side compaction the effect of the clay fraction on the resilient modulus is relatively small due to existence of less water in the micropores. Figure 9.15 also suggests that changes in the clay fraction in the solls with higher fraction of clay has greater influence on the modulus values, particularly at higher water contents.

\subsection{Replication of Field compacted Fabric}

Based on the pilot study (Section 8) on the medium plastic clay (A-6), a match between the laboratory and field compacted fabric for the soils included in the present study can be determined. The pilot study included different molding water contents, compaction energies, and compaction methods for both the laboratory and the field compaction. For the present study, each site has a target value of water content and is compacted with specific equipment with a certain number of passes to achieve the specified value of dry density. Results of the pilot study can be used as a guide to arrive at a laboratory compaction method that will replicate the field compacted fabric.

Replication analyses on the medium plastic clay (A-6) suggested that dry side compaction with energies less than the standard proctor is less likely to simulate the field compacted fabric. Extending these findings, fabric studies for laboratory compaction were mainly performed at the standard 
Proctor energy to cover the entire range of molding water contents. Further, laboratory compaction using Modified Proctor energies at about optimum water contents was also performed.

As shown in Figures 9.16 through 9.20, field compacted fabrics show a higher variability than the laboratory compacted fabric shown in Figures 9.4 through 9.8. High variability in the field compacted fabrics is mainly due to the non-uniformity in the as-compacted water contents and compaction energies.

Field compacted fabric for the Bedford site shows high variability in the total intruded volume, and the microporosity is relatively uniform compared to the large variation in the macroporosity as shown in Figure 9.16. Laboratory compacted samples at about one percent wet of optimum using Standard Proctor energy give a closer match to the field fabric data shown in Figure 9.16. Application of the Modified Proctor energy improves the comparison in the macropore zone but the total intruded volume disagrees considerably with the field compacted fabric. Hence, it is concluded that the laboratory impact compaction at standard Proctor energy and at about 1 percent wet of optimum water content gives a comparable match with the field compacted fabric for the Bedford site.

similar to the Bedford site, the Bloomington site shows uniformity in the macropore zone and large variability in the micropore zorie for the pore-size data on the field compacted samples shown in Figure 9.17. Unlike the Bedford site, there is a sudden decrease in the total intruded volume beyond a certain value of water content. The dry and the wet side compaction create distinctly different pore-size curves as shown in Figures 9.5 and 9.17 . Laboratory compaction at about 1.5-2 percent wet of optimum using standard Proctor energy gives a comparable match for the Bloomington field fabric shown in Figure 9.17. As shown in Figure 9.17, application of 


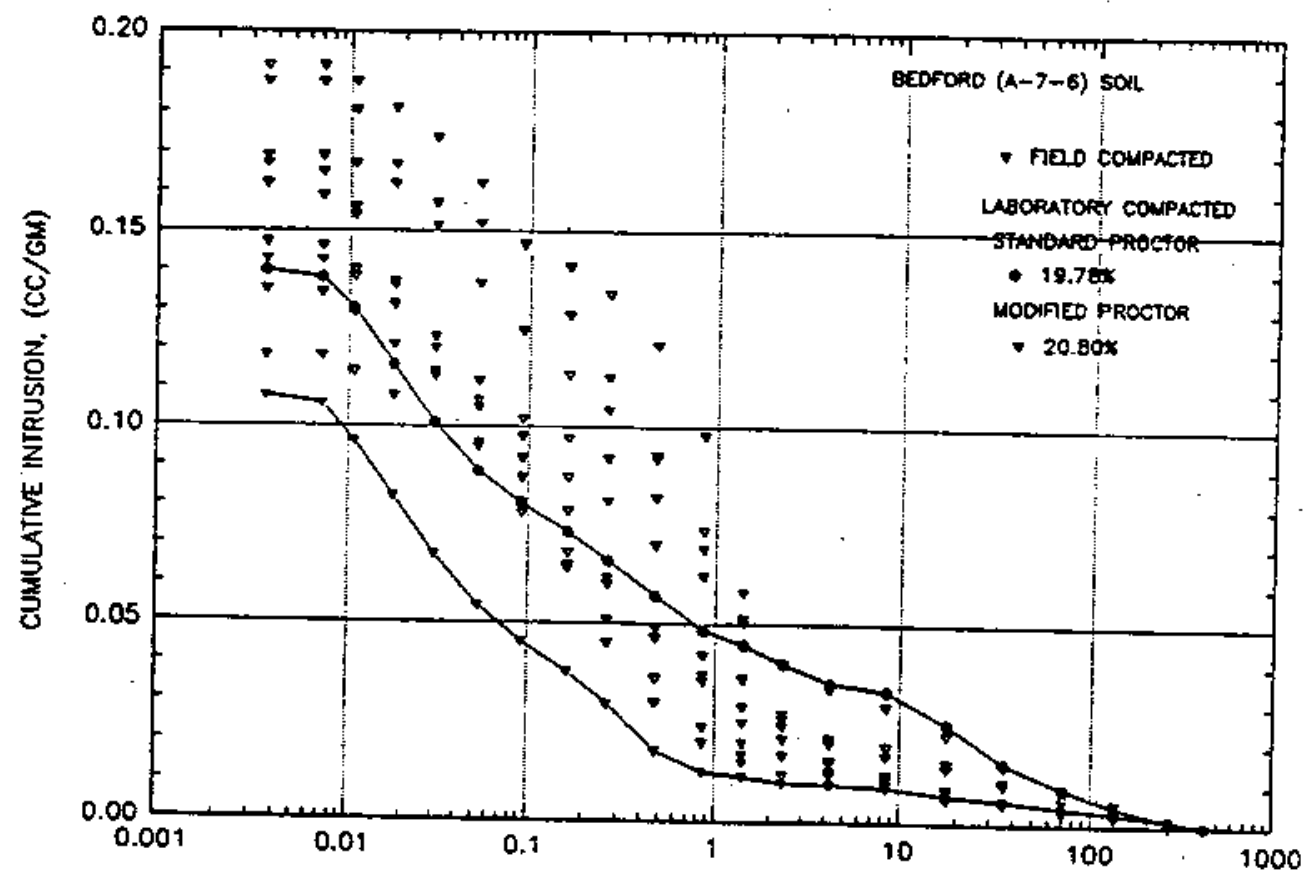

LIMITING PORE OLAMETER, (MICRON)

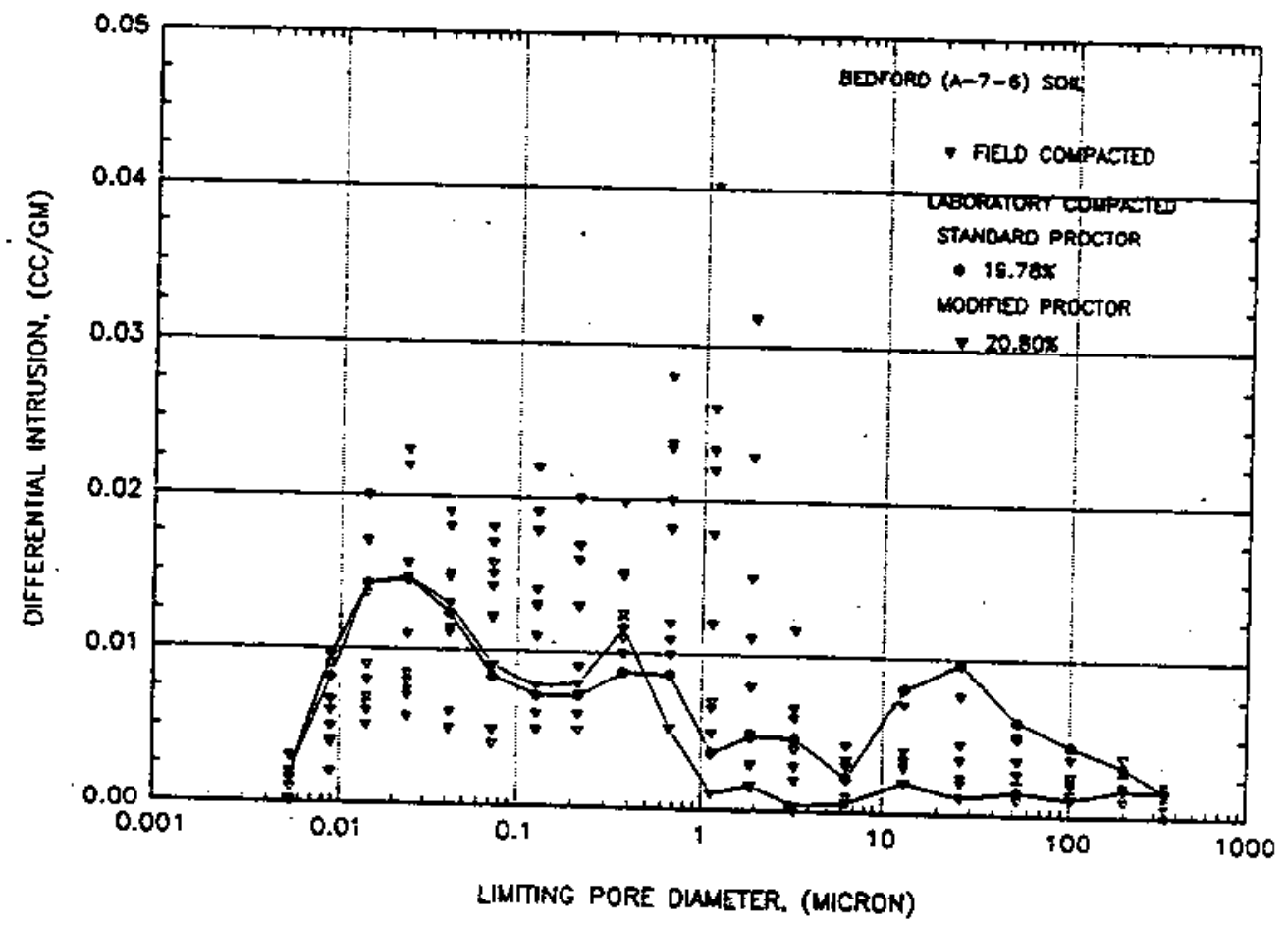

Figure 9.16 Replication of Bedford Field Compacted Fabric 

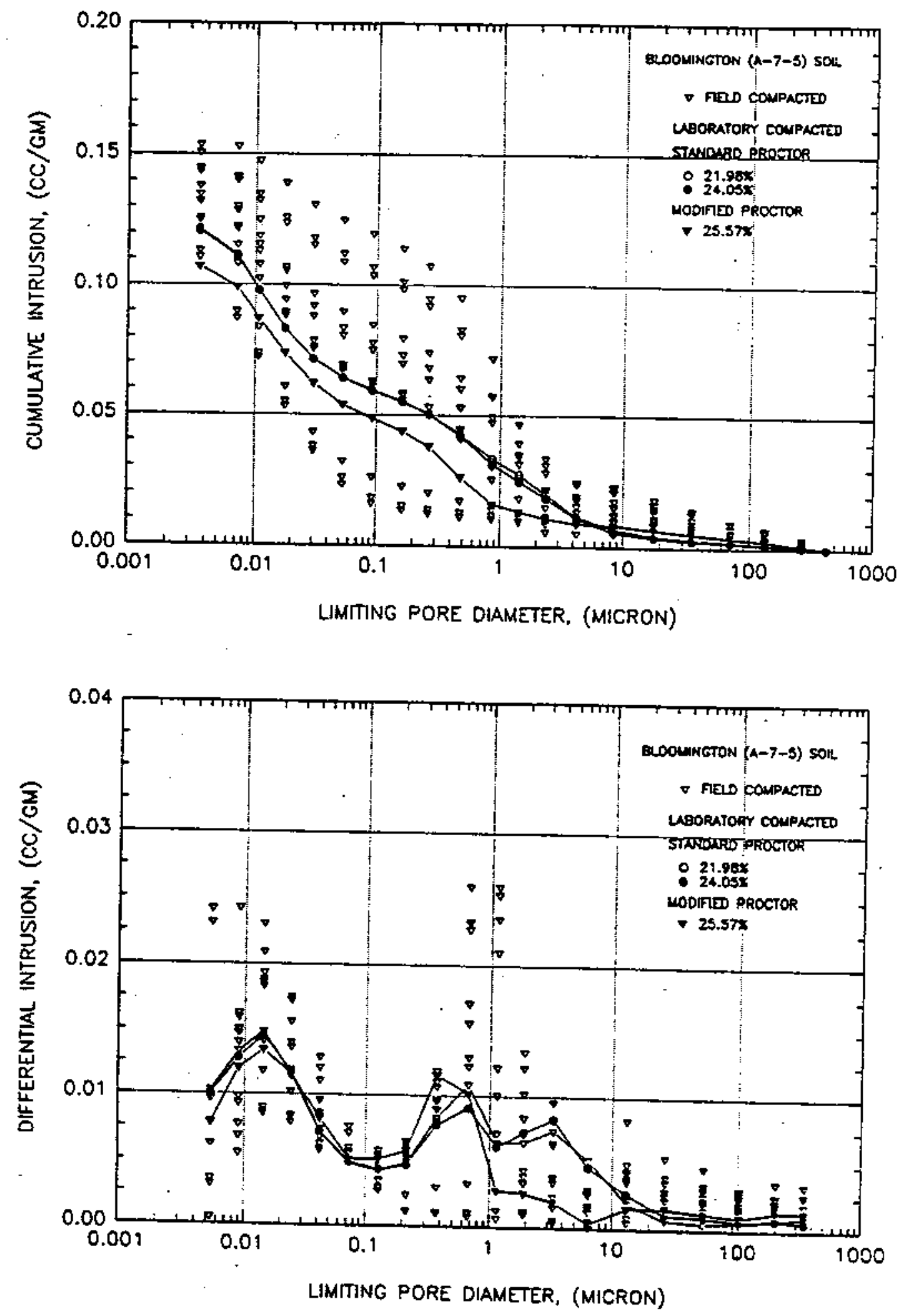

Figure 9.17 $\begin{aligned} & \text { Replication of Bloomington Field compacted } \\ & \text { Fabric }\end{aligned}$ 
the Modified Proctor energy does not improve the match between the field and laboratory compacted fabric.

Pore-size curves for the field compacted fabric for the Fort Wayne site are shown in Figure 9.18. Vaxiability in the pore-size data for the Fort Wayne site increases towards the finer pore diameters as shown in Figure 9.18. However, compared to the Bedford and Bloomington sites, the Fort Wayne site shows smaller variability in the pore-size data. Comparable laboratory match representing compaction at about optimum water content and standard Proctor energy is also shown in Figure 9.18. Increasing compaction energy to Modified proctor results in a slight decrease in the total intruded volume. Laboratory compacted fabric, using standard Proctor energy, at about optimum water content is considered as a match for the field compacted fabric for the Fort Wayne site.

The South Bend site shows the minimum total intruded volume among all the five sites considered in the present study. The smaller total intruded volume for this site results in the highest maximum dry density at the optimum water content. Clay fraction (30\%) and Plasticity Index (7) for the South Bend soil are comparatively low. Comparable laboratory compacted matches shown in Figure 9.19 show a higher macroporosity with respect to the field compacted samples. An increase in the compactive effort does not reduce the macroporosity to the level of the field compacted fabric. Examination of the laboratory compacted pore-size data in Figure 9.7 shows that the dry and the wet side compacted fabric fall in a comparatively narrow band suggesting that molding water content does not change macroporosity effectively. Based on these observations, it is concluded that the macroporosity of the laboratory compacted south Bend sample is not reduced to the field compacted level. Laboratory compaction using standard Proctor energy at about one percent wet of optimum gives a closer match, compared to the other laboratory compacted samples, with the field compacted fabric. 

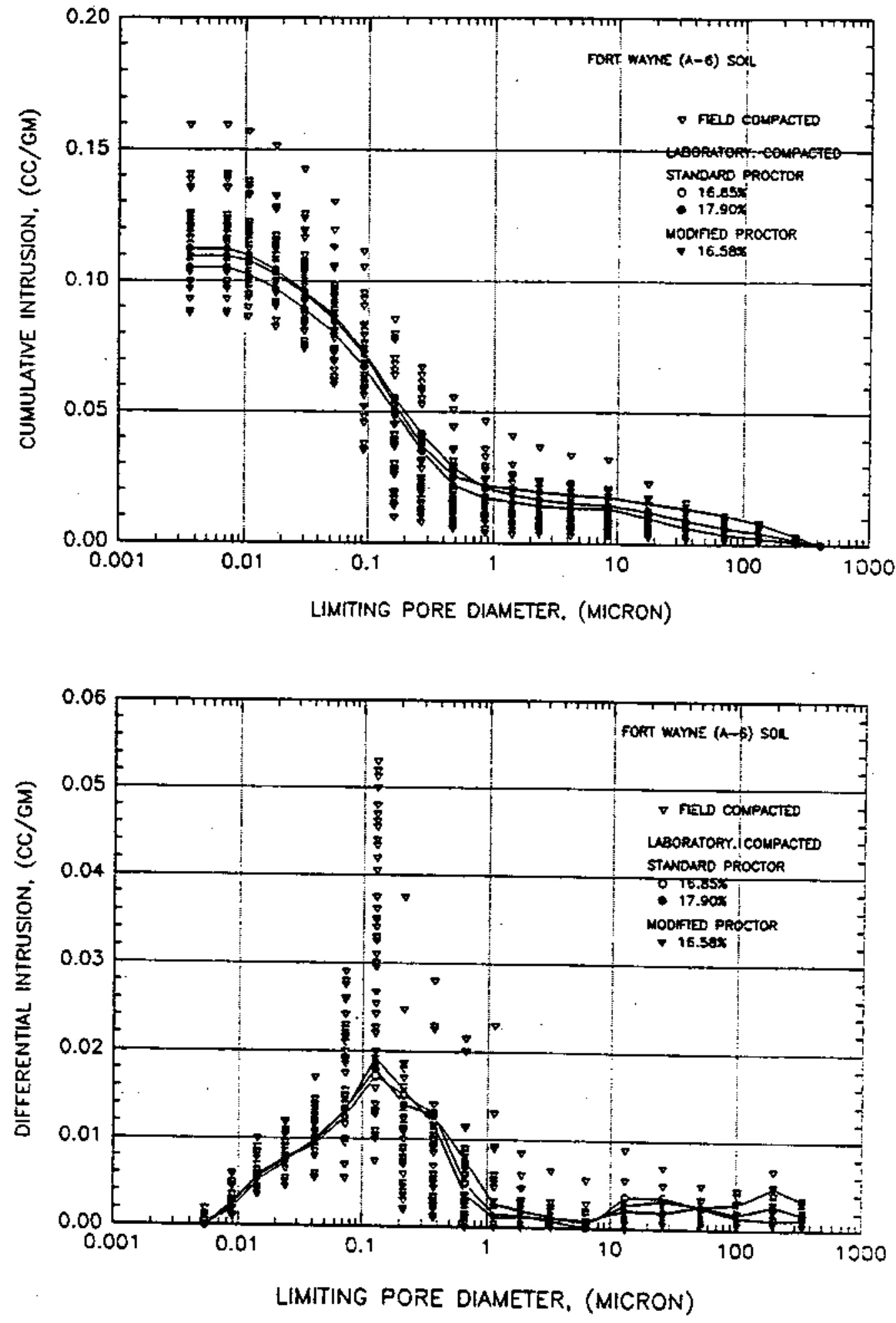

Figure 9.18 Replication of Fort Wayne Field Compacted Fabric 

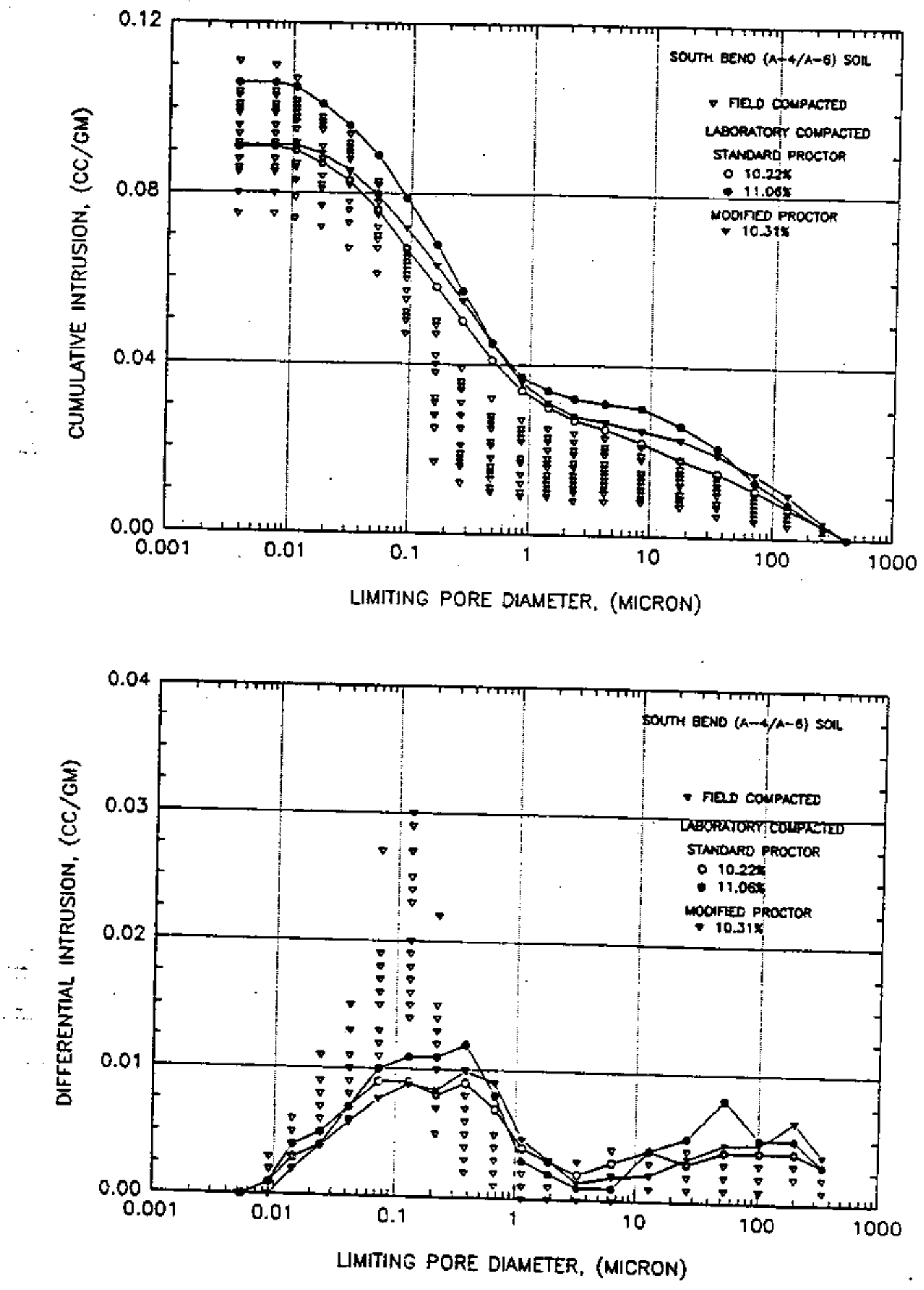

Figure 9.19 Replication of South Bend Field Compacted Fabric 
Similar to the South Bend site, washington site shows a higher macroporosity for the laboratory compacted samples. In addition, the total intruded volume for the laboratory compacted best match is higher than almost all of the field compacted values shown in Figure 9.20. Similar to the South Bend site, laboratory compacted samples for the Washington site are also insensitive to molding water content and compaction energy. This is partly due to relatively lower values of clay fraction (20\%) and Plasticity Index (9) at the Washington site (Table 9.1). Modified Proctor energy gives a slightly better match in the macropore zone but the difference between the fabrics created with the Standard and the Modified Proctor vanishes in the micropore zone. Data on the South Bend and Washington sites suggest that a comparative fabric match for soils with a decreasing percent of clay content and Plasticity Index shows larger differences between the laboratory and the field compacted matches. Considering the differences shown in Figure 9.20, the washington field compacted samples cannot be considered as a satisfactory match for the field compacted fabric.

In summary, for the majority of the sites, replication of the field compacted fabric can be achieved by preparing laboratory samples using standard Proctor energy and water contents ranging from optimum to 1-2 percent wet of optimum. However, the degree of agreement between the matched fabrics decreases as the clay fraction present in a soil decreases. In other words, matching of the fabric of soils with a smaller fraction of clay is relatively less reliable using the suggested laboratory replication method.

\subsection{Concluding Remarks}

Findings based on the experimental studies on the fabric and resilient modulus of the laboratory and field compacted samples are summarized as follows: 

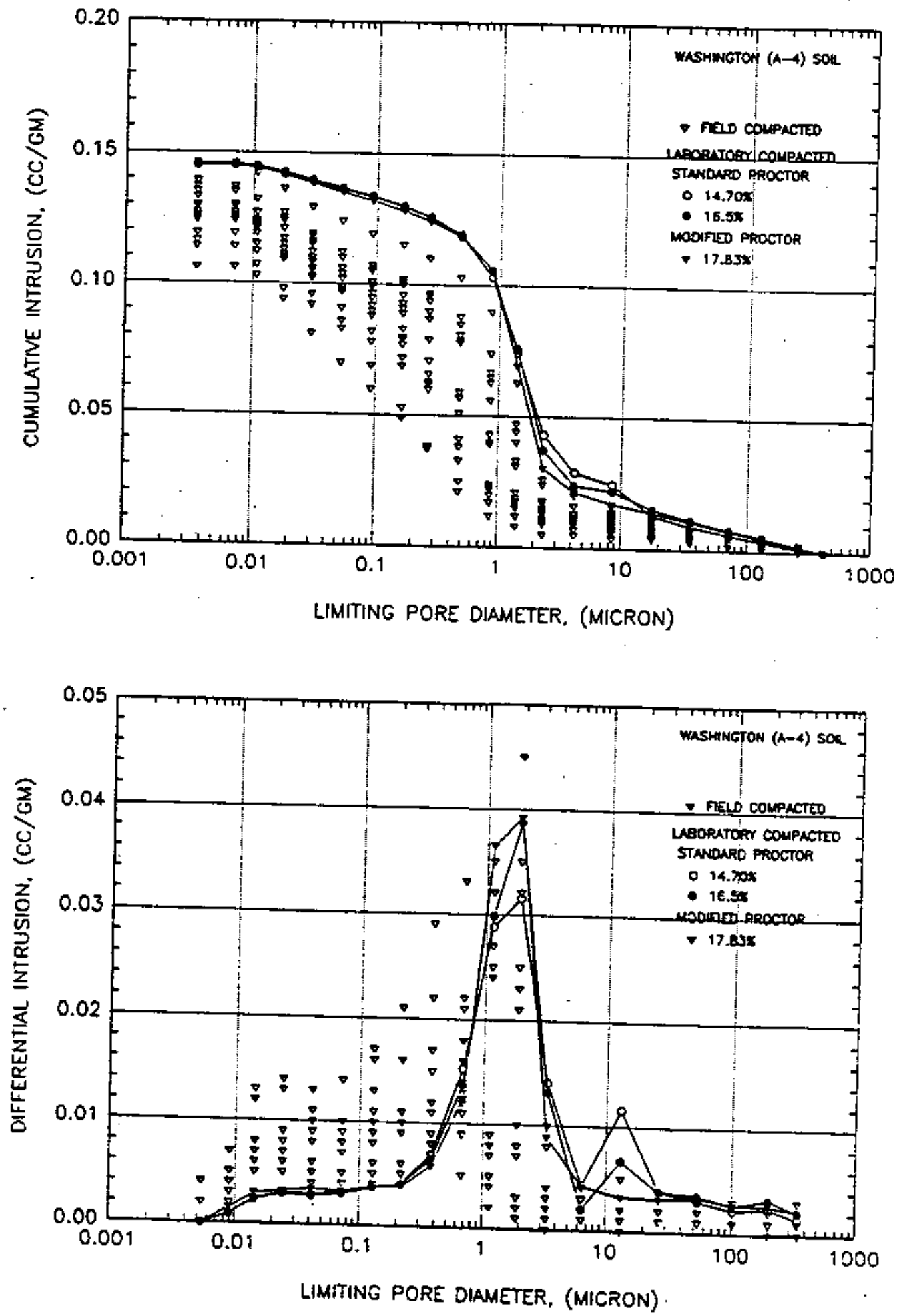

Figure 9.20 Replication of Washington Field Compacted Fabric 
(a) Fabric of an uncompacted soil can be used to characterizes inherent soil properties such as clay fraction, plasticity, and the maximum attainable dry unit
weight at certain energy level;

(b) From the fabric point of view, compaction can be defined as a process which mainly reduces the frequency of the macropores and progressively shifts the macropores
towards the finer pores;

(c) Microporosity of the high plastic soils (A-7-6, A-7-5) practically remains unchanged with the molding water content;

(d) Fabric and pore water (molding $t$ post compacted changes) can be used to predict the resilient behavior of

(e) Increase in water content after construction does not affect the as-compacted fabric but reduces the resilient modulus. It is important to minimize any increase in water content of a subgrade to prevent decrease in the resilient modulus value. Variation of resilient modulus with the increase in water content after construction is covered in

(f) Use of the fabric parameters for the prediction of resilient modulus can only be done for solls with high plasticity. The reliability of the predicted resilient modulus decreases for soils with smaller clay fraction;

(g) Fabric parameters alone cannot predict resilient response of a soil to the applied loading. Location and amount of pore water are important factors needed to be
incorporated for this prediction;

(h) Fabric and pore water can not reliably predict behavior of soil with smaller clay fraction or soils with. low
plasticity;

(1) For the majority of the solls-particularly for soils with high clay fraction-the fleld compacted fabric can be replicated by preparing laboratory samples using standard Proctor energy and water contents at about optimum and 1-2
percent wet of optimum. 
10. DEVELOPMENT OF SIMPLIFIED DESIGN PROCEDURE

\subsection{Introduction}

Evaluation of resilient response on each component of pavement system is required for analysis and design of the pavement structure. Resilient response of pavement materials - generally can be determined by repeated-load triaxial test in the laboratory. For this test, expensive equipment and data acquisition system are required and the testing procedure is time-consuming. It is, therefore, valuable to develop empirical correlations between $K_{R}$ and parameters which can be obtained with ease from simple routine test.

Previous prediction equations for $M_{k}$ on cohesive soils were mainly based on index properties, molsture content, dry density, degree of saturation, matrix suction, clay content, $C B R$ value, and $R$ value. Most of these equations were developed from test results on specimens compacted in the laboratory and they may only be used for estimating as-compacted resilient modulus. For many of these equations, the stress level corresponding to estimated resilient modulus is not clear. Few attempts have been made to include the effects of confining stress and changes of condition after construction.

Previous studies on resilient response of granular soils indicated the state of stress as the most important factor affecting resilient modulus. Since the effects of density, degree of saturation, and gradation on resilient response of granular soil appears not significant, compared with state of stresses, few attempts to include these factors into prediction equations have been made by previous researchers. 
It is not possible to offer single magnitudes for resilient modulus, soil by soil, since there are too many interacting variables. This study offers a procedure with which a resilient modulus may be determined for a given soil created in a given manner and subjected to specific environmental conditions. The procedure is based on unconfined compression test data. Influence of change in water content after construction and freeze-thaw on the resilient modulus can also be estimated from the proposed procedure. Also developed is the procedure to estimate the resilient modulus for different levels of confining stress and repeated deviator stress. Prediction equation and design charts for resilient modulus for dune sand are developed, based on state of stresses and dry density. Seasonal moduli estimated from proposed procedures are then incorporated into 1986 AASHTO Guide to establish an effective roadbed soil resilient modulus.

10.2 Procedure for Cohesive Soil

\subsubsection{Newly Constructed Pavement}

In the design stage for new pavement, the data available for the subgrade are probably the specified range of compaction conditions and the plan for earthwork. Therefore, the procedure for estimation of the resilient modulus must be based on the specified compaction conditions or on the test results from laboratory compacted soil. since the moisture condition of as-compacted subgrade is expected to change and approach an equilibrium watex content after some time, it is necessary to estimate the equilibrium water content and the corresponding resilient modulus.

For preliminary evaluation, as-compacted resilient modulus can be determined from the contour of resilient modulus by specifying the desired range of water content and 
dry density. As shown in Figures 4.24 and 4.25 , resilient modulus contours for laboratory compacted south Bend and Washington soils have been developed in present study as an example of what is possible. Estimation of resilient modulus from such curves would be very convenient if they were available for the given soil. However, contours of resilient modulus are dependent on soil type and manner of compaction; and development of these contours requires resilient modulus tests on at least 16 to 20 specimens compacted at different water contents and with several levels of compactive efforts. since there is the possibility that the line of optimums for laboratory and field compaction are different, a direct use of the resilient modulus contours developed by laboratory compaction can cause significant error in the estimated ascompacted resilient modulus.

Because of difficulties in developing the contour of field resilient modulus, another procedure to estimate ascompacted resillent modulus has been developed; a relationship has been found between resilient modulus and the stress causing 1 percent axial strain in an unconfined compression test. Once the relationship between $\mathrm{k}_{\mathrm{z}}$ and $\mathrm{s}_{\mathrm{u1.0}}$ is developed, the resilient modulus is estimated from the unconfined compression test result from the specimen; either laboratory compaction may be used to create the specimen having similar field compacted fabric or the specimen may be sampled from a test embankment.

The analysis on pore-size distribution data shows that the field soil fabric is replicated by laboratory impact compaction at about 1 percent wet of optimum for Bedford, at about 1.5 to 2 percent wet of optimum for Bloomington, and at about optimum for Fort Wayne soil-using standard Proctor energy. Field fabric of South Bend soll can be replicated using laboratory impact compaction with standard proctor energy and water content between optimum and 1 percent wet of optimum. Laboratory impact compaction with standard proctor 
energy and at water content close to optimum may be considered as a match for Washington soil. However, there are comparatively larger differences for the replicated fabric for the Washington soil. Based on this results, a criterion on laboratory compaction for replicating field soil fabric is suggested in Table 10.1.

Table 10.1 Laboratory Compaction Criteria for Replication of Field Compacted Fabric

\begin{tabular}{c|c}
\hline Site & Laboratory Compaction Method \\
\hline South Bend & $\begin{array}{c}\text { Impact compaction at oMc to 18 wet of } \\
\text { ome with Standard Proctor energy }\end{array}$ \\
\hline Fort Wayne & $\begin{array}{c}\text { Impact compaction at about oMc with } \\
\text { Standard Proctor energy }\end{array}$ \\
\hline Washington & $\begin{array}{c}\text { Impact compaction at about oMc with } \\
\text { Standard Proctor energy }\end{array}$ \\
\hline Bedford & $\begin{array}{c}\text { Impact compaction at about 18 wet of } \\
\text { OMC with Standard Proctor energy }\end{array}$ \\
\hline Bloomington & $\begin{array}{c}\text { Impact compaction at about 1.5-28 wet } \\
\text { of OMC with Standard Proctor energy }\end{array}$ \\
\hline Larger differencea exist between the replicated fabric
\end{tabular}

In this study, the relationship between $M_{R}$ and $S_{u t .0 x}$ applicable to all five sites has been developed and it may be used for estimation of as-compacted resilient modulus:

$$
M_{R}=-1599.66+833.83 S_{u 1.04}-6.9683 S_{u 1.08^{2}}
$$

where $M_{R}$ is the resilient modulus (psi) at $\sigma_{3}=3$ psi and $\sigma_{d}=6$ psi; and $s_{\text {ul.ox }}$ is the stress (psi) causing 1 percent strain in 
the unconfined compression test on the specimen compacted in the laboratory. with the specified water content and compactive effort in Table 10.1. Since the relationship between $M_{R}$ and $S_{u 1.0 x}$ is only slightly different between laboratory compacted soil and field compacted soil, the use of Equation 4.8 causes a negligible error in predicted as-compacted resilient modulus for the field condition.

The following steps are recommended to estimate the resilient modulus for new construction of pavement:

1. Determine the range of water content and dry density to be achieved in the field, based on the requirements in the specification;

2. Compact disturbed soil in 2.8-inch diameter and at least 5.6-inch high mold, following the criteria given in Table 10.1. If test embankment is constructed, sample the specimen by pushing a shelby tube;

3. Perform the unconfined compression test with a strain rate of 1 percent per minute. Calculate the stress in psi at 1 percent axial strain $\left(S_{u 1.0 x}\right)$;

4. Determine as-compacted $M_{R}$ using Equation 4.8;

5. Estimate the resilient modulus at equilibrium water content by the procedure described in section 10.3. This estimated resilient modulus is the in-service $M_{R}$ for normal condition of subgrade $\left(\mathrm{M}_{\mathrm{RN}}\right)$;

6. Estimate the resilient modulus after thawing $\left(M_{k T}\right)$ by the procedure described in Section 10.4;

7. Estimate the resilient modulus for frozen subgrade $\left(M_{R p}\right)$ by the procedure described in section 10.5 ;

8. The in-service resilient modulus, $M_{R N}$, is then obtained from step 5 , or as $M_{R T}$ obtained in step 6 , or as $M_{R P}$ obtained in step 7 for conditions present at the time in the life of the subgrade being considered;

9. Estimate the $M_{R}$ values for each month following the procedure described in Section 10.6;

10. Seasonal moduli estimated from the above steps are, then, incorporated into 1986 AASHTO Guide to establish an effective resilient modulus. 


\subsubsection{Reconstructed Pavement}

For the reconstruction of existing pavement, undisturbed samples can be obtained from the in-service subgrade; therefore, the procedure for estimation of the resilient modulus developed is based on the unconfined compression test result from undisturbed sample. Since the in-service subgrade is expected to have been subjected to change in its moisture condition, the $M_{R}$ determined from undisturbed in-service sample may be considered as an equilibrium resilient modulus.

The relationship between $M_{R}$ and $S_{u 1.0 \pi}$ for field compacted soils from five different sites was expressed by a single unified relationship which seems applicable to any soil type. The following equation can be used for estimation of resilient modulus if an unconfined compression test is performed on the undisturbed specimen obtained from subgrade:

$$
M_{R}=-1599.66+833.83 S_{u 1.08}-6.9683 S_{u 1.0 t^{2}}
$$

where $M_{R}$ is the resilient modulus at $\sigma_{3}=3$ psi and $\sigma_{d}=6$ psi (psi); and $s_{u 1.0 x}$ is the stress causing 1 percent strain in the unconfined compression test on undisturbed specimen obtained from the subgrade.

The following steps are recommended for estimation of resilient modulus for the pavement to be reconstructed: 1. Sample existing subgrade by pushing a shelby tube, and bring the sealed tube to the laboratory;

2. Cut the shelby tube and trim a specimen of about 5.6 to 6 inch height;

3. Perform the unconfined compression test with a strain rate of 1 percent per minute. Calculate the stress in psi causing 1 percent axial strain $\left(S_{\mathrm{w1.0x}}\right)$;

4. Determine the resilient modulus $\left(M_{\mathrm{RN}}\right)$ using Equation 4.8 ; 5. Estimate the resilient modulus after thawing $\left(M_{\mathrm{RT}}\right)$ by the procedure described in section $10.4 ;$ 6. Estimate the resilient modulus for frozen subgrade $\left(M_{R P}\right)$
by the procedure in section $10.5 ;$ 
7. In-service resilient modulus, $M_{R N}$, is then obtained in step 4 , or as $M_{\mathrm{RT}}$ obtained in step 5 , or as $M_{\mathrm{RF}}$ obtained in step 6 for conditions present at the time in the life of the subgrade being considered;

8. Estimate the $u_{\mathrm{q}}$ values for each month following the procedure described in Section 10.6;

9. Seasonal moduli estimated from above steps are, then, incorporated into 1986 AASHTO Guide to establish an effective resilient modulus.

10.3 Procedure for Cohesive Soil Subjected to Change in Water content after construction

The moisture condition of newly constructed subgrade changes after construction and is expected to approach an equilibrium after some time. The $M_{R}$ at equilibrium can be considered as in-service $M_{R}$ for normal condition of subgrade $\left(M_{R N}\right)$. In order to predict the decrease in $M_{R}$ due to the increase in water content after construction, a simplified procedure was developed. The results are based on the laboratory test results reported in section 5.3. However, it should be noted that further research is required to study the influence of change in subgrade moisture conditions on resilient behavior.

The moisture condition of the subgrade is affected by climate, permeability through the pavement profile, soilmoisture characteristics, type of ground cover and surrounding vegetation, the local topography, surface runoff from the pavement, free water table position, and pavement edge conditions. A reliable estimation of equilibrium moisture condition may be obtained by the programs developed by Dempsey and Elzeftawy (1977) or Espinoza et al. (on-going JHRP profect). The use of these models requires the soll-moisture characteristic curve and extensive input parameters. Since it is time consuming and not easy to obtain the soil-moisture characteristic curve, those for each site were prepared by 
using the pore-size distributions of the field compacted samples, as shown in Figures 10.1 through 10.5. These Figures also provide the variation of resilient modulus with the moderate increase in water content. These results are based on tests performed on small samples (about 1 cubic $\mathrm{cm}$ ) and may not adequately represent actual subgrade moisture conditions. Details about the prediction of the moisture characteristic curve from the pore-size data can be found in Prapaharan et al. (1985). Water content corresponding to the 100 percent saturation for a site may vary considerably due to the inherent variabllity in the field compacted subgrade. To account for different values of the saturation water content, three moisture characteristic curves are provided for each site.

For five sites examined in this study, it was found that the subgrade solis approach 90 to 100 percent saturation, regardless of the as-compacted condition. Therefore, if a reliable method for estimation of the equilibrium subgrade condition is not available, the equilibrium water content may be assumed as that corresponding to the degree of saturation between 90 to 100 percent as estimated in Table 10.2.

The following steps are suggested to estimate the change in resilient modulus due to the increase in the water content after construction:

1. Estimate the equilibrium water content of the subgrade. This can be done by using the programs developed by Dempsey and Elzeftawy (1977) or Espinoza et al. (on-going. JHRP project). If these programs are not-available, make a best estimate of equilibrium water content for range between 90 to 100 percent degree of saturation;

2. Determine the reduction of resilient modulus corresponding to the increase in water content, using the relationship between $\Delta \mathrm{M}_{\mathrm{R}}$ and $\Delta \mathrm{w} / \mathrm{c}$ given in Figures 10.1 to 10.5. The resilient modulus at the equilibrium condition $\left(\mathrm{M}_{\mathrm{RN}}\right)$ is the as-compacted $M_{R}$ minus $\Delta M_{R}$. As-compacted $M_{R}$ is determined in step 4 of Section 10.2 .1 or 10.2 .2 as appropriate for the case
considered; 

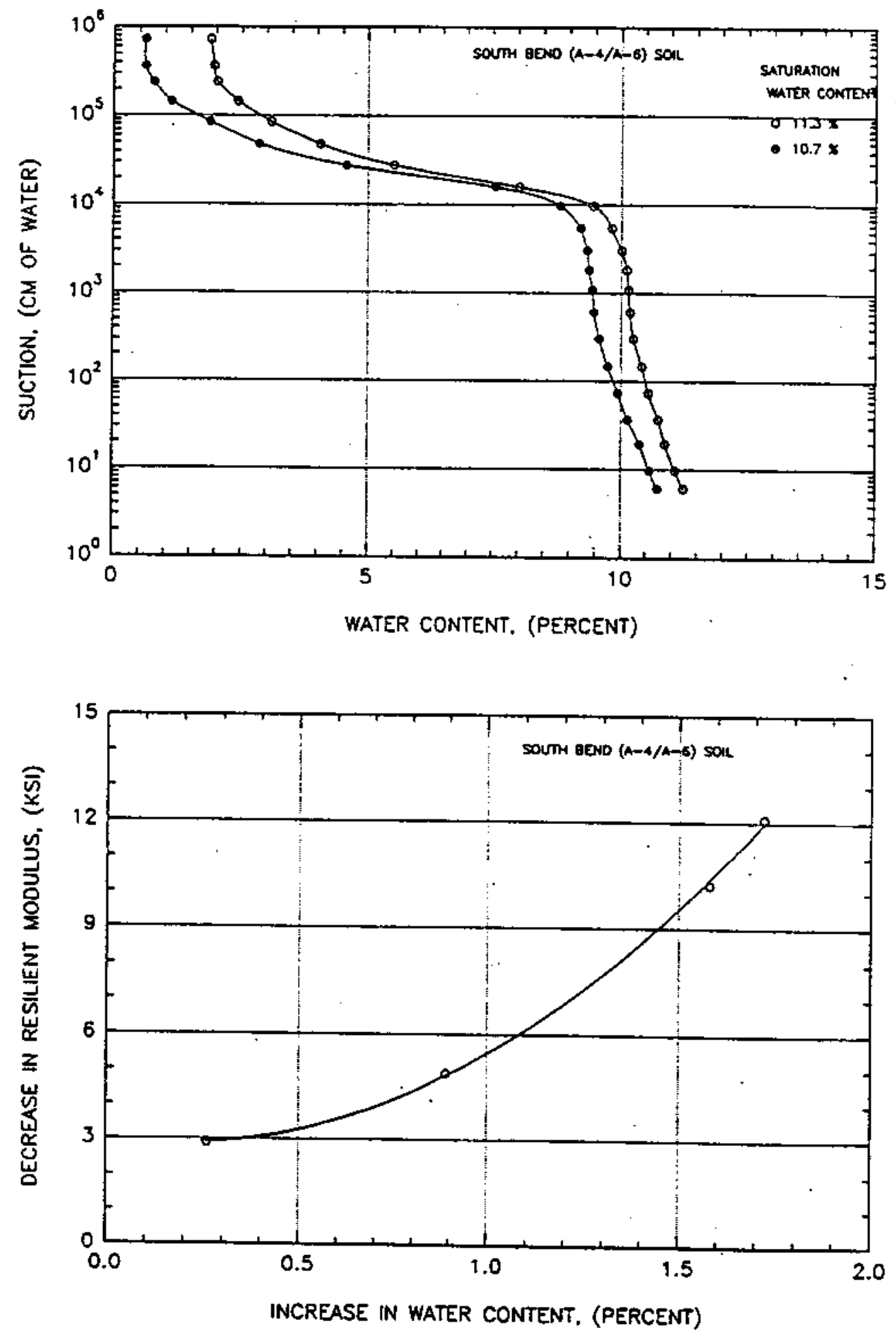

Figure 10.1 Moisture Characteristic Curve and Relationship Between $\Delta \mathrm{M}_{\mathrm{R}}$ and $\Delta \mathrm{w} / \mathrm{c}$ for South Bend site 

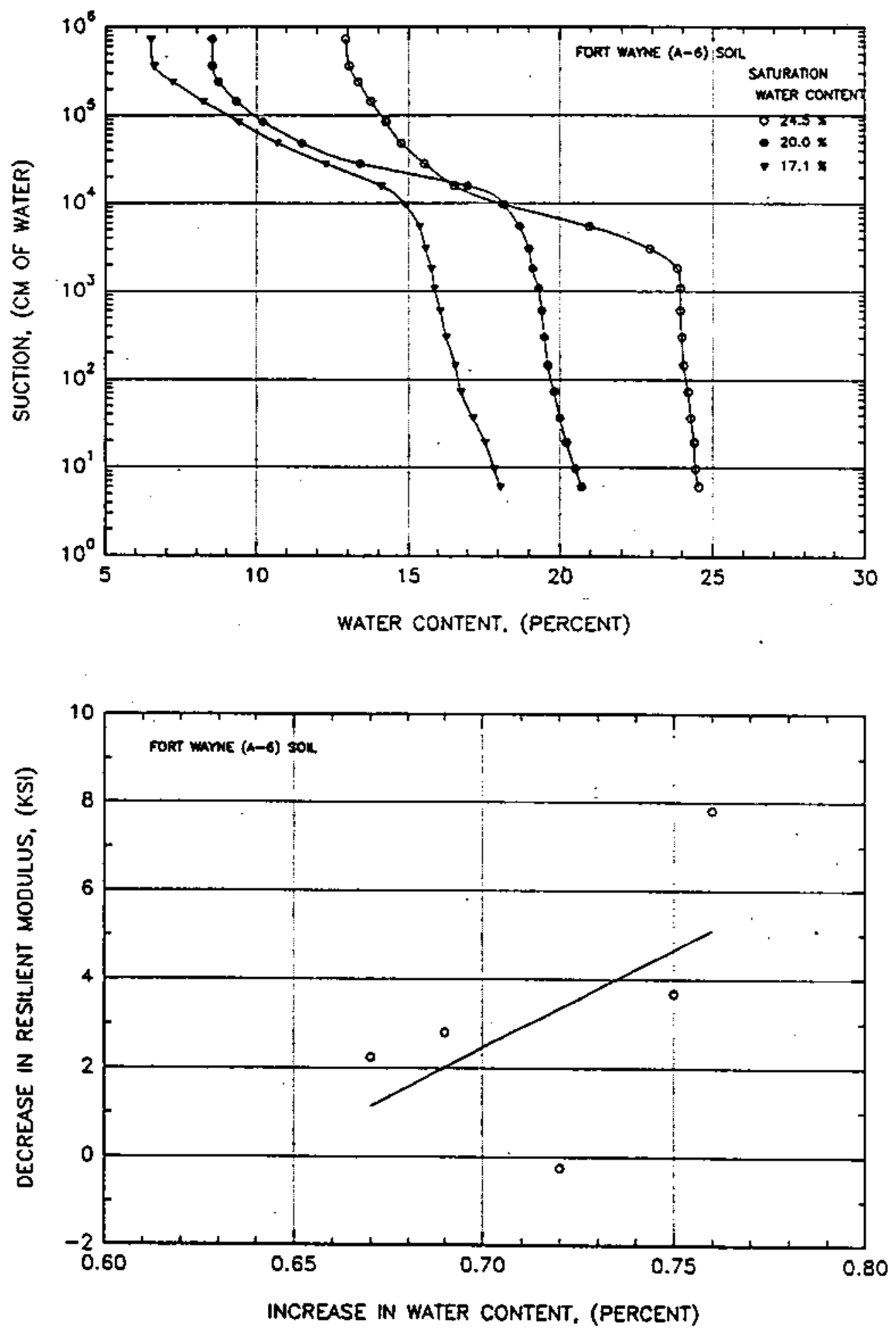

Figure 10.2 Molsture Characteristic curve and Relationship between $\Delta \mathrm{u}_{\mathrm{k}}$ and $\Delta \mathrm{w} / \mathrm{c}$ for Fort wayne site 

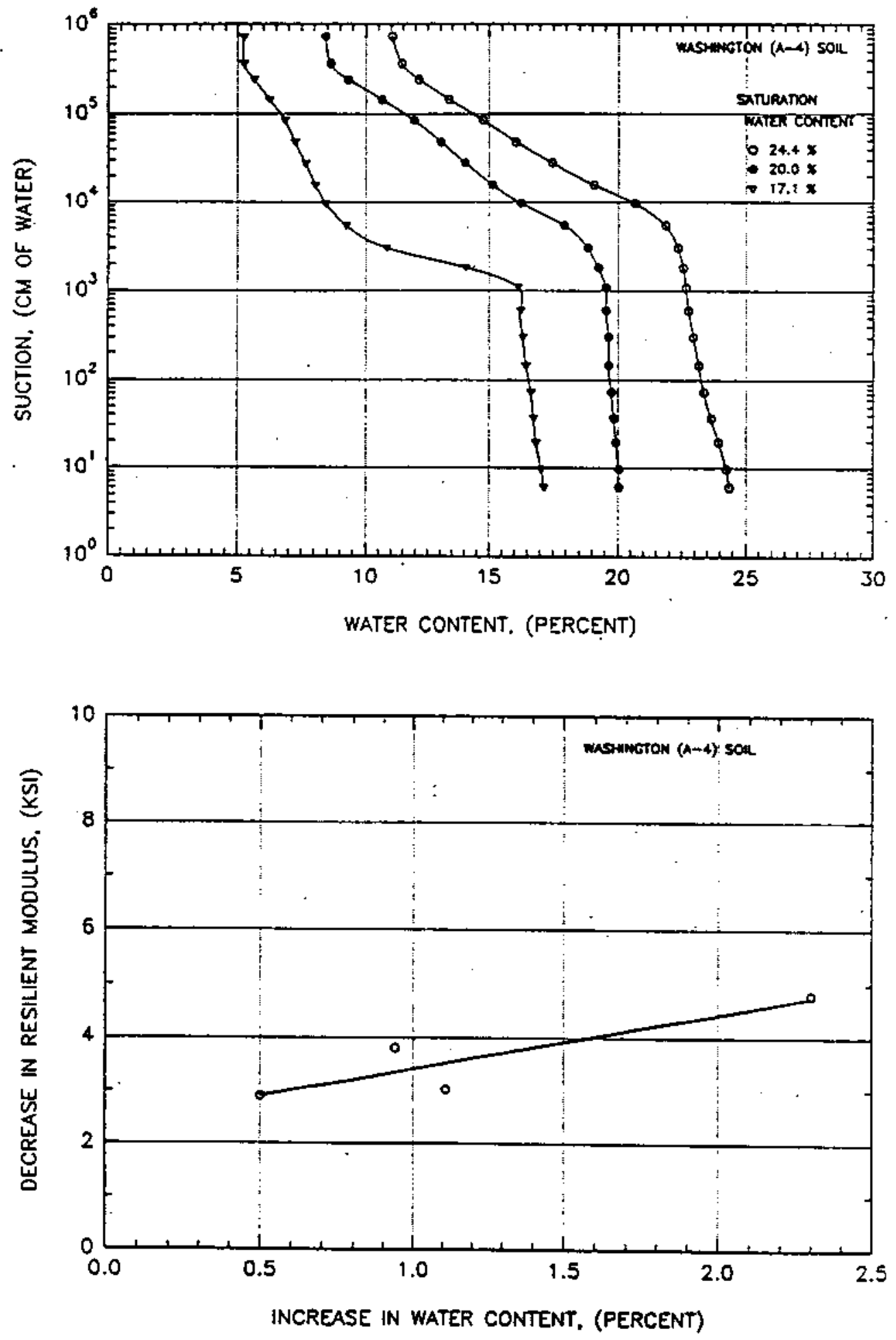

Figure 10.3 Moisture Characteristic Curve and Relationship between $\Delta \mathrm{Y}_{2}$ and $\Delta w / c$ for Washington Site 

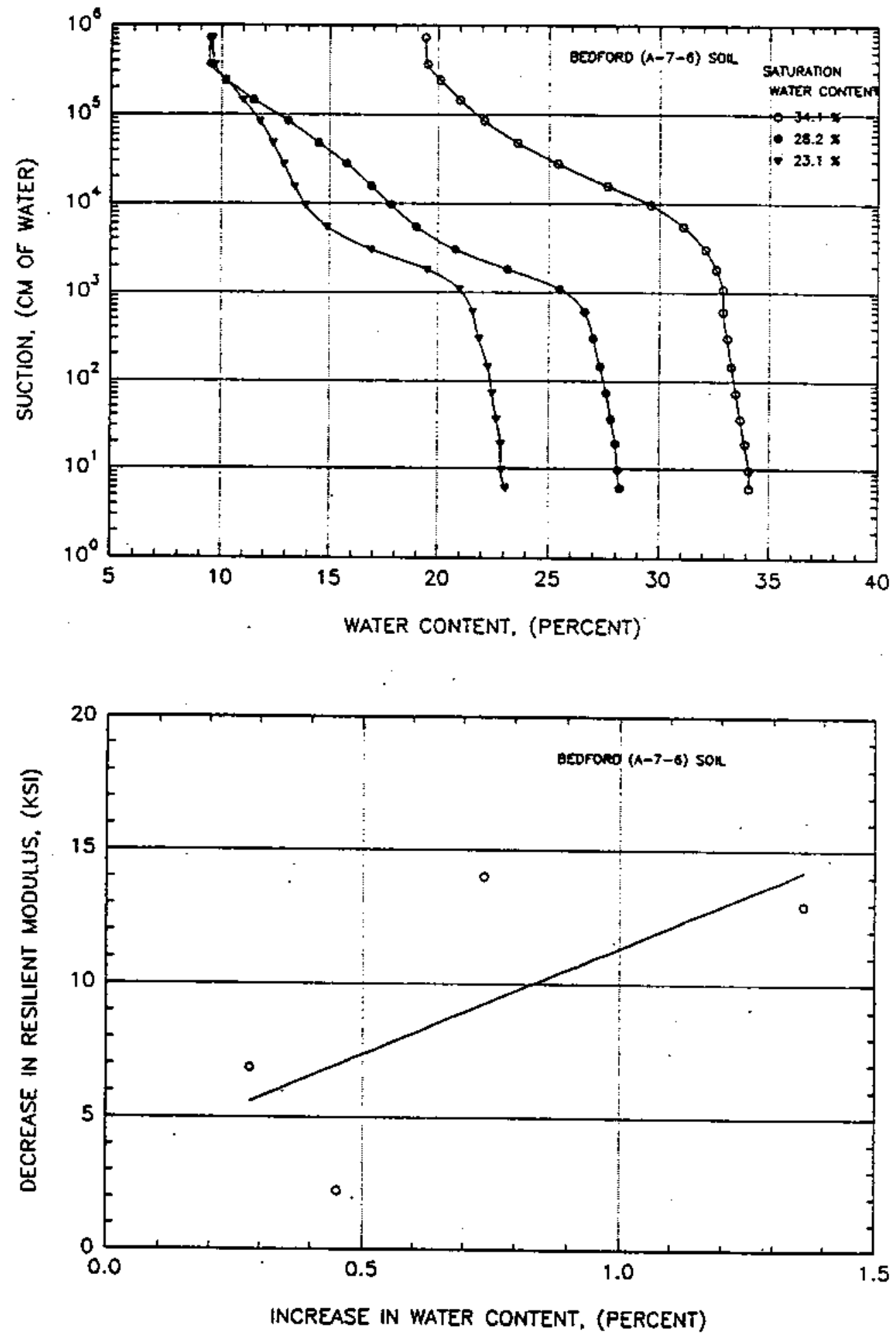

Figure 10.4 Moisture Characteristic Curve and Relationship between $\Delta \mathrm{M}_{\mathrm{R}}$ and $\Delta \mathrm{w} / \mathrm{c}$ for Bedford site 

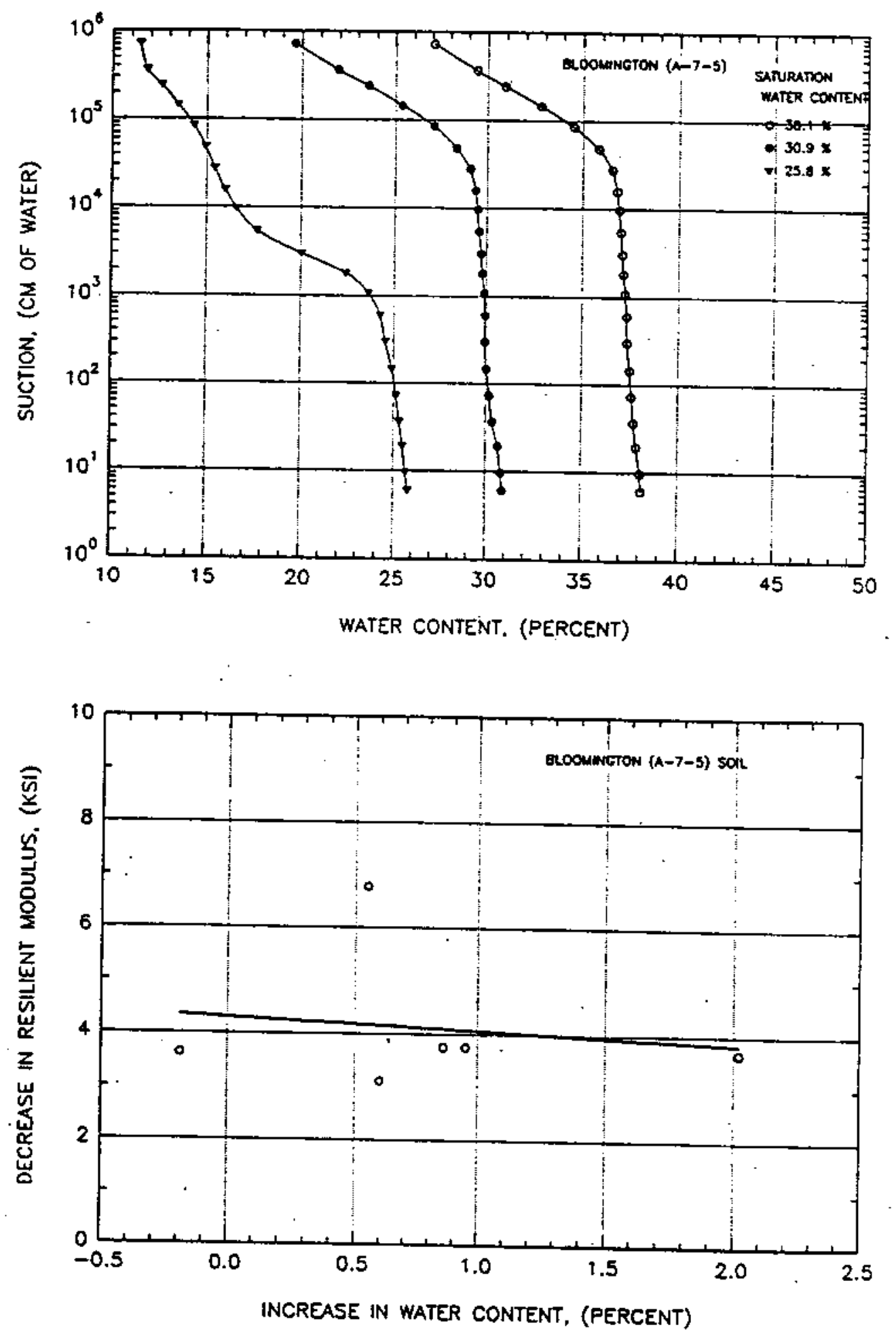

Figure 10.5 Moisture Characteristic Curve and Relationship. between $\Delta \mathrm{M}_{\mathrm{R}}$ and $\Delta w / c$ for Bloomington site 
3. When the difference in water content between the equilibrium and as-compacted condition is larger than 2.5 percent or when the soil is different from those considered in this study, develop the relationship between $\Delta \mathrm{M}_{\mathrm{k}}$ and $\Delta \mathrm{w} / \mathrm{c}$. Compact three or four identical specimens in the laboratory following the procedure outlined in section 10.2.1. Increase water content of specimens by back-pressure saturation technique with different saturating period, and perform unconfined compression tests. Calculate $M_{R}$ from $S_{u 1.0 x}$ using Equation 4.8 and develop the relationship between $M_{R}$ and $\Delta w / c$. Then, estimate $M_{R N}$ at the equilibrium water content following step 1 and 2 .

10.4 Procedure for Frozen-Thawed Cohesive Soil

The most critical time throughout year for the pavement structure is the spring thaw season because the freeze-thaw causes a significant decrease in subgrade resilient modulus. In order to predict thawed resilient modulus, the relationship between thawed $M_{R}$ and the $S_{u 1.0 x}$ for field compacted soils from five sites is expressed by an unified equation. Since the addition of extra terms in the prediction equation improves the predictability only slightly, the following simple equation is recommended for practical use:

$$
M_{R T}=2453.48+130.24 S_{\mathrm{u} 1.0 *}
$$

where $M_{R T}$ is the thawed resilient modulus at $\sigma_{3}=3$ psi and $\sigma_{d}=6$ psi (psi); and $S_{u 1.0 x}$ is the stress causing 1 percent strain in an unconfined compression test performed before the freezethaw of the specimen (psi). This equation may be used for both as-compacted and in-service conditions.

It should be noted that $S_{w 1.0 \%}$ is obtained from the unconfined compression test performed before freeze-thaw while $M_{\mathrm{RT}}$ is measured after freeze-thaw. Therefore, Equation 5:3 already includes the effect of freeze-thaw on the resilient modulus. The procedure proposed is only adequate for the situation in which there occurs no change in water content during the freeze-thaw process since it is based on the 
results of closed-system freeze-thaw test. If the estimated resilient modulus of thawed soil is larger than that of non freeze-thaw soil, the latter should be used as the thawed resilient modulus in the design process.

If it is possible to obtain an undisturbed specimen from thawed subgrade, the thawed resilient modulus can be obtained by substituting the magnitude of $s_{u i .0 x}$ obtained from that specimen into the Equation 4.8.

\subsection{Procedure for Frozen Cohesive Soil}

It was shown in section 5.4 that the resilient modulus of frozen cohesive soil is independent of the repeated deviator stress. Five specimens from three sites showed approximately identical resilient modulus though they had wide range of

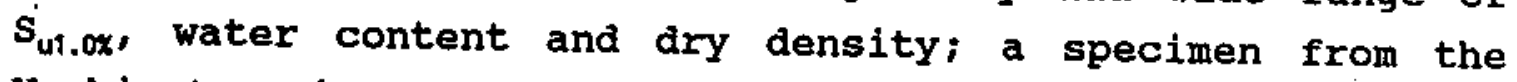
washington site showed a higher modulus than the other soils. Therefore, the resilient modulus is considered constant for deviator stress ranging from 1 psi to $10 \mathrm{psi}$, and the recommended resilient modulus for frozen subgrade $\left(M_{R F}\right)$ for each site is presented in Table 10.2.

Table 10.3 Resilient Modulus for Frozen Soils and Estimate of water Contents after Construction

\begin{tabular}{l|cccc}
\hline Sites & $M_{R F}(p s i)$ & $\begin{array}{c}\text { As-compacted } \\
\text { opt.w/C, }\end{array}$ & $\begin{array}{c}\text { Estimate of } \\
\text { w/C at }\end{array}$ \\
\hline South Bend & 27000 & $s_{c}=90 \%$ & $S_{r}=95 \%$ \\
Fort Wayne & 27000 & 9.8 & 10.8 & 11.4 \\
Washington & 46000 & 16.8 & 17.2 & 18.2 \\
Bedford & 27000 & 15.0 & 16.4 & 17.4 \\
Bloomington & 27000 & 19.5 & 20.0 & 21.1 \\
\hline
\end{tabular}

According to previous studies by Johnson et al. (1979), the resilient modulus of frozen soil is a function of temperature. Table 10.2 corresponds to temperature data estimated as bounds for Indiana locations. Therefore, further study is necessary to investigate the change of resilient modulus of typical 
soils in Indiana. It is also necessary to establish the data base on the variation of temperature beneath the pavement structure.

10.6 Seasonal Variation of Resilient Modulus

Since environmental conditions vary in the subgrade season by season through the year, it is necessary to determine subgrade $\boldsymbol{M}_{\mathrm{R}}$ changes for varlous times throughout the year. Elliott and Thompson (1985) estimated the seasonal variation of resilient modulus from the deflection data for the first spring, summer, and fall of the AAsHo Road Test subgrade. They showed the subgrade $M_{R}$ increases approximately linearly from the spring thaw value to the normal value in Fall. The Asphalt Institute (1982) presented subgrade $M_{R}$ variation throughout the year from a study of climatic data. This gave specific information, both with respect to freeze and thaw periods, and to the thaw recovery period, for three selected sites having different mean annual air temperature conditions.

Based on the above studies and the climatic data for Indiana, the tentative representation of subgrade $M_{R}$ throughout the year is proposed as shown in Figure 10.6. The resilient modulus starts to increase in December and a high modulus is maintained until March. $M_{2}$ decreases abruptly in April due to thawing, being followed by five months of a thaw recovery period. The resilient modulus for normal subgrade $\left(M_{\mathrm{RN}}\right)$ is estimated by the procedure described in section 10.2 . Resilient moduli for frozen subgrade $\left(M_{R P}\right)$ and thaw subgrade $\left(M_{\mathrm{RT}}\right)$ can be determined by section 10.5 and section 10.4, respectively. Assuming that the resilient modulus between these values varies linearly, the resilient moduli for each month throughout the year can be determined. Once the seasonal variation in $M_{R}$ is estimated, these values can be incorporated into the 1986 AASHTO Guide for estimation of effective 


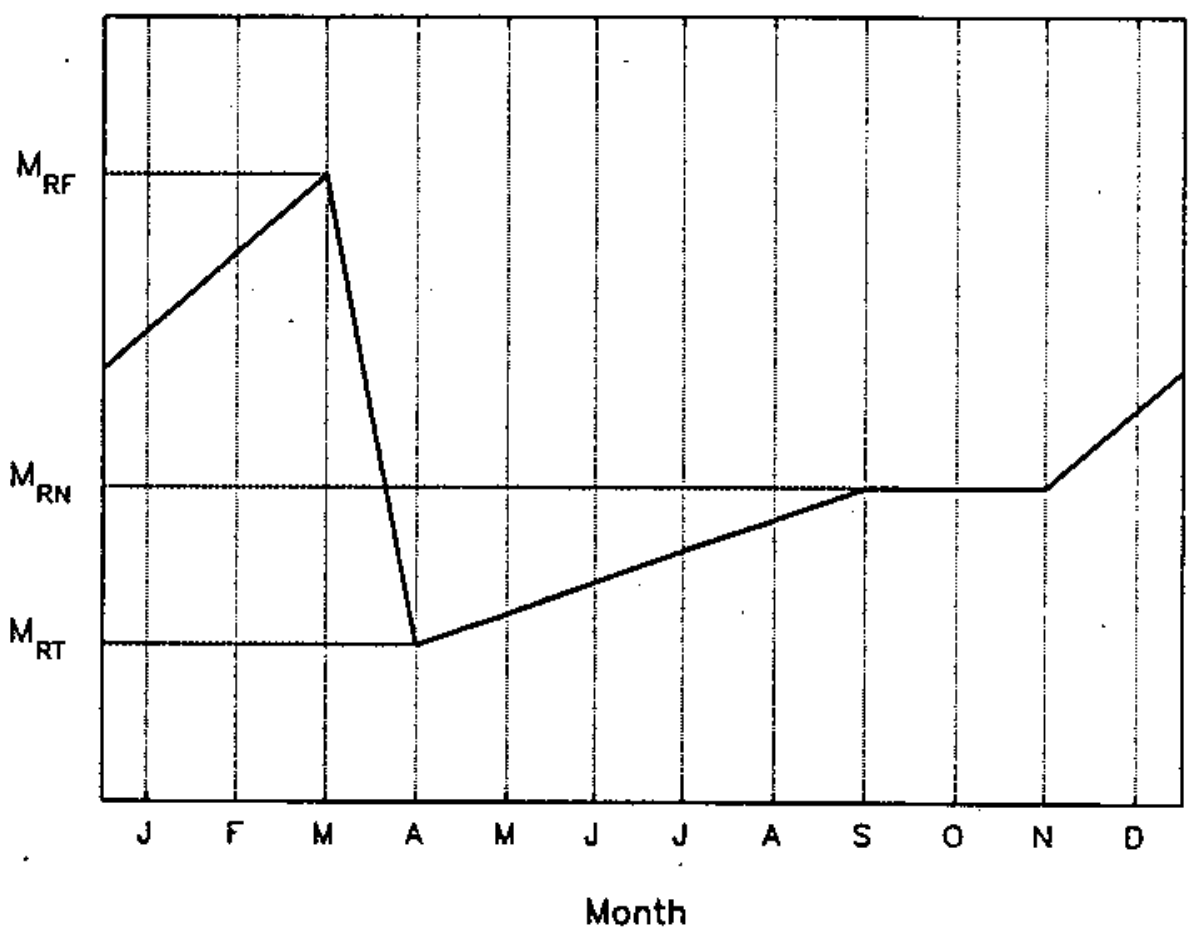

Figure 10.6 Seasonal Variation of Resilient Modulus 
resilient modulus.

A better representation of the variation in subgrade resilient modulus can be obtained by sampling and testing the subgrade soil, or by measuring the deflection of pavement and backcalculating the subgrade resilient modulus throughout the year. Until reliable data on the seasonal variation of resilient modulus are obtained, a tentative representation shown in Figure 10.6 will give a reasonable estimation of seasonal change in $\mathrm{M}_{\mathrm{R}}$.

\subsection{Resilient Modulus at Different Level of Stresses}

\subsubsection{Procedure for Normal Cohesive Soil}

For mechanistic analysis of pavement system, it is necessary to express $M_{R}$ in terms of deviator stress and confining stress since the level of stresses due to traffic loading is again a function of resilient characteristics of pavement materials. From regression analysis on resilient moduli at different levels of confining stress and repeated deviator stress, $M_{R}$ of cohesive soil can be expressed as;

$$
M_{R}=a+b S_{\mathrm{u1.08}}+c S_{\mathrm{u1.0t^{2 }}}
$$

where $M_{R}$ is the resilient modulus at the specific level of stresses (psi); $s_{\text {ul.0x }}$ is the stress causing 1 percent strain in unconfined compression test (psi); $a, b$, and $c$ are regression parameters. Since parameters $a, b$, and $c$ are a function of stresses, charts to evaluate these parameters corresponding to given confining stress and deviator stress are developed as shown in Figure 10.7. The resilient modulus at specific deviator stress and confining stress can, then, be obtained by estimating regression parameters from Figure 10.7 and substituting estimated values for these parameters into Equation 4.4. By plotting calculated resilient moduli with corresponding deviator stress and confining stress, the 

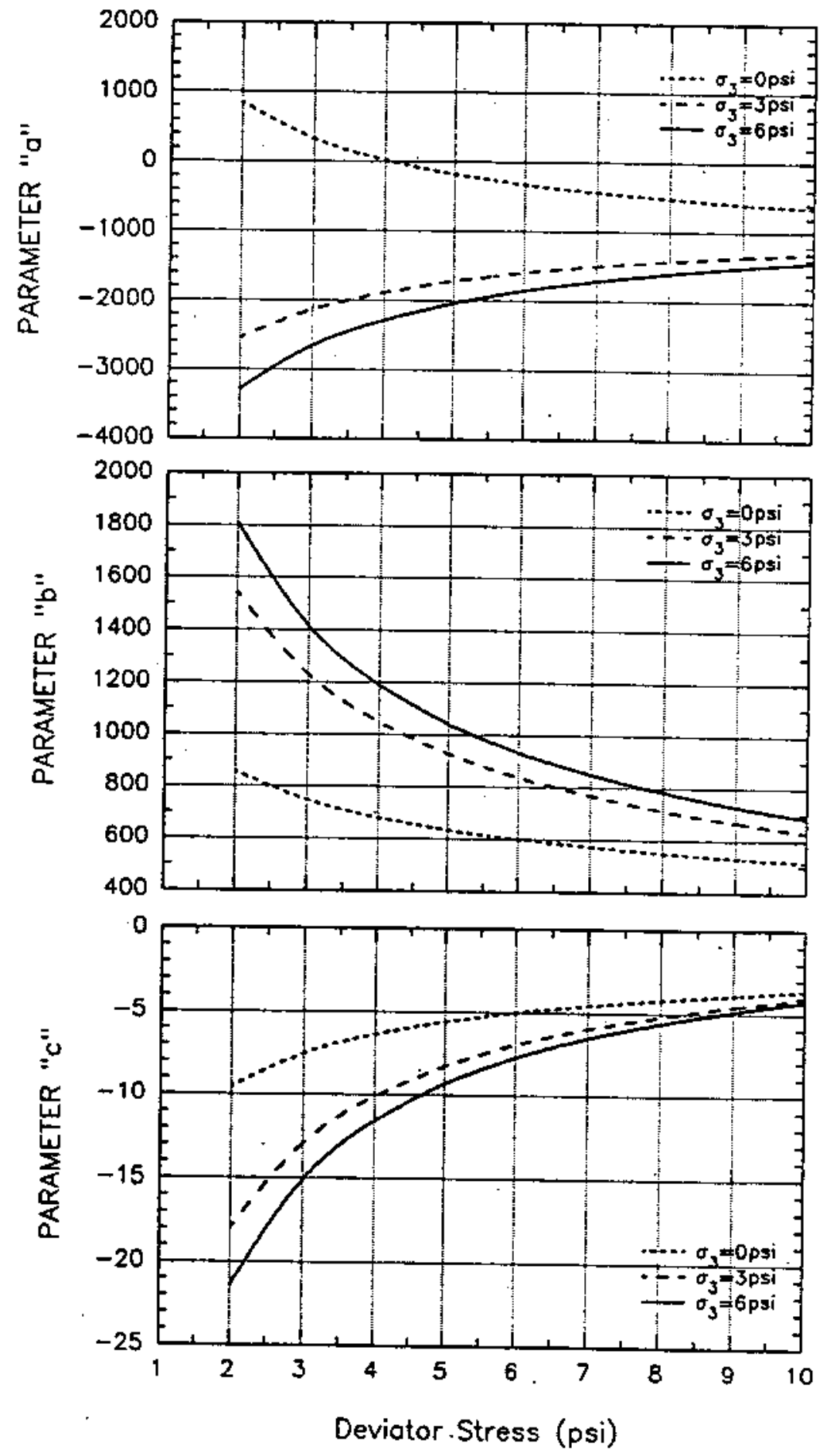

Figure 10.7 Chart for Estimation of Parameters in Equation 
relationship of resilient modulus with deviator stress for each confining stress level is established.

Another chart to determine the relationship between $M_{R}$ and $\sigma_{d}$ is developed for more convenient use. All the test results from field compacted soils are sorted and grouped by means of $S_{u 1.0 x}$, and the average value of $A$ and $B$ in the expression of $M_{R}=A \sigma_{d}{ }^{B}$ are obtained for each group. Since $S_{u 1.0 x}$ is the most influential factor on resilient modulus, this approach would provide reasonably reliable relationship between resilient modulus and deviator stress. Plots of $M_{R}$ at $\sigma_{3}$ of 3 psi for each group are shown in Figure 10.8. Table 10.3 can be used to estimate the resilient modulus for other values of $\sigma_{3}$ for the appropriate group of $s_{\mathrm{vl} .0 x}$.

For both approaches proposed above, the parameters at a deviator stress less than 2 psi are assumed constant and identical to those at deviator stress of 2 psi.

\subsubsection{Procedure for Thawed Cohesive Soil}

Resilient moduli of thawed cohesive subgrade at specific levels of confining stress and repeated deviator stress are estimated using following equation;

$$
M_{R}=a+b S_{u 1.0 *}
$$

where $M_{R}$ is the thawed resilient modulus at specific level of stresses ( $p s i$ ); and $S_{u 1,0 x}$ is stress causing 1 percent strain in unconfined compression test performed before the freeze-thaw of specimen (psi). Regression parameters $a$ and $b$ are $a$ function of the deviator stress and confining stress, and charts to estimate $a$ and $b$ are shown in Figure 10.9. Once $S_{\text {ul.ox }}$ is determined from the unconfined compression test on non freeze-thaw soil, the post-thaw $M_{R}$ is estimated using charts in Figure 10.9 and Equation 5.1. By repeating the process for various levels of stresses, the relationship for thawed $M_{R}$ with $\sigma_{d}$ at each level of confining stress is obtained and can 


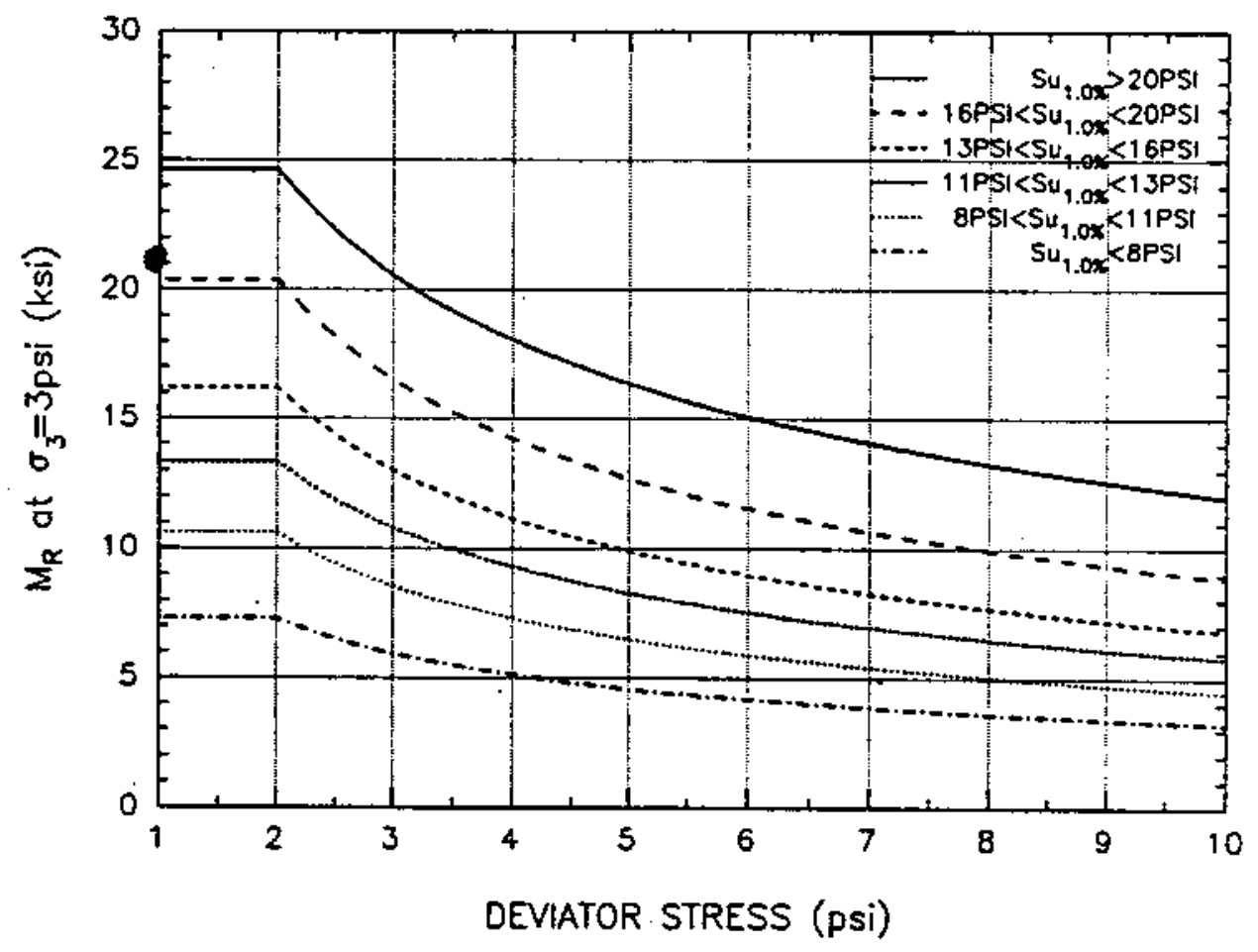

Figure 10.8 Relationships between $M_{R}$ and $\sigma_{d}$ for Each Group of $S_{41.0 \%}$ 
Table 10.3 Parameters for Estimation of $M_{R}$ for Each Group of $S_{u 1.0 \%}$

\begin{tabular}{|c|c|c|c|c|c|c|c|}
\hline Group & $A_{6}$ & $\mathbf{A}_{3}$ & $A_{0}$ & $\mathbf{B}_{6}$ & $\mathbf{B}_{\mathbf{3}}$ & $\mathrm{B}_{0}$ & * of tests \\
\hline$S_{w 1.0 \pi}>20$ & 39027 & 33623 & 19508 & -0.44895 & -0.41710 & -0.22904 & 17 \\
\hline $16<S_{u 1.0 x}<20$ & 34735 & 29218 & 18193 & -0.55268 & -0.51989 & -0.36225 & 12 \\
\hline $13<S_{u 1.0 x}<16$ & 28195 & 23534 & 16617 & -0.58155 & -0.53972 & -0.44108 & 15 \\
\hline $11<S_{n 1.0 x}<13$ & 22159 & 19118 & 12101 & -0.55045 & -0.52138 & -0.37992 & 11 \\
\hline $8<S_{\mathrm{vt} .0 x}<11$ & 18117 & 15399 & 11549 & -0.57934 & -0.53894 & -0.45585 & 18 \\
\hline$S_{\text {ut } .0 x}<8$ & 11712 & 10356 & 8248 & -0.53840 & -0.50971 & -0.44371 & 19 \\
\hline
\end{tabular}

$A_{6}, A_{3}$, and $A_{0}$ are constant $A$ in Equation (4.2) at $\sigma_{3}$ of $6,3,0$ psi, respectively. $B_{6}, B_{3}$, and $B_{0}$ are constant $B$ in Equation (4.2) at $\sigma_{3}$ of 6,3 , o psi, respectively. 

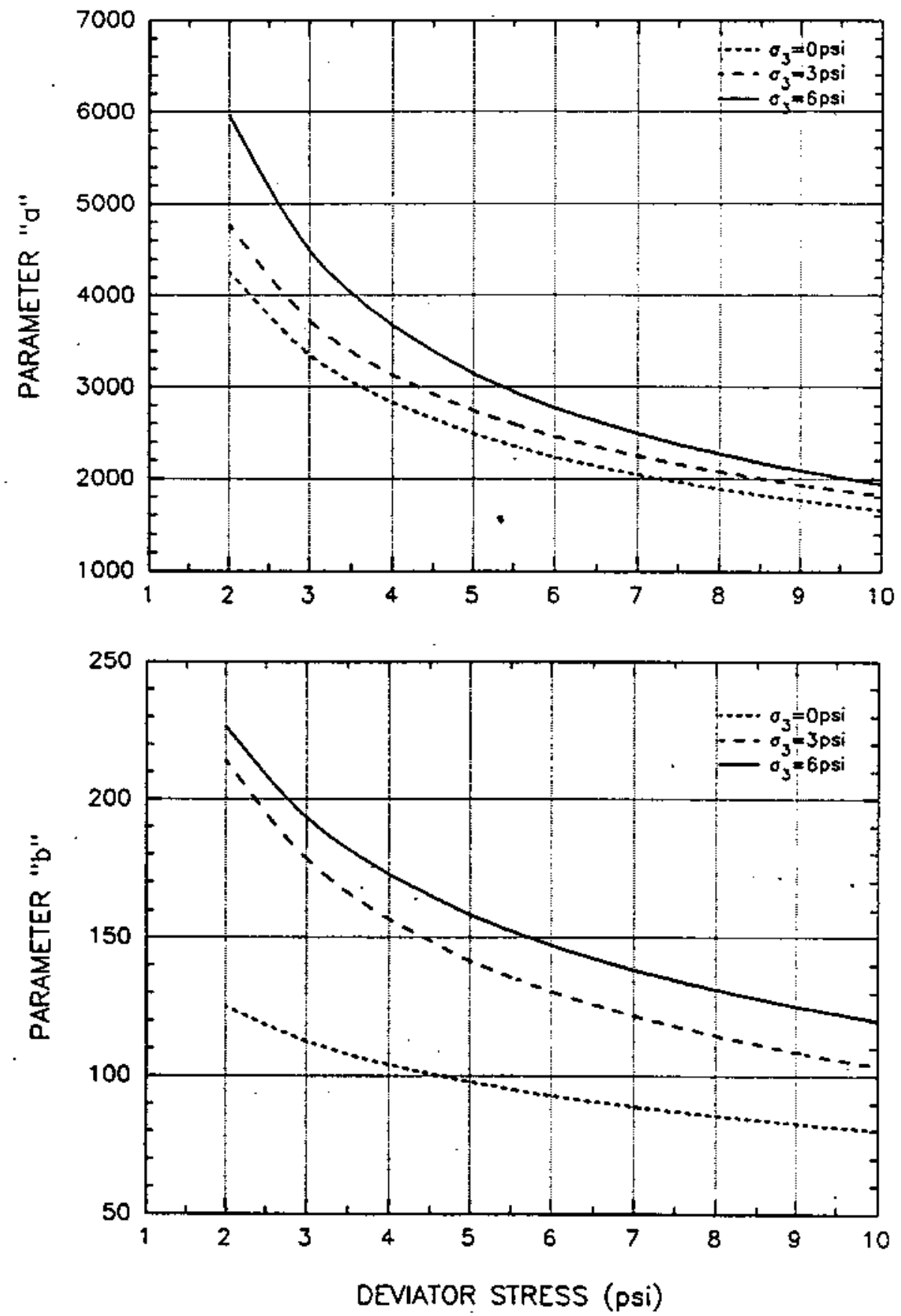

Figure 10.9 Chart for Estimation of Parameters in Equation 5.1 
be used for mechanistic analysis of pavement system.

10.8 Development of Specification to Control In-Service Subgrade Deformability

To establish limiting criteria for resilient deformability, data on what can be considered excessive resilient deformation of the subgrade materials used for typical pavement systems are required. Recommendations for the magnitude of limiting value of the $M_{R}$ are beyond the scope of the present work. In this section, a schematic procedure to control in-service subgrade deformability is presented. Once the desirable limiting resilient modulus of the subgrade is determined, proposed Equation 4.4 or 4.8 can be used during construction to control the resilient property of subgrade. From the Equation 4.4 or 4.8 , the limiting resilient modulus can be converted to the limiting $S_{v 1.0 x}$. If $S_{u l .0 x}$ obtained from the unconfined compression test on the specimen taken from constructed subgrade is larger than the limiting $s_{41.0 \%}$, the subgrade is considered to have the resilient modulus larger than the limiting resilient modulus. This will help field engineers better control the resilient characteristics of the subgrade, and will reduce pavement cracking and early deterioration. The unconfined compression test is recommended to be run on the undisturbed specimen of 2.8-inch diameter and height between 5.6 and 6 inches with 1 percent strain per minute. Time and effort can be reduced by performing the unconfined compression test only up to 1 percent axial strain.

When it is possible to establish rationally that the resilient modulus must be constrained to a limiting value, the procedures developed in this study may be followed to assure the presence of limiting value in-service. The following is recommended to help the control of in-service subgrade deformability: 
1. Set the limiting value of resilient modulus from consideration of pavement analysis;

2. Estimate the decrease in resilient modulus due to change in water content after construction by the procedure recommended in Section 10.3;

3. Add the decrease in resilient modulus estimated in step 2 to the limiting value of resilient modulus, and get the target value of as-compacted resilient modulus;

4. Since the decrease in resilient modulus due to freeze-thaw is significant, it is very difficult to maintain the thawed resilient modulus larger than the limiting resilient modulus. Therefore, the best way to reduce the damage on pavement due to freeze-thaw is not allow the subgrade to freeze; $\because$

5. Convert the target resilient modulus obtained in step 3 into a target value of $S_{u 1.0 \pi}$ using Equation 4.4 ;

6. Compact the soil in the laboratory with varying water content and compaction energy, and perform the unconfined compression test on compacted specimens. Determine the range of water content and dry density which gives the $s_{u 1.0 x}$ larger than the target value of $s_{u l .0 x}$ obtained in step 5 ;

7. Specified range of water content and dry density can be verified by performing the unconfined compression test on the specimens taken from test embankment;

8. During construction, target value of $S_{v 1.0 \%}$ can be used to help control the resilient characteristics of the subgrade.

\subsection{Procedure for Granular soil}

For granular soils prepared by vibratory compaction which produces the similar dry density with standard impact compaction, the resilient response is almost independent of compaction water content. The resilient modulus of dune sand compacted with vibratory energy corresponding to standard Proctor energy, can be expressed as follows;

$$
M_{R}=3126 \theta^{0.595}
$$

where $M_{R}$ is the resilient modulus in psi; $\theta$ is sum of principal stresses in psi. This should, however, be used with 
caution at the site where there is possibility of porepressure built up due to a poor drainage.

To consider the effect of dry density on $M_{R}$, relative compaction is defined as the ratio of as-compacted dry density to that obtained from 5 layer compaction by 5 minute vibration per layer. The prediction equation developed is;

$$
M_{R}=(-20163+232.886 R C) \theta^{0.595}
$$

where $M_{R}$ is the resilient modulus in psi; $R C$ is relative compaction in percentage. A chart to estimate the resilient modulus directly from water content and dry density is shown in Figure 10.10. Due to limitations of tested dry densities, proposed equation and chart are suggested to be used in the range of relative compaction between 93 to 105 percent.

\subsection{Numerical Examples}

\subsubsection{Newly Constructed Pavement}

This section shows how to use the proposed procedure to estimate the subgrade resilient modulus for newly constructed pavement. Assume that the unconfined compression test was performed on the South Bend soil compacted in the laboratory following the procedure described in section 10.2.1, and that the stress causing 1 percent strain $\left(S_{\text {di.0x }}\right)$ was 20.0 psi. The as-compacted resilient modulus is, then, calculated by Equation 4.8;

$$
M_{R}=-1599.66+833.83 \times 20.0-6.9683 \times(20.0)^{2}=12,290 p s i
$$

The estimated increase in water content from the ascompacted condition to an equilibrium condition is assumed to be 1.0 percent. Then, the reduction in $M_{q}$ can be estimated from Figure 10.1 and it is about $5400 \mathrm{psi}$. The resilient modulus at the equilibrium condition is considered as the $M_{R}$ for normal subgrade $\left(\mathrm{M}_{\mathrm{RN}}\right)$ and its magnitude is; 


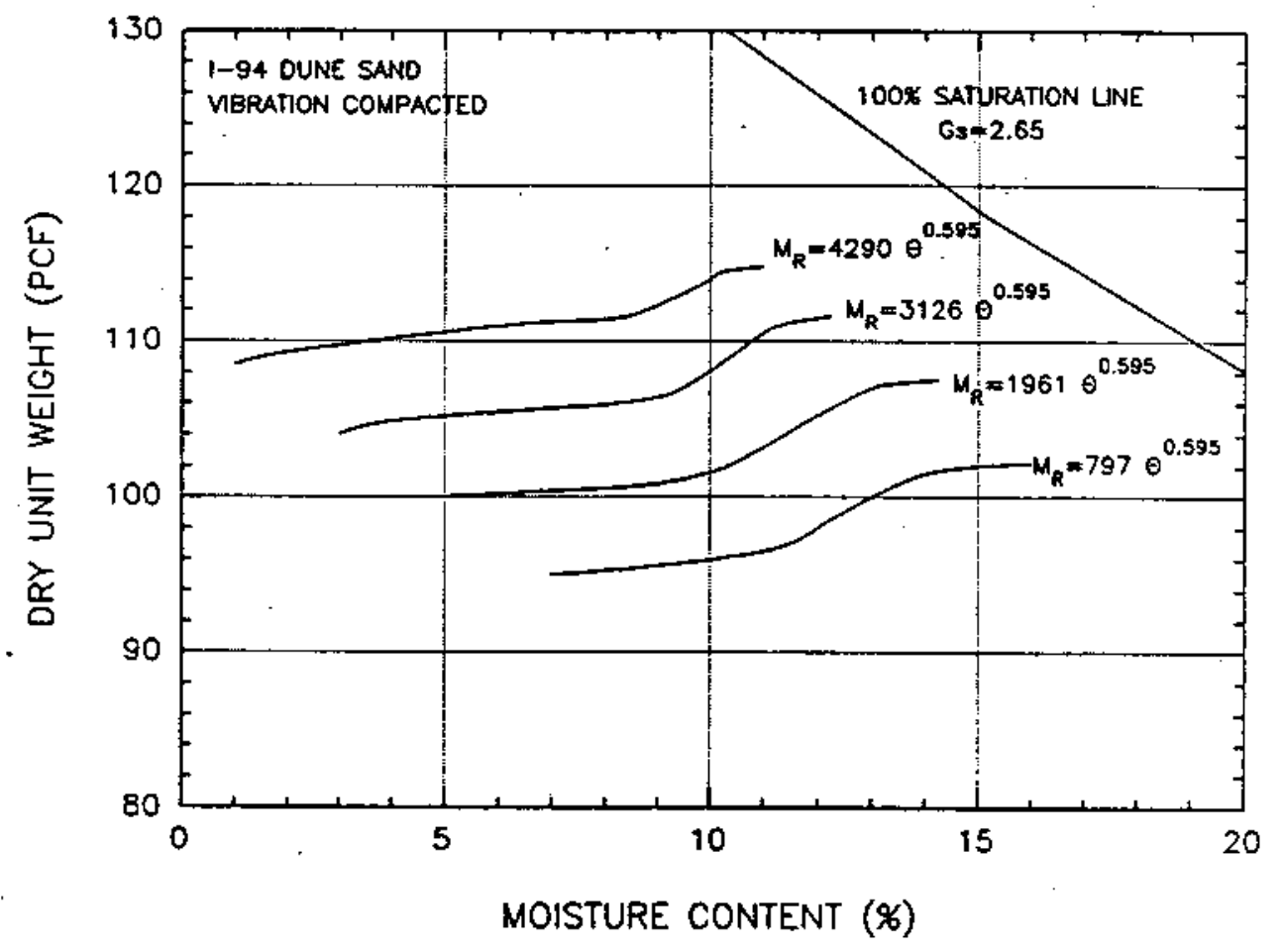

Figure 10.10 Chart for Estimation of Resilient Modulus of vibratory Compacted Dune sand 


$$
M_{R N}=12,290-5,400=6,890 p s i
$$

The $S_{\text {ut.ox }}$ corresponding to $\mathrm{M}_{\mathrm{RN}}$ can be obtained from Equation 4.8 and it is $11.24 \mathrm{psi}$. The resilient modulus for thawed subgrade $\left(\mathrm{M}_{\mathrm{RT}}\right)$ is, then, determined from Equation 5.3;

$$
M_{R T}=2453.48+130.24 \times 11.24=3,920 p s i
$$

Another way to determine $M_{R T}$ from the estimated $M_{R N}$ is shown in Figure 10.11. The resilient modulus for frozen subgrade $\left(M_{R P}\right)$ estimated from Table 10.2 is 27,000 psi.

From these seasonal moduli obtained above, the resilient moduli for each month of the year can be estimated from Figure 10.12. Estimated resilient moduli for each month are, then, incorporated in 1986 AASHTO Guide to determine the effective roadbed resilient modulus, as shown in Figure 10.13. It is shown that the effective resilient modulus determined is about 6300 psi.

10.10.2 Reconstructed Pavement

To show how to utilize the proposed procedure of estimation of resilient modulus, the test result of FWO5 is used as an example. As shown in Appendix B, the stress causing 1 percent strain in an unconfined compression test performed with a strain rate of 1 percent per minute is $18.4 \mathrm{psi}$. This value of $s_{\text {u1.0x }}$ is substituted into Equation 4.8 and the resilient modulus predicted is;

$$
M_{R N}=-1599.66+833.83 \times 18.4-6.9683 \times(18.4)^{2}=11,383 p s i
$$

since the subgrade is considered to have reached its equilibrium condition and was sampled in september, the resilient modulus obtained is the equilibrium resilient modulus $\left(\mathrm{M}_{\mathrm{RN}}\right)$ of normal subgrade. As a comparison, the resilient modulus measured during the test was 14417 psi at $\sigma_{3}=3 \mathrm{psi}$ and $\sigma_{d}=4 \mathrm{psi}$, and was $9032 \mathrm{psi}$ at $\sigma_{3}=3 \mathrm{psi}$ and $\sigma_{d}=8$ psi. Therefore, the predicted value is reasonably close to the 


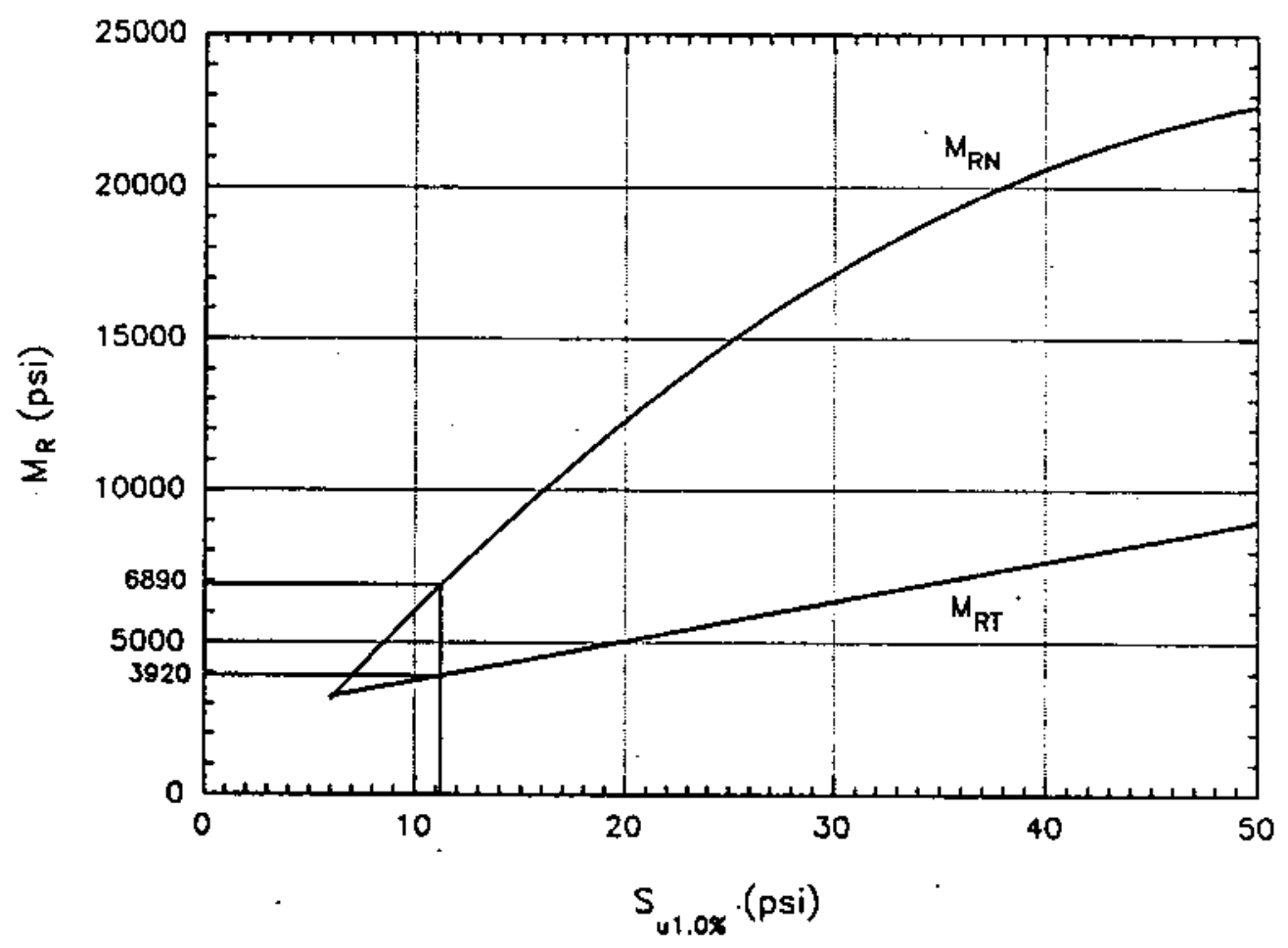

Figure 10.11 Estimation of $M_{\mathrm{RT}}$ for Example in section 10.10 .1 


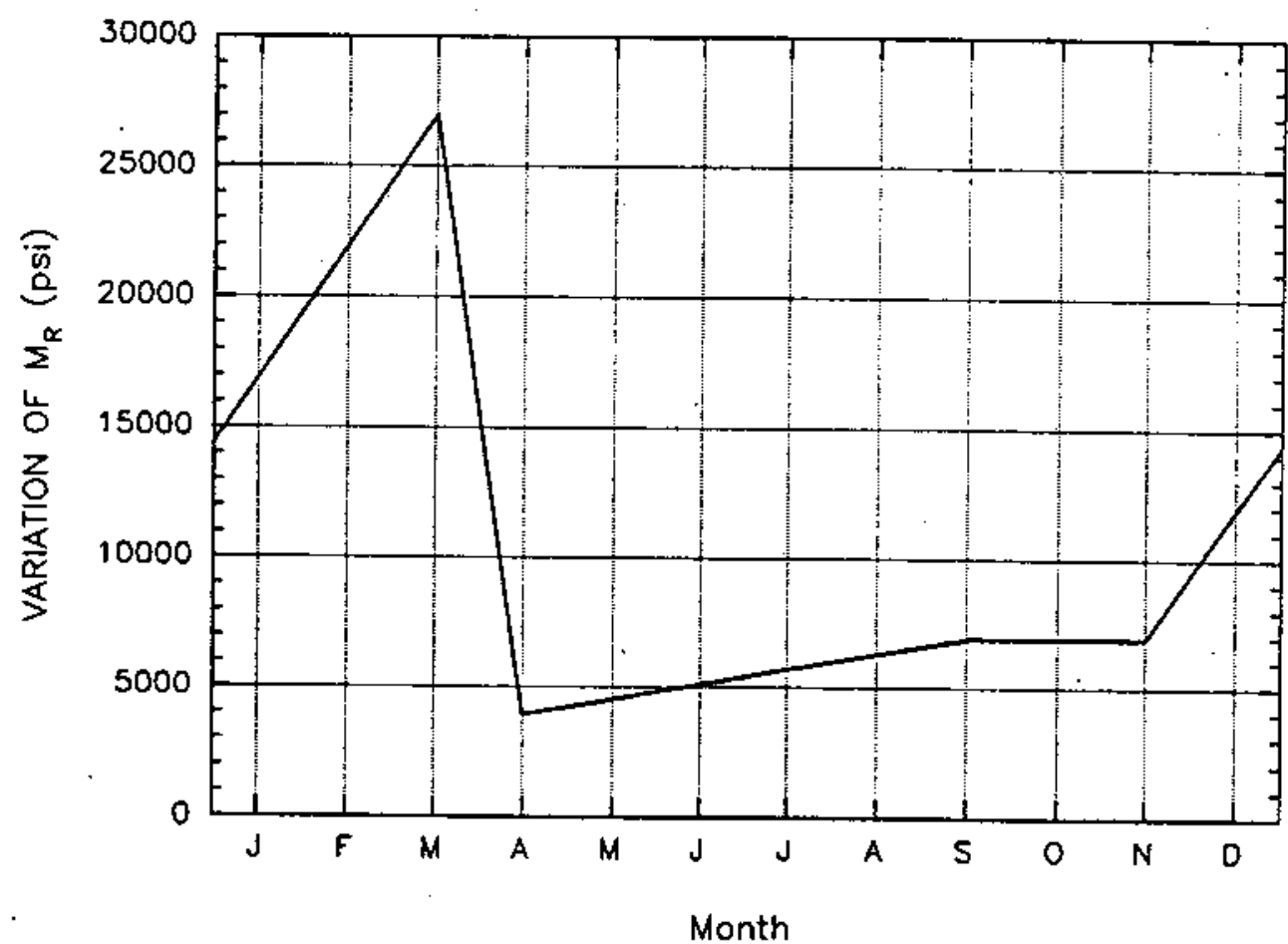

Figure 10.12 Seasonal Variation of Resilient Modulus for Example in Section 10.10.1. 


\begin{tabular}{|c|c|c|}
\hline Month & $\begin{array}{c}\text { Subgrade } \mathbf{M}_{\mathrm{R}} \\
\text { (psi) }\end{array}$ & $\begin{array}{c}\text { Relative Damage } \\
\mathbf{u}_{\mathrm{f}}\end{array}$ \\
\hline Jan. & 16950 & 0.018 \\
Feb. & 21970 & 0.010 \\
Mar. & 27000 & 0.006 \\
Apr. & 3920 & 0.550 \\
Mar. & 4500 & 0.395 \\
June & 5100 & 0.295 \\
July & 5700 & 0.228 \\
Aug. & 6300 & 0.181 \\
Sep. & 6890 & 0.147 \\
Oct. & 6890 & 0.147 \\
Nov. & 6890 & 0.147 \\
Dec. & 11920 & 0.041 \\
\hline
\end{tabular}

$$
\begin{gathered}
u_{f}=1.18 \times 10^{8} \times M_{R}^{-2.32} \\
\bar{u}_{f}=\frac{\Sigma u_{t}}{n}=\frac{2.165}{12}=0.1804 \\
M_{R E}=\sqrt{\frac{1.18 \times 10^{8}}{\bar{u}_{f}}}-6.310 p s i
\end{gathered}
$$
Figure 10.13 Estimation of Effective Subgrade $M_{R}$ for Example
in Section 10.10.1. 
measured value.

The resilient modulus for thawed subgrade $\left(M_{\mathrm{RT}}\right)$ is obtained from Equation 5.3 or from Figure 10.14;

$$
M_{R T}=2453,48+130.24 \times 18.4=4,850 p s i
$$

From Table 10.2, the resilient modulus for frozen subgrade $\left(M_{R P}\right)$ is $27,000 \mathrm{psi}$. From these seasonal moduli obtained above, the monthly change in the resilient modulus can be estimated as shown in Figure 10.15. Resilient moduli for each month are; then, incorporated into 1986 AAsHro Guide to determined the effective roadbed resilient modulus as shown in Figure 10.16 . The effective subgrade resilient modulus obtained is $8880 \mathrm{psi}$.

10.10.3 Resilient Modulus for Different Level of stresses

This section shows how to establish the relationship between resilient modulus with deviator stress. The test result of FWO5 is used as an example. Values of parameters a, $b$, and $c$ for each deviator stress and confining stress are determined from Figure 10.7. These regression parameters and $S_{\text {ul.0x }}$ are put into the Equation 4.4. Estimated regression parameters and the predicted resilient moduli are shown in Figure 10.17 .

The other approach to establish the relationship is from Table 10.3. Since $S_{\text {w.0\% }}$ of $18.4 \mathrm{psi}$ is included in the second group (16 psi $<s_{\mathrm{ul.0x}}<20 \mathrm{psi}$ ), the relationships for $\sigma_{3}=6,3$, 0 psi are $M_{R}=34735 \sigma_{d}^{-0.55268}, M_{R}=29218 \sigma_{d}^{-0.51989}$, and $M_{R}=18193 \sigma_{d}^{-0.36225}$ respectively. Predicted resilient moduli are also shown in Figure 10.17.

To compare the predicted values with measured values, predicted results by Equation 4.4 and Table 10.3 are plotted with measured resilient modulus in Figure 10.18 . It can be seen that the predicted relationships by both methods are similar and agree reasonably well with the measured values. 


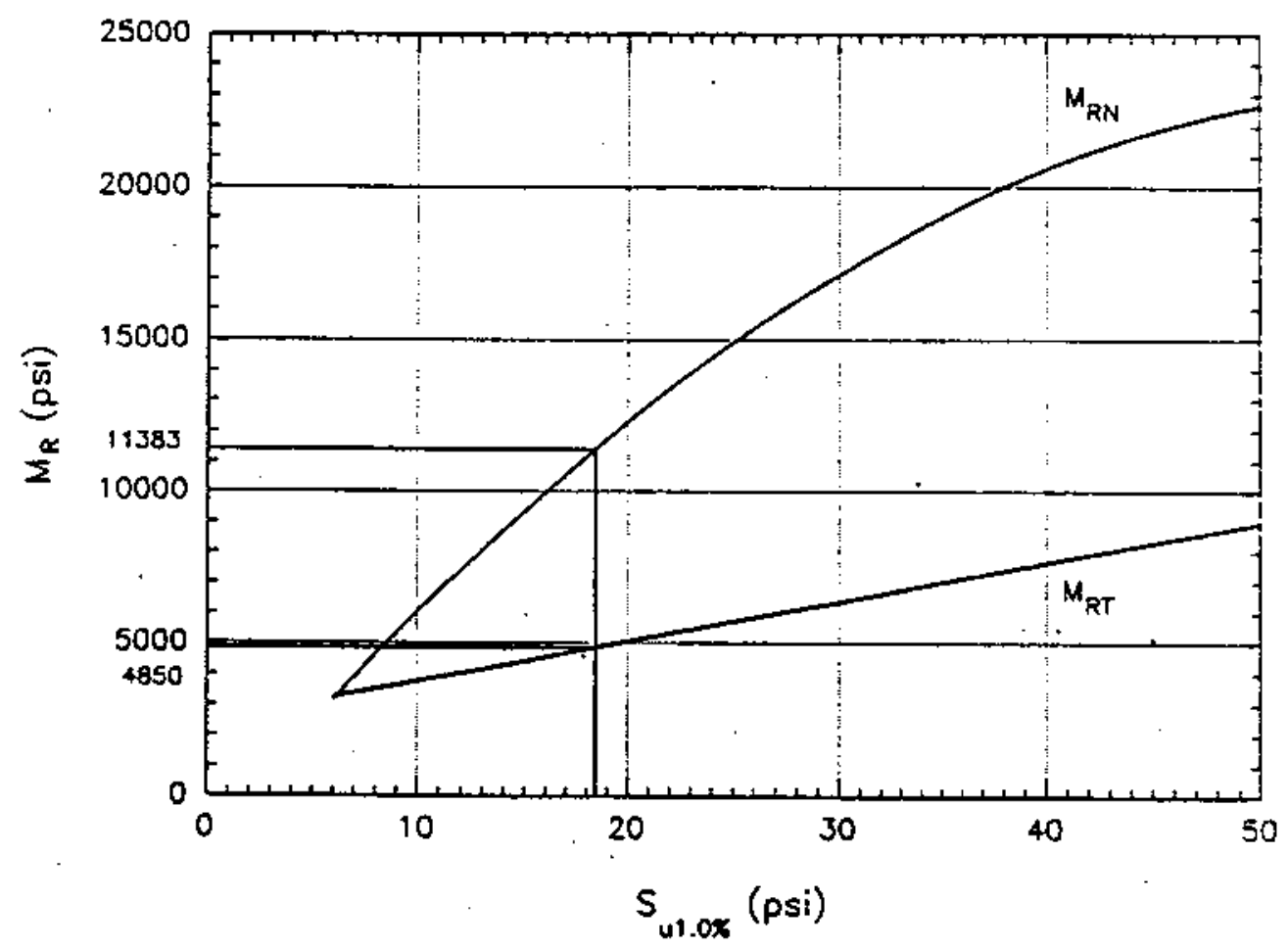

Figure 10.14 Estimation of $K_{\mathrm{RT}}$ for Example in section 10.10 .2 


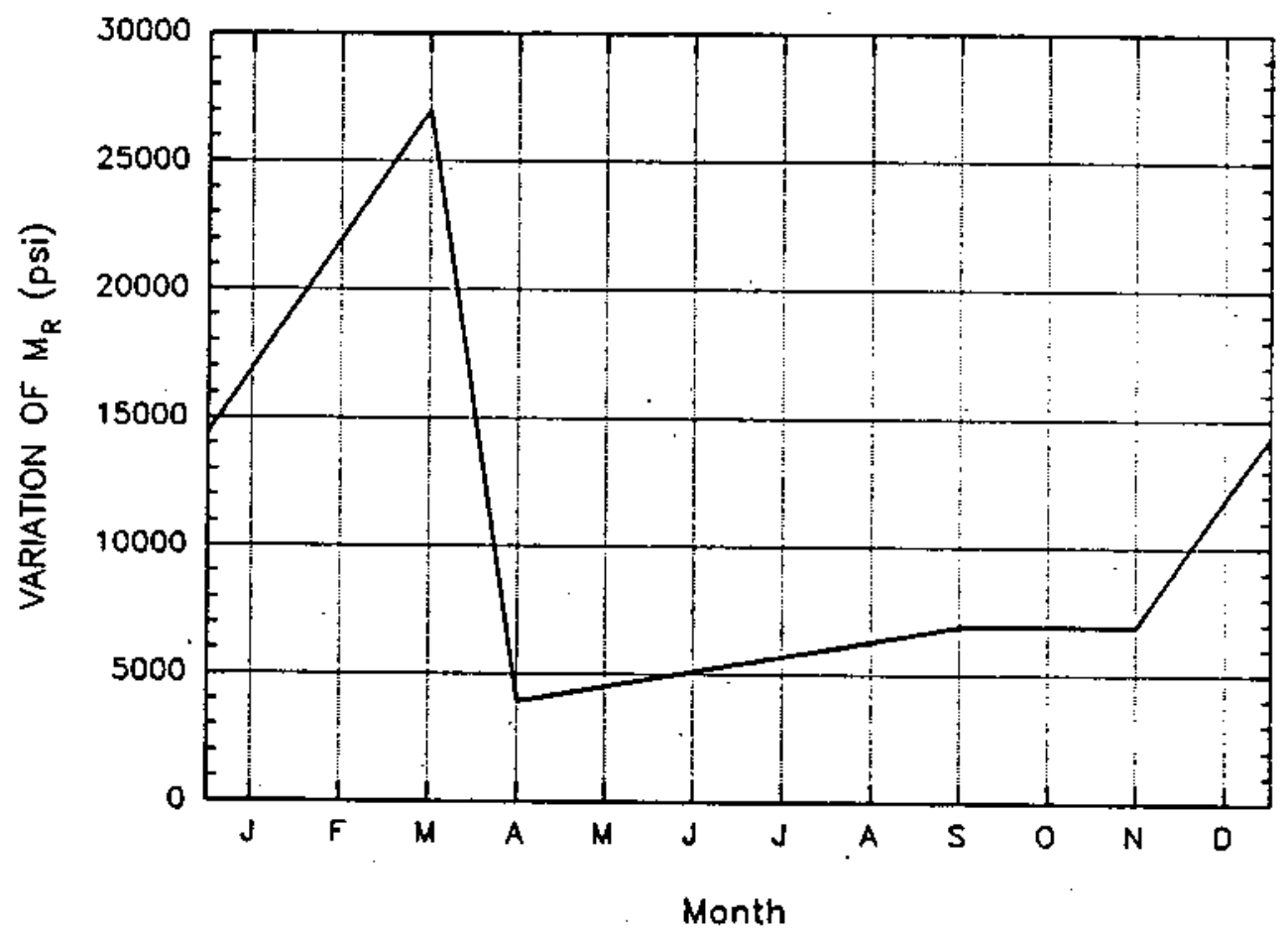

Figure 10.15 Seasonal Variation of Resilient Modulus for Example in Section 10.10.2 


\begin{tabular}{|c|c|c|}
\hline Month & $\begin{array}{c}\text { Subgrade } M_{\mathrm{R}} \\
\text { (psi) }\end{array}$ & $\begin{array}{c}\text { Relative Damage } \\
\mathbf{u}_{\mathrm{f}}\end{array}$ \\
\hline Jan. & 19190 & 0.014 \\
Feb. & 23100 & 0.009 \\
Mar. & 27000 & 0.006 \\
Apr. & 4850 & 0.332 \\
Mar. & 6160 & 0.191 \\
June & 7460 & 0.121 \\
July & 8770 & 0.084 \\
Aug. & 10070 & 0.061 \\
Sep. & 11380 & 0.046 \\
Oct. & 11380 & 0.046 \\
Nov. & 11380 & 0.046 \\
Dec. & 15290 & 0.023 \\
\hline
\end{tabular}

$$
\begin{gathered}
u_{f}=1.18 \times 10^{8} \times M_{R}^{-2.32} \\
\bar{u}_{f}=\frac{\Sigma u_{t}}{n}=\frac{0.980}{12}=0.0817 \\
M_{R E}=\sqrt{\frac{1.18 \times 10^{8}}{\bar{u}_{f}}}=8,880 p s i
\end{gathered}
$$

Figure 10.16 Estimation of Effective Subgrade $M_{R}$ for Example
in Section 10.10 .2 


\begin{tabular}{|c|c|c|c|c|c|c|}
\hline \multirow{2}{*}{$\begin{array}{r}\sigma_{3} \\
\text { (psi) }\end{array}$} & \multirow{2}{*}{$\begin{array}{r}\sigma_{d} \\
(p g i)\end{array}$} & \multicolumn{3}{|c|}{ Regression parameters } & \multicolumn{2}{|c|}{ Predicted $M_{R}$} \\
\hline & & $\mathbf{a}$ & b & c & Eqn. 4.4 & $\mathrm{Tbl} 1.10 .3$ \\
\hline 6 & 2 & -3289.92 & 1808.998 & -21.4597 & 22730 & 23681 \\
\hline 6 & 3 & -2658.54 & 1413.663 & -15.0910 & 18244 & 18927 \\
\hline 6 & 4 & -2287.64 & 1187.214 & -11.5754 & 15638 & 16144 \\
\hline 6 & 5 & -2038.04 & 1037.146 & -9.3147 & 13892 & 14271 \\
\hline 6 & 6 & -1856.14 & 928.921 & -7.7260 & 12620 & 12903 \\
\hline 6 & 7 & -1716.41 & 846.421 & -6.5427 & 11643 & 11849 \\
\hline 6 & 8 & -1604.98 & 781.012 & -5.6239 & 10862 & 11006 \\
\hline 6 & 9 & -1513.59 & 727.612 & -4.8881 & 10220 & 10313 \\
\hline 6 & 10 & -1436.98 & 683.016 & -4.2847 & 9680 & 9729 \\
\hline 3 & 2 & -2545.68 & 1542.024 & -17.9856 & 19738 & 20377 \\
\hline 3 & 3 & -2138.68 & 1227.384 & -12.9396 & 16064 & 16504 \\
\hline 3 & 4 & -1892.92 & 1044.798 & -10.1121 & 13908 & 14212 \\
\hline 3 & 5 & -1724.34 & 922.624 & -8.2730 & 12451 & 12655 \\
\hline 3 & 6 & -1599.66 & 833.832 & -6.9683 & 11384 & 11511 \\
\hline 3 & 7 & -1502.75 & 765.705 & -5.9885 & 10559 & 10624 \\
\hline 3 & 8 & -1424.67 & 711.390 & -5.2223 & 9897 & 9912 \\
\hline 3 & 9 & -1360.08 & 666.828 & -4.6048 & 9351 & 9323 \\
\hline 3 & 10 & -1305.52 & 629.449 & -4.0953 & 8890 & 8826 \\
\hline 0 & 2 & 842.19 & 854.758 & -9.5604 & 13333 & 14153 \\
\hline 0 & 3 & 319.24 & 744.848 & -7.5743 & 11460 & 12220 \\
\hline 0 & 4 & 18.57 & 678.014 & -6.4021 & 10327 & 11011 \\
\hline 0 & 5 & -181.51 & 631.725 & -5.6084 & 9543 & 10156 \\
\hline 0 & 6 & -326.44 & 597.153 & -5.0265 & 8959 & 9506 \\
\hline 0 & 7 & -437.42 & 570.019 & -4.5769 & 8501 & 8990 \\
\hline 0 & 8 & -525.81 & 547.966 & -4.2165 & 8129 & 8566 \\
\hline 0 & 9 & -598.29 & 529.566 & -3.9195 & 7819 & 8208 \\
\hline 0 & 10 & -659.10 & 513.901 & -3.6695 & 7554 & 7900 \\
\hline
\end{tabular}

Figure 10.17 Estimated Regression Parameters and Predicted Resilient Modulus 


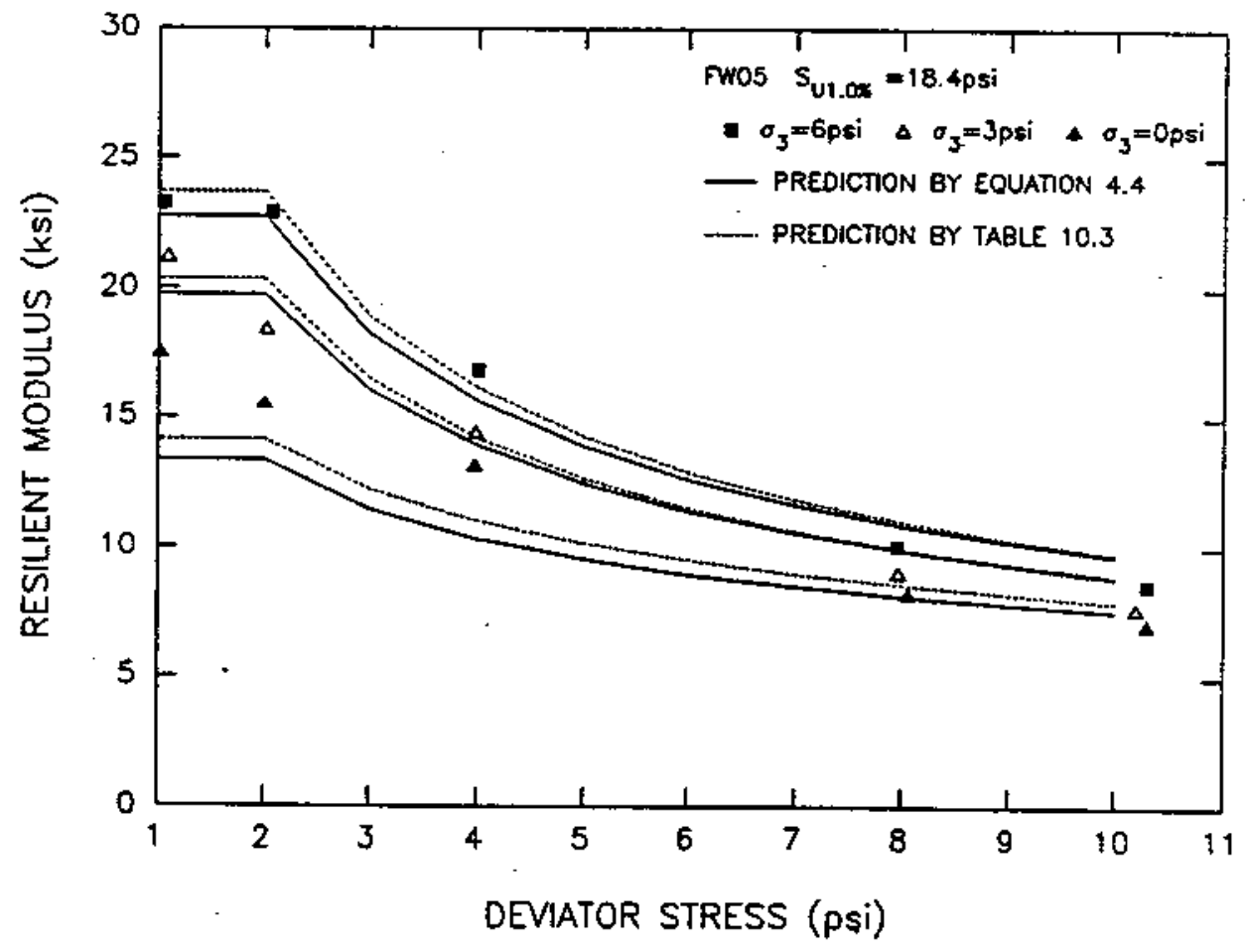

Figure 10.18 Measured and Predicted Relationship between $M_{R}$ and $\sigma_{d}$ for Specimen FW05 


\section{CONCLUSIONS AND RECOMMENDATIONS}

11. 1 Conclusions

From the test results on five cohesive soils and a granular soil typically used as subgrade materials in Indiana, following conclusions are drawn:

(1) The developed system and procedure for resilient modulus test are reliable and give good repeatability of test results.

(2) The in-service condition of cohesive subgrade approaches the 90 percent to 100 percent degree of saturation after several years of service, regardless of as-compacted condition.

(3) Field compaction for the South Bend and Washington sites produces dry densities lower than that by laboratory Standard Proctor compaction, while the Fort Wayne site displays similar ary densities by field and laboratory standard Proctor compaction.

(4) Parameters from. unconfined compression test are good indicators of resilient modulus while the in-service water content and dry density show a scattered relationship with the resilient modulus.

(5) For laboratory compacted cohesive soils, the relationship between $M_{R}$ and $S_{w 1.0 *}$ for a given soil is unique regardless of compaction water content and compactive effort. The relationship is similar for three sites tested and is believed similar for all five sites. The prediction equation developed is presented in equation 4.7.

(6) In-service resilient modulus of the fleld compacted finegrained soils. is uniquely related to $s_{u 1.0 \%}$, regardless of 
soil type. The effect of confining pressure on the resilient modulus increases as $S_{\mathrm{ul} .0 \%}$ increases. The relationship between $M_{R}$ and $S_{v 1.0 x}$ for in-service subgrade is given in equation 4.4 and 4.8 .

(7) The resilient modulus of in-service subgrade can not be estimated from that of laboratory compacted soil without considering the effect of water content change after construction. The as-compacted resilient modulus may be represented by that of laboratory compacted soil.

(8) The relationship itself between $k_{R}$ and $S_{v 1.0 \%}$ is believed not to be affected by the change of water content and dry density after construction. The relationship is slightly different between laboratory and field compacted soils. Therefore, the equation 4.4 and 4.8 may be used for the estimation of as-compacted resilient modulus as well as in-service resilient modulus.

(9) An addition of $S_{u 1.0 x}$ to the current practice of compaction specification will allow field engineers to better control the resilient property of the constructed subgrade. This will reduce fatigue cracks of pavement.

(10) The closed-system freeze-thaw testing procedure developed simulates the freeze-thaw occurring in the field reasonably well.

(11) The resilient modulus of cohesive soil is significantly reduced by only one cycle of freeze-thaw process. There is a negligible effect of freeze-thaw for soils having $S_{u 1.0 x}$ less than $8 \mathrm{psi}$, while the effect of freeze-thaw becomes larger as $s_{u 1.0 x}$ increases.

(12) Relationships between thaw-weakened resilient modulus and $S_{\text {vi.0x }}$ for five sites tested fit into single one. Equations 5.1 and 5.3 can be used to predict the thawed resilient modulus using $S_{w 1.0 x}$ from the unconfined compression test performed before the freeze-thaw of specimen.

(13) The relationship of $M_{R}$ with $S_{w 1.0 x}$ of thaw-weakened soil is similar with that between $M_{q}$ and $S_{v 1.0 \pi}$ of soil which has 
not been subjected to freeze-thaw. Therefore, the thawed resilient modulus can be estimated from equations 4.4 and 4.8 if the unconfined compression test is carried out on the specimen obtained from thawed subgrade.

(14) The cohesive soils show a decrease in resilient modulus with increase in water content after construction. The change in resilient modulus depends on the soil type.

(15) The resilient modulus of frozen cohesive soil is independent of the deviator stress and is constant.

(16) At same water content and dry density, the resilient modulus of vibratory compacted dune sand is larger than that of impact compacted dune sand. The permanent strain of vibratory compacted specimen is far smaller than that of impact compacted specimen.

(17) The resilient property of dune sand compacted with same compactive effort is similar, regardless of compaction water content, while the permanent strain increases as the water content increases.

(18) For dune sand having relative compaction between 95 and 103 percent, the resilient modulus can be estimated using equation 6.9 or Figure 10.5.

(19) Pore-size distribution gives a measure of fabric as a non-vector quantity and good reproducibility can be achieved on pore-size data.

(20) Fabric of compacted cohesive soil is significantly influenced by the molding water content. Dry and wet side fabrics are significantly different.

(21) Pore-size data can be used to compare the fabric of two different cohesive solls. If the pore-size distribution of two cohesive soils is different than their fabric is also different.

(22) Fabric parameters of an uncompacted cohesive soil can be correlated with the maximum attainable dry unit weight (at certain energy level) and the clay fraction present in the soil. 
(23) For cohesive soils, impact compaction gives less variability in the pore size data compare to the kneading compaction.

(24) For A-7-6, A-7-5, and A-6 soils, laboratory impact compaction at standard Proctor energy and optimum or slightly higher (about 1 percent wet) water content is capable of creating the field compacted fabric. similarity between the fabrics created by this method becomes less pronounced as the clay fraction present in the soil decreases.

(25) Field compacted fabric for the A-4 and A-6 soils can not be replicated by the method suggested in (24). Application of Modified Proctor energy reduces the differences between two fabrics. However, a greater degree of similarity can not be achieved even with the Modified Proctor energy.

(26) Resilient modulus of the compacted subgrade for soils with higher clay fraction can be predicted by the fabric parameters and the molding water content (Equations 9.1 and 9.2).

(27) Increase in water content after construction does not affect the as-compacted fabric and the equilibrium water content can be estimated from the field compacted pore size curve.

(28) Resilient modulus of the laboratory compacted cohesive soils is significantly influenced by the clay fraction (or microporosity).

\subsection{Recommendations for Future Research}

(1) It is recommended to explore the possibility of developing an equipment and technique capable of increasing water content after compaction beyond the moderate increase achieved by the present technique, and to study further the effect of change in water content after construction on the resilient modulus. 
(2) It is potentially useful to obtain the field data on the change of temperature in pavement subgrades to establish if refinements are required to the data of this study.

(3) An effort should be made to encourage pavement analysts to study the viability of a rational basis for what is the limiting deflection of pavement surface that best relates to good pavement performance.

(4) Further fabric studies are required to prepare compaction specifications based on the difference in the fabric of the uncompacted and the compacted soil. A field compaction may be targeted to achieve a certain fabric rather than the conventional dry unit weight criterion. 
LIST OF REFERENCES 
LIST OF REFERENCES

AASHTO Guide for Design of Pavement structures. (1986). Amer. Assoc. of State HWY, and Transp. Officials, Washington, D.C.

Ahmed, S., Lovell, C. W., and Diamond, S. (1974). "Pore size and strength of Compacted Clay." J. of Geotech. Engrg., ASCE, vol. 100, No. 4, 407-425.

Allen, J. J., and Thompson, M. R. (1974). "Resilient Response of Granular Materials Subjected to Time Dependent Lateral Stresses." Transp. Res. Record 510, 1-13.

The American Geological Institute. (1957) . Glossary of Geology and Related Sciences, $104 \mathrm{pp}$.

Andersland, O. B., and Anderson, D. K. (1978). Geotechnical Engineering for Cold Regions, McGraw-Hill Co.

Baladi, G. Y., Vallejo, L. E., and Goitom, T. (1983). "Normalized Characterization Model of Pavement Materials." Properties of Flexible Pavement Materials, ASTM STP 807, ASTM,
55-64.

Barden, L., and sides, G. R. (1970) . "Engineering Behavior and Structure of Compacted clay." J. Soil Mech. Found. Engrg., ASCE, Vol. 96, No. 4, 1171-1200.

Barden, L. (1972). "The Relation of soll structure to the Engineering Geology of Clay Soll." Quart. J. of Engrg. Geol.,

Barksdale, R. D. (1984) . "Performance of Crushed-stone Base Courses." Transp. Res. Record 954, 78-87.

Barksdale, R. D. (1986). "Performance of Base courses." Proc. on Solutions for Pavement Rehabilitation Problems, ASCE, 97-
112 .

Barksdale, R. D. (1971). "Compressive stress Pulse Times in Flexible Pavements for Use in Dynamic Testing." HwY. Res. Baver, I. D. (1964). Soll Physics, 2nd Ed., John wiley and
Sons, N.Y. 
Bergan, A. T., and Fredlund, D. G. (1973). "Characterization of Freeze-Thaw Effects on Subgrade Soils." Symp. on Frost Action on Roads, Organization for Economic cooperation and Development, Oslo, Norway, Vol.2, 119-143.

Bergan, A. T., and Monismith, C. L. (1973). "Characterization of Subgrade soils in cold Regions for Pavement Design Purposes." Hwy. Res. Record 431, 25-37.

Bergan, A. T., and culley, R. W. (1973). "Some Fatigue Considerations in the Design of Asphalt Concrete Pavements." Symp. on Frost Action on Roads, Organization for Economic Cooperation and Development, Oslo, Norway, Vol. 2, 37-77.

Boateng-Poku, Y., and Drumm, E. C. (1989). "Hyperbolic Model for the Resilient Modulus Response of Fine-Grained Subgrade Soll." Resilient Moduli of Soils: Laboratory Conditions, Geotechnical special Publication 24, ASCE, 1-14.

Bohra, N. C. (1993). "Use of Pore-size Distribution-The Control of Subgrade Resilient Modulus." PhD Thesis, purdue University, West Lafayette, Indiana.

Brakel, J. V., Modry, S., and svata, M. (1981). "Mercury Porosimetry: State of the Art." Powder Technology, Vol. 29, 112 .

Brewer, R. (1964). Fabric and Mineral Analysis of Sojls, John Wiley, New York.

Brodsky, N. S. (1989). "Resilfent Modulus Measurements on Cohesive Soils as a Function of Deviator Stress and Confining Pressure." Resilient Moduli of Solis: Laboratory Conditions, Geotechnical Special Publication 24, ASCE, 15-30.

Brown, S. F., and Pell, P. S. (1967). "Subgrade stress and Deformation under Dynamic Load." $\mathrm{J}$. of Soil Mech. Found. Engrg., ASCE, Vol. 93, No. 1, 17-46.

Brown, S. F., Lashine, A. R. F., and Hyde, A. F. L. (1975). "Repeated Load Triaxial Testing of a silty clay." Geotechnique, Vol. 25, No. 1, 95-114.

Brown, S. F., and Selig, E. T. (1991). "Chap. 6. The Design of Pavement and Rail Track Foundations." in cyclic Loading of Solls: from theory to design, Edited by o'Reilly, M. P., and Brown, s. F., Van Nostrand Reinhold, 249-305.

Brown, S. F. (1974). "Repeated Load Testing of a Granular Material." J. Geotech. Engrg., ASCE, Vol. 100, No. 7, 825-841. 
Brown, S. F., and Hyde, A. F. L. (1974). "Significance of Cyclic Confining stress in Repeated-Load Triaxial Testing of Granular Material." Transp. Res. Record 510, 49-58.

Carmichael III, R. F., and stuart, E. (1985). "Predicting Resilient Modulus: A study to Determine the Mechanical Properties of Subgrade Soils." Transp. Res. Record 1043, 145-
148 .

Casagrande, A. (1932). "The structure of clay and Its Importance in Foundation Engineering." $\mathrm{J}$. Boston Society of Civ. Engrs., Vol. 19, No. 4, 168-209.

Chamberlain, E. J. (1987). "A Freeze-Thaw Test to Determine the Frost susceptibility of Soils." U. S. Army Cold Regions Research and Engineering Laboratory, CRREL Special Report 87-

Chamberlain, E. J. (1986). "Evaluation of Selected FrostSusceptibility Test Methods." U. S. Army Cold Regions Research and Engineering Laboratory, CRREL Report 86-14.

Chamberlain, E. J., Cole, D. M., and Johnson, T. C. (1979). "Resilient Response of Two Frozen and Thawed Soils." J. Geote. Engrg., ASCE, Vol. 105, No. 2, 257-271.

Chamberlain, E. J.., Gow,w. J. (1979). "Effect of Freezing and Thawing on the Permeability and structure of Soils." Engrg.
Geol., vol. 13, 7.3-92.

Chamberlain, E. J. (1981) . "Frost susceptibility of soil." $U$. S. Army Cold Regions Research and Engineering Laboratory.

Chamberlain, E. J. (1973). "A Hodel for predicting the influence of closed system Freeze-Thaw on the Strength of Economic Cooperation Frost Action on Roads, Organization for Chisoln, 94-97.

Chisolm, E. E., and Townsend, F. C. (1976). "Behavioral Characteristics of Gravelly Sand and crushed Limestone for Aviation Admint" U. S. Department of Transportation, Federal A

Clark, J. I., and Gillott, J. E. (1985). "The Role of Composition and Fabric of solis in Selected Geotechnical Elsevier science Publication, 173-191. 
claros, G., Hudson, W. R., and stokoe, K. H. (1989). "Modifications to the Resilient Modulus Testing Procedure and the Use of Synthetic Samples for Equipment Calibration." 69 th Annual Meeting, TRB.

Cole, D. M., Durell, G., and Chamberlain, E. (1985) . "Repeated Load Triaxial Testing of Frozen and Thawed Soils." Geotech. Test. J., ASTM, Vol. 8, No. 4, 166-170.

Cole, D., Bentley, D., Durell, G., and Johnson, T. (1987). "Resilient Modulus of Freeze-Thaw Affected Granular Soils for Pavement Design and Evaluation. Part 3: Laboratory Tests on Soils from Albony County Airport." U. S. Army Cold Regions Research and Engineering Laboratory, CRREL Report 87-2.

Cole, D. M., Irwin, L. H., and Johnson, T. C. (1981). "Effect of Freezing and Thawing on Resilient Modulus of a Granular Soil Exhibiting Nonlinear Behavior." Transp. Res. Record 809, 19-26.

Cole, D., Bentley, D., Durell, G., and Johnson, T. (1986). "Resilient Modulus of Freeze-Thaw Affected Granular Soils for pavement Design and Evaluation. Part 1: Laboratory Tests on Soils from winchendon, Massachusetts, Test Sections." U. S. Army Cold Regions Research and Engineering Laboratory, CRREL Report 86-4.

Collins, K., and McGown, A. (1974). "The Form and Function of Microfabric in a Variety of Natural Soils." Geotechnique, Vol. 24, No. $2,223-254$.

Croney, D. (1977). "Chap. 13, The Behavior of Road Materials Under Repeated Loading." in The Design and Performance of Road Payements, FMso, London, 367-428.

Culley, R. w. (1971). "Effect of Freeze-Thaw Cycling on Stress-strain Characteristics and volume Change of $a$ Till Subjected. to Repetitive Loading." Can. Geotech. J., Vol. 8, No. $3,359-371$.

Dehlen, G. L. (1969). "The Effect of Non-linear Material in the Behavior of Pavements subjected to Traffic Loads." Ph.D. Thesis, University of California, Berkeley.

Dempsey, B. J., and Elzeftawy, A. (1977). "Mathematical Model for Predicting Moisture Movement in Pavement Systems." Transp. Res. Record 612, 48-55.

Diamond, S. (1970). "Pore size Distributions in clays." clays and Clay Minerals, Vol. 18, 7-23.

Diamond, S. (1971). "Microstructure and Pore structure of Impact-Compacted Clays." Clays and Clay Minerals, Vol. 19, 239-249. 
Drumm, E. C., Boateng-Poku, Y. and Pierce, T. J. (1990). "Estimation of Subgrade Resilient Modulus from standard Tests." J. Geotech. Engrg., ASCE; Vol. 116, No. 5, 774-789.

Dudley, J. H. (1970). "Review of Collapsing soils" J. Soil Mech. Found. Engrg., ASCE, Vol. 96, No. 3, 925-947.

Duncan, J. M., and Chang, C. Y. (1970). "Nonlinear Analysis of Stress and strain in Soils." J. Soil Mech. Found. Engrg., ASCE, Vol. 96, No. 5, 1629-1653.

Edris, E. V., and Lytton, R. L. (1976). "Dynamic Properties of Subgrade Solls, Including Environmental Effects." Texas Transp. Institute Research Report No. 164-3.

Elliott, R. P., and Thompson, M. R. (1985). "Mechanistic Design Concepts for Conventional Flexible Pavements. "Transp. Engrg. Series No. 42, Dept. of Civil Engrg., Univ. of Illinois, Unbana Champaign.

Elliott, R. P., and Thornton, S. I. (1988) . "Simplification of Subgrade Resilient Modulus Testing. " Transp. Res. Record 1192,
1-7.

Elliott, R. P., and Thornton, S. I. (1988). "Resilient Modulus and AASHTO Pavement Design." Transp. Res. Record 1196, 116-

Elllott, R. P., and David, L. (1989). "Improved Characterization Model for Granular Bases." Transp. Res. Record 1227, 128-133.

Espinoza, R. D., Bourdeau, P. L., and white, T. D. (1993). "Pavement Drainage and Pavement shoulder Joint Evaluation and Rehabilitation: Analytical and Numerical Modeling of Moisture Infiltration and Drainage." Draft Final Report for JHRP Project No. 2018, Purdue Univ., West Lafayette, IN.

Farrar, M. J., and Turner, J. P. (1991). "Resilient Modulus of Wyoming Subgrade Soils." Mountain-Plains Consortium Report No.
$91-1$.

Finn, $F$, N., Nair, K., and Monismith, c. L. (1972). "Application of Theory in the Design of Asphait Pavements." Third Int'l Conf. on the structural Design of Asphait Pavements, Vol. 1, 392-409.

Fredlund, D. G., Bergan, A. T., and sauer, E. K. (1975). "Deformation Characterization of subgrade Soils for Highways and Runways in Northern Environments." Can. Geotech. J., Vol. 
Fredlund, D. G., Bergan, A. T., and wong, P. K. (1977). "Relation Between Resilient Modulus and Stress Conditions for Cohesive Subgrade Soils." Transp: Res, Record 642, 73-81.

Garcia-Bengochea I., Lovell, C. W:, and Altschaeffl, A. G. (1979) . "Pore Distribution and Permeability of silty clays." J. Geotech. Engrg., ASCE, Vol. 105, No. 7, 839-856.

Gaskin, P. N., Raymond, G. P., and Addo-Abedi, F. Y. (1979) . "Repeated Compressive Loading of a Sand." Can. Geotech. $\mathrm{J}$., vol. 16, 798-802.

George, K. P. (1992) " Resilient Testing of Soils Using Gyratory Testing Machine." 71st Annual Meeting, TRB.

Gibbons, J. D. (1985) . Nonparametric Hethods for Quantitative Analysis, Second Ed., American Sciences Press, Syracuse, NY.

Grainger, G. D., and Lister, N. W. (1962). "A Laboratory Apparatus for studying the Behavior of Soils under Repeated Loading." Geotechnique, Vol. 12, No. 1, 3-14.

Griffiths, F. J., and Joshi, R. C. (1989) "Change in Pore Size Distribution Due to Consolidation of clays." Geotechnique, Vol. 39, No. 1, 159-167.

Hamilton, A. B. (1966). "Freezing Shrinkage in Compacted Clays." Can. Geotech. J., vol. 3, No. 1, 1-17.

Haynes, J. H., and Yoder, E. J. (1963). NEffects of Repeated Loading on Gravel and Crushed Stone Base Course Materials Used in the AASHTO Road Test." Hwy. Res. Record 39, 82-96.

Heuklelom, W., and Klopm, A. J. G. (1962). "Dynamic Testing as a Means of Controlling Pavement During and After Construction." Proc. 1st. Int'1. Conf. on Structural Design of Asphalt Pavement, Univ. of Michigan, Ann Arbor.

Hicks, R. G., and Monismith, c. L. (1971). "Factors Influencing the Resilient Response of Granular Materials." Highway Res. Record 345, 15-31.

Highway Research Board. (1962). The AASHO Road Test. Report 6 . Special studies. Special Report 61F.

Hodek, R. J., and Lovell, C. H. (1979). "A New Look at Compaction Process in Filis." Bull. Association of Engrg. Geol., Vol. 16, No. 4, 487-499.

Holtz R. D.; and Kovacs W. D. (1981). An Introduction to Geotechnical Englneering, Prentice-Hall, Inc., Englewood cliffs, NJ. 
Hsu, S. Y., Vinson, T. S., and Hicks, R. G. (1983). "Determination of Resilient Properties of Unbound Materials with Diametral and Cyclic Triaxial Test systems." Properties of Flexible Pavement Materials, ASTM STP 807, ASTM, 65-83.

Indiana Department of Transportation. (1993). "standard Specifications." Section 203.23 and 203.24.

Janbu, N. (1963). "Soil Compressibility as Determined by Oedometer and Triaxial Tests." European Conf. On Soil Mech. and Found. Engrg., Wiesbaden, Germany, Vol. 1, 19-25.

Jenny, H. (1941) Factors of Soil Formation: A system of Quantitative Pedology, First Ed., McGraw-Hill Book Company,

Jin, Myung-Sub, Lee, K. W., and Kovacs, D. (1992). "Field Instrumentation and Laboratory study to Investigate Seasonal Variation of Resilient Modulus of Granular Soils." 71 st Annual Meeting, TRB.

Johnson, T. C., Berg, R. L., and Dimillio, A. (1986). "Frost Action Predictive Techniques: An Overview of Research Results." Transp. Res. Record 1089, 147-161.

Johnson, T. C., Cole, D. M., and Chamberlain, E. J. (1979). "Effect of Freeze-Thaw Cycles on Resilient properties of FineGrained Solis." Engrg. Geol., Vol. 13, 247-276.

Johnson, A. W., and Sallberg, j. R. (1960). "Factors that Influence Field Compaction of solis: compaction Characteristics of Field Equipment." Hwy. Res. Board Bull. 272, HRB.

Johnson, A. W., and sallberg, J. R. (1962). "Factors Influencing Compaction Test Results." Hwy. Res. Board Bull. 319, HRB.

Jones, M. P., and witczak, H. W. (1977). "Subgrade Modulus on the San Diego Test Road." Transp. Res. Record 641, 1-6.

Juang, C. H. and Holtz, R. D. (1986). "A Probabilistic Permeability Model and the Pore size Density Function." Int'l $J$. of Numerical and Analytical Methods in Geomechanics, Vol. 10, 543-553.

Juang, C. H., and Holtz, R. D. (1986). "Fabric, Pore size Distribution, and Permeability of Sandy Soils." J. Geotech. Engrg., ASCE, Vol. 112, No. 9, 855-868.

Kalcheff, I. V., and Hicks, R. G. (1973). "A Test Procedure for Determining the Resilient Properties of Granular Materials." J. of Testing and Evaluation, ASTM, Vol. 1, No. 6, 
Kallas, B. F., and Riley, J. C. (1967). "Mechanical Properties of Asphalt Pavement Materials." Proc. 2nd Int' 1 Conf. on the structural Design of Asphalt Pavements, Univ, of Michigan.

Kaplax, C. W. (1970). "Phenomenon and mechanism of Frost Heaving." Hwy. Res. Record 304, 1-13.

Khedr, S. (1985). "Deformation Characteristics of Granular Base Course in Flexible Pavements." Transp. Res. Record 1043, 131-138.

Kim, w. H., and Daniel, D. E. (1992). "Effects of Freezing on Hydraulic Conductivity of Compactive Clay." $\mathrm{J}$. of Geotech. Engrg., ASCE, Vol. 118, No. 7, 1083-1097.

Knutson, R. M., Thompson, M. R. (1977). "Resilient Response of Railway Ballast." Transp. Res. Record 651, 31-39.

Kondner, R. L. (1963) "Hyperbolic stress-strain Response: Cohesive Soils." J. Soil Mech. Found. Engrg., ASCE, Vol. 89, No. 1, 115-143.

Konrad, J. M. (1989). "Physical Process During Freeze-Thaw Cycles in clayey Silts." Cold Regions Science and Technology,

Konrad; J. M. (1991) . "Chap. 18. The Physics of Freezing Soils and an Engineering Frost Heave Approach." in Freezing and Melting Heat Transfer in Engineering-Selected Topics on IceWater systems and Welding and Casting Processes, Edited by Cheng, K. C., and Seki, N., Hemisphere Publishing Corporation, New York, 581-612.

Lambe, T. W. (1958). "The structure of compacted clay." J. Soil Mech. Found. Engrg., ASCE, Vol. 84, No. 2.

Lambe, T. W. (1958). "The Engineering Behavior of Compacted Clay." $J$. Soil Hech. Found. Engrg., ASCE, Vol. 84, No. 2.

Lambrechts, J. R., and Leonards, G. A. (1978). "Effects of Stress History on Deformation of Sand." $\mathrm{J}$. Geotech. Engrg., ASCE, Vol. 104, No. 11, 1371-1387.

Larew, H. G., and Leonards, G. A. (1962). "A strength Criterion for Repeated Loads." Proc., Hwy. Res. Board, Vol.
41, 529-556.

Lary, J. A., and Mahoney, J. P. (1984). "Seasonal Effects on the Strength of Pavement Structures." Transp. Res. Record 954 ,
88-93.

Lee, R. L., and Haley, S. C. (1968). "strength of compacted clay at High Pressure." J. Soil Mech. Found. Engrg., ASCE, 
Lee, W. J. ' Bohra, N. C., and Altschaeffl, A. G. (1992). standard Tests." J. Geotech of Subgrade Resilient Modulus from 352-354.

Lenker, S. E. (1992). Final Report on the Analysis of Data Received From an Interlaboratory study of the proposed Standard Method for Resilient Modulus of Subgrade Soils and Untreated Base/Subbase Materials, Prepared for the AASHTO Highway Subcomittee on Materials Technical Section 1b: Engineering Geology, structural Soils, Soil practices.

Leroueil, S., Tardif, J., Roy, M., La Rochelle, P., and Konrad, J. M. (1991). "Effects of Frost on the Mechanical 690-697.

Leshchinsky, D., and Rawlings, D. L. (1988). "Stress Path and Permanent Deformations in sand Subjected to Repeated Load." Geotech. Test. J., ASTM, Vol. 11, No. 1, 36-43.

Ii, J. C., Baladi, G.. Y., and Andersland, O. B. (1979). "Cyclic Triaxial Tests on Frozen Sand." Engrg. Geol., Vol. 13,

Lotfi, H., and Witczak, M. W. (1985). "Dynamic Materials." Transp. Res. Cement-Treated Base and subbase

Marshall, T. J. (1958). "A Relation Between Permeability and Size Distribution of Pores." J. of Soil Sciences, Vol. 9, No.
$1,1-8$.

McVay, M., and Taesiri, Y. (1985). "Cyclic Behavior of Pavement Base Materials." J. Geotech. Engrg., ASCE, Vol. 111,

Mickleborough, B. W. (1970). "An Experimental study of the -Effects of Freezing on Clay Subgrades." MSCE Thesis, Univ. of Mikhailov, G. D., and Bredyuk, G. P. (1971). "Shear Strength of clayey Ground during Thawing." U.S. Army Cold Reg. Res.

Mitchell, J. K. (1960). "Fundamental Aspects of Thixotropy in 52.

Mitchell, J. K. (1976) Fundamentals of Soil Behavior, John
Wiley and Sons, Inc., $422 \mathrm{pp}$. 
Modry, S., Svata, M., and Brakel, J. V. (1981). "Thematic Bibliography of Mercury Porosimetry. " Powder Technology, Vol.
29 , No. 13, 13-43.

Monismith, C. L., Hicks, R. G., and Salam, Y. M. (1971). "Basic Properties of Pavement Components." U. S. Department of FHWA-RD-72-19.

Monismith, C. L., Seed, H. B., Mitry, F. G., and Chan, C. K. (1967): "Prediction of Pavement Deflections from Laboratory Tests." 2nd Int'l Conf. on the structural Design of Asphalt

Moossazadeh, J., and Witczak, M. W. (1981). "Prediction of Subgrade Moduli for Soil that Exhibits Nonlinear Behavior."
Transp. Res. Record 810, 9-17.

Nagpal, N. K., Boersma, L., and DeBaker, I. W. (1972) . "Pore Size Distributions for soils from Mercury Intrusion 36, 264-267.

Nataatmadja, A., and Parkin, A. K. (1989). "Characterization of Granular Materials for Pavements." Can. Geotech. J., Vol. National Lime Association. (1987). Lime stabilization
Construction Manual. Bulletin 326.

Nwaboukei, S. O. and Lovell, C. H. (1984). "Comparisons of Shear Characteristics of Laboratiry and Field-Compacted Soil."
Trans. Res. Record $998,63-71$.

O'Brien, N. R. (1971). "Fabric of Raolinite and rilite Floccules." Clay and Clay Minerals, Vol. 19, 353-359.

Olson, R. E. (1963). "Effective stress Theory of soil Compaction." J. Soll Hech. Found. Engrg., ASCE, Vol. 89, No.
2, 27-45.

Osipov, V. I. (1982). "Practical Application of the clay Microfabric studies." Proc. Fourth Congress Int'l Assoc. of Engineering Geology, Vol. II, New Delhi, II 239-II 251.

Othman, M. A., and Benson, C. H. (1992) . "Effect of FreezeThaw on the Hydraulic Conductivity of Three Compacted clays

Pagen, C. A., and Khosla, V. K. (1975). "Engineering Freeze-Thaw of Compacted clay conditioned by Saturation and Freeze-Thaw Cycles." Transp. Res. Record 532, 14-29. 
Penner, E., Johnston, G. H., and Goodrich, L. E. (1975). "Thermal Conductivity Laboratory studies of Some Mackenzie Highway Soils." Can. Geotech. J.; Vol. 12, No. 3, 271-289.

Pezo, R. F., Claros, G., and Hudson, w. R. (1992). "An Efficient Resilient Modulus Testing Procedure for subgrade and Non-Granular Subbase Materials." 71st Annual Meeting, TRB.

Prapaharan, S., White, D. M., and Altschaeffl, A. G. (1991). "Fabric of Field- and Laboratory-Compacted Clay," J. Geotech. Engrg., ASCE, Vol. 117, No. 12, 1934-1940.

Prapaharan, S., Altschaeffl, A. G., and Dempsey, B.J. (1985). "Molsture Curve of Compacted Clay: Mercury Intrusion Method." J. Geotech. Engrg., ASCE, Vol. 111, No. 9, 1139-1143.

Rada, G., and Witczak, K. W. (1981). "Comprehensive Evaluation of Laboratory Resilient Moduli Results for Granular Material." Transp. Res. Record 810, 23-33.

Raymond, G. P., Gaskin, P. N., and Addo-Abedi, F. Y. (1979). "Repeated Compressive Loading of Leda Clay." Can. Geotech. J.,
Vol. 16, No. 1, 1-10.

Reed, M. A., Lovell, C. W., Altschaeffl, A. G. and wood, I. E. (1979). "Frost Heaving Rate Predicted from The Pore Size Distribution." Canadian Geotech. J., Vol. 16, 463-472.

Ritter, H. L, and Drake, L. C. (1945). "Pore size Distribution in Porous Materials-Pressure Porosimeter and Determination of Engineering Macropore size Distribution." Industrial and Society, Vol. 17 ,

Robnett, Q. I. and Thompson, H. R. (1976). "Final ReportResilient Properties of Subgrade Soils. "Transp. Engrg. Series No. 14, Univ, of Illinois at Urbana-Champaign, Urbana, IL.

Robnett, Q. L., and Thompson, M. R. (1973) . "Interim ReportResilient Properties of Subgrade Soils: Phase 1-Development of Testing Procedure." Transp. Engrg. Series No. 5, Univ, of

Robnett, Q. L., and Thompson, M. R. (1976). "Effect of Lime Treatiment on the Resilient Behavior of Fine-Grained Soils."
Transp. Res. Record 560, 11-20.

Robnett, Q. L. (1986). "Subgrade Sţabilization." Proc. on Solutions for Pavement Rehabilitation Problems, ASCE, 119-134. 
Roy, M. , Crispin, J., Konrad, J. M., and Larose, G. (1992) . "A Field Study of Two Road Sections During a Freeze-Thaw Cycle."

Sangrey, D. A., Henkel, D. J., and Esrig, M. I. (1969). "The Effective Stress Response of a Saturated Clay soil to Repeated
Loading." Can. Geotech. J., vol. 6, 241-252.

Schmertmann, J. H. (1991). "The Mechanical Aging of Soils." J. of Geotech. Engrg., ASCE, Vol. 117, No. 9, 1288-1330.

Seed, H. B., Chan, C. K., and Lee, C. E. (1962), "Resilience Fatigue fharistics of subgrade Soils and Their Relation to Conf. on the Stru in Asphalt Pavements." Proc., First Int. Michigan, 611-636.

Seed, H. B., Chan, C. K., and Monismith, C. L. (1955). "Effects of Repeated Loading on the Strength and Deformation of Compacted clay." Proc., Hwy. Res. Board, Vol. 34, 541-558.

Seed, H. B., Mitry, F. G., Monismith, C. L., and Chan, C. K. (1967) "Prediction of Flexible Pavement Deflections From Laboratory Repeated-Load Tests." NCHRP Report 35, Highway
Research Board.

Seed, H. B. A and Chan, C. K. (1961). "Effect of Duration of Ltress Application on soil Deformation Under Repeated ISSMFE, Vol. 1, 341-345.

Seed, H. B., and Fead, J. W. N. (1960). MApparatus for Repeated Load Tests on Solls." Time Rates of Loading in soil

Seed, H. B., and Chan, C. K. (1959): "Undrained strength of Compacted clays After Soaking." J. Soll Mech. Found. Engrg., Seed, H. B.' and chan, c. K. (1957). "Thixotropic Engrg., ASCE, Vol. 83, No. 4. Clays:" J. Soll Mech. Found.

Seed, H. B., and Chan, C. K. (1958). "Effect of stress History and Frequency of stress Application on Deformation of Clay Vol. 37, 555-575.

Seed, H. B., and Chan, C. K. (1959). "structure and strength Characteristics of Compacted Clays," J. Soil Mech. Found.
Engrg., ASCE, vol. 85, No. 5, 87-128. 
Selig, E. T. (1971). "Unified system for Compactor Performance Specification." Paper No. 710727, National Farm, Construction and Industrial Machinery Meeting, Society of Automotive

Shaw, P., and Brown, S. F. (1988). "Behavior of Dry Granular Materials under Repeated Load Biaxial and Triaxial Stress Conditions." Geotechnique, vol. 38, No. 4, 627-634.

Smith, W. S., and Nair, K. (1973). "Development of Procedures for Characterization of Untreated Granular Base Course and Asphalt-Treated Base Course Materials" U. S. Department of Transportation, Federal Highway Administration, Report No.

Soll Survey Manual. (1951). U.S. Department of Agriculture, -Washington D.C., 503pp.

Sridharan, A., Altschaeffl, A. G., and Diamond, S. (1971). "Pore Size Distribution studies." J'. Soil Mech. Found. Engrg.,
AscE, Vol. 97, No. 5, 771-787.

Sweere, G. T. H., and Galjaard, P. J. (1988). "Repeated static Loading Triaxial Tests for Determination of Resilient Properties of Sands." Transp. Res. Record 1192, 8-15.

Tanimoto, K.' and Nishi, M. (1970). "On Resilience Characteristics of Some Soils Under Repeated Loading." Soils and Foundations, JSSMFE, Vol. 10, No. 1, 75-92.

Terdich, G. M. (1981). "Prediction and Control of Field swell Pressures of Compacted Medium Plastic clay." MSCE Thesis,
Purdue University, West Lafayette, IN.

Terzaghi, K. (1925). "Modern Conceptions Concerning Foundation Engineering." $J$. Boston Society of Civ. Engrs., Vol. 12, No.

The Asphalt Institute. (1982). Research and Development of the Asphalt Institute's Thickness Design Manual (MS-1) Ninth Edition., Research Report No. 82-2.

Thompson, M. R. (1989). "Factors Affecting the Resilient Moduli of Soils and Granular Materials." Workshop on Resilient Modulus Testing, Oregon State Univ., Corvaliis, OR.

Thompson, M. R. (1984). "Important Properties of Base and Subgrade Materials." Conf. on Crushed stone for Road and street Construction and Reconstruction, Arlington, vA.

Thompson, M. R.; and Smith, K. L. (1989). "Repeated Triaxial Characterization of Granular Bases." 69 th Annual Meeting, TRB. 
Thompson, M. R., and Robnett, Q. L. (1979). "Resilient Properties of Subgrade Soils." J. Transp. Engrg., ASCE, Vol. 105, No. 1, 71-89.

Thompson, M. R., and Dempsey, B. J. (1970) " "Quantitative Characterization of Cyclic Freezing and Thawing in stabilized Pavement Materials." Hwy. Res. Record 304, 38-51.

Transportation Research Board (1975). Test Procedures for Characterizing Dynamic stress-strain properties of Pavement Materials, Special Report 162.

Transportation Research Board (1987). Lime stabilization; Reactions, Properties, Design, and Construction, state of the Art Report 5, 59pp. Tsytovich, N. A. (1975). The Mechanics of Frozen Ground,
McGraw-Hill Co.

Uzan, J. (1985). "Characterization of Granular Material." Transp. Res. Record 1022, 52-59.

van olphen, H. (1977). An Introduction to clay colloid Chemistry, John-wiley and Sons, New York.

Vinson, T. (1989). "Fundamentals of Resilient Modulus Testing." Workshop on Resilient Modulus Testing, Oregon State Univ., Corvallis, OR.

Wadsworth, H. M. (1990). Handbook of Statistical Methods for Engineers and scientists, McGraw Hill Publishing company.

Wahls, H. E., Fisher, C. P., and Langfelder, L. J. (1966). "The Compaction of Soil and Rock Materials for Highway Purposes." U. S. Department of Transportation, Federal Highway

Waterways Experimental station (1949). "Soil compaction Investigation: Compaction studies on Silty clay." Tech. Memo. No. 3-271, U.S. Corps of Engineers, Vicksburg, Mississippi.

Washburn, E. พ. (1921). "Note on a Method of Determining the Distribution of Pore size in a porous Material." Proc. National Academy of Sciences, Vol. 7, 115-116.

Washburn, E. H. (1921). "The Dynamics of Capillary Flow." The Physical Review, Second series, Vol. 17, No. 3, 273-283.

White; D. M. (1980). "The Fabric of a Medium Plastic clay Compacted in the Laboratory and in the Field." MSCE Thesis, 
Winslow, D. N. (1984). "Advances in Experimental technique for Mercury Intrusion Porosimetry," Surface and colloid science,

Yoder, E. J., and witczak, H. W. (1975). Principles of Pavement Design, Second Ed., John Wiley and Sons, Inc., New

Yong, R. N.", and Sheeran, D. E. (1973). "Fabric Unit Interaction and Soil Behavior." Proc. Int'l Symp. Soil
Structure, Sweden, 176-183.

Yong, R. N., Boonsinsuk, P., and Murphy, D. (1982). "ShortSensitive clay." Proc. . Third Int'l symp. Hanover, Germany, 97-104.

Zimmie, T. F., and La Plante, C. (1990). "The Effects of Freeze/Thaw Cycles on the Permeability of a Fine-Grained Univ., Philadelphia, Pa., 580-593. 


\section{APPENOICES}


APPENDIX A

Information on Tested specimens 
Table A.1 Information on Specimens for Normal Test

\begin{tabular}{|c|c|c|c|c|c|c|}
\hline $\begin{array}{l}\text { Test } \\
\text { ID }\end{array}$ & Location & $\begin{array}{l}w / c \\
(z)\end{array}$ & $\begin{array}{c}\mathbf{Y}_{d} \\
(p \subset f)\end{array}$ & $\begin{array}{l}w_{L} \\
(8)\end{array}$ & $\begin{array}{l}w_{p} \\
(8)\end{array}$ & $\begin{array}{l}P I \\
\text { (8) }\end{array}$ \\
\hline SBO1 & $142+00, \mathrm{CL}-1,27-33$ & 11.25 & 129.94 & 25.7 & 12.2 & 13.5 \\
\hline $\mathrm{SBO2}$ & $142+00, \mathrm{CL}-2,13-19$ & 9.77 & 131.03 & 22.2 & 13.0 & 9.2 \\
\hline $\mathrm{SBO3}$ & $142+00, \mathrm{CL}-2,5-11$ & 9.77 & 132.04 & 20.5 & 10.7 & 9.8 \\
\hline SBO4 & $142+00, C L-3,13-19$ & 9.82 & 131.67 & 21.5 & 12.6 & 8.9 \\
\hline SBO5 & $142+00, \mathrm{CL}-3,6-12$ & 9.94 & 131.12 & 20.5 & 11.3 & 9.2 \\
\hline SB06 & $141+00, R-1,8-14$ & 9.82 & 127.48 & 23.7 & 12.8 & 10.9 \\
\hline SBO7 & $141+00, R-1,14-20$ & 9.73 & 130.24 & 23.1 & 10.3 & 12.8 \\
\hline SBO8 & $142+00, \mathrm{CL}-3,24-30$ & 10.74 & 128.30 & 26.1 & 13.7 & 12.4 \\
\hline sBo9 & $141+00, R-2,20-26$ & 10.66 & 128.44 & 22.8 & 13.8 & 9.0 \\
\hline SB10 & $141+00, R-2,26-32$ & 10.66 & 127.52 & 22.3 & 10.8 & 11.5 \\
\hline SB11 & $141+00, L-2,6-13$ & 10.22 & 129.81 & 24.1 & 10.0 & 14.1 \\
\hline SB12 & $142+00, C L-2,22-28$ & 11.22 & 129.32 & 25.6 & 13.5 & 12.1 \\
\hline SB13 & $141+00, R-2,13-19$ & 9.75 & 129.88 & 22.5 & 15.6 & 6.9 \\
\hline SB14 & $141+00, R-3,18-24$ & 10.33 & 128.56 & 22.5 & 11.3 & 11.2 \\
\hline $\mathbf{S B 1 5}$ & $141+00, \mathrm{~L}-1,28-36$ & 9.51 & 132.65 & 21.0 & 13.8 & 7.2 \\
\hline SB16 & $141+00,2-3,36-43$ & 9.58 & 126.67 & 20.6 & 9.7 & 10.9 \\
\hline SB17 & $141+00, \mathrm{~L}-3,43-50$ & 8.62 & 132.05 & 20.2 & 12.1 & 8.1 \\
\hline SB18 & $141+00, L-3,28-34$ & 10.52 & 125.58 & 21.2 & 10.8 & 10.4 \\
\hline FWO1 & $56+00,5-1,3-9$ & 20.75 & 107.55 & 30.6 & 18.2 & 12.4 \\
\hline FW04 & $56+00, s-1,30-36$ & 14.93 & 122.72 & 31.6 & 17.4 & 14.2 \\
\hline FWO3 & $56+00,5-2,0-6$ & 18.80 & 109.29 & 28.4 & 15.9 & 12.5 \\
\hline FWO2 & $56+00, s-2,12-18$ & 21.62 & 108.71 & 30.7 & -16.7 & 14.0 \\
\hline Fw05 & $56+00, s-2,30-37$ & 16.67 & 116.73 & 31.6 & 28.3 & 13.3 \\
\hline EW06 & $56+00, s-2,38-44$ & 15.28 & 120.11 & 32.0 & 16.0 & 16.0 \\
\hline FW07 & $56+00, s-2,46-52$ & 16.62 & 117.66 & 31.9 & 17.5 & 14.4 \\
\hline FW26 & $56+00, p-1,4-10$ & 20.70 & 108.86 & 35.2 & 19.4 & 15.8 \\
\hline Eพ27 & $56+00, P-1,14-20$ & 17.82 & 111.62 & 36.5 & 20.1 & 16.4 \\
\hline FW28 & $56+00, P-1,27-34$ & 22.43 & 103.21 & 44.6 & 22.6 & 22.0 \\
\hline FW29 & $56+00, P-1,42-48$ & 18.08 & 115.96 & 35.3 & 19.6 & 15.7 \\
\hline FW30 & $56+00, D-1,17-23$ & 24.72 & 100.27 & 44.0 & 23.0 & 21.0 \\
\hline FH31 & $56+00, D-1,31-37$ & 19.48 & 113.32 & 32.5 & 19.3 & 13.2 \\
\hline Eพ32 & $56+00, D-1,45-51$ & 15.44 & 118.74 & 31.4 & 19.0 & 12.4 \\
\hline Fw09 & $73+00,5-1,9-15$ & 16.66 & 113.85 & 31.8 & 18.2 & 13.6 \\
\hline EW10 & $73+00, s-1,17-25$ & 16.73 & 114.31 & 33.1 & 18.8 & 14.3 \\
\hline FW11 & $73+00, s-1,35-41$ & 24.13 & 101.57 & 42.2 & 21.6 & 20.6 \\
\hline
\end{tabular}




\begin{tabular}{|c|c|c|c|c|c|c|}
\hline $\begin{array}{c}\text { Test } \\
\text { ID }\end{array}$ & Location & $\begin{array}{l}w / c \\
(8)\end{array}$ & $\begin{array}{c}Y_{d} \\
(p c f)\end{array}$ & $\begin{array}{l}w_{L} \\
\text { (8) }\end{array}$ & $\begin{array}{l}w_{p} \\
(8)\end{array}$ & $\begin{array}{l}\text { PI } \\
(8)\end{array}$ \\
\hline FW12 & $73+00, s-1,43-49$ & 18.83 & 112.83 & 33.3 & 19.3 & 14.0 \\
\hline Fพ16 & $73+00,5-2,2-8$ & 18.62 & 111.54 & 36.2 & 19.0 & 17.2 \\
\hline FW17 & $73+00, s-2,8-14$ & 17.91 & 115.87 & 28.2 & 17.2 & 11.0 \\
\hline FW13 & $73+00, s-2,36-42$ & 16.95 & 115.88 & 32.0 & 17.6 & 14.4 \\
\hline FW14 & $73+00, s-2,44-50$ & 24.54 & 101.74 & 42.7 & 21.9 & 20.8 \\
\hline FW15 & $73+00, s-2,51-57$ & 18.75 & 113.71 & 31.3 & 18.3 & 13.0 \\
\hline FW18 & $73+00, P-1,2-8$ & 15.81 & 115.53 & 31.0 & 18.5 & 12.5 \\
\hline EW19 & $73+00, P-1,16-22$ & 15.84 & 117.27 & 31.2 & 18.7 & 12.5 \\
\hline Eस21 & $73+00, P-1,32-39$ & 17.73 & 113.85 & 31.0 & 17.9 & 13.1 \\
\hline FW43 & $73+00, P-2,33-39$ & 15.60 & 115.21 & 38.0 & 18.9 & 19.1 \\
\hline FW44 & $73+00, p-2,47-53$ & 16.00 & 117.67 & 36.0 & 20.3 & 15.7 \\
\hline FW22 & $73+00, D-2,1-7$ & 19.20 & 112.20 & 31.2 & 19.1 & 12.1 \\
\hline FW23 & $73+00, D-2,17-23$ & 21.49 & 110.10 & 33.6 & 17.6 & 16.0 \\
\hline FW24 & $73+00, D-2,34-40$ & 23.01 & 108.70 & 34.4 & 18.6 & 15.8 \\
\hline FW25 & $73+00, D-2,47-53$ & 16.35 & 114.39 & 34.2 & 17.8 & 16.4 \\
\hline FW37 & $181+00, s-2,36-42$ & 14.47 & 120.71 & 29.8 & 17.9 & 11.9 \\
\hline Eพ38 & $181+00, s-2,44-50$ & 18.65 & 112.42 & 33.0 & 18.6 & 14.4 \\
\hline FW39 & $181+00, s-2,51-57$ & 23.83 & 101.64 & 41.4 & 25.3 & 16.1 \\
\hline FW40 & $181+00, \mathrm{p}-1,2-8$ & 14.62 & 121.30 & 31.1 & 17.6 & 13.5 \\
\hline FW41 & $181+00, P-1,8-14$ & 19.21 & 109.34 & 34.5 & 20.1 & 14.4 \\
\hline FW42 & $181+00, P-1,21-27$ & 18.84 & 108.73 & 36.3 & 20.7 & 15.6 \\
\hline รพ36 & $181+00, D-1,2-8$ & 9.74 & 129.45 & 22.6 & 14.8 & 7.8 \\
\hline FW34 & $181+00, D-1,12-18$ & 13.53 & 124.16 & 28.6 & 16.3 & 12.3 \\
\hline FW35 & $181+00, D-1,21-27$ & 17.58 & 114.54 & 30.8 & 17.7 & 13.1 \\
\hline WAO1 & $290+00, P-1,52-58$ & 24.35 & 101.33 & & & \\
\hline WAO2 & $290+00, P-1,60-66$ & 18.59 & 111.14 & 37.0 & 19.8 & 17.2 \\
\hline WAO3 & $290+00, P-1,1-7$ & 17.50 & 113.99 & & & \\
\hline MAO4 & $290+00, P-1,7-13$ & 19.20 & 107.66 & 35.0 & 21.2 & 13.9 \\
\hline WAOS & $290+00, P-1,34-40$ & 20.76 & 108.75 & & & \\
\hline พAO6 & $290+00, \mathrm{P}-1,40-46$ & 17.14 & 115.28 & & & \\
\hline KAO7 & $290+00, D-1,2-8$ & 17.66 & 111.82 & & & \\
\hline WA08 & $290+00, D-1,14-20$ & 20.71 & 104.87 & & & \\
\hline WA09 & $290+00, D-1,49-55$ & 19.89 & 108.55 & & & \\
\hline พA10 & $290+00, D-1,63-69$ & 23.40 & 104.36 & & & \\
\hline WA11 & $290+00, D-1,30-36$ & 19.39 & 109.71 & & & \\
\hline WA12 & $290+00, D-1,38-44$ & 20.03 & 109.42 & 35.0 & 18.0 & 17.0 \\
\hline WA13 & $290+00, s-1,2-7$ & 17.12 & 113.47 & & $\cdot$ & \\
\hline MA14 & $290+00, s-1,25-31$ & 17.87 & 111.08 & 33.0 & 18.4 & 14.6 \\
\hline
\end{tabular}




\begin{tabular}{|c|c|c|c|c|c|c|}
\hline $\begin{array}{c}\text { Tegt } \\
\text { ID }\end{array}$ & Location & $\begin{array}{l}w / c \\
(8)\end{array}$ & $\begin{array}{c}\gamma_{d} \\
(p c f)\end{array}$ & $\begin{array}{l}w_{L} \\
(8)\end{array}$ & $\begin{array}{l}w_{p} \\
\text { (t) }\end{array}$ & $\begin{array}{l}P I \\
(8)\end{array}$ \\
\hline WA15 & $290+00, s-1,32-38$ & 21.39 & 104.77 & & & \\
\hline WA16 & $290+00, s-1,41-47$ & 21.80 & 105.16 & & & \\
\hline WA17 & $290+00, s-1,48-54$ & 19.25 & 109.97 & 40.2 & 22.4 & $17: 8$ \\
\hline WA18 & $290+00, s-1,58-64$ & 18.16 & 111.97 & & & \\
\hline LA01 & $530+90, s-1,21-27$ & 24.89 & 100.53 & & & \\
\hline LAO2 & $530+90, s-1,27-33$ & 24.43 & 101.85 & 34.3 & 21.1 & 13.2 \\
\hline LAO3 & $531+00, P-1,42-48$ & 28.13 & 96.90 & & & \\
\hline LAO4 & $531+00, P-1,21-27$ & 22.09 & 105.92 & & & \\
\hline LAOS & $531+00, \mathrm{P}-1,28-34$ & 31.93 & 93.51 & & & \\
\hline LA06 & $531+00, D-1,20-26$ & 23.08 & 103.66 & & & \\
\hline LAO7 & $531+00, D-1,50-56$ & 18.65 & 108.19 & 30.2 & 20.5 & 9.7 \\
\hline LA08 & $531+90, s-2,12-18$ & 33.90 & 89.98 & 66.5 & 32.9 & 33.6 \\
\hline LAO9 & $531+90,5-2,19-25$ & 28.70 & 94.60 & & & \\
\hline LA10 & $531+90, s-2,30-36$ & 34.10 & 89.24 & 57.5 & 25.1 & 32.4 \\
\hline LA11 & $531+90, s-2,102-108$ & 37.93 & 81.76 & 50.4 & 25.1 & 25.3 \\
\hline LA12 & $531+90, s-3,22-28$ & 28.18 & 96.02 & & & \\
\hline $\mathbf{L A 1 3}$ & $531+90,5-3,31-37$ & 33.56 & 90.17 & 55.0 & 43.4 & 11.6 \\
\hline LA14 & $531+90, s-4,8-14$ & 31.25 & 92.06 & & & \\
\hline LA15 & $531+90, s-4,15-21$ & 33.05 & 89.44 & . & & \\
\hline LA16 & $531+90, s-4,62-68$ & 29.30 & 92.30 & & & \\
\hline LA17 & $531+90,5-4,31-37$ & 22.26 & 103.36 & & & \\
\hline LA18 & $531+90, s-4,38-44$ & 31.54 & 93.74 & & & \\
\hline LA19 & $531+90, s-4,45-51$ & 29.62 & 93.33 & & & \\
\hline BLO1 & $849+00,5-1-1,7-13$ & 32.30 & 89.75 & & & \\
\hline BLO2 & $849+00, s-1-1,15-21$ & 28.50 & 93.59 & 72.0 & 28.7 & 43.3 \\
\hline BL03 & $849+00, s-3-1,7-13$ & 30.90 & 91.52 & & & \\
\hline BL 04 & $849+00, s-3-1,16-22$ & 27.40 & 96.19 & & & . \\
\hline BLO5 & $849+00, s-2-1,17-23$ & 38.14 & 85.57 & & & \\
\hline BL06 & $849+00, P-1-1,8-14$ & 23.20 & 102.30 & & & \\
\hline BL07 & $849+00, P-1-1,14-20$ & 28.00 & 96.71 & 48.0 & 22.0 & 26.0 \\
\hline BLOB & $849+00, P-1-2,5-11$ & 25.80 & 99.73 & $43.8^{\circ}$ & 19.3 & 24.5 \\
\hline BLO9 & $849+00, P-1-3,2-8$ & 26.80 & 97.60 & & & \\
\hline BL10 & $849+00, P-1-3,8-16$ & 23.30 & 102.30 & 42.0 & 23.1 & 18.9 \\
\hline BL11 & $849+00, P-2-2,6-12$ & 27.80 & 93.49 & 52.0 & 22.9 & 29.1 \\
\hline BL12 & $849+00,8-2-1,14-20$ & 19.30 & 107.00 & & & \\
\hline BL13 & $849+00, D-2-1,1-7$ & 37.00 & 86.26 & & & \\
\hline BL14 & $849+00, D-2-1,8-14$ & 33.30 & 89.92 & & & \\
\hline BL15 & $849+00, D-2-1,14-20$ & 22.20 & 101.41 & & & \\
\hline
\end{tabular}


Table A.2 Information on Specimens for Freeze-Thaw Test

\begin{tabular}{|c|c|c|c|c|c|c|}
\hline $\begin{array}{c}\text { Tegt } \\
\text { ID }\end{array}$ & Location & $\begin{array}{l}w / c \\
(8)\end{array}$ & $\begin{array}{c}\gamma_{d} \\
(p \subset f)\end{array}$ & $\begin{array}{l}w_{L} \\
(8)\end{array}$ & $\begin{array}{l}w_{p} \\
(z)\end{array}$ & $\begin{array}{l}\text { PI } \\
\text { (8) }\end{array}$ \\
\hline SBFT01 & $142+00, R-1,18-30$ & 14.33 & 122.60 & 20.4 & 13.7 & 6.7 \\
\hline SBFT02 & $142+00, R-2,30-40$ & 8.94 & 131.28 & 18.5 & 12.2 & 6.3 \\
\hline SBFT03 & $141+00, R-4,12-18$ & 8.95 & 130.07 & 19.9 & 12.7 & 7.2 \\
\hline SBFT04 & $142+00, \mathrm{~L}-1,36-42$ & 14.07 & 120.43 & 26.2 & 13.8 & 12.4 \\
\hline SBFTO5 & $142+00, R-1,16-23$ & 10.48 & 128.07 & 20.9 & 12.1 & 8.8 \\
\hline SBETO6 & $142+00, \mathrm{R}-1,30-42$ & 8.97 & 128.94 & 20.3 & 11.3 & 9.0 \\
\hline SBET07 & $142+00, C L-4,30-36$ & 10.49 & 126.54 & 24.9 & 15.3 & 9.6 \\
\hline SBFT08 & $141+00,2-4,35-41$ & 8.46 & 133.39 & 21.5 & 10.9 & 10.6 \\
\hline S88T09 & $141+00, R-4,24-36$ & 10.15 & 126.57 & & & \\
\hline SEFT10 & $141+00, I-4,6-18$ & 8.35 & 131.67 & 19.2 & 11.4 & 7.8 \\
\hline SBET11 & $141+00, C L-1,36-42$ & 11.51 & 126.78 & 25.2 & 13.5 & 11.7 \\
\hline SBFT12 & $141+00, C L-1,43-49$ & 12.16 & 124.28 & & & \\
\hline SBFT13 & $141+00, \mathrm{CL}-1,49-55$ & 13.42 & 124.59 & 24.0 & 12.9 & 11.1 \\
\hline FWFTO1 & $73+00, D-1,1-7$ & 18.97 & 112.05 & & & \\
\hline FWFT02 & $73+00, D-1,7-13$ & 14.32 & 119.31 & & & \\
\hline EWFT03 & $73+00,0-1,17-23$ & 16.22 & 114.66 & & & \\
\hline EWTTO4 & $73+00, D-1,25-31$ & 16.49 & 116.52 & 35.8 & 17.3 & 18.5 \\
\hline EพFT05 & $73+00, p-2,7-13$ & 18.07 & 112.57 & & & \\
\hline FWFTÓ6 & $73+00, p-2,16-22$ & 14.76 & 118.23 & & & \\
\hline FWFT07 & $73+00, P-2,24-30$ & 17.78 & 112.90 & & & \\
\hline FWFT08 & $73+00, P-2,40-46$ & 14.66 & 119.99 & & & \\
\hline FWFT09 & $181+00,5-2,4-10$ & 10.94 & 128.90 & & & \\
\hline FWFT10 & $181+00,5-2,11-17$ & 11.37 & 124.38 & & & \\
\hline FWFT11 & $181+00, s-2,18-24$ & 13.50 & 120.39 & & & \\
\hline FWIT12 & $181+00, s-2,25-31$ & 14.59 & 119.94 & 32.4 & 16.8 & 15.6 \\
\hline PWFT13 & $56+00, P-2,3-9$ & 17.87 & 110.20 & & & \\
\hline FWFT14 & $56+00, P-2,9-15$ & 20.30 & 109.48 & & & \\
\hline FWFT15 & $56+00, P-2,15-21$ & 18.53 & 111.46 & & & \\
\hline FWFT16 & $56+00, P-2,31-37$ & 16.05 & 117.46 & 34.2 & 18.8 & 15.4 \\
\hline FWET17 & $56+00, D-2,1-7$ & 19.69 & 110.58 & & & \\
\hline FWFT18 & $56+00, D-2,9-15$ & 16.24 & 112.92 & & & \\
\hline FWFT19 & $56+00, D-2,16-22$ & 28.26 & 97.56 & 53.0 & 27.1 & 25.9 \\
\hline FWFT20 & $56+00, D-2,22-28$ & 17.61 & 113.99 & 39.8 & 21.5 & 18.3 \\
\hline WAFTOI & $290+00, s-2,30-36$ & 19.62 & 108.21 & & & \\
\hline พAFTO2 & $290+00, s-2,36-42$ & 21.84 & 104.05 & 43.0 & 23.6 & 19.4 \\
\hline
\end{tabular}




\begin{tabular}{|c|c|c|c|c|c|c|}
\hline $\begin{array}{c}\text { Test } \\
\text { ID }\end{array}$ & Location & $\begin{array}{l}w / c \\
(8)\end{array}$ & $\begin{array}{c}Y_{d} \\
\text { (pсf) }\end{array}$ & $\begin{array}{l}W_{L} \\
(8)\end{array}$ & $\begin{array}{l}w_{p} \\
(z)\end{array}$ & $\begin{array}{l}\text { PI } \\
\text { (8) }\end{array}$ \\
\hline WAFTO3 & $290+00, s-2,1-7$ & 27.43 & 112.81 & 33.0 & 17.4 & 15.6 \\
\hline WAFTO4 & $290+00, s-2,8-14$ & 18.71 & 109.79 & & & \\
\hline WAFT05 & $290+00, D-2,4-10$ & 17.61 & 111.14 & & & \\
\hline WAFT06 & $290+00, D-2,27-33$ & 18.53 & 111.06 & & & \\
\hline WAFT07 & $290+00, D-2,39-45$ & 18.91 & 111.50 & & & \\
\hline WAFT08 & $290+00, P-2,4-10$ & 19.06 & 110.20 & 33.6 & 19.4 & 14.2 \\
\hline WAFTO9 & $290+00, P-2,13-19$ & 17.27 & 109.70 & 34.0 & 19.9 & 14.1 \\
\hline WAFT10 & $290+00, p-2,32-38$ & 17.50 & 112.53 & & & \\
\hline WAFT11 & $290+00, P-2,38-44$ & 21.07 & 107.58 & & & \\
\hline EAFTO1 & $530+00, s-1,9-15$ & 31.80 & 92.70 & & & \\
\hline LAFT02 & $530+00, s-2,11-17$ & 27.80 & 97.89 & & & \\
\hline LAFTO3 & $531+00, P-2-1,2-8$ & 22.50 & 104.22 & 47.8 & 19.3 & 28.5 \\
\hline LAFTO4 & $531+00, P-2-2,1-7$ & 23.30 & 102.19 & & & \\
\hline LAFT05 & $530+00, D-1,7-13$ & 33.70 & 89.93 & 68.0 & 29.1 & 38.9 \\
\hline LAFT06 & $530+00, D-1,13-19$ & 32.70 & 90.98 & & & \\
\hline LAFT07 & $531+00, P-3,4-10$ & 20.70 & 106.56 & & & \\
\hline LAFT08 & $530+00, \mathrm{D}-1,19-25$ & 28.30 & 95.63 & 58.0 & 27.7 & 30.3 \\
\hline IAFT09 & $530+00,0-1,26-32$ & 33.30 & 88.31 & & & \\
\hline LAFT10 & $530+00, D-1,33-39$ & 32.50 & 87.598 & & & \\
\hline LAFT11 & $531+00, p-4,5-11$ & 21.30 & 105.64 & & & \\
\hline BLPT01 & $849+00, s-2-1,6-12$ & 32.41 & 91.00 & & & \\
\hline BLETO2 & $849+00,5-2-1,18-24$ & 40.50 & 82.96 & & & \\
\hline BLFT03 & $849+00, s-2-2,5-11$ & 29.68 & 93.68 & & & \\
\hline BLFTO4 & $849+00, s-2-2,11-17$ & 32.50 & 90.39 & & & \\
\hline BLFT05 & $849+00, P-2-1,2-8$ & 30.31 & 94.05 & & & \\
\hline BLFT06 & $849+00, P-2-1,8-14$ & 27.76 & 94.47 & 62.0 & 21.6 & 40.4 \\
\hline BLFTO7 & $849+00, P-2-3,2-8$ & 28.87 & 95.03 & & & \\
\hline BLFT08 & $849+00, P-2-3,8-14$ & 30.04 & 92.57 & 52.5 & 24.9 & 27.6 \\
\hline BLFTO9 & $849+00, D-1-1,13-20$ & 31.53 & 92.41 & & & \\
\hline BLFT10 & $849+00, D-1-2,1-7$ & 25.40 & 97.36 & & & \\
\hline BLFT11 & $849+00, D-1-2,7-13$ & 33.30 & 89.29 & & & \\
\hline BLFT12 & $849+00, D-1-2,13-19$ & 30.75 & 92.12 & & & \\
\hline
\end{tabular}

SBFTO1 - SBFT10; frozen-thawed specimen from the field. All others; closed-system freeze-thaw in the laboratory. 
APPENDIX B

Unconfined Compression Test Results 
Table B.1 Unconfined Compression Test Results of Specimens for Normal Test

\begin{tabular}{|c|c|c|c|c|c|c|c|c|c|c|}
\hline Test & $\begin{array}{c}s_{\text {ta.ss: }} \\
\text { (psi) }\end{array}$ & $\begin{array}{r}S_{10.58} \\
\left(P^{81}\right)\end{array}$ & $\begin{array}{c}S_{\text {ta.zsx }} \\
\text { (pei) }\end{array}$ & $\begin{array}{r}S_{\mathrm{ut.0x}} \\
(\mathrm{pg} i \mathrm{i})\end{array}$ & $\begin{array}{c}E_{2 \times i} \\
(p s 1)\end{array}$ & $\begin{array}{c}E_{\text {dpol }} \\
(p B i)\end{array}$ & $\begin{array}{c}E_{\text {cpes }} \\
\text { (p=i) }\end{array}$ & $\begin{array}{c}E_{10 p a i} \\
\text { (psi) }\end{array}$ & $\mathbf{E}_{\mathbf{i}}$ & $1 / \mathrm{b}$ \\
\hline FW01 & 2.0 & 3.8 & 5.5 & 6.9 & 288.8 & 221.1 & 151.2 & 35.3 & 362.4 & 17.9 \\
\hline FW04 & 11.9 & 17.9 & 21.5 & 23.8 & 7107.7 & 6663.7 & 6196.8 & 5017.5 & 7535.3 & 36.9 \\
\hline FW03 & 3.2 & 5.0 & 6.4 & 7.5 & 1251.4 & 999.1 & 746.8 & 270.9 & 1509.4 & 16.1 \\
\hline FWO2 & 3.3 & 5.6 & 7.4 & 8.9 & 1156.3 & 992.3 & 825.5 & 497.1 & 1324.3 & 18.2 \\
\hline FW05 & 8.9 & 13.5 & 16.4 & 18.4 & 4909.6 & 4542.2 & 4160.2 & 3252.3 & 5268.9 & 29.7 \\
\hline Fพ06 & 10.1 & 18.4 & 25.3 & 30.5 & 4135.4 & 4071.0 & 3984.4 & 3613.2 & 4188.3 & 77.7 \\
\hline FW07 & 9.8 & 16.6 & 21.5 & 25.0 & 4563.3 & 4385.1 & 4187.1 & 3605.1 & 4730.5 & 49.8 \\
\hline FW26 & 2.3 & 3.6 & 4.7 & 5.6 & 728.8 & 482.5 & 238.6 & & 983.2 & 10.4 \\
\hline FW27 & 13.3 & 20.7 & 25.3 & 28.4 & 7413.3 & 7048.8 & 6656.7 & 5583.8 & 7758.3 & 46.9 \\
\hline Fพ28 & 3.6 & 5.1 & 6.1 & 7.1 & 1740.1 & 1353.1 & 968.5 & 243.7 & 2133.9 & 11.0 \\
\hline FW29 & 16.7 & 30.0 & 40.0 & 47.4 & 7579.9 & 7446.7 & 7276.4 & 6562.0 & 7687.4 & 110.6 \\
\hline FW30 & 6.1 & 8.2 & 9.5 & 10.4 & 4029.5 & 3416.8 & 2803.2 & 1562.8 & 4643.8 & 14.1 \\
\hline FW31 & 7.6 & 12.6 & 16.1 & 18.7 & 3503.6 & 3304.1 & 3090.9 & 2546.6 & 3697.5 & 35.6 \\
\hline FW32 & 17.3 & 28.7 & 37.1 & 46.4 & 8893.1 & 8623.2 & 8314.7 & 7293.0 & 9134.4 & 77.3 \\
\hline FWọ & 10.2 & 15.7 & 19.3 & 21.7 & 5535.4 & 5192.2 & 4830.3 & 3918.8 & 5867.0 & 35.9 \\
\hline Fพ10 & 9.2 & 14.8 & 18.6 & 21.3 & 4687.5 & 4420.0 & 4135.4 & 3402.4 & 4945.4 & 37.6 \\
\hline FW11 & 6.3 & 8.3 & 9.2 & 10.0 & 4545.3 & 3823.5 & 3102.2 & 1652.3 & 5269.0 & 13.1 \\
\hline FW12 & 8.4 & 14.0 & 17.9 & 20.7 & 4012.7 & 3803.2 & 3577.7 & 2982.8 & 4214.5 & 39.1 \\
\hline FW16 & 3.7 & 7.2 & 10.1 & 12.7 & 1067.2 & 1041.4 & 1008.0 & 906.4 & 1094.4 & 35.3 \\
\hline FW17 & 7.1 & 11.5 & 14.7 & 16.9 & 3365.5 & 3137.6 & 2897.9 & 2317.6 & 3589.1 & 31.2 \\
\hline FW13 & 8.7 & 13.6 & 16.9 & 19.1 & 4459.7 & 4163.9 & 3853.3 & 3090.4 & 4747.7 & 32.8 \\
\hline FW14 & 6.5 & 10.5 & 13.5 & 15.6 & 3016.7 & 2793.8 & 2560.5 & 2011.8 & 3236.6 & 28.7 \\
\hline
\end{tabular}




\begin{tabular}{|c|c|c|c|c|c|c|c|c|c|c|}
\hline Test & $\begin{array}{c}S_{10.25 \pi} \\
(P=1)\end{array}$ & $\begin{array}{c}s_{\operatorname{mos} \pi} \\
(p=1)\end{array}$ & $\begin{array}{c}s_{\infty .7 s x} \\
\text { (pBi) }\end{array}$ & $\begin{array}{r}S_{\mathrm{ul}, 0 *} \\
\text { (pB1) }\end{array}$ & $\begin{array}{c}E_{2 \times 1} \\
(p B 1)\end{array}$ & $\begin{array}{c}E_{\text {tol }} \\
(p \& 1)\end{array}$ & $\begin{array}{c}E_{\text {cped }} \\
\left(p^{\prime} B 1\right)\end{array}$ & $\begin{array}{c}E_{100 \mathrm{~m}} \\
\text { (psi) }\end{array}$ & $\mathbf{E}_{\mathrm{i}}$ & $I / b$ \\
\hline FW15 & 10.8 & 18.1 & 23.2 & 26.8 & 5160.6 & 4960.1 & 4737.6 & 4079.5 & 5347.9 & 52.1 \\
\hline FW18 & 5.7 & 8.3 & 9.9 & 12.0 & 3183.0 & 2760.1 & 2333.5 & 1453.8 & 3606.9 & 17.0 \\
\hline EW19 & 12.6 & 22.5 & 29.9 & 35.4 & 5643.2 & 5521.0 & 5371.3 & 4805.7 & 5748.1 & 80.4 \\
\hline FW21 & 6.0 & 8.4 & 9.8 & 10.8 & 3610.4 & 3103.1 & 2593.1 & 1549.4 & 4118.7 & 15.7 \\
\hline FW43 & 10.7 & 15.0 & 17.4 & 18.9 & 6856.1 & 6266.7 & 5677.2 & 4498.2 & 7445.6 & 25.3 \\
\hline FW44 & 8.9 & 12.8 & 15.1 & 16.5 & 5223.3 & 4732.1 & 4240.8 & 3258.3 & 5714.6 & 23.3 \\
\hline FW22 & 1.6 & 3.3 & 5.3 & 7.2 & & & & & & 24.1 \\
\hline FW23 & 5.2 & 8.7 & 11.3 & 13.3 & 2154.1 & 1983.6 & 1805.3 & 1398.8 & 2324.3 & 26.5 \\
\hline FW24 & 4.3 & 6.5 & 8.0 & .9 .1 & 2030.7 & 1711.0 & 1389.5 & 752.7 & 2354.4 & 15.2 \\
\hline FW25 & 8.9 & 12.7 & 15.0 & 16.4 & 5558.9 & 5040.5 & 4509.8 & 3316.5 & 6069.9 & 24.0 \\
\hline FW37 & 11.2 & 25.4 & 18.8 & 22.2 & 7669.5 & 7062.9 & 6436.4 & 4959.2 & 8261.2 & 29.1 \\
\hline FW38 & 6.9 & 11.0 & 13.7 & 15.8 & 3423.5 & 3151.2 & 2868.4 & 2213.2 & 3692.1 & 27.3 \\
\hline FW39 & 6.3 & 9.4 & 11.4 & 12.9 & 3358.9 & 2991.1 & 2616.9 & 1813.5 & 3725.4 & 20.5 \\
\hline FW40 & 8.7 & 14.3 & 18.2 & 20.8 & 4222.3 & 3994.6 & 3750.6 & 3112.5 & 4441.7 & 38.6 \\
\hline FW41 & 7.6 & 11.4 & 13.9 & 15.6 & 4121.7 & 3756.0 & 3379.7 & 2527.9 & 4482.8 & 25.1 \\
\hline FW42 & 8.7 & 13.0 & 15.9 & 17.7 & 4811.5 & 4432.5 & 4039.7 & 3119.7 & 5183.3 & 28.3 \\
\hline FW36 & 15.2 & 21.8 & 26.4 & 32.0 & 10506.1 & 9891.1 & 9241.8 & 7558.9 & 11093.5 & 41.4 \\
\hline FW34 & 20.9 & 14.8 & 17.5 & 19.4 & 6680.1 & 6133.4 & 5569.7 & 4255.2 & 7214.8 & 27.7 \\
\hline 5พ35 & 7.9 & 11.5 & 13.8 & 15.6 & 4695.3 & 4241.8 & 3777.9 & 2746.6 & 5143.9 & 23.1 \\
\hline WA01 & 4.7 & 6.1 & 6.4 & 6.6 & 3158.1 & 2443.1 & 1773.0 & 390.4 & 3881.0 & 8.9 \\
\hline WAO2 & 8.7 & 14.1 & 17.8 & 20.6 & 4178.3 & 3949.1 & 3703.9 & 3066.5 & 4399.5 & 38.0 \\
\hline พंAO3 & 6.7 & 10.8 & 13.6 & 15.7 & 3186.3 & 2944.7 & 2692.7 & 2102.7 & 3424.5 & 28.3 \\
\hline WAO4 & 5.3 & 9.1 & 11.6 & 14.0 & 2214.1 & 2055.8 & 1889.0 & 1498.0 & 2371.6 & 28.4 \\
\hline WA05 & 4.2 & 6.7 & 8.8 & 10.0 & 1653.4 & 1456.2 & 1254.8 & 839.5 & 1853.2 & 19.8 \\
\hline
\end{tabular}




\begin{tabular}{|c|c|c|c|c|c|c|c|c|c|c|}
\hline Tevt & $\begin{array}{l}s_{0.25 \pi} \\
(p 8 i)\end{array}$ & $\begin{array}{l}s_{\text {s.ss }} \\
\text { (psi) }\end{array}$ & $\begin{array}{l}s_{\mathrm{su.7ss}} \\
\text { (psi) }\end{array}$ & $\begin{array}{r}S_{u 1.0 x} \\
(p 8 i)\end{array}$ & $\begin{array}{c}E_{2 p a l} \\
(p=1)\end{array}$ & $\begin{array}{c}E_{\text {tad }} \\
(p=i)\end{array}$ & $\begin{array}{c}E_{\text {God }} \\
\text { (psi) }\end{array}$ & $\begin{array}{c}E_{10 \mathrm{ped}} \\
(p=1)\end{array}$ & $\mathbf{E}_{\mathbf{1}}$ & $1 / b$ \\
\hline WA06 & 9.3 & 14.7 & 18.3 & 20.8 & 4801.2 & 4511.4 & 4204.6 & 3428.2 & 5081.6 & 36.0 \\
\hline พA07 & 7.2 & 10.0 & 12.3 & 14.7 & 3736.5 & 3374.1 & 3002.9 & 2182.4 & 4095.9 & 23.0 \\
\hline พA08 & 6.3 & 10.3 & 13.2 & 15.2 & 2894.6 & 2680.1 & 2455.7 & 1928.6 & 3106.5 & 28.6 \\
\hline พA09 & 3.3 & 5.4 & 6.8 & 8.2 & 1214.3 & 1004.8 & 793.7 & 388.4 & 1428.9 & 15.6 \\
\hline WA1O & 2.0 & 3.8 & $5.2^{\circ}$ & 6.3 & 678.4 & 467.0 & 257.0 & & 897.3 & 11.6 \\
\hline WA11 & 5.3 & 8.2 & 10.5 & 12.4 & 1768.5 & 1647.0 & 1518.0 & 1215.9 & 1890.4 & 28.3 \\
\hline WA12 & 6.1 & 9.9 & 12.4 & 14.3 & 2869.7 & 2628.5 & 2378.3 & 1810.0 & 3109.3 & 25.9 \\
\hline WA13 & 5.0 & 8.1 & 10.0 & 11.8 & 1976.9 & 1802.4 & 1621.2 & 1220.4 & 2152.1 & 24.5 \\
\hline WA14 & 5.9 & 9.2 & 11.3 & 13.4 & 2550.3 & 2332.8 & 2107.3 & 1598.9 & 2766.8 & 25.4 \\
\hline$\omega \times 15$ & 6.3 & 9.2 & 10.9 & 12.4 & 3073.2 & 2749.7 & 2419.3 & 1706.0 & 3395.9 & 21.3 \\
\hline WA16 & 9.1 & 13.1 & 15.5 & 17.2 & 5541.6 & 5054.3 & 4553.7 & 3411.1 & 6020.9 & 25.5 \\
\hline WA 17 & 6.5 & 10.8 & 13.8 & 15.9 & 2999.2 & 2790.6 & 2571.1 & 2046.3 & 3204.8 & 30.0 \\
\hline MA18 & 6.4 & 9.1 & 11.2 & 13.0 & 3219.6 & 2881.5 & 2536.3 & 1789.6 & 3556.5 & 21.4 \\
\hline LAOI & 3.0 & 5.9 & 7.4 & 8.3 & 1407.0 & 1169.0 & 929.5 & 465.4 & 1650.0 & 15.3 \\
\hline $\mathrm{LAO} O 2$ & 3.4 & 6.1 & 8.4 & 10.4 & 1080.9 & 982.7 & 879.7 & 661.8 & 1181.8 & 23.6 \\
\hline LAO03 & 6.4 & 9.4 & 11.3 & 12.6 & 3634.1 & 3215.6 & 2790.9 & 1887.6 & 4051.4 & 19.4 \\
\hline LAO4 & 6.9 & 10.9 & 13.6 & 15.6 & 3422.2 & 3145.5 & 2858.4 & 2196.1 & 3695.5 & 27.0 \\
\hline LA05 & 12.0 & 16.4 & 18.7 & 20.2 & 8704.3 & 7979.2 & 7233.2 & 5496.2 & 9412.6 & 27.6 \\
\hline LAO6 & 6.0 & 8.6 & 10.7 & 11.6 & 3315.9 & 2897.6 & 2474.9 & 1593.6 & 3734.3 & 17.8 \\
\hline LAOT & 9.5 & 13.7 & 16.2 & 16.8 & 5911.8 & 5406.6 & 4886.6 & 3690.4 & 6407.7 & 26.4 \\
\hline LA08 & 4.7 & 6.6 & 7.7 & 8.6 & 2630.1 & 2165.4 & 1701.5 & 792.4 & 3099.3 & 12.8 \\
\hline LAOS & 3.2 & 5.0 & 6.4 & 7.5 & 1241.4 & 993.0 & 744.6 & 275.5 & 1495.3 & 13.7 \\
\hline LA10 & 4.2 & 5.7 & 6.6 & 7.4 & 2391.4 & 1887.2 & 1386.4 & 430.4 & 2902.1 & 10.6 \\
\hline LA11 & 1.6 & 2.2 & 2.7 & 3.3 & 444.2 & 84.8 & & & 815.7 & 5.3 \\
\hline
\end{tabular}




\begin{tabular}{|c|c|c|c|c|c|c|c|c|c|c|}
\hline Test & $\begin{array}{r}S_{\text {wa.2ss }} \\
\text { (psi) }\end{array}$ & $\begin{array}{r}S_{0.5 x} \\
(p 81)\end{array}$ & $\begin{array}{l}s_{\infty 0.75 x} \\
\text { (psi) }\end{array}$ & $\begin{array}{r}S_{\text {u1.0x }} \\
\text { (pei) }\end{array}$ & $\begin{array}{c}E_{2+1} \\
(p a 1)\end{array}$ & $\begin{array}{c}E_{\text {4pol }} \\
\text { (p81) }\end{array}$ & $\begin{array}{c}E_{\text {tpai }} \\
(p s i)\end{array}$ & $\begin{array}{c}E_{1 q_{\mathrm{pal}}} \\
(\mathrm{psi})\end{array}$ & $\mathrm{E}_{i}$ & $1 / b$ \\
\hline LA12 & 5.8 & 8.8 & 10.9 & 12.4 & 2886.2 & 2572.8 & 2253.3 & 1568.8 & 3199.3 & 20.8 \\
\hline LA13 & 4.6 & 6.1 & 7.1 & 7.9 & 2779.1 & 2229.4 & 1682.7 & 627.5 & 3334.7 & 11.0 \\
\hline LA14 & 4.2 & 6.9 & 9.1 & 10.8 & 1565.5 & 1402.7 & 1234.7 & 880.4 & 1730.5 & 21.9 \\
\hline LA15 & 3.7 & 6.4 & 8.5 & 10.4 & 1274.8 & 1146.3 & 1013.0 & 733.2 & 1406.1 & 22.4 \\
\hline LA16 & 3.5 & 5.4 & 6.7 & 7.9 & 1452.6 & 1179.8 & 906.6 & 384.1 & 1730.6 & 13.9 \\
\hline LAI7 & 2.2 & 3.7 & 4.9 & 6.0 & 597.6 & 408.8 & 221.0 & & 793.6 & 12.1 \\
\hline LA18 & 4.5 & 7.9 & 10.7 & 13.0 & 1598.6 & 1497.5 & 1389.0 & 1131.3 & 1700.2 & 29.2 \\
\hline LA19 & 4.6 & 7.8 & 10.4 & 12.4 & 1754.4 & 1612.7 & 1464.1 & 1130.6 & 1897.1 & 25.7 \\
\hline BLO1 & 3.5 & 4.8 & 5.6 & 6.3 & 1906.5 & 1420.5 & 939.8 & 45.8 & 2400.7 & 9.0 \\
\hline BLO2 & 4.5 & 5.5 & 5.9 & 6.4 & 3461.1 & 2616.7 & 1782.9 & 210.2 & 4315.2 & 7.7 \\
\hline BLO3 & 6.8 & 9.0 & 10.1 & 10.8 & 4629.1 & 3817.1 & 3005.1 & 1381.1 & 5441.1 & 13.4 \\
\hline $\mathrm{BLO} 4$ & 5.1 & 7.6 & 9.0 & 10.0 & 2712.1 & 2283.5 & 1854.9 & 997.7 & 3140.7 & 14.7 \\
\hline 8C.05 & 3.2 & 4.2 & 4.7 & 5.0 & 1860.2 & 944.7 & 29.1 & & 2775.8 & 6.1 \\
\hline BL06 & 4.1 & 6.3 & 7.7 & 9.0 & 1997.7 & 1654.5 & 1311.3 & 624.8 & 2341.0 & 13.6 \\
\hline BL07 & 4.9 & 7.4 & 8.9 & 9.9 & 2560.7 & 2166.0 & 1771.3 & 981.9 & 2955.4 & 15.0 \\
\hline BL08 & 2.7 & 4.5 & 5.9 & 6.9 & 1117.6 & 944.3 & 771.1 & 424.6 & 1290.8 & 14.9 \\
\hline BLO9 & 3.3 & 5.0 & $6: 1$ & 6.8 & 1551.6 & 1186.4 & 821.1 & 90.5 & $1916.9^{\circ}$ & 10.5 \\
\hline BL10 & 4.5 & 7.1 & B. 8 & 10.0 & 2128.1 & 1848.7 & 1569.3 & 1010.5 & 2407.5 & 17.2 \\
\hline BL11 & 4.9 & 8.0 & 10.2 & 11.8 & 2290.1 & 2062.3 & 1834.4 & 1378.7 & 2518.0 & 22.1 \\
\hline BLI2 & 7.1 & 12.1 & 15.8 & 18.7 & 3253.8 & 3087.5 & 2921.3 & 2588.9 & 3420.0 & 41.2 \\
\hline BL13 & 4.0 & 5.0 & 5.5 & 5.8 & 2694.3 & 1586.5 & 478.7 & & 3802.0 & 6.9 \\
\hline BL14 & 3.7 & 4.8 & 5.3 & 5.6 & 2350.8 & 1356.3 & 361.8 & & 3345.0 & 6.7 \\
\hline BL15. & 4.5 & 7.1 & 8.8 & 10.3 & 2168.0 & 1878.4 & 1588.8 & 1009.6 & 2457.6 & 17.0 \\
\hline
\end{tabular}


Table B.2 Unconfined Compression Test Results of Specimens for Freeze-Thaw Test

\begin{tabular}{|c|c|c|c|c|c|c|c|c|c|c|}
\hline Test & $\begin{array}{l}S_{\infty 0.25 x} \\
(p=1)\end{array}$ & $\begin{array}{r}s_{\operatorname{sis\pi }} \\
(\mathrm{psi})\end{array}$ & $\begin{array}{l}s_{\text {s.rsx }} \\
\text { (psi) }\end{array}$ & $\begin{array}{r}S_{\text {ut.os }} \\
(p=1)\end{array}$ & $\begin{array}{c}E_{2 \times a l} \\
\text { (Poi) }\end{array}$ & $\begin{array}{c}E_{\text {topl }} \\
\text { (poi) }\end{array}$ & $\begin{array}{c}E_{\text {6pot }} \\
(p \& 1)\end{array}$ & $\begin{array}{c}E_{10 p o l} \\
\text { (pe1) }\end{array}$ & $E_{i}$ & $1 / b$ \\
\hline FWETO1 & 10.0 & 14.1 & 16.4 & 17.8 & 6239.8 & 5675.2 & 5110.5 & 3981.2 & 6804.5 & 24.1 \\
\hline FWFTO2 & 9.5 & 13.7 & 16.0 & 17.4 & 5778.5 & 5256.0 & 4733.5 & 3688.6 & 6301.0 & 24.1 \\
\hline FWFT03 & 5.2 & 7.6 & 9.0 & 9.9 & 2824.0 & 2362.2 & 1900.3 & 976.6 & 3285.9 & 14.2 \\
\hline EWFTO4 & 6.8 & 10.2 & 13.2 & 16.1 & 3643.3 & 3299.6 & 2955.8 & 2268.3 & 3987.0 & 23.2 \\
\hline FWFT05 & 9.9 & 16.3 & 21.4 & 25.2 & 4977.1 & 4757.4 & 4537.3 & 4098.3 & 5196.8 & 47.3 \\
\hline FWFT06 & 9.6 & 14.9 & 19.2 & 22.3 & 5136.4 & 4837.8 & 4539.3 & 3942.2 & 5434.9 & 36.4 \\
\hline FWFT07 & 12.7 & 19.2 & 23.2 & 25.9 & 7087.3 & 6710.4 & 6333.5 & 5579.7 & 7464.1 & 39.6 \\
\hline FWFT08 & 8.0 & 11.0 & 12.6 & 13.7 & 5227.6 & 4555.9 & 3884.3 & 2540.9 & 5899.3 & 17.6 \\
\hline FWFTO9 & 11.5 & 18.8 & 23.9 & 27.6 & 5678.4 & 5450.2 & 5221.9 & 4765.5 & 5906.7 & 51.8 \\
\hline EWFT10 & 13.1 & 20.9 & 26.0 & 29.6 & 6776.0 & 6499.5 & 6223.1 & 5670.2 & $7052.4^{\circ}$ & 51.0 \\
\hline FWFT11 & 12.8 & 21.1 & 27.0 & 31.4 & 6245.2 & 6033.6 & 5822.0 & 5398.8 & 6456.8 & 61.0 \\
\hline FWFT12 & 14.7 & 23.8 & 30.0 & 34.5 & 7411.5 & 7167.9 & 6924.2 & 6437.0 & 7655.1 & 62.9 \\
\hline FWFT13 & 2.0 & 3.3 & 4.1 & 4.8 & 816.8 & 568.2 & 319.6 & & 1065.4 & 8.6 \\
\hline FWFT 24 & 4.2 & 6.7 & 8.8 & 10.6 & 1942.2 & 1717.1 & 1492.0 & 1041.8 & 2167.4 & 19.3 \\
\hline FWFT15 & 8.4 & 13.4 & 16.6 & 18.9 & 4291.3 & 4006.9 & 3722.6 & 3154.0 & 4575.6 & 32.2 \\
\hline FWFT16 & 14.4 & 24.7 & 32.3 & 38.3 & 6794.9 & 6631.2 & 6467.4 & 6140.0 & 6958.7 & 85.0 \\
\hline FWFT17 & 4.0 & 5.2 & 5.9 & 6.7 & 2329.1 & 1560.9 & 792.7 & & 3097.3 & 8.1 \\
\hline FWFT18 & 9.1 & 15.5 & 21.5 & 27.0 & 4053.4 & 3934.1 & 3814.7 & 3576.0 & 4172.8 & 69.9 \\
\hline FWFT19 & 15.5 & 21.9 & 24.5 & 25.5 & 10718.3 & 10053.5 & 9388.7 & 8059.1 & 11383.1 & 34.3 \\
\hline FWFT20 & 15.2 & 23.5 & 28.9 & 32.5 & 8201.9 & 7877.8 & 7553.6 & 6905.3 & 8526.1 & 52.6 \\
\hline WAFT01 & 6.3 & 9.4 & 11.3 & 12.6 & 3356.8 & 2958.5 & 2560.2 & 1763.6 & 3755.1 & 18.9 \\
\hline WAFTO2 & 10.1 & 17.3 & 22.7 & 26.9 & 4685.5 & 4524.6 & 4363.6 & 4041.8 & 4846.5 & 60.2 \\
\hline
\end{tabular}




\begin{tabular}{|c|c|c|c|c|c|c|c|c|c|c|}
\hline Test & $\begin{array}{l}s_{\infty} \cdot s s \pi \\
\text { (pai) }\end{array}$ & $\begin{array}{r}s_{\infty .3 x} \\
\text { (psi) }\end{array}$ & $\begin{array}{l}S_{\infty .75 x} \\
(p s i)\end{array}$ & $\begin{array}{r}S_{n 1,0 x} \\
(p s i)\end{array}$ & $\begin{array}{c}E_{2 \mathrm{pal}} \\
(\mathrm{psi})\end{array}$ & $\begin{array}{c}E_{4 \times i} \\
(p=i)\end{array}$ & $\begin{array}{c}E_{6 \mathrm{pad}} \\
(p \in i)\end{array}$ & $\begin{array}{c}\mathbb{E}_{10+1} \\
(\mathrm{pg} i \mathrm{i})\end{array}$ & $\mathbf{E}_{\mathbf{l}}$ & $1 / \mathrm{b}$ \\
\hline WAFTO3. & 4.6 & 6.8 & 8.2 & 10.0 & 2334.7 & 1936.6 & 1538.5 & 742.2 & 2732.9 & 13.7 \\
\hline WAPT05 & 5.7 & 8.3 & 9.8 & 10.8 & 3167.0 & 2691.4 & 2215.8 & 1264.6 & 3642.6 & 15.3 \\
\hline WAFTO6 & 6.4 & 10.7 & 13.8 & 16.2 & 2980.3 & 2788.0 & 2595.7 & 2211.1 & 3172.5 & 33.0 \\
\hline พAFT07 & 6.8 & 12.1 & 16.4 & 19.9 & 2973.5 & 2863.7 & 2754.0 & 2534.5 & 3083.2 & 56.2 \\
\hline พAFTO8 & 4.6 & 7.1 & 8.6 & 9.7 & 2330.8 & 1974.4 & 1618.0 & 905.2 & 2687.2 & 15.1 \\
\hline WAFTO9 & 6.7 & 9.9 & 12.9 & 13.2 & 3635.6 & 3220.4 & 2805.2 & 1974.8 & 4050.8 & .19 .5 \\
\hline WAFT10 & 6.3 & 10.8 & 14.2 & 16.8 & 2859.9 & 2700.1 & 2540.4 & 2220.8 & 3019.7 & 37.8 \\
\hline WAFT11 & 6.1 & 9.4 & 11.5 & 12.9 & 3120.1 & 2784.0 & 2447.9 & 1775.8 & 3456.2 & 20.6 \\
\hline LAFTO1 & 7.2 & 11.8 & 15.0 & 17.3 & 3475.6 & 3247.5 & 3019.4 & 2563.1 & 3703.8 & 32.5 \\
\hline LAFTO2 & 10.1 & 17.7 & 23.6 & 28.3 & 4582.3 & 4449.2 & 4316.2 & 4050.1 & 4715.3 & 70.9 \\
\hline LAFTO3 & 3.3 & 5.2 & 6.4 & 7.3 & 1549.2 & 1238.8 & 928.5 & 307.8 & 1859.5 & 12.0 \\
\hline LAFTO 4 & 2.2 & 3.4 & 4.1 & 4.8 & 910.1 & 577.1 & 244.0 & & 1243.1 & 7.5 \\
\hline LAFTOS & 5.5 & 8.9 & 11.2 & 12.9 & 2608.5 & 2366.7 & 2124.9 & 1641.4 & 2850.2 & 23.6 \\
\hline LAFTO6 & 10.2 & 16.5 & 20.8 & 24.0 & 5052.4 & 4810.4 & 4568.5 & 4084.7 & 5294.3 & 43.8 \\
\hline LAFTO 7 & 3.0 & 5.3 & 7.1 & 8.6 & 1241.0 & 1123.7 & 1006.4 & 771.9 & 1358.2 & 23.2 \\
\hline LAFT08 & 6.0 & 8.7 & 10.3 & 11.3 & 3333.3 & 2862.0 & 2390.6 & 1448.0 & 3804.6 & 16.1 \\
\hline LAFTO9 & 5.1 & 7.8 & 9.6 & 10.7 & 2569.9 & 2226.7 & 1883.5 & 1197.1 & 2913.0 & 17.0 \\
\hline LAFP10 & 5.7 & 8.3 & 9.9 & 10.9 & 3084.5 & 2634.3 & 2184.0 & 1283.4 & 3534.8 & 15.7 \\
\hline LAFT11 & 2.4 & 3.5 & 4.3 & 4.7 & 1023.1 & 617.7 & 212.4 & & 1428.4 & 7.1 \\
\hline BLFT01 & 3.2 & 4.3 & 4.8 & 5.1 & 1817.8 & 981.1 & 144.4 & & 2654.4 & 6.4 \\
\hline BLFTO2 & 3.6 & 4.9 & 5.6 & 6.0 & 1938.2 & 1274.7 & 611.2 & & 2601.6 & 7.8 \\
\hline BLFTO3 & 3.4 & 4.5 & 5.0 & 5.3 & 1962.2 & 1109.1 & 256.0 & & 2815.2 & 6.6 \\
\hline BLFT0 4 & 3.4 & 4.5 & 5.1 & 5.5 & 1898.7 & 1116.8 & 335.0 & & 2680.5 & 6.9 \\
\hline BLFTOS & 4.7 & 6.3 & 7.1 & 7.7 & 2851.5 & 2114.3 & 1377.1 & & 3588.8 & 9.7 \\
\hline
\end{tabular}




\begin{tabular}{|c|c|c|c|c|c|c|c|c|c|c|}
\hline Test & $\begin{array}{r}s_{\infty .25 x} \\
\text { (psi) }\end{array}$ & $\begin{array}{r}s_{\operatorname{s.5}} \\
(p \times 1)\end{array}$ & $\begin{array}{r}s_{0.75 \pi} \\
(p=1)\end{array}$ & $\begin{array}{r}s_{u 1.0 x} \\
\text { (psi) }\end{array}$ & $\begin{array}{c}E_{2+1} \\
(p=1)\end{array}$ & $\begin{array}{c}E_{\text {pal }} \\
\text { (pai) }\end{array}$ & $\begin{array}{c}E_{\text {Gpi }} \\
(p \mathrm{gi})\end{array}$ & $\begin{array}{c}\text { Elopal }_{10} \\
\text { (pai) }\end{array}$ & $\mathbf{E}_{\mathbf{i}}$ & $1 / b$ \\
\hline BLFT06 & 5.5 & 9.2 & 11.8 & 13.8 & 2512.2 & 2321.5 & 2130.8 & 1749.3 & 2702.9 & 28.3 \\
\hline BLFTO7 & 7.0 & 20.7 & 13.0 & 14.6 & 3692.9 & 3336.5 & 2980.1 & 2267.3 & 4049.3 & 22.7 \\
\hline BLFTOB & 6.1 & 9.4 & 11.4 & 12.8 & 3142.0 & 2798.6 & 2455.2 & 1768.4 & 3485.4 & 20.3 \\
\hline BLPT09 & 2.4 & 3.8 & 4.9 & 5.9 & 1025.2 & 781.0 & 536.7 & 48.2 & 1269.5 & 10.4 \\
\hline BLFT10 & 4.1 & 5.9 & 7.0 & 7.7 & 2129.1 & 1653.0 & 1176.8 & 224.6 & 2605.2 & 10.9 \\
\hline BLFT11 & 3.5 & 4.6 & 5.1 & 5.4 & 2053.5 & 1172.3 & 291.1 & & 2934.7 & 6.6 \\
\hline BLFT12 & 3.9 & 5.6 & 6.6 & 7.2 & 2021.2 & 1518.8 & 1016.4 & 11.7 & 2523.6 & 10.0 \\
\hline
\end{tabular}


APPENDIX C

Resilient Modulus Test Results 
Table C.1 Resilient Modulus Test Results of Normal Cohesive Solls

\begin{tabular}{|c|c|c|c|c|c|c|c|c|c|c|c|}
\hline \multirow{2}{*}{$\begin{array}{c}\text { Test } \\
\text { ID }\end{array}$} & \multirow{2}{*}{$\begin{array}{c}Y_{k} \\
\sigma_{3}=6\end{array}$} & \multirow{2}{*}{$\begin{array}{r}M_{2} \\
\sigma_{3}=3\end{array}$} & \multirow{2}{*}{$\begin{array}{r}M_{K} \\
a_{3}=0\end{array}$} & \multicolumn{3}{|c|}{ Parameter A } & \multicolumn{3}{|c|}{ Parameter B } & \multirow[t]{2}{*}{$\mathbf{k}$} & \multirow[t]{2}{*}{$\mathbf{n}$} \\
\hline & & & & $\sigma_{3}=6$ & $\quad \sigma_{3}=3$ & $\sigma_{3}=0$ & $\sigma_{3}=6$ & $\sigma_{3}=3$ & $\sigma_{3}=0$ & & \\
\hline SBO1 & 20562 & 16547 & 10634 & 31556 & 22750 & 9324 & -0.23904 & -0.17769 & 0.07337 & & \\
\hline $\mathrm{SBO} 2$ & 18508 & 15371 & 10939 & 30187 & 22362 & 11578 & -0.27303 & -0.20920 & -0.03165 & & \\
\hline $\mathrm{SBO3}$ & 20892 & 16190 & 9430 & 38368 & 20639 & 9145 & -0.33925 & -0.13551 & 0.01709 & & \\
\hline SBO4 & 24083 & 21117 & 14626 & 54178 & 40092 & 27520 & -0.45249 & -0.35781 & -0.10078 & & \\
\hline SBOS & 8106 & 7516 & 6627 & 16890 & 13290 & 9029 & -0.40971 & -0.31809 & -0.17266 & & \\
\hline SBO6 & 29087 & 24735 & 17178 & 49971 & 39546 & 17994 & -0.30203 & -0.26190 & -0.02591 & & \\
\hline SB07 & 40121 & 34042 & 21049 & 43950 & 33670 & 15316 & -0.05087 & 0.00614 & 0.17747 & & \\
\hline SB08 & 33843 & 28252 & 16461 & 104152 & 69869 & 26659 & -0.62738 & -0.50534 & -0.26906 & & \\
\hline sB09 & 28881 & 26674 & 22858 & 52205 & 51993 & 29759 & -0.33041 & -0.37250 & -0.14725 & & \\
\hline SB10 & 34032 & 21725 & 11044 & 36154 & 20705 & 8369 & -0.03376 & 0.02682 & 0.15481 & & $\cdot$ \\
\hline sB11 & 20957 & 18579 & 14762 & 51972 & 38460 & 26316 & -0.50689 & -0.40608 & -0.32263 & & \\
\hline SB12 & 36346 & 28812 & 15255 & 41862 & 33045 & 10454 & -0.07887 & -0.07651 & 0.21093 & & \\
\hline SE13 & 23616 & 18624 & 9461 & 30965 & 22707 & 7933 & -0.15121 & -0.11064 & 0.09831 & & \\
\hline SB14 & 18386 & 16274 & 11466 & 35236 & 33309 & 14589 & -0.36306 & -0.40319 & -0.13447 & & \\
\hline SB15 & 46213 & 40012 & 33821 & $55940^{\circ}$ & 38499 & 32400 & -0.10661 & 0.02152 & 0.02397 & & \\
\hline SB16 & 22275 & 18882 & 13584 & 51324 & 38862 & 22936 & -0.46586 & -0.40285 & -0.29233 & & \\
\hline SB17 & 40038 & 30472 & 14335 & 101597 & 66150 & 22384 & -0.51970 & -0.43261 & -0.24873 & & \\
\hline SB18 & 9759 & 7819 & 5716 & 25884 & 21009 & 15859 & -0.54439 & -0.55161 & -0.56955 & & \\
\hline FWO1 & 2506 & 2252 & 2291 & 6146 & 4606 & 3726 & -0.50062 & -0.39938 & -0.27142 & 2246 & 0.25132 \\
\hline IWO4 & 13978 & 12800 & 12517 & 28699 & 21174 & 21312 & -0.40149 & -0.28092 & -0.29703 & 12379 & 0.31502 \\
\hline FW03 & 3724 & 3243 & 2998 & 10309 & 7073 & 5629 & -0.56833 & -0.43517 & -0.35162 & 2963 & 0.62657 \\
\hline FW02 & 3167 & 2891 & 2822 & 7784 & 6086 & 4989 & -0.50191 & -0.41550 & -0.31795 & 2790 & 0.33003 \\
\hline
\end{tabular}




\begin{tabular}{|c|c|c|c|c|c|c|c|c|c|c|c|}
\hline \multirow{2}{*}{$\begin{array}{c}\text { TeBt } \\
\text { ID }\end{array}$} & \multirow{2}{*}{$\begin{array}{c}M_{\mathbf{k}} \\
\sigma_{3}=6\end{array}$} & \multirow{2}{*}{$\begin{array}{r}\mathbf{M}_{R} \\
\sigma_{3}=3\end{array}$} & \multirow{2}{*}{$\begin{array}{c}M_{1} \\
\sigma_{3}=0\end{array}$} & \multicolumn{3}{|c|}{ Parameter A } & \multicolumn{3}{|c|}{ Parameter B } & \multirow[t]{2}{*}{$\mathbf{k}$} & \multirow[t]{2}{*}{$\mathbf{n}$} \\
\hline & & & & $a_{3}=6$ & $a_{3}=3$ & $\sigma_{3}=0$ & $\sigma_{3}=6$ & $\sigma_{3}=3$ & $\sigma_{3}=0$ & & \\
\hline FW05 & 12497 & 11111 & 9870 & 45996 & 39689 & 29772 & -0.72725 & -0.71056 & -0.61620 & 9841 & 0.68778 \\
\hline FW06 & 22191 & 18729 & 10218 & 47028 & 33134 & 8400 & -0.41917 & -0.31839 & 0.10933 & 10810 & 2.29595 \\
\hline FW07 & 16179 & 15019 & 10343 & 40567 & 38550 & 12926 & -0.51305 & -0.52608 & -0.12441 & 10761 & 1.32879 \\
\hline FW26 & 2648 & 2455 & 2368 & 8270 & 6781 & 5703 & -0.63553 & -0.56711 & -0.49066 & 2350 & 0.32342 \\
\hline FW27 & 17284 & 16600 & 12859 & 33058 & 32282 & 18217 & -0.36192 & -0.37119 & -0.19439 & 13236 & 0.87950 \\
\hline FW28 & 4799 & 4601 & 4395 & 19921 & 18672 & 15480 & -0.79442 & -0.78174 & -0.70274 & 4392 & 0.25670 \\
\hline FW29 & 20214 & 18952 & 15952 & 38453 & 36934 & 27432 & -0.35890 & -0.37238 & -0.30256 & 16167 & 0.69881 \\
\hline FW30 & 8327 & 7597 & 6455 & 28438 & 23008 & 15850 & -0.68548 & -0.61847 & -0.50134 & 6504 & 0.74782 \\
\hline FW31 & 11637 & 10721 & 7887 & 38286 & 32752 & 14253 & -0.66466 & -0.62327 & -0.33026 & 8121 & 1.15189 \\
\hline EW32 & 26357 & 22600 & 15449 & 46250 & 36241 & 17470 & -0.31385 & -0.26357 & -0.06862 & 15880 & 2.57526 \\
\hline FW09 & 15711 & 14620 & 12598 & 45437 & 43480 & 32958 & -0.59269 & -0.60830 & -0.53672 & $12710^{\circ}$ & 0.64980 \\
\hline FW10 & 16079 & 14932 & 13091 & 36734 & 35257 & 25802 & -0.46110 & -0.47950 & -0.37870 & 13171 & 0.60387 \\
\hline FW12 & 8135 & 7494 & 7015 & 32742 & 31163 & 24921 & -0.77714 & -0.79540 & -0.70753 & 6984 & 0.43056 \\
\hline FW12 & 12242 & 11315 & 9802. & 47039 & 41192 & 27912 & -0.75126 & -0.72115 & -0.58405 & 9871 & 0.65316 \\
\hline Fw16 & 8261 & 7415 & 5368 & 33213 & 25701 & 10421 & -0.77656 & -0.69374 & -0.37021 & 5516 & 1.27378 \\
\hline FW17 & 14683 & 13209 & 11453 & 46576 & 42081 & 37998 & -0.64427 & -0.64667 & -0.66933 & 11480 & 0.72702 \\
\hline FW13 & 25238 & 14123 & 11061 & 46515 & 42175 & 24573 & -0.62284 & -0.61058 & -0.44552 & 11301 & 0.94756 \\
\hline FW14 & 11554 & 11190 & 10057 & 34718 & 35118 & 25517 & -0.61403 & -0.63831 & -0.51964 & 10154 & 0.41055 \\
\hline FW15 & 19586 & 18070 & 15030 & 49667 & 47493 & 33957 & -0.51933 & -0.53931 & -0.45488 & 15217 & 0.78000 \\
\hline FW18 & 9623 & 8809 & 7371 & 34801 & 33485 & 23447 & -0.71744 & -0.74525 & -0.64584 & 7447 & 0.78432 \\
\hline FW19 & 20333 & 18795 & 16315 & 52577 & 45064 & 33619 & -0.53023 & -0.48805 & -0.40353 & 16425 & 0.64672 \\
\hline FW21 & 10088 & 9117 & 7576 & 47827 & 37737 & 23444 & -0.86856 & -0.79282 & -0.63043 & 7645 & 0.84120 \\
\hline FW43 & 12347 & 10671 & 7605 & 29086 & 22298 & 10346 & -0.47823 & -0.41133 & -0.27181 & 7782 & 1.42796 \\
\hline FW44 & 9384 & 8175 & 6247 & 25978 & 20157 & 11374 & -0.56827 & -0.50369 & -0.33440 & 6340 & 1.19647 \\
\hline
\end{tabular}




\begin{tabular}{|c|c|c|c|c|c|c|c|c|c|c|c|}
\hline \multirow{2}{*}{$\begin{array}{c}\text { Teat } \\
\text { ID }\end{array}$} & \multirow{2}{*}{$\begin{array}{c}\mathrm{K}_{\mathbf{k}} \\
\sigma_{3}=6\end{array}$} & \multirow{2}{*}{$\begin{array}{r}k_{k} \\
\sigma_{3}=3\end{array}$} & \multirow{2}{*}{$\sigma_{3}=0$} & \multicolumn{3}{|c|}{ Parameter A } & \multicolumn{3}{|c|}{ Parameter B } & \multirow[t]{2}{*}{ k } & \multirow[t]{2}{*}{$\mathbf{n}$} \\
\hline & & & & $\sigma_{3}=6$ & $\sigma_{3}=3$ & $\sigma_{3}=0$ & $\sigma_{3}=6$ & $\sigma_{3}=3$ & $\sigma_{3}=0$ & & \\
\hline FW22 & 3104 & 3005 & 3004 & 9156 & 8290 & 7147 & -0.60379 & -0.56628 & -0.4837 .7 & 2988 & 0.09235 \\
\hline FW23 3 & 6592 & 6262 & 5644 & 21560 & 19693 & 15807 & -0.66131 & -0.63942 & -0.57474 & 5678 & 0.45683 \\
\hline Fw24 & 5983 & 5640 & 5214 & 24735 & 21775 & 15995 & -0.79213 & -0.75398 & -0.62556 & 5220 & 0.40232 \\
\hline IW25 & 14281 & 12386 & 8599 & 42476 & 34306 & 12932 & -0.60836 & -0.56858 & -0.22772 & 8837 & 1.49635 \\
\hline FW37 & 16955 & 15301 & 10332 & 43260 & 38389 & 16363 & -0.52276 & -0.51338 & -0.25663 & 10729 & 1.46747 \\
\hline FW38 & 10672 & 9788 & 8267 & 29675 & 24748 & 21006 & -0.57076 & -0.51767 & -0.52047 & 8344 & 0.75101 \\
\hline FW39 & 9933 & 8915 & 5691 & 27300 & 24878 & 7668 & -0.56428 & -0.57279 & -0.16640 & 5951 & 1.65075 \\
\hline FW40 & 14921 & 12508 & 6748 & 49440 & 36193 & 7865 & -0.66860 & -0.59297 & -0.08549 & 7142 & 2.34861 \\
\hline FW41 & 14939 & 13371 & 107995 & 39405 & 30235 & 20937 & -0.54131 & -0.45536 & -0.36969 & 10920 & 0.95510 \\
\hline FW42 & 12114 & 11460 & 10000 & 30041 & 28069 & 20704 & -0.50688 & -0.49998 & -0.40615 & 10099 & 0.56530 \\
\hline Eพ36 & 27098 & 24065 & 17147 & 33670 & 28657 & 17578 & -0.12120 & -0.09747 & -0.01387 & 17626 & 1.35166 \\
\hline FW34 & 15549 & 13955. & 10888 & 48568 & 38118 & 22211 & -0.63567 & -0.56083 & -0.39787 & 11071 & 1.04979 \\
\hline FH35 & 14521 & 13119 & 10069 & 49323 & .33689 & 19941 & -0.68247 & -0.52636 & -0.38134 & 10273 & 1.08021 \\
\hline mo1 & 7395 & 6911 & 6143 & 23953 & 22502 & 17533 & -0.65591 & -0.65884 & -0.58535 & 6175 & 0.54497 \\
\hline WA02 & 11365 & 10050 & 6257 & 24544 & 19477 & 7321 & -0.42973 & -0.36929 & -0.08763 & 6550 & 1.76769 \\
\hline WAO3 & 8960 & 8005 & 6782 & 22209 & 18244 & 13676 & -0.48089 & -0.45977 & -0.39146 & 6812 & 0.81614 \\
\hline พA04 & 9186 & 8033 & 6369 & 26612 & 21138 & 13978 & -0.59367 & -0.53996 & -0.43867 & 6434 & 1.07504 \\
\hline พAOS & 6000 & 5569 & 4742 & 15662 & 14515 & 11432 & -0.53548 & -0.53463 & -0.49116 & 4790 & 0.69313 \\
\hline พA06 & 11304 & 9840 & 8627 & 22692 & 16464 & 14777 & -0.38890 & -0.28729 & -0.30036 & 8587 & 0.78694 \\
\hline WA07 & 11813 & 10528 & 8139 & 26914 & 26322 & 15898 & -0.45959 & -0.51144 & -0.37365 & 8227 & 1.09710 \\
\hline MAOS & 9703 & 7992 & 6475 & 20017 & 16122 & 12491 & -0.40413 & -0.39166 & -0.36675 & 6457 & 1.18026 \\
\hline WA09 & 5045 & 4385 & 3941 & 15097 & 12850 & 11732 & -0.61177 & -0.60001 & -0.60878 & 3909 & 0.71651 \\
\hline พA10 & 4075 & 3757 & 3354 & 13030 & 11751 & 9671 & -0.64874 & -0.63648 & -0.59099 & 3362 & 0.56990 \\
\hline WA11 & 7550 & 6745 & 5988 & 18978 & 16589 & 13991 & -0.51442 & -0.50227 & -0.47360 & 5976 & 0.67572 \\
\hline
\end{tabular}




\begin{tabular}{|c|c|c|c|c|c|c|c|c|c|c|c|}
\hline \multirow{2}{*}{$\begin{array}{c}\text { Test } \\
\text { ID }\end{array}$} & \multirow{2}{*}{$\begin{array}{c}M_{\lambda} \\
\sigma_{3}=6\end{array}$} & \multirow{2}{*}{$\begin{array}{r}M_{k} \\
\sigma_{3}=3\end{array}$} & \multirow{2}{*}{$\begin{array}{r}M_{k} \\
\sigma_{3}=0\end{array}$} & \multicolumn{3}{|c|}{ Parameter A } & \multicolumn{3}{|c|}{ Parameter B } & \multirow[t]{2}{*}{$\mathbf{k}$} & \multirow[t]{2}{*}{$\mathrm{n}$} \\
\hline & & & & $\sigma_{3}=6$ & $\sigma_{3}=3$ & $\sigma_{3}=0$ & $\sigma_{3}=6$ & $\sigma_{3}=3$ & $\sigma_{3}=0$ & & \\
\hline WA12 & 9044 & 7778 & 6514 & 28378 & 19497 & 13232 & -0.63821 & -0.51290 & -0.39553 & 6513 & 0.95832 \\
\hline HA13 & 7310 & 6628 & 5820 & 22150 & 16730 & 12516 & -0.61874 & -0.51678 & -0.42735 & 5831 & 0.66687 \\
\hline WA14 & 8896 & 7615 & 6446 & 27474 & 23318 & 17828 & -0.62932 & -0.62457 & -0.56774 & 6430 & 0.93951 \\
\hline WA15 & 9004 & 8030 & 6965 & 20063 & 18711 & 13736 & -0.44715 & -0.47209 & -0.37903 & 6971 & 0.75060 \\
\hline WA16 & 13015. & 11359 & 9907 & 30396 & 22320 & 18227 & -0.47338 & -0.37700 & -0.34024 & 9873 & 0.79513 \\
\hline WA17 & 10270 & 9111 & 8101 & 32905 & 24227 & 15698 & -0.64988 & -0.54582 & -0.36923 & 8073 & 0.69102 \\
\hline WA18 & 10494 & 9278 & 8111 & 26434 & 22785 & 17518 & -0.51561 & -0.50144 & -0.42972 & 8098 & 0.75134 \\
\hline LAO1 & 4724 & 4305 & 3822 & 13000 & 9522 & 6800 & -0.56500 & -0.44300 & -0.32160 & 3827 & 0.61935 \\
\hline LAO2 & 4601 & 4047 & 3606 & 10111 & 8476 & 5822 & -0.43940 & -0.41260 & -0.26739 & 3588 & 0.70927 \\
\hline LAO3 & 9619 & 8825 & 6359 & 21397 & 16807 & 7968 & -0.44620 & -0.35952 & -0.12588 & 6562 & 1.22597 \\
\hline LAO4 & 7664 & 7131 & 6314 & 18796 & 18279 & 11295 & -0.50069 & -0.52533 & -0.32461 & 6346 & 0.56886 \\
\hline LA05 & 14795 & 13531 & 9298 & 24349 & 21614 & 7732 & -0.27805 & -0.26140 & 0.10292 & 9653 & 1.37696 \\
\hline LA06 & 8369 & 7702 & 6730 & 18275 & 16115 & 11750 & -0.43588 & -0.41201 & -0.31104 & 6764 & 0.63943 \\
\hline LA07 & 8770 & 6819 & 4952 & 15404 & 12160 & 6838 & -0.31435 & -0.32281 & -0.18009 & 4967 & 1.67110 \\
\hline LAO8 & 6024 & 5758 & 5382 & 20051 & 16682 & 12935 & -0.67113 & -0.59364 & -0.48943 & 5392 & 0.33057 \\
\hline LAO9 & 3646 & 3376 & 3046 & 10420 & 9486 & 7431 & -0.58601 & -0.57659 & -0.49779 & 3051 & 0.52656 \\
\hline LA10 & 4030 & 3839 & 3491 & 10546 & 8556 & $7175^{\circ}$ & -0.53687 & -0.44727 & -0.40200 & 3510 & 0.42174 \\
\hline LA11 & 2651 & 2304 & 2078 & 5270 & 4712 & 3864 & -0.38345 & -0.39929 & -0.34622 & 2060 & 0.70652 \\
\hline LA12 & 5322 & 4940 & 4639 & 12207 & 10373 & 9055 & -0.46330 & -0.41401 & -0.37323 & 4623 & 0.39917 \\
\hline LA13 & 3633 & 3426 & 3211 & 8524 & 7858 & 6772 & -0.47590 & -0.46339 & -0.41652 & 3208 & 0.36087 \\
\hline LA14 & 4340 & 4071 & 3641 & 11068 & 9422 & 7555 & -0.52251 & -0.46829 & -0.40742 & 3659 & 0.51592 \\
\hline LA15 & 4469 & 3943 & 3394 & 11833 & 10064 & 8310 & -0.54348 & -0.52301 & -0.49973 & 3395 & 0.80366 \\
\hline LA16 & 4318 & 3964 & 3390 & 10959 & 9606 & 7933 & -0.51978 & -0.49402 & -0.47449 & 3416 & 0.71087 \\
\hline LA17 & 2554 & 2277 & 2119 & 6150 & 5079 & 4418 & -0.49055 & -0.44783 & -0.40996 & 2101 & 0.53916 \\
\hline
\end{tabular}




\begin{tabular}{|c|c|c|c|c|c|c|c|c|c|c|c|}
\hline $\begin{array}{c}\text { Test } \\
\text { ID }\end{array}$ & $\begin{array}{c}M_{1} \\
\sigma_{3}=6\end{array}$ & $\begin{array}{r}M_{R} \\
\sigma_{3}=3\end{array}$ & $\begin{aligned} & M_{k} \\
& \sigma_{3}=0\end{aligned}$ & $\sigma_{3}=6$ & $\begin{array}{c}\text { Paramete } \\
\qquad \sigma_{3}=3\end{array}$ & $\sigma_{3}=0$ & $\sigma_{3}=6$ & $\begin{array}{l}\text { Parameter } \\
\qquad \sigma_{3}=3\end{array}$ & $\sigma_{3}=0$ & $k$ & $\mathrm{n}$ \\
\hline LA18 & $5755^{\circ}$ & 5534 & 4989 & 19509 & 19599 & 14435 & -0.68134 & -0.70574 & -0.59295 & 5029 & 0.42152 \\
\hline LAI9 & 6203 & 5705 & 4985 & 22277 & 19476 & 13741 & -0.71354 & -0.68525 & -0.56595 & 5009 & 0.64167 \\
\hline BLO1 & 5687 & 5313 & 4610 & 14421 & 136.70 & 9631 & -0.51936 & -0.52748 & -0.41114 & 4650 & 0.61735 \\
\hline BLO2 & 6258 & 5781 & 5122 & 16586 & 14246 & 11931 & -0.54402 & -0.50334 & -0.47190 & 5142 & 0.58675 \\
\hline BL03 & 5805 & 5509 & 4973 & 15213 & 14093 & 10737 & -0.53768 & -0.52425 & -0.42958 & 5001 & 0.45493 \\
\hline BLب4 & 6489 & 5890 & 5287 & 16976 & 14767 & 12388 & -0.53669 & -0.51302 & -0.47527 & 5281. & 0.59834 \\
\hline BLO5 & 5345 & 4869 & 4264 & 12249 & 10757 & 8962 & -0.46281 & -0.44244 & -0.41459 & 4277 & 0.66181 \\
\hline BLO6 & 5384 & 4668 & 4005 & 13853 & 10813 & 8736 & -0.52746 & -0.46884 & -0.43521 & 3997 & 0.86251 \\
\hline BL07 & 6556 & 6231 & 5255 & 15451 & 14976 & 11793 & -0.47847 & -0.48940 & -0.45117 & 5336 & 0.65464 \\
\hline BLOB & 4258 & 3786 & 3322 & 10677 & 10196 & 8045 & -0.51304 & -0.55289 & -0.49360 & 3318 & 0.72442 \\
\hline BLO9 & 4152 & 3705 & 3320 & 10793 & 9634 & 7572 & -0.53314 & -0.53339 & -0.46017 & 3308 & 0.65158 \\
\hline BL10 & 5667 & 5128 & 4247 & 13871 & 11582 & 7782 & -0.49955 & -0.45473 & -0.33803 & 4288 & 0.84820 \\
\hline BL11 & 6899 & 5984 & 4788 & 13090 & 11435 & 8814 & -0.35742 & -0.36142 & -0.34060 & 4824 & 1.07146 \\
\hline BL12 & 8729 & 8002 & 6051 & 17493 & 16486 & 9095 & -0.38799 & -0.40343 & -0.22745 & 6201 & 1.08360 \\
\hline BL13 & 5726 & 5358 & 4409 & 13595 & 12221 & 8428 & -0.48262 & -0.46025 & -0.36156 & 4418 & 0.77156 \\
\hline BE14 & 5437 & 4933 & 4141 & 10713 & 9363 & 6810 & -0.37855 & -0.35766 & -0.27770 & 4175 & 0.80016 \\
\hline BL15 & 6808 & 5649 & 4594 & 12391 & 9658 & 6664 & -0.33422 & -0.29936 & -0.20764 & 4584 & 1.14810 \\
\hline
\end{tabular}

$\mathrm{M}_{\mathrm{k}}$ is at $\sigma_{\mathrm{d}}=6 \mathrm{ps} 1$.

Unit of $\sigma_{3}$ is psi. 
Table C. 2 Resilient Modulus Test Results of Frozen-Thawed Cohesive Soils

\begin{tabular}{|c|c|c|c|c|c|c|c|c|c|c|c|}
\hline \multirow{2}{*}{$\begin{array}{c}\text { Test } \\
\text { ID. }\end{array}$} & \multirow{2}{*}{$\begin{array}{c}M_{R} \\
\sigma_{3}=6\end{array}$} & \multirow{2}{*}{$\begin{array}{r}M_{k} \\
\sigma_{3}=3\end{array}$} & \multirow{2}{*}{$\begin{array}{r}M_{\lambda} \\
\sigma_{3}=0\end{array}$} & \multicolumn{3}{|c|}{ Parameter A } & \multicolumn{3}{|c|}{ Parameter B } & \multirow[t]{2}{*}{$\mathbf{k}$} & \multirow[t]{2}{*}{$\mathbf{n}$} \\
\hline & & & & $\sigma_{3}=6$ & $\sigma_{3}=3$ & $\sigma_{3}=0$ & $\sigma_{3}=6$ & $\sigma_{3}=3$ & $\sigma_{3}=0$ & & \\
\hline SBET01 & 3778 & 3335 & 3085 & 6720 & 4736 & 3405 & -0.32141 & -0.19569. & -0.05495 & & \\
\hline SBFTO2 & 9220 & 7999 & 5412 & 14665 & 13868 & 8777 & -0.25904 & -0.30713 & -0.26987 & & \\
\hline SBFT03 & 11283 & 9825 & 6632 & $1605 ?$ & 15892 & 7182 & -0.19691 & -0.26836 & -0.04442 & & \\
\hline SBFT04 & 2675 & 2510 & 2234 & 6341 & 5044 & 3556 & -0.48162 & -0.38939 & -0.25941 & & \\
\hline SBFTO5 & 13231 & 11277 & 7748 & 23718 & 19623 & 9126 & -0.32575 & -0.30918 & -0.09133 & & \\
\hline SBFT06 & 6557 & 5514 & 4447 & 10049 & 7720 & 4859 & -0.23829 & -0.18776 & -0.04948 & & \\
\hline SBFT07 & 13077 & 10716 & 7136 & 18482 & 14032 & 7597 & -0.19309 & -0.15048 & -0.03496 & & \\
\hline SBFTO8 & 20734 & 18812 & 12180 & 36170 & 33910 & 16751 & -0.31055 & -0.32883 & -0.17784 & & \\
\hline SBFT09 & 13011 & 12918 & 9451 & 17824 & 25322 & 15652 & -0.17567 & -0.37564 & -0.28153 & & \\
\hline SBFT10 & 10556 & 9034 & 7479 & 19189 & 17393 & 8920 & -0.33358 & -0.36561 & -0.09834 & & \\
\hline SBFT11 & 4843 & 4331 & 3590 & 12468 & 9680 & 6803 & -0.52775 & -0.44890 & -0.35680 & 3617 & 0.87893 \\
\hline SBFT12 & 3949 & 3574 & 3204 & 10083 & 9383 & 6696 & -0.52319 & -0.53862 & -0.41142 & 3200 & 0.60971 \\
\hline SBFT13 & 9868 & 8346 & 5795 & 17237 & 14449 & 8265 & -0.31127 & -0.30633 & -0.19819 & 5930 & 1.56748 \\
\hline FWFTO1 & 4876 & 4394 & 3825 & 13880 & 12961 & 11294 & -0.58384 & -0.60369 & -0.60432 & 3833 & 0.71055 \\
\hline FWFTO2 & 3875 & 3541 & 3223 & 21288 & 15596 & 11314 & -0.95082 & -0.82743 & -0.70092 & 3217 & 0.53746 \\
\hline FWFT03 & 4942 & 4432 & 3705 & 16174 & 12025 & 8757 & -0.66174 & -0.55713 & -0.48001 & 3731 & 0.84498 \\
\hline FWFT04 & 4328 & 4026 & 3414 & 14073 & 12486 & 8863 & -0.65811 & -0.63170 & -0.53240 & 3452 & 0.69869 \\
\hline FHFT05 & 8570 & 7509 & 5467 & 17929 & 16062 & 9108 & -0.41198 & -0.42434 & -0.28492 & 5590 & 1.32530 \\
\hline FWFTO6 & 5878 & 5075 & 4339 & 19144 & 16791 & 11641 & -0.65901 & -0.66780 & -0.55077 & 4328 & 0.88538 \\
\hline FWFT07 & 6350 & 5568 & 4487 & 14834 & 12607 & 8703 & -0.47354 & -0.45612 & -0.36973 & 4524 & 1.01895 \\
\hline FWFT08 & 1576 & & & 5313 & & & -0.67811 & & & & \\
\hline FWFTO9 & 9856 & 8754 & 5931 & 22991 & 19157 & 8641 & -0.47273 & -0.43712 & -0.21001 & 6140 & 1.50205 \\
\hline
\end{tabular}




\begin{tabular}{|c|c|c|c|c|c|c|c|c|c|c|c|}
\hline \multirow{2}{*}{$\begin{array}{c}\text { Test } \\
\text { ID }\end{array}$} & \multirow{2}{*}{$\begin{array}{c}k_{k} \\
\sigma_{3}=6\end{array}$} & \multirow{2}{*}{$\begin{array}{r}\mathbf{M}_{\mathrm{R}} \\
\sigma_{\mathbf{3}}=\mathbf{3}\end{array}$} & \multirow{2}{*}{$\begin{aligned} M_{k} \\
\sigma_{3}=0\end{aligned}$} & \multicolumn{3}{|c|}{ Parameter A } & \multicolumn{3}{|c|}{ Parameter $B$} & \multirow[t]{2}{*}{$\mathbf{k}$} & \multirow[t]{2}{*}{$\mathbf{n}$} \\
\hline & & & & $\sigma_{3}=6$ & $\sigma_{3}=3$ & $\sigma_{3}=0$ & $\sigma_{3}=6$ & $\sigma_{3}=3$ & $\sigma_{3}=0$ & & \\
\hline EWET10 & 8517 & 7473 & 5865 & 19553 & 17594 & 12700 & -0.46382 & -0.47790 & -0.43116 & 5937 & 1.09596 \\
\hline FWFT11 & 7610 & 6685 & 5483 & 24626 & 18248 & 12975 & -0.65542 & -0.56047 & -0.48079 & 5517 & 0.96107 \\
\hline FWET12 & 5630 & 5240 & 4486 & 18875 & 17532 & 12132 & -0.67517 & -0.67399 & -0.55524 & 4530 & 0.66863 \\
\hline FWFT13 & 1797 & & & 6451 & & & -0.71324 & & & & \\
\hline FWFT14 & 4068 & 3662 & 3247 & 18795 & 16760 & 13913 & -0.85411 & -0.84893 & -0.81214 & 3245 & 0.65848 \\
\hline FWFT15 & 5103 & 4451 & 3635 & 13838 & 11100 & 8043 & -0.55678 & -0.51006 & -0.44331 & 3655 & 0.99414 \\
\hline FWFT16 & 8468 & 7688 & 5902 & 20508 & 19394 & 12096 & -0.49363 & -0.51643 & -0.40053 & 6026 & 1.06616 \\
\hline FWFT17 & 3877 & 3577 & 3353 & 16233 & 13922 & 11489 & -0.79922 & -0.75852 & -0.68740 & 3338 & 0.42223 \\
\hline FWFT18 & 10127 & 8492 & 5967 & 15795 & 13881 & 8258 & -0.24810 & -0.27426 & -0.18138 & 6087 & 1.55594 \\
\hline FWFT19 & 6220 & 5575 & 4720 & 14579 & 13856 & 9461 & -0.47540 & -0.50814 & -0.38806 & 4744 & 0.80846 \\
\hline FWFT2O & 4938 & 4426 & 3609 & 15336 & 13166 & 10011 & -0.63248 & -0.60836 & -0.56945 & 3646 & 0.92162 \\
\hline WAFTO1 & 5747 & 4831 & 4018 & 15327 & 11444 & 8096 & -0.54752 & -0.48129 & -0.39095 & 4006 & 1.04313 \\
\hline WAFT02 & 7822 & 6888 & 5486 & 15952 & 13809 & 11519 & -0.39772 & -0.38820 & -0.41400 & 5545 & 1.04211 \\
\hline MAFTO3 & 5053 & 4361 & 3500 & 13288 & 9997 & 7111 & -0.53965 & -0.46305 & -0.39569 & 3522 & 1.07607 \\
\hline WAFT04 & 5273 & 4158 & 3196 & 10576 & 7122 & 4823 & -0.38844 & -0.30030 & -0.22965 & 3188 & 1.46106 \\
\hline MAFT05 & 3474 & & & 11719 & & & -0.67860 & & & & \\
\hline พंAFT06 & 5002 & 4294 & 3459 & 13872 & 11084 & 7381 & -0.56932 & -0.52927 & -0.42298 & 3476 & 1.07987 \\
\hline WAFT07 & 5512 & 4897 & 3996 & 16891 & 13328 & 8865 & -0.62504 & -0.55882 & -0.44465 & 4032 & 0.94377 \\
\hline WAFTOB & 3352 & 2961 & 2567 & 8271 & 5945 & 4213 & -0.50401 & -0.38907 & -0.27650 & 2565 & 0.77955 \\
\hline WAFTO9 & 6238 & 4994 & 3995 & 13567 & 10342 & 7409 & -0.43364 & -0.40629 & -0.34476 & 3972 & 1.29888 \\
\hline WAFT10 & 4143 & 3707 & 3157 & 12297 & 9347 & 7214 & -0.60721 & -0.51609 & -0.46124 & 3170 & 0.79624 \\
\hline WAFT11 & 4767 & 4252 & 3584 & 17465 & 13431 & 9343 & -0.72473 & -0.64192 & -0.53482 & 3601 & 0.83620 \\
\hline LAFT01 & 2318 & & & 6070 & & & -0.53715 & & & & \\
\hline LAFTO2 & 5534 & 5145 & 4410 & 15554 & 14299 & 11378 & -0.57673 & -0.57053 & -0.52898 & 4452 & 0.66842 \\
\hline
\end{tabular}




\begin{tabular}{|c|c|c|c|c|c|c|c|c|c|c|c|}
\hline \multirow{2}{*}{$\begin{array}{c}\text { Test } \\
\text { ID }\end{array}$} & \multirow{2}{*}{$\begin{array}{c}M_{k} \\
\sigma_{3}=6\end{array}$} & \multirow{2}{*}{$\begin{array}{r}M_{k} \\
\sigma_{3}=3\end{array}$} & \multirow{2}{*}{$\begin{array}{r}u_{k} \\
\sigma_{3}=0\end{array}$} & \multicolumn{3}{|c|}{ Parameter A } & \multicolumn{3}{|c|}{ Parameter B } & \multirow[t]{2}{*}{$\mathbf{x}$} & \multirow[t]{2}{*}{$\mathrm{n}$} \\
\hline & & & & $\sigma_{3}=6$ & $\sigma_{3}=3$ & $\sigma_{3}=0$ & $\sigma_{3}=6$ & $\sigma_{3}=3$ & $\sigma_{3}=0$ & & \\
\hline LAFTO3 & 2804 & 2477 & 2164 & 9310 & 6944 & 5017 & -0.66966 & -0.57534 & -0.46940 & 2160 & 0.75726 \\
\hline LAFT0 4 & 1860 & & & 6568 & & & -0.70423 & & & & \\
\hline LAFT05 & 2625 & 2585 & 2312 & 11840 & 10588 & 7982 & -0.84072 & -0.78702 & -0.69161 & 2342 & 0.37820 \\
\hline LAFT06 & 4449 & 3964 & $3388^{\circ}$ & 15036 & 12759 & 8705 & -0.67965 & -0.65238 & -0.52663 & 3398 & 0.79712 \\
\hline LAFT07 & 2317 & 2079 & 1806 & 6587 & 5222 & 4153 & -0.58313 & -0.51394 & -0.46464 & 1810 & 0.72786 \\
\hline LAFTO8 & 6504 & 5858 & 4321 & 14223 & 12826 & 11285 & -0.43672 & -0.43739 & -0.53573 & 4413 & 1.20766 \\
\hline LAFTOS & 5083 & 4610 & 3765 & 12820 & 11367 & 8841 & -0.51634 & -0.50364 & -0.47642 & 3811 & 0.88310 \\
\hline LAFT1O & 4068 & 3694 & 3096 & 11975 & 10283 & 8820 & -0.60264 & -0.57139 & -0.58424 & 3123 & 0.80142 \\
\hline LAFT11 & 2410 & 2078 & 1692 & 7329 & 5353 & 3971 & -0.62078 & -0.52821 & -0.47611 & 1699 & 1.03498 \\
\hline BLFT01 & 2917 & & & 6692 & & & -0.46343 & & & & \\
\hline BLFT02 & 3395 & & & 10054 & & & -0.60586 & & & & \\
\hline BLFTO3 & 2692 & & & 9359 & & & -0.69550 & & & & . \\
\hline BLFTO4 & 4355 & 4152 & 3556 . & 11391 & 10467 & 8740 & -0.53657 & -0.51603 & -0.50189 & 3605 & 0.59974 \\
\hline BLFTO5 & $\mathbf{4 7 7 4}$ & 4293 & 3662 & 11516 & 9323 & 7437 & -0.49144 & -0.43284 & -0.39533 & 3679 & 0.77696 \\
\hline BLFTO6 & 5168 & 4449 & 3456 & 11878 & 10052 & 6974 & -0.46441 & -0.45495 & -0.39183 & 3492 & 1.18101 \\
\hline BLFTO7 & 5014 & 4515 & 3747 & 13281 & 10938 & 8517 & -0.54370 & -0.49384 & -0.45823 & 3780 & 0.85453 \\
\hline BLFT08 & 4491 & 3825 & 3213 & 13350 & 10977 & 8163 & -0.60800 & -0.58843 & -0.52038 & 3206 & 0.97709 \\
\hline BLFTO9 & 2359 & & & 5201 & & & -0.44114 & & & . & \\
\hline BLET10 & 3102 & & & 9325 & $\cdot$ & & -0.61430 & & & & \\
\hline BLFT11 & 3025 & & & 10113 & & & -0.67360 & & & & \\
\hline BLFT12 & 3195 & & & 6683 & & & -0.41189 & & & & \\
\hline
\end{tabular}

$M_{\mathrm{g}}$ is at $\sigma_{\mathrm{d}}=6 \mathrm{pgi}$.

Unit of $\sigma_{3}$ is $\mathrm{psi}$. 


\begin{tabular}{|c|c|c|c|c|c|c|c|c|c|c|c|c|c|c|c|c|c|c|c|c|c|c|c|}
\hline & $\mathbf{F 1 3}$ & & F14 & & F15 & & F16 & & F17 & & FI8 & & F19 & & F20 & & F21 & & 122 & & F23 & & F24 \\
\hline 210 & 0.0234 & 126 & 0.0202 & $\mathbf{L . 1 2}$ & 0.0412 & L3 & 0.0272 & LS & 0.0207 & $126^{\circ}$ & 0.0213 & L10 & 0.0197 & 126 & 0.0197 & $\mathbf{L}$ & $0.0310^{\circ}$ & 126 & 0.0352 & L12 & 0.0309 & L12 & 0.0347 \\
\hline 121 & 0.0272 & 15 & 0.0206 & L10 & 0.0412 & L12 & 0,0279 & 126 & 0.0209 & L3 & 0.0226 & LS & 0.0220 & LS & 0,0241 & Leto & 0.0321 & L10 & 0.0363 & 19 & 0.0376 & IS & 0.0381 \\
\hline 124 & 0.0285 & L10 & 0.0245 & L9 & 0.0417 & 120 & 0.0320 & Lto & 0.0215 & 120 & 0.0231 & $L 21$ & 0.0236 & 120 & 0.0213 & 221 & 0.0340 & LS & 0.0365 & I3 & 0.0124 & I3 & 0.0389 \\
\hline LS & 0.0295 & 121 & 0,0273 & L3 & 0.0440 & 29 & 0.0333 & 121 & 0.0234 & ts & 0.0265 & 126 & 0.0246 & $\mathbf{L}$ & 0.0308 & 126 & 0,0361 & L & 0.0366 & 12 & 0.0152 & 120 & 0.0431 \\
\hline 126 & 0,0339 & 120 & 0.0274 & 16 & 0.0455 & 126 & 0.0318 & 624 & 0.0249 & 12 & 0.0306 & 224 & 0.0255 & L10 & 0.0308 & 16 & 0.0374 & L2 & 0.0366 & 120 & 0.0455 & $\mathbf{L 2}$ & 0.0433 \\
\hline 17 & 0.0343 & 23 & 0.0290 & 120 & 0.0464 & 16 & 0.0390 & $L 7$ & 0.0291 & 16 & 0.0320 & $L$ & 0.0313 & 12 & 0,0332 & 120 & 0.0408 & $\mathbf{L 2 0}$ & 0.0967 & 126 & 0.0500 & $L 26$ & 0.0436 \\
\hline 120 & 0.0447 & 124 & 0.0321 & 126 & 0.0489 & LS & 0.0405 & L20 & 0.0308 & $\mathbf{L}$ & 0,0321 & 1.20 & 0.0327 & $\mathbf{L A}$ & 0.0364 & $\mathbf{u}$ & 0.0428 & L4 & 0.0413 & L10 & 0.0520 & LuO & 0.0437 \\
\hline 13 & 0.0454 & Is & 0.0324 & LS & 0.0496 & 12 & 0.0419 & w & 0.0333 & L.10 & 0.0338 & $\mathbf{L 3}$ & 0.0357 & 121 & 0.0364 & 124 & 0.0429 & 16 & 0.0441 & L) & 0.0523 & $\mathbf{u}$ & 0.0490 \\
\hline 16 & 0.0473 & M & 0.0362 & 4 & 0.0543 & $\mathbf{M}$ & 0.0454 & LA & 0.0363 & 19 & 0.0339 & L & 0.0379 & 16 & 0.0384 & $\mathbf{L}$ & 0.0474 & L12 & 0.0456 & L22 & 0.0528 & L6 & 0.0511 \\
\hline LA & 0.0484 & $\mathbf{L 2}$ & $0.03 n$ & 12 & 0.0552 & $\mathbf{4 7}$ & 0.0464 & 12 & 0.0386 & L12 & 0.0345 & $L 2$ & 0.0392 & 124 & 0.0412 & 12 & 0.0500 & 19 & 0.0457 & LS & 0.0538 & LS & 0.0514 \\
\hline 12 & 0.0516 & L7 & 0.0395 & 121 & 0.0573 & L10 & 0.0481 & 16 & 0.0405 & 121 & 0.0431 & 16 & 0.0397 & E & 0.0459 & 19 & 0.0504 & L21 & 0.0464 & $\mathbf{L A}$ & 0.0538 & IS & 0.0570 \\
\hline 10 & 0.0529 & 19 & 0.0402 & L17 & 0.0637 & 122 & 0.0483 & 19 & 0.0461 & 524 & 0.0479 & L9 & 0.0488 & $\mathrm{Lt}$ & 0.0178 & LT & 0.0514 & 124 & 0.0515 & L17 & 0.0564 & $\ln$ & 0.0574 \\
\hline$L 12$ & 0.0572 & L.2 & 0.0427 & 124 & 0.0648 & L8 & 0.0499 & L12 & 0.0483 & $\mathbf{1 4 7}$ & 0.0453 & L.12 & 0.0514 & $\mathbf{L 1 2}$ & 0.0480 & L.12 & 0.0532 & 17 & $0.05 n$ & 123 & 0.0564 & 123 & $0.05: 6$ \\
\hline$L 17$ & 0.0775 & L17 & 0.0569 & $\ln$ & 0.0651 & 219 & 0.0510 & $L 17$ & 0.0639 & L7 & 0.0554 & L17 & 0.0652 & L17 & 0.0610 & $\mathrm{L17}$ & 0.0680 & L17 & 0.0589 & Ls & $0.057 !$ & L17 & $0.062 t$ \\
\hline $\mathbf{L 2}$ & 0.0357 & $L .19$ & 0.0679 & L8 & 0.0734 & 203 & 0.0569 & L19 & 0.0747 & Li9 & 0.0593 & L19 & 0.0762 & 123 & 0.0697 & L19 & 0.0419 & 1.23 & 0.0644 & L19 & 0.0624 & 1.21 & 0,0601 \\
\hline 123 & 0.0867 & 2.23 & 0.0707 & L7 & 0.0734 & 221 & 0.0573 & L23 & 0.0759 & 123 & 0.0615 & 1.23 & 0.0762 & L19 & 0.0700 & $12 n$ & 0.0860 & LA & 0.0681 & Li1 & 0.0692 & L19 & 0.0703 \\
\hline L19 & 0.0880 & 122 & 0.0736 & LAS & 0.0749 & 124 & 0.0632 & 122 & 0.0083 & $\mathbf{L s}$ & 0.061: & $\mathrm{L} 22$ & 0.0877 & L & 0.0749 & .123 & 0.0900 & 122 & 0.0687 & 214 & 0.0709 & L24 & 0.0735 \\
\hline L & 0.0945 & L & 0.0756 & 123 & 0.0783 & $\mathbf{L 1 1}$ & 0.0645 & w & 0.0887 & 122 & 0.0619 & $L$ & 0.0893 & 122 & 0.0794 & L14 & 0.0963 & Lt9 & 0,0717 & L21 & 0.0743 & L11 & 0.0738 \\
\hline L.14 & $0.107 t$ & 214 & 0.0260 & 114 & 0.0854 & L14 & 0.0659 & L14 & 0.0923 & L14 & $0.074 t$ & L14 & 0.0941 & 214 & 0.0895 & $\mathbf{L}$ & 0.0968 & L14 & 0.0768 & 224 & 0.0788 & L7 & 0.0799 \\
\hline LII & 0.1120 & L1 & 0.0932 & L11 & 0.0905 & L7 & 0.0709 & L1t & 0.1054 & Lt1 & 0.0717 & L11 & 0.1064 & L11 & 0.0959 & $\mathbf{L}$ & 0.1119 & L11 & 0.0851 & 125 & 0.0203 & 214 & 0.0451 \\
\hline LI & 0.1239 & $\mathbf{u}$ & 0.1037 & Ls: & 0,1032 & L25 & 0.0764 & $\mathbf{L}$ & 0.1098 & $L 25$ & 0.0910 & $\omega$ & 0.1112 & L25 & $0.107 \mathrm{~s}$ & L11 & 0.1141 & L18 & 0.0884 & L7 & 0.0857 & 125 & 0.0852 \\
\hline Lt8 & 0.1279 & t.1. & 0.1067 & $\mathbf{L}$ & 0.1038 & L.t8 & 0.0834 & Lit & 0.1129 & L) & 0.0915 & L18 & 0.1150 & Lit: & 0.1075 & L.ts & $0.11 \mathrm{as}$ & Ls & 0.0957 & L18 & 0.0864 & Lis & 0.0993 \\
\hline 1.25 & 0.1312 & 125 & 0.1077 & 125 & 0.1056 & $\mathbf{u}$ & $0.0 \mathrm{n} 42$ & 125 & 0.1172 & $\boldsymbol{u}$ & 0.0944 & 225 & 0.1182 & LA & $0.107 \%$ & L16 & 0.1235 & 125 & 0.0971 & L & 0.0911 & $\mathbf{L l}$ & 0.1040 \\
\hline L.16 & 0.1341 & 216 & 0.1135 & L16 & 0.1157 & 216 & 0.0937 & L16 & 0.1202 & LES & 0.1002 & L16 & 0.1216 & L16 & 0.1180 & 125 & 0.1288 & $\mathbf{u}$ & 0.1002 & 215 & 0.0965 & LIS & 0.1044 \\
\hline L15 & 0.1414 & L.t5 & 0.1194 & L15 & 0.114 & LS & 0.0940 & L15 & 0.1261 & L16 & 0.1043 & 1.15 & 0.1284 & Lis & 0.1189 & LIS & 0.1339 & L16 & 0.1125 & Li6 & 0.1010 & L16 & 0.1147 \\
\hline 2.3 & 0.1659 & $\mathbf{4 3}$ & 0.1447 & L13 & 0.1471 & $\mathbf{L 1 3}$ & 0.1248 & $\mathbf{L 1 3}$ & 0.1510 & $\mathbf{L 1 3}$ & 0.1349 & 213 & 0.1525 & L13 & 0.1491 & L13 & 0.1536 & $L 13$ & 0.1389 & L.3 & 0.1313 & LI3 & 0.1456 \\
\hline
\end{tabular}




\begin{tabular}{|c|c|c|c|c|c|c|c|c|c|c|c|c|c|c|c|c|c|c|c|}
\hline & L11 & & L12 & & $\mathbf{L 3}$ & & 214 & & L15 & & L16 & & Lt7 & & L18 & & L19 & & L20 \\
\hline F16 & 0.0645 & F16 & 0.0279 & $\mathbf{F} 2$ & 0.1120 & $\mathbf{F 2}$ & 0.0506 & $\mathbf{F 2}$ & 0.0872 & F2 & 0.0893 & $\mathbf{F} 2$ & 0.0413 & $\mathbf{F} 2$ & 0.0709 & F16 & 0.0510 & F3 & 0.0214 \\
\hline F23 & 0.0692 & F12 & 0.0302 & 76 & 0.1157 & F6 & 0.0549 & F5 & 0.0925 & F6 & 0.0917 & F3 & 0.0447 & F6 & 0.0766 & $\mathbf{F} 2$ & 0.0566 & F27 & 0.0221 \\
\hline F12 & 0.0698 & F23 & 0.0309 & F16 & 0.1248 & F16 & 0.0659 & F6 & 0.0928 & F16 & 0.0937 & F6 & 0.0457 & F5 & 0.0825 & F18 & 0.0593 & F18 & 0.0231 \\
\hline F29 & 0.0718 & $\mathbf{F} 2$ & 0.0325 & $\mathbf{F 3}$ & 0.1279 & $\mathbf{F 3}$ & 0.0663 & F16 & 0.0940 & F29 & 0.0979 & F16 & 0.0464 & F16 & 0.0834 & F6 & 0.0596 & $\mathbf{F !}$ & 0.0234 \\
\hline F24 & 0.0738 & F11 & 0.0327 & F29 & 0.1285 & FS & 0.0678 & $\mathbf{F} 22$ & 0.0957 & $\mathbf{F 2 3}$ & 0.1010 & F18 & 0.0403 & $\mathbf{F 3}$ & 0.0838 & F27 & 0.0604 & $\mathbf{F 7}$ & 0.0256 \\
\hline FS & 0.0739 & F5 & 0.0328 & FS & 0.1294 & F29 & 0.0689 & $\mathbf{F 3}$ & 0.0963 & $\mathbf{F 3}$ & 0.1010 & F27 & 0.0485 & $\mathbf{F 2 3}$ & 0.0864 & $\mathbf{F 3}$ & 0.0606 & F33 & 0.0258 \\
\hline Fo & $0.0739^{\circ}$ & F30 & 0.0329 & F23 & 0.1313 & F23 & 0.0709 & F23 & 0.0965 & F5 & 0.1022 & FS & 0.0495 & $\mathbf{F 2 2}$ & 0.0884 & Fs & & F14 & 0.0274 \\
\hline F35 & 0.0748 & F29 & 0.0342 & F35 & 0.1331 & F7 & $0.0729^{\prime}$ & F29 & 0.1001 & F30 & 0.1031 & F7 & 0.0496 & $F 29$ & 0.0884 & F30 & 0.0623 & F20 & 0.0283 \\
\hline $\mathbf{F 2}$ & 0.0749 & F18 & 0.0345 & F30 & 0.1338 & F30 & 0.0740 & F3S & 0.1014 & F27 & 0.1036 & F1 & 0.0508 & F7 & 0.0913 & F23. & 0.0624 & F26 & 0.0295 \\
\hline FI1 & 0.0768 & F24 & 0.0347 & F7 & 0.1343 & F18 & 0.0748 & Fl8 & 0.1022 & F18 & 0.1043 & F33 & 0.0538 & F18 & 0.0915 & F1 & 0.0633 & F2 & 0.0306 \\
\hline F18 & 0.0787 & F6 & 0.0366 & $\mathbf{F 2 7}$ & 0.1346 & $\mathbf{F 2 7}$ & 0.0761 & F30 & 0.1027 & F35 & 0.1045 & F30 & & F3O & 0.0917 & F29 & & F17 & 0.0308 \\
\hline F6 & 0.0808 & $\mathbf{F}$ & 0.0374 & F18. & 0.1349 & $F 22$ & 0.0768 & F7 & 0.1052 & F7 & 0.1062 & $\mathbf{F 2 3}$ & 0.05 & $\mathbf{F 1}$ & 0.0955 & F7 & & F5 & 0.0312 \\
\hline F3 & 0.0812 & $\mathbf{F I}$ & 0.0376 & F1 & 0.1372 & F! & 0.0770 & F1 & 0.1075 & $\mathbf{F I}$ & 0.10 & F14 & 0.0 & F27 & 0.09 & F12 & 0.0643 & F16 & 320 \\
\hline F1 & 0.0813 & $\mathbf{F 2 7}$ & 0.0388 & $F 22$ & 0.1388 & F12 & 0.0800 & F24 & 0.1084 & F12 & 0.10 & $\mathbf{F} 29$ & 0.0574 & F12 & 0.0978 & Ft1 & 0.0 & F19 & 0.0327 \\
\hline 222 & 0.0051 & F35 & 0.0405 & F12 & 0.1397 & F33 & 0.06 & F12 & 0.1 & F33 & 0.1107 & F12 & & F35 & & F33 & & F34 & 339 \\
\hline FIS & 0.09 & F15 & 0.0412 & 33 & 0.14 & FI1 & 0.0 & F27 & & F11 & 0.1 & FI 1 & & F24 & & F14 & & $\mathbf{F} 12$ & 344 \\
\hline $\mathbf{F 2 7}$ & 0.0912 & F14 & 0.04 & 11 & 0.14 & F24 & 0.0851 & F11 & 0.11 & F22 & 0.1125 & F22 & 0.0 & F11 & 0.1 & F24 & & F25 & \\
\hline F7 & 0.0923 & F7 & 0.04 & 14 & 0.1447 & FI5 & 0.0854 & F15 & 0.1164 & F14. & 0.1135 & F20 & 0.0 & F33 & & F2O & & F22 & 367 \\
\hline F14 & 0.0932 & F22 & 0.0456 & F2A & 0.1456 & F14 & 0.0860 & F33 & 0.1173 & $\mathbf{F 2 4}$ & 0.1 & P24 & 0.0621 & & & $F 22$ & & F6 & 370 \\
\hline F20 & 0.0959 & F26 & 0.0458 & F15 & 0.1471 & 35 & 0.00 & $\mathbf{F 2 0}$ & 0.11 & F15 & 0.1157 & F34 & 0.0 & F14 & 0.1 & F26 & & F11 & \\
\hline F26 & 0.1006 & 33 & 0.0468 & F20 & 0.1491 & F20 & 0.0895 & F14 & 0.1194 & 20 & 0.1180 & F26 & 0.0 & F20 & 0.1 & F17 & & $F 21$ & 0.0408 \\
\hline F33 & 0.1037 & F20 & 0.0480 & F34 & 0.1498 & P34 & 0.0913 & F26 & 0.1259 & F34 & 0.1 & F1s & & F34 & 0.1 & F1s & & F30 & 0.0416 \\
\hline F17 & 0.1054 & F17 & 0.0483 & F17 & 0.1510 & Fi7 & 0.0923 & F17 & 0.12 & F26 & 0.11 & F17 & 0.0639 & FI7 & & P34 & 0.0 & F28 & 0.0418 \\
\hline F25 & 0.1064 & F34 & & F26 & 0.1513 & $F 26$ & 0.0928 & F34 & 0.1277 & FM & 0.1 & Ft9 & 0.0652 & F26 & 0.1 & F19 & 0.0 & F24 & 0.0431 \\
\hline$F 19$ & 0.1064 & F19 & 0.0514 & F19 & 0.1525 & F19 & 0.0941 & Ft9 & & F19 & 0.1216 & F21 & 0.0 & F19 & 0.1150 & F35 & 0.0 & F4 & 0.0440 \\
\hline F34 & 0.1080 & F2s & 0.0530 & F21 & 0.1536 & F21 & 0.0963 & F25 & 0.1317 & $\mathbf{F} 21$ & 0.1235 & F2S & 0.0707 & F21 & & F25 & 0.0801 & FI3 & 0.0447 \\
\hline F13 & 0.1120 & F2t & 0.0532 & F31 & 0.1562 & F31 & 0.0992 & F21 & 0.1339 & F4 & 0.1271 & F4 & 0.0718 & F25 & 0.1196 & F21 & & F23 & 0.0455 \\
\hline F28 & 0.1133 & F28 & 0.0551 & F4 & 0.1562 & F4 & 0.0995 & F4 & & F2S & 0.1275 & F31 & 0.0730 & F3t & 0.1226 & F28 & & F15 & \\
\hline F21 & 0.1141 & $\cdot \mathbf{F 4}$ & 0.0570 & F25 & 0.1588 & F2s & 0.0997 & F28 & & F31 & 0.1278 & $\mathrm{~F} 28$ & 0.0732 & F4 & 0.1226 & F4 & 0.0861 & F31 & 0.0472 \\
\hline$F 4$ & 0.1200 & F13 & 0.0572 & F28 & 0.1599 & F28 & 0.1021 & F3I & 0.1390 & F28 & 0.1292 & F35 & 0.0755 & F28 & & F13 & 0.0880 & $\mathbf{F 9}$ & 0.0509 \\
\hline F31 & 0.1250 & F31 & 0.0594 & $\mathbf{F 8}$ & & F8 & 0.1032 & F13 & 0.1414 & F13 & 0.1341 & F13 & & F8 & 0.1265 & F31 & 0.0886 & Fo & 0.0595 \\
\hline $\mathbf{F B}$ & 0.1377 & $\mathbf{F 8}$ & 0.0643 & F13 & 0.1659 & F13 & 0.1071 & F8 & 0.1456 & $\mathbf{F} 8$ & 0.1358 & $\mathbf{F 8}$ & 0.0836 & F13 & 0.1279 & F8 & 0.0998 & $F 9$ & 0.0605 \\
\hline F9 & 0.1382 & $F$ & 0.0676 & F9 & 0.1693 & F9 & 0.1091 & F9 & 0.1491 & F9 & 0.1397 & F9 & 0.0865 & $\mathbf{F 9}$ & 0.1314 & F9 & 0.1016 & F35 & 0.0657 \\
\hline F10 & 0.1503 & F10 & 0.0725 & & 0.1766 & F10 & & F10 & 0.1533 & F10 & 0.1456 & F10 & & FIO & 0.1333 & F10 & 0.1115 & F10 & 0.0768 \\
\hline $\mathbf{F 3 2}$ & 0.1824 & F32 & 0.0936 & $\mathbf{F 3 2}$ & 0.2091 & F32 & 0.1426 & $\mathbf{F 3 2}$ & 0.1837 & $\mathbf{F 3 2}$ & 0.1768 & $\mathbf{F 3 2}$ & 0.1281 & F32 & 0.1629 & F32 & 0.1430 & F32 & 0.1043 \\
\hline
\end{tabular}




\begin{tabular}{|c|c|c|c|c|c|c|c|c|c|c|c|}
\hline & 2.21 & & $L .22$ & & 123 & & L24 & & 125 & & L26 \\
\hline F33 & 0.0205 & F1! & 0.0468 & F23 & 0.0564 & F31 & 0.01 t & F16 & 0.0764 & F26 & 0.0181 \\
\hline F3t & 0.0207 & F29 & 0.0472 & F16. & 0.0569 & F28 & 0.0224 & $\mathbf{F 2 3}$ & 0.0803 & F1 & 0.0191 \\
\hline F4 & 0.0219 & F16 & 0.0483 & F24 & 0.0586 & F4 & 0.0241 & F35 & 0.0830 & F27 & 0.0192 \\
\hline F28 & 0.0231 & F12 & 0.0527 & F12 & 0.0589 & F17 & 0.0249 & F12 & 0.0840 & F2O & 0.0197 \\
\hline F26 & 0.0232 & F23 & 0.0528 & FS & 0.0590 & F19 & 0.0255 & F24 & 0.0852 & Fi4 & 0.0202 \\
\hline F34 & 0.0233 & F30 & 0.0538 & F18 & 0.0615 & F26 & 0.0262 & F2 & 0.0852 & F33 & 0.0206 \\
\hline F17 & 0.0234 & $\mathbf{F} 2$ & 0.0554 & F1 & 0.0623 & $\mathbf{P 1 3}$ & 0.0285 & F5 & 0.0854 & F17 & 0.0209 \\
\hline F19 & 0.0236 & $\mathbf{F} \mathbf{5}$ & 0.0567 & F2 & 0.0625 & F33 & 0.0288 & F30 & 0.0875 & F18 & 0.0213 \\
\hline F27 & 0.0250 & F24 & 0.0574 & $\mathbf{F}$ & 0.0626 & F34 & 0.0290 & F29 & 0.0877 & $\mathbf{F 3}$ & 0.0218 \\
\hline F13 & 0.0272 & F6 & 0.0591 & F22 & 0.0644 & F14 & 0.0321 & F18 & 0.0910 & F25 & 0.0220 \\
\hline F14 & 0.0273 & FI8 & $0: 0619$ & F3o & 0.0655 & F27 & 0.0322 & F6 & 0.0917 & F19 & 0.0246 \\
\hline F7 & 0.0302 & $\mathbf{F 3}$ & $0.063 !$ & F27 & 0.0679 & F9 & 0.0341 & $\mathbf{F 3}$ & 0.0918 & F7 & 0.0257 \\
\hline F8 & 0.0318 & F35 & 0.0643 & F6 & 0,0685 & $\mathrm{FB}^{8}$ & 0.0365 & $\mathbf{F 1}$ & 0.0940 & F34 & 0.0265 \\
\hline F25 & 0.0327 & F! & 0.0645 & F7 & 0.0689 & F25 & 0.0374 & Fl1 & 0.0948 & F12 & 0.0318 \\
\hline F9 & 0.0334 & F1s & 0.0651 & F20 & 0.0697 & F? & 0.0384 & 122 & 0.0971 & F2s & 0.0322 \\
\hline F21 & 0.0340 & F22 & 0.0687 & F14 & 0.0707 & $\mathbf{F 2 0}$ & 0.0112 & F) & 0.1030 & FS & 0.0326 \\
\hline $\mathbf{F 3}$ & 0.0350 & F27 & 0.0724 & F29 & 0.0713 & F21 & 0.0429 & F27 & 0.1039 & F16 & 0.0338 \\
\hline F20 & 0.0368 & F14 & 0.0736 & F11 & 0.0725 & F1 & 0.0431 & F15 & 0.10 & F13 & 0.0339 \\
\hline F1 & 0.0379 & F7 & 0.0737 & F26 & 0.0735 & $\mathbf{F 3}$ & 0.0436 & F2O & 0.1073 & F22 & 0.0352 \\
\hline F18 & 0.0431 & F20 & 0.0794 & F25 & 0.0751 & F18 & 0.0479 & F14 & 0.1077 & $\mathbf{F 2 1}$ & 0.0361 \\
\hline $\mathbf{F 2 2}$ & 0.0464 & F26 & 0.0815 & F33 & 0.0753 & F22 & 0.0515 & F33 & 0.1138 & $\mathbf{F 4}$ & 0.0378 \\
\hline F6 & 0.0518 & F33 & 0.0852 & F17 & 0.0759 & F12 & 0.0570 & F26 & 0.1145 & $\mathbf{F} 2$ & 0.0392 \\
\hline $\mathbf{F} 2$ & 0.0521 & F13 & 0.0857 & Ft9 & 0.0762 & F10 & 0.0592 & F17 & 0.1172 & F31 & 0.0424 \\
\hline F12 & 0.0527 & F2I & 0.0860 & F3S & 0.0763 & F5 & 0.0594 & F25 & 0.1178 & F24 & 0.0436 \\
\hline Fs & 0.0547 & F34 & 0.0867 & F15 & 0.0783 & $\mathbf{F} 2$ & & PI9 & 0.1182 & F6 & 0.0448 \\
\hline F15 & 0.0573 & Ft9 & 0.0877 & F34 & 0.0810 & F6 & 0.0625 & F34 & 0.1218 & F11 & 0.0451 \\
\hline F16 & 0.0578 & F17 & 0.0883 & F28 & 0.0828 & F16 & 0.0632 & F21 & 0.1288 & F30 & 0.0462 \\
\hline F10 & 0.0583 & F25 & 0.0900 & F13 & 0.0867 & F15 & 0.0648 & F28 & 0.1291 & F15 & 0.0489 \\
\hline FII & 0.0632 & F28 & 0.0929 & $F 4$ & 0.0871 & F32 & 0.0679 & F13 & 0.1312 & F23 & 0.0500 \\
\hline F30 & 0.0673 & F4 & 0.0991 & F3t & $0.08 \times 2$ & FI1 & 0.0723 & $\mathbf{F} 4$ & 0.1328 & Fs & 0.0541 \\
\hline F24 & 0.0581 & F31 & 0.1043 & F21 & 0.0900 & F24 & 0.0735 & F3! & 0.1364 & F9 & 0.0552 \\
\hline F32 & 0.0726 & F8 & 0.1178 & F9 & 0.1009 & $\mathbf{F 3 0}$ & 0.0749 & F8 & 0.1475 & F29 & 0.0609 \\
\hline F23 & 0.0743 & F9 & 0.1184 & F8 & 0.1018 & F23 & 0.0788 & $F 9$ & 0.1488 & F35 & 0.0669 \\
\hline F29 & 0.0768 & F10 & 0.1324 & F10 & 0.1153 & F29 & 0.0878 & Fio & 0.1597 & F10 & 0.0743 \\
\hline F35 & 0.0844 & F32 & 0.1658 & F32 & 0.1394 & F3S & 0.0888 & F32 & 0.1918 & F32 & 0.0974 \\
\hline
\end{tabular}

